\title{
Penergies
}

Automation,

Control and Energy

Efficiency in

complex Systems

Edited by

Hamid Khayyam

Printed Edition of the Special Issue Published in Energies 


\section{Automation, Control and Energy Efficiency in Complex Systems}





\section{Automation, Control and Energy Efficiency in Complex Systems}

Editor

Hamid Khayyam

MDPI • Basel • Beijing • Wuhan $\bullet$ Barcelona $\bullet$ Belgrade $\bullet$ Manchester $\bullet$ Tokyo $\bullet$ Cluj $\bullet$ Tianjin

\section{MDPI}


Editor

Hamid Khayyam

Department of Mechanical and

Automotive Engineering,

School of Engineering,

RMIT University

Australia

Editorial Office

MDPI

St. Alban-Anlage 66

4052 Basel, Switzerland

This is a reprint of articles from the Special Issue published online in the open access journal Energies (ISSN 1996-1073) (available at: https:/ /www.mdpi.com/journal/energies/special_issues / Automation_Control_Energy_Efficiency_Complex_Systems).

For citation purposes, cite each article independently as indicated on the article page online and as indicated below:

LastName, A.A.; LastName, B.B.; LastName, C.C. Article Title. Journal Name Year, Volume Number, Page Range.

ISBN 978-3-03943-627-9 (Hbk)

ISBN 978-3-03943-628-6 (PDF)

(C) 2020 by the authors. Articles in this book are Open Access and distributed under the Creative Commons Attribution (CC BY) license, which allows users to download, copy and build upon published articles, as long as the author and publisher are properly credited, which ensures maximum dissemination and a wider impact of our publications.

The book as a whole is distributed by MDPI under the terms and conditions of the Creative Commons license CC BY-NC-ND. 


\section{Contents}

About the Editor $\ldots \ldots \ldots \ldots \ldots \ldots \ldots \ldots \ldots$ vii

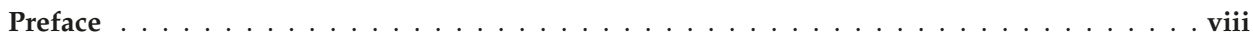

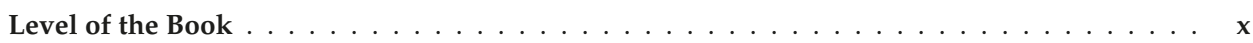

Jie Tian, Jun Tong and Shi Luo

Differential Steering Control of Four-Wheel Independent-Drive Electric Vehicles

Reprinted from: Energies 2018, 11, 2892, doi:10.3390/en11112892 . . . . . . . . . . . . . 1

Hongwei Liu, Chantong Wang, Xin Zhao and Chong Guo

An Adaptive-Equivalent Consumption Minimum Strategy for an Extended-Range Electric Bus

Based on Target Driving Cycle Generation

Reprinted from: Energies 2018, 11, 1805, doi:10.3390/en11071805 . . . . . . . . . . . . 19

Shuang Gao, Jianzhong $\mathrm{Wu}$ and Bin $\mathrm{Xu}$

Controllability Evaluation of EV Charging Infrastructure Transformed from Gas Stations in Distribution Networks with Renewables

Reprinted from: Energies 2019, 12, 1577, doi:10.3390/en12081577 . . . . . . . . . . . . . 45

Jie Tian, Jie Ding, Yongpeng Tai and Ning Chen

Hierarchical Control of Nonlinear Active Four-Wheel-Steering Vehicles

Reprinted from: Energies 2018, 11, 2930, doi:10.3390/en11112930 . . . . . . . . . . . . 65

Mo Chen, Zhuang Xiao, Pengfei Sun, Qingyuan Wang, Bo Jin and Xiaoyun Feng

Energy-Efficient Driving Strategies for Multi-Train by Optimization and Update Speed Profiles Considering Transmission Losses of Regenerative Energy

Reprinted from: Energies 2019, 12,3573, doi:10.3390/en12183573 . . . . . . . . . . . . . . . 79

Guang-Hui Xu, Meng Xu, Ming-Feng Ge, Teng-Fei Ding, Feng Qi and Meng Li

Distributed Event-Based Control of Hierarchical Leader-Follower Networks with Time-Varying Layer-To-Layer Delays

Reprinted from: Energies 2020, 13, 1808, doi:10.3390/en13071808 . . . . . . . . . . . . . 105

Aniela Kaminska and Andrzej Ożadowicz

Lighting Control Including Daylight and Energy Efficiency Improvements Analysis

Reprinted from: Energies 2018, 11, 2166, doi:10.3390/en11082166 . . . . . . . . . . . . . . 119

Mohammed Al-Azba, Zhaohui Cen, Yves Remond and Said Ahzi

An Optimal Air-Conditioner On-Off Control Scheme under Extremely Hot Weather Conditions

Reprinted from: Energies 2020, 13, 1021, doi:10.3390/en13051021 . . . . . . . . . . . . . 137

Farinaz Behrooz, Rubiyah Yusof, Norman Mariun, Uswah Khairuddin and Zool Hilmi Ismail

Designing Intelligent MIMO Nonlinear Controller Based on Fuzzy Cognitive Map Method for Energy Reduction of the Buildings

Reprinted from: Energies 2019, 12, 2713, doi:10.3390/en12142713 . . . . . . . . . . . . . . 159

Srinivas Nunna, Maxime Maghe, Seyed Mousa Fakhrhoseini, Bhargav Polisetti and Minoo Naebe

A Pathway to Reduce Energy Consumption in the Thermal Stabilization Process of Carbon Fiber Production

Reprinted from: Energies 2018, 11, 1145, doi:10.3390/en11051145 . . . . . . . . . . . . . 191 
Fardila Mohd Zaihidee, Saad Mekhilef and Marizan Mubin

Robust Speed Control of PMSM Using Sliding Mode Control (SMC)—A Review

Reprinted from: Energies 2019, 12, 1669, doi:10.3390/en12091669 . . . . . . . . . . . . . . . 201 


\section{About the Editor}

Hamid Khayyam received his B.Sc. degree (Hons.) from the University of Isfahan, his M.Sc. degree from the Iran University of Science and Technology, and his Ph.D. degree in mechanical engineering from Deakin University, Australia. Dr. Khayyam has worked in automation and energy productivity in various industrial companies for more than ten years. In his previous position, he was leading the efforts on modelling, control and optimization of energy systems in the carbon fiber production line at Carbon Nexus, Deakin University. Dr. Khayyam is currently a Senior Lecturer in the Department of Mechanical and Automotive Engineering, School of Engineering at the RMIT University, Australia. He has contributed more than 100 articles to professional journals and currently serves on several Editorial Boards of ISI journals. Dr. Khayyam's research is focused on instituting new technologies in support of to develop distinctive approaches for the integrating artificial intelligence and machine learning, for solving complex energy systems, towards developing simple and procedural approaches for end-users of these technologies. Dr. Khayyam is an academic member of Intelligent Automation Research Group (IARG) at RMIT in Australia and The Materials and Manufacturing Research Institute (MMRI) at The University of British Columbia in Canada. Dr. Khayyam is a Senior Member of IEEE and actively involved in Power and Energy and Intelligent Transportation Systems Societies. 


\section{Preface}

A complex system is a system composed of many components (elements) which interrelate with each other, and the collective behaviour of these elements results in the emergence of properties that can hardly, if not at all, be inferred from properties of the elements alone.

Complex systems are intrinsically complicated, and difficult to model or control, due to inherent nonlinearity, coupling, chaotic behaviour, uncertainty, embedded stochastic patterns and parameter sensitivities, under multi-scale responses. Complex systems are pervasive in today's world, yet visions into their unanticipated behaviour remains limited, severely reducing the ability to design and control them for particular desired responses.

The general approaches that can be used to simplify complex systems are: (i) divide and conquer, (ii) shift complexity, (iii) simplified commands, and (iv) structural methodologies.

From an application and technological development perspective, the engineering world is in great need of methods and tools for identifying complex systems' behaviour, as well as tractable methods for their design and analysis. Therefore, any new methods of computation and/or processing (e.g. using novel machine learning-based operations) that leads to successfully applying automation, automated control and optimized energy efficiency will be appreciated by academia and engineering communities.

This book covers some significant impacts from recent research contributions, in both the private and public sectors of engineering complex systems, in which automation, control, energy analysis, energy modelling, energy management, and energy efficiency are outcomes. This book is also a collection of eleven different crucial complex systems arranged in three groups: Transportation Systems, Building Systems, and Manufacturing Systems, which are focused on applied engineering problems.

The first group; Transportation Systems complex challenges, which are: capacity, transfer, reliability and integration to reduce time and energy consumption. Chapter 1,2, and 3 cover the automated controls for operating electric vehicles, including hybrid electric vehicle and plug-in hybrid electric vehicles and charging infrastructure, as part of transportation systems. Chapter 4 provides a study of an active controller for four-wheeled steering vehicles. Chapter 5 investigates an energy consumption model for multi-train urban rail transit systems. Chapter 6 explores a switching coordination of multi-agent systems for transportation networks, which permits rapid and safe.

The second group; Building Systems complex challenges, which are: mechanical systems (involving topics of energy consumption, heating, air conditioning, boiler systems automatic temperature controls) and electrical systems (such as: electrical power service). Chapter 7 and 8 extensively studies energy reductions of buildings through the modelling and control of lighting and air-conditioning systems. Chapter 9 introduces a new designing method of an intelligent Fuzzy Cognitive Map (FCM) controller for the energy reduction of the building systems.

The third group; Manufacturing Systems complex challenges, which are: improving production processes, control and optimized energy efficiency. Chapter 10 begins with an extensive article on how to reduce energy consumption in the carbon fiber production industry. Chapter 11 is a comprehensive review of some robust speed control methods of Permanent Magnet Synchronous Motors (PMSM) for industrial automation applications.

Due to the nonlinearity and robustness of complex systems, the ability to apply automation and automated control with minimal human assistance would provide us with a great ability and 
motivation to control dynamic energy systems. Artificial intelligence may be defined as the branch of computer science that is concerned with the automation of intelligent behaviour. Artificial intelligence techniques learn about the data they are trained on, and learning algorithms are designed to generalize from that data.

Artificial intelligence in automation, uses intelligent control techniques such as fuzzy logic systems, neural networks, machine learning, and optimization algorithms, which are deployed to achieve energy efficiency in many spheres of engineering complex systems. The positive aspects of intelligent controllers are their simplicity, having the benefit of independence from models, not requiring extensive knowledge of the problem field, reduced cost and environmental impact, and solvability for several energy reduction strategies of engineering systems.

Hamid Khayyam Editor 


\section{Level of the Book}

This book is amid to serve researchers, engineers, scientists, and engineering graduate and $\mathrm{PhD}$ students of engineering and physical science, together with the individuals generally interested in engineering, and science. In particular, the book can be used for training graduate students, $\mathrm{PhD}$ students as well as senior undergraduate students to enhance their knowledge by taking a graduate or advanced undergraduate course in the areas of complex systems, control systems, energy systems, and engineering applications. The covered research topics are also of interest to engineers and academia who are seeking to expand their expertise in these areas. This book focuses on the application of engineering methods to complex systems including transportation, building, and manufacturing, with approaches representing a wide variety of disciplines of engineering and science. Throughout the book, great emphases are placed on engineering applications of complex systems, as well as the methodologies of automation including artificial intelligence, automated and intelligent control, energy analysis, energy modelling, energy management, and optimized energy efficiency. The significant impact of the recent researches that have been selected are of high interest in engineering complex systems. An attempt has been made to expose the reading audience of engineers and researchers to a broad range of theoretical and practical topics. The topics contained in the following book are of specific interest to engineers who are seeking expertise in transportation, building and manufacturing technologies as well as mathematical modelling of complex systems, engineering approaches to engineering complex problems, automation via artificial intelligence methods, automated and intelligent control, and energy systems.

\section{Organization of the Book}

The main structure of the book consists of three parts: Transportation Systems, Building Systems, and Manufacturing Systems including eleven chapters. Each of the chapters covers an independent topic along the automation, automated control approaches for engineering of complex systems. All the necessary concepts, proofs, mathematical background, solutions, methodologies, and references are supplied except for some fundamental knowledge that is well-known in the general fields of engineering. The readers may therefore gain the main concepts of each chapter, with as little of a need as possible, to refer to the concepts of the other chapters and references. The readers may hence start to read one or more chapters of the book for their own interests.

\section{Acknowledgements}

This book has been made possible through the effective collaborations of all the enthusiastic chapter author contributors, who have the expertise and experience in various disciplines in the engineering of complex system. They deserve the sincerest gratitude for the motivation of creating such a book, for the encouragement in completing the book, for the scientific and professional attitude in constructing each of the chapters of the book, and for the continuous efforts toward improving the quality of the book. Without the collaboration and consistent efforts of the chapter contributors 
including authors and anonymous reviewers, the completion of this book would have not been possible. It has been gratifying to work with the staff of MDPI publisher through the development of this book. The assistance provided by the staff members has been valuable and efficient. I thank MDPI publisher specially Ms. Kristy Zhang and Ms. Vivian Lu for their production of a stylish book. Finally, the greatest thanks to Professor Reza N. Jazar and Mr. Bryn Crawford for their support and useful discussions.

Hamid Khayyam 

Article

\title{
Differential Steering Control of Four-Wheel Independent-Drive Electric Vehicles
}

\author{
Jie Tian ${ }^{1, *}$, Jun Tong ${ }^{1}$ and Shi Luo ${ }^{2}$ \\ 1 College of Automobile \& Traffic Engineering, Nanjing Forestry University, Nanjing 210037, China; \\ tongjun37@njfu.edu.cn \\ 2 College of Automobile \& Traffic Engineering, Jiangsu University, Zhengjiang 212013, China; \\ luoshi@ujs.edu.cn \\ * Correspondence: tianjie@njfu.com.cn; Tel.: +86-158-5187-8088
}

Received: 8 August 2018; Accepted: 22 October 2018; Published: 24 October 2018

check for updates

\begin{abstract}
This paper investigates the skid steering of four-wheel independent-drive (4WID) electric vehicles (EV) and a differential steering of a 4WID EV with a steer-by-wire (SBW) system in case of steering failure. The dynamic models of skid steering vehicle (SSV) and differential steering vehicle (DSV) are established and the traditional front-wheel steering vehicle with neutral steering characteristics is selected as the reference model. On this basis, sideslip angle observer and two different sliding mode variable structure controllers for SSV and DSV are designed respectively. Co-simulation results of CarSim and Simulink show that the designed controller for DSV not only controls the yaw rate and sideslip angle of DSV to track those of the reference model exactly, but also ensures the robustness of the controlled system compared with the designed controller for SSV. And the differential driving torque needed to realize the differential steering is much smaller than that for skid steering, which indicates the possibility of the differential steering in case of steering failure.
\end{abstract}

Keywords: four-wheel independent-drive; electric vehicle; skid steering; differential steering; sliding mode variable structure control; robustness

\section{Introduction}

The vehicle steering system has experienced several stages, such as manual steering, hydraulic steering, electro-hydraulic steering, electric power steering and by-wire steering. However, in order to achieve the latter two, the structure becomes more complicated because one or two extra motors are required [1]. The appearance of four-wheel independent-drive (4WID) electric vehicles (EVs) opens up the possibility of differential steering system (DSS) by coupled control of left and right in-wheel motors (IWM), which eliminates the restrictions of a traditional steering system completely [2]. With the emergence of intelligent vehicle systems (IVS), the 4WID system can also be used to solve the path tracking problem [3,4]. However, there are three functions of the DSS: (1) Steering the vehicle without the lateral turning of the wheel, i.e., skid steering [5,6]; (2) Assisting the driver to steer the vehicle, that is, differential drive assisted steering (DDAS) $[1,2,7,8]$; (3) Steering the vehicle instead of the regular steering system [9-13]. Skid steering was realized by giving a tire speed differential between the left and right tires $[5,6]$. And the wheel torque difference between the left and right tires, which controlled the tire velocity difference, was defined as a function of the steering wheel angle. In addition, a stability compensator for the adhesion limit of tire/load and the yaw rate and yaw acceleration are used as control variables. However, the needed differential driving torque is not given in the paper [5].

A closed loop control method of differential drive assisted steering (DDAS) was proposed, which includes a reference steering wheel torque (RSWT) design module and an integral anti-windup variable PI control module. The former was to design a three-dimensional characteristic curve of torque 
and steering wheel angle at different vehicle speeds, and the latter was aiming to address the saturation of motor's output torque. The simulation results showed that the RSWT can be tracked perfectly by the DDAS, drivers' handling efforts were reduced and the vehicle steering performance was improved [1]. A multidiscipline collaborative optimization model of the differential steering system was built with the steering economy as the main system, and the steering flexibility, the steering road feel and the mechanic character of the steering sensors as the subsystems. And the main system and the subsystems were optimized by the multi-island algorithm and the sequential quadratic programming algorithm. The simulation results show that the differential steering system can have good economy, good steering road feel, good steering flexibility and good mechanic character of the steering sensors [2]. The DDAS control system, the drive torque distribution and the compensation control system were designed. The proportional-integral (PI) feedback control loop was employed to track the reference steering effort. In addition, the traction control subsystem and the direct yaw moment control subsystem were both employed to make the DDAS work as well as wished [7]. The structure and basic theory of the DSS were discussed and its dynamic model was built. $\mathrm{A}_{\infty}$ mixed sensitivity controller is designed to suppress the model uncertainties and road disturbance. The simulation results verified the efficacy of the DSS with the designed controller [8]. However, they are aimed at mitigating the driver's steering efforts.

By regulating the four wheels to the desired differential speed base on the reference vehicle velocity, kinematic model of the distributed wheels, combined with Ackermann-Jeant and steering model, was introduced to achieve electrical differential steering for 4WID EVs. The effectiveness of the proposed control strategy was demonstrated by the simulation and experimental study [9]. A continuous steering stability controller based on energy-saving torque distribution algorithm was proposed for four-wheeled built-in motor independent driving electric vehicle. The simulation results showed that, compared with the traditional servo controller and the ordinary continuous controller, the proposed controller can significantly reduce the energy consumption and improve the steering stability of the vehicle [10]. The literature [11-13] investigates the DSS in the case of the complete failure of the regular steering system. To achieve the yaw stabilization, a robust $\mathrm{H}_{\infty}$ output-feedback controller of the DSS was designed and parametric uncertainties for the cornering stiffness and the external disturbances were considered to guarantee the vehicle robustness [11]. A multiple-disturbances observer-based composite nonlinear feedback (CNF) approach was proposed to improve the transient performance of the fault-tolerant control with the DDAS, and the disturbance observer was designed to estimate the external disturbances with unknown bounds. CarSim-Simulink joint simulation results verified the efficacy of the proposed controller [12]. To realize the yaw control when the active front steering entirely breaks down and guarantee the transient control performance, a disturbance observer based integral sliding mode control (ISMC) strategy was designed to deal with the unknown mismatched disturbances, which was addressed by an adaptive super-twisting control approach. And the composite nonlinear feedback technique was applied to design the controller's nominal part to depress overshoots and avoid steady-state errors considering the tire force saturations. The simulation results verified the effectiveness of the proposed control approach in the case of the steering failure [13].

The innovation points are as follows: (1) Based on the reference model, the steering function of SSV and DSV are realized by the differential driving torque between the two sides of the front wheels instead of the normal desired differential speed; (2) An simple and practicable observer is constructed to estimate the actual sideslip angle; (3) The vehicle has parametric uncertainties, such as tire stiffness perturbation and external disturbances, and there is no direct relationship between handling wheel angle and differential driving torque of the left and right side wheels, thus two kinds of SMC controller are designed; (4) Contrast and analysis of the SSV and the DSV with controllers is carried out, such as the response curves, the needed differential torque and robustness.

The article structure is as follows: The SSV and DSV models, and reference model are described in Section 2. Sideslip angle observer and two kinds of SMC controller based on the reference model are designed in Section 3. Section 4 is the joint simulation based on CarSim (MATLAB, R2012a, mathworks, 
Natick, MA, USA) and Simulink. (CarSim, 8.02, MSC software, Los Angeles, CA, USA) Section 5 is the conclusion.

\section{Vehicle Models and Problem Formulation}

In this section, we will first present three kinds of vehicle models, including an SSV model, a DSV model and a reference model. Here vehicle models are based on the following assumptions: (1) The lateral acceleration is small and the roll motion can be ignored; (2) The left and right tire slip angles are equal; (3) The front and rear tire lateral forces are proportional to the tire slip angles. Then the problem formulation will be proposed.

The difference between SSV and DSV is that the former has no mechanical steering mechanism. However, both of them depend on the differential driving torque between the two sides of front wheels, but for the latter, the torque will also contribute to the generation of the front wheel angles. The SSV obtains steering yaw moment by increasing the speed of outer wheels and decreasing the speed of inner wheels, other than swinging the steered wheels.

\subsection{Skid Steering Vehicle Model}

The forces acting on the vehicle body are shown in Figure 1, where $F_{x i j}(i=f, r, j=l, r)$ is the tire longitudinal force of the left/right front/rear wheel, $F_{y i j}(i=f, r, j=l, r)$ is the tire lateral force of the left/right front/rear wheel.

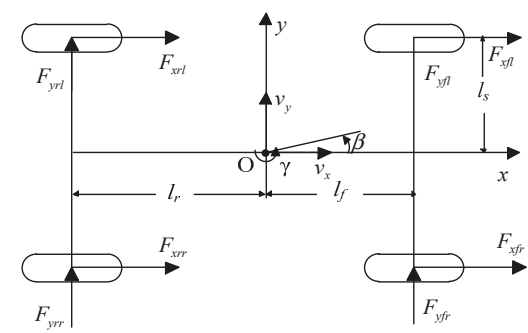

Figure 1. Skid Steering Vehicle Model.

The lateral and yaw motion of the EV are modeled as:

$$
\left\{\begin{array}{l}
m u_{x}(\dot{\beta}+\gamma)=F_{y f l}+F_{y f r}+F_{y r l}+F_{y r r} \\
I_{Z} \dot{\gamma}=l_{s} \frac{\Delta M}{R}+l_{f}\left(F_{y f l}+F_{y f r}\right)-l_{r}\left(F_{y r l}+F_{y r r}\right)
\end{array}\right.
$$

where

$$
F_{y f l}=F_{y f r}=k_{f}\left(\beta+l_{f} \gamma / u_{x}\right), F_{y r l}=F_{y r r}=k_{r}\left(\beta-l_{r} \gamma / u_{x}\right), \Delta M=T_{f r}-T_{f l}=\left(F_{x f r}-F_{x f l}\right) R,
$$

$m$ is the total vehicle mass, $u_{x}$ is the longitudinal velocity at the CG point, $\beta$ is the sideslip angle, $\gamma$ is the yaw rate, $I_{z}$ is the yaw moment of inertia, $l_{s}$ is the half of wheel track, $R$ is the radius of front wheel, $l_{f}$ and $l_{r}$ are the distances from the center of gravity (CG) to the front and rear axles, $\Delta M$ is the differential driving torque between the two sides of the front wheels, $T_{f r}$ and $T_{f l}$ are the right and left driving torques of the front wheel.

Here a static wheel model is adopted and wheel rotational dynamics are not considered. Define the state variable and system input as $x(t)=[\beta, \gamma]^{T}, u(t)=\Delta M$, the state equation of the two degree-of-freedom (2-DOF) model can be given as: 


$$
\left\{\begin{array} { l } 
{ \dot { x } = A x + B u } \\
{ y = C x }
\end{array} \left\{\begin{array}{l}
\dot{x}=A x+B u \\
y=C x
\end{array}\right.\right.
$$

where

$$
A=\left[\begin{array}{cc}
\frac{2\left(k_{f}+k_{r}\right)}{m u_{x}} & -1+\frac{2 l_{f} k_{f}-2 l_{r} k_{r}}{m u_{x}^{2}} \\
\frac{2 l_{f} k_{f}-2 l_{r} k_{r}}{I_{Z}} & \frac{2 l_{f}{ }^{2} k_{f}+2 l_{r}{ }^{2} k_{r}}{I_{z} u_{x}}
\end{array}\right], B=\left[\begin{array}{c}
0 \\
\frac{l_{s}}{I_{Z} R}
\end{array}\right], C=\left[\begin{array}{cc}
1 & 0 \\
0 & 1
\end{array}\right] .
$$

Considering that the tire cornering stiffness always fluctuates due to the change of the road conditions, they can be expressed as:

$$
k_{f}=k_{f 0}+\Delta k_{f}, k_{r}=k_{r 0}+\Delta k_{r} .
$$

where $k_{f 0}$ and $k_{r 0}$ are the nominal values of the front and rear tire cornering stiffness, $\Delta k_{f}$ and $\Delta k_{r}$ are the corresponding perturbation values.

Then Equation (3) can be written as:

$$
\left\{\begin{array}{l}
\dot{x}=\left(A_{0}+\Delta A\right) x+B u \\
y=C x
\end{array}\right.
$$

where

$$
A_{0}=\left[\begin{array}{cc}
\frac{2\left(k_{f 0}+k_{r 0}\right)}{m u_{x}} & -1+\frac{2\left(l_{f} k_{f 0}-l_{r} k_{r 0}\right)}{m u_{x}^{2}} \\
\frac{2\left(l_{f} k_{f 0}-l_{r} k_{r 0}\right)}{I_{Z}} & \frac{2\left(l_{f}{ }^{2} k_{f 0}+l_{r}^{2} k_{r 0}\right)}{I_{z} u_{x}}
\end{array}\right], \Delta A=\left[\begin{array}{cc}
\frac{2\left(\Delta k_{f}+\Delta k_{r}\right)}{m u_{x}} & \frac{2\left(l_{f} \Delta k_{f}-l_{r} \Delta k_{r}\right)}{m u_{x}^{2}} \\
\frac{2\left(l_{f} \Delta k_{f}-l_{r} \Delta k_{r}\right)}{I_{Z}} & \frac{2\left(l_{f}{ }^{2} \Delta k_{f}+l_{r}{ }^{2} \Delta k_{r}\right)}{I_{z} u_{x}}
\end{array}\right]
$$

\subsection{Differential Steering Vehicle Model}

As we all know, when the braking force between the left and right sides is different, the vehicle will turn to the side with larger braking force. Similarly, the different driving force between the left and right sides will drive the steering wheel to generate the steering motion and the vehicle will turn to the side with smaller driving force. Differential steering system of the 4WID EV equipped with an SBW system and the force acting on the vehicle body are shown in Figures 2 and 3, respectively.

The driver's intention is provided to the electronic control unit (ECU), which gives instructions to the steering mechanism and achieves the steering according to the collected signals and internal control procedures. However, when the SBW system fails suddenly, the steering can only be realized by the differential driving torque, which can also generate the front wheel angles simultaneously.

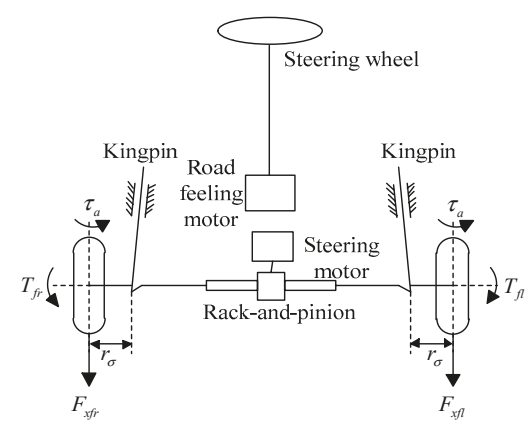

Figure 2. Differential steering system of DSV. 


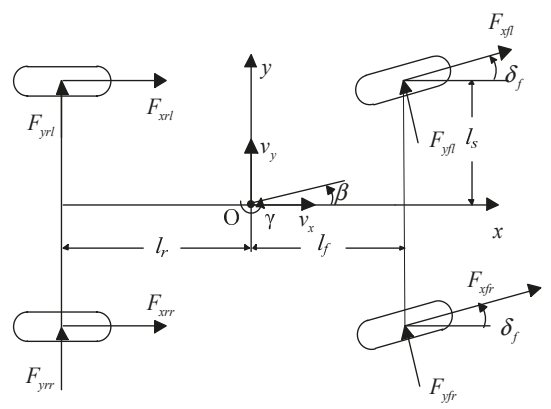

Figure 3. Dynamic model of DSV.

The dynamic equation of the steering system (shown in Figure 2) can be written as [11]:

$$
\begin{gathered}
J_{e} \ddot{\delta}_{f}+b_{e} \dot{\delta}_{f}=\tau_{a}+\frac{\Delta M}{R} r_{\sigma}-\tau_{f}, \\
\tau_{a}=k_{f} \alpha_{f} l^{2} / 3 \\
\alpha_{f}=\beta+l_{f} \gamma / u_{x}-\delta_{f},
\end{gathered}
$$

where $J_{e}$ and $b_{e}$ are the effective moment of inertia and damping of the SBW system, $\delta_{f}$ is the front wheel steering angle, $\tau_{a}$ is the tire self-aligning moment, $r_{\sigma}$ is the scrub radius, $\tau_{f}$ is the friction torque, $\alpha_{f}$ is the tire slip angle of the front wheel, $l$ is the half of the tire contact length. In addition, $\ddot{\delta}_{f}$ and $\tau_{f}$ can be assumed as bounded disturbances.

The lateral and yaw motion (shown in Figure 3) of the EV can be expressed as:

$$
\left\{\begin{array}{l}
m u_{x}(\dot{\beta}+\gamma)=\left(F_{y f l}+F_{y f r}\right) \cos \delta_{f}+\left(F_{x f l}+F_{x f r}\right) \sin \delta_{f}+F_{y r l}+F_{y r r} \\
I_{Z} \dot{\gamma}=\left(l_{f} \sin \delta_{f}+l_{s} \cos \delta_{f}\right) \frac{\Delta M}{R}+\left(l_{f} \cos \delta_{f}-l_{s} \sin \delta_{f}\right)\left(F_{y f l}+F_{y f r}\right)-l_{r}\left(F_{y r l}+F_{y r r}\right)
\end{array},\right.
$$

where

$$
F_{y f l}=F_{y f r}=k_{f}\left(\beta+l_{f} \gamma / u_{x}-\delta_{f}\right), F_{y r l}=F_{y r r}=k_{r}\left(\beta-l_{r} \gamma / u_{x}\right) .
$$

Define $X(t)=\left[\begin{array}{lll}\beta & \gamma & \delta_{f}\end{array}\right]^{T}, U(t)=\Delta M$, the state equation can be obtained as

$$
\dot{X}=A_{s} X+B_{s} U
$$

where

$$
A_{s}=\left[\begin{array}{ccc}
\frac{2 k_{f}+2 k_{r}}{m u_{x}} & \frac{2 k_{f} l_{f}-2 k_{r} l_{\mathrm{r}}}{m u_{x}^{2}}-1 & \frac{2 k_{f}}{m u_{x}} \\
\frac{2 k_{f} l_{f}-2 k_{r} l_{r}}{I_{Z}} & \frac{2 k_{f} l_{f}^{2}-2 k_{r} l_{r}^{2}}{I_{Z} u_{x}} & -\frac{2 k_{f} l_{f}}{I_{Z}} \\
\frac{k_{f} l^{2}}{3 b_{e}} & \frac{k_{f} l^{2} l_{f}}{3 b_{e} u_{x}} & -\frac{k_{f} l^{2}}{3 b_{e}}
\end{array}\right], B_{s}=\left[\begin{array}{c}
0 \\
\frac{l_{s}}{I_{Z} R} \\
\frac{r_{\sigma}}{R b_{e}}
\end{array}\right] .
$$

Note: From Equations (1) and (11), it is not difficult to see that they are similar to each other and there are so many $\delta_{f}$ emerged in Equation (11). However, here $\delta_{f}$ is not the external input of the system, but the steering angle of front wheels produced by the differential driving torque between the two sides of the front wheels. 


\subsection{Reference Model}

Here the 2DOF vehicle model with neutral steering characteristics, which can be easily obtained by adjusting the position of the CG, is employed to calculate the reference side-slip angle and yaw rate, and to estimate the actual side-slip angle.

Define $x_{d}(t)=\left[\beta_{d}, \gamma_{d}\right]^{T}$ and $u_{d}=\delta$, the corresponding state equation is expressed as:

$$
\begin{gathered}
\left\{\begin{array}{l}
\dot{x}_{d}=A_{d} x_{d}+B_{d} u_{d} \\
y_{d}=C_{d} x_{d}
\end{array}\right. \\
A_{d}=\left(\begin{array}{cc}
\frac{2\left(k_{f 0}+k_{r 0}\right)}{m u_{x}} & -1+\frac{2 l_{f d} k_{f 0}-2 l_{r d} k_{r 0}}{m u_{x}^{2}} \\
\frac{2 l_{f d} k_{f 0}-2 l_{r d} k_{r 0}}{I_{Z}} & \frac{2 l_{f d}{ }^{2} k_{f 0}+2 l_{r d} k_{r 0}}{I_{z} u_{x}}
\end{array}\right), B_{d}=\left(\begin{array}{c}
-\frac{2 k_{f 0}}{m u_{x}} \\
2 l_{f d} k_{f 0} \\
I_{Z}
\end{array}\right), C_{d}=\left(\begin{array}{ll}
1 & 0 \\
0 & 1
\end{array}\right) .
\end{gathered}
$$

where $\beta_{d}$ and $\gamma_{d}$ are the sideslip angle and the yaw rate of the reference model, $l_{f d}$ and $l_{r d}$ are the distances from the CG to the front and rear axles, respectively.

\subsection{Problem Formulation}

For the reference model, the normal input is the front wheel steering angle, which is proportional to the steering angle commanded by the driver. But for the SSV and DSV in the case of the steering failure, both of their inputs are the differential drive torque between the left and right front wheels, which should be controlled to drive the sideslip angle and yaw rate of the vehicles to their desired ones calculated by Equation (15). The sensors to measure the sideslip angle are usually very expensive. Consequently, the state observer should be firstly designed to estimate the actual sideslip angle. In addition, there is no direct relationship between the steering wheel angle and differential drive torque. To obtain the desired vehicle performance, sliding mode variable structure control strategy is applied. The diagram of control design is depicted in Figure 4.

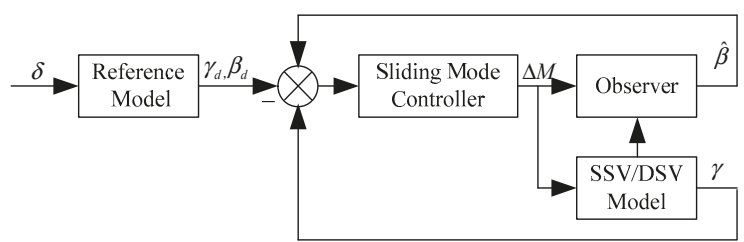

Figure 4. Structure of control system.

\section{Controllers Design}

\subsection{Sideslip Angle Observer}

The designs of the reduced-order observers for the SSV and the DSV are similar. This subsection takes the DSV as an example to illustrate the method. According to the Equation (13), the following equation can be obtained:

$$
\left\{\begin{array}{l}
{\left[\begin{array}{c}
\dot{\bar{X}}_{1} \\
\dot{\bar{X}}_{2}
\end{array}\right]=\left[\begin{array}{ll}
\bar{A}_{11} & \bar{A}_{12} \\
\bar{A}_{21} & \bar{A}_{22}
\end{array}\right]\left[\begin{array}{l}
\bar{X}_{1} \\
\bar{X}_{2}
\end{array}\right]+\left[\begin{array}{c}
\bar{B}_{1} \\
\bar{B}_{2}
\end{array}\right] U,} \\
\bar{Y}=\left[\begin{array}{ll}
0 & I
\end{array}\right]\left[\begin{array}{l}
\bar{X}_{1} \\
\bar{X}_{2}
\end{array}\right]=\bar{X}_{2}
\end{array}\right.
$$


where

$$
\begin{aligned}
& \bar{X}_{1}=[\beta], \bar{X}_{2}=\left[\begin{array}{ll}
\gamma & \delta_{f}
\end{array}\right], \bar{A}_{11}=\left[\frac{2 k_{f}+2 k_{r}}{m u_{x}}\right], \bar{A}_{12}=\left[\begin{array}{ll}
\frac{2 k_{f} l_{f}-2 k_{r} l_{\mathrm{r}}}{m u_{x}^{2}}-1 & \frac{2 k_{f}}{m u_{x}}
\end{array}\right], \\
& \bar{A}_{21}=\left[\begin{array}{cc}
\frac{2 k_{f} l_{f}-2 k_{r} l_{r}}{I_{Z}} & \frac{2 k_{f} l_{f}^{2}-2 k_{r} l_{r}^{2}}{I_{Z} u_{x}} \\
\frac{k_{f} l^{2}}{3 b_{e}} & \frac{k_{f} l^{2} l_{f}}{3 b_{e} u_{x}}
\end{array}\right], \bar{A}_{22}=\left[\begin{array}{r}
-\frac{2 k_{f} l_{f}}{I_{Z}} \\
-\frac{k_{f} l^{2}}{3 b_{e}}
\end{array}\right], \bar{B}_{1}=[0] .
\end{aligned}
$$

Equation (15) can be rewritten as:

$$
\left\{\begin{array}{l}
\dot{\bar{X}}_{1}=\bar{A}_{11} \bar{X}_{1}+v \\
\mathrm{Z}=\bar{A}_{21} \bar{X}_{1}
\end{array}\right.
$$

where

$$
v=\bar{A}_{12} \bar{Y}+\bar{B}_{1} U, Z=\dot{\bar{Y}}-\bar{A}_{22} \bar{Y}-\bar{B}_{2} U .
$$

The dynamic equation of the observer is as follows,

$$
\left\{\begin{array}{l}
\dot{\hat{X}}_{1}=\bar{A}_{11} \bar{X}_{1}+v-H(\hat{Z}-Z) \\
Z=\bar{A}_{21} \hat{X}_{1}
\end{array} .\right.
$$

Substituting the Equation (20) and the second expression of Equation (21) into the first one of Equation (21), the following can be obtained:

$$
\dot{\hat{X}}_{1}=\left(\bar{A}_{11}-H \bar{A}_{21}\right) \hat{X}_{1}+\left(\bar{A}_{12} \bar{Y}+\bar{B}_{1} U\right)+H\left(\dot{\bar{Y}}-\bar{A}_{22} \bar{Y}-\bar{B}_{2} U\right) .
$$

where, $\left(\bar{A}_{11}-H \bar{A}_{21}\right)$ is the coefficient matrix of the observer. And the pole of the reduced-order observer is determined by the following characteristic equation.

$$
\left|\lambda I-\left(\bar{A}_{11}-H \bar{A}_{21}\right)\right|=0 .
$$

Since Equation (22) contains a derivative term $\dot{\bar{Y}}$, it is necessary to obtain the derivative signal of the output, which will affect the uniqueness of the estimated state $\hat{X}_{1}$. Alternative state variables are allowed to be selected so that the state equation does not contain the derivative signal.

Define

$$
W=\hat{X}_{1}-H \bar{Y}
$$

then,

$$
\dot{\hat{X}}_{1}=\dot{W}+H \dot{\bar{Y}}=\left(\bar{A}_{11}-H \bar{A}_{21}\right)(W+H \bar{Y})+\left(\bar{A}_{12} \bar{Y}-\bar{B}_{1} U\right)+H \dot{\bar{Y}}-H \bar{A}_{22} \bar{Y}-H \bar{B}_{2} U .
$$

And the following expression can be obtained from

$$
\left\{\begin{array}{l}
\hat{X}_{1}=W+H \bar{Y} \\
\dot{W}=\left(\bar{A}_{11}-H \bar{A}_{21}\right) W+\left(\bar{B}_{1}-H \bar{B}_{2}\right) U+\left[\left(\bar{A}_{11}-H \bar{A}_{21}\right) H+\bar{A}_{12}-H \bar{A}_{22}\right] \bar{Y} .
\end{array}\right.
$$

Subtracting Equation (22) from the first expression of Equation (19), and

$$
\dot{\bar{X}}_{1}-\dot{\hat{X}}_{1}=\left(\bar{A}_{11} \bar{X}_{1}+\bar{A}_{12} \bar{Y}+\bar{B}_{1} U\right)-\left(\bar{A}_{11}-H \bar{A}_{21}\right) \hat{X}_{1}-\left(\bar{A}_{12} \bar{Y}+\bar{B}_{1} U\right)-H\left(\dot{\bar{Y}}-\bar{A}_{22} \bar{Y}-\bar{B}_{2} U\right),
$$


then it can be simplified as follows

$$
\dot{\bar{X}}_{1}-\dot{\hat{X}}_{1}=\bar{A}_{11} \bar{X}_{1}-\left(\bar{A}_{11}-H \bar{A}_{21}\right) \hat{X}_{1}-H \bar{A}_{21} \bar{X}_{1}=\left(\bar{A}_{11}-H \bar{A}_{21}\right)\left(\bar{X}_{1}-\hat{X}_{1}\right) .
$$

This equation is homogeneous. So long as $H$ is properly selected, the poles of the reduced-order observer can be disposed arbitrarily [14], so that the satisfactory attenuation speed of $\left(\bar{X}_{1}-\hat{X}_{1}\right)$ can be obtained and the state estimation $\hat{X}_{1}$ can approach $\bar{X}_{1}$ as soon as possible.

\subsection{Sliding Mode Controller of Skid Steering Vehicle}

For the SSV there is no direct relationship between the steering wheel angle and differential drive torque, the traditional vehicle with neutral steering characteristic is used as the reference model. And the SMC controller based on uncertain parameters is designed to make sure that the state variables of skid steering vehicle to track those of the reference model.

Define the state variables of error system as $e=x_{d}-x$, and the error model is as follows:

$$
\dot{e}=A_{d} e+\left(A_{d}-A_{0}\right) x+B_{d} u_{d}-B u-\Delta A x .
$$

The nominal model of the error system can be expressed as:

$$
\dot{e}=A_{d} e+\left(A_{d}-A_{0}\right) x+B_{d} u_{d}-B u .
$$

In order to control the output of SSV completely tracking that of the reference model, $u$ should satisfy the following equation for arbitrary state $x$, external reference input $u_{d}$ and various uncertainties.

$$
\left(A_{d}-A_{0}\right) x+B_{d} u_{d}-B u-\Delta A x=0 .
$$

According to the linear system theory, it can be drawn that the necessary and sufficient conditions which the controlled object completely tracks the reference model are as follows [15]:

$$
\begin{aligned}
& \operatorname{rank}[B]=\operatorname{rank}\left[\begin{array}{cc}
B & \Delta A
\end{array}\right]=\operatorname{rank}\left[\begin{array}{ll}
B & C
\end{array}\right], \\
& \operatorname{rank}[B]=\operatorname{rank}\left[\begin{array}{cc}
B & A_{d}-A
\end{array}\right]=\operatorname{rank}\left[\begin{array}{ll}
B & B_{d}
\end{array}\right] .
\end{aligned}
$$

where Equation (32) is the matching condition as well as Equation (33) is the uncertainty matching condition.

At this point, the controlled system can be rewritten as:

$$
\left\{\begin{array}{l}
\dot{x}=A_{0} x+B(u+\Delta \widetilde{A} x+\Delta \widetilde{B} u) \\
y=C x \\
\Delta A=B \Delta \widetilde{A}, \Delta B=B \Delta \widetilde{B}
\end{array}\right.
$$

The complete compensation for uncertainties can be achieved by designing the control law. The full-scale sliding mode switching surface for the error model is selected as:

$$
s=G e,
$$

where, $G$ is the sliding mode parameter matrix to be designed and $G=\left[\begin{array}{ll}G_{1} & G_{2}\end{array}\right]$.

The structure of the variable structure control law can be expressed as:

$$
u=u_{m}+u_{v}
$$

where, $u_{m}$ is the matching control law for the model reference closed-loop control system, $u_{v}$ is the variable structure control law. 
As long as the matrix $B$ is full rank, i.e., $\operatorname{rank}(B)=m$, there must be a non-singular linear transformation matrix $T$ to make $\widetilde{B}_{1}=T_{1} B=0$, then the Equation (29) can be transformed into

$$
\dot{z}=\widetilde{A}_{d} z+A_{d p} x+\widetilde{B}_{d} \delta-\widetilde{B} u-\Delta \widetilde{A} x,
$$

where

$$
\widetilde{A}_{d}=T A_{d} T^{-1}, A_{d p}=T\left(A_{d}-A\right), \widetilde{B}_{d}=T B_{d}, \widetilde{B}=T B, \Delta \widetilde{A}=T \Delta A
$$

In order to get the appropriate $\widetilde{B}$, select

$$
T=\left[\begin{array}{cc}
I_{n-m} & -B_{1} B_{2}^{-1} \\
0 & I_{m}
\end{array}\right]=\left[\begin{array}{l}
T_{1} \\
T_{2}
\end{array}\right]
$$

then

$$
z=\left[\begin{array}{c}
z_{1} \\
z_{2}
\end{array}\right], \widetilde{A}_{d}=T A_{d} T^{-1}=\left[\begin{array}{cc}
\widetilde{A}_{d}^{11} & \widetilde{A}_{d}^{12} \\
\widetilde{A}_{d}^{21} & \widetilde{A}_{d}^{22}
\end{array}\right], \widetilde{B}=T B=\left[\begin{array}{c}
\widetilde{B}_{1} \\
\widetilde{B}_{2}
\end{array}\right]=\left[\begin{array}{c}
0 \\
\widetilde{B}_{2}
\end{array}\right],
$$

where $z_{1} \in R^{n-m}, z_{2} \in R^{m}, \widetilde{A}_{d}^{11} \in R^{(n-m) \times(n-m)}, \widetilde{A}_{d}^{22} \in R^{m \times m}, \widetilde{B}_{2} \in R^{m \times m}$

And the switching surface becomes

$$
s=G e=\widetilde{G} T e=\widetilde{G} z=\left(\widetilde{G}_{1}, \widetilde{G}_{2}\right) z
$$

The pole assignment method is used to compute $G$, which makes the final sliding mode have the pre-given pole set $\Lambda=\left\{\lambda_{i}, i=1,2, \ldots, n-m\right\}$ (where all of the poles have negative real parts and imaginary parts appear in pairs). Since $\left(\widetilde{A}_{d}^{11}, \widetilde{A}_{d}^{12}\right)$ is a controllable matrix, there is matrix $K$ to ensure that pole set of $\widetilde{A}_{d}^{11}-\widetilde{A}_{d}^{12} K$ is $\Lambda$.

In addition, since

$$
\widetilde{G}=\left[\widetilde{G}_{1}, \widetilde{G}_{2}\right]=\left[\widetilde{G}_{2} K, \widetilde{G}_{2}\right]=\widetilde{G}_{2}[K, I],
$$

where $\widetilde{G}_{2}$ is a non-singular matrix of $m \times m$, and usually $\widetilde{G}_{2}=I_{m}$. And $\widetilde{G}=[K, I]$ can be confirmed uniquely, and the switching function $s=G e$ is obtained.

According to the matching condition of fully matched model, the matching control law is as follows.

$$
u_{m}=\widetilde{B}_{2}^{-1}\left[\begin{array}{ll}
0 & I_{m}
\end{array}\right] T\left(A_{d}-A\right) x+\widetilde{B}_{2}^{-1}\left[\begin{array}{ll}
0 & I_{m}
\end{array}\right] B_{d} \delta .
$$

Substituting Equations (36) and (43) into the Equation (37), the error equation becomes:

$$
\dot{z}=\widetilde{A}_{d} z-\widetilde{B} u_{v}-\Delta \widetilde{A} x
$$

where the variable structure control law $u_{v}$ can be chosen to ensure that the system is stable and reliably maintained in the sliding mode, which means that $u_{v}$ can make the sliding mode reach the condition, $\frac{d}{d t}\left(s^{T} Q s\right)<0$, where $Q$ is the symmetric positive definite matrix and $Q>0$. So $u_{v}$ should make the following equation hold.

$$
\dot{w}=s^{T} \dot{s}<0 .
$$

Let

$$
u_{v}=g(t)(G \widetilde{B})^{-1} \operatorname{sgn}(s),
$$

where $g(t)$ is the scalar control coefficient to be determined and $g(t)>0$, then

$$
\dot{w}=s^{T}\left[C\left(A_{0}+\Delta A\right) x+C B u\right] .
$$


Substituting Equation (46) into Equation (47), then

$$
\dot{w}=-g(t) s^{T} \operatorname{sgn}(s)+s^{T}\left[G \widetilde{A}_{m} z-G \Delta \widetilde{A} x\right] \leq-g(t)\left\|s^{T}\right\|+\left\|s^{T}\right\|\left[\left\|G \widetilde{A}_{m}\right\|\|z\|+\phi_{a}\|G\|\|x\|\right] .
$$

And the control law is

$$
g(t)=a_{1}\|z\|+a_{2}\|x\|+a_{3}+\varepsilon, a_{1}=\left\|G \widetilde{A}_{m}\right\|, a_{2}=\phi_{a}\|G\| .
$$

where $\varepsilon$ is a small positive number. After obtaining the above control law, the variable structure control law of the full-scale sliding mode model can be got by transforming $z$ into $e$.

\subsection{Sliding Mode Controller of Differential Steering Vehicle}

Here the sliding surface of SMC can be defined as the following to make sure that the real sideslip angle and yaw rate approach the ideal ones.

$$
s=\gamma-\gamma_{d}+\xi\left(\hat{\beta}-\beta_{d}\right)
$$

where, $\xi$ is the weight coefficient, which is used to enhance or reduce the influence of sideslip angle. Take the derivative of Equation (50) and substitute Equations (11) and (26) into Equation (50), the following equation can be obtained:

$$
\dot{s}=d_{1}+d_{2}+\frac{l_{s}}{I_{Z} R} \Delta M+D_{1}(t),
$$

where

$$
\begin{gathered}
d_{1}=-\frac{2 k_{f} l_{f}}{I_{Z}} \delta_{f}+\frac{2 k_{f} l_{f}-2 k_{r} l_{r}}{I_{Z}} \beta+\frac{2 k_{f} l_{f}^{2}-2 k_{r} l_{r}^{2}}{I_{Z} u_{x}} \gamma, \\
d_{2}=\xi\left[\frac{2 k_{f}}{m u_{x}} \delta_{f}+\frac{2 k_{f}+2 k_{r}}{m u_{x}} \beta+\left(\frac{2 k_{f} l_{f}-2 k_{r} l_{\mathrm{r}}}{m u_{x}^{2}}-1\right) \gamma\right], \\
D_{1}(t)=-\left[\dot{\gamma}_{d}+\xi \dot{\beta}_{d}-\xi\left(\bar{A}_{11}-H \bar{A}_{21}\right)(\beta-\hat{\beta})\right] .
\end{gathered}
$$

Note that the sideslip angle observer is convergent, that is, the observer error $\beta-\hat{\beta}$ converges to zero in a finite time. And from the Equation (11), it can be drawn that $\dot{\gamma}_{d}, \dot{\beta}_{d}$ is bounded. Then the following inequality holds.

$$
\left|D_{1}(t)\right| \leq \bar{D}_{1}
$$

where $\bar{D}_{1}$ is a constant.

In order to weaken the chattering phenomenon of sliding mode control, the exponential reaching law with saturation function is applied, and the controller can be designed as

$$
\Delta M=\frac{I_{Z} R}{l_{s}}\left[-k_{1} \operatorname{sat}(s)-k_{2} s-d_{1}-d_{2}\right],
$$

with $k_{1}>\bar{D}_{1}, k_{2}>0$, and the sliding variable $s$ will converge to the origin in a finite time.

Substitute Equation (54) into Equation (51), and

$$
\dot{s}=\frac{1}{I_{Z}}\left[-k_{1} \operatorname{sat}(s)-k_{2} s+D_{1}(t)\right] .
$$

Select the Lyapunov function as $V(s)=\frac{1}{2} s^{2}$, take the derivative of $V(s)$ and we get

$$
\dot{V}=\frac{1}{I_{Z}}\left[-k_{1} \operatorname{sat}(s)-k_{2} s+D_{1}(t)\right] s \leq \frac{1}{I_{Z}}\left(-k_{1}+\bar{D}_{1}\right)|s| .
$$


Note $k_{1}>\bar{D}_{1}$, and we can get

$$
\dot{V}<\sqrt{2} \frac{k_{1}-\bar{D}_{1}}{I_{Z}} V^{\frac{1}{2}}
$$

According to the finite-time Lyapunov stability theory, the sliding mode variable $s$ will converge to the origin in a finite time.

\section{Simulation and Analysis}

In this section, Simulink and CarSim are used to confirm the effectiveness of the control systems designed in this paper. The parameters used in the simulation are as follows: $m=1111 \mathrm{~kg}$, $I_{z}=2031.4 \mathrm{~kg} \mathrm{~m}^{2}, l_{f}=1.04 \mathrm{~m}, l_{r}=1.56 \mathrm{~m}, l_{f d}=1.04 \mathrm{~m}, l_{r d}=1.56 \mathrm{~m}, l_{s}=0.7405 \mathrm{~m}, R=0.304 \mathrm{~m}$, $k_{f 0}=-98,202.8 \mathrm{~N} / \mathrm{rad}, k_{r 0}=-63,947.18 \mathrm{~N} / \mathrm{rad}$. The simulation results are explained in the following subsections.

\subsection{Simulation in J-Turn Manoeuvre}

In this simulation, $u_{x}=10 \mathrm{~m} / \mathrm{s}$ and the steering wheel steering angle with a maximum steering angle of $3.5 \mathrm{rad}$ as shown in Figure $5 \mathrm{a}$ is used as the input of the reference model. Figure 5b-l illustrate the simulation results of reference model, SSV and DSV with different SMC controllers.

Figure $5 b, c$ are the calculated and the estimated sideslip angles of the SSV and the DSV with SMC controller. It can be drawn that the designed sideslip angle observer is effective to track the calculated sideslip angle. Figure 5d,e are the yaw rate and sideslip angle curves of the three models. It can be seen that both of the yaw rate and sideslip angle of DSV with SMC controller can follow the desired ones, while only the yaw rate of SSV with SMC controller can follow the desired one. Generally speaking, the side slip angle and the yaw rate are coupled. It is often difficult to track two state variables simultaneously by a single control variable-differential torque. Here the desired yaw rate is tracked very well.

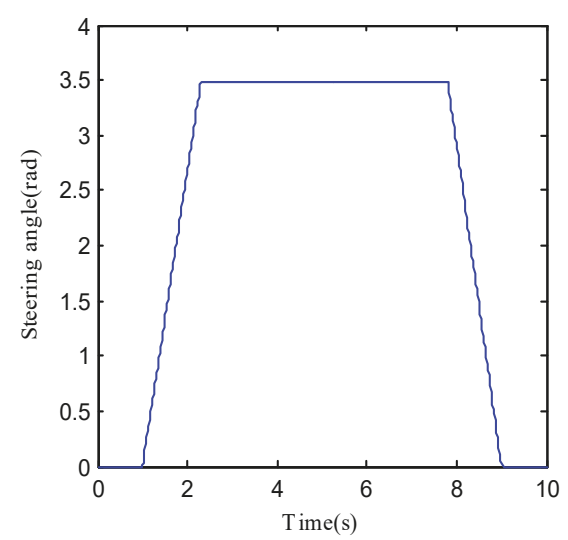

(a)

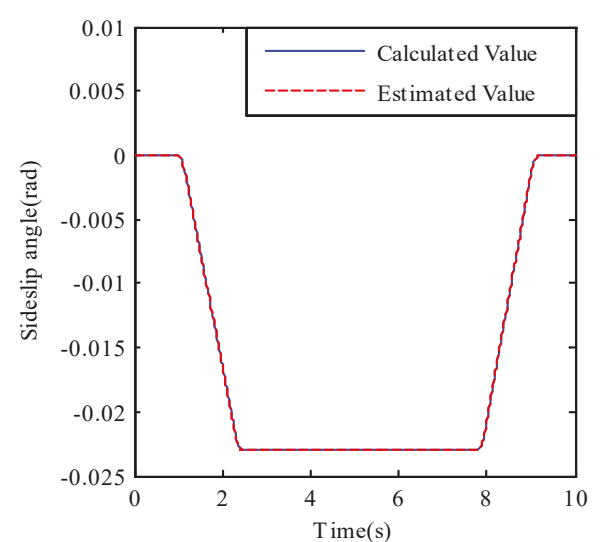

(b)

Figure 5. Cont. 


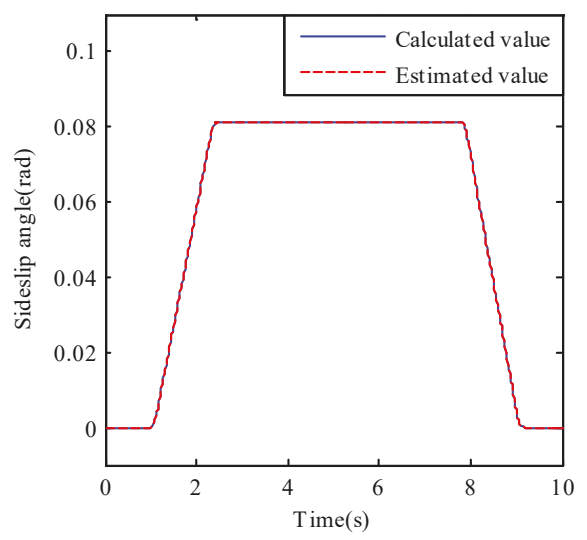

(c)

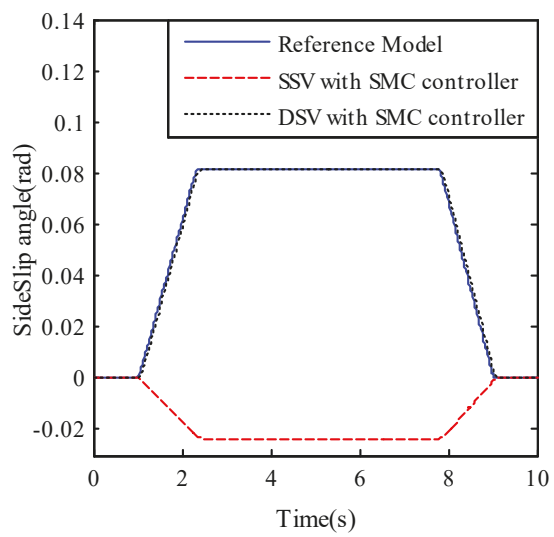

(e)

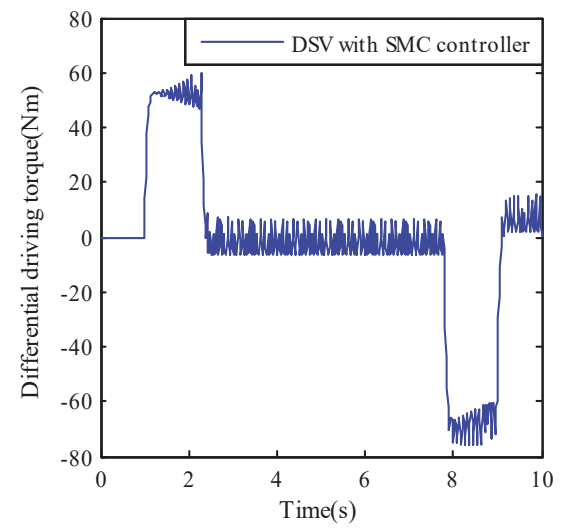

(g)

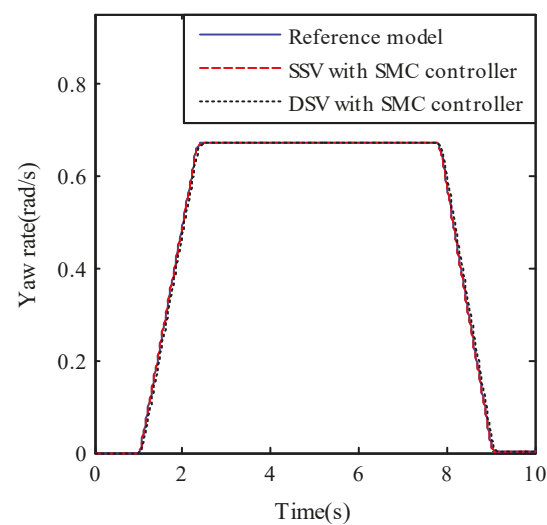

(d)

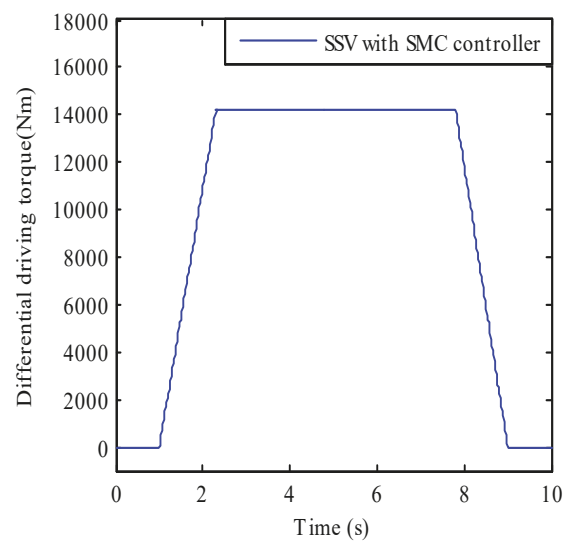

(f)

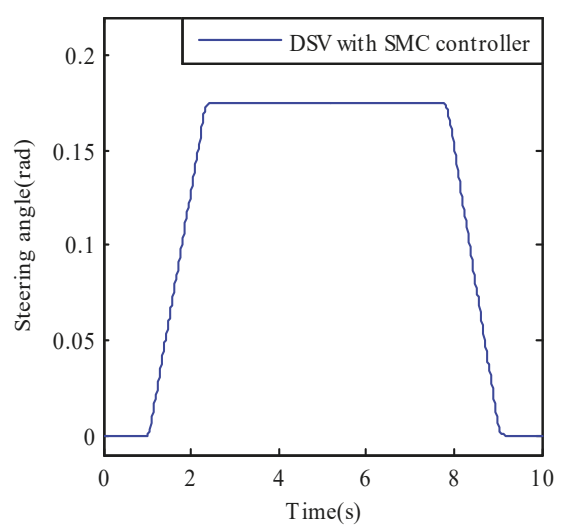

(h)

Figure 5. Cont. 


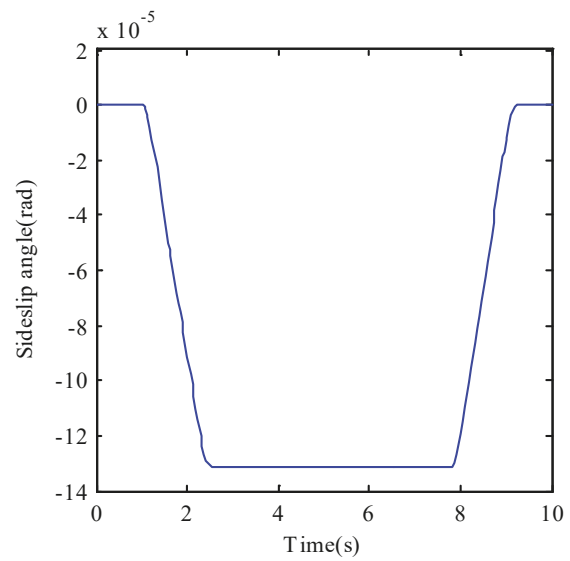

(i)

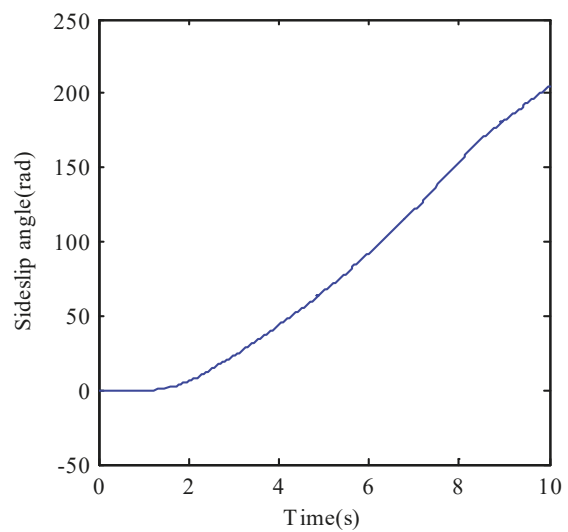

(k)

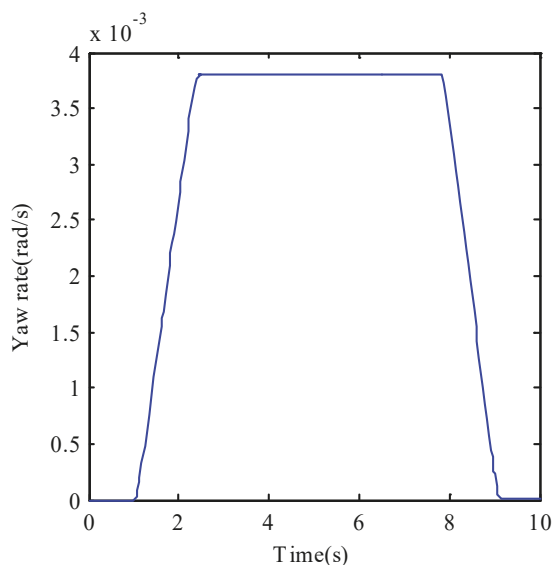

(j)

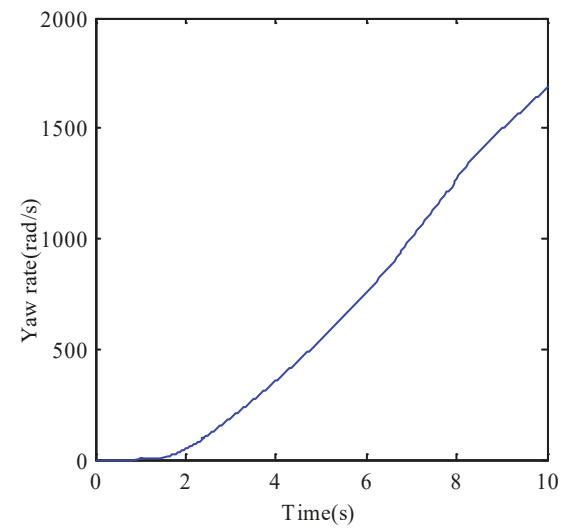

(l)

Figure 5. J-turn simulation at $10 \mathrm{~m} / \mathrm{s}$ (a) Steering wheel steering angle curve; (b) Sideslip angle curves of skid steering vehicle (SSV); (c) Sideslip angle curves of DSV; (d) Yaw rate curves; (e) Sideslip angle curves; (f) Differential driving torque for skid steering; (g) Differential driving torque for differential steering; (h) Front wheel steering angle; $(\mathbf{i}, \mathbf{j})$ Response curves of SSV without sliding mode control (SMC) controller; (k,1) Response curves of DSV without SMC controller.

Figure 5f,g show that the differential driving torques for the controllers of SSV and DSV respectively, and their absolute maximum are $142,210 \mathrm{Nm}$ and $75.86 \mathrm{Nm}$. Obviously, there is too large a difference between the two needed differential driving torques. The reason is for the DSV the differential torque also produces a front wheel steering angle at the same time, which decreases the needed differential torque in turn. And the maximum front wheel angle produced by the differential driving torque for the controller of DSV are 0.1745 rad as shown in Figure $5 \mathrm{~h}$. Figure $5 \mathrm{i}-1$ are the response curves of SSV and DSV without SMC controller. It can be seen that they are so different from the desired ones.

In conclusion, the simulation results show that the designed observer is effective and the responses of DSV with SMC controller can exactly track the ideal ones. Although the yaw rate of SSV with SMC controller can also exactly track that of the reference model, the differential driving torque needed for SSV is too much larger than that needed for DSV, which obviously exceeds the maximum torque provided by the in-wheel motor. 


\subsection{Simulation in Double Lane Change Maneuver}

Double lane change maneuver is another important method to examine the maneuvering stability of the vehicle. In this simulation, $u_{x}=20 \mathrm{~m} / \mathrm{s}$ and Figure 6 a shows the input of the reference model, which can guarantee the vehicle to achieve the double lane change maneuver. Figure $6 \mathrm{~b}-1$ illustrate the simulation results of reference model, SSV and DSV with different SMC controllers.

Figure $6 b, c$ are the calculated and the estimated sideslip angles of the SSV and the DSV with SMC controller, which show that the designed observer can track the calculated sideslip angle very well. Figure $6 \mathrm{~d}$ is the yaw rate curves of the three models and their responses are almost the same. Figure $6 \mathrm{e}$ is the sideslip angles of the three models, which illustrates that only the sideslip angle of the SSV with controller can follow the desired one. The differential drive torques between the left and right front wheels required for the SSV and the DSV with SMC controller are shown in Figure 6f,g and their absolute maximums are $3150 \mathrm{Nm}$ and $46.4 \mathrm{Nm}$. In addition, the external steering angle of the front wheel is as shown in Figure 6h. Figure 6i-l are the response curves of the SSV and the DSV without SMC controller. It can be seen that they are so different from the desired ones.

In conclusion, both of the differential driving torque and the external steering angle of the front wheel produced by the torque contribute to the steering of DSV with SMC controllers, which leads to a substantial reduction in the required differential driving torque. Therefore, the differential driving torque needed for the DSV is much smaller than that needed for the SSV.

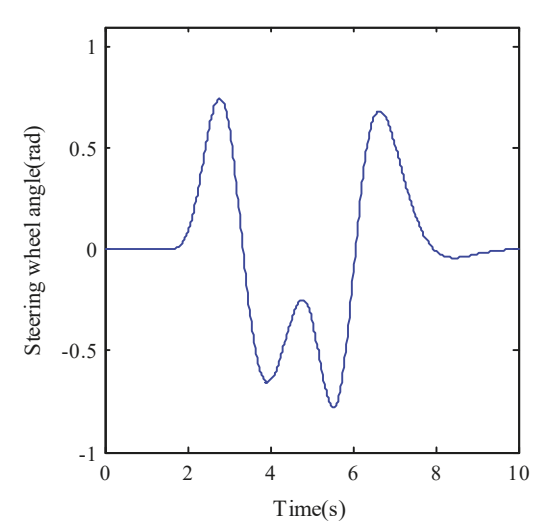

(a)

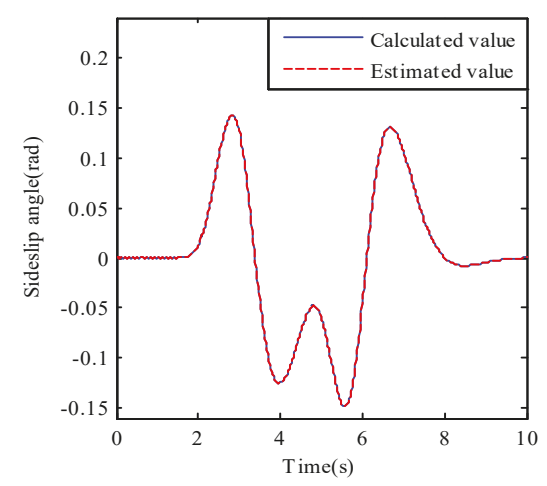

(c)

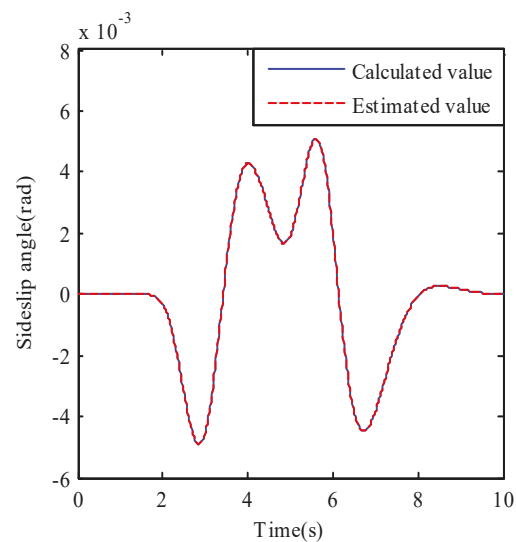

(b)

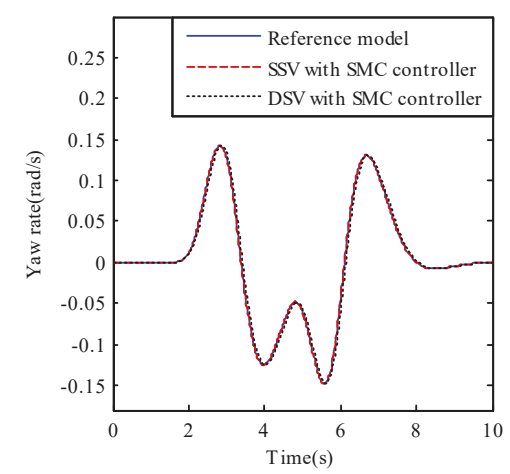

(d)

Figure 6. Cont. 


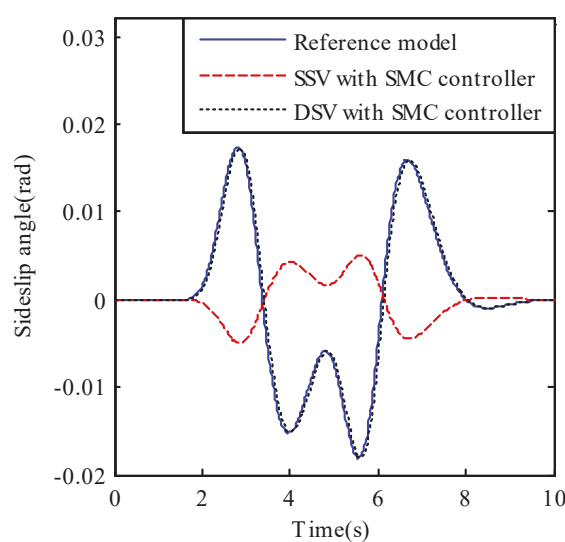

(e)

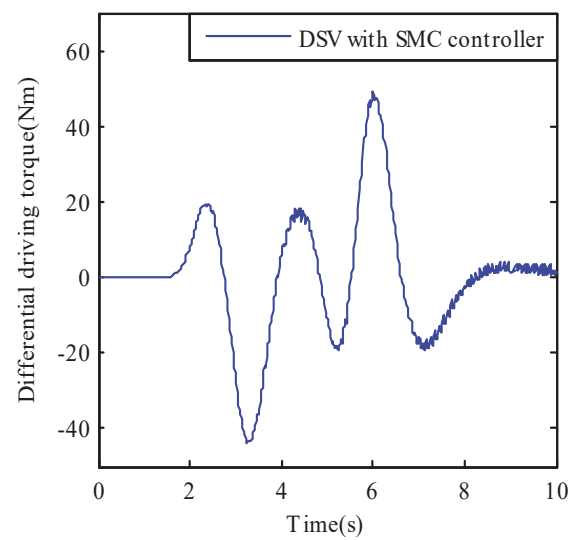

(g)

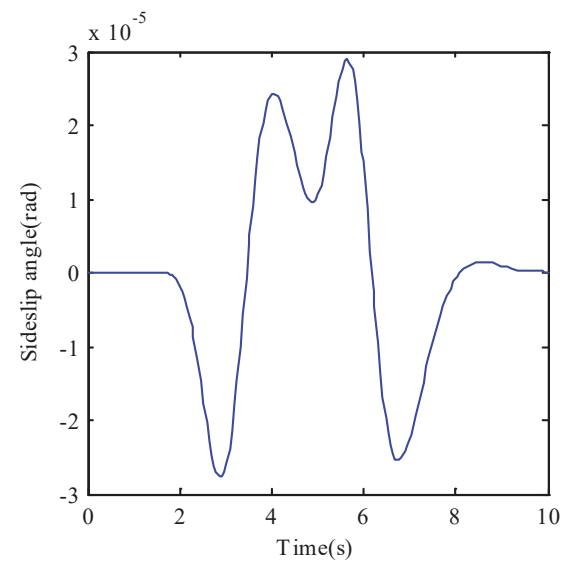

(i)

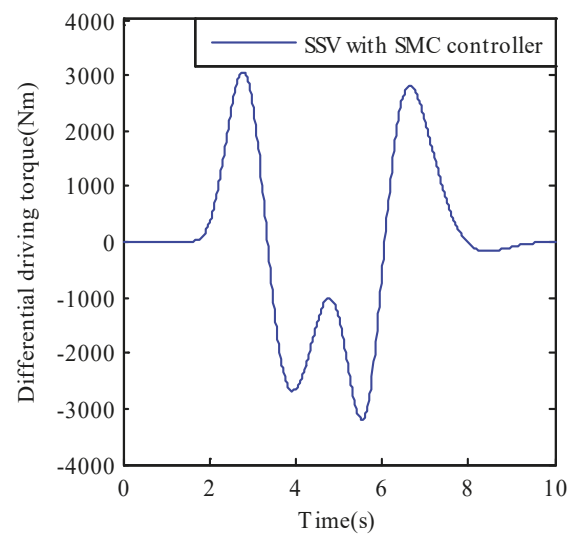

(f)

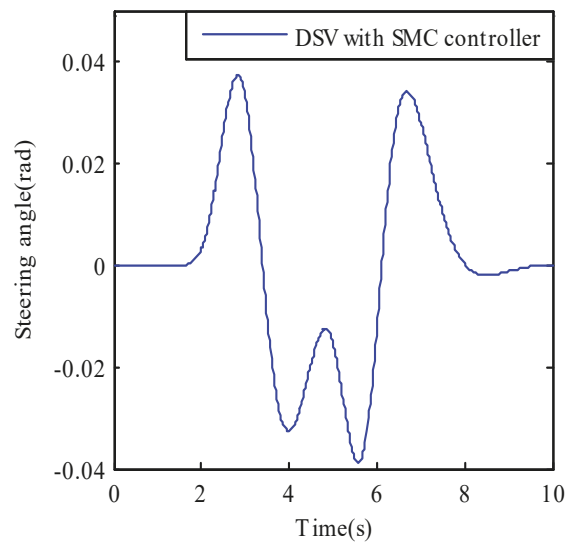

(h)

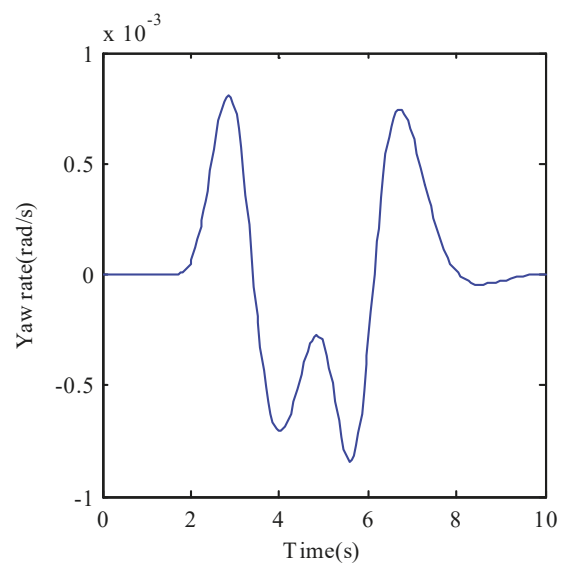

(j)

Figure 6. Cont. 


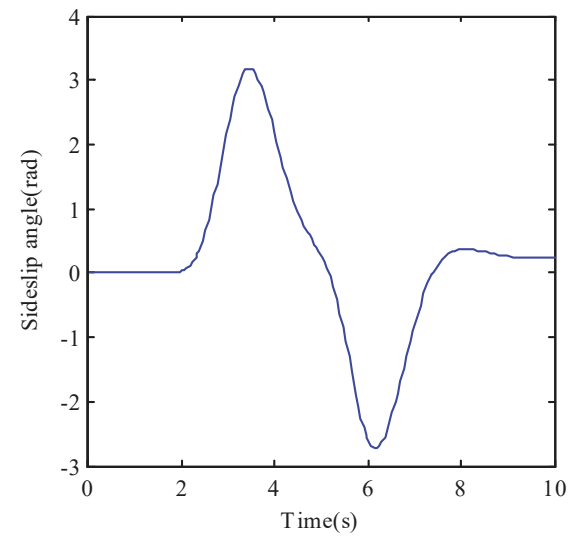

(k)

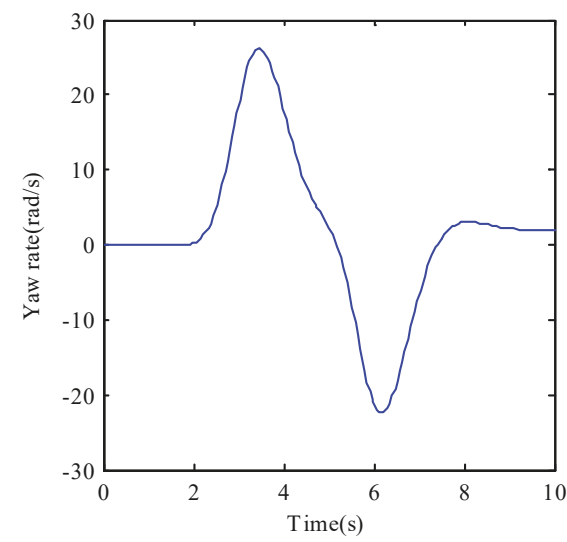

(1)

Figure 6. Double lane change simulation at $20 \mathrm{~m} / \mathrm{s}$ (a) Steering wheel steering angle curve; (b) Sideslip angle curves of SSV; (c) Sideslip angle curves of DSV; (d) Yaw rate curves; (e) Sideslip angle curves; (f) Differential driving torque for skid steering; (g) Differential driving torque for differential steering; (h) Front wheel steering angle; $(\mathbf{i}, \mathbf{j})$ Response curves of SSV without SMC controller; $(\mathbf{k}, \mathbf{l})$ Response curves of DSV without SMC controller.

\subsection{Robustness Analysis}

When the cornering stiffness of the front and rear tires are decreased and increased by $5 \%$ respectively as other parameters remain unchanged, yaw rate curves of reference model, SSV and DSV with different SMC controllers are shown in Figures 7 and 8, respectively.

From Figures 7 and 8, it can be seen that the yaw rate curve of DSV with SMC controller can completely track that of the reference model when front or rear tire cornering stiffness is changed. However, there are always a little difference between the yaw rates of SSV with SMC controller and the reference model. It can be drawn that the robustness of DSV with SMC controller is better than that of SSV with SMC controller.

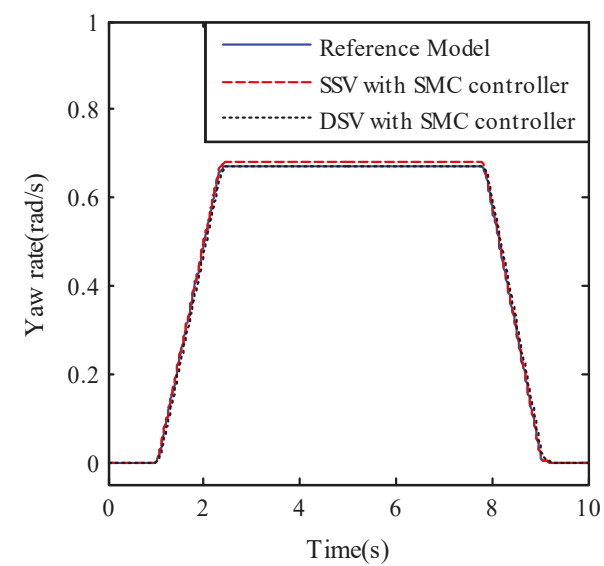

Figure 7. Yaw rate curves with $k_{f 0}$ reduced by $5 \%$. 


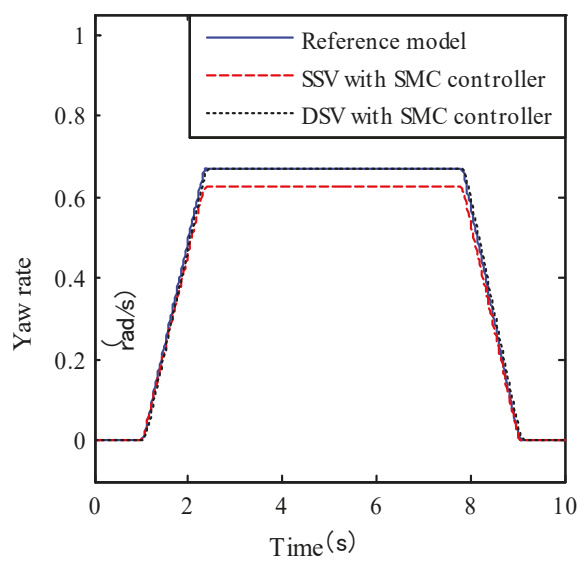

Figure 8. Yaw rate curves with $k_{r 0}$ increased by $5 \%$.

\section{Conclusions}

The realizations of skid steering and differential steering of IWM EVs with the failure of SBW system are studied:

(1) Simulation results show that the skid steering of SSV can be realized by the differential drive torque between the two sides of the front wheels. However, the needed differential drive torque is so large and far beyond the maximum torque generated by the in-wheel motor. Therefore, the skid steering cannot be used in practice.

(2) The differential steering of DSV in case of steering failure also can be achieved by the differential drive torque between the two sides of the front wheels. Because this torque generates an external vehicle yaw moment and at the same time an additional steering angle of the front wheel is also produced, so its value can be greatly reduced.

(3) The structure of differential steering is relatively simple. It not only can be used as the backup steering system, but also can be used for the active steering of front and rear wheels and the improvement of vehicle handling stability, and even can be applied as a sole steering system for future pilotless vehicles.

Author Contributions: Conceptualization, J.T. (Jie Tian) and S.L.; Formal analysis, J.T. (Jie Tian); Funding acquisition, J.T. (Jie Tian); Investigation, S.L.; Methodology, J.T. (Jie Tian) and S.L.; Project administration, J.T. (Jie Tian); Resources, J.T. (Jie Tian).; Software, J.T. (Jun Tong); Supervision, S.L.; Validation, J.T. (Jun Tong) and S.L.; Visualization, J.T. (Jun Tong); Writing—original draft, J.T. (Jie Tian); Writing—review and editing, S.L.

Acknowledgments: This research was funded by National Natural Science Foundation of China grant number 11272159, and National Youth Foundation of China grant number 51305207.

Conflicts of Interest: The authors declare no conflict of interest.

\section{References}

1. Leng, B.; Xiong, L.; Jin, C.; Liu, J.; Zhuo, P.Y. Differential Drive Assisted Steering Control for an In-wheel Motor Electric Vehicle. SAE Int. J. Passeng. Cars Electron. Electr. Syst. 2015, 8, 433-441. [CrossRef]

2. Sun, C.Y.; Zhang, X.; Xi, L.H.; Tian, Y. Design of a path-tracking steering controller for autonomous vehicles. Energies 2018, 11, 1451. [CrossRef]

3. To, C.N.; Hormoz; Dai, V.Q.; Simic, M.; Khayyam, H.; Marzbani; Jazar, R.N. Autodriver autonomous vehicles control strategy. Procedia Comput. Sci. 2018, 126, 870-877. [CrossRef]

4. Zhao, W.Z.; Xu, X.H.; Wang, C.Y. Multidiscipline collaborative optimization of differential steering system of electric vehicle with motorized wheels. Sci. China-Technol. Sci. 2012, 55, 3462-3468. [CrossRef] 
5. Li, W.; Potter, T.; Jones, R.P. Steering of $4 \mathrm{WD}$ vehicles with independent wheel torque control. Veh. Syst. Dyn. 1998, 29, 205-218. [CrossRef]

6. Shuang, G.; Cheung, N.C.; Cheng, K.W.E.; Dong, L.; Liao, X. Skid Steering in 4-Wheel-Drive Electric Vehicle. In Proceedings of the 7th International Conference on Power Electronics and Drive Systems, Bangkok, Thailand, 27-30 November 2007; pp. 1548-1553.

7. Wang, J.; Wang, Q.; Jin, L.; Song, C. Independent wheel torque control of 4WD electric vehicle for differential drive assisted steering. Mechatronics 2011, 21, 63-76. [CrossRef]

8. Zhao, W.Z.; Li, Y.J.; Wang, C.Y.; Zhang, Z.Q.; Xu, C.L. Research on control strategy for differential steering system based on H mixed sensitivity. Int. J. Automot. Technol. 2013, 14, 913-919. [CrossRef]

9. Wu, X.D.; Yang, L.; Xu, M. Speed Following Control for Differential Steering of 4WID Electric Vehicle. In Proceedings of the 40th Annual Conference of the IEEE-Industrial-Electronics-Society, Dallas, TX, USA, 29 October-1 November 2014; pp. 3054-3059.

10. Zhai, L.; Hou, R.F.; Sun, T.M.; Steven, K. Continuous Steering Stability Control Based onan Energy-Saving Torque Distribution Algorithm for a Four in-Wheel-Motor Independent-Drive Electric Vehicle. Energies 2018, 11, 350. [CrossRef]

11. Wang, R.R.; Jing, H.; Hu, C.; Chadli, M.; Yan, F.J. Robust $\mathrm{H}_{\infty}$ output-feedback yaw control for in-wheel motor driven electric vehicles with differential steering. Neurocomputing 2016, 173, 676-684. [CrossRef]

12. Hu, C.; Wang, R.R.; Yan, F.J.; Karimi, H.R. Robust Composite Nonlinear Feedback Path Following Control for Independently Actuated Autonomous Vehicles with Differential Steering. IEEE Trans. Transp. Electrif. 2016, 2, 1-10. [CrossRef]

13. Hu, C.; Wang, R.R.; Yan, F.J.; Huang, Y.J.; Wang, H.; Wei, C.F. Differential Steering Based Yaw Stabilization Using ISMC for Independently Actuated Electric Vehicles. In Proceedings of the Symposium of Mechatronic and Embedded Technologies in Intelligent Transporation Systems at the 12th IEEE/ASME International Conference on Mechatronic and Embedded Systems and Applications, Auckland, New Zealand, 29-31 August 2016; Volume19, pp. 627-638.

14. Xie, H.W.; Zou, F.X.; Zhang, M.; Li, P.B.; Li, Q. Morden Control Systems, 8th ed.; Higher Education Press: Beijing, China, 2006; pp. 201-204, ISBN 7-04-009643-9.

15. Li, Y.J.; Zhang, K. Theory and Application of Adaptive Control; Northwestern Polytechnical University Press: Xi'an, China, 2005; pp. 168-170, ISBN 7-5612-1875-3.

(C) 2018 by the authors. Licensee MDPI, Basel, Switzerland. This article is an open access article distributed under the terms and conditions of the Creative Commons Attribution (CC BY) license (http:/ / creativecommons.org/licenses/by/4.0/). 
Article

\title{
An Adaptive-Equivalent Consumption Minimum Strategy for an Extended-Range Electric Bus Based on Target Driving Cycle Generation
}

\author{
Hongwei Liu, Chantong Wang, Xin Zhao and Chong Guo * \\ State Key Laboratory of Automotive Dynamic Simulation and Control, Jilin University, Changchun 130022, \\ China; asgroup@163.com (H.L.); wangct17@mails.jlu.edu.cn (C.W.); njauclzx@163.com (X.Z.) \\ * Correspondence: guochong@jlu.edu.cn; Tel.: +86-135-0445-4886
}

Received: 5 June 2018; Accepted: 7 July 2018; Published: 10 July 2018

\begin{abstract}
Energy management strategies based on instantaneous optimization have been widely used in hybrid/plug-in hybrid electric vehicles (HEV/PHEV) in order to improve fuel economy while guaranteeing vehicle performance. In this study, an adaptive-equivalent consumption minimum strategy (A-ECMS) based on target driving cycle (TDC) generation was proposed for an extended-range electric bus (E-REB) operating on fixed routes. Firstly, a Hamilton function and a co-state equation for E-REB were determined according to the Pontryagin Minimum Principle (PMP). Then a series of TDCs were generated using Markov chain, and the optimal solutions under different initial state of charges (SOCs) were obtained using the PMP algorithm, forming the optimal initial co-state map. Thirdly, an adaptive co-state function consisting of fixed and dynamic terms was designed. The co-state map was interpolated using the initial SOC data and the vehicle driving data obtained by an Intelligent Transport System, and thereby the initial co-state values were solved and used as the fixed term. A segmented SOC reference curve was put forward according to the optimal SOC changing curves under different initial SOCs solved by using PMP. The dynamic term was determined using a PI controlling method and by real-time adjusting the co-states to follow the reference curve. Finally with the generated TDCs, the control effect of A-ECMS was compared with PMP and Constant-ECMS, which was showed A-ECMS provided the final SOC closer to the pre-set value and fully used the power of the batteries. The oil consumption solutions were close to the PMP optimized results and thereby the oil depletion was reduced.
\end{abstract}

Keywords: extended-range electric bus; adaptive-equivalent consumption minimum strategy; Markov chain; target driving cycles; SOC reference curve; energy management system

\section{Introduction}

\subsection{Literature Review}

Environmental deterioration and the increasing shortage of petroleum resources have greatly increased the demand for energy-saving and environmental protective vehicles. The new energy vehicle technology is regarded as an excellent way to simultaneously address the energy crisis and insecurity and reduce environmental impacts [1]. As a type of plug-in hybrid electric vehicle (PHEV), extended-range electric buses (E-REBs) can coordinate the energy allocation between batteries and the auxiliary power unit, and prolong the mileage of pure electric vehicles while improving the fuel economy. Thus, extended-range electric buses have gained growing attention from vehicle manufacturers and customers [2,3].

Energy management is still a technical puzzle faced by hybrid electric vehicles because it not only aims at the minimum energy consumption, but also needs to take into consideration the vehicle 
dynamic performance, emission performance and the characteristic of each component. Due to the presence of multiple power sources, the reasonability of energy allocation directly affects the dynamic performance and fuel economy of vehicles [4]. However, the conventional energy management strategy only considers a single performance index and cannot achieve an overall optimization. Thus deeper research shows the energy management has gradually transited from the initially single goals of fuel depletion or emission to the currently multi-goal real-time intelligent integrated control. So far, the control strategies can be divided into rule-based and optimization-based energy allocation strategies [5].

In the first category, the vehicle working states are firstly divided according to pre-set control rules and then controlled separately. The rules are set to make the engine, generator and batteries work within the pre-set high-efficiency zones, but are only slightly dependent on specific working conditions and thereby operate in real-time. The two main directions of this category are the control strategies based on logic thresholds or fuzzy rules [6]. Firstly, the logic thresholds are usually the state of charge (SOC) of battery power or the speed signals of vehicles, which allow for switching between different operation modes [7]. Its disadvantage is that the dynamic performance of the vehicle will be greatly reduced when the work mode enters the charging-sustaining phase. The control strategies based on fuzzy rules control energy allocation via the use of fuzzy algorithms. Firstly, the control parameters are fuzzified into a power allocation factor, and thereby the driving system is controlled $[8,9]$. The common problem of rule-based strategies is that energy can only be allocated according to fixed rules without considering optimization, so the fuel consumption is relatively high.

The energy management of extended-range electric vehicles is essentially aimed to solve a multi-objective nonlinear optimization problem. Since the major objective is the minimization of systematic energy consumption, the energy control strategies based on global optimization and instantaneous optimization algorithms have been widely studied. As one global optimization algorithm, PMP constructs a Hamilton function, and when the Hamilton function reaches the minimum value under constraints, the target function is also minimized [10]. Dynamic programming (DP), another global algorithm, divides the whole working condition into several segments, and starting from the final state, reversely calculates the initial state and finally selects the controlling rule that makes the target function reach the minimum value as the optimal strategy [11,12]. However, both PMP and DP can get the optimal solution only when the whole driving cycles are known. Since the road conditions and driving behaviors are all unknown in real driving, global optimization algorithms are unfeasible in reality, but can be used as the benchmark for real-time energy management strategies. With the same hybrid electric vehicle model, Yuan compared DP and PMP and found their control effects were similar, but PMP was faster [13].

The energy management based on instantaneous optimization does not need any information about driving cycles and can be controlled in real-time according to the real driving conditions. The DP-developed stochastic dynamic programming (SDP) control strategies utilize DP algorithms to solve a number of working conditions, and apply the datasets as-obtained into neural network training [14]. In real applications, the real-time working conditions are substituted into the classifier to form energy distribution relations. Similarly, the artificial intelligent algorithms for energy management also include neural network control [15], particle swarm optimization [16], and genetic algorithm $[17,18]$. These Artificial Intelligence-strategies are faced with the problem of large amount of data training and too much computation in real-time operation. Model prediction control is aimed to model the controlled object, predict the output according to the vehicle state as-collected, optimize the predicted value, and input the resulting optimal energy allocation into the control system $[19,20]$. Borhan built an energy optimization control strategy based on model prediction and the simulations showed in the recycled working conditions of UDDS, the MPC control strategy reduced the oil consumption by $4.7 \%$ than the rule-based control strategy [21]. ECMS originating from PMP finds the minimum value through real-time solving the target function, and obtains the instantaneous energy distribution relation between batteries and APU [22]. However, the co-state of ECMS is constant 
and cannot well adapt to different working conditions in real tests. Thus, researchers have proposed adaptive-ECMS (A-ECMS) which is developed on the basis of ECMS. It can adjust the co-state value of Hamilton function in real time according to the operation state of the vehicle. The strategy can well adapt to the actual operation state and make the important vehicle performances reach the ideal value. Gu put forward an adaptive ECMS based on driving pattern recognition, and by identifying the information of working conditions, it adjusted the value of co-state to adapt to different working conditions [23]. Mahyar proposed to use GPS and ITS to predict the working conditions and built an A-ECMS control strategy based on reference SOC, so that the real SOC could decline along with the reference SOC curve [24]. According to the optimal co-state values under different driving conditions, Onori et al. plotted a co-state map and used SOC feedback to build a linear co-state function, which performed well in simulations $[25,26]$. Because A-ECMS has the characteristics of strong adaptability, good real-time performance and excellent control effect, it is selected as the energy management strategy of this paper.

\subsection{Motivation}

The objective of energy management strategy for an E-REB is to guarantee the dynamic performance of the E-REB during operation. Meanwhile the strategy ensures the SOC is always greater than the pre-set value and makes the final SOC close to the pre-set value, which not only protects the battery pack, but also fully uses the battery pack power.

In order to meet the above performance requirements, the co-state value needs to be adjusted in real time according to the vehicle status. The motivation for this is explained as follows: (1) to solve the optimal co-state value, the Hamilton function and co-state equation for E-REB should be developed based on PMP; (2) the co-state is affected by driving distance and working conditions, so in order to establish the relationship between the co-state and its influencing factors, the target driving cycles are needed, however, there is often a lack of TDCs in practice. In order to solve this problem, the Markov chain based generation technology is proposed; (3) by the way of making the fuel consumption close to the optimal control result, and the final SOC value is similar to the pre-set value, SOC reference curve should be reasonably designed; (4) taking the SOC deviation value as the independent variable, the co-state adaptive function is established by PI control technology; (5) also, the initial value of SOC also has a significant impact on the co-state, thus the change of co-state function should be considered under different initial values.

\subsection{Major Contribution}

During formulation of A-ECMS, the key is to build a co-state adaptive function, which fully considers the effects of initial SOC, driving distance, and working conditions on the co-state. Since this study was targeted at extended-range electric buses operating on fixed routes, the driving distance could be ignored. The co-state function consisting of fixed term and dynamic term was designed. To determine the fixed term, we first had to get the optimal initial co-state map, which could be determined by solving multiple target driving cycles by using PMP. However, during control strategy research, there are always few working conditions suitable for the exploitation goal, which largely hinders the determination of concrete control strategy parameters and the simulation of control effect. For this problem, a goal condition generation method based on Markov chain was proposed. The working condition was gradually generated through the formation of a highway and city transition probability matrix. Furthermore, with the ITS-acquired vehicle information, the average vehicle speed was determined by weighted averaging. Together with the initial SOCs of vehicles, the fixed terms could be determined by interpolating the co-state map. The role of the dynamic term was to make the SOC at termination be equal to the set value, so as to make full use of the electrical energy. It usually can be realized by following the reference SOC. However, the common SOC reference curve is a linear function of SOC and distance, which totally disobeys the ideal solution. A segmented SOC reference curve was put forward according to the optimal SOC changing curves under different initial 
SOC conditions solved using PMP. When the initial SOC was large or small, an exponential reference curve and a linear reference curve were selected, respectively, which better fitted the variation of the optimal SOC. With the introduction of PI, the deviation of the real SOC from the reference curve was regarded as the input to real-time adjust the co-state dynamic term, so as to follow the reference curve. During the research, A-ECMS was simulated under different working conditions and different initial SOCs. Results showed A-ECMS could meet the design requirements and was well adaptive.

\subsection{Outline}

This study is organized as below: Section 2 introduced the mathematical model and concrete parameters of E-REB; In Section 3, the PMP algorithm targeting at E-REB is developed and thus the co-state differential equation is achieved. In Section 4, Markov chain was used to generate the target driving cycles; In Section 5, the co-state map and SOC reference curve were acquired, and A-ECMS was designed through PI control. In Section 6, the A-ECMS, ECMS and PMP were simulated and comparatively analyzed under different target driving cycles and different initial SOCs. Finally, the conclusions are summarized in Section 7.

\section{E-REB Model Description}

\subsection{Powertrain and Parameters}

The extended-range electric bus studied here is the inter-city passenger car travelling between Changchun and Shenyang, and its powertrain structure is showed in Figure 1. The E-REB is powered by an auxiliary power unit (APU) and a battery pack. The APU consists of the engine and the generator, while the engine drives the generator to generate power. The output power of the APU is coupled with that of batteries, and the electric power is converted by a driving generator to machinery power, and then the main reducer and the differential further transfer the energy to the wheels. The parameters of main vehicle components are listed in Table 1.

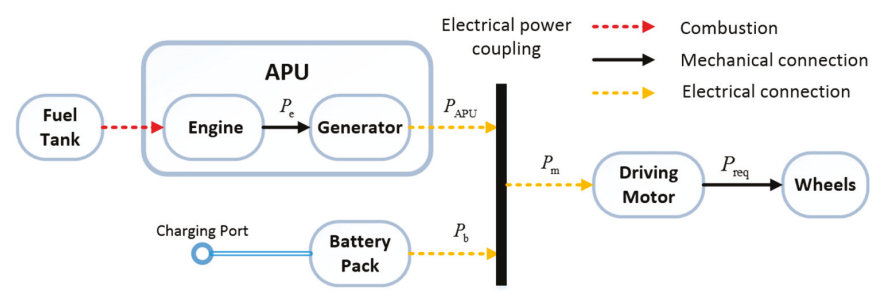

Figure 1. Extended-range electric bus powertrain.

Table 1. Main component parameters of E-REB.

\begin{tabular}{|c|c|c|}
\hline Component & Parameter & Value \\
\hline \multirow{3}{*}{ Engine } & Max speed & $4000 \mathrm{rpm}$ \\
\hline & Max power & $95 \mathrm{~kW}$ \\
\hline & Max torque & 311 Nm @ 2200 rpm \\
\hline \multirow{3}{*}{ Generator } & Max speed & $5000 \mathrm{rpm}$ \\
\hline & Rated power & $95 \mathrm{~kW}$ \\
\hline & Rated torque & 420 Nm @ 0 2160 rpm \\
\hline \multirow{3}{*}{ Battery pack } & Type & Lithium-ion \\
\hline & Capacity & $300 \mathrm{Ah}$ \\
\hline & Rated voltage & $576 \mathrm{~V}$ \\
\hline \multirow{3}{*}{ Driving motor } & Max speed & $2000 \mathrm{rpm}$ \\
\hline & Peak / Rated power & $180 / 120 \mathrm{~kW}$ \\
\hline & Peak/Rated torque & 1800/1200 Nm @ 0 955 rpm \\
\hline
\end{tabular}




\subsection{Key Component Model}

\subsubsection{Battery Model}

The battery pack consists of 156 battery cells in series, the total capacity of which is 300 Ah and the rated voltage is $576 \mathrm{~V}$. Then ten battery cells in series are selected and tested at ambient temperature $25^{\circ} \mathrm{C}$. The data of charge/discharge resistance, open circuit voltage (OCV) and SOC from the ten cells are fitted in Figure 2.

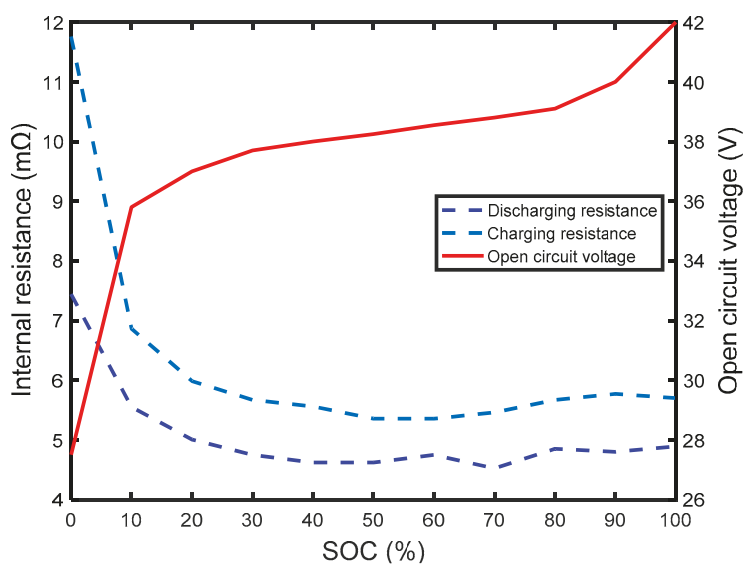

Figure 2. The internal resistance and OCV relative to SOC (10 cells).

The batteries are constructed using the internal resistance equivalent model (Rint model). At the discharge stage, the total power of the batteries $P_{b a t}$ is equal to the external power $P_{b}$ and internal resistance $R_{\text {int }}$ depletion. At this moment, the internal resistance is discharge resistance $R_{d c}$. At the charge stage, the external power $P_{b}$ charges the batteries, where the internal resistance is charging resistance $R_{c}$. The open circuit voltage $U_{o c v}$ and the internal resistance $R_{\text {int }}$ are both correlated with SOC. It is supposed the batteries are maintained at $25^{\circ} \mathrm{C}$, or namely the effect of temperature on internal resistance is ignored:

$$
P_{b a t}=U_{o c v}(S O C) \cdot I=I^{2} \cdot R_{i n t}(S O C)+P_{b}
$$

The current can be solved by Equation (1) as follows:

$$
I=\frac{U_{o c}(S O C)-\sqrt{U_{o c}(S O C)^{2}-4 P_{b} \cdot R_{i n t}(S O C)}}{2 R_{i n t}(S O C)}
$$

The changing rate of SOC can be expressed as follows:

$$
S \dot{O C}=d\left(\frac{Q_{b}-\int_{0}^{t} I d t}{Q_{b}}\right) / d t=-\frac{I}{Q_{b}}
$$

where $Q_{b}$ is the capacity of the battery pack.

\subsubsection{Driving Motor Model}

This extended-range electric bus is driven by a permanent magnet synchronous motor, and its efficiency map at the rated power is showed in Figure 3. The driving motor efficiency at given 
rotating speed and torque could be determined by the lookup-table interpolation method. When the driving motor works as a generator (braking energy recovery stage), the efficiency map is symmetrical. The efficiency of the driving motor is:

$$
\eta_{m}=\eta_{m}\left(n_{m}, T_{m}\right)
$$

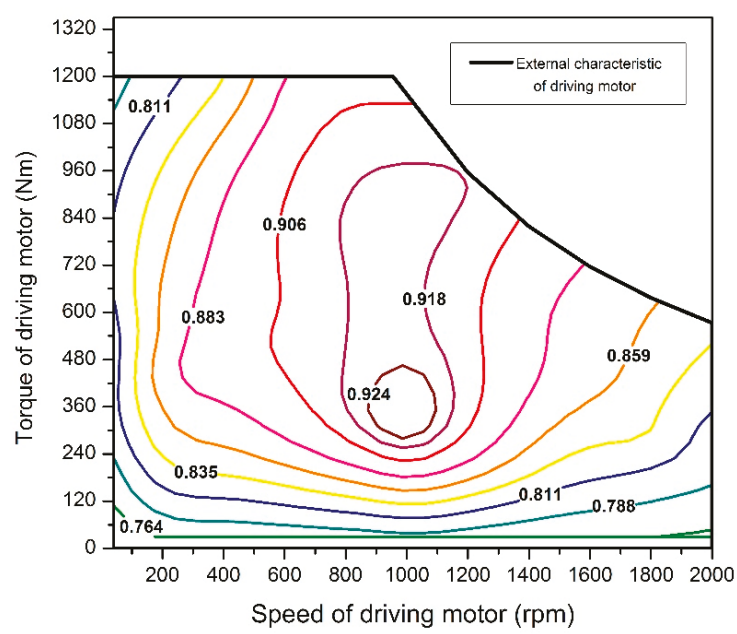

Figure 3. The efficiency map of driving motor.

The coupling total power $P_{m}$ supplies to the driving motor, the motor output power is delivered by the transmission to the wheels, so as to meet the driving demanded power $P_{\text {req }}$, and its power transfer is expressed as:

$$
P_{r e q}=P_{m} \cdot \eta_{m} \cdot \eta_{T}
$$

where $\eta_{T}$ is the transmission efficiency.

\subsubsection{APU Model}

As for an instantaneous energy management strategy, the optimal working point of each demanded power is computed offline in advance, which largely reduced the computational amount and favored the timeliness of the strategy. The optimal working point of APU is defined as to meet the demanded output power of APU and to minimize the fuel consumption. The optimal working points at different APU power values is connected, forming the optimal operation curve of APU. Since the output power and fuel consumption rate of APU are affected jointly by the engine and the generator, the characteristics of these two parts should be integrated to solve the optimal working curve. The output power of APU was expressed as:

$$
P_{A P U}=P_{e} \cdot \eta_{g}\left(n_{g}, T_{g}\right)=P_{e} \cdot \eta_{g}\left(n_{e}, T_{e}\right)
$$

where $\eta_{g}$ is the generator efficiency, $n_{e}$ is the rotation speed and $T_{e}$ is the torque. The fuel consumption rate of APU was calculated as:

$$
b e=b e\left(n_{e}, T_{e}\right)
$$

The universal characteristics and APU optimal operation curve of the engine are shown in Figure 4. Unlike the optimal working curve of the engine, that of the APU is integrated the generator efficiency. The minimum fuel consumption of each disperse power was plotted in Figure 5. Since the maximum 
output power of APU is $85 \mathrm{~kW}$, the fuel consumption rate is very low when the output power of APU is $40-50 \mathrm{~kW}$. Since the optimal working point of each discrete power is only and known, the rotating speed and torque of a working point can be determined if the allocation power of APU in the energy management algorithm is known.

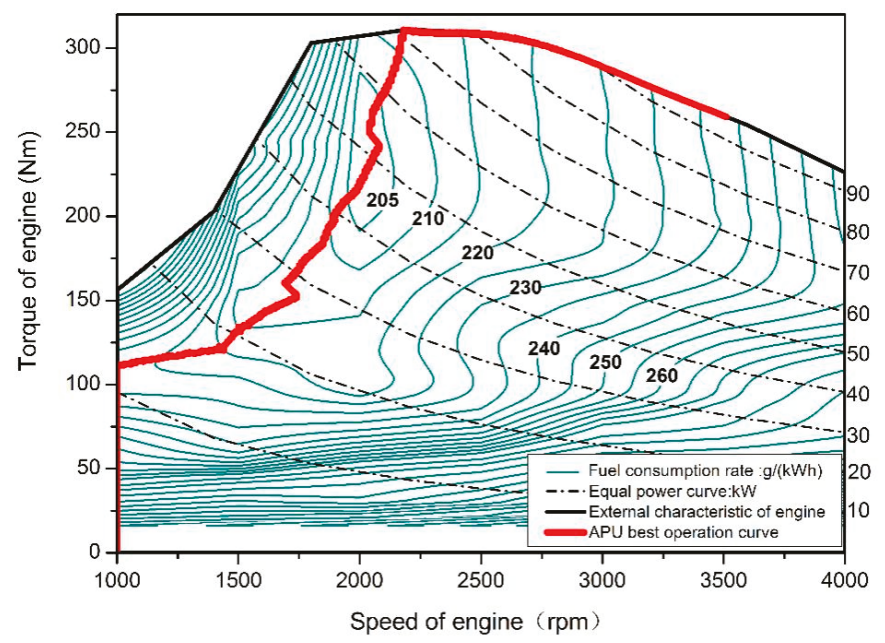

Figure 4. The APU best operation curve (red) and engine efficiency map.

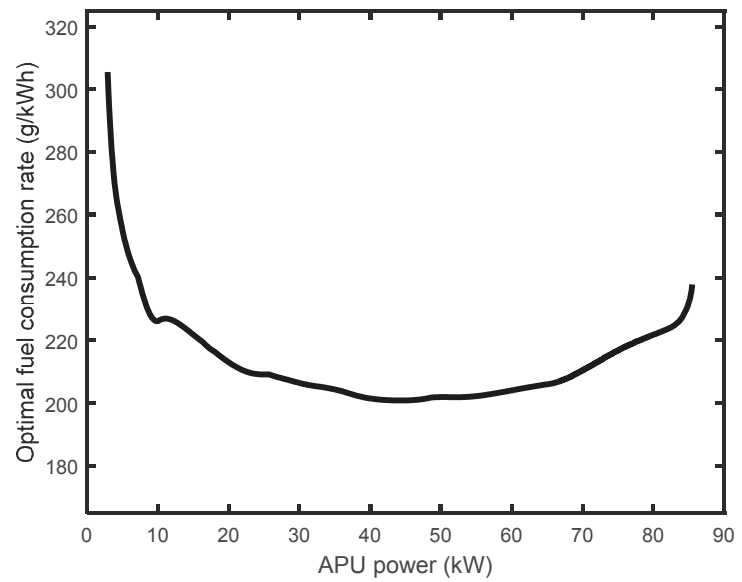

Figure 5. Relationship between APU power and fuel consumption rate in best operation curve.

\subsection{Dynamic Model}

According to the driving dynamic equation of a vehicle, the driving force can be expressed as:

$$
\begin{gathered}
F_{t}=m g \cdot f+\frac{C_{D} A v^{2}}{21.15}+\sigma m \frac{d v}{d t} \\
P_{r e q}=\frac{v}{3600} \cdot F_{t}
\end{gathered}
$$


The symbols and their values are shown in Table 2.

Table 2. E-REB model parameters.

\begin{tabular}{ccc}
\hline Symbol & Parameter & Value \\
\hline$m$ & Vehicle mass & $11,718 \mathrm{~kg}$ \\
$f$ & Rolling resistance coefficient & 0.01 \\
$C_{D}$ & Air resistance coefficient & 0.7 \\
$A$ & Windward area & $8.6 \mathrm{~m}^{2}$ \\
$\sigma$ & Rotational inertia coefficient & 1.1 \\
$i_{o}$ & Main ratio & 4.875 \\
$r$ & Rolling Radius & $0.511 \mathrm{~m}$ \\
$\eta_{T}$ & Transmission efficiency & 0.9 \\
\hline
\end{tabular}

\section{PMP Algorithm Formulation}

Since PMP is the basis of ECMS, complete driving cycles are needed to solve the optimal control rate under the constraint conditions. The goal of the optimal energy management of a hybrid electric vehicle is that: the power of APU and the battery pack is allocated to minimize the fuel consumption while meeting the driver's required power $P_{\text {req }}$ [27]. For extended-range vehicles, since the APU and transmission system are decoupled, the working points of APU can be randomly selected under the premise of meeting the constraint conditions. At the end of driving, $\mathrm{SOC}$ reached the set minimum value $S C_{f}$ so as to fully utilize electric energy and reduce oil consumption. During the whole driving process, $\mathrm{SOC}$ is maintained at the reasonable range $\left[\mathrm{SOC}_{f}, \mathrm{SOC}_{\text {initial }}\right]$ so as to prolong the service life of batteries. Meanwhile, the output power of APU should never surpass the demanded power and thereby enter the mode of APU charging batteries, which avoided the secondary energy transfer and increased the energy use rate. Taken together, the target function and constraint conditions of energy management of E-REB can be expressed as:

$$
\begin{gathered}
\text { Minimize } J(t)=\int_{0}^{t_{f}} \dot{m}_{f}(u(t)) \cdot d t=\int_{0}^{t_{f}} \frac{b e(u(t)) \cdot u(t)}{3600} \cdot d t \\
\text { subject to SOCC }(t)=-\frac{I(t)}{Q_{b}} \\
\operatorname{SOC}\left(t_{0}\right)=S O C_{\text {initial }}, \operatorname{SOC}\left(t_{f}\right)=\operatorname{SOC}_{f} \\
\operatorname{SOC}(t) \in\left[S O C_{f}, S_{\text {SOC }} \text { initial }\right] \\
u(t)=P_{\text {APU }}(t) \in\left[P_{\text {min }}, P_{\max }\right] \text { and } u(t) \leq P_{\text {req }}(t)
\end{gathered}
$$

Here $m_{f}$ is fuel consumption rate $(\mathrm{g} / \mathrm{s}) ; u(t)$ is the APU allocation power, as the control variable, which varies within $[0,85] \mathrm{kW}$ according to Section 2.2.3. SOC is a state variable and its termination value is usually $\mathrm{SOC}_{f}=30 \%$. The energy management Hamilton function is:

$$
H=\frac{b e(u) \cdot u}{3600}+\lambda \cdot S \dot{O C}
$$

where $\lambda$ is the co-state, which is time-variant during the solving process of PMP and can be expressed as:

$$
\dot{\lambda}=-\frac{\partial H}{\partial S O C}=-\lambda \cdot \frac{\partial S O \dot{C}}{\partial S O C}
$$


Since the partial derivatives in this equation cannot be directly solved, it can be further transformed according to Equation (3):

$$
-\frac{\partial S \dot{O C}}{\partial S O C}=\frac{1}{Q_{b}} \cdot \frac{\partial I}{\partial U_{o c}(S O C)} \cdot \frac{\partial U_{o c}(S O C)}{\partial S O C}
$$

According to the characteristics of battery pack in Figure 2, when $S O C_{f}=30 \%$, the charge/discharge resistance nearly does not change with voltage. Namely under the restrictions of SOC, the battery internal resistance can be regarded as constant:

$$
\frac{\partial R_{i n t}}{\partial S O C}=\frac{\partial U_{o c}}{\partial S O C} \cdot \frac{\partial R_{i n t}}{\partial U_{o c}} \approx 0
$$

On this basis, the partial derivative of Equation (2) over $U_{o c}$ can be determined:

$$
\frac{\partial I}{\partial U_{o c}(S O C)}=-\frac{I}{\sqrt{U_{o c}(S O C)^{2}-4 \cdot P_{b} R_{i n t}}}
$$

The partial derivative of voltage over SOC can be determined from the battery characteristics in Figure 2, marked as $k(S O C)$. Taken together, the state equation of the co-state can be expressed as:

$$
\dot{\lambda}=\frac{\lambda}{Q_{b}} \cdot \frac{\sqrt{U_{o c}(S O C)^{2}-4 \cdot P_{b} R_{i n t}}-U_{o c}(S O C)}{2 R_{i n t} \sqrt{U_{o c}(S O C)^{2}-4 \cdot P_{b} R_{i n t}}} \cdot k(S O C)
$$

After the state equations of SOC and $\lambda$ are determined, the PMP algorithm can be solved using the Shooting method [28]. However, for PMP as a global optimization algorithm, all information about driving cycles should be acquired in advance, and thus, PMP cannot be directly applied in a real environment. The instantaneous optimization algorithms ECMS and A-ECMS based on PMP will be introduced in Section 5. The driving cycle generation method based on Markov chain will be introduced in Section 4. With the PMP algorithm, the optimal initial value of $\lambda$ was solved, forming the optimal initial $\lambda$ maps at different vehicle speeds and different initial SOCs.

\section{Markov Chain Based Target Driving Cycles Generation}

During research on control strategies, usually real driving cycles suitable for the development goal are insufficient. For instance, this study on the energy management strategies for the fixed route from Changchun to Shenyang required similar simulation conditions of several driving cycles. However, the shortage of driving cycles largely hindered the determination of concrete control strategy parameters and the simulation of control effect. To achieve this goal, we put forward a target driving cycle generation method based on Markov chain. The Markov chain method is widely used in speed prediction. Xie adopted it to forecast velocity sequences at every current state, with post-processing algorithms to moderate fluctuations of the prediction results like average filtering [29]. In addition to forecasting the vehicle speed, Liu forecasted the demand power accurately through a Markov chain approach. According to the current vehicle running state, the probability transfer matrix is used to estimate the demand power at the next time [30,31]. In fact, the speed prediction is intrinsically linked to the demand power prediction. According to the vehicle dynamics equation, the conversion of vehicle speed and demand power can be performed. The above methods all use Markov chain for short-term prediction. In contrast, this paper uses it to generate complete driving cycles. The main purpose is to perform optimal co-state calculations, reference curve solving and verification, rather than real-time forecasting. Based on the collected data and the data of typical driving cycles, a probability transition matrix is formed, and thereby the simulated driving cycles close to real data are generated. 
During a Markov process, the future state and the past state of the system are mutually independent. The current state of the system is known, and the system state at any time is related with the current state, but not with the past state [32]. Then the vehicle speed $v_{k+1}$ at step $k+1$ of discrete time is decided by the vehicle speed $v_{k}$ at step $k$ of discrete time. Thus, the complete driving cycle can be generated stepwise starting from the initial status $v_{1}=0 \mathrm{~km} / \mathrm{h}$. The Markov status space consists of the discrete vehicle speed and acceleration; let the discrete vehicle speed be $v_{i}(i=1,2, \Lambda, p)$, the discrete acceleration be $a_{j}(i=1,2, \Lambda, q)$, and the acceleration at step $k+1$ be $a_{k+1}$. Then starting from the current state $v_{k}=v_{i}$, the conversion probability reaching the next state $a_{k+1}=a_{j}$ can be expressed as:

$$
p\left(a_{k+1}=a_{j} \mid v_{k}=v_{i}\right)=p_{i j}=\frac{N_{i j}}{\sum_{j=1}^{q} N_{i j}}
$$

where $p_{i j}$ is the transition probability from the current vehicle speed $v_{i}$ to the acceleration $a_{j}$ of the next state; $N_{i j}$ is the number of occurrences of the event where the current vehicle speed $v_{i}$ reaches the acceleration $a_{j}$ of the next state; $\sum_{j=1}^{q} N_{i j}$ is the total number of event occurrences of the current vehicle speed $v_{i}$ reaching the acceleration of the any next state.

Then the target driving cycle from Changchun to Shenyang is analyzed. Using Google Map, the driving cycle from Changchun to Shenyang can be generally divided into three segments: (1) from city of Changchun to the highway junction: $5.4 \mathrm{~km}$; (2) highway: $277 \mathrm{~km}$; (3) from the highway junction to the city of Shenyang: $12.6 \mathrm{~km}$. Of them, (1) and (3) correspond to city conditions, while (2) is a highway condition. Thus, the probability transition matrix should be divided into a city-matrix and a highway-matrix, to separately calculate the data statistics and matrix generation, respectively. The city road collected data, representing driving cycles such as FTP75 and ChinaCityBus are used to generate the city-matrix; for the highway collected data, representative driving cycles such as US_SC03 and Highway are used to generate the highway-matrix. The transition probability maps are shown in Figures 6 and 7 .

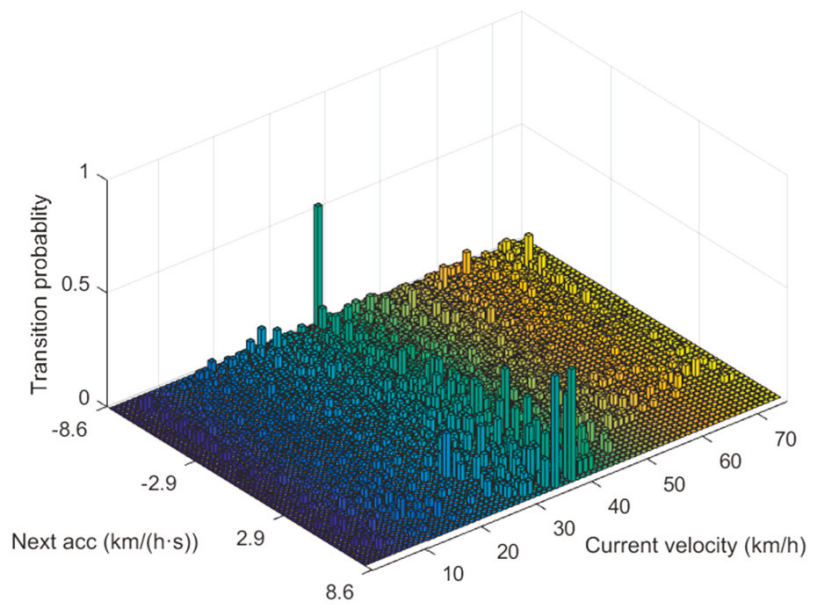

Figure 6. Transition probability map of city conditionz. 


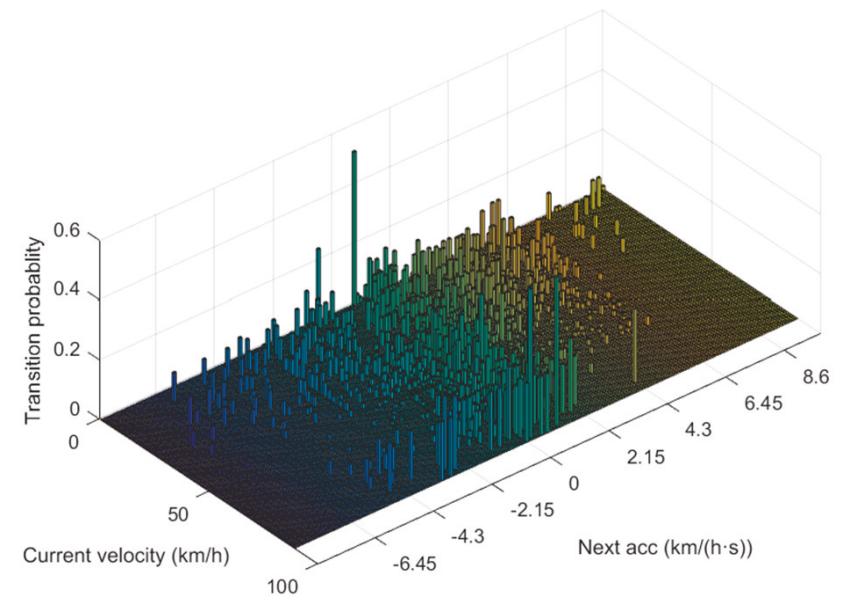

Figure 7. Transition probability map of highway conditions.

After the probability transition matrix is determined and under the known current vehicle speed, the acceleration at the next state is determined according to sampling by probability, and the vehicle speed at the next state is further determined:

$$
v_{k+1}=v_{k}+a c c_{k+1} \cdot d t
$$

Taken together, the driving cycles are generated segment-wise by using the Markov probability transition matrix. To simulate the variation of each driving distance during real driving, a random error $\delta \in[0,5 \%]$ is introduced. When each segment of driving cycle is generated, the effect of random error is considered. Based on the above conditions, in Figure 8, 10 simulated driving cycles (Cycle 01 10) are formed and used to determine the energy management strategy parameters and validate the control effect. The specific statistics of driving cycles and the solution results of PMP will be introduced in Section 5, and the solving process of PMP will be illustrated in Section 6.
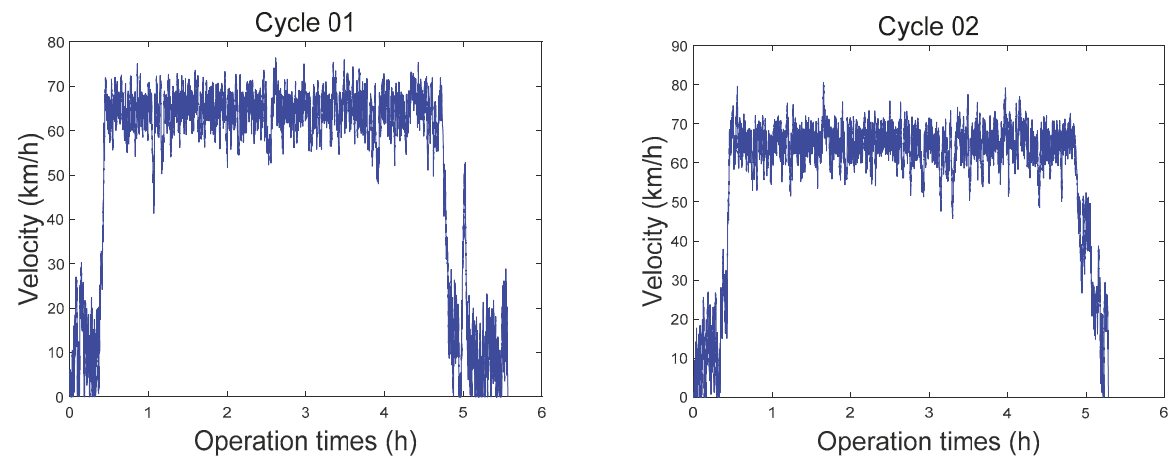

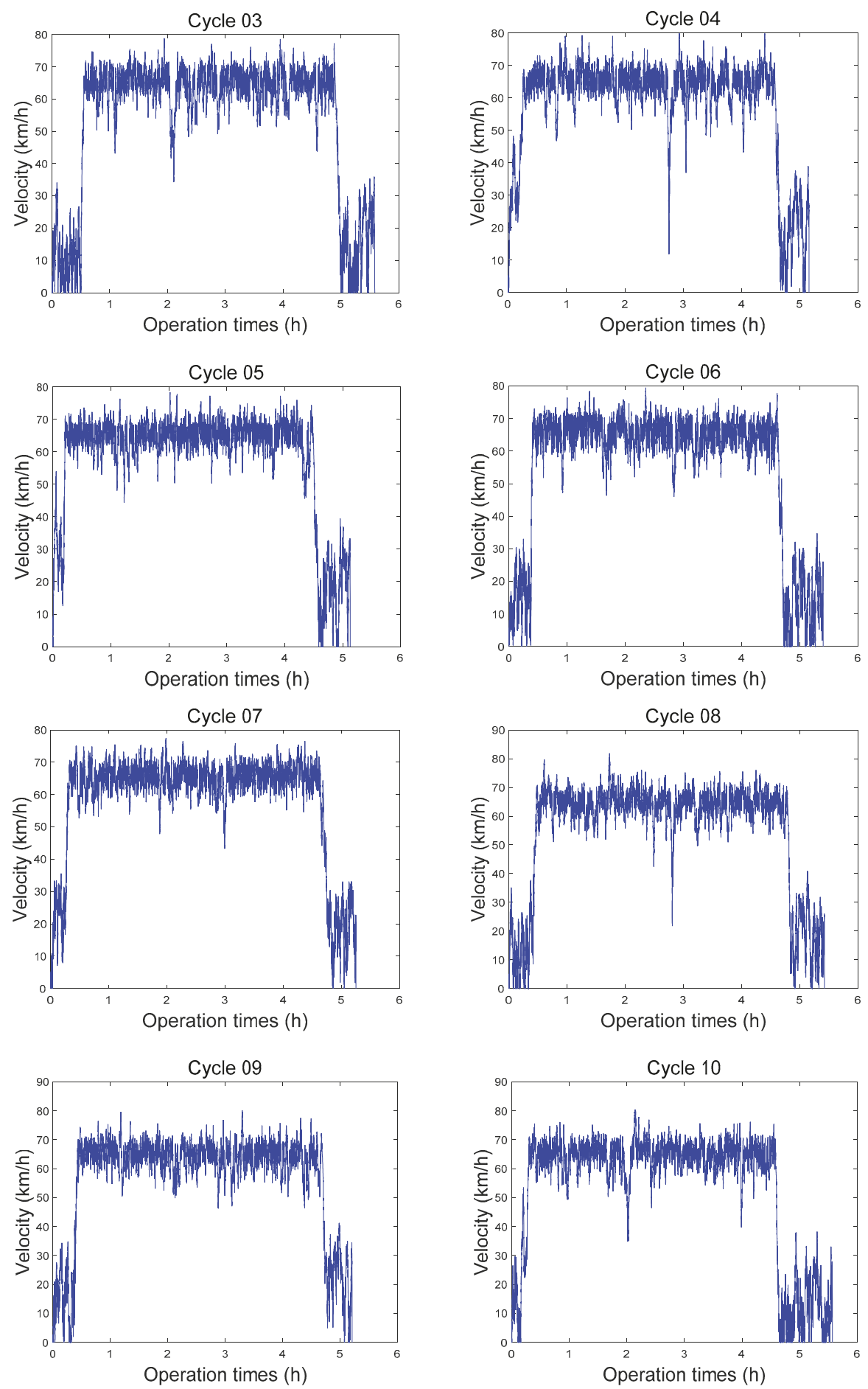

Figure 8. 10 target driving cycles generated by Markov chain. 


\section{Adaptive-ECMS}

\subsection{Co-State Map Generated for ECMS}

ECMS is an extension of PMP and an instantaneous optimization algorithm that does not need the information of whole driving cycles. Based on Equation (15), ECMS continually finds the optimal solution at each single step $k$. The target functions at stage $k$ can be expressed as:

$$
J(k)=\frac{b e(u(k)) \cdot u(k)}{3600}+\lambda(k) \cdot \operatorname{SOC}(k)
$$

In the basic ECMS, the value of co-state is equivalent to the optimal initial value $\lambda_{0}$ of the co-state, and thus, this strategy is also called Constant-ECMS (C-ECMS). $\lambda_{0}$ is mainly affected by the driving distance, the initial value of SOC, and the working conditions [33]. In this study, since the driving route is fixed, the effect of driving distance can be ignored.

The 10 as-generated driving cycles are used in PMP training; let the initial value of SOC be $S O C_{\text {initial }}$, and the average vehicle speed $v_{\text {average }}$ is used to characterize the working condition. The maps of $\lambda_{0}$ with $S O C_{\text {initial }}$ and $v_{\text {average }}$ are plotted. During the generation of driving cycles, the error $\delta$ of each driving distance is considered, which would affect the accuracy of the map. Thus, the smallest distance $d_{\min }$ of the 10 driving cycles is selected for equidistance processing; the data when the driving distance is $d_{\text {min }}$ under all driving cycles are used into PMP training. The information of PMP training under the 10 driving cycles after equidistance are summarized in Table 3. The table is converted to 3D maps (Figure 9) for ECMS interpolation to determine $\lambda_{0}$. Due to the limitation of training driving cycles, the average vehicle speed coverage is very narrow, so $\lambda_{0}$ in the parts beyond the range is solved through outward interpolation.

Table 3. $\lambda_{0}$ solving results by using the equal distance process.

\begin{tabular}{|c|c|c|c|c|c|c|c|}
\hline \multirow{2}{*}{ Number } & \multirow{2}{*}{$\begin{array}{l}v_{\text {average }} \\
(\mathrm{km} / \mathrm{h})\end{array}$} & \multicolumn{6}{|c|}{$S O C_{\text {initial }}$} \\
\hline & & 1.00 & 0.95 & 0.90 & 0.85 & 0.80 & 0.75 \\
\hline Cycle09 & 51.6853 & -33.399 & -34.5494 & -35.4932 & -36.2315 & -36.9346 & -37.7542 \\
\hline Cycle08 & 51.9578 & -33.407 & -34.5503 & -35.4777 & -36.229 & -36.9626 & -37.8124 \\
\hline Cycle01 & 53.2634 & -33.413 & -34.5773 & -35.5221 & -36.2579 & -36.9967 & -37.8462 \\
\hline Cycle03 & 54.8937 & -33.522 & -34.7074 & -35.6664 & -36.4219 & -37.2319 & -38.2886 \\
\hline Cycle10 & 55.0385 & -33.496 & -34.6745 & -35.6334 & -36.3932 & -37.1821 & -38.2179 \\
\hline Cycle05 & 58.8617 & -33.530 & -34.7165 & -35.689 & -36.429 & -37.2883 & -38.4523 \\
\hline Cycle04 & 59.6607 & -33.559 & -34.7494 & -35.7163 & -36.4997 & -37.339 & -38.4693 \\
\hline Cycle02 & 60.1133 & -33.639 & -34.8513 & -35.8389 & -36.6909 & -37.6289 & -38.9175 \\
\hline Cycle06 & 61.6337 & -33.654 & -34.9023 & -35.9111 & -36.7447 & -37.7832 & -39.0079 \\
\hline Cycle07 & 62.5894 & -33.663 & -34.888 & -35.867 & -36.6936 & -37.6759 & -38.949 \\
\hline
\end{tabular}

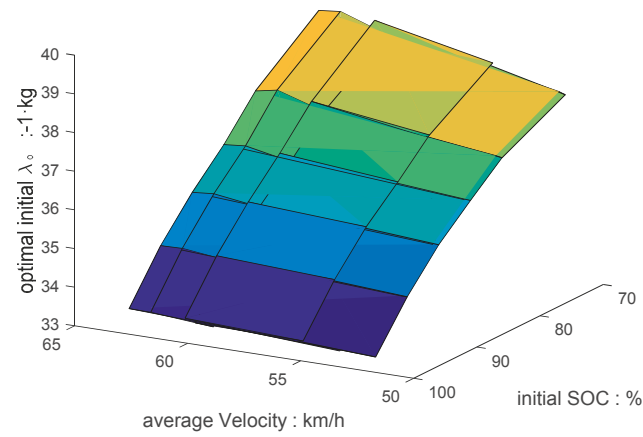

Figure 9. $\lambda_{0}$ map solving by equal distance process. 


\subsection{A-ECMS and SOC Reference Curve}

Under the whole driving cycle, $\lambda$ is constant and invariable during the solving process of C-ECMS, but $\lambda$ is continually updated according to Equation (20) during PMP training. This means $S O C_{\text {max }} \neq S O C_{f}=0.3$ may occur at the end of the cycle. When $S O C_{k m a x}>S O C_{f}, \lambda$ is small and the electricity is not completely used, leading to excessive fuel consumption; when $S O C_{k m a x}<S O C_{f}$, $\lambda$ is large and the electricity is excessively used, which affects the service life of batteries. Thus, Adaptive-ECMS (A-ECMS) has been widely studied, so as to make $S O C_{k m a x}$ at the end of working conditions close to the preset value. As for A-ECMS, the co-state $\lambda$ is varying during the operation, and the adaptive $\lambda$ as-designed can be expressed as:

$$
\left\{\begin{array}{l}
\lambda(t)=\lambda_{0}+k_{p} \cdot \Delta S O C(t)+k_{i} \cdot \int_{0}^{t} \Delta S O C(t) \cdot d t \\
\Delta S O C(t)=S O C(t)-S_{\text {SOC }}(t)
\end{array}\right.
$$

where $k_{p}$ and $k_{i}$ are the adjustment coefficients of the proportion step and the integral step, and $S O C_{r e f}$ is the SOC reference curve. By following the reference curve, the $S O C_{k m a x}$ at the end of driving cycle is close to the preset value $S O C_{f}$ During the operation, the SOC is higher than the reference curve, indicating the electricity use is little and $\lambda$ should be enlarged, so that the energy management strategy is leaning to electricity use; and vice versa.

The commonly-used SOC reference curve is SOC-distance linear curve [34]. However, in real situations, the SOC-distance is not completely linear, and thus this reference curve is blind to some extent. A segmented SOC reference curve is designed according to the changing curves under different initial SOCs of 10 cycles solved using PMP:

$$
\begin{gathered}
S O C_{\text {ref }}(t)= \begin{cases}S O C_{\text {initial }} \cdot e^{-b \cdot d_{\text {now }}(t)} & \text { if } S O C_{\text {initial }} \geq 0.85 \\
S O C_{\text {initial }}+\frac{d_{\text {now }}(t)}{D} \cdot\left(S O C_{\mathrm{f}}-S O C_{\text {initial }}\right), & \text { if } S O C_{\text {initial }}<0.85\end{cases} \\
b=\frac{1}{D}\left(\ln \left(S O C_{\text {initial }}\right)-\ln \left(S O C_{\mathrm{f}}\right)\right) \\
d_{\text {now }}(t)=\int_{0}^{t} v(t) d t
\end{gathered}
$$

where $D$ is the total driving distance $(\mathrm{km})$, and $d_{\text {now }}(t)$ is the driven distance $(\mathrm{km})$.

With different initial SOCs, the optimal changing curves of the 10 driving cycles and the SOC reference curves are determined (Figure 10). It should be noted during each solving process, the 10 whole driving cycles are used, rather than the cycles after equalizing them. The SOC changing curve of the optimal solution is arc-shaped (Figure 10). When SOC is large, $\lambda$ is large, and the energy management strategy is leaning to electricity use, so the declining slope of SOC is very large. As the driving distance increased, $\lambda$ was updated according to Equation (20) and thus declined, so the energy management strategy is leaning to oil use, and thus the declining slope of SOC gradually decrease. Moreover, as the initial value of SOC decreased, the radian of SOC changing curve declined and when $S O C_{\text {initial }}$ is $<0.85$, it is close to a line. This is because the whole vehicle does not have enough electricity, leading to the relatively small initial value $\lambda_{0}$ of optimal co-state from the solving process. The strategy is leaning to oil use compared to the case of large initial SOC, so the declining slope of SOC at the early stage is small. Taking the above simulated data and the analysis together, for A-ECMS with initial SOC $\geq 0.85$ or $<0.85$, an exponential reference curve or a linear reference curve are used, respectively. Together with Figure 10, it is clear that the segmented reference curve of SOC has very good fitting results 


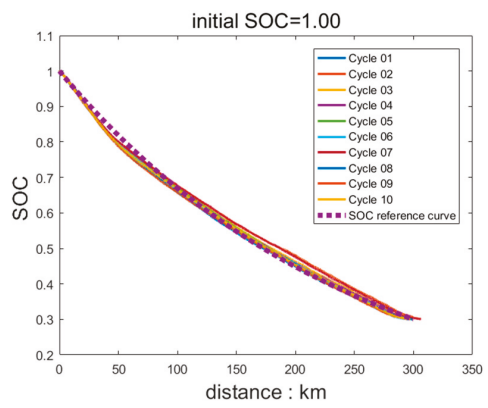

(a)

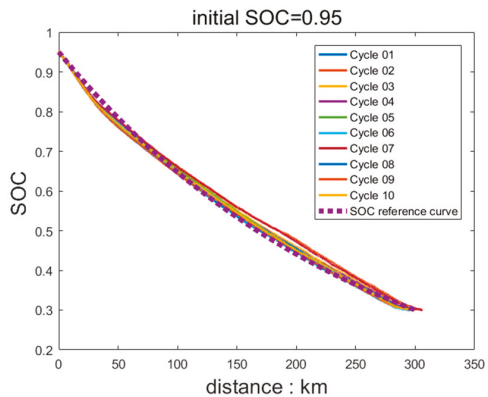

(c)

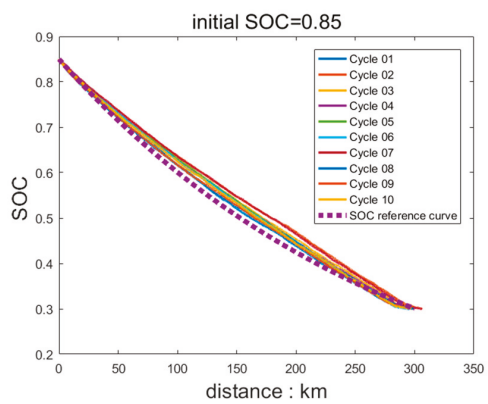

(e)

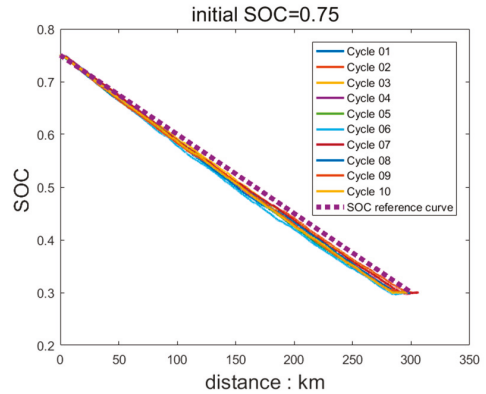

(g)

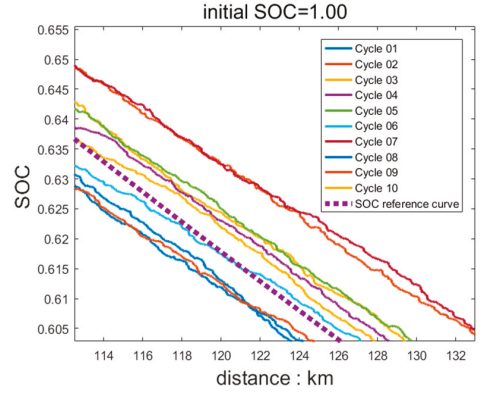

(b)

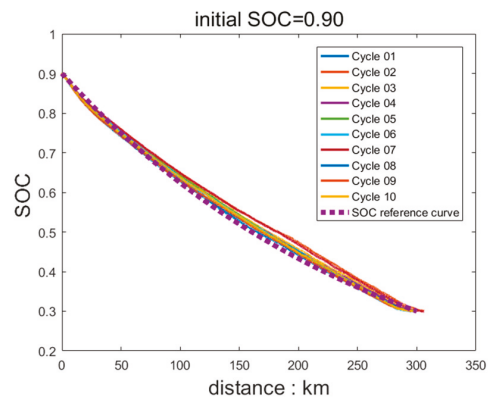

(d)

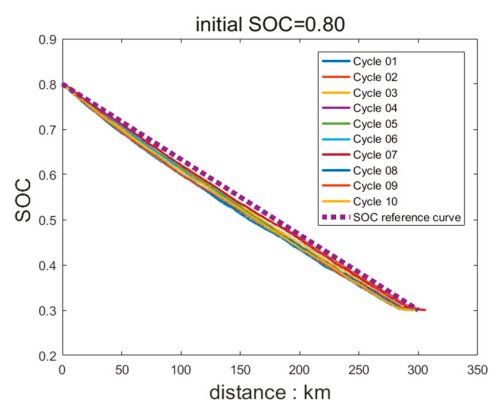

(f)

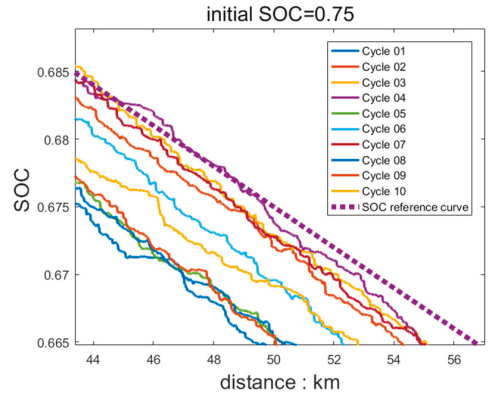

(h)

Figure 10. The SOC change curve of 10 cycles under optimal solution by PMP in different initial SOC: Among them, (b) and (h) are (a) and (g) partially enlarged image, respectively. 


\subsection{Average Velocity Obtained from Traffic Information}

The adaptive co-state function (24) can be divided into a dynamic term and a constant term. Specifically, the PI-adjusted part is the dynamic term and is variable during the driving process; while the constant term $\lambda_{0}$ can be determined from the interpolation of the map. Thus, the $S O C_{\text {initial }}$ and average vehicle speed $v_{\text {average }}$ are needed to determine $\lambda_{0}$. In particular, the initial SOC can be determined from the battery management system. The average vehicle speed can be determined by statistically analyzing the information of the driving vehicle, with the use of ITS.

The route from Changchun to Shenyang is already divided as mentioned above. The three sections from Changchun city to the highway junction, highway, and from the highway junction to Shenyang city are marked as 1,2 and 3 , respectively. The distances of three sections are marked as $d_{1}, d_{2}$ and $d_{3}$ with the total distance of $D$. To calculate the average vehicle speed $\bar{v}_{i} \in\{1,2,3\}$ of section $i$, we have to calculate the average of ITS-acquired vehicle data of section $i$ :

$$
\bar{v}_{i}=\frac{1}{N} \sum_{j=1}^{N} v_{i, j}
$$

where $N$ is the number of vehicles in section $i$, and $v_{i, j}$ is the vehicle speed of vehicle $j$ in section $i$. It should be noted since most of the vehicles recorded by the ITS are passenger vehicles and their speeds may surpass that of the extended-range bus, the data disobeying the driving requirements of the bus should be excluded. Furthermore, the average vehicle speed at each section is weight-averaged to get the total average vehicle speed:

$$
\begin{gathered}
\bar{v}=\sum_{i=1}^{3} \bar{v}_{i} \cdot \omega_{i} \\
\left\{\begin{array}{l}
\omega_{i}=\frac{d_{i}}{D} \\
\omega_{1}+\omega_{2}+\omega_{3}=1
\end{array}\right.
\end{gathered}
$$

where $\omega_{i}$ is the weight of section $i$, which reflects the effect of section distance on the total average vehicle speed. Since the highway section is the longest, the total average vehicle speed is also very large.

\subsection{Architecture of A-ECMS}

As a summary of the above flowchart, the architecture of A-ECMS is shown in Figure 11. Firstly, data of representative driving cycles and collected data are used to generate a city-highway probability transition matrix, and with Markov chain, the target driving cycles are formed. The equidistance driving cycles are solved by PMP, forming the optimal co-state initial values under each driving cycles with different initial SOC, forming a map. In real-time operation, the ITS data are weight-averaged, and together with the initial SOCs provided by the BMS system, $\lambda_{0}$ is determined from interpolation. A vehicle provides information of real-time driving distance, demanded power, SOC and its changing rate. With the segmented SOC reference curve established from real-time follow-up as the goal, the adaptive $\lambda$ is adjusted through PI control. Within the range of constraint conditions, the optimal working curve of APU is traversed so as to solve the minimum value of the target function J. Finally, the optimal APU and the battery allocation power are determined, and substituted into the whole-vehicle model for control. 


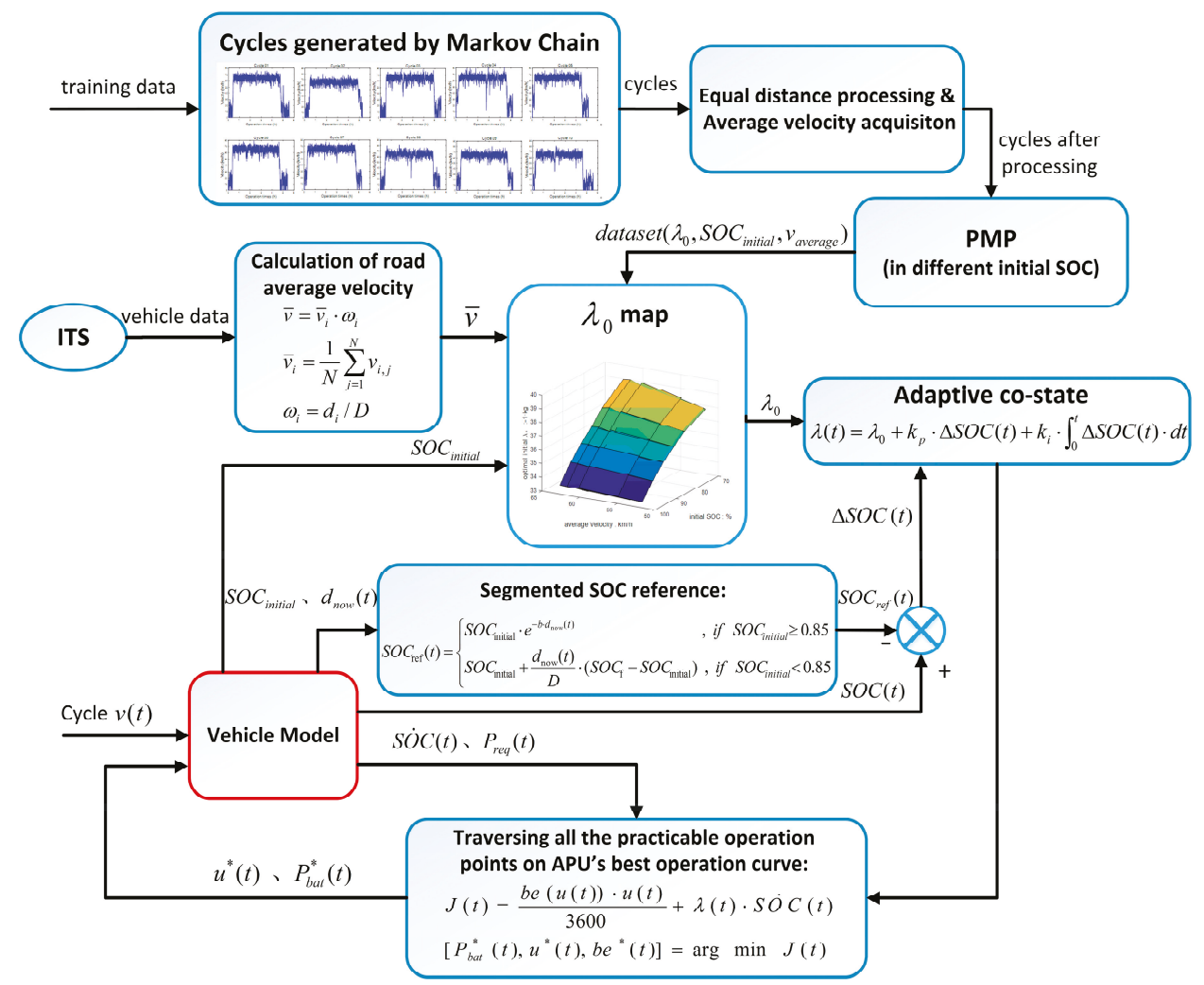

Figure 11. The architecure of A-ECMS.

\section{Validation and Discussion}

\subsection{Optimal Initial Value of Co-State Solved by PMP}

With PMP, for the 10 driving cycles as-generated, the optimal initial values of co-state are solved under different initial SOCs. With the solutions of equidistance driving cycles (Table 3), the map of $\lambda_{0}$ is plotted. In this Section, the complete information of driving cycles are utilized to compare PMP, C-ECMS and A-ECMS. The initial co-state to be substituted into PMP is gradually adjusted via the Shooting method until the difference between the final SOC and the preset value after the PMP meets the required precision. The initial co-state from the final searching is selected as the optimal initial value. With Cycle 10 for instance, the optimal initial co-state is solved at the initial SOC of 1.0, 0.95, 0.9, $0.85,0.8$ and 0.75 (Figure 12). 


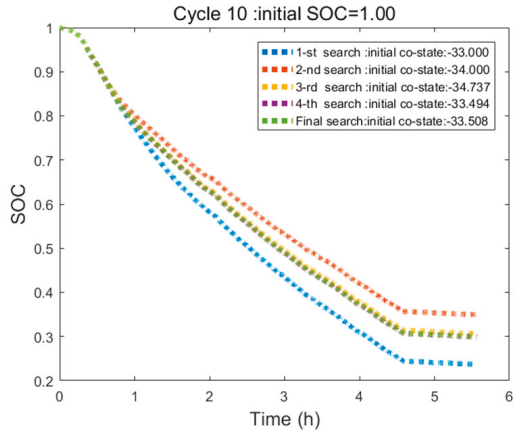

(a)

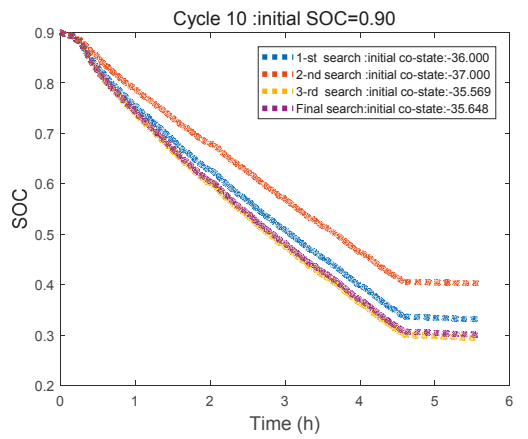

(c)

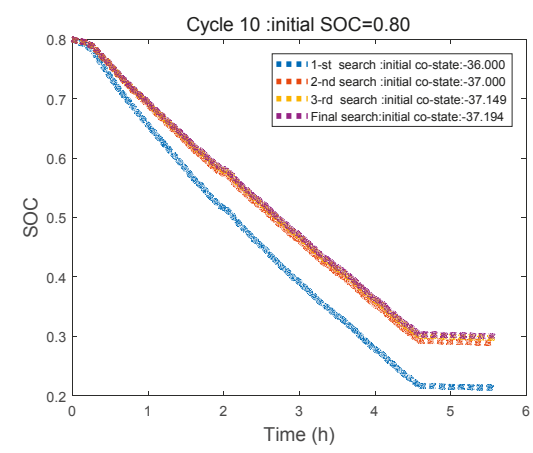

(e)

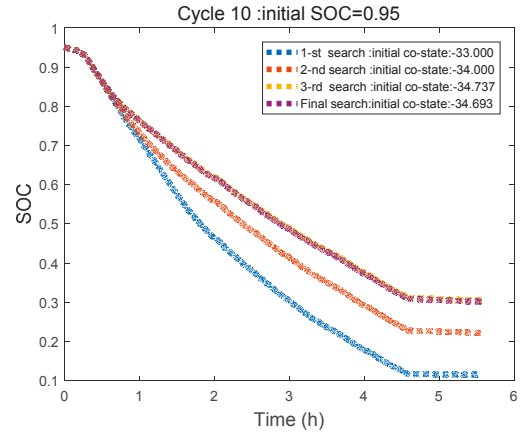

(b)

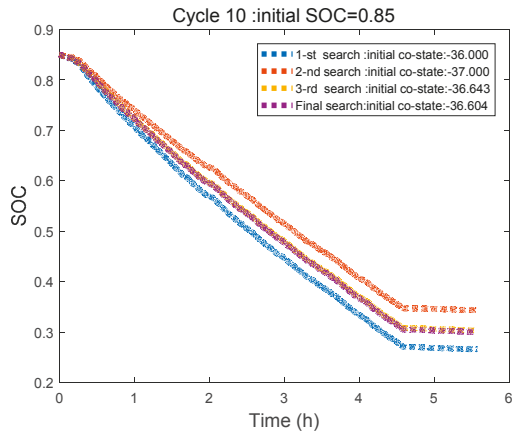

(d)

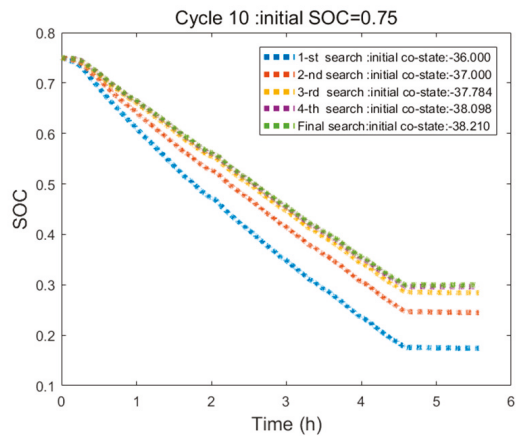

(f)

Figure 12. The $\lambda_{0}$ search process of Cycle 10 under different initial SOC.

The initial value for the first searching can be selected empirically, and the value selected closer to the real value would make the searching faster. In this study, when the initial SOC is 1.0 or 0.95 , the initial value of the first searching is $-33 \mathrm{~kg}$; under other conditions, it is $-36 \mathrm{~kg}$. Similarly, the optimal initial co-state under different initial values of SOC for the 10 driving cycles is solved (Table 4). During the PMP solving process, the co-state continuously varied according to the updating Equation (20). When the different initial SOCs under the 10 driving cycles are outputted, the co-state changing curve under the optimal initial co-state is selected (Figure 13). Clearly, the co-state gradually declined with time. The changing range of co-state in (a) is about $-3.5 \mathrm{~kg}$, but is $-1.5 \mathrm{~kg}$ in (f), 
indicating with a smaller initial SOC, the changing amplitude of co-state decreases. At the initial stage of (a), the changing rate of co-state is very large, but at the distance of 40-50 km, an evident turning point appeared and the changing rate of co-state decreased. This is because the electricity consumption led to the platform stage of SOC, as shown in the $20 \%-80 \%$ stage of SOC in Figure 2. At this moment, the changing degree of voltage $U_{o c}$ with SOC is smaller, or namely the $k(S O C)$ of updating equation and thereby the changing rate decreased.

Table 4. $\lambda_{0}$ solving results and statistical data of 10 cycles.

\begin{tabular}{|c|c|c|c|c|c|c|c|c|c|c|c|}
\hline \multirow{2}{*}{ Number } & \multirow{2}{*}{$\begin{array}{l}v_{\text {average }} \\
(\mathrm{km} / \mathrm{h})\end{array}$} & \multirow{2}{*}{$\begin{array}{c}d_{1} \\
(\mathrm{~km})\end{array}$} & \multirow{2}{*}{$\begin{array}{c}d_{2} \\
(\mathrm{~km})\end{array}$} & \multirow{2}{*}{$\begin{array}{c}d_{3} \\
(\mathrm{~km})\end{array}$} & \multirow{2}{*}{$\begin{array}{c}D \\
(\mathrm{~km})\end{array}$} & \multicolumn{6}{|c|}{$S O C_{\text {initial }}$} \\
\hline & & & & & & 1.00 & 0.95 & 0.90 & 0.85 & 0.80 & 0.75 \\
\hline Cycle01 & 53.1239 & 5.540 & 276.721 & 13.210 & 295.471 & -33.413 & -34.565 & $4-35.5$ & -36.2 & -36 & -37.8421 \\
\hline & 8.0003 & & & 3.1 & & -33.753 & -34.9 & 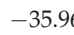 & -3 & & -39.0112 \\
\hline Cycle03 & 53.7017 & .563 & 1.166 & 2.795 & 532 & -33.5429 & $9-34.7$ & $3-35$ & -3 & & -38 . \\
\hline Cycle04 & 58.0786 & 5.537 & 81.048 & 2.867 & 9.456 & -33.5874 & $4-34$ & 35.7 & -3 & & -38 \\
\hline Cycle05 & 58.0706 & 5.450 & 79.188 & 13.127 & 7.769 & -33.55 & $5-34$ & -35 & -36 & & -38 \\
\hline Cycle06 & 54.8340 & 5.566 & 277.619 & 3.020 & 296.210 & -33.6 & & $3-35$ & -36 & -3 & -38.9854 \\
\hline Cycle07 & 58.3364 & 5.412 & 288.078 & 12.670 & 306.162 & -33.7263 & $3-34.9$ & $9-35$ & $3-36$ & $6-37$ & $4-38.8759$ \\
\hline Cycle08 & 55.0633 & 5.532 & 280.125 & 13.103 & 298.761 & -33.4223 & $3-34.574$ & $5-35.4$ & -36.2 & $6-36.9$ & $1-37.7948$ \\
\hline Cycle09 & 56.6853 & 5.633 & 276.396 & 13.125 & 295.153 & -33.3999 & $9-34.560$ & $1-35.4$ & $8-36.2$ & $4-36.9$ & $9-37.7511$ \\
\hline Cycle10 & 53.3712 & 5.449 & 278.652 & 13.024 & 297.129 & -33.5077 & $7-34.692$ & $8-35.6$ & -36.4 & $1-37$ & $3-38.2107$ \\
\hline
\end{tabular}

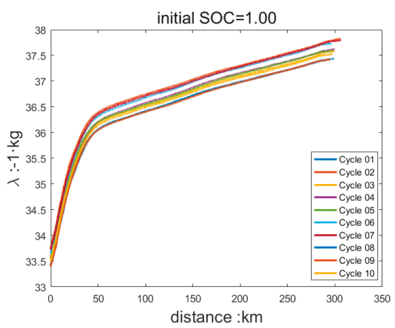

(a)

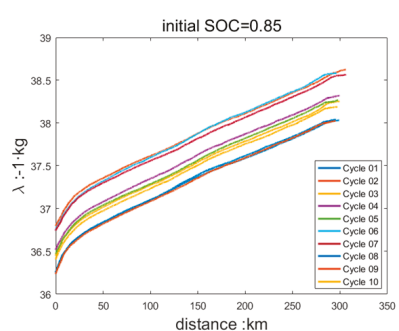

(d)

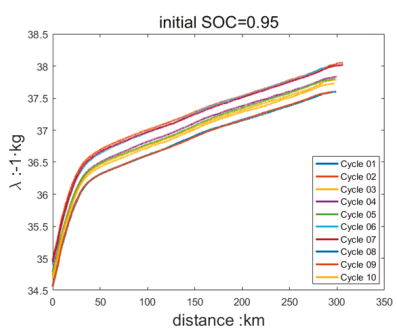

(b)

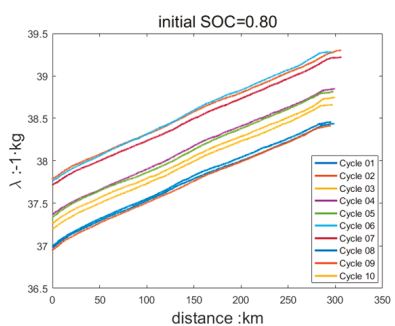

(e)

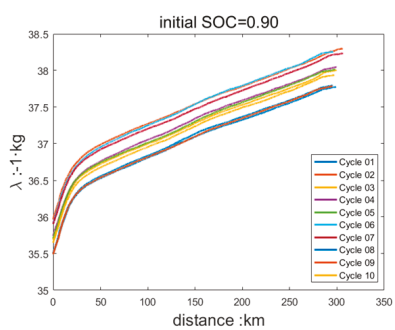

(c)

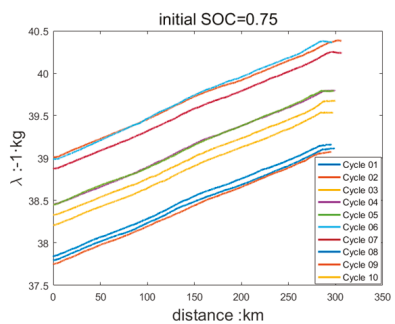

(f)

Figure 13. The change curve of $\lambda$ when $\lambda_{0}$ is taken.

As the initial SOC decreased, the turning point moved forward, which is because the insufficiency of initial electricity led to the earlier entrance to the SOC platform stage. There is no turning point in (e) or (f), which is because SOC at the initial stage of the operation has already entered the platform stage.

\subsection{Comparison of Different Energy Management Strategies}

In this study, the global optimization algorithm PMP, and its derivative instantaneous algorithms C-ECMS and A-ECMS are introduced. In this section, the three energy management strategies under different initial SOCs and the 10 generated driving cycles are compared. As shown in Section 6.1, 
during the solving process of PMP, when the initial SOC is 1.00, the changing range of co-state is about $-3.5 \mathrm{~kg}$; when the initial SOC decreases, the changing range of co-state becomes narrower; when the initial SOC is 0.75 , the changing range is about $-1.5 \mathrm{~kg}$. In C-ECMS, the co-state is always the initial value. To illustrate the effect of the initial value of co-state on the effect of C-ECMS, we select two initial values with the bias of $-2 \mathrm{~kg}$. In the adaptive co-state function, the proportionality factor $k_{p}$ is 0.2 , and the integral coefficient $k_{i}$ is 0.004 . For Cycle 10 for instance, the simulation map is shown in Figure 14.

Clearly, the adjustment by PI makes the variation of SOC follow the SOC reference curve well, and at the end of driving, the final SOC is very close to the pre-set value (0.3). The SOC changing curves of A-ECMS and PMP are overlapped well, indicating the instantaneous optimization result is very close to the global optimized result. When the initial SOC is $<0.85$, the degree of overlap decreases slightly. This is because the reference curve of SOC is switched from an exponential function, when the initial SOC is large, to a linear function, when the initial SOC is low. The middle stage of the linear reference curves in Figure 10f,g are leaning to oil use, leading to the deviation of the SOC curves of A-ECMS and PMP at the middle stage in Figure 14e,f. Generally, however, when the initial SOC is low, the linear reference curve of SOC is closer to the SOC curve of PMP, which better ensures the lower oil consumption. Neither of the two types of C-ECMS meets the condition of making the final SOC close to the pre-set value. The co-state of unbiased C-ECMS is excessively large, leading to excessive discharge from the batteries; the co-state of the C-ECMS with bias of $-2 \mathrm{~kg}$ is too small, so the electricity of batteries is not fully used.

The comparison of SOC changing curves of four energy management strategies show though the bias of two types of C-ECMS is constantly $-2 \mathrm{~kg}$, the difference between the SOC changing curves vary under different initial SOCs. Specifically, at very large initial SOC, the SOC changing curves of these two types of C-ECMS are largely different; at very small initial SOC, the differences decreases. It is indicated at very low initial SOC, the co-state determined from map interpolation is very small, which largely limits the changing rate of SOC. At this moment, with further decrease of the initial co-state, the effect of SOC on the whole driving cycle decreases.

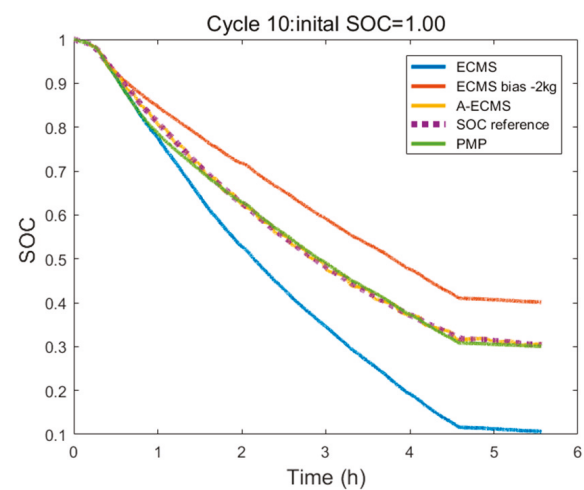

(a)

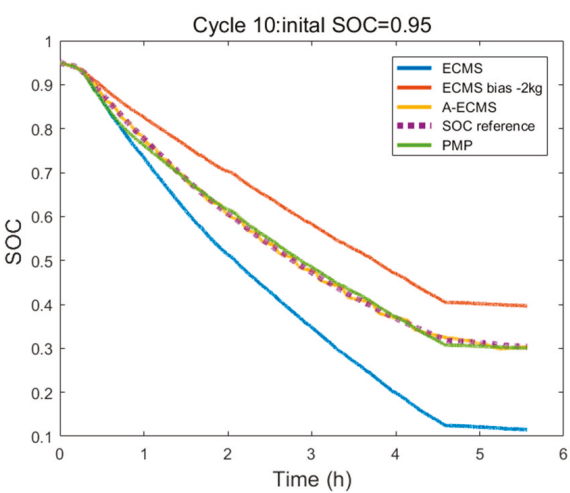

(b) 


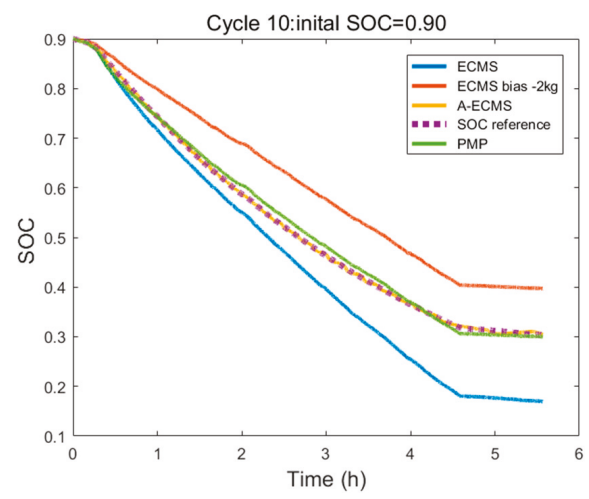

(c)

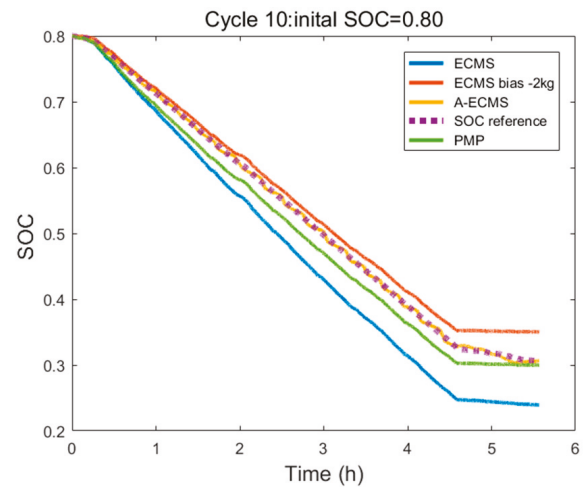

(e)

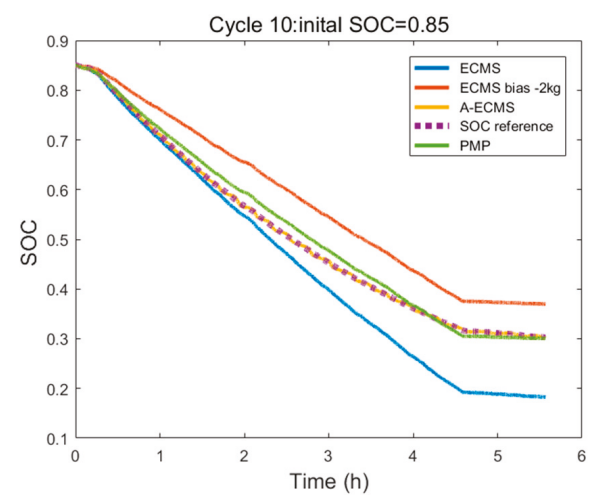

(d)

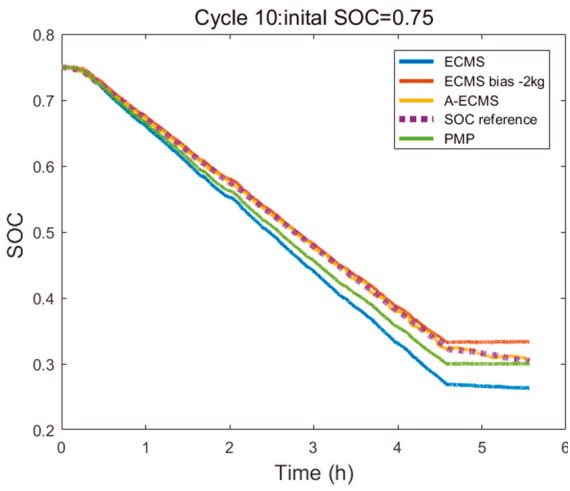

(f)

Figure 14. The strategies comparison under different initial SOC (Cycle 10): (a) SOC = 1.00; (b) $\mathrm{SOC}=0.95 ;(\mathbf{c}) \mathrm{SOC}=0.90 ;(\mathbf{d}) \mathrm{SOC}=0.85 ;(\mathbf{e}) \mathrm{SOC}=0.80 ;(\mathbf{f}) \mathrm{SOC}=0.75$.

In addition, the effectiveness of the system is verified by the standard driving cycles WLTP and US_US06. Due to the short simulation distance of these cycles, it is necessary to extend the distance to the same distance as the target driving cycles $(290-300 \mathrm{~km})$. The simulation results are shown in Figure 15. In the standard cycles, the A-ECMS can still make the final SOC converge to the pre-set SOC, and ensure the electric energy is fully utilized and the solution of oil consumption is close to that of PMP. In the US_US06 cycle, the results of A-ECMS and PMP are different in the first stage of operation $(<1.5 \mathrm{~h})$, but the results of the latter stage are very close. However, in the operation of the WLTP cycle, the SOC of A-ECMS is always greater than the SOC of PMP. This is because the difference between the standard driving cycle WLTP and the target driving cycle of the paper is too large, so that the SOC reference curve cannot be well adapted to this cycle. This shows that the A-ECMS can make the final SOC reach the expected value in different driving cycles. As long as the reference curve is adjusted according to the cycles, the result of the oil consumption solution can be close to the global optimization result. 


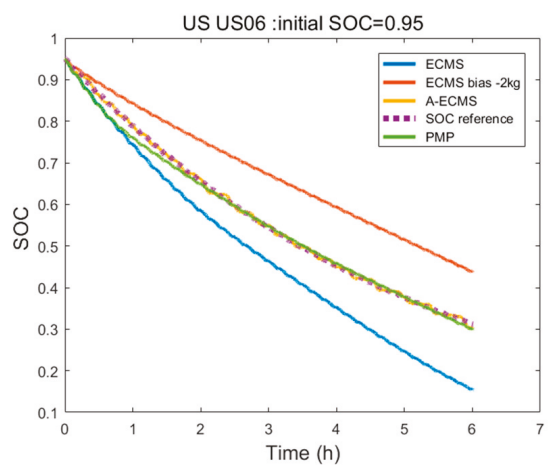

(a)

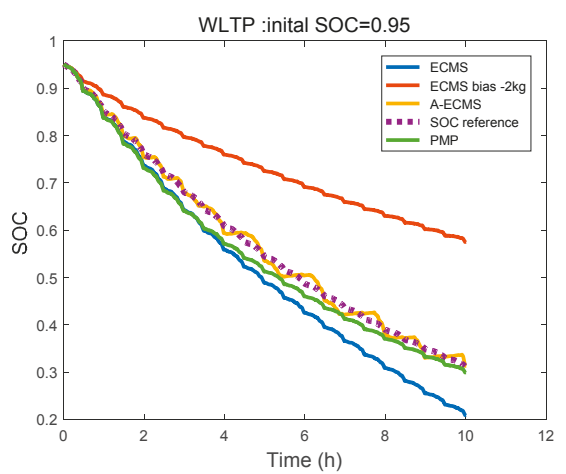

(b)

Figure 15. The strategies verified in standard driving cycles :(a) US_US06; (b) WLTP.

The simulation results of Cycles 01 10 are statistically analyzed (Tables 5-10). As a global optimization algorithm, PMP requires the information of whole driving cycles; though it does not apply to real vehicle environments, it has good optimization effect and is a suitable benchmark for comparison with other energy management strategies. As the initial SOC decreases, the oil consumption increases since the set value is constantly 0.3. Statistics show the largest control deviation of A-ECMS over the final SOC is 0.0076 and the control precision is within $2.33 \%$, indicating it could control SOC to reach the preset value regardless the initial SOC. At different initial conditions, the largest oil consumption deviations of A-ECMS relative to PMP are 6.97\%, 5.52\%, 5.10\%, 4.37\%, 5.01\% and $5.07 \%$, respectively, which are very close to the global optimization results. On the contrary, C-ECMS could not balance well the relationship between the final SOC and oil consumption. Taken together, A-ECMS as an instantaneous optimization algorithm can be applied into real vehicles, fully utilize the electricity of batteries, and well improve the fuel economy while protecting the batteries. When the initial SOCs differ, the control results are very close, indicating A-ECMS could adapt well.

Table 5. Comparison statistics with initial SOC $=1.00$.

\begin{tabular}{lcccccccc}
\hline \multirow{2}{*}{ Number } & \multicolumn{4}{c}{ Final SOC } & \multicolumn{3}{c}{ Fuel Consumption (kg) } \\
\cline { 2 - 8 } & C-ECMS & C-ECMS(-2) & A-ECMS & PMP & C-ECMS & C-ECMS(-2) & A-ECMS & PMP \\
\hline Cycle 01 & 0.1141 & 0.4121 & 0.2999 & 0.3004 & 17.9110 & 28.2983 & 25.5074 & 24.3694 \\
Cycle 02 & 0.0788 & 0.3700 & 0.2975 & 0.3009 & 19.2109 & 29.2575 & 27.9867 & 26.8491 \\
Cycle 03 & 0.1004 & 0.3957 & 0.2906 & 0.3008 & 18.2269 & 28.4817 & 26.5841 & 25.1481 \\
Cycle 04 & 0.0969 & 0.3907 & 0.3019 & 0.3007 & 18.4140 & 28.6073 & 26.8725 & 25.4510 \\
Cycle 05 & 0.1171 & 0.4060 & 0.3069 & 0.3004 & 18.8644 & 28.9469 & 25.9820 & 25.2288 \\
Cycle 06 & 0.1128 & 0.3862 & 0.3022 & 0.2996 & 18.9374 & 28.458 & 27.1953 & 25.4218 \\
Cycle 07 & 0.1047 & 0.3854 & 0.2963 & 0.3004 & 19.8999 & 29.6612 & 27.8810 & 26.6793 \\
Cycle 08 & 0.1293 & 0.4268 & 0.3042 & 0.3000 & 18.1772 & 28.5881 & 25.6125 & 24.1148 \\
Cycle 09 & 0.1273 & 0.4261 & 0.3044 & 0.3002 & 17.6741 & 28.1196 & 25.0490 & 23.6817 \\
Cycle 10 & 0.1065 & 0.4010 & 0.3064 & 0.3006 & 17.9894 & 28.2285 & 26.1526 & 24.7016 \\
\hline
\end{tabular}


Table 6. Comparison statistics with initial SOC $=0.95$.

\begin{tabular}{lcccccccc}
\hline \multirow{2}{*}{ Number } & \multicolumn{4}{c}{ Final SOC } & \multicolumn{3}{c}{ Fuel Consumption (kg) } \\
\cline { 2 - 8 } & C-ECMS & C-ECMS(-2) & A-ECMS & PMP & C-ECMS & C-ECMS(-2) & A-ECMS & PMP \\
\hline Cycle 01 & 0.1211 & 0.4076 & 0.3035 & 0.2997 & 20.0642 & 30.0979 & 27.3669 & 26.2766 \\
Cycle 02 & 0.0843 & 0.3643 & 0.2965 & 0.3007 & 21.3064 & 31.0145 & 30.3512 & 28.7851 \\
Cycle 03 & 0.1064 & 0.3911 & 0.2919 & 0.3003 & 20.3453 & 30.2729 & 28.0594 & 27.0690 \\
Cycle 04 & 0.1046 & 0.3859 & 0.2994 & 0.3008 & 20.5835 & 30.3917 & 28.7220 & 27.3925 \\
Cycle 05 & 0.1262 & 0.4023 & 0.3022 & 0.3003 & 21.0894 & 30.7718 & 27.9162 & 27.1597 \\
Cycle 06 & 0.1208 & 0.3795 & 0.3025 & 0.2993 & 21.1224 & 30.1799 & 28.7759 & 27.3507 \\
Cycle 07 & 0.1121 & 0.3795 & 0.2962 & 0.3002 & 22.0665 & 31.4124 & 29.4395 & 28.6175 \\
Cycle 08 & 0.1400 & 0.4210 & 0.3050 & 0.3008 & 20.4566 & 30.3412 & 27.4689 & 26.0750 \\
Cycle 09 & 0.1408 & 0.4217 & 0.2988 & 0.3008 & 20.0433 & 29.9250 & 26.9613 & 25.6372 \\
Cycle 10 & 0.1150 & 0.3966 & 0.3030 & 0.3005 & 20.1883 & 30.0272 & 28.1068 & 26.6365 \\
\hline
\end{tabular}

Table 7. Comparison statistics with initial SOC $=0.90$.

\begin{tabular}{ccccccccc}
\hline \multirow{2}{*}{ Number } & \multicolumn{4}{c}{ Final SOC } & \multicolumn{4}{c}{ Fuel Consumption (kg) } \\
\cline { 2 - 8 } & C-ECMS & C-ECMS(-2) & A-ECMS & PMP & C-ECMS & C-ECMS(-2) & A-ECMS & PMP \\
\hline Cycle 01 & 0.1750 & 0.4099 & 0.3068 & 0.2996 & 23.7514 & 32.1257 & 29.5778 & 28.1679 \\
Cycle 02 & 0.1379 & 0.3663 & 0.2968 & 0.3007 & 24.9463 & 33.0341 & 31.8291 & 30.6878 \\
Cycle 03 & 0.1597 & 0.3939 & 0.2985 & 0.3001 & 23.9973 & 32.3222 & 30.4358 & 28.9580 \\
Cycle 04 & 0.1575 & 0.3869 & 0.2984 & 0.3000 & 24.2220 & 32.3742 & 30.3386 & 29.2607 \\
Cycle 05 & 0.1876 & 0.3992 & 0.3038 & 0.3001 & 25.0373 & 32.6126 & 30.1988 & 29.0491 \\
Cycle 06 & 0.1734 & 0.3763 & 0.3020 & 0.3001 & 24.7708 & 32.0144 & 30.7227 & 29.2799 \\
Cycle 07 & 0.1675 & 0.3782 & 0.2854 & 0.2994 & 25.8026 & 33.3165 & 30.9027 & 30.4900 \\
Cycle 08 & 0.2006 & 0.4224 & 0.2998 & 0.3002 & 24.3828 & 32.3437 & 29.2079 & 27.9447 \\
Cycle 09 & 0.2008 & 0.4232 & 0.3076 & 0.3001 & 23.9537 & 31.9299 & 28.2447 & 27.4992 \\
Cycle 10 & 0.1696 & 0.3970 & 0.3046 & 0.3000 & 23.8905 & 31.9899 & 29.4823 & 28.5116 \\
\hline
\end{tabular}

Table 8. Comparison statistics with initial SOC $=0.85$.

\begin{tabular}{lcccccccc}
\hline \multirow{2}{*}{ Number } & \multicolumn{4}{c}{ Final SOC } & \multicolumn{3}{c}{ Fuel Consumption (kg) } \\
\cline { 2 - 8 } & C-ECMS & C-ECMS(-2) & A-ECMS & PMP & C-ECMS & C-ECMS(-2) & A-ECMS & PMP \\
\hline Cycle 01 & 0.1910 & 0.3837 & 0.3024 & 0.2996 & 26.1578 & 33.0950 & 31.179 & 30.0414 \\
Cycle 02 & 0.1532 & 0.3379 & 0.2921 & 0.3003 & 27.3240 & 33.9269 & 33.0170 & 32.5639 \\
Cycle 03 & 0.1754 & 0.3673 & 0.2957 & 0.2998 & 26.3919 & 33.2790 & 31.5416 & 30.8269 \\
Cycle 04 & 0.1734 & 0.3589 & 0.3017 & 0.2998 & 26.6208 & 33.2789 & 32.3868 & 31.1340 \\
Cycle 05 & 0.1979 & 0.3666 & 0.3048 & 0.3004 & 27.2541 & 33.3523 & 32.0638 & 30.9375 \\
Cycle 06 & 0.1844 & 0.3466 & 0.3043 & 0.3000 & 27.0162 & 32.8624 & 32.5282 & 31.1648 \\
Cycle 07 & 0.1792 & 0.3478 & 0.2880 & 0.3000 & 28.0668 & 34.1402 & 33.2943 & 32.4012 \\
Cycle 08 & 0.2144 & 0.3931 & 0.2987 & 0.3000 & 26.7266 & 33.2017 & 30.5049 & 29.8154 \\
Cycle 09 & 0.2173 & 0.3945 & 0.3049 & 0.3005 & 26.3865 & 32.8099 & 30.3681 & 29.3865 \\
Cycle 10 & 0.1825 & 0.3697 & 0.3009 & 0.3004 & 26.1897 & 32.9222 & 31.5386 & 30.4019 \\
\hline
\end{tabular}


Table 9. Comparison statistics with initial SOC $=0.80$.

\begin{tabular}{lcccccccc}
\hline \multirow{2}{*}{ Number } & \multicolumn{4}{c}{ Final SOC } & \multicolumn{4}{c}{ Fuel Consumption (kg) } \\
\cline { 2 - 9 } & C-ECMS & C-ECMS(-2) & A-ECMS & PMP & C-ECMS & C-ECMS(-2) & A-ECMS & PMP \\
\hline Cycle 01 & 0.2481 & 0.3638 & 0.3029 & 0.3000 & 30.0416 & 34.3067 & 32.9472 & 31.9411 \\
Cycle 02 & 0.2046 & 0.3175 & 0.2947 & 0.3001 & 30.9870 & 35.1225 & 35.9526 & 34.4765 \\
Cycle 03 & 0.2320 & 0.3457 & 0.2965 & 0.3002 & 30.2468 & 34.4266 & 34.3192 & 32.7390 \\
Cycle 04 & 0.2271 & 0.3389 & 0.3007 & 0.3000 & 30.3779 & 34.4914 & 33.5825 & 33.0420 \\
Cycle 05 & 0.2547 & 0.3467 & 0.3028 & 0.3007 & 31.1545 & 34.5756 & 34.1150 & 32.8512 \\
Cycle 06 & 0.2317 & 0.3234 & 0.2980 & 0.3001 & 30.5680 & 33.9585 & 34.7231 & 33.0893 \\
Cycle 07 & 0.2320 & 0.3281 & 0.2928 & 0.3003 & 31.8128 & 35.3712 & 35.1544 & 34.3285 \\
Cycle 08 & 0.2734 & 0.3719 & 0.3062 & 0.2996 & 30.7096 & 34.3694 & 32.5991 & 31.6819 \\
Cycle 09 & 0.2745 & 0.3742 & 0.3014 & 0.3003 & 30.3059 & 34.0108 & 32.8297 & 31.2640 \\
Cycle 10 & 0.2392 & 0.3501 & 0.3065 & 0.2996 & 30.0533 & 34.1472 & 33.7634 & 32.2688 \\
\hline
\end{tabular}

Table 10. Comparison statistics with initial $\mathrm{SOC}=0.75$.

\begin{tabular}{lcccccccc}
\hline \multirow{2}{*}{ Number } & \multicolumn{4}{c}{ Final SOC } & \multicolumn{4}{c}{ Fuel Consumption (kg) } \\
\cline { 2 - 8 } & C-ECMS & C-ECMS(-2) & A-ECMS & PMP & C-ECMS & C-ECMS(-2) & A-ECMS & PMP \\
\hline Cycle 01 & 0.2764 & 0.3464 & 0.3012 & 0.3001 & 32.9633 & 35.6346 & 35.7002 & 33.8629 \\
Cycle 02 & 0.2308 & 0.3024 & 0.2927 & 0.3002 & 33.8258 & 36.5494 & 37.0121 & 36.4756 \\
Cycle 03 & 0.2600 & 0.3281 & 0.2924 & 0.3001 & 33.1545 & 35.7507 & 35.7310 & 34.6719 \\
Cycle 04 & 0.2515 & 0.3223 & 0.3058 & 0.2997 & 33.1557 & 35.8551 & 36.1694 & 34.9837 \\
Cycle 05 & 0.2724 & 0.3490 & 0.3031 & 0.2995 & 33.7165 & 36.6957 & 36.5232 & 34.7612 \\
Cycle 06 & 0.2487 & 0.3211 & 0.2961 & 0.3002 & 33.0974 & 35.9002 & 36.5854 & 35.0874 \\
Cycle 07 & 0.2506 & 0.3265 & 0.2953 & 0.3004 & 34.3992 & 37.3358 & 37.4909 & 36.3196 \\
Cycle 08 & 0.2956 & 0.3675 & 0.3071 & 0.2990 & 33.4331 & 36.2207 & 34.8088 & 33.5721 \\
Cycle 09 & 0.2977 & 0.3671 & 0.3072 & 0.2997 & 33.0643 & 35.7570 & 34.4008 & 33.1546 \\
Cycle 10 & 0.2632 & 0.3335 & 0.3048 & 0.3000 & 32.8231 & 35.5104 & 35.2473 & 34.2196 \\
\hline
\end{tabular}

\section{Conclusions}

To ensure the sufficient use of electric energy and reduce fuel consumption while ensuring the performances of an extended-range electric bus, an adaptive-equivalent consumption minimum energy management strategy is proposed based on target driving cycles generation:

(1) With the collected data and representative driving cycles, the target driving cycles are generated by a Markov chain approach and used to train the optimal initial co-state map and validate the simulations.

(2) The equidistant target driving cycles are solved via the PMP algorithm, forming the optimal changing curves of SOC under different initial SOCs. Based on the solutions, a segmented SOC reference curve is obtained: an exponential reference curve is used at $S O C \geq 0.85$, and a linear reference curve is adopted at SOC $<0.85$.

(3) An adaptive co-state function consisting of a fixed term and a dynamic term is established. The vehicle information is acquired via ITS, and the weight-averaged vehicle speed is determined. Together with the initial SOC data, the co-state map is interpolated, forming the optimal initial co-state, which is used as the fixed term. The dynamic term is obtained using PI method to control the co-state so as to follow the SOC reference curve.

(4) With the ten target driving cycles and different initial SOCs, the simulations of A-ECMS, PMP and biased ECMS are validated. It is found A-ECMS could make the final SOC converge to the preset SOC, and ensures the electric energy is fully utilized and the oil consumption solution is close to that of PMP, while improving fuel economy.

Author Contributions: H.L. managed the project and conceptualized scheme; C.W. designed the energy management strategy, completed the modeling and simulation, and wrote the manuscript; X.Z. collected and processd the data; C.G. contributed to validations and analyses of the results and reviewed the writing. 
Funding: This research was funded by 1. Key Science and Technology Projects in Jilin Province (20170204085GX); 2. Jilin Province Science and Technology Development Funds (20170101139JC).

Conflicts of Interest: The authors declare no conflict of interest.

\section{References}

1. Chau, K.T.; Wong, Y.S. Overview of power management in hybrid electric vehicles. Energy Convers. Manag. 2002, 43, 1953-1968. [CrossRef]

2. Chen, J.; Wu, J.; Du, J. Real-time optimal energy management strategy for range- extended electric bus in Harbin urban bus driving cycle. In Proceedings of the 28th International Electric Vehicle Symposium and Exhibition, Goyang, Korea, 3-6 May 2015.

3. Rizzoni, G.; Onori, S. Energy management of hybrid electric vehicles: 15 years of development at the Ohio State University. Oil Gas Sci. Technol. 2015, 70, 41-54. [CrossRef]

4. Tate, E.; Harpster, M.O.; Savagian, P. The electrification of the automobile: From conventional hybrid, to plug-in hybrids, to extended-range electric vehicles. SAE Int. J. Passeng. Cars-Electron. Electr. Syst. 2009, 1, 156-166. [CrossRef]

5. Salmasi, F.R. Control strategies for hybrid electric vehicles: Evolution, classification, comparison, and future trends. IEEE Trans. Veh. Technol. 2007, 56, 2393-2404. [CrossRef]

6. Wirasingha, S.G.; Emadi, A. Classification and review of control strategies for plug-in hybrid electric vehicles. IEEE Trans. Veh. Technol. 2011, 60, 111-122. [CrossRef]

7. Bang, J.H.; Yoon, H.W.; Won, K.M. Experiment and simulation to improve key on/off vehicle vibration quality. In Proceedings of the SAE Noise and Vibration Conference and Exhibition, St. Charles, IL, USA, 15-17 May 2007.

8. Roumila, Z.; Rekioua, D.; Rekioua, T. Energy management based fuzzy logic controller of hybrid system wind/photovoltaic/diesel with storage battery. Int. J. Hydrog. Energy 2017, 42, 19525-19535. [CrossRef]

9. Ming, L.; Ying, Y.; Liang, L.; Yao, L.; Zhou, W. Energy management strategy of a plug-in parallel hybrid electric vehicle using fuzzy control. Energy Proced. 2017, 105, 2660-2665. [CrossRef]

10. Sharma, O.P.; Onori, S.; Guezennec, Y. Analysis of Pontryagin's Minimum Principal-Based Energy Management Strategy for PHEV Applications. In Proceedings of the ASME 5th Annual Dynamic Systems and Control Conference joint with the JSME 2012 11th Motion and Vibration Conference, Fort Lauderdale, FL, USA, 17-19 October 2012.

11. Chen, B.C.; Wu, Y.Y.; Tsai, H.C. Design and analysis of power management strategy for range extended electric vehicle using dynamic programming. Appl. Energy 2014, 113, 1764-1774. [CrossRef]

12. Chen, Z.; Mi, C. An adaptive online energy management controller for power-split HEV based on Dynamic Programming and fuzzy logic. In Proceedings of the Vehicle Power and Propulsion Conference, Dearborn, MI, USA, 7-10 September 2009.

13. Yuan, Z.; Teng, L.; Fengchun, S.; Peng, H. Comparative study of dynamic programming and Pontryagin's minimum principle on energy management for a parallel hybrid electric vehicle. Energies 2013, 6, 2305-2318. [CrossRef]

14. Xie, S.; Peng, J.; He, H. Plug-in hybrid electric bus energy management based on stochastic model predictive control. Energy Proced. 2017, 105, 2672-2677. [CrossRef]

15. Tian, H.; Lu, Z.; Wang, X.; Zhang, X.; Huang, Y. A length ratio based neural network energy management strategy for online control of plug-in hybrid electric city bus. Appl. Energy 2016, 177, 71-80. [CrossRef]

16. Litchy, A.J.; Nehrir, M.H. Real-time energy management of an islanded microgrid using multi-objective Particle Swarm Optimization. In Proceedings of the IEEE PES General Meeting I Conference \& Exposition, National Harbor, MD, USA, 27-31 July 2014.

17. C, Z.; Mi, C.; Xiong, R.; Xu, J.; You, C. Energy management of a power-split plugin hybrid electric vehicle based on genetic algorithm and quadratic programming. Power Sour. 2014, 248, 416-26.

18. Khayyam, H.; Alireza, B. Adaptive intelligent energy management system of plug-in hybrid electric vehicle. Energy 2014, 69, 319-335. [CrossRef]

19. He, H.; Zhang, J.; Li, G. Model predictive control for energy management of a plug-in hybrid electric bus. Energy Proced. 2016, 88, 901-907. [CrossRef] 
20. Borhan, H.; Vahidi, A.; Phillips, A.; Kuang, M.; Kollmanovsky, I.V. MPC-based energy management of a power-split hybrid vehicle. IEEE Trans. Control Syst. Technol. 2012, 20, 593-603. [CrossRef]

21. Borhan, H.A.; Vahidi, A.; Phillips, A.; Kuang, M.; Kollmanovsky, I.V. Predictive energy management of a power-split hybrid electric vehicle. In Proceedings of the American Control Conference, St. Louis, MO, USA, 10-12 June 2009.

22. Schacht, E.; Bezaire, B.; Cooley, B.; Bayar, K.; Kruckenberg, J.W. Addressing Drivability an Extended Range Electric Vehicle Running an Equivalent Consumption Minimization Strategy (ECMS). In Proceedings of the SAE World Congress \& Exhibition, Detroit, MI, USA, 12 April 2011.

23. Bo, G.; Rizzoni, G. An adaptive algorithm for hybrid electric vehicle energy management based on driving pattern recognition. In Proceedings of the ASME International Mechanical Engineering Congress and Expositon, Chicago, IL, USA, 5-10 November 2006.

24. Vajedi, M.; Taghavipour, A.; Azad, N.L.; Mcphee, J. A comparative analysis of route-based power management strategies for real-time application in plug-in hybrid electric vehicles. In Proceedings of the American Control Conference, Portland, OR, USA, 4-6 June 2014.

25. Onori, S.; Serrao, l.; Rizzoni, G. Adaptive equivalent consumption minimization strategy for hybrid electric vehicles. In Proceedings of the ASME Dynamic Systems and Control Conference, Cambridge, MA, USA, 12-15 September 2010.

26. Onori, S.; Tribioli, L. Adaptive Pontryagin's Minimum Principle supervisory controller design for the plug-in hybrid GM Chevrolet Volt. Appl. Energy 2015, 147, 224-234. [CrossRef]

27. Jiang, Q.; Ossart, F.; Marchand, C. Comparative study of real-time HEV energy management strategies. IEEE Trans. Veh. Technol. 2017, 66, 10875-10888. [CrossRef]

28. Xie, S.; Li, H.; Xin, Z.; Liu, T.; Wei, L. A Pontryagin Minimum Principle-based adaptive equivalent consumption minimum strategy for a plug-in hybrid electric bus on a fixed route. Energies 2017, 10, 1379. [CrossRef]

29. Xie, S.; He, H.; Peng, J. An energy management strategy based on stochastic model predictive control for plug-in hybrid electric buses. Appl. Energy 2017, 196, 279-288. [CrossRef]

30. Liu, T.; Hu, X. A bi-level control for energy efficiency improvement of a hybrid tracked vehicle. IEEE Trans. Ind. Inform. 2018, 14, 1616-1625. [CrossRef]

31. Liu, T.; Hu, X.; Li, S.E.; Cao, D. Reinforcement learning optimized look-ahead energy management of a parallel hybrid electric vehicle. IEEE/ASME Trans. Mechatron. 2017, 22, 1497-1507. [CrossRef]

32. Schlote, A.; Crisostomi, E.; Kirkland, S.; Shorten, R. Traffic modelling framework for electric vehicles. Int. J. Control 2012, 85, 880-897. [CrossRef]

33. Zheng, C.H.; Xu, G.Q.; Cha, S.W.; Liang, Q. Numerical comparison of ECMS and PMP-based optimal control strategy in hybrid vehicles. Int. J. Automot. Technol. 2014, 15, 1189-1196. [CrossRef]

34. Tian, H.; Wang, X.; Lu, Z.W.; Huang, Y.; Tian, G.Y. Adaptive fuzzy logic energy management strategy based on reasonable soc reference curve for online control of plug-in hybrid electric city bus. IEEE Trans. Intell. Trans. Syst. 2017, 19, 1607-1617. [CrossRef]

(C) 2018 by the authors. Licensee MDPI, Basel, Switzerland. This article is an open access article distributed under the terms and conditions of the Creative Commons Attribution (CC BY) license (http:/ / creativecommons.org/licenses/by/4.0/). 
Article

\title{
Controllability Evaluation of EV Charging Infrastructure Transformed from Gas Stations in Distribution Networks with Renewables
}

\author{
Shuang Gao ${ }^{1, *}$, Jianzhong $\mathrm{Wu}^{2, *}$ and $\mathrm{Bin} \mathrm{Xu}^{3}$ \\ 1 School of Electrical and Information Engineering, Tianjin University, Tianjin 300072, China \\ 2 School of Engineering, Cardiff University, Cardiff CF24 3AA, UK \\ 3 State Grid Anhui Electric Power Science Research Institute, Hefei 230061, China; xubin1980@sina.com \\ * Correspondence: sgao@tju.edu.cn (S.G.); WuJ5@cardiff.ac.uk (J.W.)
}

Received: 13 March 2019; Accepted: 22 April 2019; Published: 25 April 2019

check for updates

\begin{abstract}
A considerable market share of electric vehicles (EVs) is expected in the near future, which leads to a transformation from gas stations to EV charging infrastructure for automobiles. EV charging stations will be integrated with the power grid to replace the fuel consumption at the gas stations for the same mobile needs. In order to evaluate the impact on distribution networks and the controllability of the charging load, the temporal and spatial distribution of the charging power is calculated by establishing mapping the relation between gas stations and charging facilities. Firstly, the arrival and parking period is quantified by applying queuing theory and defining membership function between EVs to parking lots. Secondly, the operational model of charging stations connected to the power distribution network is formulated, and the control variables and their boundaries are identified. Thirdly, an optimal control algorithm is proposed, which combines the configuration of charging stations and charging power regulation during the parking period of each individual EV. A two-stage hybrid optimization algorithm is developed to solve the reliability constrained optimal dispatch problem for EVs, with an EV aggregator installed at each charging station. Simulation results validate the proposed method in evaluating the controllability of EV charging infrastructure and the synergy effects between EV and renewable integration.
\end{abstract}

Keywords: vehicle-to-grid; EV charging infrastructure; optimal dispatch; oil-to-electricity transformation for automobiles

\section{Introduction}

The high penetration of electric vehicles and renewable energy sources in the electric power networks provides a promising solution to the energy crisis and environmental stress. If properly controlled, EVs can act as mobile energy storage to facilitate the integration of intermittent renewable power generation, further alleviating the dependency on fossil fuels [1]. The feasibility of an electrified transportation system for zero or low carbon emission in the near future has been studied [2,3]. China Energy Administration announced the goal to have 120 thousand EV charging stations and 4.8 million charging piles in the power network by 2020. With increasing market share of electric vehicles (EVs), the gas stations are expected to be replaced with EV charging facilities supplied by electric power systems. However, as the EV charging introduces additional load demand, new challenges will be placed on the power system, consisting of reliability and power quality issues [4]. The charging load distribution transformed from the fuel consumption at the gas stations needs to be precisely quantified to evaluate the impact on the power system. Then the possible solutions can be suggested, such as upgrading the existing power system or applying appropriate control techniques in order to maintain the reliable and economic operation of the power system. 
As the fuel consumption at the gas stations is transformed to electric energy consumption at various charging facilities, the temporal and spatial distribution of energy demand in the electric power network needs to be quantified. The economic benefits have been studied by using a lifetime cycle model for oil-to-electricity transformation in the transportation sector across the city $[5,6]$. However, the mapping relation between gas station and charging station for computing the distribution of charging load in the power network, based on the accessible service data of the facilities, has not been studied so for in the literature. Unlike gas stations, where the service time is usually within several minutes, the charging period of EV takes several hours, which means that the charging usually occurs at the parking lots. The real-time information of parking lots and gas stations are accessible from the internet in the E-map (e.g., google map) or the online service website of the refueling and parking facilities. Taking the advantage of real-time data from the internet, a practical computation of energy distribution of EV charging load is developed and; therefore, the impact on the power network and the controllability of this additional charging load can be estimated. Load computation of EV charging stations has been reported extensively in the literature. Monte Carlo simulation is used to model the stochastic process of EV charging considering the driving habits and trip distances, etc., and hence to formulate the daily charging power profile of a charging station by summing up the energy consumption of EVs [7,8]. Queuing theory has also been adopted to model the operation of parking lots $[9,10]$ and EV charging demand in $[11,12]$, where the charging processes are represented by an $M / M / \infty$ queuing system and the arrival rates of EVs are generated as a Poisson process. Vehicle mobility statistics extracted from the 2009 (US) National Highway Travel Survey (NHTS) data is used in $[11,12]$ to define the parameters of the queuing model for the output profiles, for the number of EVs charged and the times EVs arrive and depart from the charging station. However, the stochastic process of EV travel patterns in various countries receives the same input from fixed statistics of a certain area, such as the NHTS dataset. Moreover, the Poisson process in the queuing model assumes EVs have several constant arrival rates and the charging time is typically modeled by Gaussian distribution with given upper and lower limits, which are randomly assigned to each EV. In the proposed queuing theory-based modeling of EV charging stations, the stochastic process of EV arrival and parking can be expressed as a probability function and the parameters can be calculated according to the statistical data accessible from E-map and the service website of facilities.

The modeling and management of EV charging stations are integrated in to the power system analysis and optimal dispatch problem. In the state of art control framework for high-penetration EVs in the power system (i.e., vehicle-to-grid (V2G) technology), the EV aggregator (EVA) is usually introduced as the intermediate control entity between EVs and the distribution system operator (DSO) $[13,14]$. Since a group of EVs are aggregated at the charging stations, an aggregator is assigned to monitor and control the chargers. The interactive control among EV aggregators and the autonomous control within each aggregator are performed in the typical V2G control framework to relieve the computation load of DSO for the regional distribution network. If properly controlled, the negative impact of the additional charging load can be mitigated and ancillary services can be provided, such as cost minimization, peak shaving, and power quality improvement [12,15]. However, EV parking behavior and energy demand are usually modeled by typical distribution function with the fixed parameters or historical statistics. But the random distribution function and historical statistics of a certain region cannot reflect the diverse characteristics of service facilities for automobiles in other places and; therefore, the results may be very different from the actual condition of the power network with massive EVs.

The control method is the key technique to explore the controllability of charging load for functioning as a virtual energy storage and coordinating with renewable energy sources. There has been a lot of existing research on the optimal dispatching of EV charging power in the power system [16]. From the perspective of an EV aggregator, the location and size of charging aggregations need to be found, the differential evolution and particle swarm optimization (PSO) algorithms are used to solve the optimization problem with the objective of total cost minimization [17]. The location of the 
charging stations and the optimal number of charging spots were given in the optimal solution [18]. However, the power network has not been taken into account in the model [19]. Other objective functions were investigated for the optimal placement and sizing of an EV aggregator, particularly the profit maximization of an EV aggregator, but some practical issues from the side of users were not described, such as the satisfaction of mobile needs and power quality deterioration caused by recharging massive EVs at peak hours [20]. The optimal dispatch by using EVs as mobile energy storage in the power grid is also studied in the recent literature [21]. In the optimal control problem of EV charging stations modeled in this paper, both EV aggregator configuration at available parking lots and EV charging power regulation should be solved. Therefore, a combined optimization of EV aggregation configuration and operation should be derived based on the modeling of EV charging stations in the optimal dispatch problem.

The main objectives of this paper are to propose a quantitative evaluation of EV charging load and the controllability in the power network, based on the assumption that the fuel consumption at the gas stations is transformed to EV charging facilities covering the same region. The main contributions of the work presented in this paper are as follows:

- The mapping relation between gas stations and EV charging stations is proposed to estimate the distribution of EV charging load in the power network, by using the online accessible data from E-map and service website of the facilities. These features render the proposed charging station model more realistic and accurate than the existing models, in which mobility statistics for vehicles in a certain area is adopted in the charging load computation.

- A novel operational model of EV charging stations is formulated by using membership function between EVs and parking places and queuing theory. The arrival and parking behavior of EVs are captured from the online data and used for estimating the 24-hour electric power demand. So far, there is no reported work that examines how the operation of an EV charging station can be modeled as a function of input parameters and control variables integrated into the optimal dispatch of the power system. In the proposed modeling of EV charging stations, the parameters and boundaries of control variables are calculated for the reliability constrained optimal dispatch of the distribution network.

- The optimal control of EV charging stations is devised under the typical V2G control framework, where the EV aggregator performs as the intermediate controller for EVs at each charging station. The optimal dispatch problem is composed of both EV charging station configuration and EV charging power regulation. The optimal setting of the EV charging station and the optimal charging plan of each individual EV are given by the optimal dispatch. A two-stage hybrid algorithm is developed to reduce the complexity of the optimization that evaluates the controllability of the charging stations in the oil-to-electricity transformation.

The operational model of EV charging stations changed from gas stations is presented in Section 2. The optimal configuration and control of charging stations integrated into the optimal dispatch of the power system are explained in detail in Section 3. Section 4 describes the two-stage hybrid optimization algorithm devised for the optimal charging strategy in Section 3. Section 5 presents the analysis and discussion of controllability evaluation for charging facilities in the test 123-bus test network. Section 6 outlines the conclusion drawn based on the numerical results.

\section{Modeling of EV Charging Stations Transformed from Gas Stations}

As vehicles served by the gas stations will be replaced by EVs in the future, EV charging facilities will be built to meet this energy demand. The EV charging stations are established with both fast charging and slow charging devices installed at the parking lots. EV charging load changed from gas stations was calculated based on the data extracted from accessible E-map or the online service website of the facilities. For instance, the location and real-time occupancy rate of gas stations and parking lots across the certain area of the town, as shown in Figure 1. The key parameters of the modeling 
were extracted from the online graphic data to characterize the parking of EVs at different places. The mathematical model of the charging process was formulated considering the energy consumption at gas stations and the EV parking period at parking lots. The weekly occupancy rate of the parking lot, as laid out on the map of Figure 1, shows that each day of the week had a different service pattern. Therefore, a daily occupancy rate was extracted for modeling the operation of EV charging stations, as shown in the right side of the figure. The EV-to-carpark membership function and the queuing theory were also adopted to calculate the parking time and charging service pattern. The temporal and spatial distribution of the EV charging can be calculated according to the online data and the control strategies can be developed based on the adjustable variables, with boundaries defined by modeling the operation of EV charging stations. The workflow of the proposed modeling of the EV charging station and the integrated optimal charging algorithm in the power network is illustrated in Figure 2.

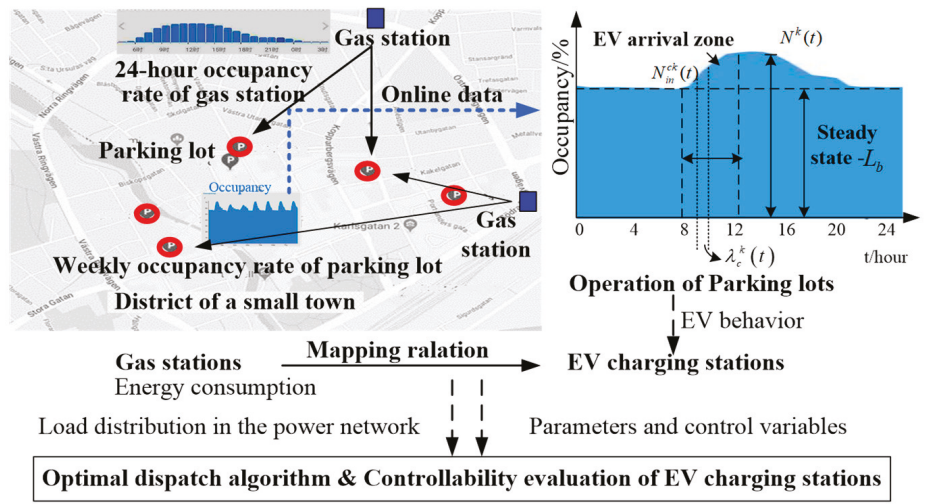

Figure 1. Modeling of the electric vehicle (EV) charging facilities changed from gas stations based on online data analysis.

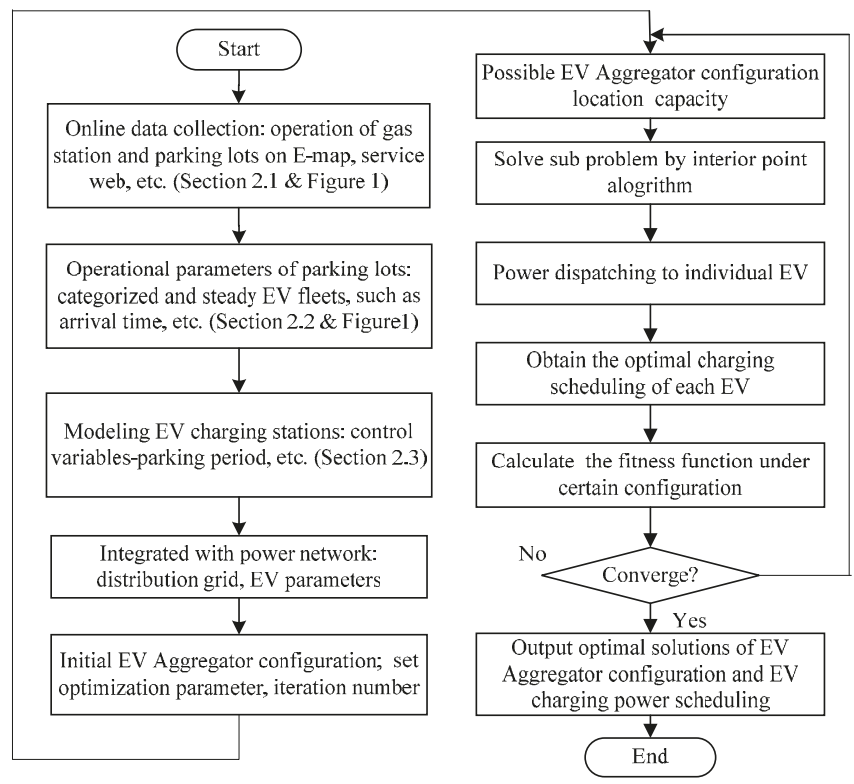

Figure 2. Flowchart of the proposed optimal control algorithm for EV charging facilities. 


\subsection{Oil-To-Electricity Transformation with Membership Function between EVs and Parking Lots}

It was assumed that the fuel consumption at the gas station was transformed to the electricity supplied by EV charging stations during the electrification of transportation system. The energy consumption taken over by the regional EV charging stations can be expressed by:

$$
\begin{gathered}
F_{e}=\sum_{t=1}^{T} \sum_{j=1}^{G} C_{f}^{j}(t)=\sum_{t=1}^{T} \sum_{k=1}^{M} C_{e}^{k}(t) \\
C_{e}^{k}(t)=\sum_{i=1}^{N^{k}(t)} P_{i}^{k}(t) \cdot \Delta t
\end{gathered}
$$

where $M$ is the number of parking lots, $G$ is the number gas of stations covering the same region, $t$ denotes $t^{\text {th }}$ hour, and $T$ represents the planned time period, which is a typical day in the simulation, as shown in Figure 1. $j$ denotes $j^{\text {th }}$ gas station, $k$ denotes the $k^{\text {th }}$ parking lot, and $i$ is the $i^{\text {th }} \mathrm{EV}$. $C_{f}$ represents the fuel consumption at the gas station, which is measured by heat based on the quantity $(\mathrm{mL})$ and density $(\mathrm{kg} / \mathrm{mL})$ of oil traded at the gas station and fuel heating value $(42 \mathrm{MJ} / \mathrm{kg})$, and then converted into the unit of $\mathrm{kWh} . C_{e}$ is the equivalent electricity consumption in the unit of $\mathrm{kWh} . P(t)$ is the charging power at each time step, and $N^{k}(t)$ is the number of EVs at $k^{t h}$ parking lot. Because, at each time step, EVs can be at any parking lots in the region, the membership function is defined to represent the possibility and the duration of each EV at the $k^{\text {th }}$ parking lot. The membership function between EVs to parking lots can be expressed by:

$$
\begin{aligned}
& \alpha_{m}=\left[\vec{\alpha}^{1} \cdots \vec{\alpha}^{k} \cdots \vec{\alpha}^{M}\right] \\
& \vec{\alpha}^{k}=\left[\alpha_{1}^{k} \cdots \alpha_{i}^{k} \cdots \alpha_{N}^{k}\right]^{T}
\end{aligned}
$$

where $N_{p}$ is the number of all parked vehicles, $\alpha_{m}$ is the matrix of membership degree, and $\alpha_{i}^{k}$ is the membership degree of $i^{\text {th }} \mathrm{EV}$ at $k^{\text {th }}$ parking lot. $\alpha_{m}$ subjects to the following conditions:

$$
\begin{gathered}
\sum_{k=1}^{M} \alpha_{i}^{k}=1 \quad \forall i \in N \\
\sum_{k=1}^{M} N^{k}(t)=N \quad \forall t \in T \\
\sum_{k=1}^{N} \alpha_{i}^{k} \cdot T=\sum_{t=1}^{T} N^{k}(t)
\end{gathered}
$$

These conditions included that the summation of membership degrees for each EV to all the parking lots should be 1 . Based on the same assumption that all the vehicles served by the gas stations were changed to parking lots in the same region, the summation of EVs at the $M$ parking lots should be equal to the total number of EVs. Furthermore, the total service time of the parking lots can be calculated by the summation of membership degree. It can also be derived from occupancy and capacity of parking lots which can be read from online graphic data as shown in Figure 1. The temporal and spatial distribution of energy consumed at gas stations to the parking lots was calculated by the membership functions in the following section. 


\subsection{Operation of EV Charging Stations Based on Queuing Theory and Accessible E-Map Database}

The arrival and departure times of EVs at parking lots, which are essential for daily profile of EV load, were derived by the parking data analysis, whereby queuing theory applies. The condition of a typical queuing theory model is that the arrival intensity is constant over the time. But the arrival of EVs for commuting can be concentrated on several hours. Moreover, the service time of parking, which usually takes several hours, is much longer than that of gas station on the time scale of minutes. The steady state of a queuing system cannot be reached in a short time, which is consistent with the real data shown in Figure 1. Thus, in the proposed model, the number of EV was composed of two portions, the steady state and the dynamic state. The steady state was defined as basic EV flow, where the arrival intensity was constant over the time. The dynamic state was defined as categorized EV flow, where EVs were used for a certain purpose and show a fixed pattern, such as household charging for EVs that started charging once coming back from work.

The operation of parking lots was modeled based on queuing theory and the online data. The variation of the parked vehicles can be expressed by:

$$
\begin{aligned}
N^{k}(t)= & L_{s}^{k}(t)=L_{b}^{k}+N_{c}^{k}(t) \quad \forall k \in M \\
\overline{N_{c}(t)} & =\overline{L_{s}(t)}-\overline{L_{b}}
\end{aligned}
$$

where $L_{b}^{k}$ is the expected number of basic EV fleet, which is also the queuing length in the steady state; and $N_{c}^{k}$ is the number of categorized EV fleet, which is varying over the time according to the arrival intensity. These numbers can be read from online service data for each parking lots, as shown in Figure 1. The accessible data is described as $\overline{N_{c}(t)}, \overline{L_{s}(t)}$, and $\overline{L_{b}}$ in Equation (9) for the varying number of EVs parked at each time slot.

According to queuing theory, the arrival of EVs at parking lots is Poisson distribution, denoted by $\lambda_{b}$ and $\lambda_{c}$ for steady and categorized EV fleets, respectively. The queuing length $\left(L_{b}\right)$ in the steady state can be calculated by applying the result of queuing theory, where the parking lots can be modeled as a $\mathrm{M} / \mathrm{M} / \mathrm{Z} / \mathrm{Z}$ queuing system $[7,8]$.

$$
\begin{aligned}
& \lambda_{z}=\lambda_{b}, z=1,2, \cdots, z^{k} \\
& \mu_{z}=z \mu, z=1,2, \cdots, z^{k}
\end{aligned}
$$

where $Z^{k}$ is the capacity of $k^{\text {th }}$ parking lot, and $z$ is the number of EVs at a parking lot. The service time in this case is the parking period, which complies with negative exponent distribution $1 / \mu$ in the queuing model. The service intensity of the parking lot is defined by the queuing theory, and the number of EVs at the parking lot can be written as a function of $\rho$ :

$$
\begin{gathered}
\rho=\frac{\lambda_{b}}{Z^{k} \mu} \\
L_{b}^{k}=\sum_{z=0}^{z^{k}} z P_{z}=z^{k} \rho\left(1-P_{z}\right)
\end{gathered}
$$

The complete modeling of parking lots is described in Appendix A. The arrival rate of a basic EV fleet can be derived if the queuing length is given:

$$
\lambda_{b}=Z^{k} \mu \rho\left(\overline{L_{b}^{k}}\right)
$$

where $\overline{L_{b}^{k}}$ is the average number of basic EVs at a parking that can be collected from online data.

Similarly, the categorized EV fleet in the queuing model can be quantified by assuming that the arrival rate at each time step is Poisson distribution. The arrival of categorized EVs is concentrated on several hours, which can be easily identified from the occupancy variation of the parking lots. 
As shown in Figure 1, the rising zone is assumed to be arrival period of categorized EVs as explained in Appendix A. Therefore, the number of arrival EVs $N_{i n}^{c k}$ can be calculated from $N_{c}^{k}(0)$ and $N_{c}^{k}(t)$, as shown in Appendix A, and can also be calculated by:

$$
\begin{gathered}
E\left(N_{i n}^{c k}(t)\right)=\sum_{y=0}^{\infty} y \frac{(\lambda t)^{k}}{k !} e^{-\lambda t}=\lambda_{c}^{k}(t) \cdot \Delta t \\
\lambda_{c}^{k}(t)=\frac{\overline{N_{i n}^{c k}(t)}}{\Delta t}=\frac{\overline{\Delta N_{c}^{k}(t)}}{\Delta t}
\end{gathered}
$$

where $\overline{\Delta N_{c}^{k}(t)}$ is the difference of categorized EV number at $t^{t h}$ hour, which can be read from the online data shown in Figure 1. The arrival rate at each time slot can be calculated by Equations (14) and (16), and used to determine the parking time period of EVs at parking lots.

The membership degree of each individual EV to parking lot is correlated with the parking period in the queuing theory:

$$
\alpha_{i}^{k} \cdot T=\tau_{i} \sim \frac{1}{\mu}, \frac{1}{\mu}=\overline{\tau_{i}}
$$

where $\tau_{i}$ is the parking period of $t^{\text {th }} \mathrm{EV}$ at one parking lot. The service time complies with negative exponential distribution $[7,8] . \overline{\tau_{i}}$ is the average EV parking period that can be collected from online data. As all the other parameters are calculated, the arrival and the time duration of EVs at the parking lot can be completely quantified.

\subsection{Parameter Configuration of EV Charging Facilities}

In order to analyze the temporal and spatial distribution of EV charging energy at parking lots covering the same region of gas stations, the number of EVs charged at the parking lot and the energy consumption were calculated according to the fuel consumption at gas stations. Based on the formulation of EV charging facilities installed at parking lots by using membership function, the total number of served EVs can be calculated by:

$$
\begin{gathered}
\sum_{k=1}^{M} \sum_{i=1}^{N_{i n}^{k}} \alpha_{i}^{k} \cdot T=\sum_{i=1}^{N} \sum_{k=1}^{M} \alpha_{i}^{k} \cdot T=\sum_{i=1}^{N} T=N T \\
\sum_{k=1}^{M} \alpha_{a}^{k} \cdot N_{i n}^{k}=N^{P} \\
\alpha_{i}^{k}=\left\{\begin{array}{cc}
\alpha_{a}^{k} & \alpha_{i}^{k} \neq 0 \\
0 & \alpha_{i}^{k}=0
\end{array}\right.
\end{gathered}
$$

where $N^{P}$ is the number of served EVs at all parking lots during $T$ hours. The membership degree of EVs at the same parking lot was assumed to be the same in Equation (20), since the customers of a parking lot usually had the same purpose for working in an office building or for staying at home in the residential area. The number of EVs charged at the parking lot can be calculated according to the energy consumption of gas stations:

$$
\begin{gathered}
F_{e}=\sum_{k=1}^{M} \sum_{t=1}^{T} \sum_{i=1}^{N^{k}(t)} P_{i}^{k}(t) \cdot \Delta t=\sum_{k=1}^{M} \sum_{i=1}^{N^{k}(t)} \sum_{t=1}^{T} P_{i}^{k}(t) \cdot \Delta t \\
=\sum_{k=1}^{M} \sum_{i=1}^{N_{i n}^{k}} E C_{i}\left(S O C_{\text {out }}^{k}-S O C_{i n}^{k}\right)
\end{gathered}
$$


where $E C_{i}$ is the battery energy capacity of $i^{\text {th }} \mathrm{EV} . S O C_{\text {out }}^{k}$ is the SOC of EV battery at departure and $S O C_{i n}^{k}$ is the SOC of EV battery at arrival, respectively. The initial SOC and the target SOC (defined as above $90 \%$ ) are also random variables that are defined as normal distribution:

$$
\operatorname{SOC}_{i n^{\prime}}^{k} \operatorname{SOC}_{\text {out }}^{k} \sim N\left(\mu, \sigma^{2}\right)
$$

The parameters of normal distribution are given for estimating the EV charging load, and the variety of different types of EVs was taken into account:

$$
\overline{E C_{i}}=\sum_{r=1}^{R} E C_{r} \cdot \eta_{r}
$$

$\overline{E C_{i}}$ is the average battery capacity. $E C_{r}$ is the battery capacity of $r$ type $\mathrm{EV}$, and $\eta_{r}$ is the ratio of $r$ type EVs to all EVs served at the regional charging stations.

$$
\begin{gathered}
F_{e}=\sum_{k=1}^{M} \sum_{i=1}^{N_{\text {in }}^{k}} \overline{E C_{i}} \cdot\left(\overline{S O C_{\text {out }}^{k}}-\overline{S O C_{\text {in }}^{k}}\right) \\
\bar{N}=\frac{F_{e}}{\overline{E C_{i}} \cdot\left(\overline{S O C_{\text {out }}^{k}}-\overline{S O C_{\text {in }}^{k}}\right)} \\
\eta_{E C}=\frac{\bar{N}}{N^{P}}
\end{gathered}
$$

where $\bar{N}$ is the average number of charged EVs, and $\eta_{E C}$ is the ratio of charged EVs to parked EVs.

\subsection{Mathematical Formulation of EV Charging Load and Controllable EV Charging Variables}

In order to analyze the impact of EV charging transferred from gas stations and to develop the control strategy for the EV charging process, the calculation of EV charging load and the control variables were derived from the operational model of aforementioned charging facilities incorporated with parking lots.

$$
\begin{gathered}
F_{e}^{u n}=\sum_{k=1}^{M} \sum_{t=1}^{T} C_{e}^{k}(t)=\sum_{k=1}^{M} \sum_{i=1}^{N} \alpha_{m} \cdot \vec{P}_{r} \cdot T \\
\vec{P}_{r}=\left[P_{r}^{1}, \cdots, P_{r}^{k}, \cdots, P_{r}^{M}\right]^{T}
\end{gathered}
$$

$F_{e}^{u n}$ denotes the energy consumption calculated in an uncontrolled case, and $P_{r}^{k}$ is the rated power of EV charger at $k^{\text {th }}$ parking lot. The matrix of membership degree of EV to parking lots can also be expressed as $\vec{\alpha}_{i}=\left[\alpha_{i}^{1}, \alpha_{i}^{2}, \cdots, \alpha_{i}^{k}, \cdots, \alpha_{i}^{M}\right]^{T}$.

$$
\begin{gathered}
\alpha_{i}^{k} \cdot P_{r}^{k}=\left\{\begin{array}{cc}
\alpha_{i}^{k} \cdot P_{r}^{k} & \alpha_{i}^{k} \leq \frac{T_{i}^{k r}}{T} \\
\frac{P_{r}^{k} \cdot T_{r}^{k r}}{T} & \alpha_{i}^{k}>\frac{T_{i}^{k r}}{T}
\end{array}\right. \\
T_{i}^{k r}=\frac{E C_{i} \cdot\left(\operatorname{soc}_{\text {out }}^{k}-s o c_{\text {in }}^{k}\right)}{P_{r}^{k}}
\end{gathered}
$$

$T_{i}^{k r}$ is the charging period of EV to reach target SOC at the departure time with rated power.

$$
P_{\mathrm{var}}^{\vec{k}}(t)=\left[P_{1}^{k}(t), \cdots, P_{i}^{k}(t), \cdots, P_{N}^{k}(t)\right]
$$




$$
\begin{gathered}
F_{e}^{c o n}=\sum_{k=1}^{M} \sum_{t=1}^{T} C_{e}^{k}(t)=\sum_{k=1}^{M} \sum_{i=1}^{N} \sum_{t=1}^{T_{\alpha i}} P_{i}^{k}(t) \Delta t=\sum_{k=1}^{M} \sum_{i=1}^{N} \sum_{t=1}^{T_{\alpha i}} P_{v a r}^{k}(t) \Delta t \\
T_{\alpha i}=\alpha_{i}^{k} \cdot T
\end{gathered}
$$

$F_{e}^{c o n}$ denotes the energy consumption calculated in controlled case, and $P_{\mathrm{var}}^{\vec{k}}$ is the vector of variables (charging power) in a controlled case.

$$
\sum_{t=1}^{T_{\alpha i}} P_{i}^{k}(t) \cdot \Delta t=E C_{i} \cdot\left(S O C_{\text {out }}^{k}-S O C_{i n}^{k}\right)
$$

$T_{\alpha i}$ is the time period of $i^{t h} \mathrm{EV}$ parked at $k^{\text {th }}$ parking lot.

\section{Coordinated Integration of EV Charging Stations into the Electric Distribution Grid}

As temporal and spatial distribution of EV charging demand and controllable variables are given from the above quantitative analysis of oil-to-electricity transformation for regional automobiles, the charging facilities can be integrated into the electric distribution network. The impact of EV charging load on the power system operation is quantified and the control strategy is developed by adjusting the control variables within the boundaries that are given in the above section. In the vehicle-to-grid operation framework, an EV aggregator is usually defined as the control entity between power grid and EVs, which offers services to aggregate EV charging power (or discharging defined as V2G) from a group of EVs, and acts towards the grid to provide considerable power regulation capacity. In this context, each parking lot is correlated with one EV aggregator, which can perform real-time monitoring and regulation on the charging behavior of each individual EV as long as it is plugged into the power grid. Since the power feedback from EV to grid results in serious battery degradation, which may prevent EV owners from participating in the vehicle-to-grid interaction, only the charging adjustment is taken into account in this paper to provide the optimal charging plan for EVs.

In the impact analysis of EV charging load, EVs were assumed to start charging as soon as they are parked, which is the direct charging used as the uncontrolled case in the simulation. In this case, EVs are charged at rated power of the charging facility or on-board battery charger. The daily charging profile in the impact analysis was defined as the reference to be compared with the controlled case in the simulation. A two-stage optimization was formulated for managing the charging load based on the control variables and the boundaries defined in the above section.

\subsection{Objective Function of the Optimal Control Strategy}

The multi-period optimal dispatch with EV charging infrastructure, formulated in considering network constraints, is a mixed integer nonlinear programming (MINLP) problem [20-22]. A two-stage optimization algorithm was derived from the basic formulation of the MINLP optimal charging problem. The optimal configuration of the EV aggregator and the operation of the EV charging infrastructure for regulating the charging rate of individual EVs were modelled in the optimal dispatch problem. The overall objective in this work is to minimize the operating cost. In the objective function, the expenditure on supplying electricity to the load, including charging EVs and the total power loss, were calculated in Equation (35):

$$
\min \sum_{t=0}^{T} \sum_{i=1}^{n} \rho(t)\left(P_{L d}(t)+P_{E V A i}(t)+P_{L s}(t)\right) \Delta t
$$

where $\rho(t)$ is the electricity price at time $t ; P_{L d}(t)$ is the net power load of the original distribution system; $P_{E V A i}(t)$ is the accumulated EV charging power at bus $i ; P_{L S}(t)$ is the total power loss at time $t$; and $\Delta t$ is the time interval, which was defined as one hour in the proposed day-ahead optimal dispatch 
of EV charging infrastructure, and it was also consistent with the above charging station model. The load of the EV charging station was calculated by:

$$
P_{E V A i}(t)=\sum_{m=1}^{M i} P_{E V A i}^{m}(t)
$$

$P_{E V A i}^{m}(t)$ is the charging rate of individual EVs in the control realm of the EV aggregator installed at bus $i$.

$$
P_{L S}(t)=\sum_{(i, j) \in B} R_{i j} \frac{P_{i j}^{2}+Q_{i j}^{2}}{\left|V_{i}\right|^{2}}
$$

$P_{i j}$ and $Q_{i j}$ are the active and reactive power, respectively, transmitted on the branch between bus $i$ and $j ;\left|V_{i}\right|$ is the voltage magnitude at bus $i$, and $R_{i j}$ is the resistance of branch $i j$.

\subsection{Constraints of EV Charging Optimization}

The mathematical model was subject to constraints from each participant in the EV charging optimization, including limits on the reliable operation of power system, EV charging station, and the requirements of EV users. The power flow balance was expressed by:

$$
I_{i j}(t)^{2}=\left(G_{i j}^{2}+B_{i j}^{2}\right)\left[U_{i}^{2}(t)+U_{j}^{2}(t)-2 U_{i}(t) \cdot U_{j}(t) \cos \theta_{i j}(t)\right] \leq I_{i j \max }^{2}
$$

$I_{i j}(t)$ is the branch current; $G_{i j}$ and $B_{i j}$ denote the admittance matrix of the power network; $U_{i}$ and $U_{j}$ are the bus voltage; and $I_{i j \max }$ is the maximum current of the transmission line.

$$
\left(V_{i}^{\min }\right)^{2} \leq V_{i}^{r}(t)^{2}+V_{i}^{i m}(t)^{2} \leq\left(V_{i}^{\max }\right)^{2}
$$

$V_{i}^{\min }$ and $V_{i}^{\max }$ are the lower and upper limits of bus voltage, respectively; $V_{i}^{r}(t)$ and $V_{i}^{i m}(t)$ are the real and imaginary parts of the bus voltage, respectively.

The adjustable EV charging power was limited within the capacity of the charging facility:

$$
P_{E V A i, \min } \leq P_{E V A i}^{m}(t) \leq \min \left\{P_{m, \max }, P_{E V A i, \max }\right\}
$$

$P_{E V A i, m i n}$ and $P_{E V A i, m a x}$ are the lower and upper power boundaries of charging facilities, respectively; $P_{m \text { max }}$ is the rated power specified by the EV on-board charger.

The target SOC set by EV users must be achieved till the departure of the parking lots:

$$
S O C_{t a r}^{m} \leq \sum_{t=1}^{T} P_{E V A i}^{m}(t) \eta_{c h r} / C_{b a t}^{m}+S O C_{i n i}^{m} \leq 1
$$

where $C_{b a t}^{m}$ is the battery capacity of the $m^{\text {th }} \mathrm{EV}$; $S O C_{i n i}^{m}$ and $S O C_{t a r}^{m}$ are the initial and target SOC, respectively; and $\eta_{c h r}$ denotes the efficiency of battery charging.

\section{Two-Stage Hybrid Optimization Algorithm}

In the proposed EV charging optimization model, the decision variables included the setting of a charging station associated with its EV aggregator and the multi-period charging power control under the given configuration parameters. For the time-series simulation, the increased number of variables made it even harder to solve the MINLP problem. Thus, a two-stage algorithm was formulated to separate the variables into two categories, which were EV aggregator configuration and optimal charging plan for individual EVs. The original problem was converted to a master problem of optimal 
configuration and a sub-problem of optimal charging strategy, so as to reduce the complexity of the nonlinear constrained optimization modelled in the above section.

\subsection{Solution Procedure for Optimal Integration of EV Charging Stations into the Power Grid}

A two-stage hybrid optimization algorithm based on PSO and sequential quadratic programming (SQP) was derived to accelerate the calculation speed, as depicted in Figure 2. As shown in the flowchart, the problem solution of the hybrid optimization algorithm required an iterative process between the master problem of optimal configuration and the sub-problem of optimal charging strategy. In the master problem, the optimal locations of EV charging stations with the power level limits were found by applying PSO algorithm, and the charging plans for each individual EV were embedded as a sub-problem solved in the lower stage. In this way, the aggregated power of EVs connected to the bus of the distribution network was provided so that the PSO algorithm was implemented on mid-voltage level with a limited number of variables. The optimal charging stations were chosen from the candidate locations that were all the parking lots in the region of oil-to-electricity transformation. The capacity of the charging station must be lower than the parking lots:

$$
\begin{gathered}
L C_{E V A i} \in\left\{L P_{V 1}, \ldots L P_{V j}, \ldots, L P_{V m}\right\} \\
C P_{E V A i} \leq\left(N_{i n}^{c k}+L_{b}^{k}\right) P_{E V r i}
\end{gathered}
$$

$L C_{E V A i}$ denotes the location of charging station at bus $i$, which is selected from the candidate locations of all parking lots, and the bus number is denoted by $L P_{V j}$. $C P_{E V A i}$ denotes the capacity of the charging station at bus $i$, and $P_{E V r i}$ is the rated charging power of charging facility at this charging station.

The capacity of the installed charging facilities was also subject to the operation limits of the power grid considering each EV charging station connected to a bus of the network, as described in the above constraints Equations (38) and (39). All served EVs calculated in Equation (25) were distributed proportionally to each charging station based on its capacity, as expressed by:

$$
\begin{gathered}
N_{E V A i}=\bar{N} \cdot C P_{E V A i} / \sum_{i=1}^{n} C P_{E V A i} \\
P_{i, \min } \leq P_{L D i}(t)+\sum_{m=1}^{N_{E V A i}} P_{E V A i}^{m}(t) \leq P_{i, \max }
\end{gathered}
$$

$N_{E V A i}$ is the number of EVs distributed to a charging station according to the proportion of capacities of all charging stations. $P_{L D i}(t)$ is the base load at bus $i, P_{i, \min }$ and $P_{i, \max }$ are the minimum and maximum power allowed to be connected to bus $i$ by the power system, respectively.

The optimal solution of the master problem was provided to the sub-problem as input parameters, including the bus where the EV charging station connected to the network and the charging power limits. The sub-problem without any integer variables can be solved by SQP within the feasible region given in the master problem. The constraints from the power network operation and EV mobile needs were also checked to ensure that the charging plan of each individual EV can fulfill the overall objective.

\subsection{Two-Stage Hybrid Optimization Algorithm}

The objective function of the master problem is described in Equation (35), with the location and power capacity of EV charging station in the power network as decision variables and subject to:

$$
0 \leq P_{E V A i}(t) \leq P_{E V A i}^{\max }(t)
$$


where $P_{E V A i}^{\max }(t)$ is the upper limit of the overall charging power, that is the power capacity of each EV aggregator.

$$
P_{E V A i}^{\max }(t)=\sum_{m=1}^{M i} P C_{E V A i}^{m}(t)
$$

$P C_{E V A i}^{m}(t)$ is the power capacity of individual $\mathrm{EV}$ at bus $i$.

$$
L C_{E V A i} \in\{0,1\}
$$

$L C_{E V A i}$ is the location of EV aggregation in the charging station, and in this algorithm is defined as a binary variable.

$$
N_{A}^{\min } \leq \sum_{i=1}^{n} L C_{E V A i} \leq N_{A}^{\max }
$$

$N_{A}^{\min }$ and $N_{A}^{\max }$ are the minimum and maximum number of EV aggregators planned to be installed in the power system depending on the construction budget of the EV charging infrastructure.

The sub-problem implemented the optimal dispatch of EV charging power under the configuration parameters of the EV aggregator given in the master problem. Accordingly, the sub-problem was formulated as:

$$
\min S P\left(\left.P_{E V A i}^{m}(t)\right|_{P_{E V A i}^{\max }(t), L C_{E V A i}}\right)=\sum_{t=0}^{T} \sum_{i=1}^{n} P(t)\left(P_{L d}(t)+P_{E V A i}^{m}(t)+P_{L s}(t)\right) \Delta t
$$

where $\left.P_{E V A i}^{m}(t)\right|_{P_{E V A i}^{\max }(t), L C_{E V A i}}$ represents the charging rate of individual $\mathrm{EV}$, which is the decision variable of the sub-problem that is to be optimized under the parameters given in the master problem.

$$
\mathrm{f}_{m}(L)=\frac{1}{\sqrt{2 \pi} \sigma_{\mathrm{m}} L} \exp \left(\frac{-\left(\ln L-\mu_{m}\right)^{2}}{2 \sigma_{m}^{2}}\right)
$$

$\mathrm{f}_{m}(L)$ is the probability distribution function of the distance $L$ travelled by each $\mathrm{EV} ; \sigma_{m}$ and $\mu_{m}$ are the parameters of exponential distribution that is used to simulate the stochastic travelled distance of EV. In this paper, it can be set to $\ln L \sim N\left(3.46,0.95^{2}\right)$.

On the basis of travel distance, the required power recharged of each EV was calculated by:

$$
S O C_{i n i}^{m}=S O C_{t a r}^{m}-\frac{\lambda L}{C_{b a t}^{m}}
$$

where $\lambda$ is the energy consumption per unit of distance.

The other commonly used constraints that describe the limits on the charging rate and EV battery SOC boundaries during the charging process under the control of EV aggregator can be found in existing research work [20-23].

\section{Simulation Results of the EV Charging Station in Oil-To-Electricity Transformation}

The simulation model built for oil-to-electricity transformation for regional automobiles was mainly composed of EV charging facilities changed from gas stations and the regional distribution network across a small town, as shown in Figures 1 and 3.

\subsection{Case Study}

The IEEE 123-bus distribution network with three-phase unbalanced load was used for simulation studies [24], as depicted in Figure 3. For time-series simulation, active and reactive loads were calculated by multiplying the coefficient that reflected the daily load curve, as shown in Figure 4a. 
The net load profile of the power network was calculated based on the wind power and solar power generation and average daily load of South Australia in [25]. The original load of the test power network was equivalent to the load level indicated by coefficient 0.8 . The two-tariff pricing was adopted for daily electric energy price including two main periods. The electricity prices applied to charging stations during the peak hours and the off-peak hours were set to 1.01 and $0.25 \mathrm{CNY} / \mathrm{kWh}$, according to the trial operation of EV charging stations in Shenzhen, Guangdong province of China. It can be seen in Figure 4a that the integration of solar and wind power generation exacerbated the load variation. EV charging load can be controlled for load leveling as shown in Figure 4b, thus ensuring the reliability of the power network. The minimal number and the maximal number of EV charging stations planned to be installed in the test network were set to 5 and 8 , considering existing parking lots in the region where automobiles were served by gas stations. The operating cost of the EV aggregator was assumed to be zero in the objective function.

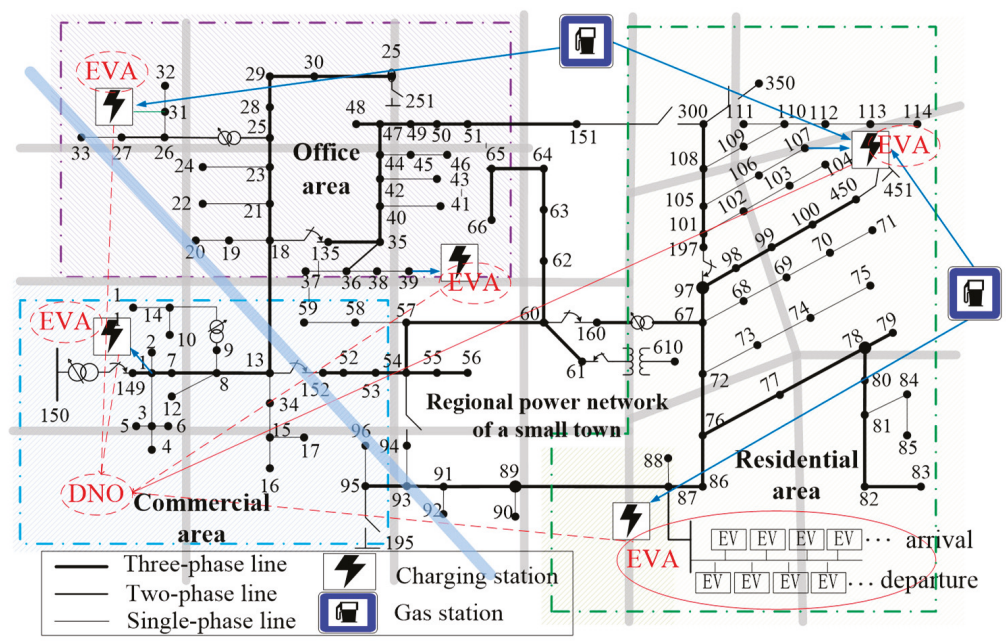

Figure 3. EV charging facilities integrated into the 123-bus distribution network.

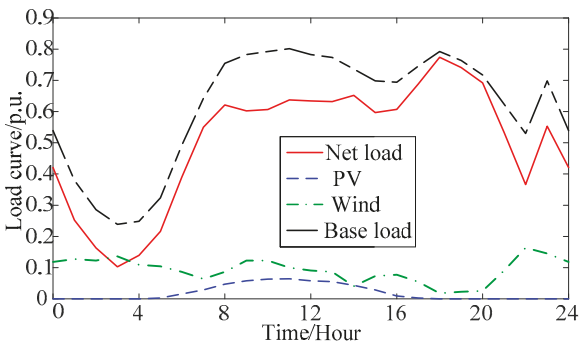

(a)

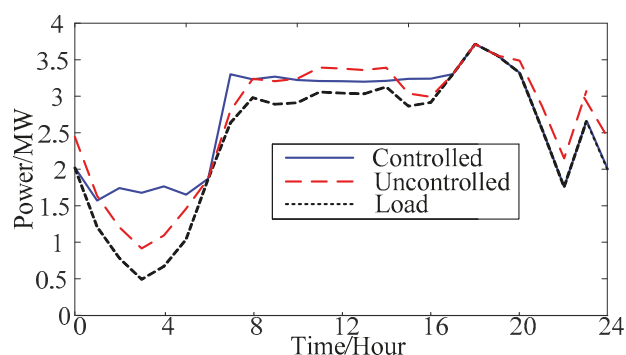

(b)

Figure 4. Power load profiles in the distribution network with renewable energy sources: (a) Load profiles of 123-bus power network; (b) EV charging load with and without control.

Charging stations transferred from two gas stations, as shown in Figure 1, were integrated into the regional distribution network represented by the test 123-node systems shown in Figure 3. Two of the charging stations were assumed to be the parking lots located in the residential area. The characteristics of EV arrival and parking period were identified as the home charging scenario. Two of the charging stations were assumed to be located among office buildings where most of the customers came to 
work. The last charging station was assumed to be in the commercial area, and composed of EVs parked for shopping and EVs parked overnight. By integrating charging stations into the regional distribution network, the two home charging stations were connected to bus 87 and bus 107 in the 123-node power system. The two office charging stations were connected to bus 31 and bus 39 , and the complex charging station was connected to bus 1 . The capacity of charging stations, which also means the charging load distribution among the five stations, will be calculated in the proposed EV charging optimization algorithm. In this paper, the acceptable range of bus voltage was set from 0.95 to 1.05 p.u. as the constraints of optimal configuration for EV charging stations. Four different types of EVs were adopted in the case study. As shown in Table 1, the battery capacity and charging rate varied among different EV models. The power electronic interfaced chargers were installed to meet the fast and slow charging needs of EVs, which ranged from 2.2 to $44 \mathrm{~kW}$, and the efficiency of energy conversion process was set to 0.9 in the simulation. As type I and II were designed to be capable of fast charging, these two types of EVs were assigned to office and commercial charging stations. Type III and IV were designed for slow charging and can be connected to standard household outlets. Therefore, these two types of EVs were assigned to home and also the complex charging station where both fast and slow charging facilities were installed. The parameters of EV charging scenario for computing battery energy and charging load are given in Tables 1 and 2.

Table 1. Simulation parameters for EV type and EV charging infrastructure.

\begin{tabular}{|c|c|c|c|c|c|c|}
\hline & EV Type & $\begin{array}{c}\text { Charging } \\
\text { Power }(\mathbf{k W})\end{array}$ & $\begin{array}{c}\text { Battery } \\
\text { Capacity (kWh) }\end{array}$ & $\begin{array}{c}\text { Target Stage of } \\
\text { Charge (SOC) }(\%)\end{array}$ & $\begin{array}{l}\text { No. of } \\
\text { EVs }\end{array}$ & $\begin{array}{l}\text { Distance per } \\
\text { Charge }(\mathbf{k m})\end{array}$ \\
\hline Fast & I: Tesla Model X & 13 & 60 & $90-95$ & 60 & 355 \\
\hline Slow & III: Chevrolet VOLT & 2.2 & 13.2 & $90-95$ & 150 & 80 \\
\hline Charging & IV: Changan EADO & 3.75 & 30 & $90-95$ & 150 & 200 \\
\hline
\end{tabular}

Table 2. Optimal configuration of EV charging stations.

\begin{tabular}{|c|c|c|c|c|c|}
\hline $\begin{array}{c}\text { Bus no./ } \\
\text { Type of Parking }\end{array}$ & Phase & $\begin{array}{l}\text { Power Level of } \\
\text { Charging Station }\end{array}$ & $\begin{array}{l}\text { Ratio of Charging } \\
\text { Station Capacities }\end{array}$ & $\begin{array}{c}\text { EV } \\
\text { Types }\end{array}$ & $\begin{array}{c}\text { Ratio of EV } \\
\text { Types (\%) }\end{array}$ \\
\hline 1/Complex & $\mathrm{A}$ & $\begin{array}{l}\text { Fast }+ \text { Slow } \\
\text { charging }\end{array}$ & $33 \%$ & I/II/III/IV & $14 / 14 / 36 / 36$ \\
\hline 31/Office & C & Fast charging & $13 \%$ & $\mathrm{I} / \mathrm{II}$ & $50 / 50$ \\
\hline 39/Office & B & Fast charging & $38 \%$ & I/II & $50 / 50$ \\
\hline 87/Home & B & Slow charging & $6 \%$ & III/IV & $50 / 50$ \\
\hline 107/Home & B & Slow charging & $10 \%$ & III/IV & $50 / 50$ \\
\hline
\end{tabular}

\subsection{Results and Discussion}

In the formulation of EV charging stations in oil-to-electricity transformation, charging load distributed at certain charging stations was equivalent to energy consumption for the automobiles served at the gas stations. The optimal configuration of charging stations is given in Table 2 . The averages of normal distribution for initial SOC and target SOC at minimum were set to 0.5 and 0.9 . The total number of EVs served by the charging stations was calculated to be 420 vehicles, with the assumption that the proportion of different types of EVs at each charging station was the same. For the office charging station at bus 31, the total number of EVs for type I and II was 20. Similarly, 150 EVs of type III and IV were served by the home charging station at bus 107. Four types of EV were served by the complex charging station at bus 1: 40 EVs of type I and II and 100 EVs of type III and IV. The number of EVs at each station were calculated according to the ratio of the capacities given by the proposed optimization algorithm for all EV charging stations. As shown in Table 2 and Figure 3, five optimal locations were selected from the parking lots in the regional power network and the capacities were set to minimize the impact of the additional EV charging load. 
The load pattern and voltage deviation of the network with EVs in the uncontrolled condition and charging optimization were compared to evaluate the efficiency of the proposed method, as shown in Figures $4 \mathrm{~b}$ and $5 \mathrm{a}$. In both cases, the configuration of EV charging stations adopted the results of the optimization algorithm, thus upgrading the performance of the uncontrolled case. Consequently, the uncontrolled charging with the best configuration was picked up from other randomly configured charging stations to analyze the advantage of optimally controlled EV charging load. The charging behavior of EV aggregations was regulated to achieve the valley filling and peak shaving, as seen in Figure $4 \mathrm{~b}$. The total power loss was reduced since the power losses at the peak hours were significantly curtailed, as shown in Figure 5b, in which ten lines with largest power flow were selected. The median, minimum, and maximum values of line losses can be seen in the box plot for the ten lines. The bus voltage of the test three-phase unbalanced power network is given in Figure 6, showing the reduction in voltage deviation. For the home charging stations at bus 87 and 107, most EVs were parked overnight and charged for commuting. The comparison of load and voltage profiles indicated that EV charging load is switched to the off-peak period during 24:00 to 6:00 the next day as shown in Figure 7a. Similar results can be found in Figure $7 \mathrm{~b}$ for the office charging stations at bus 31 and 39, where the two types of EVs were charged to match the network load profile as long as the constraints on the target SOC and the parking time can be satisfied. Noted that the two-tariff pricing directed the EV charging load to off-peak hours with a lower electricity price to cut down the cost, and the reduced power loss further minimized the objective function.

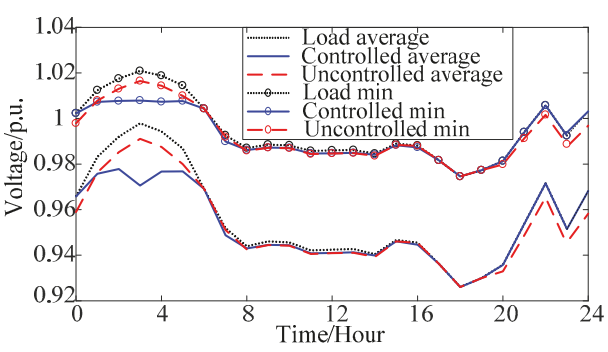

(a)

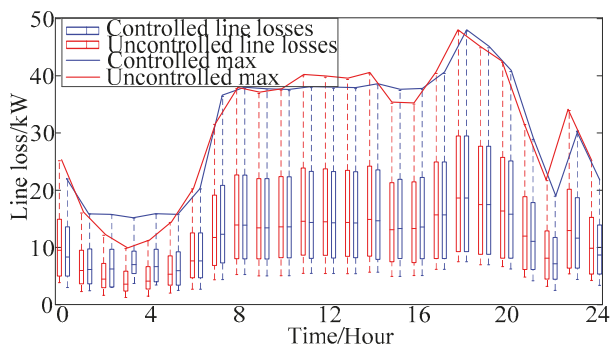

(b)

Figure 5. Power losses of distribution network with EV charging load: (a) Daily profile of total losses with and without EV charging control; (b) line losses of 123-bus power network at the peak hour.

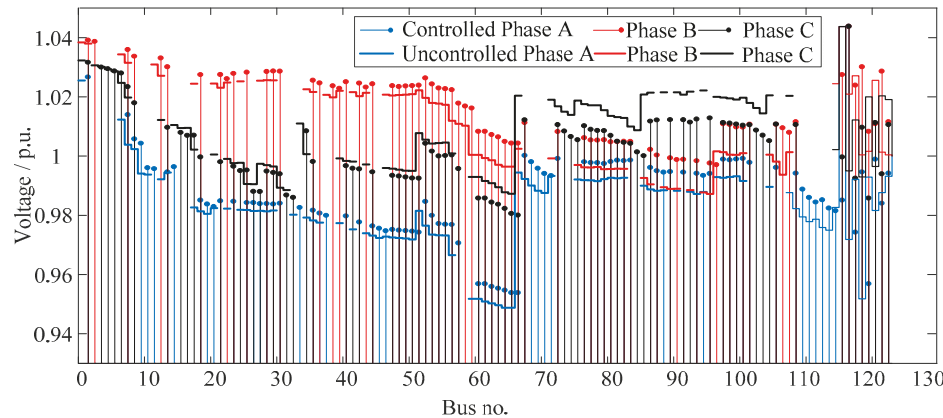

Figure 6. Bus voltage of the 123-bus test system with and without EV charging control. 


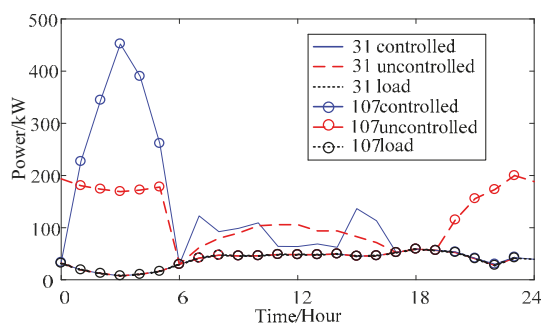

(a)

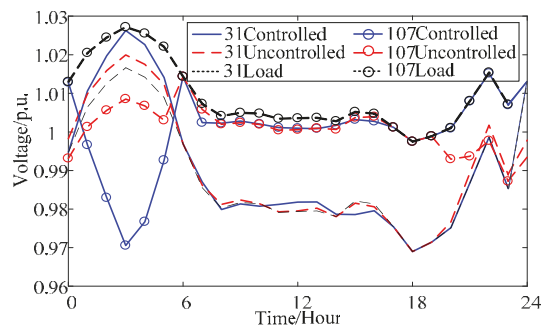

(b)

Figure 7. Load and voltage profiles at bus 31 phase-C and bus 107 phase-B: (a) Load profiles at different buses; (b) voltage profiles at different buses with and without EV charging control.

To calculate the charging plan for each EV, the capacity of the charging station fixed by the optimal configuration restricted the maximal charging power at each bus. The overall charging power during the parking period was allocated to each individual EV by the EV aggregator at a charging station. The charging plans for EV type I in uncontrolled and controlled cases are compared in Figure 8a, and the corresponding SOC curves for all EVs of type I are depicted in Figure 8b. The charging plans of EVs connected at different buses are shown in Figure 9, in which the charging profiles of two types of EVs served at the charging station connected to node- 31 phase- $\mathrm{C}$ and node- 107 phase-B are illustrated. The slow charging of type III and IV needed several hours to reach the target SOC, while the fast charging for type I and II can be finished within an hour if the parking period of the vehicle was limited. The charging rate of each individual EV at both fast charging and slow charging facilities was coordinated to enhance the economic and reliable operation of power system, without breaking the boundaries on service capacity of facilities and EV mobile needs described in Sections 2 and 3. It can be seen that EVs of different types reached the desired SOC before departure even though the charging power profile varied according to the solution given by optimal charging algorithm.

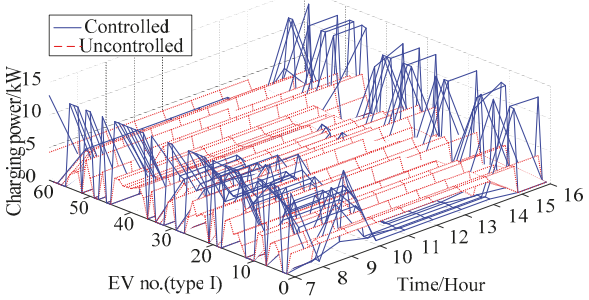

(a)

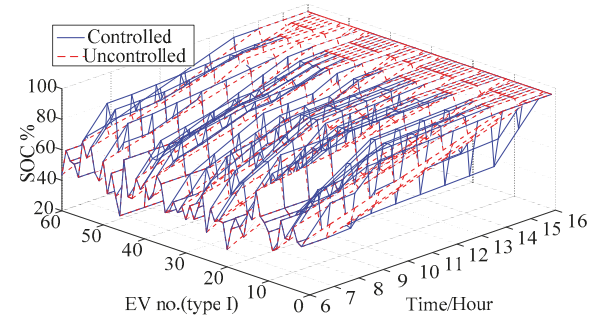

(b)

Figure 8. Charging rate and state of charge (SOC) profiles of EV type I at fast charging facilities with and without control: (a) EV charging curves (Tesla Model X); (b) SOC curves during the parking period.

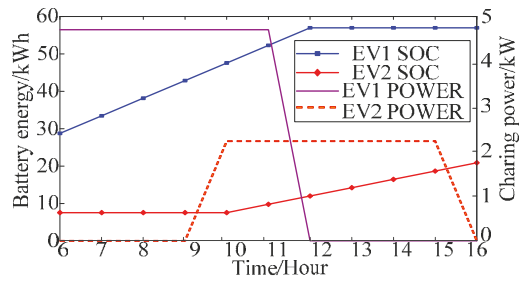

(a)

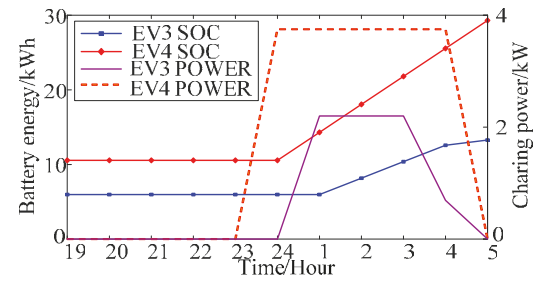

(b)

Figure 9. Charging profiles of EVs connected to facilities at different buses: (a) Charging profiles of EV type-I and type-II parked at bus 31; (b) charging profiles of EV type-III and type-IV parked at bus 107. 


\section{Conclusions}

To evaluate the impact of charging stations changed from gas stations in the oil-to-electricity transformation for automobiles, a mapping relation model between gas stations and charging stations serving vehicles in the same region was established by using membership degree function of EV and queuing model-based charging stations. A novel feature of the proposed EV charging stations was that the real-time data of gas stations and parking lots serving the regional automobiles was used to determine the stochastic EV charging time and charging demand distribution in the power network. Therefore, the controllability of EV charging load can be quantified by integrating the EV charging process with parameters and control variables in the optimal dispatch of the distribution network. With the defined boundaries and power system constraints, a combined optimization of configuration and operation of EV charging stations was developed for reducing the operating cost and risks of power system. A two-stage hybrid optimization algorithm composed of master problem and sub-problem was proposed to reduce the complexity of the combined optimization problem. The optimal setting of charging stations and the optimal charging created for each EV can improve the economic and reliable operation of the power system with flexible EV charging load. The proposed method can serve as an effective tool for evaluating the increased EV charging load and the controllability of charging stations in the oil-to-electricity transformation for automobiles across the town.

Author Contributions: Methodology and writing—original draft preparation, S.G.; writing-review and editing, J.W.; project administration, B.X.

Funding: This research was partially funded by the National Key R\&D Program of China (2016YFB0900400), and the National Nature Science Foundation of China (51707130).

Acknowledgments: This research was partially supported by the International Clean Energy Talent Programme (iCET). The three-month training in Sweden improved the research work in the paper and contributed to the data collection for modeling the regional gas station and parking lots from their online service websites and electronic map.

Conflicts of Interest: The authors declare no conflict of interest. The funders had no role in the design of the study; in the collection, analyses, or interpretation of data; in the writing of the manuscript, or in the decision to publish the results.

\section{Appendix A Detailed Modeling of Parking Lots Based on Queuing Theory}

This section presents the modeling of parking lots as a $M / M / Z / Z$ queuing system $[7,8]$. The variation of the parked vehicles given the data accessible online can be expressed by:

$$
\begin{gathered}
N^{k}(t)=L_{s}^{k}(t)=L_{b}^{k}+N_{c}^{k}(t) \quad \forall k \in M \\
\overline{N_{c}(t)}=\overline{L_{s}(t)}-\overline{L_{b}} \\
N_{b}^{k}(t+1)=N_{b}^{k}(t)+\Delta N_{b}^{k}(t+1), \quad \Delta N_{b}(t+1)=0 \quad \forall t \in T \\
N_{c}^{k}(t+1)=N_{c}^{k}(t)+\Delta N_{c}^{k}(t+1), \quad N_{c}(0)=0 \quad \forall t \in T \\
\Delta N_{b}^{k}(t)=N_{\text {in }}^{b k}(t)-N_{\text {out }}^{b k}(t) \\
\Delta N_{c}^{k}(t)=N_{\text {in }}^{c k}(t)-N_{\text {out }}^{c k}(t)
\end{gathered}
$$

where $L_{b}^{k}$ is the expected number of the basic EV fleet, which is also the queuing length in the steady state, and $N_{c}^{k}$ is the number of categorized EV fleet, which is varying over the time according to the arrival intensity.

The total number of both EV fleet can be read from the online data, but the arrival number of EVs at each time needs to be calculated. According to queuing theory, the arrival of EVs at parking lots is Poisson distribution:

$$
N_{\text {in }}^{c}(t) \sim \lambda_{c}
$$




$$
N_{i n}^{b}(t) \sim \lambda_{b}
$$

where $\lambda_{b}$ and $\lambda_{c}$ are the average arrival rate of the basic and categorized EVs respectively. The queuing length $L_{b}$ in the steady state can be calculated by applying the result of queuing theory, the parameters of a $\mathrm{M} / \mathrm{M} / \mathrm{Z} / \mathrm{Z}$ queuing system are adopted for parking lots:

$$
\begin{aligned}
& \lambda_{z}=\lambda_{b}, z=1,2, \cdots, z^{k} \\
& \mu_{z}=z \mu, z=1,2, \cdots, z^{k}
\end{aligned}
$$

where $Z^{k}$ is the capacity of $k^{\text {th }}$ parking lot, and $z$ is the number of EVs at a parking lot. The service time in this case is the parking period, which complies with negative exponent distribution $\frac{1}{\mu}$ in the queuing model.

$$
\begin{gathered}
P_{0}=\left(\sum_{i=0}^{z^{k}} \frac{\left(z^{k} e\right)^{i}}{i !}\right) \quad \mathrm{z}=1,2, \cdots, Z^{k} \\
P_{z}=\frac{\left(z^{k} \rho\right)^{z}}{z !} P_{0} \\
\rho=\frac{\lambda_{b}}{Z^{k} \mu}
\end{gathered}
$$

$P_{0}$ denotes the probability of no EV at parking, and $P_{z}$ denotes the probability of $z$ EVs at parking. $\rho$ is the service intensity of the parking lot defined by the queuing theory. Consequently, the number of EVs at the parking lot can be written as a function of $\rho$, and the arrival rate of basic EV fleet can be derived if the queuing length is given.

$$
\begin{gathered}
L_{b}^{k}=\sum_{z=0}^{z^{k}} z P_{z}=z^{k} \rho\left(1-P_{z^{k}}\right) \\
L_{b}^{k}=\overline{L_{b}^{k}}
\end{gathered}
$$

where $\overline{L_{b}^{k}}$ is the average number of basic EVs at parking that can be collected from online data.

Similarly, the categorized EV fleet in the queuing model can be quantified by assuming that the arrival rate at each time step is Poisson distribution. The arrival of categorized EVs is concentrated on several hours which can $b$ easily identified from the occupancy variation of the parking lots. As shown in Figure 1, the rising zone is assumed to be arrival period of categorized EV. Therefore, the arrival EV number $N_{i n}^{c k}$ can be calculated from $N_{c}^{k}(0)$ and $N_{c}^{k}(t)$ as shown in Equations (A2)-(A6), and can also be calculated by:

$$
\begin{gathered}
N_{i n}^{c k}(t)=N^{k}(t)-L_{b}^{k} \\
N_{i n}^{c k}=\sum_{t=1}^{T_{c}} N_{i n}^{c k}(t), \quad N_{i n}^{c k}(t) \sim \lambda_{c}^{k}(t) \\
\overline{N_{i n}^{c k}(t)}=\lambda_{c}^{k}(t) \cdot \Delta t \quad t \in\left[0, T_{c}\right]
\end{gathered}
$$

where $\overline{N_{i n}^{c k}(t)}$ is the average number of categorized EVs arriving at $t^{\text {th }}$ hour, which can be collected from online data as shown in Figure 1. $\lambda_{c}^{k}(t)$ is the Poisson distribution of categorized EVs arriving at $t^{\text {th }}$ hour. $T_{c}$ is the time period of categorized EV arrival. Following the queuing model of each parking lot, the arrival rate of categorized EV fleet at each time step can be derived by:

$$
P\left(N_{i n}^{c k}(t)=\mathrm{y}\right)=\frac{(\lambda t)}{y !} e^{-\lambda t} \quad \lambda=\lambda_{c}^{k}, \quad \mathrm{y}=0,1,2, \cdots
$$




$$
N_{i n}^{k}(t)=N_{i n}^{c k}(t)+N_{i n}^{b k}(t)
$$

$N_{i n}^{k}$ is total number of EVs parked at $k^{\text {th }}$ parking lot within $T_{c}$ hours, and $N_{i n}^{k}(t)$ is the number of EVs arriving at $k^{\text {th }}$ parking lot during each time interval. As described above, it is composed of two portions. $N_{i n}^{b k}(t)$ denotes the number of basic EVs arriving at $k^{t h}$ parking lot at $t^{\text {th }}$ hour, and $N_{i n}^{c k}(t)$ denotes the number of categorized EVs arriving at $k^{\text {th }}$ parking lot at $t^{\text {th }}$ hour. Based on the queuing theory, the number of EVs arriving at each time step is given by:

$$
\begin{gathered}
E\left(N_{i n}^{c k}(t)\right)=\sum_{y=0}^{\infty} y \frac{(\lambda t)^{k}}{k !} e^{-\lambda t}=\lambda_{c}^{k}(t) \cdot \Delta t \\
\lambda_{c}^{k}(t)=\frac{\overline{N_{i n}^{c k}(t)}}{\Delta t}=\frac{\overline{\Delta N_{c}^{k}(t)}}{\Delta t}
\end{gathered}
$$

where $\Delta N_{c}^{k}(t)$ is the difference of categorized EV number at $t^{t h}$ hour that can be read from the online data shown in Figure 1.

The membership degree of each individual EV to parking lot is correlated with the parking period in the queuing theory:

$$
\alpha_{i}^{k} \cdot T=\tau_{i}, \quad \tau_{i} \sim \frac{1}{\mu}
$$

where $\tau_{i}$ is the parking period of $i^{\text {th }} \mathrm{EV}$ at one parking lot. The service time complies with negative exponential distribution:

$$
\begin{gathered}
P\left(\tau_{i} \leq t\right)=\left\{\begin{array}{cc}
1-e^{-\mu t} & t \geq 0 \\
0 & t<0
\end{array}\right. \\
E\left(\tau_{i}\right)=\int \begin{array}{c}
+\infty \\
0
\end{array} \mu+e^{-\mu t} d t=\frac{1}{\mu} \\
\frac{1}{\mu}=\overline{\alpha_{i}^{k}} \cdot T=\bar{\tau}_{i}
\end{gathered}
$$

where $\overline{\tau_{i}}$ is the average EV parking period that can be collected from online data. As all the other parameters are calculated, the arrival and the duration of EVs at the parking lot can be completely quantified.

\section{References}

1. Tang, W.; Bi, S.; Zhang, Y.J. Online charging scheduling algorithms of electric vehicles in smart grid: An overview. IEEE Commun. Mag. 2016, 54, 76-83. [CrossRef]

2. Bloomberg New Energy Finance. Electric Vehicle Outlook 2017. July 2017. Available online: https: //data.bloomberglp.com/bnef/ (accessed on 10 March 2019).

3. Farid, A.M. A hybrid dynamic system model for multimodal transportation electrification. IEEE Trans. Control Syst. Technol. 2017, 25, 940-951. [CrossRef]

4. Farzin, H.; Moeini-Aghtaie, M.; Fotuhi-Firuzabad, M. Reliability studies of distribution systems integrated with electric vehicles under battery-exchange mode. IEEE Trans. Power Deliv. 2016, 31, 2473-2482. [CrossRef]

5. Ying, Z.M.; Wu, X.; Yang, W. Carbon emission benefit accounting of Hangzhou bus oil-to-electricity project. Acta Ecol. Sin. 2018, 38, 6452-6464.

6. Krishna, K.M.; Pandey, N.K.; Thimmalapura, S. Break-even analysis and economic viability of powertrain electrification-An analytical approach. In Proceedings of the IEEE Transportation Electrification Conference, Pune, India, 13-15 December 2017.

7. Shaaban, M.F.; Atwa, Y.M.; El-Saadany, E.F. PEVs modeling and impacts mitigation in distribution networks. IEEE Trans. Power Syst. 2013, 28, 1122-1131. [CrossRef] 
8. Shojaabadi, S.; Abapour, S.; Abapour, M. Optimal planning of plut-in hybrid electric vehicle charging station in distribution network considering demand response programs and uncertainties. IET Gener. Trans. Distrib. 2016, 10, 3330-33340. [CrossRef]

9. Gong, Q.; Midlam-Mohler, S.; Serra, E.; Marano, V. PEV charging control for a parking lot based on queuing theory. In Proceedings of the American Control Conference, Washington, DC, USA, 17-19 June 2013; pp. 1124-1129.

10. Chukwu, U.C.; Mahajan, S.M. V2G parking lot with PV rooftop for capacity enhancement of a distribution system. IEEE Trans. Sustain. Energy 2014, 5, 119-127. [CrossRef]

11. Bae, S.; Kwasinski, A. Spatial and temporal model of electric vehicle charging demand. IEEE Trans. Smart Grid 2012, 3, 3394-3403.

12. Albarbi, W.; Bhattacharya, K. Electric vehicle charging facility as a smart energy microhub. IEEE Trans. Sustain. Energy 2017, 8, 616-628.

13. Ko, K.S.; Han, S.; Sung, D.K. Performance-Based settlement of frequency regulation for electric vehicle aggregators. IEEE Trans. Smart Grid 2018, 9, 866-875. [CrossRef]

14. Wenzel, G.; Negrete-Pincetic, M.; Olivares, D.E. Real-Time charging strategies for an electric vehicle aggregator to provide ancillary services. IEEE Trans. Smart Grid 2018, 9, 5141-5151. [CrossRef]

15. Vagropoulos, S.I.; Balaskas, G.A.; Bakirtzis, A.G. An investigation of plug-In electric vehicle charging impact on power systems scheduling and energy costs. IEEE Trans. Power Syst. 2017, 32, 1902-1912. [CrossRef]

16. DeForest, N.; MacDonald, J.S.; Black, D.R. Day ahead optimization of an electric vehicle fleet providing ancillary services in the Los Angeles Air Force Base vehicle-to-grid demonstration. Appl. Energy 2018, 210, 987-1001. [CrossRef]

17. Bessa, R.J.; Matos, M.A. Optimization models for EV aggregator participation in a manual reserve market. IEEE Trans. Power Syst. 2013, 28, 3085-3095. [CrossRef]

18. Wei, W.; Liu, F.; Mei, S. Charging strategies of EV aggregator under renewable generation and congestion: A normalized Nash equilibrium approach. IEEE Trans. Smart Grid 2016, 7, 1630-1641. [CrossRef]

19. Shafie-khah, M.; Heydarian-Forushani, E.; Golshan, M.E.H. Optimal trading of plug-in electric vehicle aggregation agents in a market environment for sustainability. Appl. Energy 2016, 162, 601-612. [CrossRef]

20. Ko, K.S.; Sung, D.K. The effect of EV aggregators with time-varying delays on the stability of a load frequency control system. IEEE Trans. Power Syst. 2018, 33, 669-680. [CrossRef]

21. Li, W.J.; Tan, X.; Sun, B. Optimal power dispatch of a centralised electric vehicle battery charging station with renewables. IET Commun. 2018, 12, 579-585.

22. Perez-Diaz, A.; Gerding, E.; McGroarty, F. Coordination and payment mechanisms for electric vehicle aggregators. Appl. Energy 2018, 212, 185-195. [CrossRef]

23. Floch, C.L.; Kara, E.C.; Moura, S. PDE modeling and control of electric vehicle fleets for ancillary services: A discrete charging case. IEEE Trans. Smart Grid 2018, 9, 573-581. [CrossRef]

24. Liu, Z.; Wen, F.; Ledwich, G. Optimal planning of electric-vehicle changing stations in distribution systems. IEEE Trans. Power Deliv. 2013, 28, 102-110. [CrossRef]

25. Mosadeghy, M.; Yan, R.; Saha, T.K. A time-dependent approach to evaluate capacity value of wind and solar PV generation. IEEE Trans. Sustain. Energy 2016, 7, 129-138. [CrossRef]

(C) 2019 by the authors. Licensee MDPI, Basel, Switzerland. This article is an open access article distributed under the terms and conditions of the Creative Commons Attribution (CC BY) license (http://creativecommons.org/licenses/by/4.0/). 


\title{
Article \\ Hierarchical Control of Nonlinear Active Four-Wheel-Steering Vehicles
}

\author{
Jie Tian ${ }^{1}$, Jie Ding ${ }^{1}$, Yongpeng Tai ${ }^{1}$ and Ning Chen ${ }^{2, *}$ \\ 1 College of Automobile \& Traffic Engineering, Nanjing Forestry University, Nanjing 210037, China; \\ tianjie@njfu.com.cn (J.T.); dingj@njfu.edu.cn (J.D.); tai@njfu.edu.cn (Y.T.) \\ 2 College of Mechanical and Electronic Engineering, Nanjing Forestry University, Nanjing 210037, China \\ * Correspondence: chenning@njfu.com.cn; Tel.: +86-138-515-02589
}

Received: 17 September 2018; Accepted: 23 October 2018; Published: 26 October 2018

check for updates

\begin{abstract}
A new type of hierarchical control is proposed for a four-wheel-steering (4WS) vehicle, in which both the sideslip angle and yaw rate feedback are used, and the saturation of the control variables (i.e., the front and rear steering angles) is considered. The nonlinear three degrees of freedom (3DOF) 4WS vehicle model is employed to describe the uncertainties originating from the operating situations. Further, a normal front-wheel-steering (2WS) vehicle with a drop filter of the sideslip angle is selected as the reference model. The inputs for the rear and front steering angles of the linear 2DOF 4WS, required to achieve the performances described by the reference model, are obtained and controlled by the upper controller. Further, the lower controller is designed to eliminate the state error between the linear 2DOF and nonlinear 3DOF 4WS vehicle models. The simulation results of several vehicle models with/without the controller are presented, and the robustness of the hierarchical control system is analyzed. The simulation results indicate that using the proposed hierarchical controller yields the same performance between the nonlinear 4WS vehicle and the reference model, in addition to exhibiting good robustness.
\end{abstract}

Keywords: active 4WS system; hierarchical control; decoupling; fractional sliding mode control

\section{Introduction}

The active four-wheel-steering (4WS) system is studied widely to improve the handling stability at high speeds, and the maneuverability at low speeds. With the emergence of intelligent vehicle systems (IVS), the 4WS system can also be used to solve the path tracking problem to ensure that the vehicle can follow the scheduled route, and its algorithm is mainly about determining the required steering angle to adjust the dynamic turning point on the road curvature center [1-3]. Hitherto, some control strategies and methods have been proposed to improve the aforementioned control goals, such as fuzzy, adaptive, feedforward, feedback, optimal, $\mathrm{H}_{\infty}$, sliding mode, decoupling, and neural network controls, as well as $\mu$ synthesis.

A yaw stability controller based on fuzzy logic was proposed, which took the yaw angular velocity error, steering angle given by driver, and side slip angle as input, calculated the additional steering angle as output, and compared with the existing fuzzy control system with two inputs of the yaw angle and yaw angular velocity. The results showed that the proposed fuzzy yaw controller had better performance [4]. Considering the rear wheel steering resistance moment, the $\mathrm{H}_{2} / \mathrm{H}_{\infty}$ mixed robust controller was designed to track the desired yaw rate, and can be obtained according to the variable transmission ratio strategy [5]. Applying the yaw rate tracking strategy to the 4WS vehicle and considering the resistance moment of rear wheel steering, the $\mathrm{H}_{2} / \mathrm{H}_{\infty}$ infinity hybrid robust controller was designed and the stability control of $4 \mathrm{WS}$ vehicle was studied [6]. A $\mu$ synthesis robust controller was designed to overcome the model uncertainty [7]. Considering the velocity changing, a linear 
parameter varying (LPV) controller was designed to improve the handling stability, safety, and comfort of the 4WS vehicle [8]. A high-level control redistribution method based on LPV control framework was adopted to realize torque vectorization and steering, which can ensure the speed and path tracking of the four-wheel independently-actuated (4WIA) vehicle in the case of one or even several wheeled motor failures or performance degradation [9]. Using the preview theory, robust theory, and adaptive path following, the 4 WS vehicle can exhibit a good path tracking capability in both the longitudinal and lateral directions [10]. Based on the singular boundary theory and Lyapunov stability theory, the uncertainty of the 4 WS vehicle was debated [11]. Combined with the linear programming algorithm and the improved sliding mode algorithm, a joint control strategy combining the exponential reaching law with the saturation function was proposed. The strategy can control the side slip angle, yaw speed, and yaw moment of the vehicle well to improve the handling and stability performance of the vehicle significantly [12]. Based on sliding mode control, an integrated control system with 4WS and direct yaw moment control (DYC) was proposed to improve vehicle handling stability [13]. A fast terminal sliding mode controller was designed to suppress the external disturbance of the steer-by-wire (SBW) system. Considering the unstructured and structural uncertainties, a robust controller was designed by using the synthesis method; the controller order reduction was realized based on Hankel-Norm approximation, and the extended Kalman filter was used to estimate the sideslip angle [14].

In addition, relative investigations have shown that the decoupling control of the vehicle steering characteristics can improve the vehicle's safety, driving, and even exhibit accurate trajectory tracking because of the vehicle's individual control of the lateral and yaw motions [15]. A robust triangular decoupling control strategy was proposed to decouple the front axle acceleration from the yaw rate [16]. A lateral-force observer based on the disturbance observer theory was developed, aiming to overcome the fragile robustness of the decoupling control for road conditions, and used along with the yaw-moment observer in a small-scale electric vehicle. Further, the robustness against parameter variation was improved [17]. The necessary and sufficient conditions for the solution of diagonal decoupling were presented, and the vehicle model (lateral plus roll dynamics) was implemented to control the steering angle and sideslip angle of the vehicle independently, using a static state feedback law with a static pre-compensator [18]. An asymptotic decoupling control for the 4WS vehicle based on an easy measurement feedback was introduced. Its control inputs were the front wheel steering angle, and the torques of front and rear wheels; further, the $\mathrm{H} \infty$ optimization method was used to derive the desired eigenvalues [19]. Pre-compensation decoupling with $\mathrm{H}_{\infty}$ performance control for a 3DOF nonlinear 4WS vehicles was studied to consider the influence of disturbances on the output under the $\mathrm{H}_{\infty}$ index. The results showed that the longitudinal velocity, lateral velocity, and yaw rate can be controlled independently, and the drivers can steer rapidly [20]. A simple decoupling subsystem consisting of longitudinal, lateral, and yaw dynamics is derived by choosing the combination of longitudinal acceleration/braking force and steering angle of front and rear wheels as the virtual control input [21].

However, compared with diagonal decoupling, triangular decoupling is more complicated, particularly for uncertain systems [22]. Further, it is difficult to build fuzzy rules in all situations for fuzzy control algorithms, and the adaptive control is suitable for the slow time-varying system that is vastly different from the actual steering system whose parameters may change quickly [23]. In addition, the control strategies for a 4WS system can be divided into two types: one is to make the sideslip angle zero, corresponding to trajectory preserving problem, the other one is to track the desired yaw rate. Most studies on the control of 4WS vehicles usually aim to determine the rear wheel steering angle based on the certain front wheel steering angle, and compare the response of 4WS vehicle under the inputs of the front and rear wheel steering angles with that of the 2WS vehicle.

The novelties of this study are as follows: (1) The reference model is obtained by filtering the sideslip angle of a normal 2WS vehicle, which is very different from past practices. (2) According to the $2 \mathrm{WS}$ reference model, the front and rear wheel steering angles of linear 4WS vehicle are determined simultaneously by the decoupling controller, and its response is consistent with that of the 2WS 
reference model completely. (3) Considering that it is very difficult to design controllers directly by using nonlinear models, a new hierarchical controller is proposed in this paper. Firstly, the upper controller controls the linear 4WS model to have the same response as the 2WS reference model. Then the lower controller controls the nonlinear 4WS model to have exactly the same response as the linear 4WS. (4) The upper control strategy, including the decoupling controller and asymptotic tracking controller, is designed to provide the standard input for the linear $4 \mathrm{WS}$ vehicle model. The former is to decouple the sideslip angle and yaw rate from each other, which can obtain the required front and rear wheel steering angles. The latter is to control the linear 4WS vehicle model to track the reference model completely. (5) The lower control strategy, including the optimal controller and fractional sliding mode controller, is designed to remove the adverse effects of the state errors between the linear 2DOF and nonlinear 3DOF 4WS vehicle models. The former is to eliminate the state error caused by the different initial states. The latter is to eliminate the state error caused by the uncertainty.

The subsequent parts of this paper are organized as follows: In Section 2, the vehicle models, including the nonlinear 3DOF 4WS/2WS model, the linear 2DOF 4WS/2WS model, and the reference model are presented. The hierarchical control strategies, including the upper control strategy (i.e., the decoupling and the asymptotic tracking control) and the lower control strategy (i.e., the optimal control and the fractional sliding mode control), are described in Section 3. The comparison of the simulation results of the linear 2DOF 2WS vehicle, reference model, nonlinear 3DOF 2WS vehicle, real vehicle with the hierarchical controller and linear $4 \mathrm{WS}$ with linear quadratic regulator (LQR) controller are reported in Section 4. Finally, the conclusion is summarized in Section 5.

\section{Model Description}

We consider three types of vehicle models for the hierarchical control system. The first one is a nonlinear 3DOF 4WS/2WS vehicle model used to describe the system uncertainty, which is controlled by a lower controller to ensure the system robustness. The second one is a linear 2DOF $4 \mathrm{WS} / 2 \mathrm{WS}$ vehicle model controlled by an upper controller, which can supply the standard inputs to 4WS vehicles. The last one is a reference model to provide the desired yaw rate and sideslip angle.

\subsection{Nonlinear 3DOF 4WS/2WS Vehicle Model}

The 4 WS vehicle model considering the lateral, yaw, and roll motions is given by

$$
\left\{\begin{array}{l}
I_{z} \dot{\gamma}-I_{x z} \ddot{\phi}=2 a F_{f}-2 b F_{r} \\
m u_{x}(\dot{\beta}+\gamma)+m_{s} h_{s} \ddot{\phi}=2 F_{f}+2 F_{r} \\
I_{x} \ddot{\phi}-I_{x z} \dot{\gamma}+m_{s} h_{s} u_{x}(\dot{\beta}+\gamma)=\left(m_{s} g h_{s}-k_{\phi}\right) \phi-c_{\phi} \dot{\phi}
\end{array},\right.
$$

where $I_{z}$ is the moment of inertia of the vehicle about the z-axis, $I_{x}$ is the roll moment of inertia of the vehicle body about the x-axis, $I_{x z}$ is the moment of the inertia product, $\gamma$ is the yaw rate, $\phi$ is the roll angle of the vehicle body, $a$ and $b$ are the distances from the centroid to the front and rear axle, $F_{f}$ and $F_{r}$ are the lateral forces of the front and rear wheel respectively, $m$ is the vehicle mass, $u_{x}$ is the longitudinal speed of the vehicle, $\beta$ is the sideslip angle, $m_{s}$ is the mass of the vehicle body, $h_{s}$ is the distance of the body centroid to the roll center axis, $g$ is the gravitational acceleration, $k_{\phi}$ is the sum of the roll stiffness of the front and rear suspensions, and $c_{\phi}$ is the sum of the roll damping of the front and rear suspensions.

Considering the nonlinearity of the tires, the lateral forces of the front and rear wheels can be expressed as follows [17]:

$$
F_{i}=\varphi D_{i} \sin \left(C_{i} \arctan \left(B_{i}\left(1-E_{i}\right) \frac{\alpha_{i}}{\varphi}+E_{i} \arctan \left(B_{i} \frac{\alpha_{i}}{\varphi}\right)\right)\right) \quad i=f, r,
$$


where

$$
\alpha_{f}=\beta+a \gamma / u_{x}-R_{f} \phi-\delta_{f}, \alpha_{r}=\beta-b \gamma / u_{x}-R_{r} \phi-\delta_{r},
$$

$\varphi$ is the road adhesion coefficient (ranging from 0.2 to 1 ), $B_{i}, C_{i}, D_{i}, E_{i}$ are the coefficients, $\alpha_{i}$ is the slip angle of the wheel. $R_{f}$ and $R_{r}$ are the roll deflection coefficients of the front and rear axles, respectively; $\delta_{f}$ and $\delta_{r}$ are the steering angles of the front and rear wheels, respectively.

Equation (2) can also be expressed as

$$
F_{y i}=k_{i} \alpha_{i}+f_{y i}\left(\alpha_{i}, \varphi\right) i=f, r,
$$

where $k_{i}$ is the cornering stiffness of the front or rear wheel and $k_{i}=B_{i} C_{i} D_{i}$. Then, according to Equations (2) and (4), the following expression can be obtained:

$$
f_{y i}\left(\alpha_{i}, \varphi\right)=\varphi D_{i} \sin \left(C_{i} \arctan \left(B_{i}\left(1-E_{i}\right) \frac{\alpha_{i}}{\varphi}+E_{i} \arctan \left(B_{i} \frac{\alpha_{i}}{\varphi}\right)\right)\right)-k_{i} \alpha_{i} .
$$

Substituting (4) into (1), we define $\widetilde{x}=\left[\begin{array}{lll}\gamma & \beta & \dot{\phi}\end{array}\right]^{T}, u=\left[\begin{array}{ll}\delta_{f} & \delta_{r}\end{array}\right]^{T}$; thus, the state space model can be expressed by

$$
\widetilde{E} \dot{\tilde{x}}=\widetilde{A} \widetilde{x}+\widetilde{B} u+\widetilde{F}
$$

where

$$
\begin{aligned}
& \widetilde{E}=\left[\begin{array}{ccc}
I_{z} & 0 & -I_{x z} \\
0 & m u_{x} & m_{s} h \\
-I_{x z} & m_{s} h u_{x} & I_{x}
\end{array}\right], \widetilde{A}=\left[\begin{array}{ccc}
2\left(a^{2} k_{f}+b^{2} k_{r}\right) / u_{x} & 2\left(a k_{f}-b k_{r}\right) & 0 \\
-m u_{x}+2\left(a k_{f}-b k_{r}\right) / u_{x} & 2\left(k_{f}+k_{r}\right) & 0 \\
-m_{s} h u_{x} & 0 & -c_{\phi}
\end{array}\right], \\
& \widetilde{B}=\left[\begin{array}{cc}
-2 a k_{f} & 2 b k_{r} \\
-2 k_{f} & -2 k_{r} \\
0 & 0
\end{array}\right], \widetilde{F}=\left[\begin{array}{l}
2 a f_{y f}\left(\alpha_{f}, \varphi\right)-2 b f_{y r}\left(\alpha_{r}, \varphi\right)+2\left(-a R_{f} k_{f}+\mathrm{b} R_{r} k_{r}\right) \phi \\
2 f_{y f}\left(\alpha_{f}, \varphi\right)+2 f_{y r}\left(\alpha_{r}, \varphi\right)-2\left(R_{f} k_{f}+R_{r} k_{r}\right) \phi \\
m_{s} g h \phi-k_{\phi} \phi
\end{array}\right] .
\end{aligned}
$$

When $\delta_{r}=0$, the 4WS vehicle model described by Equation (6) will become the 2WS vehicle model, which will be used in the simulation for the comparison.

\subsection{Linear 2DOF 4WS/2WS Vehicle Model}

To obtain the standard input of the $4 \mathrm{WS}$ vehicle by the upper controller, the $4 \mathrm{WS}$ vehicle model with the lateral and yaw motions is established. By defining $\bar{x}=\left[\begin{array}{ll}\gamma & \beta\end{array}\right]^{T}$, the space state equation is as follows:

$$
\dot{\bar{x}}=\bar{A} \bar{x}+\bar{B} u,
$$

where

$$
\bar{A}=\left[\begin{array}{cc}
2 \frac{a^{2} k_{f}+b^{2} k_{r}}{I_{I} u_{x}} & 2 \frac{a k_{f}-b k_{r}}{I_{z}} \\
2 \frac{a k_{f}-\bar{b} k_{r}}{m u_{x}^{2}}-1 & 2 \frac{k_{f}+k_{r}}{m u_{x}}
\end{array}\right]=\left[\begin{array}{cc}
a_{11} & a_{12} \\
a_{21} & a_{22}
\end{array}\right], \bar{B}=\left[\begin{array}{cc}
\frac{-a k_{f}}{I_{z}} & \frac{b k_{r}}{I_{z}} \\
\frac{-k_{f}}{m u_{x}} & \frac{-k_{r}}{m u_{x}}
\end{array}\right]=\left[\begin{array}{ll}
b_{11} & b_{12} \\
b_{21} & b_{22}
\end{array}\right] .
$$

When $\delta_{r}=0$, the linear 2DOF 2WS vehicle, which will also be used in the simulation for the comparison, can be described as follows:

$$
\dot{\hat{x}}=\hat{A} \hat{x}+\hat{B} \hat{u},
$$

where

$$
\hat{x}=\left[\begin{array}{ll}
\gamma & \beta
\end{array}\right]^{T}, \hat{u}=\delta_{f}, \hat{A}=\left[\begin{array}{cc}
2 \frac{a^{2} k_{f}+b^{2} k_{r}}{I_{z} u_{x}} & 2 \frac{a k_{f}-b k_{r}}{I_{z} k_{r}} \\
2 \frac{a k_{f}-b k_{r}}{m u_{x}^{2}}-1 & 2 \frac{k_{f}+k_{r}}{m u_{x}}
\end{array}\right], \hat{B}=\left[\begin{array}{c}
\frac{-a k_{f}}{I_{z}} \\
\frac{-k_{f}}{m u_{x}}
\end{array}\right] .
$$




\subsection{Reference Model}

The typical early practice is to define a first-order system as the reference model and maintain the sideslip angle as zero [5]. However, some studies have shown that 4WS vehicles with zero sideslip angle in cornering maneuvers exhibit significant under-steering characteristic and the zero sideslip angle will contribute to the driver's unadaptation [1]. Therefore, in this study, the reference model is obtained by filtering the sideslip angle of the normal 2 WS vehicle described by Equation (10). Its filtering magnitude can be adjusted in real time and depends on the drivers' requirements. The transfer function for the drop filter of the sideslip angle can be expressed as follows:

$$
G(s)=\frac{\eta \omega_{n}^{2}}{s^{2}+\sqrt{2} \omega_{n}+\omega_{n}^{2}},
$$

where $\eta$ is the gain coefficient that can be used to adjust the amplitude of the sideslip angle, $\omega_{n}$ is the cutoff frequency, and $s$ is the complex variable.

\section{Design of Hierarchical Controller}

Because the considered $4 \mathrm{WS}$ vehicle is equipped with a four-wheel steer-by-wire system, only the steering wheel angle issued by the driver is used to describe the driving intention. In fact, the real $4 \mathrm{WS}$ vehicle is affected by the parameter uncertainty, tire nonlinearity, non-modeling dynamics, and coupling disturbance caused by the roll. Under such condition, the adopted hierarchical control structure is as depicted in Figure 1. The desired yaw rate and sideslip angle, $\gamma_{d}$ and $\beta_{d}$, are generated by the reference model with only the input of the front wheel steering angle, which is proportional to the steering wheel angle. The upper controller computes the inputs of the front and rear steering angles for the linear $2 \mathrm{DOF} 4 \mathrm{WS}$ vehicle; further, $\delta_{f}$ and $\delta_{r}$, are required to obtain the required performances described by the reference model. Owing to the many differences between the linear 2DOF 4WS vehicle and the nonlinear 3DOF 4WS vehicle, we used the theory of the fractional calculus and the sliding mode control to design the lower controller to guarantee the robustness of the whole control system.

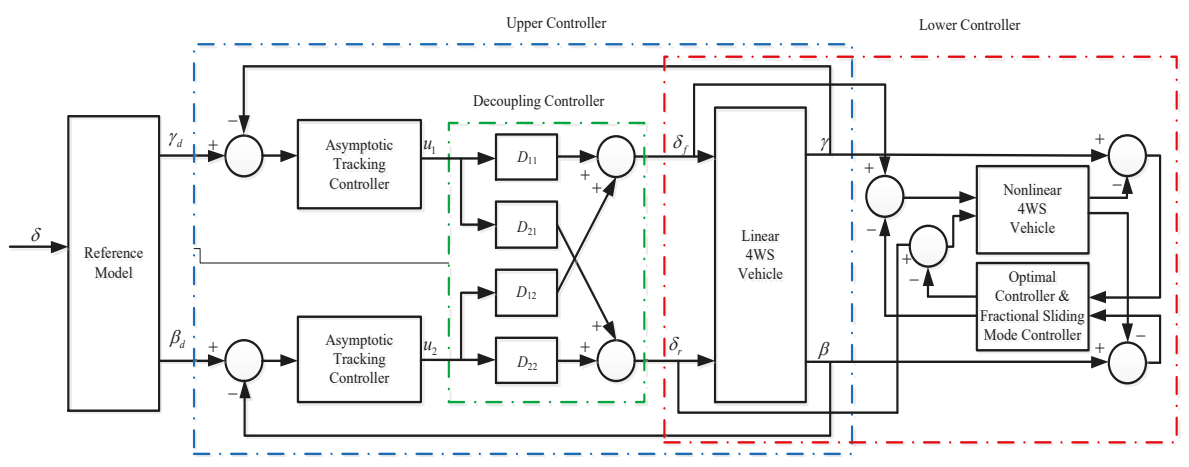

Figure 1. Hierarchical control system.

\subsection{Design of Upper Controller}

The standard input of the $4 \mathrm{WS}$ vehicle can be obtained by the upper controller according to the reference model with the single input of the front wheel steering angle. To completely follow the desired sideslip angle and yaw rate, the decoupling of the linear 2DOF 4WS system is performed, and the transfer functions between $\gamma$ and $u_{1}$, and between $\beta$ and $u_{2}$ are obtained. Subsequently, by the asymptotic tracking controller, the linear 2DOF 4WS system can yield the same response as the reference model. 


\subsubsection{Decoupling of Linear 2DOF 4WS System}

The following expression can be obtained from Equation (8):

$$
\left[\begin{array}{l}
\gamma(s) \\
\beta(s)
\end{array}\right]=G(s)\left[\begin{array}{c}
\delta_{f}(s) \\
\delta_{r}(s)
\end{array}\right]=\left[\begin{array}{ll}
G_{11}(s) & G_{12}(s) \\
G_{21}(s) & G_{22}(s)
\end{array}\right]\left[\begin{array}{c}
\delta_{f}(s) \\
\delta_{r}(s)
\end{array}\right],
$$

where

$$
\begin{aligned}
& G_{11}(s)=\frac{s b_{11}-a_{22} b_{11}+a_{12} b_{21}}{s^{2}-\left(a_{11}+a_{22} b_{1}+a_{11} a_{22}-a_{21} a_{12}\right.}, G_{12}(s)=\frac{s b_{12}-a_{22} b_{12}+a_{12} b_{22}}{s^{2}-\left(a_{11}+a_{22} 2\right) s+a_{11} a_{22}-a_{21} a_{12}}, \\
& G_{21}(s)=\frac{s b_{21}-a_{11} b_{21}+a_{21} b_{11}}{s^{2}-\left(a_{11}+a_{22}\right) s+a_{11} a_{22}-a_{21} a_{12}}, G_{22}(s)=\frac{\left(s b_{22}-a_{11} b_{22}+a_{21} b_{12}\right.}{s^{2}-\left(a_{11}+a_{22}\right) s+a_{11} a_{22}-a_{21} a_{12}} .
\end{aligned}
$$

As shown in Equation (13), both $\gamma$ and $\beta$ are controlled by both $\delta_{f}$ and $\delta_{r}$. Therefore, it is necessary to decouple $\gamma$ from $\beta$ to ensure that the linear 2DOF 4WS system can track the two variables of the reference model simultaneously. Subsequently, two independent input variables, $u_{1}$ and $u_{2}$, are introduced as shown in Figure 1.

From Figure 1, the relationship between $\delta_{f}, \delta_{r}$ and $u_{1}, u_{2}$ are as follows:

$$
\left[\begin{array}{c}
\delta_{f}(s) \\
\delta_{r}(s)
\end{array}\right]=\left[\begin{array}{ll}
D_{11}(s) & D_{12}(s) \\
D_{21}(s) & D_{22}(s)
\end{array}\right]\left[\begin{array}{l}
u_{1}(s) \\
u_{2}(s)
\end{array}\right] .
$$

Substitute Equation (15) into Equation (13), we obtain

$$
\left[\begin{array}{l}
\gamma(s) \\
\beta(s)
\end{array}\right]=\left[\begin{array}{ll}
G_{11}(s) & G_{12}(s) \\
G_{21}(s) & G_{22}(s)
\end{array}\right]\left(\begin{array}{cc}
D_{11}(s) & D_{12}(s) \\
D_{21}(s) & D_{22}(s)
\end{array}\right)\left(\begin{array}{l}
u_{1}(s) \\
u_{2}(s)
\end{array}\right) .
$$

Only when

$$
\left(\begin{array}{ll}
G_{11}(s) & G_{12}(s) \\
G_{21}(s) & G_{22}(s)
\end{array}\right)\left(\begin{array}{ll}
D_{11}(s) & D_{12}(s) \\
D_{21}(s) & D_{22}(s)
\end{array}\right)=\left(\begin{array}{cc}
G_{11}(s) & 0 \\
0 & G_{22}(s)
\end{array}\right),
$$

can $\gamma$ and $\beta$ be controlled by $u_{1}$ and $u_{2}$, respectively. That is,

$$
\left[\begin{array}{l}
\gamma(s) \\
\beta(s)
\end{array}\right]=\left[\begin{array}{cc}
G_{11}(s) & 0 \\
0 & G_{22}(s)
\end{array}\right]\left[\begin{array}{l}
u_{1}(s) \\
u_{2}(s)
\end{array}\right] .
$$

Subsequently,

$$
G_{11}(s)=\frac{\gamma(s)}{u_{1}(s)}, G_{22}(s)=\frac{\beta(s)}{u_{2}(s)}
$$

In addition,

$$
|G(s)|=\frac{(a+b) k_{f} k_{r} u_{x}}{I_{z} m u_{x}^{2} s^{2}-\left[k_{f}\left(m a^{2}+I_{z} u_{x}\right)+k_{r}\left(m b^{2}+I_{z} u_{x}\right)\right] s+(a+b)^{2} k_{f} k_{r}+m u_{x}^{2}\left(a k_{f}-b k_{r}\right)} .
$$

Obviously, $|G(s)|$ is always not zero as long as $u_{x} \neq 0$. Substituting Equation (18) into (16), we obtain

$$
\left[\begin{array}{cc}
D_{11}(s) & D_{12}(s) \\
D_{21}(s) & D_{22}(s)
\end{array}\right]=\left[\begin{array}{ll}
G_{11}(s) & G_{12}(s) \\
G_{21}(s) & G_{22}(s)
\end{array}\right]^{-1}\left[\begin{array}{cc}
G_{11}(s) & 0 \\
0 & G_{22}(s)
\end{array}\right]
$$


where

$$
\begin{aligned}
& D_{11}(s)=\left(b_{2} s^{2}+b_{1} s+b_{0}\right) /\left(a_{2} s^{2}+a_{1} s+a_{0}\right), D_{12}(s)=\left(b_{5} s^{2}+b_{4} s+b_{3}\right) /\left(a_{2} s^{2}+a_{1} s+a_{0}\right), \\
& D_{21}(s)=\left(b_{8} s^{2}+b_{7} s+b_{6}\right) /\left(a_{2} s^{2}+a_{1} s+a_{0}\right), D_{22}(s)=\left(b_{11} s^{2}+b_{10} s+b_{9}\right) /\left(a_{2} s^{2}+a_{1} s+a_{0}\right), \\
& a_{0}=\left(a_{12} b_{21}-a_{22} b_{11}\right)\left(a_{21} b_{12}-a_{11} b_{22}+a_{11} b_{12}-a_{12} b_{22}\right), a_{1}=a_{21} b_{12} b_{11}-a_{11} b_{22} b_{11}+a_{11} b_{12} b_{21}-a_{12} b_{21} b_{12}, \\
& a_{2}=b_{11} b_{22}-b_{21} b_{12}, b_{0}=\left(a_{21} b_{12}-a_{11} b_{22}\right)\left(a_{12} b_{21}-a_{22} b_{11}\right), b_{1}=a_{21} b_{12} b_{11}-a_{11} b_{22} b_{11}+a_{12} b_{21} b_{22}-a_{22} b_{11} b_{22} \\
& b_{2}=b_{22} b_{11}, b_{3}=\left(a_{11} b_{12}-a_{12} b_{22}\right)\left(a_{21} b_{12}-a_{11} b_{22}\right), b_{4}=-a_{21} b^{2}{ }_{12}+a_{11} b_{22} b_{12}+a_{11} b_{12} b_{22}-a_{12} b_{22}^{2} \\
& b_{5}=-b_{12} b_{22}, b_{6}=\left(-a_{12} b_{21}+a_{22} b_{11}\right)\left(a_{21} b_{11}-a_{11} b_{21}\right), b_{7}=-a_{12} b^{2}{ }_{21}+a_{22} b_{11} b_{21}-a_{21} b^{2} 11+a_{11} b_{11} b_{21}, \\
& b_{8}=-b_{11} b_{21}, b_{9}=\left(a_{21} b_{12}-a_{11} b_{22}\right)\left(a_{12} b_{21}-a_{22} b_{11}\right), b_{10}=a_{21} b_{12} b_{11}-a_{11} b_{22} b_{11}+a_{12} b_{21} b_{22}-a_{22} b_{11} b_{22}, \\
& b_{11}=b_{22} b_{11} .
\end{aligned}
$$

Only the decoupling of the linear 2DOF 4WS vehicle has been introduced here. Nevertheless, it is still necessary to design the corresponding controller to ensure that the decoupled linear 2DOF 4WS vehicle can obtain the required performances described by the reference model.

\subsubsection{Design of Asymptotic Tracking Controller}

The studies above show that the transfer functions between $\gamma$ and $u_{1}$, and between $\beta$ and $u_{2}$ are $G_{11}(s)$ and $G_{22}(s)$, respectively. Here, the transfer function $G_{11}(s)$ is used as an example to illustrate the design of the asymptotic tracking controller $G_{\mathrm{c} 1}(s)$. Figure 2 shows the control block diagram.

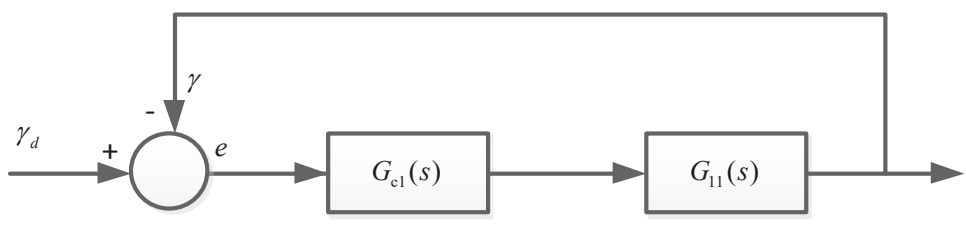

Figure 2. Asymptotic tracking control system.

From Figure 2, the following expression can be obtained:

$$
e(t)=\gamma_{d}(t)-\gamma(t)=\gamma_{d}(t)-G_{\mathrm{c} 1}(t) G_{11}(t) e(t)
$$

Its Laplace transform can be expressed as

$$
e(s)=\frac{1}{1+G_{\mathrm{c} 1}(s) G_{11}(s)} \gamma_{d}(s),
$$

where

$$
G_{\mathrm{c} 1}(s)=k_{p}+k_{d} s,
$$

$k_{p}$ and $k_{d}$ are the proportional and differential coefficients of the PD controller.

Further, the characteristic polynomial of this second-order system can be simplified as

$$
d_{2} s^{2}+d_{1} s+d_{0}=0
$$

where

$$
d_{0}=a_{11} a_{22}-a_{21} a_{12}+k_{p}\left(-a_{22} b_{11}+a_{12} b_{21}\right), d_{1}=b_{11} k_{p}+\left(-a_{22} b_{11}+a_{12} b_{21}\right) k_{d}-\left(a_{11}+a_{22}\right), d_{2}=1+b_{11} k_{d} .
$$

Its Routh Series is as follows,

$$
\begin{array}{c|cc}
s^{2} & d_{2} & d_{0} \\
s^{1} & d_{1} & 0 \\
s^{0} & e_{1} & 0
\end{array},
$$


where

$$
e_{1}=-\frac{1}{d_{1}}\left|\begin{array}{cc}
d_{2} & d_{0} \\
d_{1} & 0
\end{array}\right|=d_{0} .
$$

According to Routh-Hurwitz stability criterion, the parameters of the PD controller can be set and $k_{p}=-5, k_{d}=-2$, when the coefficients of the characteristic polynomials of the 2-order system are all positive. Similarly, the parameters of the asymptotic tracking controller for controlling the sideslip angle can also be determined.

\subsection{Design of Lower Controller}

Although the standard input of the 4WS vehicle has been obtained by the upper controller, it is still necessary to employ the lower controller such that the nonlinear 3DOF 4WS follows the linear 2DOF 4WS vehicle because many differences exist between the two vehicles. The linear 2DOF 2WS vehicle described by Equations (10) and (12) is used as the reference model.

Let $x=\left[\begin{array}{ll}\gamma & \beta\end{array}\right]^{T}$ and $u_{c}=\left[\begin{array}{ll}\delta_{f_{c}} & \delta_{r_{c}}\end{array}\right]^{T}$; subsequently, Equation (6) can be re-expressed as

$$
\dot{x}=A x+B u+B u_{c}+F+W_{\phi}
$$

where

$$
\begin{gathered}
A=E^{-1}\left[\begin{array}{cc}
\frac{2 a^{2} k_{f}+2 b^{2} k_{r}}{u_{x}}+\frac{I_{x z} m_{s} h u_{x}}{I_{x}} & 2\left(a k_{f}-b k_{r}\right) \\
\frac{\left(2 a k_{f}-2 b k_{r}\right)}{u_{x}}-m u_{x}+\frac{m_{s}^{2} h^{2} u_{x}}{I_{x}} & 2\left(k_{f}+k_{r}\right)
\end{array}\right], E=\left[\begin{array}{cc}
I_{z}-\frac{I_{x z}^{2}}{I_{x}} & \frac{I_{x z} m_{s} h u_{x}}{I_{x}} \\
\frac{I_{x z} m_{s} h}{I_{x}} & m u_{x}-\frac{m_{s}^{2} h^{2} u_{x}}{I_{x}}
\end{array}\right], \\
W_{\phi}=E^{-1}\left[\begin{array}{cc}
-\frac{I_{x z} c_{\phi} \dot{\phi}}{I_{x}}+\frac{-I_{x z} m_{s} g h \phi+I_{x z} k_{\phi} \phi}{I_{x}}+\left(-2 a k_{f} R_{f}+2 b k_{r} R_{r}\right) \phi \\
\frac{m_{s} h c_{\phi} \dot{\phi}}{I_{x}}+\left(-2 R_{f} k_{f}-2 R_{r} k_{r}\right) \phi+\frac{-m_{s}^{2} h^{2} g+m_{s} h k_{\varphi}}{I_{x}} \phi
\end{array}\right], \\
B=E^{-1}\left[\begin{array}{cc}
-2 a k_{f} & 2 b k_{r} \\
-2 k_{f} & -2 k_{r}
\end{array}\right], F=\left[\begin{array}{c}
2 a f_{y f}\left(\alpha_{f}, \varphi\right)-2 b f_{y r}\left(\alpha_{r}, \varphi\right) \\
2 f_{y f}\left(\alpha_{f}, \varphi\right)+2 f_{y r}\left(\alpha_{r}, \varphi\right)
\end{array}\right] .
\end{gathered}
$$

The state error between the linear 2DOF and nonlinear 3DOF 4WS vehicle models can be expressed as $e=\bar{x}-x$. Therefore,

$$
\dot{e}=\bar{A} e-\bar{B} u_{c}+\Delta
$$

where

$$
\Delta=(\bar{A}-A) x+(\bar{B}-B) u+(\bar{B}-B) u_{c}-F-W_{\phi}
$$

As shown, the state error between the two vehicle models is primarily from the initial state error of the state variable, the uncertainty error that includes the parameters' difference, the nonlinear term of the tires $F$, and the coupling disturbance caused by the vehicle roll $W_{\phi}$. Therefore, it is necessary to define

$$
u_{c}=u_{c o}+u_{c c}
$$

where $u_{c o}$ is the output of the optimal controller to avoid the error caused by the different initial states, and $u_{c c}$ is the output of the fractional sliding mode controller to avoid the error caused by the uncertainties.

\subsubsection{Design of Optimal Controller}

When only the initial state error of state variable is considered, that is, the uncertainty error under the nominal parameters is not considered, that is, $\Delta=0, u_{c c}=0$, Equation (32) becomes

$$
\dot{e}=\bar{A} e-\bar{B} u_{c o} .
$$


Let $u_{c o}=K e$, where $K$ is the optimal feedback gain matrix; therefore, the performance index of the vehicle system is given by

$$
J=\int_{0}^{t}\left(e^{T} Q e+u_{c}^{T} R u_{c}\right) d t
$$

Let $Q=\operatorname{diag}(100,1000)$ and $R=\operatorname{diag}(1,1)$; therefore, $K$ can be calculated by

$$
K=R^{-1} \bar{B}^{T} P
$$

where

$$
\bar{A}^{T} P+P \bar{A}-P \bar{B} R \bar{B}^{T} P+Q=0 .
$$

\subsubsection{Design of Fractional Sliding Mode Controller}

When only the uncertainty error under the nominal parameters is considered, that is, $\Delta \neq 0$, the sliding superplane can be expressed as

$$
s=\dot{e}-(\bar{A}-\bar{B} K) e .
$$

It is easy to conclude that Equation (39) is completely equivalent to Equation (35) when $s=0$. Therefore, provided that $s=0$ can be controlled, the state error of the two vehicles can fulfill the requirements of the optimal control. However, if $\Delta \neq 0$, the condition $s=0$ is not satisfied at this time. To control the $e$ move on $s=0$, the sliding mode control is required after the optimal control of the front and rear steering angles for the actual 4WS vehicle. Subsequently, Equation (32) can be transformed into

$$
\dot{e}=\bar{A} e-\bar{B}\left(u_{c o}+u_{c c}\right)+\Delta .
$$

To further improve the robustness of the system, the fractional order theory is introduced here. That is, $s$ is assumed to satisfy the fractional order decay rate [24]:

$$
{ }_{c} D_{t}^{\alpha} s=-\Lambda s-\varepsilon \operatorname{sgn}(s) \quad(0<\alpha \leq 1) .
$$

where ${ }_{c} D_{t}^{\alpha}(\cdot)$ is the $\alpha$ order Riemann-Liouvilla fractional derivative, $\Lambda$ is the positive real diagonal matrix, $\varepsilon$ is a coefficient, $\operatorname{sgn}(\cdot)$ is a sign function.

Subsequently, we obtain the $-\alpha$ order derivative on both sides of Equation (41); therefore,

$$
s=-\Lambda_{c} D_{t}^{-\alpha} s-\varepsilon_{c} D_{t}^{-\alpha} \operatorname{sgn}(s) .
$$

Substituting (39), (40) into (41), the fractional sliding model control law is expressed as follows:

$$
u_{c c}=\bar{B}^{-1} \Lambda_{c} D_{t}^{-\alpha} s-\bar{B}^{-1} \varepsilon_{c} D_{t}^{-\alpha} \operatorname{sgn}(s)-\bar{B}^{-1} \Delta .
$$

\section{Simulation Analysis}

The nominal vehicle parameters and five different parameter groups of the real $4 \mathrm{WS}$ vehicle are summarized in Tables 1 and 2, respectively. Assuming that the real 4WS vehicle (represented herein by the nonlinear 3DOF 4 WS model) traverses on a road with the adhesion coefficient of 0.8 , its partial parameters $\left(m=1700 \mathrm{~kg}, I_{z}=4100 \mathrm{kgm}^{2}\right)$ and initial state of state variable $\left(\mathbf{x}_{0}=(0,0)^{T}\right)$ are slightly different from those of the linear 2DOF 4 WS vehicle $\left(m=1818.2 \mathrm{~kg}, I_{z}=3885 \mathrm{kgm}^{2}\right.$, $\left.\bar{x}_{0}=(0.5,-1)^{T}\right)$. The system input is the step input of the front wheel steering angle with amplitude $0.045 \mathrm{rad}$. The saturation values of the front and rear steering angles are $\left|\delta_{f}\right| \leq \frac{\pi}{6} \mathrm{rad},\left|\delta_{\mathrm{r}}\right| \leq \frac{\pi}{36}$ $\mathrm{rad}$, respectively. 
Table 1. Vehicle parameters.

\begin{tabular}{cccccc}
\hline Symbol & Value & Symbol & Value & Symbol & Value \\
\hline$m(\mathrm{~kg})$ & 1818.2 & $k_{\phi}(\mathrm{Nm} / \mathrm{rad})$ & 131,380 & $c_{\phi}$ & 10,000 \\
$m_{s}(\mathrm{~kg})$ & 1200 & $R_{f}$ & -0.114 & $a(\mathrm{~m})$ & 1.4435 \\
$I_{x}\left(\mathrm{kgm}^{2}\right)$ & 729.6 & $R_{r}$ & 0 & $b(\mathrm{~m})$ & 1.6045 \\
$I_{z}\left(\mathrm{kgm}^{2}\right)$ & 3885 & $k_{f}(\mathrm{~N} / \mathrm{rad})$ & $-62,618$ & $h_{s}(\mathrm{~m})$ & 0.44 \\
$I_{x z}\left(\mathrm{kgm}^{2}\right)$ & 0 & $k_{r}(\mathrm{~N} / \mathrm{rad})$ & $-110,185$ & $u_{x}(\mathrm{~m} / \mathrm{s})$ & 25 \\
\hline
\end{tabular}

Table 2. Parameters of real four-wheel-steering (4WS) vehicle.

\begin{tabular}{ccccc}
\hline Group & $\boldsymbol{m} \mathbf{( k g )}$ & $\boldsymbol{I}_{\boldsymbol{z}}\left(\mathbf{k g m}^{\mathbf{2}}\right)$ & $\boldsymbol{a}(\mathbf{m})$ & $\boldsymbol{b}(\mathbf{m})$ \\
\hline 1 & 1800 & 3785 & 1.543 & 1.505 \\
2 & 1600 & 3685 & 1.423 & 1.625 \\
3 & 1700 & 3885 & 1.583 & 1.465 \\
4 & 1900 & 3985 & 1.343 & 1.705 \\
5 & 2150 & 4085 & 1.243 & 1.805 \\
\hline
\end{tabular}

The linear 2DOF 2WS vehicle, reference model, nonlinear 3DOF 2WS vehicle, real 4WS vehicle with the hierarchical controller, and linear 2DOF 4WS vehicle with LQR controller are simulated with the same front wheel steering angle; their response curves are shown in Figures 3-8. To investigate the robustness of the hierarchical controller system, five different groups of vehicle parameters are used in the simulation, and their deviations in yaw rate and sideslip angle from those of the real 4WS vehicle with the nominal parameters are shown in Figures 9 and 10.

As shown in Figures 3 and 4, the steady-state values of both the yaw rate and sideslip angle of the linear 2DOF 2WS vehicle, and those of the nonlinear 3DOF 2WS vehicle are different from each other. In particular, their steady-state values of sideslip angle are far from that of the reference model. This is because the nonlinear 3DOF $2 \mathrm{WS}$ vehicle considers the roll motion, and the reference model is obtained by filtering the sideslip angle of the linear 2DOF 2WS vehicle, which can also be adjusted according to the driving condition, such as the mass and speed of the vehicle. In addition, at the very beginning, the value of sideslip angle of the real $4 \mathrm{WS}$ vehicle with the hierarchical controller exhibits little fluctuation. However, their steady-state values of yaw rate and sideslip angle are almost exactly the same as the reference model. This is because both the partial parameters and the initial state are not the same, and the nonlinear 3DOF 4WS vehicle requires extra consideration for the roll motion.

It can be drawn from Figure 5 that front and rear wheel steering angles of the real 4 WS vehicle with hierarchical controller are $0.0698 \mathrm{rad}$ and $0.0215 \mathrm{rad}$, respectively, which are less than the saturation values. Further, the front and the rear wheels rotate in the same direction, thus fully meeting the requirement of the handling stability at high speed. In addition, it also indicates that although the input of the hierarchical control system is only the front wheel steering angle, the proper front and rear wheel steering angles are obtained by the hierarchical controller. Specifically, the actual front and rear steering angles come from two parts: one is the standard input achieved by the upper controller of the $4 \mathrm{WS}$ vehicle, the other is the corrected value obtained by the lower controller to eliminate the uncertainty influence.

Figure 6 shows that the roll angles of the nonlinear 3DOF $2 \mathrm{WS}$ vehicle and the real $4 \mathrm{WS}$ vehicle with hierarchical controller are $-0.031 \mathrm{rad}$ and $-0.0285 \mathrm{rad}$, respectively, thus indicating that the roll angle of the rear $4 \mathrm{WS}$ vehicle decreases to a certain extent by adopting the hierarchical controller, and its handling stability is improved compared with the nonlinear 3DOF 2WS vehicle.

It can be seen from Figures 7 and 8 that the yaw rate of the linear 2DOF 4WS vehicle with LQR controller can track that of the linear 2DOF 2WSvehicle, but the sideslip angle of the former cannot track that of the latter. Thus the upper controller of the hierarchical control strategy is effective to track both the yaw rate and sideslip angle. 
As shown in Figures 9 and 10, although almost all of the parameters of the real 4WS vehicle have changed, the maximum deviations in the yaw rate and sideslip angle of the real 4WS vehicle with the hierarchical controller are $-3.7 \times 10^{-3} \mathrm{rad} / \mathrm{s}$ and $-2.16 \times 10^{-3} \mathrm{rad}$, respectively, which approach 0 after approximately $1 \mathrm{~s}$ and $1.5 \mathrm{~s}$, respectively. Hence, even if the actual $4 \mathrm{WS}$ vehicle is traversing with little change in these parameters, the driver will hardly feel these variations and experience no uncomfortable feeling. Obviously, the hierarchical controller demonstrates good robustness.

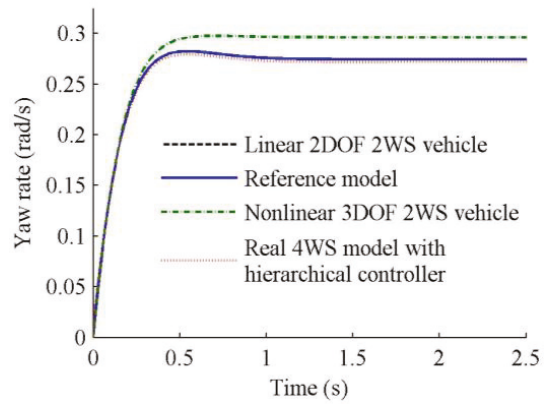

Figure 3. Curves of yaw rate.

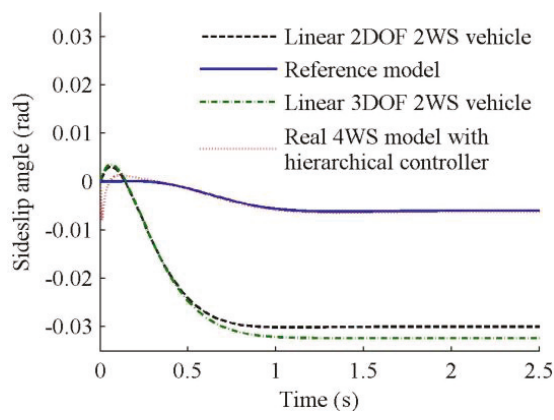

Figure 4. Curves of sideslip angle.

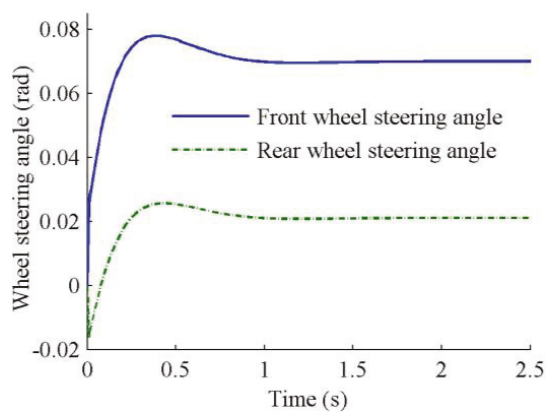

Figure 5. Steering angles of vehicle wheels. 


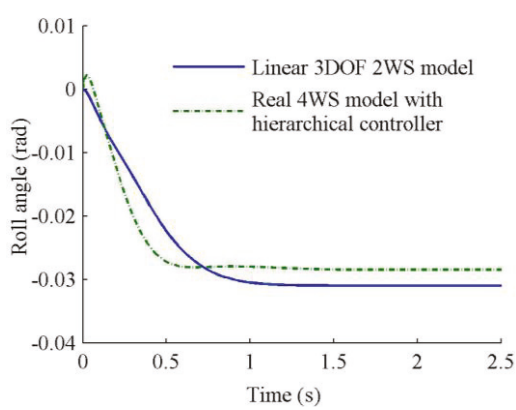

Figure 6. Roll angle curves.

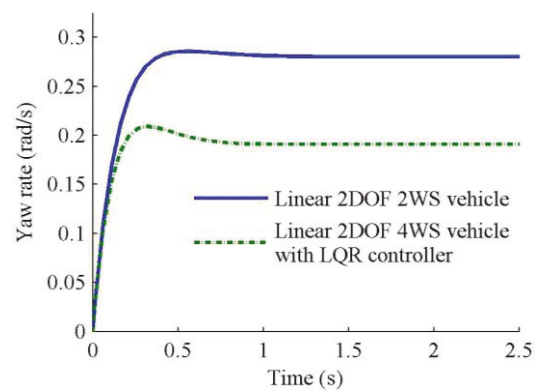

Figure 7. Curves of yaw rate.

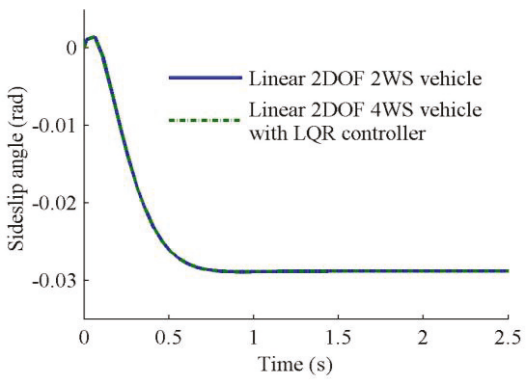

Figure 8. Curves of sideslip angle.

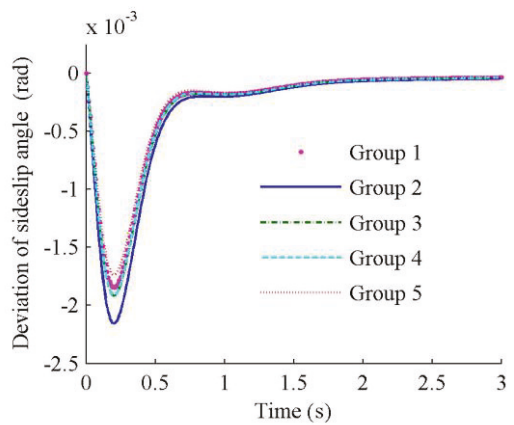

Figure 9. Deviation curves of yaw rate. 


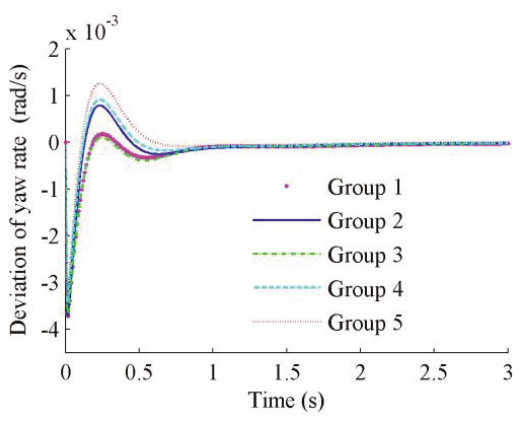

Figure 10. Deviation curves of sideslip angle.

\section{Conclusions}

The realization of a new hierarchical control strategy for the nonlinear 3DOF 4WS vehicle is studied. The upper control calculates and controls the standard input for the 4WS vehicle, thus allowing the linear 2DOF $4 \mathrm{WS}$ vehicle to track the yaw rate and sideslip angle of the $2 \mathrm{WS}$ reference model. The lower control is used primarily to solve the effects of the vehicle uncertainty, and perform the appropriate amendments to yield the required performance in the real vehicle. That is, the optimal controller is applied to control the state error caused by the different initial states of the variable; subsequently, the fractional order sliding mode controller is applied to compensate for the state error caused by the uncertainty error. And the simulation results show that this hierarchical control strategy is effective for the nonlinear 3DOF 4WS vehicles.

Author Contributions: Conceptualization, J.T. and N.C.; Data curation, N.C.; Funding acquisition, J.T. and N.C.; Methodology, J.T. and N.C.; Project administration, J.T.; Resources, J.T.; Validation, J.D.; Visualization, J.D.; Writing - original draft, J.T.; Writing - review \& editing, Y.T.

Funding: This research was funded by National Natural Science Foundation of China (Grant No. 11272159, and National Youth Foundation of China (Grant No. 51305207).

Conflicts of Interest: The authors declare no conflict of interest.

\section{References}

1. Chen, N.; Chen, N.; Chen, Y.D. On fractional control method for four-wheel-steering vehicle. Sci. China Technol. Sci. 2009, 52, 603-609. [CrossRef]

2. Sun, C.Y.; Zhang, X.; Xi, L.H.; Tian, Y. Design of a path-tracking steering controller for autonomous vehicles. Energie 2018, 11, 1451. [CrossRef]

3. Ching, N.T.; Hormoz; Dai, V.Q.; Simic, M.; Khayyam, H.; Marzbani; Jazar, R.N. Autodriver autonomous vehicles control strategy. Procedia Comput. Sci. 2018, 126, 870-877.

4. Krishna, S.; Narayanan, S.; Ashok, S.D. Fuzzy logic based yaw stability control for active front steering of a vehicle. J. Mech. Sci. Technol. 2014, 28, 5169-5174. [CrossRef]

5. Yin, G.D.; Chen, N.; Wang, J.X.; Chen, J.-S. Robust control for 4WS vehicles considering a varying tire-road friction coeffcient. Int. J. Automot. Technol. 2010, 11, 33-40. [CrossRef]

6. Zhao, W.Z.; Qin, X.X. Study on mixed $\mathrm{H}_{2} / \mathrm{H}_{\infty}$ robust control strategy of four wheel steering system. Sci. China Technol. Sci. 2017, 60, 1-10. [CrossRef]

7. Li, M.; Jia, Y.; Du, J. LPV control with decoupling performance of 4 WS vehicles under velocity-varying motion. IEEE Trans. Control Syst. Technol. 2014, 22, 1708-1724.

8. Chen, C.; Shu, M.; Liu, R. Virtual-point-based asymptotic tracking control of 4WS vehicles. Int. J. Control Autom. Syst. 2015, 13, 371-378. [CrossRef]

9. Mihaly, A.; Gaspar, P.; Nemeth, B. Multiple fault-tolerant in-wheel vehicle control based on high-level control reconfiguration. IFAC-PapersOnLine 2017, 50, 8606-8611. 
10. Huang, D.W.; Wang, H.L.; Zhu, Z.W.; Zhang, F. Stochastic stability of four-wheel-steering system. Chaos Soliton Fractals 2007, 33, 823-828. [CrossRef]

11. Ackermann, J. Robust decoupling of car steering dynamics with arbitrary mass distribution. In Proceedings of the 1994 American Control Conference-ACC '94, Baltimore, MD, USA, 29 June-1 July 1994; pp. 1964-1968.

12. Zhu, Z.; Du, R. Yaw Moment control strategy for four-wheel side driven EV. Autom. Control Comput. Sci. 2018, 52, 32-39. [CrossRef]

13. Shen, H.; Tan, Y.S. Vehicle handling and stability control by the cooperative control of 4 WS and DYC. Mod. Phys. Lett. B. 2017, 31, 19-21. [CrossRef]

14. Hang, P.; Chen, X.; Fang, S.; Luo, F. Robust control for four-wheel-independent-steering electric vehicle with steer-by-wire system. Int. J. Automot. Technol. 2017, 18, 785-797. [CrossRef]

15. Koumboulis, F.N.; Skarpetis, M.G. Robust triangular decoupling with application to 4WS cars. IEEE Trans. Autom. Control 2000, 45, 344-352. [CrossRef]

16. Fujimoto, H.; Yamauchi, Y. Advanced motion control of electric vehicle based on lateral force observer with active steering. IEEE Int. Symp. Ind. Electron. 2010, 3627-3632.

17. Skarpetis, M.G.; Koumboulis, F.N.; Barmpokas, F.S.; Chamilothoris, G.E. Decoupling control algorithms for 4ws vehicles. In Proceedings of the IEEE International Conference on Mechatronics, Budapest, Hungary, 3-5 July 2006; pp. 499-504.

18. Chen, C.; Jia, Y.; Gao, Q.; Yu, F. Asymptotic Decoupling Control with Active Front Steering and Electronic Differentials in Four Wheel Steering Vehicles. In Proceedings of the 30th Chinese Control Conference, Yantai, China, 22-24 July 2011; pp. 111-116.

19. Li, M.X.; Jia, M.Y. Precompensation decoupling control with $\mathrm{H} \infty$ performance for 4 WS velocity-varying vehicles. Int. J. Syst. Sci. 2016, 47, 3864-3875. [CrossRef]

20. Chen, C.; Tan, H. Steering Control of High Speed Vehicles: Dynamic Look Ahead and Yaw Rate Feedback. In Proceedings of the 37th IEEE Conference on Decision \& Control, Tampa, FL, USA, 18 December1998; Volume 1, pp. 1025-1030.

21. Chen, C.; Jia, Y. Nonlinear decoupling control of four-wheel-steering vehicles with an observer. Int. J. Control Autom. Syst. 2012, 10, 697-702. [CrossRef]

22. Marino, R.; Cinili, F. Input-Output decoupling control by measurement feedback in four-wheel-steering vehicles. IEEE Trans. Control Syst. Technol. 2009, 17, 1163-1172. [CrossRef]

23. Yuhara, N.; Horiuchi, S.; Arato, Y. A robust adaptive rear wheel steering control system for handling improvement of four-wheel steering vehicles. Veh. Syst. Dyn. 1992, 20, 666-680. [CrossRef]

24. Manabe, S. A suggestion of fractional-order controller for flexible spacecraft attitude control. Nonlinear Dyn. 2002, 29, 251-268. [CrossRef]

(c) 2018 by the authors. Licensee MDPI, Basel, Switzerland. This article is an open access article distributed under the terms and conditions of the Creative Commons Attribution (CC BY) license (http://creativecommons.org/licenses/by/4.0/). 
Article

\title{
Energy-Efficient Driving Strategies for Multi-Train by Optimization and Update Speed Profiles Considering Transmission Losses of Regenerative Energy
}

\author{
Mo Chen, Zhuang Xiao, Pengfei Sun *, Qingyuan Wang, Bo Jin and Xiaoyun Feng \\ School of Electrical Engineering, Southwest Jiaotong University, Chengdu 610031, China; \\ mochan@my.swjtu.edu.cn (M.C.); xiaozhuang@my.swjtu.edu.cn (Z.X.); wangqy@home.swjtu.edu.cn (Q.W.); \\ bjin@my.swjtu.edu.cn (B.J.); fengxy@home.swjtu.edu.cn (X.F.) \\ * Correspondence: pengfeisun@home.swjtu.edu.cn
}

Received: 22 August 2019; Accepted: 16 September 2019; Published: 18 September 2019

\begin{abstract}
This paper aims at minimizing the total energy consumption of multi-train in an urban rail transit (URT) system by optimizing and updating speed profiles considering regenerative braking power losses on the catenary. To make full use of regenerative energy and decrease traction energy consumption simultaneously, energy-efficient control strategies of multi-train and a corresponding solution method are proposed. The running process of multi-train is divided into several sections based on passenger stations. Speed profiles of each train in each section are collaboratively optimized by searching only one transition point from the optimized single-train speed profile, which can be worked out by searching the switching point of coasting mode, and the optimized multi-train speed profiles are updated based on departure orders of trains. Moreover, an electrical network model is established to analyze energy flows, and dynamic losses of recovered regenerative energy on the line can be calculated. Besides, an improved optimization strategy of multi-train, which contains seven motion phases, is presented for steep slope. Simulation results based on Guangzhou Metro Line 8 verify the effectiveness of the proposed methods. Total energy consumption of optimized multi-train can be decreased by $6.95 \%$ compared with multi-train adopted single-train optimal control strategy, and the energy-saving rate of $21.08 \%$ can be achieved compared with the measured data by drivers under same trip time. In addition, the influence of departure interval on total energy consumption is analyzed and the optimal departure interval can be obtained.
\end{abstract}

Keywords: URT; multi-train optimization; steep slope; electrical network model; regenerative energy dynamic losses

\section{Introduction}

Urban rail transit (URT) has achieved rapid development recent years owing to its punctuality, convenience, and comfortability. Meanwhile, energy consumption is also growing fast. Taking the Guangzhou URT as an example, the power consumption reached up to 1323 million kWh in 2017. About $40-50 \%$ of the energy is consumed by train traction system. The amount of regenerative energy accounts for more than $30 \%$ of the traction energy consumption; however, around $40 \%$ of the regenerative energy is not used. Therefore, the reduction of traction energy and improvement of braking energy recuperation are promising techniques to improve energy efficiency for the URT, which makes a lot of sense for green transportation and sustainable development [1].

Research on energy-saving optimization began early with single train, the purpose of which was to minimize traction energy consumption by optimizing the speed profile under the fixed time and operation constraints. The solution methods can be divided into three kinds-analytical method, 
numerical method, and intelligent algorithm. Analytical methods are based on the Pontryagin maximum principle [2-8], through which the optimal control strategy of single-train that contains four motion phases (namely, maximum traction (MT), cruising (CR), coasting (CO), and maximum braking (MB) by sequence) is proved theoretically, and the corresponding speed profile can usually be calculated by searching two transition points, namely the transition speed from MT mode to $\mathrm{CR}$ mode and the transition position from $\mathrm{CR}$ mode to $\mathrm{CO}$ mode. The numerical method may be used to calculate the optimal speed profile directly when the optimization problems are converted into the standard form of the corresponding model, such as dynamic programming (DP) $[9,10]$, sequential quadratic programming (SQP) [11,12], and mixed-integer linear programming (MILP) [13]. The intelligent algorithm simulates natural processes and has strong adaptability to complex objective functions [14-16].

Optimization of single train does not consider the specific utilization way of regenerative energy, and the optimization of the multi-train cooperative operation is an effective way to increase the utilization of regenerative energy $[17,18]$, which can be divided into scheduling and control of trains $[19,20]$. The former focuses on optimizing the timetable and the latter focuses on optimizing energy-efficient driving strategies for multi-train. Albrecht [21] formulated an optimization algorithm that can reduce the peak power consumption by $29 \%$. Alcaraz et al. [22] established a power flow model of the electrical network and designed a mathematical programming model to synchronize the braking trains and traction trains. Lin et al. [23] optimized train dwell time using genetic algorithms. Genetic algorithm (GA) was used to optimize the train scheduling [24,25]. A DP-based algorithm was developed for the solution of the itinerary planning [26]. However, the optimization of the timetable is limited by fixed control strategy. Optimization of the timetable and driving strategy were combined by $\mathrm{Su}$ et al. [27] to generate the globally optimal operation schedule. Optimizing the speed profile of trains with a planned timetable can further increase the energy-saving effect. Tang optimized the control strategy of the tracking train using dynamic programming (DP) and quadratic programming $(\mathrm{QP})$, respectively [28]. However, the model was complex, which makes it difficult to use for multiple trains. Goodwin et al. [29] used the GA to obtain the suboptimal condition transition points of multiple trains, but the calculation efficiency of GA was not high. Liu [30,31] discussed the two-trains and three-trains systems in turn and proposed that the optimal control strategy of the tracking train adopts four modes or five modes of movements, and the optimized solution can be obtained by a heuristic algorithm. However, the speed profile was not updated in all sections of the multi-train system. Sun et al. [32] formulated that the braking process can be predicted to supply the regenerative energy to neighboring traction trains by perturbance analysis. Lu et al. [33] accurately predicted the energy consumption and regenerative braking energy of heavy-haul trains on large long slopes by establishing a single-particle model of train dynamics. Actually, the energy-saving effect brought by active braking to generate regenerative energy $[32,33]$ is not ideal, which was proved by the authors of [28].

In conclusion, most studies separate the optimization of single-train and multi-train. The optimal control strategy of single train with $\mathrm{MT}, \mathrm{CR}, \mathrm{CO}$, and MB may not be optimal in a multi-train system [34], considering the utilization of regenerative energy. Besides, cooperative train optimization in a steep slope situation and dynamic losses of recovered regenerative energy on the catenary are rarely considered; especially, the latter is influenced by the line resistances and current, which depend on the positions of trains and vary with time, and may greatly influence the total energy of multi-train.

In this paper, energy-saving optimization of single-train and multi-train systems are combined. An energy-saving control strategy of multi-train and corresponding solution methods are proposed in the foundation of the optimal control strategy of single train to simultaneously reduce the traction energy and increase the utilization of regenerative energy. The running process of multi-train is divided into several sections based on passenger stations. The speed profile of each train in each section is collaboratively optimized by searching only one transition point from the optimized speed profile of single-train, which can be worked out by searching the switching point of coasting mode, and the optimized speed profiles of multi-train are updated based on departure orders, which greatly 
simplifies the complex problem and makes the calculation process efficient. In order to make full use of the line potential energy of steep slope and further reduce the total energy consumption, an improved optimization strategy of multi-train, which contains seven motion phases, is formulated. Moreover, an electrical network model is built to evaluate the loss of recovered regenerative energy, and the change of line resistance and current over time can be clearly observed. Finally, the optimization of control strategy and timetable are combined, the total energy of different departure interval is analyzed, and the optimal departure interval is obtained. Simulation results based on Guangzhou Metro Line 8 are shown to verify the effectiveness of proposed optimization methods.

The remainder of this paper is structured as follows. In Section 2, the energy-saving models of single-train and multi-train systems are established, as well as the electrical network model. Section 3 proposes the optimization method for the two systems and combines them, and the solving procedures are outlined. In addition, an improved energy-saving control strategy of steep slope is formulated, which can further increase the energy-saving rate. The effectiveness of the proposed methods is verified with a simulation based on Guangzhou Metro Line 8 in Section 4, and the energy consumption of the multi-train system at different departure intervals is compared. Section 5 concludes this paper.

\section{System Modelling}

\subsection{Modelling of Single-Train}

The motion process of a train can be described as follows:

$$
\begin{gathered}
\frac{d t}{d s}=\frac{1}{v}, \\
\frac{d v}{d s}=\frac{\mu_{t} F_{t}(v)-\mu_{b} F_{b}(v)-W(v)-G(s)}{(1+\rho) M v},
\end{gathered}
$$

where $s$ is the position of the train; $v(s)$ and $t(s)$ are the corresponding speed and trip time, respectively; $M$ is the total mass of the train and $\rho$ is the weighted average rotary mass coefficient, $\mu_{t}$ and $\mu_{b}$ are the coefficient of the traction force and braking force, respectively; and $F_{t}(v)$ and $F_{b}(v)$ are the maximum traction force and maximum braking force, respectively, corresponding to the speed $v(s)$. $W(v)$ is the basis running resistance, as shown in Equation (3), in which $a, b$, and $c$ are constant for a specific vehicle type. $G(s)$ is the additional resistance, as shown in Equation (4), where $g$ is the gravitational acceleration, $i$ is the angle of a slope, $r$ is the radius of the curve, and $A$ is a constant [35]. Actually, the first term on the right side of Equation (4) denotes the resistance caused by gradient, which is positive for uphill and negative for downhill, and the second term denotes the resistance caused by curve.

$$
\begin{gathered}
W(v)=a+b v+c v^{2} \\
G(s)=M g \sin i+M g \frac{A}{r}
\end{gathered}
$$

To minimize the traction energy consumption of single-train, the objective function of energy consumption is modeled as

$$
\min J=\sum_{k=1}^{N} \frac{\mu_{k} F_{k}(v)}{\eta_{t}} \Delta s,
$$

where $J$ is the energy consumption consumed by the traction system and $\eta_{t}$ denotes the conversion efficiency of the traction system. The distance of the section is $S ; \Delta s$ denotes the simulation step interval, which values $1 \mathrm{~m}$ in this paper; and the simulation count $N$ can be obtained by $N=S / \Delta s$. $F_{k}$ is the maximum traction force at simulation step $k$, and $\mu_{k}$ is the corresponding coefficient. In addition, the path constraint of the speed limit is shown in Equation (6) and the continuous control variables are 
shown in Equation (7), which means any force between 0 to the maximum traction/braking force can be taken.

$$
\begin{gathered}
0 \leq v(s) \leq \bar{V}(s) \\
\mu_{t,} \mu_{b} \in[0,1] \\
\mu_{t} \mu_{b}=0
\end{gathered}
$$

The boundary conditions are

$$
\begin{gathered}
v(0)=0, \\
v(S)=0, \\
t(S)-t(0)=T,
\end{gathered}
$$

where $\bar{V}(s)$ is the speed limit at position $s$, and $T$ is the planned trip time for the section.

\subsection{Modelling of Multi-Train}

Two trains running in the same direction and electrical substation are selected for example in this paper, shown in Figure 1. The front train denotes Train A and the latter is Train B, the length of the two sections are $L_{1}$ and $L_{2}$, respectively, $S_{1}, S_{2}$, and $S_{3}$ are the positions of the three stations by sequence.

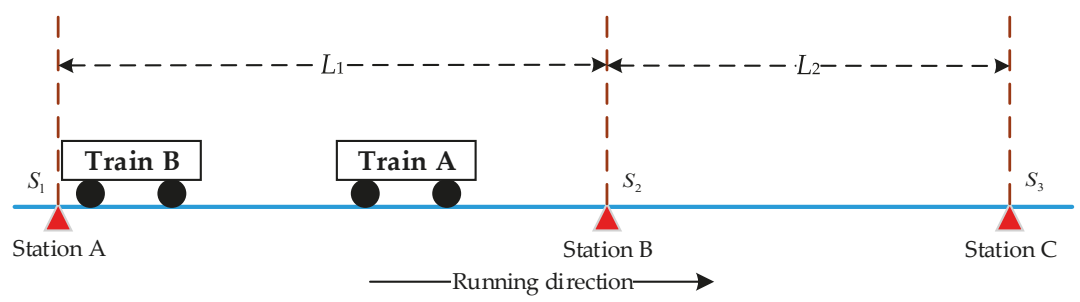

Figure 1. Running process of two trains.

In the optimization of multiple train, the utilization way of regenerative energy is taken into consideration and the model for minimizing total energy consumption of the multi-train system is formulated as below:

$$
\begin{gathered}
\min J_{\text {multi }}=\sum_{k=1}^{N} \frac{\mu_{A k} F_{A k}}{\eta_{t}} \Delta s+\sum_{k=1}^{N} \frac{\mu_{B k} F_{B k}}{\eta_{t}} \Delta s-\sum_{i=1}^{M} \eta_{t} P_{i} \Delta t, \\
P_{i}=\left\{\begin{array}{cc}
P_{A i} & P_{A i}>0, P_{B i}<0,\left|P_{A i}\right|<\left|P_{B i}\right| \\
\left|P_{B i}\right| & P_{A i}>0, P_{B i}<0,\left|P_{A i}\right|>\left|P_{B i}\right| \\
\left|P_{A i}\right| & P_{A i}<0, P_{B i}>0,\left|P_{A i}\right|<\left|P_{B i}\right| \\
P_{B i} & P_{A i}<0, P_{B i}>0,\left|P_{A i}\right|>\left|P_{B i}\right|
\end{array}\right.
\end{gathered}
$$

where $J_{\text {multi }}$ denotes the total energy consumption of multi-train system. On the right side of Equation (9), the first two terms are the traction energy consumption of Train A and Train B, respectively, which can refer to Equation (5), and the simulation count $N$ here can be obtained by $N=\left(L_{1}+L_{2}\right) / \Delta s$. The third term is the regenerative energy consumed by the multi-train system, which can be calculated by comparing the power of two trains at each moment of the regenerative energy absorption process. As the times corresponding to each simulation distance step of two trains in the absorption process are usually not equal, the functions of power and time are linearly interpolated. What needs to be explained here is that, when the train at station, the corresponding power is assumed to be $0 . \Delta t$ denotes the interpolate interval, which has a value of $0.1 \mathrm{~s}$, and the interpolate count $M=\left(T_{A}+t_{\text {stop }}+T_{B}+\Delta T\right) / \Delta t$, where $T_{A}$ and $T_{B}$ are the planned trip time of two sections and $t_{\text {stop }}$ denotes the dwell time of Station B. Assume that Train A departures from Station A at time 0, with departure interval values of $\Delta T$, 
the corresponding departure time of Train B is $\Delta T$. Therefore, the actual absorbed power $P_{i}$ can be obtained by Equation (10).

In this model, speed profiles of each train are cooperative optimized, which means no matter which train produces regenerative energy, the other train is intended to absorb it as far as possible by adopting traction mode. Therefore, speed profiles of both trains in each section are optimized and updated during their running processes, and the flow of the regenerative energy of the multi-train system throughout the optimization process consists of three main stages, as shown in Figure 2. The first stage is the braking phase of Train A in the first section, the second stage is the braking phase of Train B in the first section, and the third stage is the braking phase of Train A in the second section.

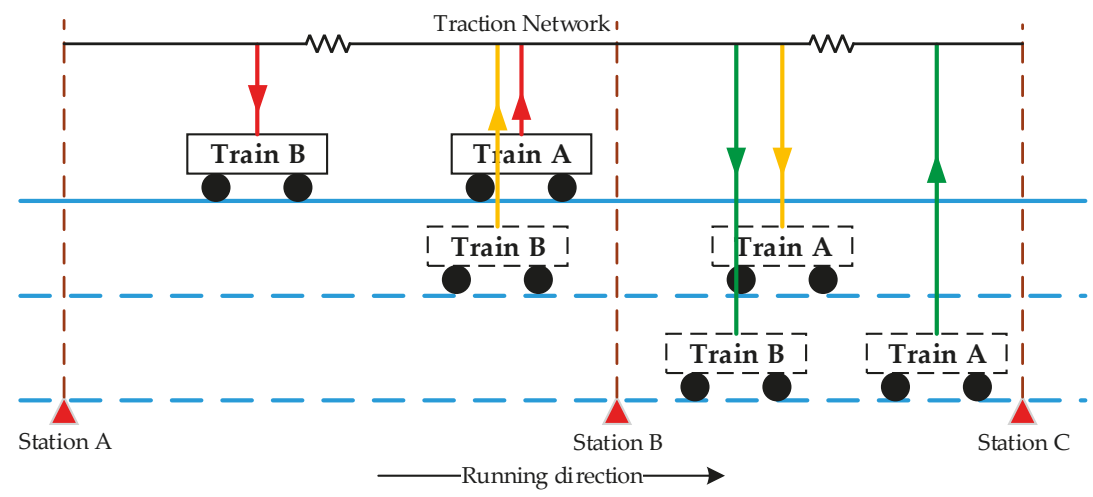

Figure 2. Power flows of regenerative energy.

\subsection{Modelling of Electrical Network}

The regenerative energy will be lost during the transmission process of the catenary, which is a factor that cannot be ignored. Therefore, an electrical network model is established and the equivalent circuit $[36,37]$ is shown in Figure 3.

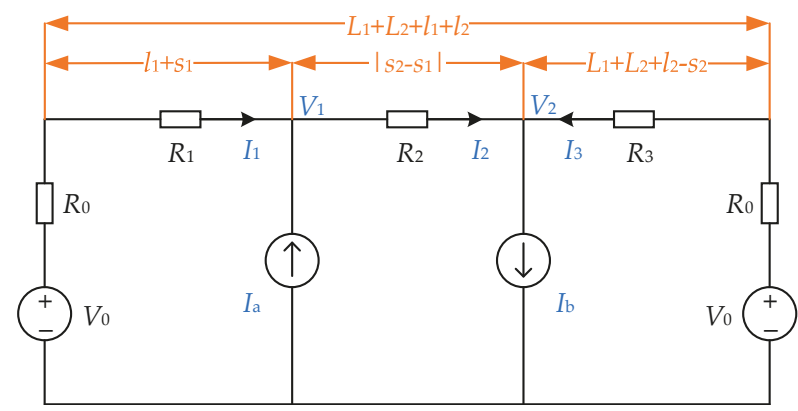

Figure 3. Equivalent circuit of the electrical network.

The traction substation is modeled as a DC voltage source $V_{0}$ and a lumped resistance $R_{0}$. Power sources $I_{\mathrm{a}}$ and $I_{\mathrm{b}}$ are used to represent the braking train and the tracking train, respectively. The catenary is regarded as a distributed parameter resistance model. The equivalent resistances $R_{1}, R_{2}$, and $R_{3}$ are decided by the position of two trains and can be calculated by Equation (11). The traction substation power $P_{0}$ and the terminal power of catenary of two trains $P_{1}, P_{2}$ can be calculated by Equation (12).

$$
R_{1}=\delta\left(l_{1}+s_{1}\right) \quad R_{2}=\delta\left|s_{2}-s_{1}\right| \quad R_{3}=\delta\left(L_{1}+L_{2}+l_{2}-s_{2}\right),
$$




$$
\begin{aligned}
& P_{0}=V_{0}\left(I_{1}+I_{3}\right)=V_{0}\left(\frac{V_{0}-V_{1}}{R_{0}+R_{1}}+\frac{V_{0}-V_{2}}{R_{0}+R_{3}}\right), \\
& P_{1}=V_{1}\left(I_{2}-I_{1}\right)=V_{1}\left(\frac{V_{1}-V_{2}}{R_{2}}-\frac{V_{0}-V_{1}}{R_{0}+R_{1}}\right), \\
& P_{2}=V_{2}\left(I_{2}+I_{3}\right)=V_{2}\left(\frac{V_{1}-V_{2}}{R_{2}}+\frac{V_{0}-V_{2}}{R_{0}+R_{3}}\right),
\end{aligned}
$$

where $\delta$ is the resistivity of the catenary, and $s_{1}$ and $s_{2}$ are the position of the braking train and the tracking train, respectively. $L_{1}$ and $L_{2}$ are the distance of two sections. $l_{1}$ and $l_{2}$ are the distance between the traction substation and Station A or Station C, respectively. $V_{1}$ and $V_{2}$ denote the voltage of catenary at the position $s_{1}$ and $s_{2}$, respectively. $I_{1}$ and $I_{3}$ are the current supplied by traction substations, and $I_{2}$ is the current from the braking train to the tracking train during the absorption process.

Then, the total lost regenerative energy $J_{\text {loss }}$ during the transmission process of the catenary can be obtained by Equation (13). As the equivalent resistance changes with the position of two trains, the function of position and time was linearly interpolated before calculating $I_{2}$ and $R_{2} . \Delta t$ and $M$ here can refer to Equation (9).

$$
J_{\text {loss }}=\sum_{i=1}^{M} I_{2}^{2} R_{2} \Delta t
$$

With the consideration of dynamic losses of regenerative energy, the objective function of minimizing total energy of the multi-train system presented in Equation (9) is transferred to Equation (14), which is more in line with the practical situation.

$$
\min J_{\text {total }}=J_{\text {multi }}+J_{\text {loss }}
$$

\section{Optimization Method}

On the basis of the models established before, the optimization method and solving procedure of single-train and multi-train are proposed in this section, and the two parts are combined to work out the energy-saving speed profiles of multi-train, as shown in Figure 4.

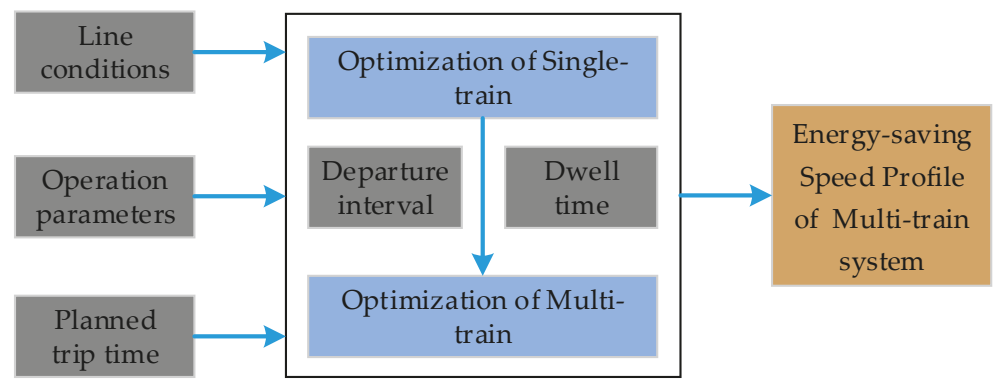

Figure 4. Combination between the optimization of single-train and multi-train.

\subsection{Optimization of Single-Train}

As the section length is usually short in the URT, the optimal control strategy of single train consists of three motion phases: MT phase, $\mathrm{CO}$ phase, and MB phase by sequence [5]. The key point to get the optimal speed profile is to find the transition speed $v^{*}$ from the MT mode to the CO mode, as shown in Figure 5. Actually, the MT phase from speed 0 to speed limit $\bar{V}$ can be easily worked out, and for each transition speed of the MT phase, there exists a unique three motion phases speed profile in order to meet the requirement of distance, and the corresponding trip time is also obtained. Therefore, $v^{*}$ can be worked out when the trip time equals to the planned trip time $T$, as well as the optimal speed profile. 


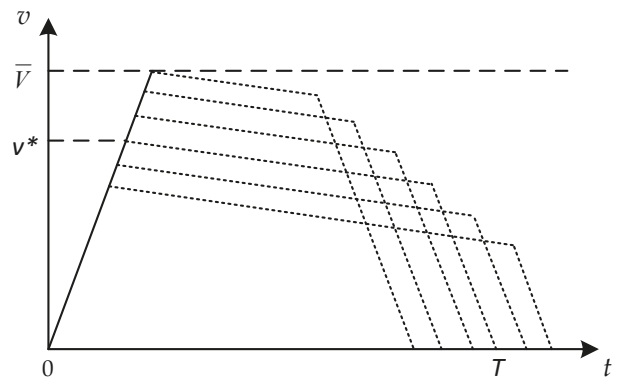

Figure 5. Searching process of $v^{*}$.

\subsection{Optimization of Multi-Train}

Aiming to minimize the total energy consumption of multi-train system, a cooperative optimization method is proposed in this part, as well as the corresponding solution algorithm. Normally, optimization of multi-train can be realized based on the optimization of single-train, and the multi-train system adopts the same single-train optimal control strategy (single-train optimal operation mode) at the beginning, as shown in Figure 6.

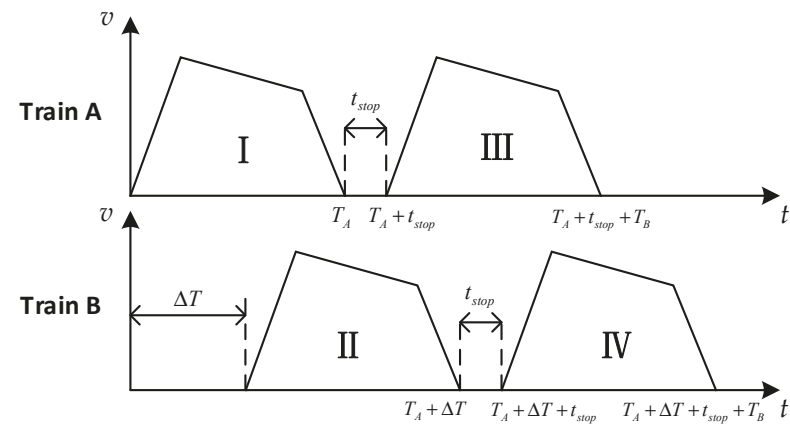

Figure 6. Single-train optimal operation curve of multi-train system.

According to the departure orders of trains, the entire running process of multi-train system can be divided into Subsection I-IV based on passenger stations, as shown in Figure 6. It is easy to observe that there exist situations in which regenerative energy cannot be recovered, where a multi-train joint optimization method needs to be proposed to make better use of regenerative energy. Subsection I still adopts the optimal control strategy of single train, as it has no regenerative energy to recover. Therefore, optimization for multi-train system starts from Subsection II and goes backwards.

Taking the optimization of Subsection II, for example, during which period only Subsection I produces regenerative energy and its speed profile has been determined. Hence, the MT phase is added to Subsection II at the MB phase of Subsection I, in order to absorb the regenerative energy generated by Subsection I, as shown in Figure 7a. 


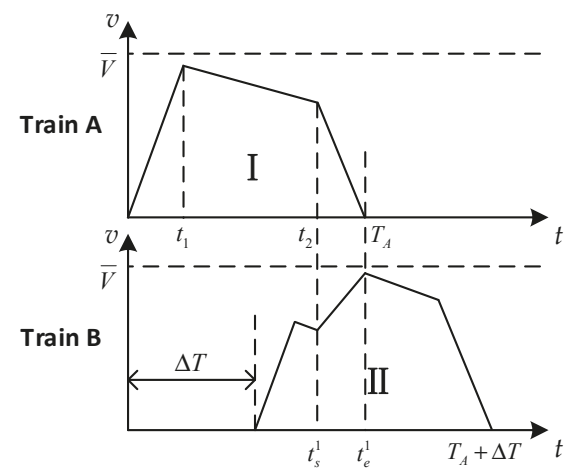

(a)

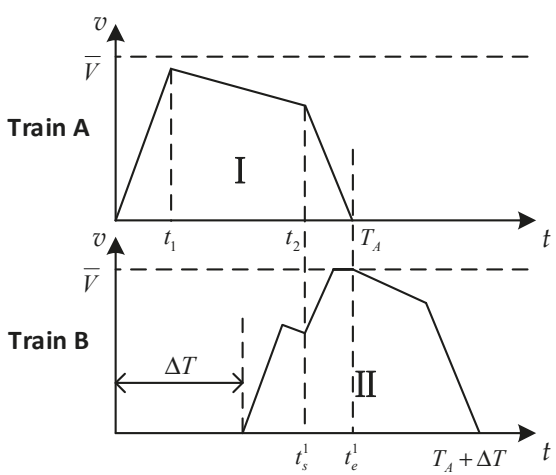

(b)

Figure 7. Optimization of Subsection II: (a) not reach speed limit in overlap phase (OP); (b) reach speed limit in OP.

Where $\bar{V}$ is the speed limit; $t_{1}$ and $t_{2}$ are the transition time of the three motion phases curve in Subsection I; $t_{s}^{1}$ and $t_{e}^{1}$ are the starting time and ending time, respectively, of the regenerative energy absorption process; and interval $\left[t_{s}^{1}, t_{e}^{1}\right]$ is called as overlap phase (OP) of Subsection II. Therefore, the optimal control strategy of multi-train consists of five motion phases: $\left[\Delta T, t_{s}^{1}\right]$ contains the MT phase and CO phase, the MT phase in OP, and $\left[t_{e}^{1}, T_{A}+\Delta T\right]$ is made up with the CO phase and MB phase. Actually, MT is not the unique operation mode in the OP; CR phase will exist when the train accelerates to $\bar{V}$, as shown in Figure $7 \mathrm{~b}$.

In order to calculate the corresponding optimal speed profile, an optimization algorithm is proposed, and the key is to find just one point, which is the transition speed $v^{*}$ from the MT phase to CO phase, as shown in Figure 8.

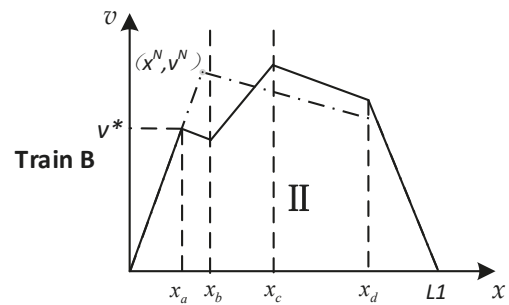

(a)

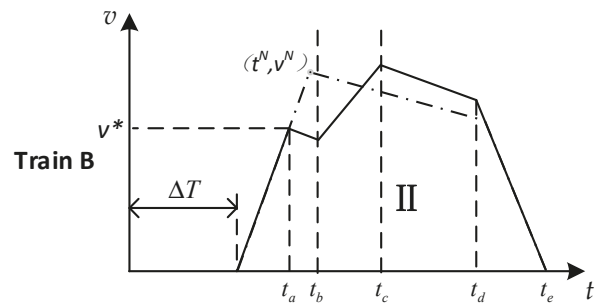

(b)

Figure 8. Transition speed of optimal operation curve: (a) the $v-x$ curve; (b) the $v-t$ curve.

Where $\left(x_{a}, x_{b}, x_{c}, x_{d}\right)$ are the transition points of the five motion phases operation curve, the corresponding times of which are $\left(t_{a}, t_{b}, t_{c}, t_{d}\right)$, obviously, $t_{b}=t_{s}^{1}, t_{c}=t_{e}^{1} .\left(x^{N}, v^{N}\right)$ is the transition point of single-train optimized speed profile, which is the input for multi-train speed profiles optimization, and the searching range (SR) of $v^{*}$ is $\left[0, v^{N}\right]$, which belongs to the MT phase. However, the SR becomes $\left[0, v^{b}\right]$ when $t_{b}<t^{N}, v^{b}$ is the speed corresponding to time $t_{b}$ in the three motion phases curve presented here. As the simulation step is distance $\Delta s$, the position set $X$ of the SR is shown in Equation (15), as well as the corresponding time set $T$ and speed set $V$.

$$
\begin{aligned}
X & =\left[S_{1}, x^{1}, x^{2}, \cdots, x^{k}, \cdots, x^{N-1}, x^{N}\right], \\
T & =\left[\Delta T, t^{1}, t^{2}, \cdots, t^{k}, \cdots, t^{N-1}, t^{N}\right], \\
V & =\left[0, v^{1}, v^{2}, \cdots, v^{k}, \cdots, v^{N-1}, v^{N}\right],
\end{aligned}
$$


where $x^{k+1}-x^{k}=x^{1}-S_{1}=\Delta s, k \in[1, N-1]$.

On the basis of the set of SR mentioned above, once the transition point $v^{k}$ is determined, the corresponding position $x_{a}$ and time $t_{a}$ are known too. As $\left[t_{b}, t_{c}\right]$ is the OP, which is known, and the operation mode of the second phase is $\mathrm{CO}, x_{b}$ can be calculated. Also, $x_{c}$ can be obtained in the same way with the MT mode in OP. Until now, the rest of the distance and trip time of the section are $\left(L_{1}-x_{c}\right)$ and $\left(T_{A}-t_{c}\right)$, respectively. Hence, the calculation of $x_{d}$ is simple. It is required to coast from $x_{c}$, and inverse calculation starts from $L_{1}$ with MB mode at the same time. When the speeds of both processes intersect, the five motion phases operation curve is completed and $x_{d}, t_{d}$, and $t_{e}$ are obtained simultaneously. Then, a time precision $\delta_{t}=1 \mathrm{~s}$ is defined, and the solution result is saved when $t_{e}$ meets Equation (16).

$$
\left|T_{A}-t_{e}\right| \leq \delta_{t}
$$

The constraints of both distance and trip time are satisfied for all saved results, which are feasible solutions of the proposed optimal control strategy. Then, the model formulated in Equation (14) is used to calculate the total energy consumption of the multi-train system, and $v^{*}$ is solved out by comparing the energy consumption of all feasible solutions, and the optimal speed profile can be obtained at the same time.

When the optimization of Subsection II is done, energy consumption of the system of Subsection I and Subsection II has been minimized. The speed profile of Subsection II is also updated, which means the braking phase of Subsection II has been updated too. Therefore, the speed profile of the first two subsections has been determined, and the speed profile of Subsection III is optimized in the same way as shown in Figure 9, as well as Subsection IV.

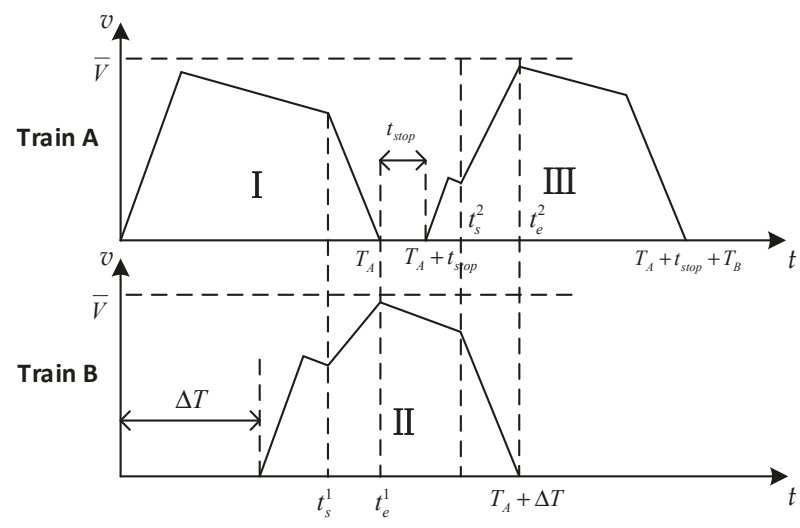

Figure 9. Optimization of Subsection III.

Actually, the proposed optimization method of multi-train system is suitable for more than two sections, with the optimization going backward until Subsection X (the last subsection) is optimized. The flow chart of the algorithm proposed above is shown in Figure 10. 


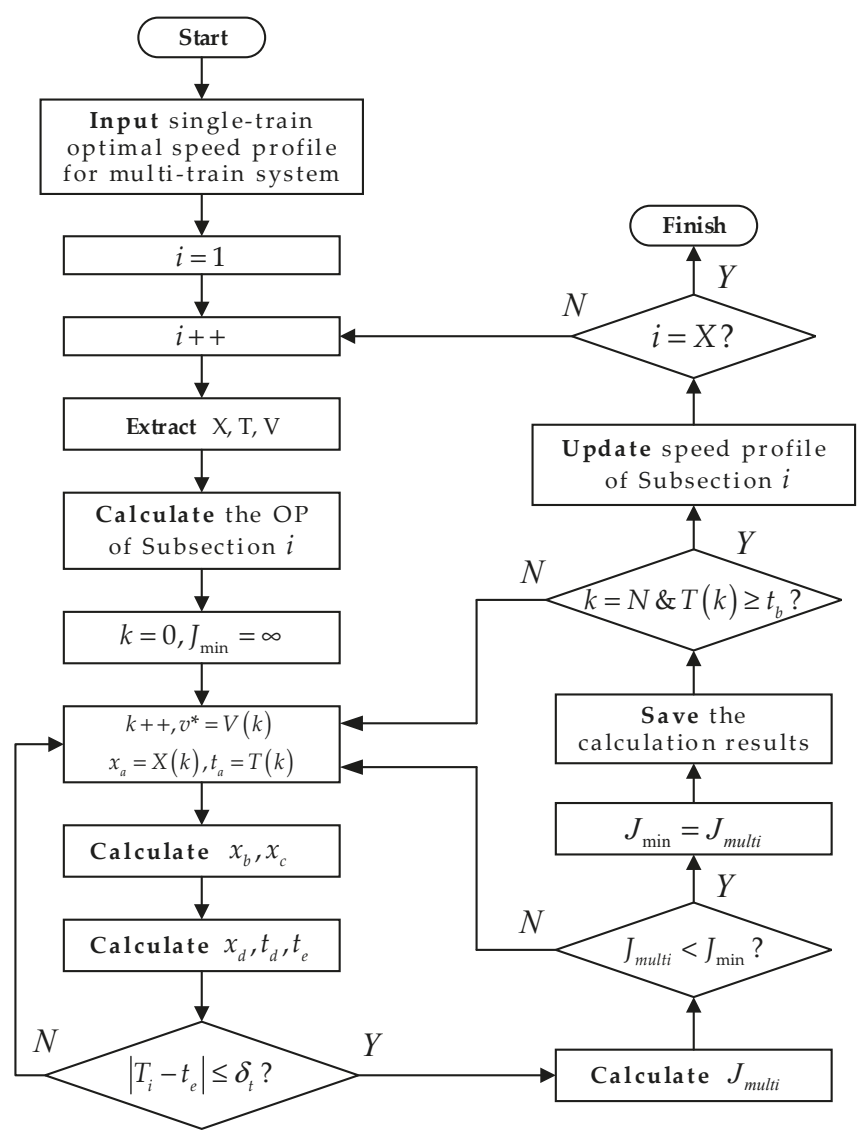

Figure 10. Flow chart of the optimization algorithm.

It is important to note that the optimal control strategy of multi-train is not always five motion phases, which means the MT phase cannot be added into the operation curve in some circumstances, and the specific situation depends on the relationship among trip time, departure interval, and dwell time. Besides, even though the optimal speed profile of a subsection is five motion phases, its OP is not always the entire MB phase of the previous subsection. Taking the optimization of Subsection II as an example, the possible situations are listed in Table $1 . t^{*}$ in Case 1 is the transition time from MT to MB, and the detailed calculation method is illustrated in Figure 11.

Table 1. Possible situations of optimal control strategy and overlap phase (OP).

\begin{tabular}{cccc}
\hline Case & Situation & Motion Phases & OP \\
\hline 1 & $T_{A}>t_{2}+\Delta T$ & 4 & {$\left[t_{2}, t^{*}\right]$} \\
2 & $t_{1}+\Delta T<T_{A}<t_{2}+\Delta T \& t_{2}>\Delta T$ & 5 & {$\left[t_{2}, T_{A}\right]$} \\
3 & $t_{1}+\Delta T<T_{A}<t_{2}+\Delta T \& t_{2}<\Delta T$ & 3 & {$\left[\Delta T, t_{1}+\Delta T\right]$} \\
4 & $\Delta T<T_{A}<t_{1}+\Delta T \& t_{2}>\Delta T$ & 3 & {$\left[t_{2}, T_{A}\right]$} \\
5 & $\Delta T<T_{A}<t_{1}+\Delta T \& t_{2}<\Delta T$ & 3 & {$\left[\Delta T, T_{A}\right]$} \\
6 & $T_{A}<\Delta T$ & 3 & NULL \\
\hline
\end{tabular}

In single-train optimization, the $\mathrm{CO}$ phase usually accounts for the most part of the three motion phases. Hence, when departure interval is less than planned trip time (namely $T_{A}>\Delta T$ ), $T_{A} \in\left[t_{1}+\Delta T\right.$, 
$\left.t_{2}+\Delta T\right]$ is satisfied for most circumstances, under which it is almost impossible for $t_{2}$ to be smaller than $\Delta T$. On the basis of the above analysis, Case 2 is the most likely situation and Case 3 basically will not appear. Therefore, the five motion phases control strategy is suitable for most circumstances, and it is valuable for the optimization of multi-train.

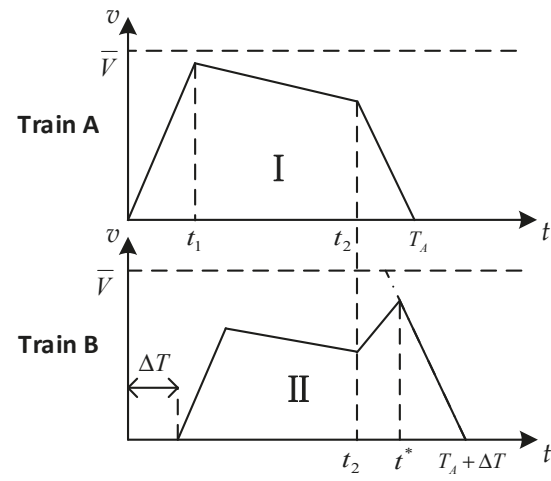

(a)

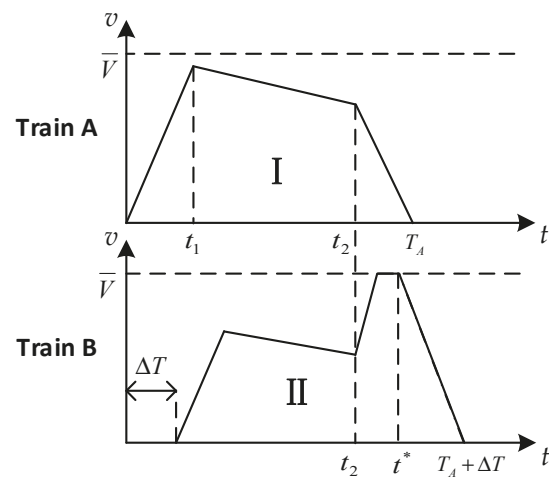

(b)

Figure 11. Optimization of Subsection II in Case 1: (a) not reach the speed limit before intersecting the maximum braking curve; (b) reach the speed limit before intersecting the maximum braking curve.

In summary, the optimal control strategy takes five motion phases only in Case 2, regenerative energy still can be recovered in the overlapping part of traction trains and braking trains in Case 3-5, and there is no regenerative energy absorption in Case 6. However, the MT phase can also be added in Case 1, according to proposed optimal control strategy, MT mode starts form $t_{2}$ and ends when intersecting the MB phase, which can be obtained by inverse calculation from $\left(T_{A}+\Delta T\right)$ to the speed limit. Therefore, the forth motion phase (namely $\mathrm{CO}$ ) will not exist in Case 1 , and there are four motion phases in Case 1, as shown in Figure 11a. Besides, $t^{*}$ is not a constant in Case 1, which depends on the intersecting time of the MT phase and MB phase. However, there may exist the CR phase in the OP if the speed reaches the speed limit before it intersects the MB phase, as shown in Figure 11b.

Actually, the starting speed of the OP is usually high when the departure interval is small, which means the demanded traction power in the OP is higher than the generated electrical braking power; hence, the energy-saving effect may not be significant for the optimization in Case 1.

\subsection{Optimization for Steep Slope}

According to the proposed optimal control strategy of multi-train, the MT phase is added to the OP and the train adopts the CR mode in the remaining time of the OP when it accelerates to $\bar{V}$. However, the partial braking (PB) mode may appear at $\bar{V}$ in the OP instead of the partial traction (PT) mode if there exists a steep slope, under which circumstance not only the power of the train is negative that cannot absorb regenerative energy, but also the gravitational potential energy of the steep slope is wasted.

On the basis of the analysis above, the energy-saving operation curve of multi-train system can be improved for a steep slope and the energy-saving rate can be further increased theoretically. In order to work out the optimal speed profile, a novel optimization method is formulated as shown in Figure 12. The basic idea is to let the train coast if there exists a steep slope in the OP. Although the CO mode cannot absorb the regenerative energy, it uses the gravitational potential energy. 


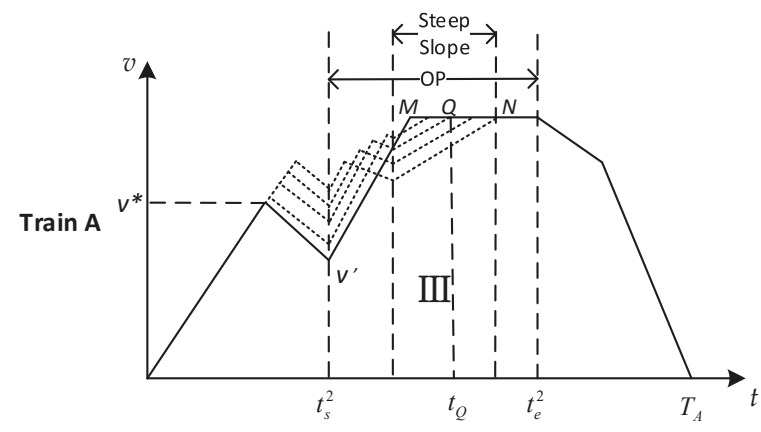

Figure 12. Optimization method for a slope steep.

Therefore, there exist three motion phases in the OP, which are the MT phase, CO phase, and CR phase by sequence. The key point is to find the transition point $Q$ from the $C O$ phase to $C R$ phase, and the searching range is the CR phase of a steep slope, namely $M N$. Once $Q$ is determined, the inverse calculation starts from $Q$ with the $\mathrm{CO}$ and MT mode by sequence, and $v^{\prime}$ can be worked out because $t_{Q}$ and $t_{s}^{2}$ are known. Then, $v^{*}$ can be obtained in the same way. Therefore, each $Q$ corresponds to a unique speed profile in order to satisfy the constraint of distance and trip time. It can be easily observed that when $Q$ is closer to $N$, the corresponding $v^{\prime}$ and $v^{*}$ are higher (for a $v$ - $t$ curve, area of the speed profile is constant under fixed distance). Assume that the position of $M$ and $N$ are $x_{m}$ and $x_{n}$, hence, the amount of searching point $Q$ and corresponding feasible solutions are $(m-n+1)$. The optimal speed profile can be obtained by comparing the energy consumption of feasible solutions.

\section{Simulation}

\subsection{Single-Train}

(a) Related Parameters

Taking the section between Chigang Station and Kecun Station of Guangzhou Metro Line 8 as the test line, the total length and planned trip time are $1.489 \mathrm{~km}$ and $96 \mathrm{~s}$, respectively. The line conditions are shown in Table 2.

Table 2. Line Conditions.

\begin{tabular}{cccc}
\hline Start Position $(\mathbf{m})$ & End Position $(\mathbf{m})$ & Gradient $\mathbf{( \% o})$ & Speed Limit $\mathbf{( k m} / \mathbf{h})$ \\
\hline 3732.0 & 3794.0 & -3 & 80 \\
3794.0 & 4075.4 & -3 & 65 \\
4075.4 & 4163.0 & -3 & 80 \\
4163.0 & 4199.0 & -3 & 75 \\
4199.0 & 4360.6 & -4 & 75 \\
4360.6 & 4489.0 & -4 & 80 \\
4489.0 & 5099.0 & 9.64 & 80 \\
5099.0 & 5221.0 & 3 & 80 \\
\hline
\end{tabular}

In addition, the metro vehicle of Guangzhou Metro Line 8 is A-Type produced by CRRC Corporation Limited, which has the best passenger capacity. The vehicle marshalling type is $4 \mathrm{M} 2 \mathrm{~T}$ (A-B-C-C-B-A) and is shown as follows:

In Figure 13, A is a trailer with driver's room, B is a motor train with pantograph, and C is a motor train, which weigh $37.3 \mathrm{t}, 40.6 \mathrm{t}$, and $40.6 \mathrm{t}$, respectively. In AW2 case, the total mass of a train is $339.6 \mathrm{t}$. 


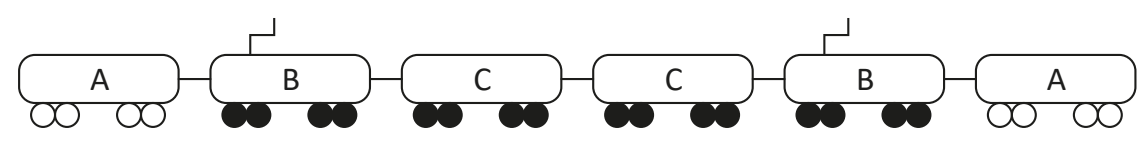

Figure 13. Marshalling structure of A-type metro vehicles.

According to the official product data sheet, the weighted average rotary mass coefficient $\rho$ and the conversion efficiency of traction system $\eta_{t}$ are given as

$$
\rho=0.08, \eta_{t}=90 \%
$$

The basis running resistance corresponding to the speed $v(s)$ is

$$
W(v)=8.907+1.334 \times 10^{-3} v^{2} .
$$

The characteristic curve of basis running resistance and the maximum traction/braking force corresponding to the speed $v(s)$ are shown in Figure 14:

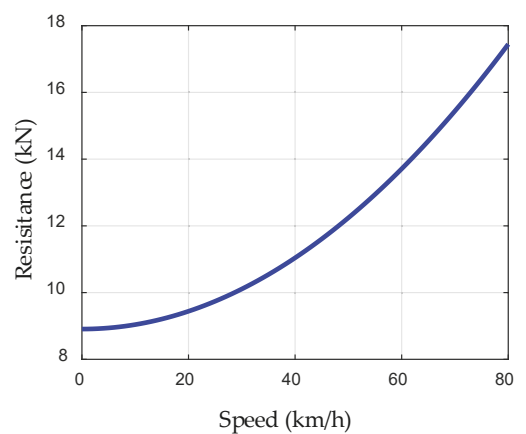

(a)

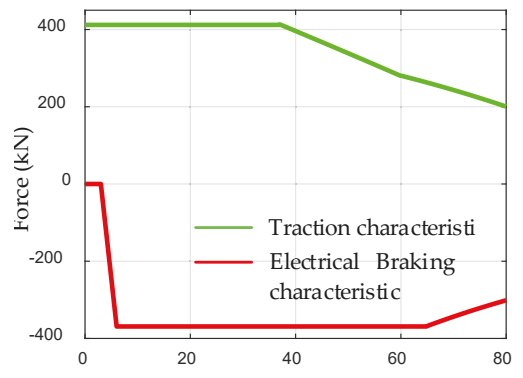

(b)

Figure 14. (a) Basis running resistance characteristic curve; (b) traction and braking characteristic curve.

\section{(b) Result}

On the basis of the proposed method, the test line mentioned above is taken for simulation and a transition speed of $76.7 \mathrm{~km} / \mathrm{h}$ is worked out. The optimal operation curve and real operation curve are shown in Figure 15. The measured data of the real operation process are collected by the onboard device of Guangzhou Metro Line 8, and the sampling interval of speed and traction/braking force is $1 \mathrm{~s}$. Besides, the total energy consumption of real running process $J_{\text {real }}$ can be obtained as follows:

$$
\begin{gathered}
J_{\text {real }}=\sum_{i=1}^{N} P_{t i} \Delta t, \\
P_{t i}=F_{t i} v_{i},
\end{gathered}
$$

where $P_{t i}$ is the traction power of train; $\Delta t$ is the sampling interval; $N$ is the sample count that can be obtained by $N=T / \Delta t$; and $F_{t i}$ and $v_{i}$ are the traction force and speed of train, respectively. 


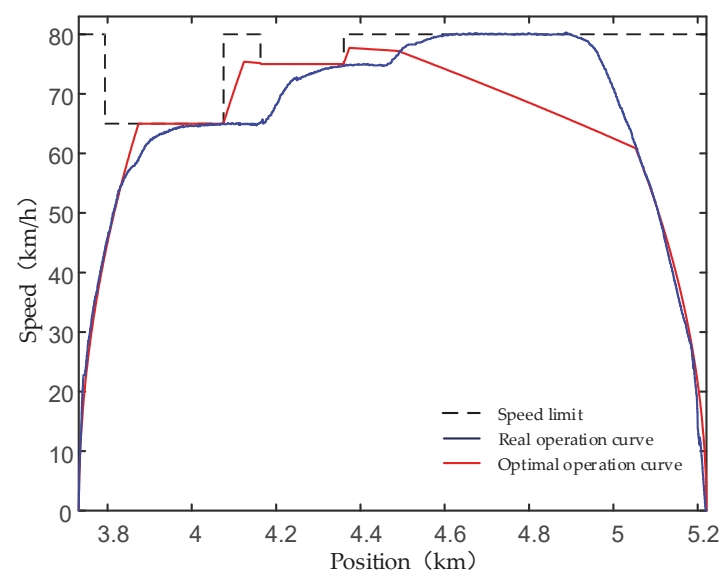

Figure 15. Optimal operation curve and real operation curve.

The comparison of energy consumption between optimal operation and real operation is shown in Table 3. The total traction energy consumption of optimal operation mode is $24.23 \mathrm{kWh}$, and a $14.08 \%$ energy-saving rate can be obtained compared with the energy consumption of real operation.

Table 3. Comparison of energy consumption.

\begin{tabular}{cc}
\hline Operation Mode & Energy Consumption (kWh) \\
\hline Optimal Operation & 24.23 \\
Real Operation & 28.20 \\
\hline
\end{tabular}

\subsection{Multi-Train}

(a) Related Parameters

The three successive stations of the test line are Wanshengwei Station, Pazhou Station, and Xingangdong Station by sequence of Guangzhou Metro Line 8. The line conditions are shown in Table 4 and the operation parameters are shown in Table 5 .

Table 4. Line Conditions.

\begin{tabular}{cccc}
\hline Start Position $(\mathbf{m})$ & End Position $(\mathbf{m})$ & Gradient $(\%$ o) & Speed Limit $(\mathbf{k m} / \mathbf{h})$ \\
\hline-1428 & -1300 & -3 & 80 \\
-1300 & -650 & -5 & 80 \\
-650 & 0 & 5.029 & 80 \\
0 & 235 & 2 & 80 \\
235 & 610 & 3 & 80 \\
610 & 780 & -24 & 80 \\
780 & 1030 & 9.352 & 80 \\
1030 & 1398 & 3 & 80 \\
\hline
\end{tabular}


Table 5. Operation Parameters.

\begin{tabular}{cccc}
\hline Parameters & Value & Parameters & Value \\
\hline$S_{1}(\mathrm{~m})$ & -1428 & $L_{1}(\mathrm{~m})$ & 1832 \\
$S_{2}(\mathrm{~m})$ & 404 & $L_{2}(\mathrm{~m})$ & 994 \\
$S_{3}(\mathrm{~m})$ & 1398 & $\Delta s(\mathrm{~m})$ & 1 \\
$T_{A}(\mathrm{~s})$ & 115 & $\Delta t(\mathrm{~s})$ & 0.1 \\
$T_{B}(\mathrm{~s})$ & 77 & $\Delta T(\mathrm{~s})$ & 65 \\
$t_{\text {stop }}(\mathrm{s})$ & 30 & $V_{0}(\mathrm{~V})$ & 1650 \\
$R_{0}(\Omega)$ & 0.054 & $\delta(\Omega / \mathrm{km})$ & 0.03 \\
\hline
\end{tabular}

\section{(b) Result for the Flat Route}

Simulation on flat route is conducted to demonstrate the effectiveness of the proposed method. The single-train optimal operation curve of the multi-train system is shown in Figure 16. The transition speed from MT to CO of the two trains in the two sections are $77.9 \mathrm{~km} / \mathrm{h}$ and $64.6 \mathrm{~km} / \mathrm{h}$, respectively. It can be observed from power curves of two trains that the regenerative energy cannot be effectively absorbed for the single-train optimal operation mode. Besides, corresponding results with dynamic losses of recovered regenerative energy on the catenary are also shown, from which the resistance $R_{2}$ and the power losses between two trains of the catenary can be clearly observed.
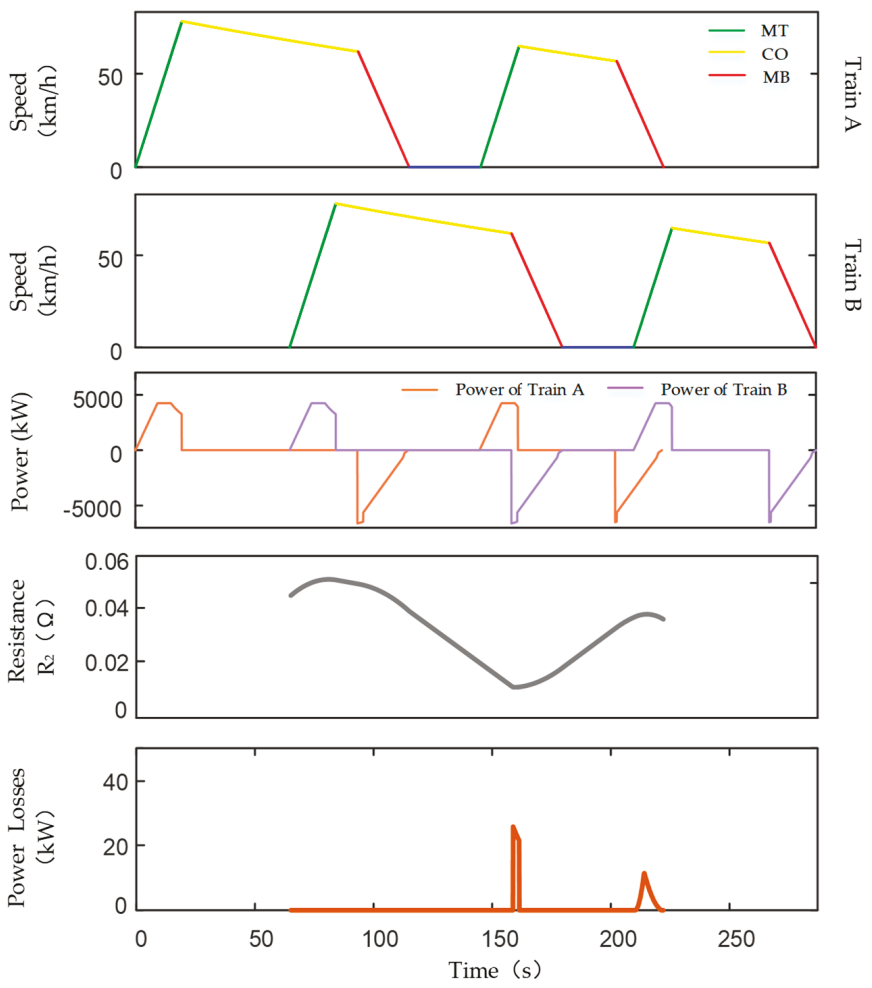

Figure 16. Single-train optimal operation curve of the multi-train system on a flat route. MT, maximum traction; $\mathrm{MB}$, maximum braking; $\mathrm{CO}$, coasting.

The energy-saving operation curve is worked out by multi-train joint optimization and is shown in Figure 17, as well as the results of regenerative energy dynamic losses. Subsection I has no regenerative 
energy to absorb and Subsection IV belongs to Case 5; both need not to be optimized, and the $v^{*}$ of Subsection II-III are $47.7 \mathrm{~km} / \mathrm{h}$ and $13.1 \mathrm{~km} / \mathrm{h}$, respectively. The power curve shows that regenerative energy is effectively absorbed. The energy consumption of two operation modes is shown in Table 6. Although multi-train joint optimization increases the traction energy, it makes better use of regenerative energy and decreases the system energy, and the total energy consumption of Figures 16 and 17 is $65.0306 \mathrm{kWh}$ and $61.7688 \mathrm{kWh}$, respectively. The energy-saving rate brought by multi-train joint optimization is $5.02 \%$ in this case.
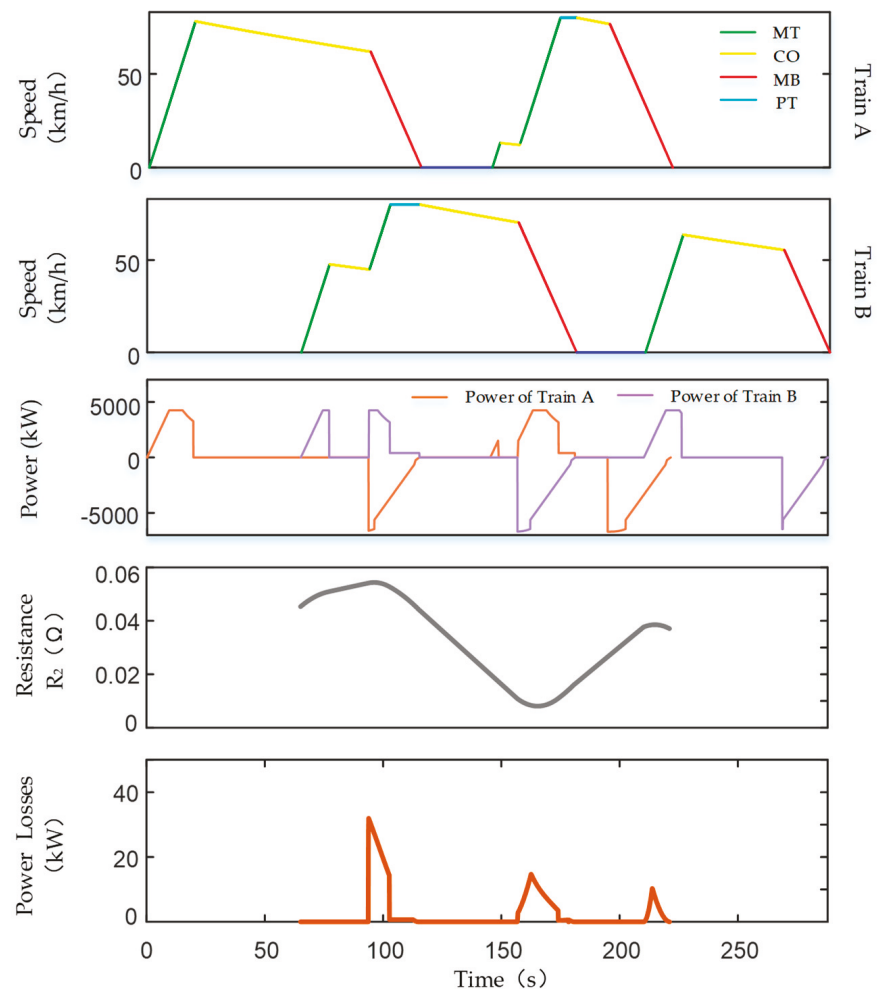

Figure 17. Optimal operation curve of multi-train system on flat route. PT, partial traction.

Table 6. Comparison of energy consumption on a flat route.

\begin{tabular}{ccccc}
\hline $\begin{array}{c}\text { Operation } \\
\text { Mode }\end{array}$ & $\begin{array}{c}\text { Traction Energy } \\
\mathbf{( k W h )}\end{array}$ & $\begin{array}{c}\text { Recovered Regenerative } \\
\text { Energy } \mathbf{( k W h )}\end{array}$ & $\begin{array}{c}\text { Lost Regenerative } \\
\text { Energy } \mathbf{( k W h )}\end{array}$ & $\begin{array}{c}\text { Total Energy } \\
\mathbf{( k W h )}\end{array}$ \\
\hline Figure 16 & 69.4219 & 4.4222 & 0.0309 & 65.0306 \\
Figure 17 & 81.2183 & 19.5612 & 0.1118 & 61.7688 \\
\hline
\end{tabular}

\section{(c) Result for the Practical Route}

The effectiveness of the proposed solution method was verified on the flat route. Now, the gradient listed in Table 4 is taken into consideration, and the single-train optimal operation curve of multi-train system and corresponding results with dynamic losses of recovered regenerative energy are shown in Figure 18. The speed in the CO phase fluctuates as the gradient changes, and regenerative energy still cannot be effectively absorbed. On the practical route, the transition speed from MT to CO of the 
two sections are $76.8 \mathrm{~km} / \mathrm{h}$ and $61.2 \mathrm{~km} / \mathrm{h}$, respectively. The detailed energy consumption is shown in Table 7.
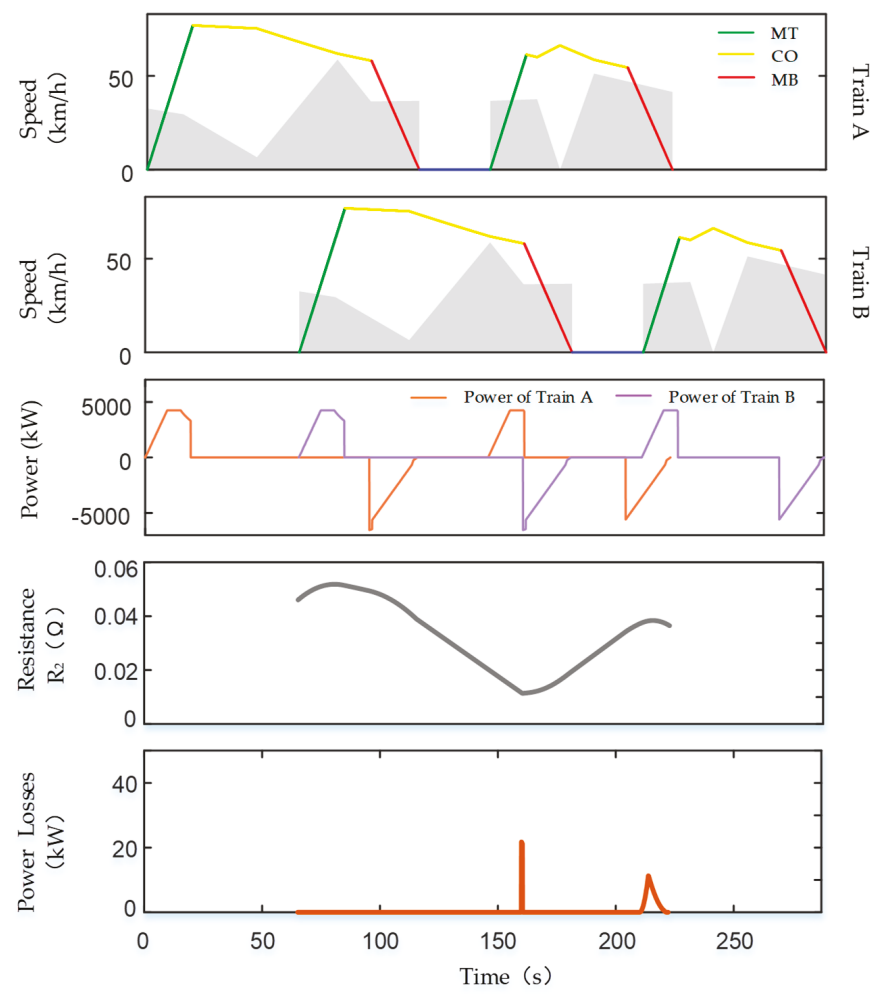

Figure 18. Single-train optimal operation curve of multi-train system on a practical route.

Table 7. Detailed energy consumption of single-train optimal operation mode.

\begin{tabular}{ccccc}
\hline Subsection & $\begin{array}{c}\text { Traction Energy } \\
\mathbf{( k W h )}\end{array}$ & $\begin{array}{c}\text { Recovered Regenerative } \\
\text { Energy } \mathbf{( k W h )}\end{array}$ & $\begin{array}{c}\text { Lost Regenerative } \\
\text { Energy (kWh) }\end{array}$ & $\begin{array}{c}\text { Total Energy } \\
\mathbf{( k W h )}\end{array}$ \\
\hline I & 19.3669 & 0.0000 & 0.0000 & 19.3669 \\
II & 19.3669 & 0.0000 & 0.0000 & 19.3669 \\
III & 12.9795 & 0.4709 & 0.0034 & 12.5120 \\
IV & 12.9795 & 2.1228 & 0.0131 & 10.8698 \\
Total & 64.6928 & 2.5937 & 0.0165 & 62.1156 \\
\hline
\end{tabular}

The energy-saving operation curve on a practical route is obtained by multi-train joint optimization and shown in Figure 19. Same as the situation on the flat route, Subsection I and Subsection IV need not be optimized. The $v^{*}$ of Subsection II-III are $53.4 \mathrm{~km} / \mathrm{h}$ and $16.9 \mathrm{~km} / \mathrm{h}$, respectively. The power curves of two trains show that the energy-saving control strategy of multi-train system has a good absorption effect for the regenerative energy. Besides, the resistance $R_{2}$ and the power losses are also shown. The power losses curve increases to the peak and then decreases in each OP; actually, the peak represents the maximum absorption capacity for regenerative energy, namely when the traction power and the braking power are equal. The electrical line resistance $R_{2}$ depends on the train positions and varies with time, as the distance of the two trains varies with time. The detailed energy consumption is shown in Table 8. 

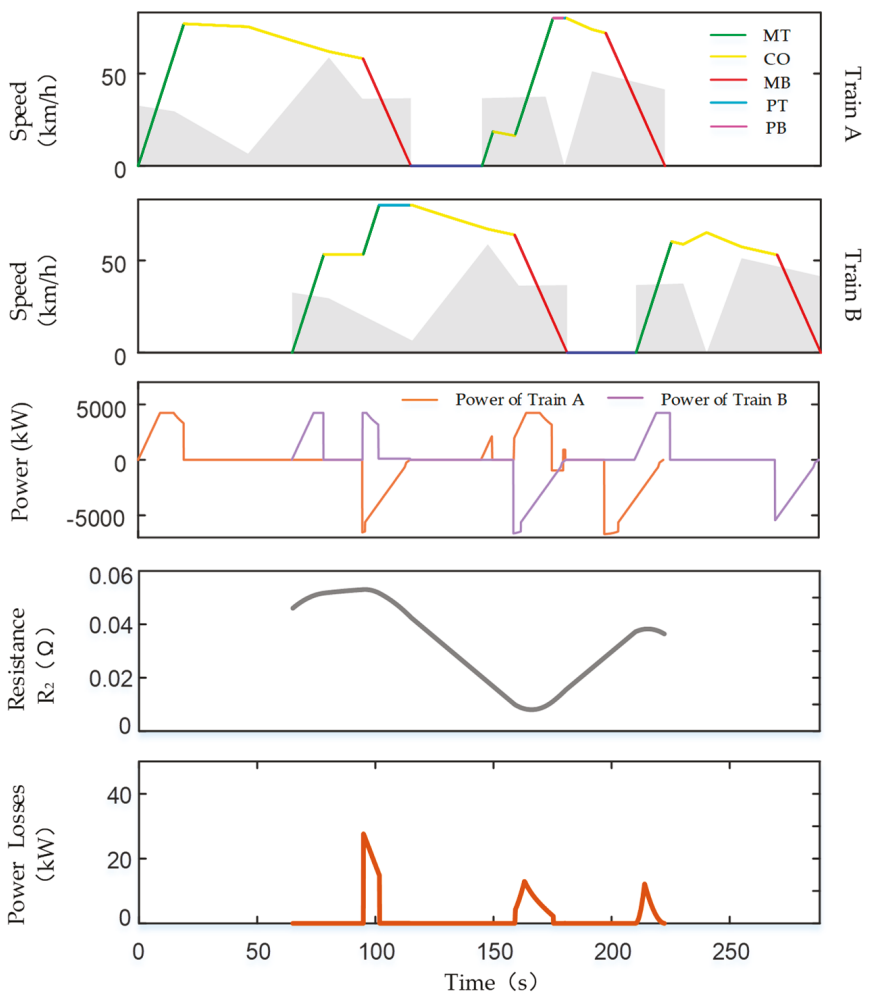

Figure 19. Optimal operation curve of the multi-train system on a practical route.

Table 8. Detailed energy consumption of multi-train optimal operation mode

\begin{tabular}{ccccc}
\hline Subsection & $\begin{array}{c}\text { Traction Energy } \\
\mathbf{( k W h )}\end{array}$ & $\begin{array}{c}\text { Recovered Regenerative } \\
\text { Energy } \mathbf{( k W h )}\end{array}$ & $\begin{array}{c}\text { Lost Regenerative } \\
\text { Energy (kWh) }\end{array}$ & $\begin{array}{c}\text { Total Energy } \\
\mathbf{( k W h )}\end{array}$ \\
\hline I & 19.3669 & 0.0000 & 0.0000 & 19.3669 \\
II & 21.5283 & 5.0768 & 0.0403 & 16.4918 \\
III & 21.3106 & 6.9397 & 0.0363 & 14.4072 \\
IV & 12.5800 & 2.2773 & 0.0146 & 10.3173 \\
Total & 74.7859 & 14.2938 & 0.0912 & 60.5832 \\
\hline
\end{tabular}

However, the influence of considering the dynamic losses of regenerative energy on the optimal operation curve of multi-train needs to be evaluated, and the difference of optimal speed profile between with and without the regenerative energy transmission losses is shown in Figure 20, in which it is easy to observe that speed profiles of Subsections I, II, and IV remain unchanged. However, the $v^{*}$ of Subsection III changes from $18.0 \mathrm{~km} / \mathrm{h}$ to $16.9 \mathrm{~km} / \mathrm{h}$ after considering the regenerative energy transmission losses. 

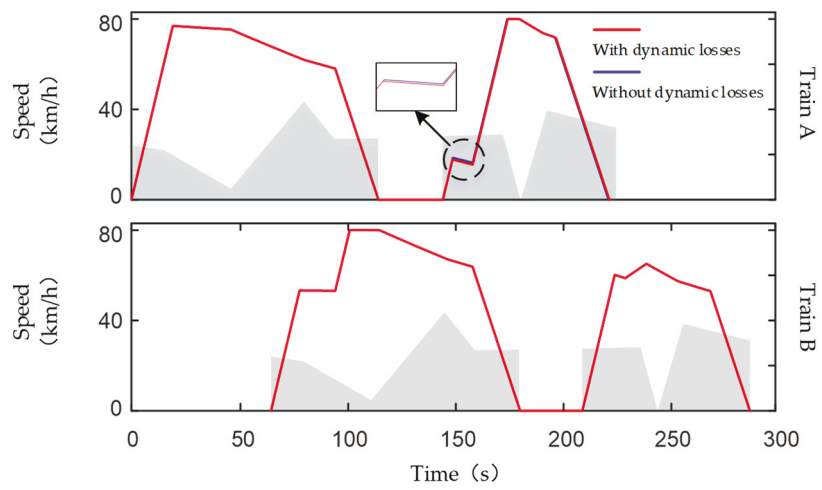

Figure 20. Difference of optimal speed profile between with and without the regenerative energy transmission losses.

The speed profile of the real running process and gradient is shown in Figure 21. Energy consumption can be obtained by Equations (19) and (20), which is $41.67 \mathrm{kWh}$. Assume that the departure interval also takes $\Delta T$, the corresponding $P$ - $t$ curve of multi-train system with real operation mode is shown in Figure 22, as well as the result of dynamic losses of recovered regenerative energy on the catenary.

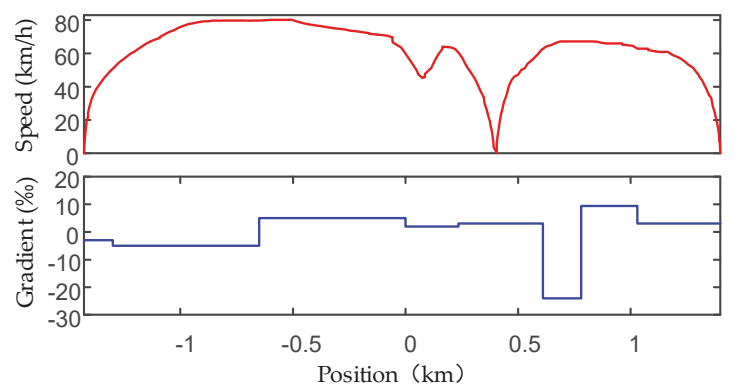

Figure 21. Real operation curve and corresponding gradient of the test line.
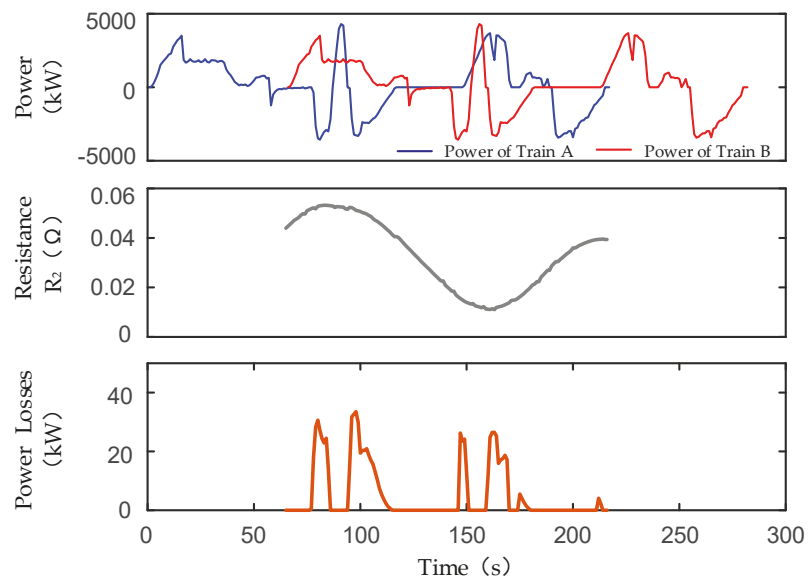

Figure 22. Results with regenerative energy dynamic losses of real operation mode. 
On this basis, absorbed regenerative energy of the real running process can be worked out by the third item of Equations (9) and (10), which is $10.33 \mathrm{kWh}$. The lost regenerative energy during the transmission process on the catenary is $0.2275 \mathrm{kWh}$. Therefore, total energy consumption of the multi-train system with the real operation mode is $73.2375 \mathrm{kWh}$. The result shows that the energy-saving rates of the optimal operation mode are $2.47 \%$ and $17.28 \%$ compared with the single-train optimal operation mode and real operation mode, respectively.

\section{(d) Energy-Saving Effect Promotion for Steep Slope}

It can be observed from Tables 7 and 8 that the energy consumption of Subsection II and IV is reduced, but is increased for Subsection III. The speed profile and corresponding power curve of Subsection III are shown in Figure 23. Obviously, PB mode appears at $\bar{V}$ in the OP, the start and end positions of this area are $675 \mathrm{~m}$ and $780 \mathrm{~m}$, respectively. It can be known from Table 4 that the corresponding gradient is -24 . Not only the power of Train A in this area is negative and cannot absorb the regenerative energy, but the line potential energy is also wasted.

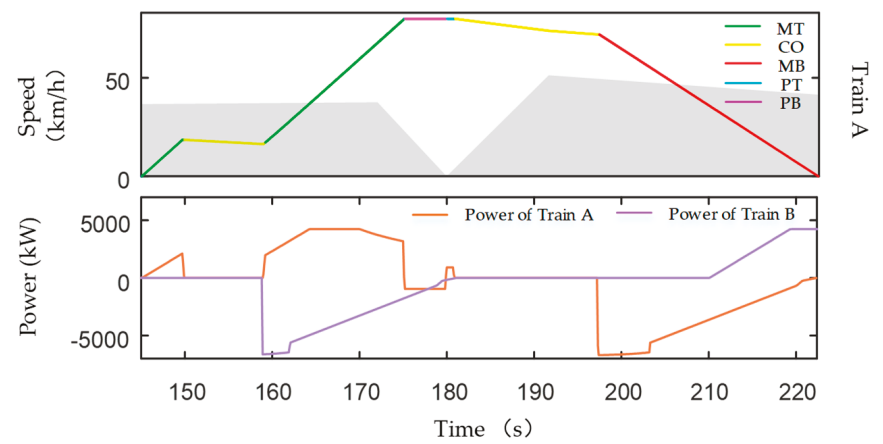

Figure 23. Speed profile and corresponding power curve of Subsection III.

The optimal operation curve is worked out by the optimization method of a steep slope and is shown in Figure 24, as well as the results with dynamic losses. The $v^{*}$ and $v^{\prime}$ of Subsection III are $17.8 \mathrm{~km} / \mathrm{h}$ and $16.4 \mathrm{~km} / \mathrm{h}$, which were $16.9 \mathrm{~km} / \mathrm{h}$ and $15.5 \mathrm{~km} / \mathrm{h}$ before improvement, respectively. In addition, there were three operation modes in the OP (namely MT, PB, and PT), and the transition speeds of three phases were the speed limit. After improvement, the OP is made of MT, CO, and PT, and the PB mode disappears. The transition speed from MT to CO is $76.1 \mathrm{~km} / \mathrm{h}$, then the train coasts to the speed limit using the line potential energy. Actually, the corresponding $Q$ is $N$, namely the transition position from $\mathrm{CO}$ to $\mathrm{PT}$ is the end of steep slope. Therefore, the optimal control strategy of multi-train for a steep slope contains seven motion phases (namely, MT, CO, MT, CO, PT, CO, and MB by sequence).

The detailed energy consumption of each subsection is shown in Table 9, and the total energy consumption of Subsection III decreases by $2.73 \mathrm{kWh}$, among which the traction energy decreases by $1.73 \mathrm{kWh}$ and the absorbed regenerative energy increases by $1.00 \mathrm{kWh}$. The former confirmed the concept of the leaving slope speed proposed by Jin and Wang [38], namely when the train reaches the speed limit at the end of a steep slope, it can make full use of the line potential energy and minimize the traction energy. As for the latter, the power of Train A corresponding speed $v^{\prime}$ increases because $v^{\prime}$ increases, which means the absorbed power increases at the beginning part of the OP. Therefore, both conditions are met simultaneously, optimization for steep slope improves the utilization of regenerative energy while making full use of the line potential energy. 

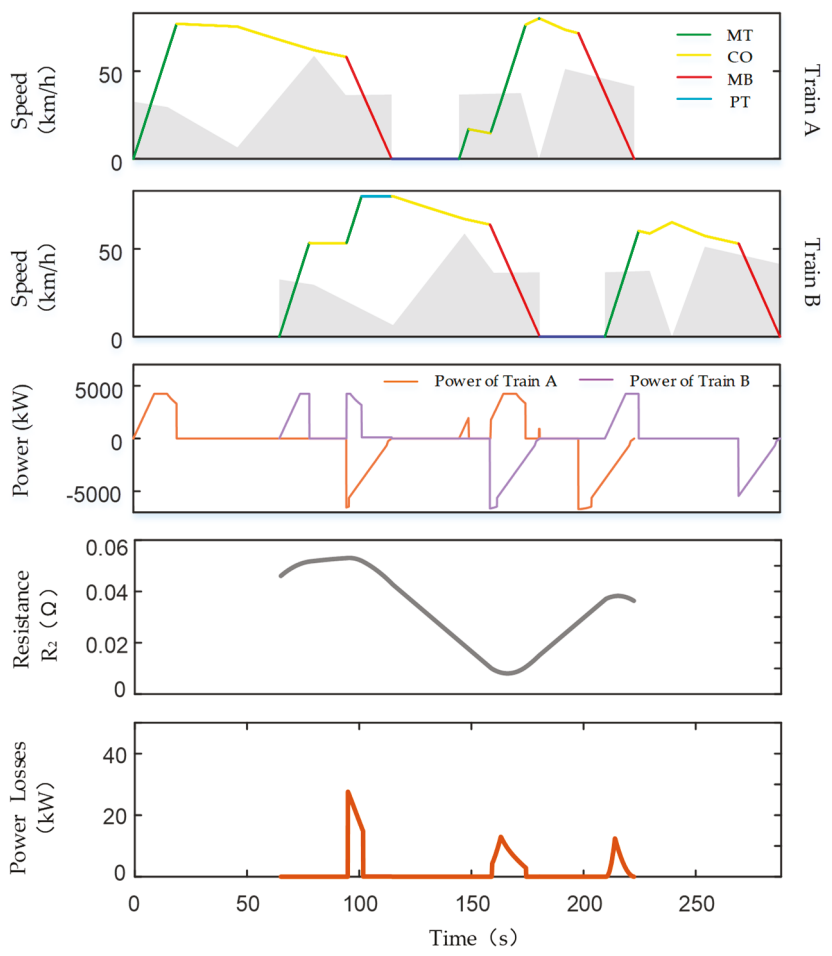

Figure 24. Optimal operation curve of the multi-train system for a steep slope.

Table 9. Detailed energy consumption after improvement for a steep slope.

\begin{tabular}{ccccc}
\hline Subsection & $\begin{array}{c}\text { Traction Energy } \\
\mathbf{( k W h )}\end{array}$ & $\begin{array}{c}\text { Recovered Regenerative } \\
\text { Energy } \mathbf{( k W h )}\end{array}$ & $\begin{array}{c}\text { Lost Regenerative } \\
\text { Energy } \mathbf{( k W h )}\end{array}$ & $\begin{array}{c}\text { Total Energy } \\
\mathbf{( k W h )}\end{array}$ \\
\hline I & 19.3669 & 0.0000 & 0.0000 & 19.3669 \\
II & 21.5283 & 5.0768 & 0.0403 & 16.4918 \\
III & 19.5833 & 7.9442 & 0.0368 & 11.6760 \\
IV & 12.5800 & 2.3317 & 0.0151 & 10.2634 \\
Total & 73.0586 & 15.3528 & 0.0923 & 57.7981 \\
\hline
\end{tabular}

The result verifies the effectiveness of the proposed optimization method for a steep slope, which brings ideal energy-saving effect and the total energy consumption of the multi-train system is $57.7981 \mathrm{kWh}$. The comparison of total energy consumption between four operation modes is shown in Table 10. The energy-saving rate after improvement for steep slope can reach up to $4.60 \%, 6.95 \%$, and $21.08 \%$, respectively, compared with the unimproved operation mode, single-train optimal operation mode, and real operation mode by sequence.

Table 10. Total energy consumption of four operation modes.

\begin{tabular}{cc}
\hline Operation Mode & Total Energy (kWh) \\
\hline After Improvement & 57.7981 \\
Before Improvement & 60.5832 \\
Single-train Optimal Operation & 62.1156 \\
Real Operation & 73.2375 \\
\hline
\end{tabular}




\section{(e) Different Departure Intervals}

The departure interval of the previous simulation is $65 \mathrm{~s}$; however, the total energy consumption and the regenerative energy utilization rate are different for different departure intervals. The relationship of which with the two operation modes is shown in Figure 25.

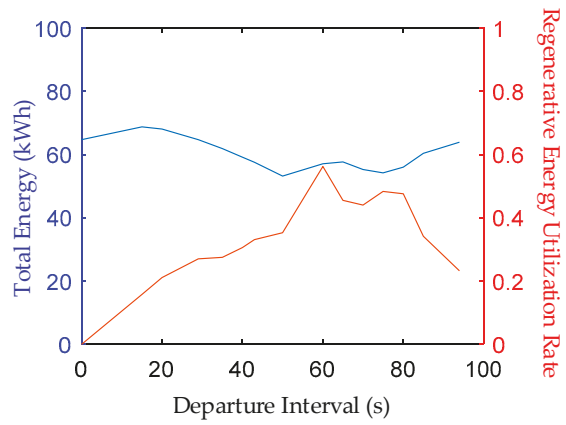

(a)

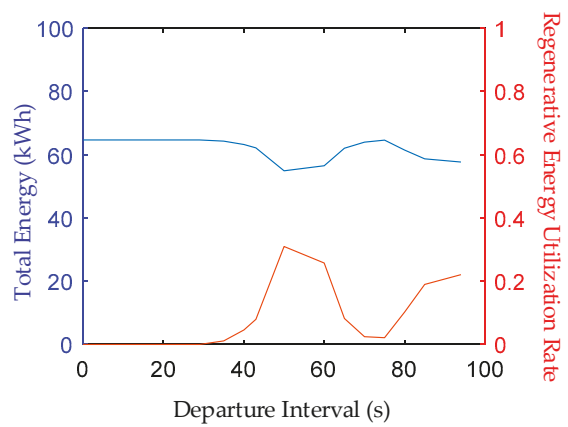

(b)

Figure 25. Total energy consumption and regenerative energy utilization rate of different departure intervals: (a) multi-train optimal operation mode; (b) single-train optimal operation mode.

It can be observed that for the single-train optimal operation mode, because the speed profile of each subsection is changeless, the MB phases are changeless, namely the total regenerative energy is a constant, hence, minimum total energy consumption corresponding to maximum regenerative energy utilization rate, and the corresponding departure interval is $50 \mathrm{~s}$, as shown in Figure 25b. However, the rule is not applicable for the multi-train optimal operation mode, because the speed profile of each subsection changes after joint optimization, which means the MB phases change and the total regenerative energy is not a constant. As shown in Figure 25a, the departure interval of the minimum total energy consumption is $50 \mathrm{~s}$, but the departure interval of the maximum regenerative energy utilization rate is $60 \mathrm{~s}$.

Besides, in the calculation of regenerative energy utilization rate, some studies that only optimize the tracking train just count the regenerative energy of the former train into total regenerative energy. In this paper, the braking phases of all subsections are taken into consideration even though the regenerative energy generated by the last subsection has no other trains to recover, otherwise the utilization rate will be much higher.

\section{Conclusions}

In this paper, an energy-efficient control method of the multi-train system is proposed in the foundation of the single-train optimal control strategy. Speed profiles of multi-train are collaborative optimized and updated based on departure orders of trains. In addition, an improved optimization strategy of multi-train that contains seven motion phases is formulated for a steep slope, which can make full use of the line potential energy and further reduce the system total energy consumption. Moreover, an electrical network model is established to evaluate the regenerative energy losses on the line, making the optimization results more realistic and convincing. The numerical simulation results indicate that the energy-saving rates can be up to $6.95 \%$ and $21.08 \%$, respectively, by multi-train joint optimization compared with single-train optimal operation mode and measured data. All proposed solution methods in this paper only need to find one transition point, and the effectiveness is verified based on Guangzhou Metro Line 8. The complex problem of multi-train cooperative optimization is greatly simplified and the calculation process is efficient.

In addition, total energy consumption of different departure intervals is analyzed, as well as the corresponding regenerative energy utilization rate. A conclusion can be obtained that the departure 
interval of minimum total energy and maximum regenerative energy utilization rate are not same as for the multi-train optimal operation mode. However, the target of multi-train optimization is to minimize the system total energy consumption; the optimal departure interval of two operation modes is $50 \mathrm{~s}$.

The combination of two parts above can work out the optimal departure interval of a multi-train system and the corresponding energy-saving speed profile, which is meaningful for practical engineering application. Moreover, the electrical network model presented in this paper is limited to dual trains on single track. The evaluation of lost regenerative energy in the situation with two trains on dual tracks deserves to be studied, which will guide our next-step research.

Author Contributions: M.C. and Z.X. contributed to the outline of the study and the model. P.S. and B.J. analyzed the data; Q.W. helped to revise the paper; X.F. provided the line, vehicle, and onboard measured data; M.C. designed the algorithm and wrote this paper.

Funding: This work was supported by the National Key Research and Development Program of China under Grant 2016YFB1200502.

Acknowledgments: The measured data used for simulation is supported by Guangzhou Metro.

Conflicts of Interest: The authors declare no conflict of interest.

\section{Abbreviations}

The following abbreviations are used in this manuscript:

$\begin{array}{ll}\text { URT } & \text { Urban rail transit } \\ \text { OP } & \text { Overlap phase } \\ \text { MT } & \text { Maximum traction } \\ \text { CR } & \text { Cruising } \\ \text { CO } & \text { Coasting } \\ \text { MB } & \text { Maximum braking } \\ \text { PT } & \text { Partial traction } \\ \text { PB } & \text { Partial braking }\end{array}$

\section{References}

1. Fouracre, P.; Dunkerley, C.; Gardner, G. Mass rapid transit systems for cities in the developing world. Transp. Rev. 2003, 23, 299-310. [CrossRef]

2. Ichikawa, K. Application of Optimization Theory for Bounded State Variable Problems to the Operation of a Train. Bull. JSME 1968, 11, 857-865. [CrossRef]

3. Howlett, P.G.; Milroy, I.P.; Pudney, P.J. Energy-Efficient Train Control; Springer Science \& Business Media: Berlin, Germany, 1994; Volume 2, pp. 193-200.

4. Howlett, P.G. Optimal strategies for the control of a train. Automatica 1996, 32, 519-532. [CrossRef]

5. Howlett, P.G. An optimal strategy for the control of a train. ANZIAM J. 1990, 31, 454-471. [CrossRef]

6. Khmelnitsky, E. On an optimal control problem of train operation. IEEE Trans. Autom. Control. 2000, 45, 1257-1266. [CrossRef]

7. Liu, R.; Golovitcher, I.M. Energy-efficient operation of rail vehicles. Transp. Res. Part A Policy Pract. 2003, 37, 917-932. [CrossRef]

8. Wang, Q.Y.; Feng, X.Y. Optimal switching for control conditions of punctual and energy efficient operation of train. China Railw. Soc. 2016, 37, 91-98. (In Chinese)

9. Miyatake, M.; Matsuda, K. Energy Saving Speed and Charge/Discharge Control of a Railway Vehicle with On-board Energy Storage by Means of an Optimization Model. IEEJ Trans. Electr. Electron. Eng. 2009, 4, 771-778. [CrossRef]

10. Lu, S.; Hillmansen, S.; Ho, T.K.; Roberts, C. Single-Train Trajectory Optimization. IEEE Trans. Intell. Transp. Syst. 2013, 14, 743-750. [CrossRef]

11. Miyatake, M.; Ko, H. Optimization of Train Speed Profile for Minimum Energy Consumption. IEEJ Trans. Electr. Electron. Eng. 2010, 5, 263-269. [CrossRef] 
12. Gu, Q.; Tang, T.; Cao, F.; Song, Y.-D. Energy-Efficient Train Operation in Urban Rail Transit Using Real-Time Traffic Information. IEEE Trans. Intell. Transp. Syst. 2014, 15, 1216-1233. [CrossRef]

13. Wang, Y.; De Schutter, B.; Boom, T.J.V.D.; Ning, B. Optimal trajectory planning for trains-A pseudospectral method and a mixed integer linear programming approach. Transp. Res. Part C Emerg. Technol. 2013, 29, 97-114. [CrossRef]

14. Chang, C.; Sim, S. Optimising train movements through coast control using genetic algorithms. IEE Proc.-Electr. Power Appl. 1997, 144, 65. [CrossRef]

15. Ke, B.-R.; Lin, C.-L.; Yang, C.-C. Optimisation of train energy-efficient operation for mass rapid transit systems. IET Intell. Transp. Syst. 2012, 6, 58. [CrossRef]

16. Song, Y.D.; Song, W.T. A Novel Dual Speed-Curve Optimization Based Approach for Energy-Saving Operation of High-Speed Trains. IEEE Trans. Intell. Transp. Syst. 2016, 17, 1-12. [CrossRef]

17. González-Gil, A.; Palacin, R.; Batty, P.; Powell, J.P. A systems approach to reduce urban rail energy consumption. Energy Convers. Manag. 2014, 80, 509-524. [CrossRef]

18. Ceraolo, M.; Giglioli, R.; Lutzemberger, G.; Bechini, A. Cost effective storage for energy saving in feeding systems of tramways. In Proceedings of the Electric Vehicle Conference, IEEE, Florence, Italy, 17-19 December 2014; pp. 1-6.

19. Yang, X.; Li, X.; Ning, B.; Tang, T. A Survey on Energy-Efficient Train Operation for Urban Rail Transit. IEEE Trans. Intell. Transp. Syst. 2015, 17, 1-12. [CrossRef]

20. Su, S.; Tang, T.; Roberts, C. A Cooperative Train Control Model for Energy Saving. IEEE Trans. Intell. Transp. Syst. 2015, 16, 622-631. [CrossRef]

21. Albrecht, T. Reducing power peaks and energy consumption in rail transit systems by simultaneous train running time control. Comput. Railw. 2004, 769, 100-107.

22. Peña-Alcaraz, M.; Fernández, A.; Cucala, A.P.; Ramos, A.; Pecharromán, R.R. Optimal underground timetable design based on power flow for maximizing the use of regenerative-braking energy. Proc. Inst. Mech. Eng. Part F J. Rail Rapid Transit 2012, 226, 397-408. [CrossRef]

23. Lin, F.; Liu, S.; Yang, Z.; Zhao, Y.; Yang, Z.; Sun, H. Multi-Train Energy Saving for Maximum Usage of Regenerative Energy by Dwell Time Optimization in Urban Rail Transit Using Genetic Algorithm. Energies 2016, 9, 208. [CrossRef]

24. Chen, J.-F.; Lin, R.-L.; Liu, Y.-C. Optimization of an MRT Train Schedule: Reducing Maximum Traction Power by Using Genetic Algorithms. IEEE Trans. Power Syst. 2005, 20, 1366-1372. [CrossRef]

25. Li, X.; Lo, H.K. An energy-efficient scheduling and speed control approach for metro rail operations. Transp. Res. Part B Methodol. 2014, 64, 73-89. [CrossRef]

26. Zografos, K.G.; Androutsopoulos, K.N. Algorithms for Itinerary Planning in Multimodal Transportation Networks. IEEE Trans. Intell. Transp. Syst. 2008, 9, 175-184. [CrossRef]

27. Su, S.; Tang, T.; Li, X.; Gao, Z. Optimization of multitrain operation in a subway system. IEEE Trans. Intell. Transp. Syst. 2014, 15, 673-684.

28. Tang, H.; Dick, C.T.; Feng, X.; Information, R. Improving Regenerative Energy Receptivity in Metro Transit Systems. Transp. Res. Rec. J. Transp. Res. Board 2015, 2534, 48-56. [CrossRef]

29. Goodwin, J.C.J.; Fletcher, D.I.; Harrison, R.F. Multi-train trajectory optimisation to maximise rail network energy efficiency under travel-time constraints. Proc. Inst. Mech. Eng. Part F J. Rail Rapid Transit 2016, 230, 1318-1335. [CrossRef]

30. Liu, J.Q.; Guo, H.L.; Yu, Y.X. Research on the Cooperative Train Control Strategy to Reduce Energy Consumption. IEEE Trans. Intell. Transp. Syst. 2017, 18, 1134-1142. [CrossRef]

31. Liu, J.; Zhao, N. Research on Energy-Saving Operation Strategy for Multiple Trains on the Urban Subway Line. Energies 2017, 10, 2156.

32. Sun, X.B.; Lu, H.; Dong, H.R. Energy-Efficient Train Control by Multi-Train Dynamic Cooperation. IEEE Trans. Intell. Transp. Syst. 2017, 18, 3114-3121. [CrossRef]

33. Lu, Q.W.; He, B.B.; Wu, M.Z.; Zhang, Z.C.; Luo, J.T.; Zhang, Y.K.; He, R.K.; Wang, K.Y. Establishment and Analysis of Energy Consumption Model of Heavy-Haul Train on Large Long Slope. Energies 2018, 11, 965. [CrossRef]

34. Gu, Q.; Tang, T.; Ma, F. Energy-Efficient Train Tracking Operation Based on Multiple Optimization Models. IEEE Trans. Intell. Transp. Syst. 2016, 17, 882-892. [CrossRef] 
35. Rochard, B.P.; Schmid, F. A review of methods to measure and calculate train resistances. Proc. Inst. Mech. Eng. Part F J. Rail Rapid Transit 2000, 214, 185-199. [CrossRef]

36. Xiao, Z.; Sun, P.F.; Wang, Q.Y.; Zhu, Y.Q.; Feng, X.Y. Integrated Optimization of Speed Profiles and Power. Split for a Tram with Hybrid Energy Storage Systems on a Signalized Route. Energies 2018, 11, 478. [CrossRef]

37. Wang, H.M.; Ge, X.L.; Liu, Y.C. An Active Damping Stabilization Scheme for the Suppression of the DC-Link Oscillation in Metro Traction Drive System. IEEE Trans. Ind. Appl. 2018, 54, 5113-5123. [CrossRef]

38. Jin, W.D.; Wang, Z.L.; Li, C.W.; Gou, X.T.; Jin, F. Study on Optimization Method of Train Operation for Saving Energy. China Railw. Soc. 1997, 19, 58-62. (In Chinese)

(C) 2019 by the authors. Licensee MDPI, Basel, Switzerland. This article is an open access article distributed under the terms and conditions of the Creative Commons Attribution (CC BY) license (http://creativecommons.org/licenses/by/4.0/). 

Article

\title{
Distributed Event-Based Control of Hierarchical Leader-Follower Networks with Time-Varying Layer-To-Layer Delays
}

\author{
Guang-Hui Xu ${ }^{1,2}$, Meng Xu ${ }^{1,2}$, Ming-Feng Ge ${ }^{3, *}$, Teng-Fei Ding ${ }^{3}$, Feng $\mathrm{Qi}^{1,2}$ and Meng Li ${ }^{1,2}$ \\ 1 School of Electrical and Electronics Engineering, Hubei University of Technology, Wuhan 430068, China; \\ xgh@hbut.edu.cn (G.-H.X.); 101710208@hbut.edu.cn (M.X.); 101810271@hbut.edu.cn (F.Q.); \\ 101810207@hbut.edu.cn (M.L.) \\ 2 Hubei Key Laboratory for High-Efficiency Utilization of Solar Energy and Operation Control of Energy \\ Storage System, Hubei University of Technology, Wuhan 430068, China \\ 3 School of Mechanical Engineering and Electronic Information, China University of Geosciences, \\ Wuhan 430074, China; dingtf@cug.edu.cn \\ * Correspondence: gemf@cug.edu.cn or fmgabc@163.com
}

Received: 12 February 2020; Accepted: 3 April 2020; Published: 9 April 2020

\begin{abstract}
Compared with the traditional multi-agent models, the hierarchical leader-follower network (HLFN) can describe some real-world multi-agent systems more precisely due to its layered properties. The distributed event-based consensus control problem of HLFNs with layer-to-layer delays, namely, communication delays among agents of different layers, is presented in this essay. In order to solve the aforementioned problem, several innovative hierarchical event-based control (HEC) algorithms are proposed. The sufficient conditions on the control parameters and event-triggered mechanism were derived to undertake the reliability of the closed-loop dynamics. Moreover, it is shown that the zeno-behaviors of the presented HEC algorithms can be excluded. Finally, there are some numerical examples that verify the availability of the results.
\end{abstract}

Keywords: event-based consensus; hierarchical leader-follower network; hierarchical event-based control; layer-to-layer delays

\section{Introduction}

In recent years, coordination problems of multi-agent systems (MAS) have attracted research interests, which cover a wide area such as flocking [1,2], formation control [3-5], consensus tracking of a robot [6], and rendezvous in distributed robotics [7-9]. On these issues, many remarkable and significant results have emerged one after another.

In recent years, the research on the consensus of the leader-following multi-agent system has been increasing, and a large number of achievements have emerged. Wang et al. [10] investigated the leader-following consensus by a self-triggered algorithm. At the same time, considering the existence of a disturbance in reality, the input delay was considered. Shen et al. [11] investigated the leader-following consensus problem of multi-agent systems with two distributed adaptive control schemes. Ning [12] investigated the problem of leader-following consensus for multiple wheeled mobile robots. Based on the above research,the multi-layer hierarchical structure on the basis of the two-layer structure was investigated. The hierarchical structure exists widely in nature. In reality, birds show a hierarchical structure in the process of migration, and each layer is both a leader layer and follow layer, in addition to the leader of the first layer and the last follow layer, this model is called a hierarchical leader-follower network (HLFN). Nagy [13] showed the hierarchical piloting behavior of a group of pigeons during a long flight for the first time. Through the way of recording the 
experimental data, it can be found that the pigeons have established a hierarchical leadership structure to interact with each other during the flight, so as to complete the whole long-distance flight mission effectively. He and Xu [14] solved the coordination problem of MAS with two-layer leaders. Based on the previous work, we investigated the consensus of HLFNs.

In the actual communication network, due to the limitations of distance, bandwidth, and sensors, time delays between nodes are inevitable. Based on the passivity theory, the state estimation of recurrent neural networks (MRNNs) with time-varying delays is studied in [15]. Li [16] investigated the leader-following consensus of MASs with time delays and switching topology. Zhao [17] studied the consensus problem for MASs with communication delays. Jiang [18] solved the consensus problem of networked multiple robotic manipulators with and without time-varying delays. However, in large hierarchical networks, the communication delays between layers (layer-to-layer delays) are often different, and the different types of delays between different layers have not been solved.

In practical applications, continuous communication will lead to high communication costs and high requirements for equipment. Therefore, the event-based control has received widespread attentions as a method which can effectively reduce the cost of communication. In event-based control, the controller update only depends on the designed trigger conditions, and the trigger conditions are often related to the state of the system. The bipartite consensus problem of multi-agent system with connected structure balanced symbol graph based on event trigger was studied in paper [19]. Yi [20] has investigated the global consensus problem for first-order continuous-time multi-agent systems with input saturation, in which the triggering law does not require any a priori knowledge of global network parameters. The paper [21] investigates the bipartite leader-following consensus of second-order multi-agent systems with signed digraph topology. The paper [22] showed that event trigger control has lower communication times and controller update times and better control effect than periodic sampling control on the premise of completing control tasks. The event-based control technology attracted much attention because of the characteristics of resource saving, and related research also achieved more results [23-30]. However, the consensus problem of HLFNs remains unsolved.

Inspired by the preceding discussions, it is desirable to design economical and efficient hierarchical event-based control (HEC) algorithms for consensus problems of HLFNs with layer-to-layer delays. The main contributions of our paper are twofold: (1) Compared with the existing research achievements [30-34], this essay deals with the consensus problem of HLFNs with layer-to-layer delays. The existing literature considers the time-delays between different agents at the same layer. In order to better study the hierarchical structure, we considered layer-to-layer delays between different layers. (2) Based on the particularity of hierarchical structure, a new HEC algorithm was proposed to solve the previous problem. The algorithm is different from the general event-based method, as the trigger conditions of each layer were different.

In the rest of this paper is structured as follows: Section 2 introduces several fundamental theories of graph and matrix, and the three-layer MAS is described. In Section 3, the convergence of the designed control law is analyzed in detail. A numerical example is given to present the effectiveness of designed event-triggered mechanism in Section 4 . Section 5 concludes this paper.

\section{Preliminaries}

\subsection{Basic Theory on Graphs and Matrices}

A directed graph $\mathcal{G}=\{\mathcal{V}, \mathcal{E}, \mathcal{A}\}$ consists of a node set $\mathcal{V}=\left\{v_{1}, v_{2}, \cdots, v_{N}\right\}$, an edge set $\mathcal{E} \in$ $(\mathcal{V} \times \mathcal{V})$, and a weighted adjacency matrix $\mathcal{A}=\left(a_{i j}\right)$. If there is a edge between nodes $i$ and $j$, then $(i, j) \in \mathcal{E} \Leftrightarrow a_{i j}>0$. There are no self-loops, i.e., $a_{i i}=0$. The neighbor set of agent $i$ is defined as $\mathcal{N}_{i}=\{j \in \mathcal{V} \mid(j, i) \in \mathcal{E}, j \neq i\}$. Besides, the Laplacian matrix $\mathcal{L}$ is defined as $\mathcal{L}=\left[l_{i j}\right]$, where $l_{i i}=\sum_{j \neq i} a_{i j}$ and $l_{i j}=-a_{i j}, i \neq j$. Further, $D_{k}=\operatorname{diag}\left(d_{1}, d_{2}, \ldots, d_{r_{k}}\right), k=2,3, \ldots, l, r_{k}$ is the number of 
nodes of $k$ th layer. $1+r_{2}+\ldots+r_{l}=N$. If the node of $k$ th layer can receive information from the $r_{k-1}$, then $d_{r_{k}}>0$, otherwise $d_{r_{k}}=0$.

\subsection{System Description}

Without loss of generality, the HLFNs contain three-layer network in this paper. The first layer contains only one leader (as node 0 ), the second layer contains $r_{2}$ minor leader, the third layer contains $m$ groups, $r_{3}$ followers, and each group can receive information from the minor leader layer. Let the topological relationship between the minor leaders be $\mathcal{G}_{2}$, its corresponding Laplacian matrix is $\mathcal{L}_{2}$. Followers are divided into $m$ subgroups, and $\mathcal{G}_{p}$ consists of $r_{p}$ number of nodes and satisfies $N=$ $1+r_{2}+r_{3}, p=1,2, \ldots, m$. The Laplacian matrix of the graph $\mathcal{G}_{p}$ is recorded as $\mathcal{L}_{p}$. Including a major leader and minor leaders, an augmented topology diagram $\overline{\mathcal{G}}_{2}$ which consists of diagram $\mathcal{G}_{2}$, node 0 , and the directed edges of some nodes to node 0 is considered. An augmented topology diagram $\overline{\mathcal{G}}_{p}$ is composed of diagram $\mathcal{G}_{p}$, nodes from minor leader layer, and the directed edges of some node-to-node from the minor leader layer. Define set $v 1=\left\{1,2, \ldots, r_{2}\right\}, v 2=\left\{r_{2}+1, r_{2}+2, \ldots, r_{2}+r_{3}\right\}$.

The linear dynamics of major leader without input can be described as

$$
\dot{x}_{0}(t)=A x_{0}(t),
$$

the dynamics of minor leaders and followers are given as

$$
\dot{x}_{i}(t)=A x_{i}(t)+B u_{i}(t), i \in v 1 \cup v 2,
$$

where $x_{0}(t) \in \mathbb{R}^{n}, x_{i}(t) \in \mathbb{R}^{n}$ are the states of major leader, minor leaders and followers, respectively, $A \in \mathbb{R}^{n \times n}$ and $B \in \mathbb{R}^{n \times m}$ are constant matrices, and $u(t) \in \mathbb{R}^{m}$ is the control input.

Firstly, some basic assumptions and lemmas are given as follows:

Assumption 1. Node 0 is globally accessible in a graph $\overline{\mathcal{G}}_{2}$ that contains a major leader and $r_{2}$ minor leaders. The node from the minor leader layer is globally accessible in a graph $\overline{\mathcal{G}}_{p}$ that contains a minor leader and some followers.

Assumption 2. The matrix pair $(A, B)$ is stabilizable.

Assumption 3. Each row sum of $l_{i j}$ in $\mathcal{L}_{l}$ is zero; each row sum of $l_{i j}$ in $\mathcal{L}_{p}$ is zero.

Lemma 1. The Laplacian matrix has a simple zero eigenvalue and all the other eigenvalues have positive real parts if and only if graph $\mathcal{G}$ contains a directed spanning tree.

Definition 1. The consensus of the HLFN is considered achieved if

$$
\lim _{t \rightarrow \infty}\left\|x_{i}(t)-x_{0}(t)\right\|=0, i \in v 1 \cup v 2 .
$$

Remark 1. These assumptions are necessary for MAS with three-layer networks. If there is no Assumption 1, some minor leaders may be isolated from the major leader and unable to receive information from the major leader, thus detaching from the entire MAS, and several isolated smaller groups or individual isolated followers may be formed in the same follower subgroup, so that some followers cannot receive control information from the corresponding minor leaders. This obviously fails to achieve the desired control objectives.

\section{Main Results}

\subsection{Event-Based Control for Consensus of HLFNs}

In this section, the consensus of uncoupled followers under the influence of dynamic major leader is analyzed. 
For the minor leaders, the control goal is to enable the states of the minor leaders follow the major leader states. In order to achieve the consensus, the event-triggered control law for agent $i(i \in v 1)$ is designed:

$$
u_{i_{1}}(t)=K\left[\sum_{j \in \mathcal{N}_{i}} a_{i j}\left(\hat{x}_{j}(t)-\hat{x}_{i}(t)\right)+d_{i}\left(x_{0}(t)-\hat{x}_{i}(t)\right)\right], i \in v 1,
$$

where $K \in \mathbb{R}^{m \times n}$ is a feedback matrix, which is described in a later section. For the follower $i(i \in v 2)$ in the follower group, the corresponding control protocol is designed as

$$
u_{i_{2}}(t)=K\left[\sum_{j \in \mathcal{N}_{i}} a_{i j}\left(\hat{x}_{j}(t)-\hat{x}_{i}(t)\right)+d_{i}\left(\hat{x}_{p}(t)-\hat{x}_{i}(t)\right)\right], i \in v 2 .
$$

where $x_{p}(t)$ is the state of the minor leader to node $i$. Here, $\hat{x}_{i}(t)$ is defined as $x_{i}\left(t_{k}^{i}\right), t \in\left[t_{k}^{i} t_{k+1}^{i}\right)$.

In order to analyze the consensus problem, we define:

$$
\begin{aligned}
& \delta_{i}(t)=x_{i}(t)-x_{0}(t), i \in v 1 \cup v 2, \\
& \varepsilon_{i}(t)=\hat{x}_{i}(t)-x_{i}(t), i \in v 1 \cup v 2 .
\end{aligned}
$$

Then the system of Equation (2) can be expressed as:

$$
\begin{aligned}
\dot{\delta}_{i 1}(t) & =\dot{x}_{i}(t)-\dot{x}_{0}(t) \\
& =A \delta_{i}(t)+B K\left[\sum_{j \in \mathcal{N}_{i}} a_{i j}\left(\varepsilon_{j}(t)+\delta_{j}(t)-\varepsilon_{i}(t)-\delta_{i}(t)\right)\right. \\
& \left.-d_{i}\left(\varepsilon_{i}(t)+\delta_{i}(t)\right)\right], i \in v 1, \\
\dot{\delta}_{i 2}(t) & =\dot{x}_{i}(t)-\dot{x}_{0}(t) \\
& =A \delta_{i}(t)+B K\left[\sum_{j \in \mathcal{N}_{i}} a_{i j}\left(\delta_{j}(t)+\varepsilon_{j}(t)-\delta_{i}(t)-\varepsilon_{i}(t)\right)\right. \\
& \left.+d_{i}\left(\varepsilon_{p}(t)+\delta_{p}(t)-\delta_{i}(t)-\varepsilon_{i}(t)\right)\right], i \in v 2 .
\end{aligned}
$$

where $\dot{\delta}_{i 1}(t)$ is the error of minor leaders, and $\dot{\delta}_{i 2}(t)$ is the error of followers. Let $\delta(t)=$ $\left(\delta_{1}^{T}(t), \delta_{2}^{T}(t), \cdots, \delta_{r}^{T}(t)\right)^{T}, \varepsilon(t)=\left(\varepsilon_{1}^{T}(t), \varepsilon_{2}^{T}(t), \cdots, \varepsilon_{r}^{T}(t)\right)^{T}$, the system of Equations (8) and (9) becomes

$$
\begin{gathered}
\dot{\delta}_{1}(t)=\left[\left(I_{r_{2}} \otimes A\right)-\left(\mathcal{L}_{2}+\mathcal{D}_{2}\right) \otimes B K\right] \delta_{1}(t) \\
-\left[\left(\mathcal{L}_{2}+\mathcal{D}_{2}\right) \otimes B K\right] \varepsilon(t), i \in v 1, \\
\dot{\delta}_{2}(t)=\left[\left(I_{r_{p}} \otimes A\right)-\left(\mathcal{L}_{p}+\mathcal{D}_{p}\right) \otimes B K\right] \delta(t)-\left[\left(\mathcal{L}_{p}\right.\right. \\
\left.\left.+\mathcal{D}_{p}\right) \otimes B K\right] \varepsilon(t)+\left(\mathcal{D}_{p} \otimes B K\right)\left(\delta_{p}(t)+\varepsilon_{p}(t)\right), i \in v 2 .
\end{gathered}
$$

Theorem 1. Suppose that Assumptions 1, 2 and 3 hold. Consider the HLFN Equations (1) and (2) with the control in Equations (4) and (5). The consensus problem can be solved if:

$$
\begin{gathered}
f_{i_{1}}(t)=\left\|\varepsilon_{i}(t)\right\|-\left\|B K q_{i}(t)\right\|-\beta e^{-\gamma\left(t-t_{0}\right)}, i \in v 1 . \\
f_{i_{2}}(t)=\left\|\varepsilon_{i}(t)\right\|-\beta e^{-\gamma\left(t-t_{0}\right)} \\
-\frac{\left\|B K q_{i}(t)\right\|-2\left\|B K \sum_{j \in \mathcal{N}_{i}} d_{i}\left(\hat{x}_{p}(t)-x_{0}(t)\right)\right\|}{2\left\|\left(L_{P}+D\right) \otimes B K\right\|}, i \in v 2 .
\end{gathered}
$$


and

$$
\begin{aligned}
& q_{i}(t)=\sum_{j \in \mathcal{N}_{i}} a_{i j}\left(\hat{x}_{j}(t)-\hat{x}_{i}(t)\right)+d_{i}\left(x_{0}(t)-\hat{x}_{i}(t)\right), i \in v 1, \\
& q_{i}(t)=\sum_{j \in \mathcal{N}_{i}} a_{i j}\left(\hat{x}_{j}(t)-\hat{x}_{i}(t)\right)+d_{i}\left(\hat{x}_{p}(t)-\hat{x}_{i}(t)\right), i \in v 2 .
\end{aligned}
$$

For any $\beta>0$, and $K=B^{T} P, P$ is a positive-definitive matrix.

Proof of Theorem 1. Using the variation of parameter formula, Equation (10) becomes

$$
\begin{aligned}
\delta(t) & =e^{\left[\left(I_{r} \otimes A\right)-\left(\mathcal{L}_{2}+\mathcal{D}_{2}\right) \otimes B K\right]\left(t-t_{0}\right)} \delta\left(t_{0}\right) \\
& +\int_{t_{0}}^{t} e^{\left[\left(I_{r} \otimes A\right)-\left(\mathcal{L}_{2}+\mathcal{D}_{2}\right) \otimes B K\right](t-\theta)} \\
& \times\left(-\left(\left(\mathcal{L}_{2}+\mathcal{D}_{2}\right) \otimes B K\right)\right) \varepsilon(\theta) d \theta .
\end{aligned}
$$

In reality, if $(A, B)$ is stabilizable and the communication topology of these agents in the leader-follower structure has a directed spanning tree, it can be obtained from Lemma 1 that all the eigenvalues of $I_{r} \otimes A-\left(\mathcal{L}_{2}+\mathcal{D}_{2}\right) \otimes B K$ have non-positive real parts. Therefore, positive constants $a$ and $\rho$ for $t \geq t_{0}$ are existed, such that (refer to [35])

$$
\left\|e^{\left[I_{r_{2}} \otimes A-\left(\mathcal{L}_{2}+\mathcal{D}_{2}\right) \otimes B K\right]\left(t-t_{0}\right)}\right\| \leq a e^{-\rho\left(t-t_{0}\right)} .
$$

Furthermore, we can get

$$
\begin{aligned}
\|\delta(t)\| & \leq \alpha_{1} e^{-\gamma_{1}\left(t-t_{0}\right)}\left\|\delta\left(t_{0}\right)\right\| \\
& +\alpha_{1} \int_{t_{0}}^{t} e^{-\gamma_{1}(t-\theta)}\left\|\left(\mathcal{L}_{2}+\mathcal{D}_{2}\right) \otimes B K\right\|\|\tilde{x}(\theta)\| d \theta,
\end{aligned}
$$

Forced by Equation (12), we can get

$$
\|\varepsilon(t)\| \leq \tilde{\alpha}\|\delta(t)\|+\tilde{\beta} e^{-\gamma\left(t-t_{0}\right)},
$$

Combine Equation (14) with Equation (15), it holds

$$
\begin{aligned}
\|\delta(t)\| & \leq \alpha_{1}\left\|\delta\left(t_{0}\right)\right\| e^{-\rho\left(t-t_{0}\right)}+\alpha_{1} \int_{t_{0}}^{t} e^{-\rho(t-\theta)} \\
& \times\left\|\left(\mathcal{L}_{2}+\mathcal{D}_{2}\right) \otimes B K\right\|\left(\tilde{\alpha}\|\delta(\theta)\|+\tilde{\beta} e^{-\gamma\left(\theta-t_{0}\right)}\right) d \theta .
\end{aligned}
$$

Then, we prove that

$$
\|\delta(t)\|<\eta \alpha_{1}\left\|\delta\left(t_{0}\right)\right\| e^{-\gamma\left(t-t_{0}\right)} .
$$

If Equation (17) does not hold, then $t^{*}>t_{0}$ for $\left\|\delta\left(t^{*}\right)\right\|=v\left(t^{*}\right)$ and $\|\delta(t)\|<v(t)$ for $t<t^{*}$. Then by Equation (16), one has 


$$
\begin{aligned}
v\left(t^{*}\right) & =\left\|\delta\left(t^{*}\right)\right\| \\
& <\eta \alpha_{1} e^{-\rho\left(t^{*}-t_{0}\right)}\left\|\delta\left(t_{0}\right)\right\|+\eta \alpha_{1} \int_{t_{0}}^{t^{*}} e^{-\rho\left(t^{*}-\theta\right)} \\
& \times\left(\tilde{\alpha}\|\delta(\theta)\|+\tilde{\beta} e^{-\gamma\left(\theta-t_{0}\right)}\right) d \theta \\
& <\eta \alpha_{1} e^{-\rho\left(t^{*}-t_{0}\right)}\left\|\delta\left(t_{0}\right)\right\|+\eta \alpha_{1}\left(\tilde{\alpha} \alpha_{1}\left\|\delta\left(t_{0}\right)\right\|+\tilde{\beta}\right) \\
& \times\left(e^{-\gamma\left(t^{*}-t_{0}\right)}-e^{-\rho\left(t^{*}-t_{0}\right)}\right) \\
& <\eta \alpha_{1}\left\|\delta\left(t_{0}\right)\right\| e^{-\gamma\left(t^{*}-t_{0}\right)} \\
& =v\left(t^{*}\right) .
\end{aligned}
$$

The conflict of Equation (18) demonstrates that Equation (17) is well-founded, which implies that the consensus of the system in Equation (1), Equation (2) can be achieved exponentially.

Then using the variation of parameter formula, Equation (11) can be rewritten as

$$
\begin{aligned}
\varepsilon(t) & =e^{\left[\left(I_{r_{p}} \otimes A\right)-\left(\mathcal{L}_{p}+\mathcal{D}_{p}\right) \otimes B K\right]\left(t-t_{0}\right)} \varepsilon\left(t_{0}\right) \\
& +\int_{t_{0}}^{t} e^{\left[\left(I_{r_{p}} \otimes A\right)-\left(\mathcal{L}_{p}+\mathcal{D}_{p}\right) \otimes B K\right](t-\theta)} \\
& \times\left[\left(-\left(\left(\mathcal{L}_{p}+\mathcal{D}_{p}\right) \otimes B K\right)\right) e(\theta)\right. \\
& \left.+\left(\mathcal{D}_{p} \otimes B K\right)(\tilde{x}(\theta)+\delta(\theta))\right] d \theta .
\end{aligned}
$$

It follows from Equation (19)

$$
\begin{aligned}
\|\varepsilon(t)\| & \leq \alpha_{2} e^{-\rho\left(t-t_{0}\right)}\left\|\varepsilon\left(t_{0}\right)\right\| \\
& +\alpha_{2} \int_{t_{0}}^{t} e^{-\rho(t-\theta)}\left[\left\|\left(\mathcal{L}_{p}+\mathcal{D}_{p}\right) \otimes B K\right\|\|\varepsilon(\theta)\|\right. \\
& \left.+\left\|\mathcal{D}_{p} \otimes B K\right\|\|\varepsilon(\theta)+\delta(\theta)\|\right] d \theta .
\end{aligned}
$$

Replacing Equation (15) in Equation (20),

$$
\begin{aligned}
\|\varepsilon(t)\| & \leq \alpha_{2} e^{-\rho\left(t-t_{0}\right)}\left\|\varepsilon\left(t_{0}\right)\right\| \\
& +\alpha_{2} \int_{t_{0}}^{t} e^{-\rho(t-\theta)}\left[\left\|\left(\mathcal{L}_{p}+\mathcal{D}_{p}\right) \otimes B K\right\|\|\varepsilon(\theta)\|\right. \\
& \left.+r \beta e^{-\gamma\left(\theta-t_{0}\right)}\right] d \theta .
\end{aligned}
$$

Then, we prove that

$$
\|\varepsilon(t)\|<\eta \alpha_{2}\left\|\varepsilon\left(t_{0}\right)\right\| e^{-\gamma\left(t-t_{0}\right)} .
$$

This part of the proof is equivalent to the previous process, which is omitted here.

To avoid zeno-behavior, we need to get the lower boundary of the positive constant for the interval between any two events $\Delta t_{i}$, suppose agent $i$ triggered at the moment $t_{i}^{*} \geq 0$, then $e_{i}\left(t_{i}^{*}\right)=0$. For between the time $t_{i}^{*}$ and the time $t_{i}$ which the next event is triggered, we have

$$
\dot{e}_{i}(t)=-\dot{x}_{i}(t)=-\left[A x_{i}(t)+B u_{i}(t)\right] .
$$

By integrating the two sides of the above equation, we can get

$$
\left|e_{i}(t)\right|=\int_{t^{*}}^{t}\left|A x_{i}(s)+B u_{i}(s)\right| d s .
$$


we know

$$
\begin{aligned}
\left|A x_{i}(t)+B u_{i}(t)\right| & \leq\left\|\left(I_{r} \otimes A\right)\right\|\|x(t)\| \\
& +\left\|\left(\mathcal{L}_{p}+\mathcal{D}_{p}\right) \otimes B K\right\|(\|\varepsilon(t)\|+\|e(t)\| \\
& +\left\|\mathcal{D}_{p} \otimes B K\right\|\|\delta(t)\| \\
& =c .
\end{aligned}
$$

where $c$ is a normal value.

By Equations (24) and (25), $\left|e_{i}(t)\right| \leq c\left(t-t^{*}\right)$ can be obtained. Assume $c\left(t-t^{*}\right)=c_{1}$, when the error vector decreases from $\left|e_{i}(t)\right|=c_{1}$ to $\left|e_{i}(t)\right|=0$, the next event trigger will occur. Therefore, there exists a strict positive lower bound $\Delta t_{i}=t_{i}-t_{i}^{*}=c_{1} / c$ between the triggering times of two events. Since there exists such a lower bound for arbitrary agent and any event time, we believe that zeno-behavior will not exist. The process of proof for Theorem 1 is thus completed.

\subsection{Event-Based Control for Consensus of HLFNs with Layer-to-Layer Delays}

In this part, we consider the result in case when the layer-to-layer delays exist. For the minor leaders, the following protocol for agent $i$ is designed:

$$
\begin{array}{r}
u_{i}(t)=K\left[\sum_{j \in \mathcal{N}_{i}} a_{i j}\left(\hat{x}_{j}(t)-\hat{x}_{i}(t)\right)+d_{i}\left(x_{0}\left(t-\tau_{1}(t)\right)-\hat{x}_{i}(t)\right)\right], \\
i \in v 1, \\
u_{i}(t)=K\left[\sum_{j \in \mathcal{N}_{i}} a_{i j}\left(\hat{x}_{j}(t)-\hat{x}_{i}(t)\right)+d_{i}\left(\hat{x}_{p}\left(t-\tau_{2}(t)\right)-\hat{x}_{i}(t)\right)\right],
\end{array}
$$

$i \in v 2$.

In this part, we consider layer-to-layer delays in communication. Define that the communication between major leader and minor leaders is subject to a time delay $\tau_{1}(t)$, which satisfies $0<\tau_{1}(t)<\mu_{1}$, the communication between minor leader and followers is subject to a time delay $\tau_{2}(t)$, which satisfies $0<\tau_{2}(t)<\mu_{2}$.

Theorem 2. Suppose that Assumptions 1, 2 and 3 hold. Consider the HLFN Equations (1) and (2) with control, (26) and (27), the consensus problem can be solved if:

$$
\begin{aligned}
f_{i}(t) & =\left\|\varepsilon_{i}(t)\right\|-\beta e^{-\gamma\left(t-t_{0}\right)} \\
& -\frac{\left\|B K q_{i}(t)\right\|-2\left\|B K \sum_{q \in \mathcal{N}_{p}} d_{i}\left(\hat{x}_{p}(t)-x_{0}\left(t-\tau_{1}(t)\right)\right)\right\|}{2\left\|\left(\mathcal{L}_{2}+\mathcal{D}_{2}\right) \otimes B K\right\|},
\end{aligned}
$$

$i \in v 1$,

$$
\begin{aligned}
f_{i}(t) & =\left\|\varepsilon_{i}(t)\right\|-\beta e^{-\gamma\left(t-t_{0}\right)} \\
- & \frac{\left\|B K q_{i}(t)\right\|-2\left\|B K \sum_{j \in \mathcal{N}_{i}} d_{i}\left(\hat{x}_{p}(t)-x_{0}\left(t-\tau_{2}(t)\right)\right)\right\|}{2\left\|\left(\mathcal{L}_{p}+\mathcal{D}_{p}\right) \otimes B K\right\|},
\end{aligned}
$$

$i \in v 2$

For any $\beta>0$, and $K=B^{T} P, P$ is a positive-definitive matrix. 
Proof of Theorem 2. In this part, we divide the proof process into two parts, for minor leader $i\left(i=1,2, \ldots r_{2}\right)$, we can get

$$
\begin{aligned}
\|\delta(t)\| & \leq \alpha_{1} e^{-\rho\left(t-t_{0}\right)}\left\|\delta\left(t_{0}\right)\right\| \\
& +\alpha_{1} \int_{t_{0}}^{t} e^{-\rho(t-\theta)}\left\|\left(\mathcal{L}_{2}+\mathcal{D}_{2}\right) \otimes B K\right\|\left\|\varepsilon_{i}(\theta)\right\| \\
& \left.-\left\|\left(\mathcal{D}_{2} \otimes B K\right)\right\|\left\|\left(e^{A \theta}-e^{A\left(\theta-\tau_{1}(\theta)\right)}\right)\right\|\right) d \theta,
\end{aligned}
$$

forced by Equation (28), one can get

$$
\|\delta(t)\|<\eta \alpha_{1} e^{-\gamma\left(t-t_{0}\right)}\left\|\delta\left(t_{0}\right)\right\|
$$

For follower $i(i \in v 2)$, it holds

$$
\begin{aligned}
\|\delta(t)\| & \leq \alpha_{2} e^{-\rho\left(t-t_{0}\right)}\left\|\delta\left(t_{0}\right)\right\|+\alpha_{2} \int_{t_{0}}^{t} e^{-\rho(t-\theta)} \\
& \times\left[\left\|\left(\mathcal{L}_{p}+\mathcal{D}_{p}\right) \otimes B K\right\|\|\varepsilon(\theta)\|+\left\|\mathcal{D}_{2} \otimes B K\right\|\right. \\
& \left.\times\left\|\delta\left(\theta-\tau_{2}(\theta)\right)+\varepsilon_{p}\left(\theta-\tau_{2}(\theta)\right)-\left(e^{A \theta}-e^{A\left(\theta-\tau_{2}(\theta)\right)}\right)\right\|\right] d \theta,
\end{aligned}
$$

It can be concluded from Equation (29) that

$$
\|\delta(t)\|<\eta \alpha_{2}\left\|\delta\left(t_{0}\right)\right\| e^{-\gamma\left(t-t_{0}\right)}
$$

The proof of zeno-behavior is equivalent to that of Theorem 1, which is omitted here. The proof of Theorem 2 is thus completed.

Remark 2. The conclusion of this paper is that, based on the three-layer hierarchical structure, agents from the minor leader layer and the follower layer only interacts with the same layer agents and direct leaders, therefore, the analysis of three-layer network can be extended to multi-layer hierarchical MAS.

\section{Simulation Results}

In this section, two numerical example are shown to demonstrate the corresponding analysis of the results gained in the previous section. Consider the event-based consensus of HLFNs with thirteen agents, a major leader, three minor leaders, and nine followers. The communication topology is described as seen in Figure 1 and the connectivity weights are given as $a_{i j}=1, d_{i j}=1$, the other weights are all equal to zero. From the topology graph and the matrix relationship given, it can be seen that node 0 and nodes from the second layer are globally reachable in $\overline{\mathcal{G}}_{2}$ and $\overline{\mathcal{G}}_{p}$, respectively. Moreover, there is a directed spanning tree in the interconnection graph. Assume that

$$
\begin{gathered}
A=\left[\begin{array}{ccc}
-6 & 3 & 3 \\
3 & -3 & 0 \\
-3 & 0 & 0
\end{array}\right], B=\left[\begin{array}{c}
0 \\
0.2 \\
0
\end{array}\right] \\
P=\left[\begin{array}{lll}
0.3492 & 0.4488 & 0.2378 \\
0.4488 & 0.9798 & 0.4697 \\
0.2378 & 0.4697 & 0.4827
\end{array}\right]
\end{gathered}
$$

Thus $(\mathrm{A}, \mathrm{B})$ is stabilizable. Consider the hierarchical leader-follower networks with the interconnection graph given in Figure 1, for any initial condition, Figures $2-4$ show the change of error of each agents. It can be seen from Figures $2-4$ that the consensus can be achieved eventually under the proposed event-triggered strategy. The enlarged part of the figure shows that the system 
reached consensus in about $11 \mathrm{~s}$. Event time instants are shown in Figure 5, in which no Zeno triggering behaviors or even dense events for any agent are observed. Figures 6-8 shows the the change of error of agents with layer-to-layer delays, which shows the consensus can be achieved eventually under the proposed event-triggered strategy with layer-to-layer delays in about $15 \mathrm{~s}$. Due to the interference of the layer-to-layer delays, the time to reach the consensus in Figures 6-8 is longer than that in Figures 2 and 3. Figure 9 shows the event time instants under the event-triggered law with layer-to-layer delays. As can be seen from Figures 5 and 9, leader 1-3 triggers less than other followers, which greatly saves the waste of system resources. From the simulation results, one can see that Theorem 2 does more controller updates than that in Theorem 1.

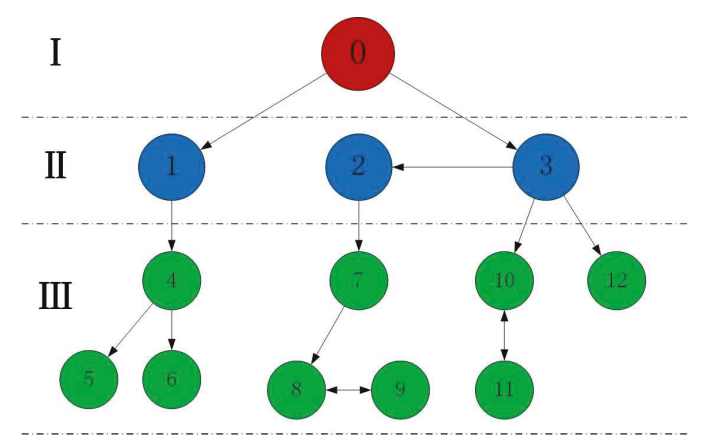

Figure 1. Topology of the hierarchical multi-agent network with uncoupled follower groups.

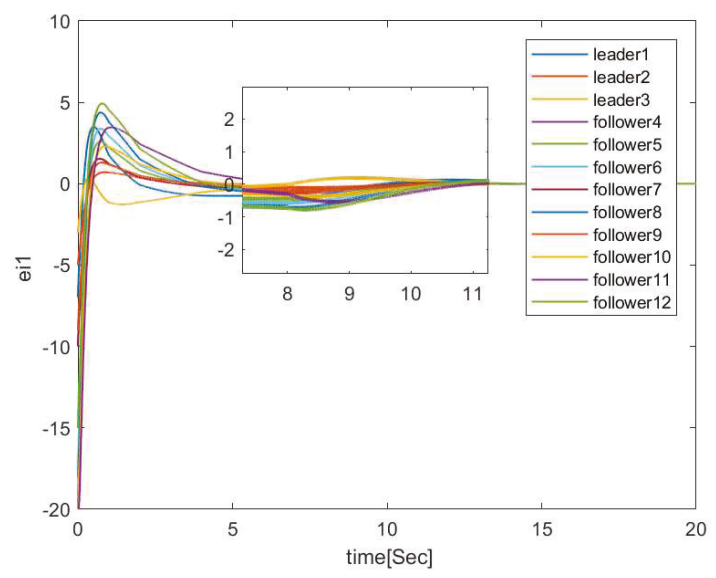

Figure 2. Position State Component 1 of Agent without delay. 


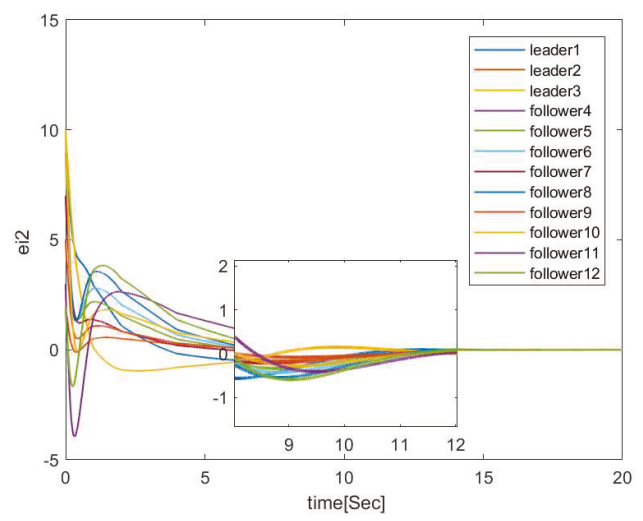

Figure 3. Position State Component 2 of Agent without delay.

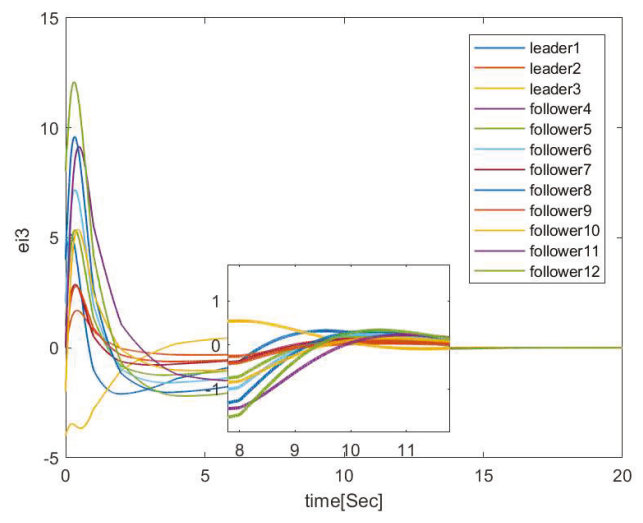

Figure 4. Position State Component 3 of Agent without delay.

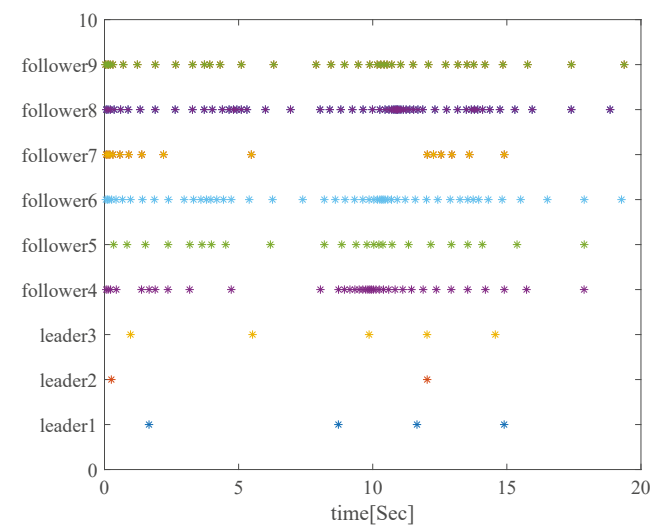

Figure 5. Event-trigger numbers under the event-based control law of Equation (4). 


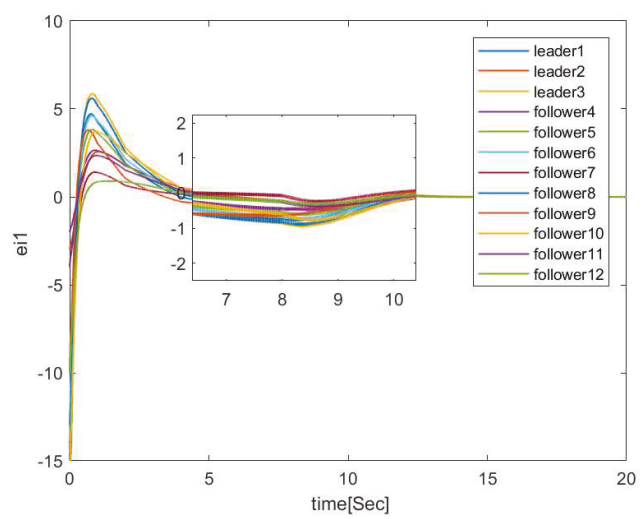

Figure 6. Position State Component 1 of Agent with hierarchical delay.

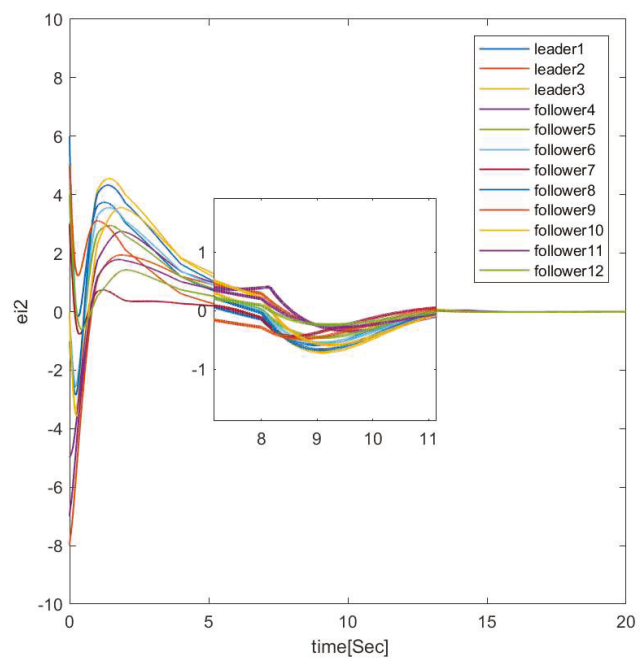

Figure 7. Position State Component 2 of Agent with hierarchical delay. 


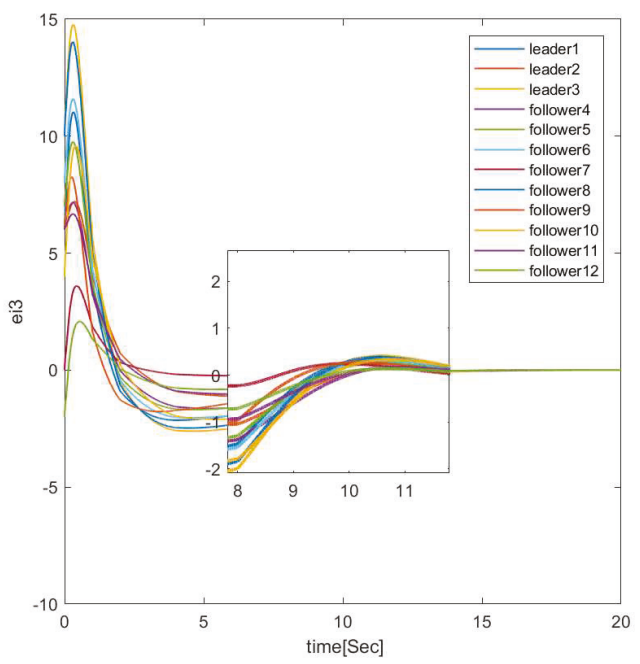

Figure 8. Position State Component 3 of Agent with hierarchical delay.

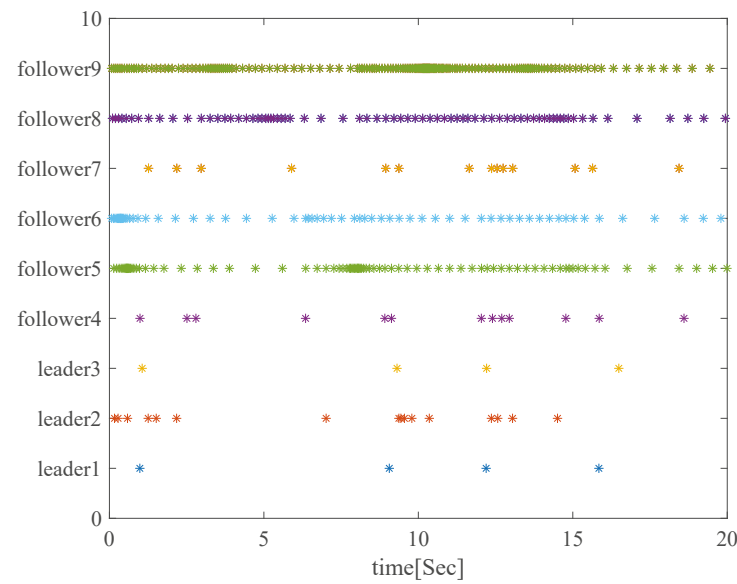

Figure 9. Event-trigger numbers under the event-based control law of Equation (5) with layer-to-layer delay.

Remark 3. The state of the agents in this paper is three-dimensional, and Figures 2-4 describe the state changes of the three dimensions, respectively.

\section{Conclusions}

In this paper, a novel hierarchical event-based control for hierarchical leader-follower networks is proposed. Based on matrix theory, algebraic graph theory, Lyapunov theory, and under the given event-triggered condition, it is proven that the protocols can realize consensus. Moreover, we show that the system can reach consensus with the layer-to-layer delays. Finally, simulation results are presented to support the theorems. In the future, more effort will be made towards relaxing the connectivity 
condition on the topology of the multi-agent network. The restrictions on the time delays may be lifted. How to extend consensus to multi-consensus is another topic in the future.

Author Contributions: Conceptualization and methodology, G.-H.X., M.X., and M.-F.G.; software, F.Q.; validation, M.L.; writing-review and editing, T.-F.D. Finally, All authors have read and agreed to the published version of the manuscript.

Funding: This work was supported by National Natural Science Foundation of China(61603127, 61703374).

Conflicts of Interest: The authors declare no conflict of interest.

\section{References}

1. Zheng, Y.; Ma, J.; Wang, L. Consensus of hybrid multi-agent systems. IEEE Trans. Neural Netw. Learn. Syst. 2017, 29, 1359-1365. [CrossRef] [PubMed]

2. Liu, X.; Zhang, K.; Xie, W.C. Consensus of multi-agent systems via hybrid impulsive protocols with time-delay. Nonlinear Anal. Hybrid Syst. 2018, 30, 134-146. [CrossRef]

3. Yan, Z.; Pan, X.; Yang, Z.; Yue, L. Formation Control of Leader-Following Multi-UUVs With Uncertain Factors and Time-Varying Delays. IEEE Access 2019, 7, 118792-118805. [CrossRef]

4. Ding, Y.; Wang, X.; Cong, Y.; Li, H. Scalability Analysis of Algebraic Graph-Based Multi-UAVs Formation Control. IEEE Access 2019, 7, 129719-129733. [CrossRef]

5. Tanner, H.G.; Christodoulakis, D.K. Decentralized cooperative control of heterogeneous vehicle groups. Robot. Auton. Syst. 2007, 55, 811-823. [CrossRef]

6. Liang, C.D.; Wang, L.; Yao, X.Y.; Liu, Z.W. Multi-target tracking of networked heterogeneous collaborative robots in task space. Nonlinear Dynam. 2019, 1-15. [CrossRef]

7. HYazdani, S.; Haeri, M. Flocking of multi-agent systems with multiple second-order uncoupled linear dynamics and virtual leadere. IET Control Theory Appl. 2016, 10, 853-860. [CrossRef]

8. Ren, W.; Beard, R.W.; Atkins, E.M. Information consensus in multivehicle cooperative control. IEEE Control Syst. Mag. 2007, 27, 71-82.

9. Olfati-Saber, R.; Fax, J.A.; Murray, R.M. Consensus and cooperation in networked multi-agent systems. Proc. IEEE 2007, 95, 215-233. [CrossRef]

10. Wang, X.; Su, H. Self-triggered leader-following consensus of multi-agent systems with input time delay. Neurocomputing 2019, 330, 70-77. [CrossRef]

11. Shen, Q.; Shi, P.; Zhu, J.; Zhang, L. Adaptive consensus control of leader-following systems with transmission nonlinearities. Int. J. Control 2019, 92, 317-328. [CrossRef]

12. Ning, B.; Han, Q.L.; Lu, Q. Fixed-Time Leader-Following Consensus for Multiple Wheeled Mobile Robots. IEEE Trans. Cybern. 2019. [CrossRef] [PubMed]

13. Nagy, M.; Akos, Z.; Biro, D.; Vicsek, T. Hierarchical group dynamics in pigeon flocks. Nature 2010, 464, 890. [CrossRef] [PubMed]

14. He, D.X.; Xu, G.H.; Guan, Z.H.; Chi, M.I. Hybrid coordination of multi-agent networks with hierarchical leaders. Commun. Nonlinear Sci. Numer. Simul. 2015, 27,110-119. [CrossRef]

15. Rakkiyappan, R.; Chandrasekar, A.; Laksmanan, S.; Park, J.H. State estimation of memristor-based recurrent neural networks with time-varying delays based on passivity theory. Complexity 2014, 19, 32-43. [CrossRef]

16. Li, Y.; Li, H.; Ding, X.; Zhao, G. Leader-Follower Consensus of Multiagent Systems With Time Delays Over Finite Fields. IEEE Trans. Cybern. 2018, 49, 3203-3208. [CrossRef]

17. Zhao, G.; Hua, C.; Guan, X. Distributed Event-Triggered Consensus of Multiagent Systems With Communication Delays: A Hybrid System Approach. IEEE Trans. Cybern. 2019. [CrossRef]

18. Jiang, Y.; Zhang, Y.; Wang, S. Distributed adaptive consensus control for networked robotic manipulators with time-varying delays under directed switching topologies. Peer-to-Peer Netw. Appl. 2019. [CrossRef]

19. Yu, H.; Chen, X.; Chen, T.; Hao, F. Event-triggered bipartite consensus for multi-agent systems: A Zeno-free analysis. IEEE Trans. Autom. Control 2019. [CrossRef]

20. Yi, X.; Yang, T.; Wu, J.; Johansson, K.H. Distributed event-triggered control for global consensus of multi-agent systems with input saturation. Automatica 2019, 310, 1-9. [CrossRef]

21. Ren, J.; Song, Q.; Lu, G. Event-triggered bipartite leader-following consensus of second-order nonlinear multi-agent systems under signed digraph. IEEE J. Frankl. Inst. 2019, 356, 6591-6609. [CrossRef] 
22. Mirollo, R.E.; Strogatz, S.H. Synchronization of pulse-coupled biological oscillators. SIAM J. Appl. Math. 1990, 50, 1645-1662. [CrossRef]

23. Strogatz, S.H. From Kuramoto to Crawford: Exploring the onset of synchronization in populations of coupled oscillators. Phys. D Nonlinear Phenom. 2000, 143, 1-20. [CrossRef]

24. Preciado, V.M.; Verghese, G.C. Synchronization in Generalized Erd ö sR é nyi Networks of Nonlinear Oscillators. In Proceedings of the 44th IEEE Conference on Decision and Control, Seville, Spain, 12-15 December 2005; pp. 4628-4633.

25. Sepulchre, R.; Paley, D.; Leonard, N. Collective motion and oscillator synchronization. Lect. Notes Control Inf. Sci. 2004, 309, 466-469.

26. Rojo-Rodriguez, E.G.; Garcia, O.; Ollervides, E.J.; Zambrano Robledo, P.C. Robust Consensus-Based Formation Flight for Multiple Quadrotors. J. Intell. Robot. Syst. 2019, 93, 213-226. [CrossRef]

27. Masroor, S.; Peng, C. Event triggered non-inverting chopper fed networked DC motor speed synchronization. COMPEL Int. J. Comput. Math. Electr. Electron. Eng. 2018, 37, 911-929. [CrossRef]

28. Zhang, H.; Yang, R.; Yan, H. Distributed event-triggered control for consensus of multi-agent systems. J. Frankl. Inst. 2015, 352, 3476-3488. [CrossRef]

29. Wang, Y.W.; Lei, Y.; Bian, T.; Guan, Z.H. Distributed Control of Nonlinear Multiagent Systems With Unknown and Nonidentical Control Directions via Event-Triggered Communication. IEEE Trans. Cybern. 2019. [CrossRef]

30. Jian, L.; Hu, J.; Wang, J. New Event-based Control for Sampled-data Consensus of Multi-agent Systems. Int. J. Control Autom. Syst. 2019, 17, 1107-1116. [CrossRef]

31. Lai, Q.; Didier Kamdem Kuate, P.; Liu, F.; Ho-Ching Iu, H. An extremely simple chaotic system with infinitely many coexisting attractors. IEEE Trans. Circuits Syst. II Express Briefs 2019. [CrossRef]

32. Lai, Q.; Akgul, A.; Li, C.; Xu, G.; Çavuşoğlu, Ü. A New Chaotic System with Multiple Attractors: Dynamic Analysis, Circuit Realization and S-Box Design. Entropy 2018, 20, 12. [CrossRef]

33. Lai, Q.; Norouzi, B.; Liu, F. Dynamic analysis, circuit realization, control design and image encryption application of an extended Lü system with coexisting attractors. Chaos Solitons Fractals 2018, 114, 230-245. [CrossRef]

34. Ge, M.F.; Liu, Z.W.; Wen, G.; Yu, X.; Huang, T. Hierarchical Controller-Estimator for Coordination of Networked Euler-Lagrange Systems. IEEE Trans. Cybern. 2019. [CrossRef] [PubMed]

35. Zhu, W.; Sun, C.; Li, H. Event-based leader-following consensus of multi-agent systems with switching topologies. In Proceedings of the Chinese Control Conference (CCC), Hangzhou, China, 28-30 July 2015; pp. 7511-7516.

(C) 2020 by the authors. Licensee MDPI, Basel, Switzerland. This article is an open access article distributed under the terms and conditions of the Creative Commons Attribution (CC BY) license (http:/ / creativecommons.org/licenses/by/4.0/). 
Article

\title{
Lighting Control Including Daylight and Energy Efficiency Improvements Analysis
}

\author{
Aniela Kaminska ${ }^{1}$ and Andrzej Ożadowicz ${ }^{2, *}$ \\ 1 Faculty of Electrical Engineering, Poznan University of Technology, ul. Piotrowo 3a, 60-965 Poznań, Poland; \\ aniela.kaminska@put.poznan.pl \\ 2 Faculty of Electrical Engineering, Automatics, Computer Science and Biomedical Engineering, \\ AGH University of Science and Technology, al. Mickiewicza 30, 30-059 Kraków, Poland \\ * Correspondence: ozadow@agh.edu.pl; Tel.: +48-12-617-50-11
}

Received: 26 July 2018; Accepted: 16 August 2018; Published: 19 August 2018

check for updates

\begin{abstract}
Energy used for lighting is one of the major components of total energy consumption in buildings. Nowadays, buildings have a great potential to reduce their energy consumption, but to achieve this purpose additional efforts are indispensable. In this study, the need for energy savings evaluation before the implementation of lighting control algorithms for a specified building is highlighted. Therefore, experimental tests have been carried out in a university building with laboratories and other rooms, equipped with KNX building automation system. A dimmable control strategy has been investigated, dependent on daylight illuminance. Moreover, a relationship between external and internal daylight illuminance levels has been evaluated as well. Based on the experimental results, the authors proposed a method for the rough estimation of electrical energy savings. Since, according to the EN 15232 standard, Building Automation and Control Systems (BACS) play an important role in buildings' energy efficiency improvements, the BACS efficiency factors from this standard have been used to verify the experimental results presented in the paper. The potential to reduce energy consumption from lighting in non-residential buildings by $28 \%$ for offices and $24 \%$ for educational buildings has been confirmed, but its dependence on specific building parameters has been discussed as well.
\end{abstract}

Keywords: building automation systems; building energy efficiency; daytime lighting; lighting control systems; EN 15232 standard

\section{Introduction}

Buildings are responsible for almost $40 \%$ of the world's energy consumption, including up to $65 \%$ of electrical energy [1-3]. Moreover, lighting is a major portion of the electrical energy consumption of non-residential and commercial buildings, especially those that are not fully retrofitted to LED lighting $[4,5]$. Hence, in recent years one of the key strategies for this sector is increasing energy efficiency by reducing the energy used for lighting. Various concepts, approaches, and technologies have been proposed to achieve these purposes. Many studies have shown that proper lighting controls can significantly reduce annual energy consumption. However, this depends on many factors, such as the climatic conditions of the country [6-8], the north-south orientation of the building [9], and its surroundings, that is, shade from trees or reflections from neighboring buildings [10]. Much attention has been paid to building construction, such as the shape of the building [11,12], the shape of the rooms-mostly the depth [13-15], and the size of windows and doors $[8,16]$.

In the last few years, advances in lighting control systems have significantly improved energy savings and visual comfort in rooms. Moreover, interest in finding new solutions for electric lighting systems and controls has been increasing due to new architectural design trends as well as the 
accessibility and lower cost of new technologies and products [17]. For years lighting control and monitoring functions have been provided in buildings by different automated devices and systems. In particular, in non-residential, public, and commercial buildings various kinds of BACS have been implemented. The most popular are three international standards of the BACS: the LonWorks, the KNX, and the BACnet $[18,19]$. However, there are numerous other technologies and applications on the market that can be installed in smart buildings. Generally, they are classified as proprietary (closed) or non-proprietary (open). The former are closed solutions, integrating equipment and software produced by the company that manufactured the system, while non-proprietary BACS integrate equipment from different manufacturers and software platforms [20,21]. Moreover, the emerging Internet of Things (IoT) affects the building automation sector as well and brings new categories of technical solutions for BACS, using various communication protocols for short-range devices such as Zigbee, EnOcean, X10, and Z-Wave [22]. Therefore, systems for lighting control in buildings can be organized in different ways, even with universal microcomputers (Raspberry Pi) and other modules (Arduino), providing full openness and integrity on the field level $[23,24]$. Nowadays, the devices and systems mentioned above allow for the implementation of even very complex lighting control algorithms. However, proper selection of the technology and algorithms used in the BACS for lighting control in a specified building is still a challenge because it requires taking into account many factors and parameters. In this context, the importance of potential energy savings estimation should be emphasized as well. Small energy savings indicates the need for simple lighting control, while large savings favors the use of complex systems. It is worth noting that the studies in this area give varying results that are difficult to generalize.

Bearing in mind all these aspects, appropriate and advanced lighting control has become an essential part of lighting systems in modern and retrofitted buildings, both residential and non-residential. For different kinds of buildings, several control strategies are commonly used in modern building design $[4,17,25]$ :

- Manual switching/dimming

- Presence/occupant detection

- Daylight harvesting

- Constant illuminance

These two last strategies are particularly interesting. They can help building occupants work in appropriate lighting conditions as well as lead to additional electrical energy savings. For their implementation, adaptive dimming schemes are proposed for the rooms [26]. A lot of studies are focused on daylight integration within control strategies and systems. In [27] an adaptive dimming scheme based on a daylight pipe integrated with an indoor lighting control platform has been analyzed, showing significant energy savings potential. In another study, Larsen et al. [28] introduce a new estimation methodology for electrical energy consumption in an office with daylight- and occupancy-controlled artificial lighting. Considering this aspect, the analysis of energy efficiency improvements for a school building has been presented and discussed in [29]. For three classrooms with different types of control systems, the total annual energy savings varied from $18 \%$ to $46 \%$; the authors concluded that this is closely related to the operation of the control systems, implemented and integrated control functions as well as the obtained illuminance levels in the classrooms. They also noticed that the energy savings due to the implementation of a lighting control can be divided into two aspects: (i) artificial lighting dimming due to daylight penetration and (ii) initial dimming to compensate for the over-dimensioning of the lighting system, taking into account constant illuminance $[29,30]$. Another important aspect is the prediction of daylight illuminance both external (outdoor) and internal (indoor), discussed in [14] as a key stage in daytime lighting designs in buildings. It also affects control strategies by taking into account daylight penetration and maintaining constant illuminance in the rooms. 
The above brief discussions indicate that the estimation of potential energy savings due to advanced, daylight-centered lighting control strategies must take into account many factors affecting daylight penetration inside a building as well as control function integration. These factors should be incorporated in the design of buildings and lighting. Additionally, the organization and design of a distributed control systems should be taken into consideration as well [31,32]. On the other hand, an evaluation of the lighting control system's behavior, well-documented energy consumption, and occupant satisfaction with building operations are necessary to evaluate the benefits of using complex lighting control systems [33]. Therefore, to permit the integration of all these factors and parameters into one control and management platform as well as to provide interoperability into the control systems, it is suggested to use a fully distributed, standardized, and open Building Automation and Control Systems (BACS) in such applications [17,34].

Since daylight-centered control strategies and their assumptions should be analyzed individually for specific buildings, in this paper the authors present results of investigations focused on a university building automation laboratory, equipped with BACS based on the KNX standard, including distributed brightens and presence sensors as well as advanced lighting controllers. A case study approach has been proposed to assess the feasibility of the dimmable lighting control method, depending on the daylight illuminance distribution in rooms. A 10-month monitoring of different parameters has been carried out in several rooms in the same university building and potential energy efficiency improvements have been estimated and discussed. A method for the rough estimation of electrical energy savings has been proposed by the authors. This is based on the measured number of hours with specified illuminance levels of daylight in the aforementioned rooms with dimmable lighting control. An additional contribution of this paper is a comparison of the calculated estimations of energy savings with the BACS efficiency factors introduced in the EN 15232 standard concerning the impact of the BACS on a building's energy efficiency.

The remainder of this paper is organized as follows. Section 2 contains information about the EN 15232 standard and its regulations as well as requirements regarding the selection and organization of the BACS control functions, providing potential energy efficiency improvements. Information about case study building and systems is presented in Section 3. Afterwards, Section 4 provides the results of the experiments, with analyses and findings. Estimated energy savings and their potential are presented and discussed in Section 5. Finally, Section 6 gives the conclusions and suggestions for future work.

\section{Potential Energy Efficiency Improvements in Buildings with BACS}

As mentioned in the previous section, BACS are more and more often considered not only as a tool providing better control of a building's infrastructure devices, users' comfort and security, but as a platform for energy management, providing additional energy savings as well. Moreover, the Energy Performance of Building Directive (EPBD) 2010/31/EU [35,36], in line with the Directive 2012/27/EU [37] as well as the Energy Roadmap 2050 [38], promotes the implementation of smart home and building technologies to reduce energy consumption in both residential and non-residential buildings. Modern BACS offer a lot of control and monitoring functions dedicated to various kinds of devices, subsystems, and services in buildings. Hence, in 2007 European Standard EN 15232 [39] was introduced, providing a list of the BACS control and Technical Building Management (TBM) functions having an impact on the energy performance of buildings [40]. They are divided into a few categories. The most significant categories of proposed advanced control functions refer to heating, ventilation, air conditioning (HVAC), cooling, and hot water generation and distribution systems. Apart from these, there are lighting and blind control functions mentioned in the standard as well. Therefore, they could be considered in the organization of lighting control strategies for BACS aimed at energy efficiency improvements. 


\subsection{BACS Efficiency Classes}

To classify possible various control strategies for the BACS, the EN 15232 standard defines four BACS efficiency classes, depending on the BACS control and TBM functions' complexity, integration level, and interoperability:

- Class A-highest level of integration: BACS and TBM systems provide high energy performance with individual control of devices and subsystems in rooms.

- Class B-advanced BACS with specific TBM functions provide additional savings in energy consumption with distributed control of devices and subsystems.

- Class C-standard BACS without TBM functions; this is a reference class for BACS.

- Class D-non-energy-efficient BACS; buildings with such systems shall be retrofitted and new buildings with such BACS shall not be built.

Since the lighting control systems and daylight availability in the rooms are considered in this paper, the authors have focused on the control functions dedicated to them and their potential for energy efficiency improvements. Similar questions have been considered in [41,42] with an experimental validation and case study analyses. Hence, lists of the specific BACS control functions with their assignment to the BACS efficiency classes from the EN 15232 are given in Table 1 [39].

Table 1. The specific BACS control and TBM functions for lighting [39].

\begin{tabular}{|c|c|c|c|}
\hline \multirow{2}{*}{ Lighting Control } & \multirow{2}{*}{ Description } & \multicolumn{2}{|c|}{ BACS Efficiency Class } \\
\hline & & Residential & Non-Residential \\
\hline \multirow{2}{*}{ Occupancy control } & Manual on/off switch & $\mathrm{C}$ & $\mathrm{D}$ \\
\hline & Automatic detection & A & A \\
\hline Daylight control & Manual & B & $\mathrm{C}$ \\
\hline
\end{tabular}

\subsection{An Expected Impact of the BACS Lighting Control Functions on Electrical Energy Efficiency}

In relation to the BACS efficiency classes, the EN 15232 standard proposes two methods for calculating the impact of the BACS and TBM functions on the building energy performance: (i) the "detailed method" with the requirement of a deep knowledge of the characteristics of the building and its installations, subsystems like HVAC, lighting, etc., as well as (ii) simplified the "BACS factors method," allowing us to roughly estimate the impact of the BACS and TBM functions on the building energy performance $[39,43]$. In this paper the authors compare the BACS efficiency factors from this simplified method with the potential energy savings estimated based on real data from an experiment. These factors have been classified in the EN 15232 standard, depending on the BACS efficiency classes and the building types $[3,39]$. Potential energy savings due to the lighting BACS control functions are expressed by BACS efficiency factors for lighting, provided in this standard. Since the case study presented and analyzed in this paper concerns a specific university building with laboratories and offices, some factors related to those kinds of buildings are presented in Table 2 [39].

Table 2. The selected BACS efficiency factors for lighting in non-residential buildings [39]

\begin{tabular}{ccccc}
\hline \multirow{2}{*}{$\begin{array}{c}\text { Non-Residential } \\
\text { Building Types }\end{array}$} & \multicolumn{4}{c}{$\begin{array}{c}\text { The BACS Efficiency Factors for Lighting } \\
\text { Related to the BACS Efficiency Classes }\end{array}$} \\
\cline { 2 - 5 } & D & C (Reference) & B & A \\
\hline Offices & 1.1 & 1 & 0.85 & 0.72 \\
Lecture hall & 1.1 & 1 & 0.88 & 0.76 \\
Education buildings (schools) & 1.1 & 1 & 0.88 & 0.76 \\
\hline
\end{tabular}


As shown in Table 2, the factors for the BACS efficiency class $C$ are defined with a value of 1 as reference class, with standard functionality of the BACS and TBM systems. The factors for other, higher classes (B and A) show significant potential for energy efficiency improvements with the BACS and TBM functions. In particular, for advanced BACS functions organized and integrated according to the guidelines and requirements of class A, the energy consumption for lighting can be reduced by almost $25 \%$ to $30 \%$. Nevertheless, it should be underlined that the real outcomes obtained from the data measured for specific buildings can show significant differences from the expected and estimated energy savings. Therefore, some research and experimental validations with real buildings as case studies have been conducted by different research teams, presenting data analyses and comparisons [44]. In [41] Beccali et al. conclude that the BACS factors method can be used with sufficient precision to evaluate the energy consumption of C or A BACS classes' lighting systems, both in residential and non-residential buildings. However, at the same time they declare a wider research aiming at determining how the BAC factor method can be improved and made more accurate.

In this paper we present a case study and experimental results aimed at verifying the soundness of this simplified BACS factor method and validating the estimated levels of the energy savings in a lighting system controlled by distributed KNX BACS modules.

\section{Case Study-Experimental Building and Control System}

This study deals with the activities of the Laboratory of KNX System and Evolution of Installation Energy Efficiency (SKNX \& EIEE Laboratory) at Poznan University of Technology in Poznan, located in the northwestern part of Poland. The laboratory is on the first floor of a two-story building situated at latitude $52^{\circ} 24^{\prime} 05.6^{\prime \prime} \mathrm{N}$ and longitude $16^{\circ} 57^{\prime} 07.6^{\prime \prime} \mathrm{E}$. The north-south orientation of the building is shown in Figure 1. The surroundings of the building do not affect daylight availability to the rooms in terms of shade or light reflection. The layout of the laboratory, the area of the rooms, and the total area of windows in each room as well as the arrangement of lamps and the power density of the installed lighting are also presented in Figure 1. In all the rooms, the windows are of the same area $\left(0.63 \mathrm{~m}^{2}\right)$ and are placed $0.3 \mathrm{~m}$ from the exterior surface of the building. There are two laboratory rooms, two offices, and a corridor on the floor considered in the case study for this paper.

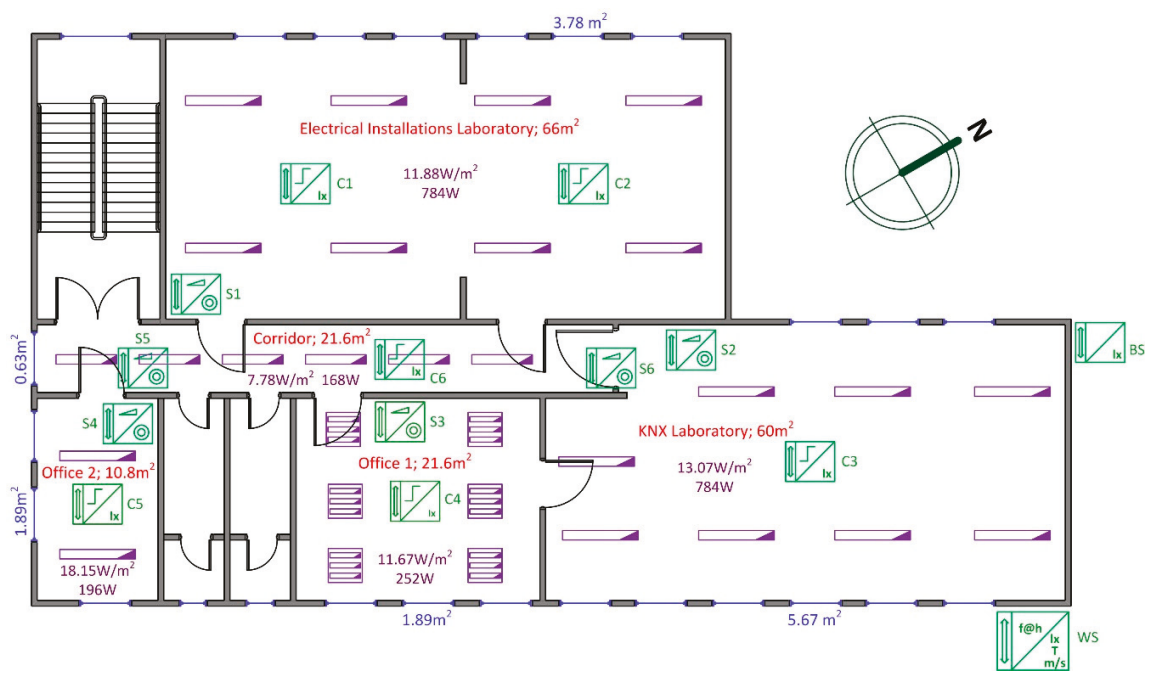

Figure 1. Layout of the SKNX \& EIEE Laboratory with arrangement of lamps, installed lighting power density, and deployment of KNX BACS devices (the numbers outside the building indicate the total area of windows in the room). 


\subsection{Lighting Installation and KNX Control System Components}

The laboratory is equipped with both a KNX standardized, distributed building automation system and SCADA solutions for the control and visualization of heating, lighting, and air conditioning. This control and monitoring platform allows us to organize and modify various control scenarios for the laboratory rooms, offices, and corridor. The structure of the KNX lighting control system of the SKNX \& EIEE Laboratory is shown in Figure 2. This structure consists of one line (KNX bus) powered by a KNX power supply unit PS (30 V DC). Each device connected to the KNX bus has its own intelligence thanks to the integrated microcontroller, operating system, and program memory. These features with a standardized communication interface enable data transmission between devices using KNX standard telegrams (messages). The design of the installation and configuration is done via a PC with the installed ETS (Engineering Tool Software) connected by a KNX/IP router.

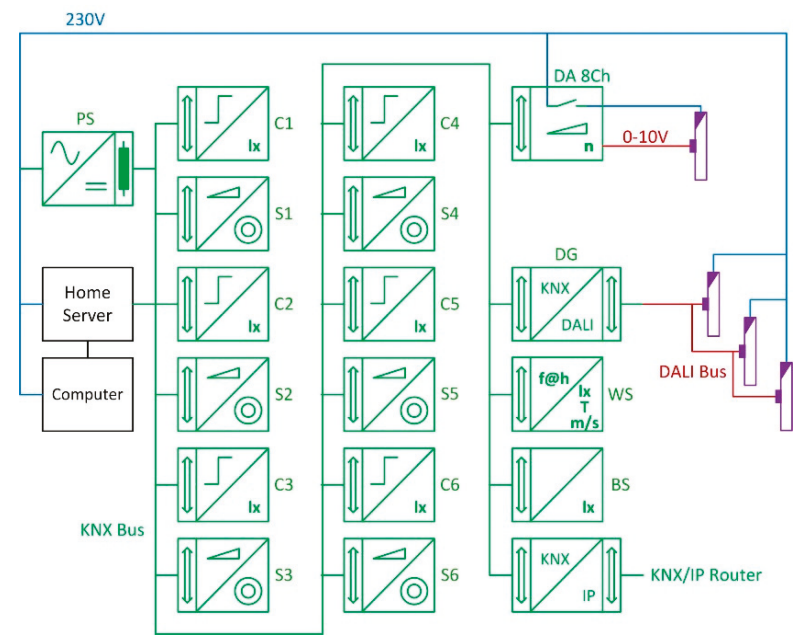

Figure 2. A structure of the KNX lighting control system in the SKNX \& EIEE Laboratory (the symbols of devices are standardized by the KNX Association).

In the KNX Laboratory room, the lighting system consists of fluorescent lamps controlled by DALI (Digital Addressable Lighting Interface) communication protocol; therefore, the KNX/DALI gateway (DG) is used to integrate the DALI lamp controllers with a KNX bus. The lighting in other rooms is controlled with the eight-channel Dimming Actuator (DA). All rooms are equipped with presence detectors with brightness sensors (C1-C6) as well as push buttons (S1-S6). All these devices allow us to control lamps manually or automatically depending on the occupancy and daylight level in the rooms. The arrangement of the sensors in the rooms is also shown in Figure 1. Moreover, a weather station with brightness sensor (WS in Figures 1 and 2) is installed on the southeastern facade of the building and an additional brightness sensor (BS in Figures 1 and 2) is mounted on the north facade to measure the outdoor daylight illuminance levels. The basic technical characteristics of the brightness sensors C1-C6, WS, and BS are presented in Table 3.

Bearing in mind all these features of the KNX BACS implemented in the SKNX \& EIEE Laboratory, the authors state that for this case study the BACS efficiency class is A, taking into account the occupancy and daylight control considered in the experiments presented in this paper. 
Table 3. Technical characteristics of the brightness sensors.

\begin{tabular}{llll}
\hline $\begin{array}{c}\text { Technical } \\
\text { Characteristics }\end{array}$ & Weather Station WS & $\begin{array}{c}\text { External Brightness } \\
\text { Sensor BS }\end{array}$ & $\begin{array}{c}\text { Presence Detector with } \\
\text { Brightness Sensor C }\end{array}$ \\
\hline Type of sensor & $\begin{array}{l}\text { KNX Basic weather station } \\
\text { Art. No. 663990 }\end{array}$ & $\begin{array}{l}\text { KNX brightness and } \\
\text { temperature sensor } \\
\text { Art. No. 663991 }\end{array}$ & $\begin{array}{l}\text { INSTABUS ARGUS Presence with } \\
\text { infrared receiver } \\
\text { Art. No. 630591 }\end{array}$ \\
\hline Brightness range & 1 to 100,000 lux & $\begin{array}{l}1 \text { to } 100,000 \text { lux } \\
( \pm 20 \% \text { or } \pm 5 \text { lux })\end{array}$ & 10 to 2000 lux \\
\hline Measurement mode & $\begin{array}{l}\text { Send brightness value } \\
\text { cyclically every } 5 \text { min }\end{array}$ & $\begin{array}{l}\text { Send brightness value } \\
\text { cyclically every } 5 \text { min }\end{array}$ & $\begin{array}{l}\text { Sensors C1-C } 5 \text { send brightness } \\
\text { value cyclically every } 5 \text { min } \\
\text { Sensor C6 sends brightness value } \\
\text { if the new value differs on more } \\
\text { than } 10 \% \text { from the last sent one }\end{array}$ \\
\hline
\end{tabular}

\subsection{Data Acquisition and Lighting Control Strategy Assumptions}

The KNX BACS field network is a key tool used for data transmission and acquisition in the case study presented in this paper. Values and parameters measured by all kinds of sensors integrated within the BACS in SKNX \& EIEE Laboratory are transmitted to the HomeServer connected to KNX bus (Figure 2). The HomeServer visualizes the results on-line, archives them, and sends the results as .csv file once a day to specified e-mail addresses. The recording format allows further processing of the results by external tools and programs. Additionally, the laboratory is protected by an Intruder Alarm System. Arming of this system automatically turns off lights in the entire space of the laboratory, while disarming this system turns on lights in the corridor. A violation of a protected zone is detected by a presence detector and a light turns on in the violated zone. The following data are recorded by the HomeServer:

- illuminance level from the weather station and brightness sensor mounted on building facades, as well as from presence detectors with brightness sensors in each room;

- $\quad$ wind speed from weather station;

- $\quad$ state of the Intruder Alarm System—armed or standby;

- on/off status of lights in each room;

- position status of the blinds in each room.

The occupancy of each room is monitored using a sensor, and lights are turned off in case of inactivity after a sufficiently long period of time and turned on instantly when activity is detected again. In the corridor, in case of inactivity for a specified period of time, lights are not turned off but dimmed to $25 \%$ illuminance. The brightness sensor monitors daylight in the room and if the presence detector is active the lighting is controlled by the daylight illuminance. When the illuminance of the daylight falls below a specified point, the lights are brightened such that the illuminance monitored by the sensor increases to the required threshold. When a set point is exceeded, the lamps are turned off.

However, it should be noted that the potential electrical energy savings achieved by using presence detectors depends strongly on the behavior of the occupants. Obviously, the lighting control method described above is favorable in rooms in which the occupancy is changing, but it is not considered in this paper. Therefore, in the estimation of energy savings possibilities, in this study only a dimmable lighting control method was investigated. This estimation was based on daylight illuminance recorded inside the rooms and the determination of the number of hours in which the illuminance exceeded the specified set-points required to turn on and increase the intensity of light.

The mentioned set points are determined by correlation between the illuminance measured by the sensor (light reaching the sensor) and the illuminance on a table surface in the rooms and on the floor surface in the corridor. This correlation takes into account daylight distribution in the depths of the room. An example of daylight illuminance distribution in Office 1 and Corridor depths are shown 
in Figure 3. The illuminance decreases rapidly away from the window and remains nearly constant as the distance from the window increases.

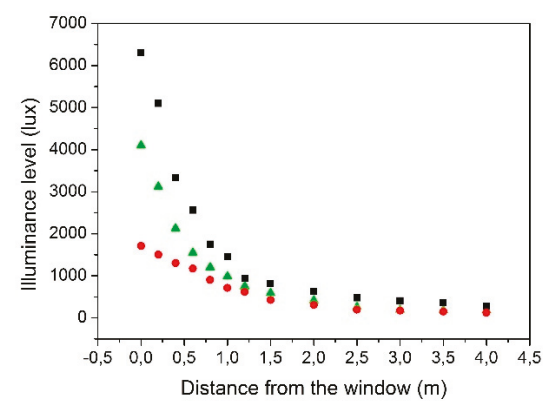

(a)

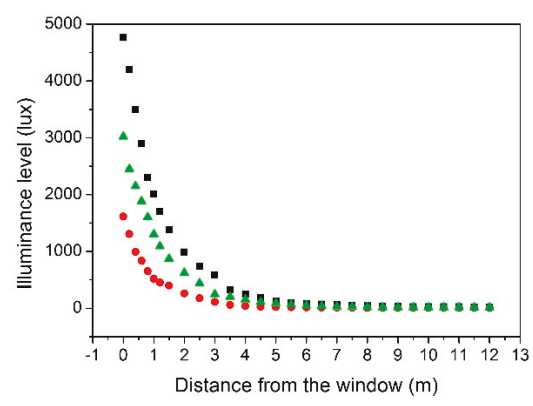

(b)

Figure 3. Daylight illuminance level distributions (a) at Office 1 depth and (b) at Corridor depth;

- —on 12 May 2016 at 14:30, •—on 13 May 2016 at 10:10, ^—on 19 May 2016 at 10:15.

Knowledge of the distributions was applied to control algorithm of the lights, such that the lamps are being switched on and their brightness increases more and more away from the windows. For example, in the corridor, the required illuminance level is assumed to be 150 lux, in accordance with the EN 12464-1 standard [45]. On 12 May 2016 at 14:30, three lamps located close to the window can be turned off; the fourth should be brightened to $50 \%$ and the rest to $100 \%$. On 19 May 2016 at $10: 15$, only the first lamp can be turned off; the second lamp should be brightened to $50 \%$ and the rest to $100 \%$. In the rooms, the lights can also be controlled by taking into account the distribution of the daylight with depth. The distribution in Office 1 shown in Figure 3a indicates that the lights in the first row from the window can be turned off, those in the second row should be brightened to $50 \%$, and those in the next row should be brightened to $100 \%$ (or other estimated values).

\section{Experimental Results, Analysis, and Findings}

As mentioned in previous sections, daylight illuminance and its distribution in the rooms are crucial for possible electrical energy savings. However, for the estimation of daylight in the rooms, there are two illuminance levels considered in research simulations and experiments: (i) external-outside the building and (ii) internal-inside the rooms. Moreover, the well-known Daylight Factor (DF) is used for indication of daylight availability in each room. The DF is defined as:

$$
D F=\frac{E_{\text {int }}}{E_{\text {ext }}},
$$

where $E_{\text {int }}$ is the internal illuminance and $E_{\text {ext }}$ is the external illuminance, simultaneously available on the horizontal plane from the whole of an unobstructed CIE overcast sky given by the International Commission on Illumination $[7,30,46]$.

\subsection{External Daylight Illuminance Measurements}

In the case study of the SKNX \& EIEE Laboratory analyzed in this paper, the external daylight illuminance levels are measured on the southeastern facade of the building by a weather station and on the north facade by a brightness sensor. During overcast days, the illuminance levels obtained from these two sensors were almost identical; however, on sunny days these values vary owing to varying sunlight at the building facades. Sample results are presented in Figure 4. Therefore, the lighting and blinds control in the room-depending on the illuminance measured by an external sensor-must consider the position of the sensor relative to the selected room. 


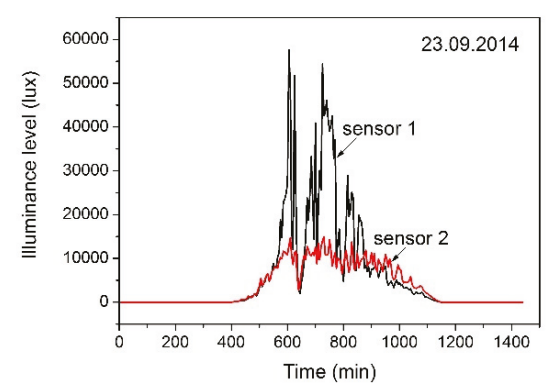

(a)

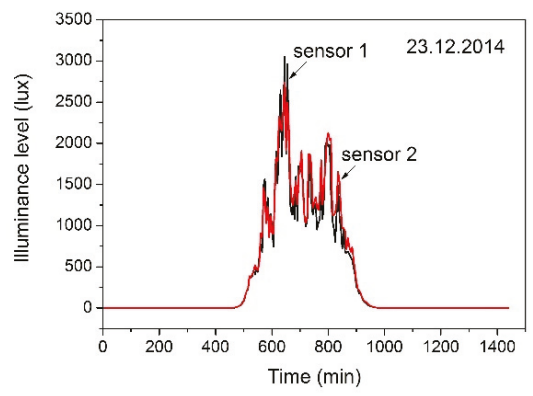

(b)

Figure 4. Illuminance levels of daylight measured by the weather station (sensor 1) and brightness sensor (sensor 2) on a sunny day (a) and an overcast day (b).

In order to estimate the feasibility of the dimmable lighting control method depending on the daylight illuminance distribution in rooms, the external daylight illuminance was measured every 5 min by the weather station during the period 1 July 2014 to 30 April 2015. Figure 5 shows the illuminance level measured each day from 1 July 2014 to 5 July 2014 and from 26 November 2014 to 30 November 2014. A value of 0 on the time axis means hour 0:00. On 1 July 2014 the sun rose at 4:33 and set at 21:17. In Figure 5a the sunrise hour corresponds to $273 \mathrm{~min}$ and the time of sunset to $1277 \mathrm{~min}$. On 23 November 2014 the sun rose at 7:29 (corresponding time in Figure 5b is $449 \mathrm{~min}$ ) and set at 15:47 (in Figure 5b-947 min). The highest illuminance of approximately 85,000 lux was recorded in the first period. Then, the illuminance level began to decrease and, in the second period, the maximum and minimum values of recorded illuminance were approximately 11,000 lux and less than 1000 lux, respectively. In the period with the shortest day of the year, the maximum value of the illuminance was approximately 14,000 lux because of the sunny day. It was observed that the recorded illuminance from 1 July to 5 July suddenly decreased owing to the position of the sun relative to the building façade where the weather station is installed and, obviously, there were more non-zero recorded values than in the period of 26 November to 30 November.

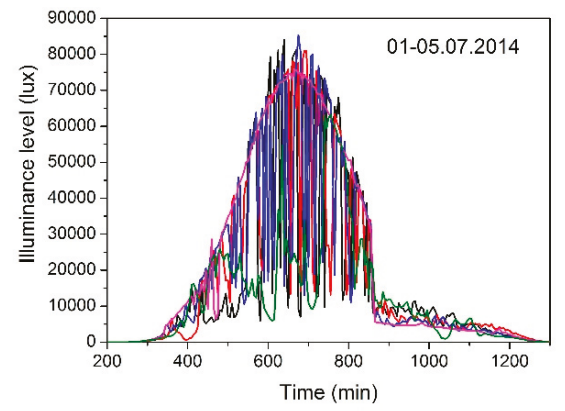

(a)

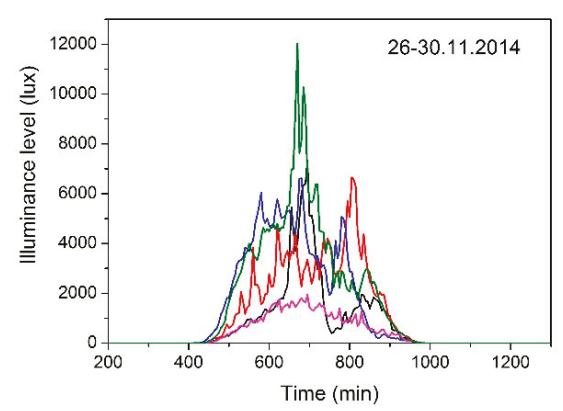

(b)

Figure 5. Daylight illuminance level recorded by the weather station mounted on the south-eastern facade of the building (a) from 1 July 2014 to 5 July 2014 and (b) from 26 November 2014 to 30 November 2014.

Since January 2015, the illuminance of the daylight increased steadily and reached a maximum value of approximately 72,000 lux in the last period investigated (16 to 30 April 2015). The minimum illuminance also increased; on cloudy days, it was less than 5000 lux. To highlight the influence of the 
sensor location on the measured illuminance, Figure 6 shows the daylight illuminance measured by the brightness sensor mounted on the north facade. The illuminance measured by this sensor was approximately 1.5 to 4 times lower than the illuminance measured by the weather station.

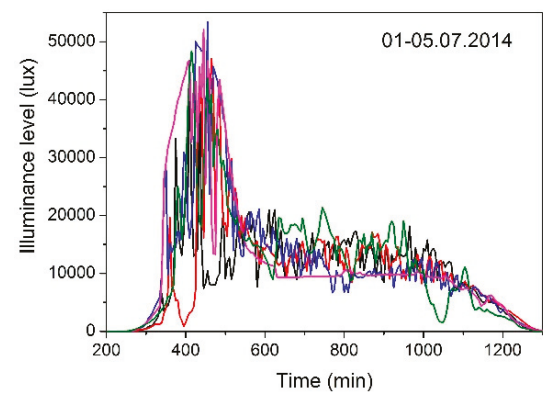

(a)

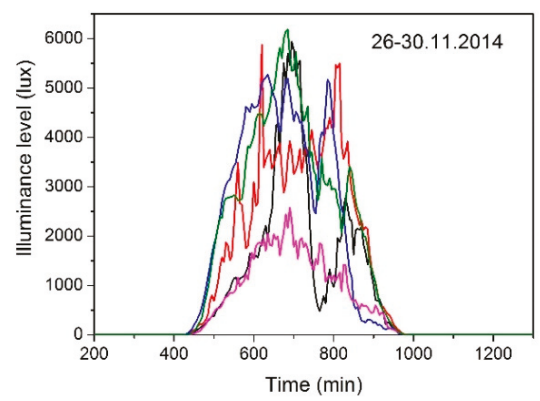

(b)

Figure 6. Daylight illuminance level recorded by the brightness sensor mounted on the north facade of the building (a) from 1 July 2014 to 5 July 2014 and (b) from 26 November 2014 to 30 November 2014.

Figures 5 and 6 indicate a very large variation of the daylight illuminance. In Figure 5, the illuminance reached approximately 85,000 lux, but the values decreased to less than 10,000 lux in a day owing to cloud cover. This variation affects the daylight variation inside the rooms, where in a short time it can change from a few times to tens of times, depending on variations in cloudiness. It should be noted that such a large variation occurs on most days of the year. These variations are similar to the results presented in $[13,14]$.

\subsection{Internal Daylight Illuminance Measurements}

Inside the laboratory rooms, the illuminance of daylight was measured by brightness sensors, sent and transmitted via KNX bus, then recorded in the HomeServer memory. Figure 7a shows the illuminance levels in rooms on a cloudy day on 23 November 2014 in which the outdoor illuminance was lower than 7000 lux. The highest illuminance was recorded in Office 2; however, it was lower than the required value of 500 lux. In the other rooms, the illuminance values were lower than 100 lux.

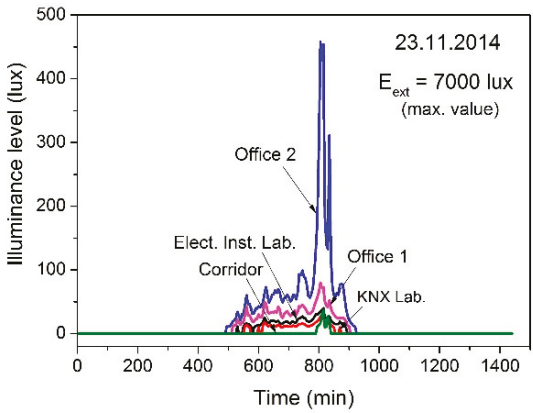

(a)

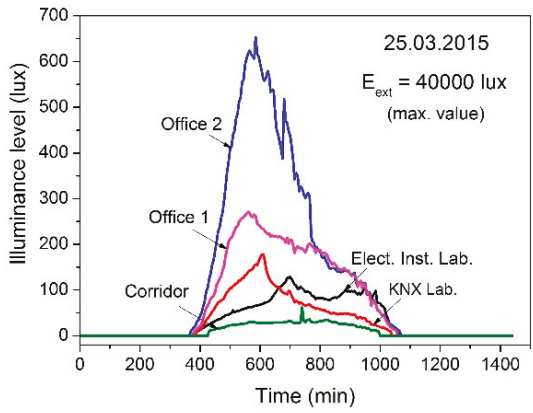

(b)

Figure 7. Daylight illuminance level in rooms (a) on a cloudy day (23 November 2014) and (b) on a sunny day (25 March 2015). 
The illuminance level on a sunny day (25 March 2015) measured inside rooms is shown in Figure $7 \mathrm{~b}$. The maximum value measured by the weather station was approximately 40,000 lux, while the value measured by the outdoor illuminance sensor was approximately 10,000 lux. In such outdoor daylight, only the illuminance in Office 2 was higher than 500 lux for about two hours, while in the other rooms the illuminance remained below 500 lux all day.

\subsection{Correlation between External and Internal Daylight Illuminance Levels}

The relationships between indoor and outdoor illuminance of daylight measured by the weather station for Offices 1 and 2 are shown in Figure 8 for a cloudy day (23 November 2014) and in Figure 9 for a sunny day (25 March 2015). On 23 November 2014 the external illuminance measured by the weather station and the external brightness sensor were practically the same. This means that on a cloudy day the illuminance does not depend on the facade where the sensors are mounted. In this case, internal illuminance $E_{i n t}$ can be approximated using a linear or polynomial function of external illuminance $E_{\text {ext }}$ :

$$
\text { for Office } 1: E_{\text {int }}=-0.37+0.0111 \cdot E_{\text {ext }} \text {; coefficient } R^{2} \text { is } 0.98301
$$

for Office $2: E_{i n t}=-0.8618+0.0403 \cdot E_{\text {ext }}-1.5342 \cdot 10^{-5} \cdot E_{\text {ext }}^{2}+2.9588 \cdot 10^{-9} \cdot E_{\text {ext }}^{3} ;$ coefficient $R^{2}$ is 0.89729 .

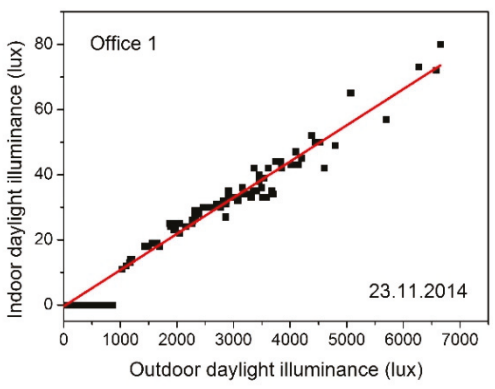

(a)

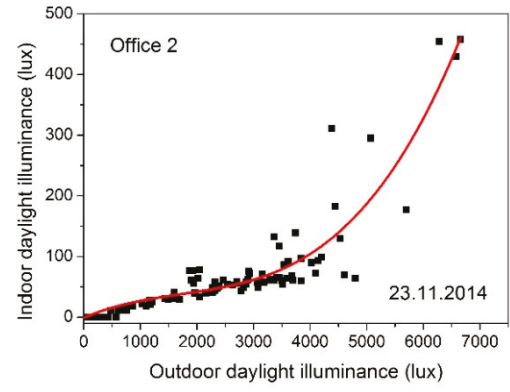

(b)

Figure 8. Relationships between internal and external illuminance of daylight measured by weather station on cloudy day on 23 November 2014 for (a) Office 1 and (b) Office 2.

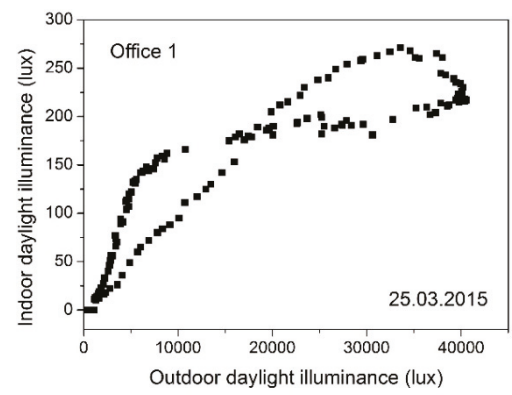

(a)

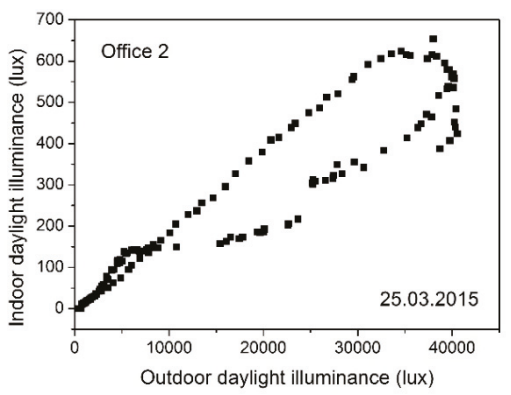

(b)

Figure 9. Relationships between internal and external illuminance of daylight measured by the weather station on a sunny day (25 March 2015) for (a) Office 1 and (b) Office 2.

The values of the $R^{2}$ coefficient show a very good correlation between indoor and outdoor illuminance levels for both functions. The form of the function depends on the shape of the room 
and daylight availability: Office 1 receives daylight from one side of the room, while Office 2 receives daylight from two sides. Offices 1 and 2 are quite small and sensors measuring illuminance are placed near the windows.

On a sunny day, the internal daylight depends on the position of the sun; therefore, for one value of the external illuminance, there are two values of the internal illuminance measured in a room, as shown in Figure 9. The more intense the sunlight, the larger the difference between these values.

The relationship between external and internal daylight illuminance levels is very complex and depends on many factors, as has already been mentioned in previous subsections. However, it should be noted that knowledge of this relationship is essential for proper selection of lighting control algorithms and evaluation of potential energy savings. If formulation of function equations is possible, these equations can be incorporated into the control algorithm to estimate daylight penetration in rooms.

\subsection{Availability of Daylight in the SKNX \& EIEE Laboratory Rooms}

In the SKNX \& EIEE Laboratory the experimental investigations were carried out in rooms with different daylight availability. The Electrical Installations Laboratory receives daylight through windows located on one side of the room, at the northwest. In the KNX Laboratory, the windows are on two sides of the room - the northwest and the northeast. The training stands are placed on two sides of the laboratory, obscuring the daylight. The depth of the two laboratories is $5.7 \mathrm{~m}$ and the presence detector with brightness sensor is mounted on the ceiling at a distance of $2.85 \mathrm{~m}$ from the wall with windows. In Office 1, the windows are at the southeast, while in Office 2 they are at the southeast and south. The corridor is in the central part of the laboratory, with daylight penetration via only one window at the south-in the narrow side of the corridor. The work plane includes practically the entire surface of each room at a height of $0.75 \mathrm{~m}$ above the floor, except for the corridor where the work plane includes the floor surface.

In order to assess the validity of dimmable lighting control and energy savings, the number of hours with illuminance levels higher than 500,400,300, 200, and 100 lux were estimated in the period from 1 July 2014 to 30 April 2015. These values were determined based on measurements by sensors placed in each room and taking into account the variation of the illuminance resulting from cloud cover. They are in accordance with EN 12464-1 standard [45]. The values for the Electrical Installation Laboratory, KNX Laboratory and two offices in July 2014 are shown in Figure 10, and the total number of hours determined during the considered 10-month period are shown in Figure 11. Results for the Corridor were not presented because the measured illuminance was less than 100 lux. Obviously, the number of hours in which the illuminance exceeds the specified values depends on the month: higher in the summer months and lower in the winter months, respectively.

Figures 10 and 11 show that the daylight availability in several rooms of one building was very different. The number of hours when the illuminance level exceeded the specified values is shown in Table 4.

Table 4. The number of hours when the illuminance level exceeded the specified values.

\begin{tabular}{cccccc}
\hline \multirow{2}{*}{$\begin{array}{c}\text { Room of } \\
\text { the Building }\end{array}$} & \multicolumn{4}{c}{ The Number of Hours When the Illuminance $E_{\text {int }}$ Expressed in Lux Was } \\
\cline { 2 - 6 } & $\mathbf{1 0 0 < E _ { \text { int } } < \mathbf { 2 0 0 }}$ & $\mathbf{2 0 0}<E_{\text {int }}<\mathbf{3 0 0}$ & $\mathbf{3 0 0}<E_{\text {int }}<\mathbf{4 0 0}$ & $\mathbf{4 0 0}<E_{\text {int }}<\mathbf{5 0 0}$ & $\boldsymbol{E}_{\text {int }}>\mathbf{5 0 0}$ \\
\hline Elect. Inst. Lab. & 762.5 & 70.3 & 0 & 0 & 0 \\
KNX Lab. & 488.6 & 7.4 & 0 & 0 & 0 \\
Office 1 & 811.1 & 196.0 & 125.2 & 128.4 & 224.0 \\
Office 2 & 602.3 & 373.2 & 281.8 & 120.2 & 552.1 \\
\hline
\end{tabular}

The largest value occurred in Office 2 owing to the location of the windows on two sides and the small dimensions of the room, and therefore the location of the illuminance sensor is near windows. Slightly less daylight availability occurred in the deeper Office 1, with one side window. Comparison of 
daylight availability in the two offices reveals that the number of hours with illuminance greater than 500 lux was 2.5 times higher in Office 2 than in Office 1 and the number of hours with illuminance between 200 and 300 lux as well as between 300 and 400 lux were approximately twice as high.

In the KNX Laboratory with windows on two sides and a depth comparable to the depth of Office 1, daylight availability is limited owing to a piece of equipment blocking access to light from the windows. Low availability also occurred in the Electrical Installations Laboratory owing to the orientation of the building relative to the northwest.

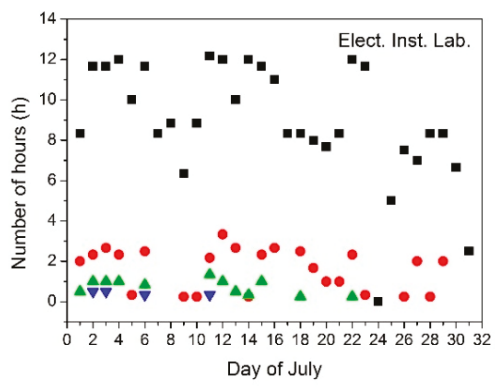

(a)

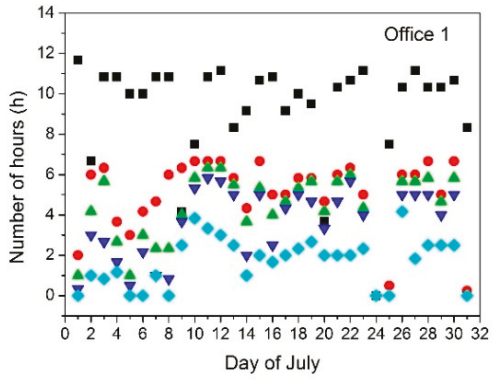

(c)

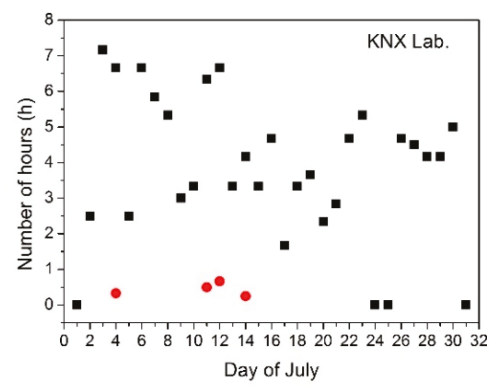

$(b)$

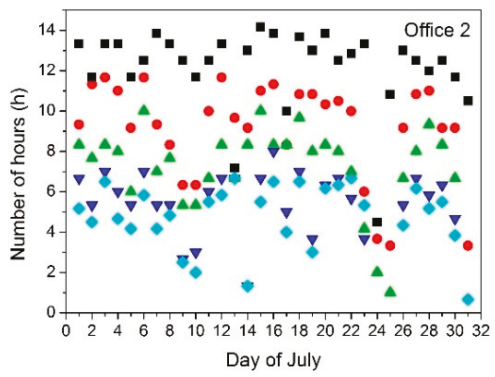

(d)

Figure 10. Number of hours in July 2014 for (a) Electrical Installations Laboratory, (b) KNX Laboratory, (c) Office 1, and (d) Office 2 with illuminance level higher than: $\mathbf{\square}-100$ lux, $\bullet-200$ lux, $\mathbf{\Delta}-300$ lux, $\nabla-400$ lux, -500 lux.

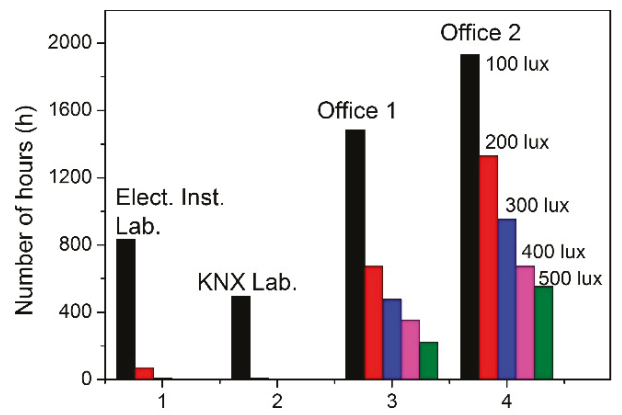

Figure 11. Number of hours from 1 July 2014 to 30 April 2015 with illuminance level higher than specified values. 


\section{Energy Savings Estimation and Discussion}

The results presented in the previous section indicate that, in the two offices, the use of dimmable lighting controls is economically reasonable unlike in the laboratories, where a simpler method giving significant energy savings is on/off lighting control. Therefore, to estimate possible energy savings, the authors assumed that in a room where the required illuminance level was 500 lux, any reduction in the daylight illuminance of 100 lux requires an increase in the lamp's brightness by $20 \%$. Knowing the installed power of the lamps in the room and the time when the daylight illuminance was less than 500 lux by 100, 200, 300, 400, and 500 lux, the energy reduction can be estimated. Moreover, it was assumed that the light was automatically controlled at all times and only dimmable lighting control was used. The rooms were occupied from 8:00 a.m. to 6:00 p.m. each day including holidays; therefore, the period from 1 July 2014 to 30 April 2015 has 304 days, and hence $3040 \mathrm{~h}$. The time in which the light should be switched on was determined separately for each day and if this time was greater than $10 \mathrm{~h}, 10 \mathrm{~h}$ was used in the calculation.

The energy consumption without control and with dimmable lighting control as well as the energy savings as a percentage of energy consumed without control are presented in Table 5. Energy consumption without control was calculated by multiplying the installed power of lamps by the overall time of activity in rooms $(3040 \mathrm{~h})$.

Table 5. Energy consumptions and energy savings in several rooms of building.

\begin{tabular}{cccc}
\hline $\begin{array}{c}\text { Room of } \\
\text { the Building }\end{array}$ & \multicolumn{2}{c}{ Energy Consumption in $\mathbf{k W h} / \mathbf{m}^{\mathbf{2}}$} & $\begin{array}{c}\text { Energy Savings as a Percentage of } \\
\text { Energy Consumed without Control }\end{array}$ \\
\cline { 2 - 3 } & Without Control & With Control & 6 \\
Elect. Inst. Lab. & 36.12 & 33.94 & 3 \\
KNX Lab. & 39.62 & 38.43 & 16 \\
Office 1 & 35.48 & 29.8 & 27 \\
Office 2 & 35.44 & 25.87 & \\
\hline
\end{tabular}

The results of the energy savings estimation for different rooms in the SKNX \& EIEE Laboratory confirmed that implementation of the BACS control and TBM functions provides additional energy savings in buildings. However, as has been noted in Section 2, these savings are closely related to the physical characteristics of specific buildings and rooms. The results of the experiment show that better energy efficiency improvements for advanced dimmable lighting control can be obtained in rooms with better daylight penetration. For rooms like KNX and Electrical Installations laboratories in this case study, the potential energy savings are not so great since the sufficient illuminance level in the rooms should be provided with artificial lighting (lamps) most of the time. For the two laboratories, there are no economic reasons for applying dimmable control; however, owing to the low availability of daylight in the rooms, on/off lighting control is recommended. In these cases, the orientation of the building relative to the south and north plays a significant role, affecting daylight penetration into the rooms. Moreover, the relationship between the indoor and outdoor illuminance levels of daylight should be determined, as has been done in Section 4.3. If this relationship is formulated by a function equation, it can be used to estimate daytime lighting in rooms by the algorithms and control functions of the BACS.

Nevertheless, the results of the experiment obtained and presented in this paper indicate that the potential energy savings on the level expected directly from the BACS factors presented and discussed in Section 2 are achievable. For this case study the BACS efficiency class is A, taking into account the occupancy and daylight control. Therefore, the expected savings from the BACS factors related to the electrical energy used for lighting in non-residential buildings are $28 \%$ for offices and $24 \%$ for educational buildings, respectively. The experimental results are similar for Office 2. For the other rooms the real savings are lower than expected from the BACS factors, but in this case study only the dependence on daylight availability has been analyzed. To achieve better energy efficiency, the impact 
of other control and TBM functions should be considered, but they were not considered in this paper. Finally, it could be expected that in Polish climatic conditions, the possible maximum energy savings obtainable using dimmable lighting control would be approximately $30 \%$ of the energy consumed without control. However, in practice these energy savings should always be analyzed individually for specific buildings and only for long periods.

\section{Conclusions and Future Works}

The study presented in this paper focuses on the feasibility of daylight-centered control strategy implementation in non-residential buildings. Experimental evaluation and validation of the dimmable lighting control strategy proposed by the authors have been carried out in a specific university building. In this case study, it is the SKNX \& EIEE Laboratory equipped with the open, distributed BACS based on the widely used KNX international standard of building automation. This KNX BACS provided a universal platform for measurements, data acquisition, and visualization from different sensors and control modules distributed in the SKNX \& EIEE Laboratory rooms. A technical analysis of the BACS infrastructure installed in the laboratories allowed the authors to implement the requirements from the EN 15232 standard. Therefore, the BACS efficiency factors method for the rough estimation of electrical energy savings due to the BACS control and TBM functions implementation has been presented and experimentally verified for the case study considered in the paper. The authors are convinced that this approach is in line with the current and suggested directions of research work in the field of using BACS in the energy efficiency improvements of buildings. The results of this experiment confirm that BACS control functions integrated in accordance with the BACS efficiency class requirements have a great impact on a building's energy efficiency. However, this impact is not always as great as expected based directly on the BACS efficiency factors. Therefore, these factors cannot be treated arbitrarily. The results of the experiment presented and discussed in the paper confirmed this statement as well. Hence, as has been motioned, the potential energy savings should always be analyzed and verified for specific buildings individually [34].

To verify the diversity of potential energy savings, a detailed analysis of the daylight impact on the proposed dimmable lighting control strategy has been presented. The authors focused on daylight illuminance changes and distributions in different rooms. The $R^{2}$ coefficient proves a strong correlation between external and internal illuminance levels, showing the possibility of formulating function equations for this correlation, which could be used in control algorithms to predict daytime lighting in the rooms. Moreover, based on long-term daylight illuminance level measurements, the authors proposed their own method of rough electrical energy savings estimation for a dimmable lighting control strategy. The results of this estimation have been compared with the BACS efficiency factors, confirming the mentioned diversity of energy savings. In this way the study presents a possible approach for estimating lighting control schemes and the prediction of energy savings. It should be noticed that the methods proposed in the paper are universal and can be considered in verifications of various control systems concepts and strategies. This is especially important since, as shown in the paper, non-residential buildings have a large potential for overall energy savings and electrical savings for lighting as well. However, the key aspects are not only the building's location and orientation, as considered in other studies, but also integrity and interoperability between various devices and subsystems of a building's infrastructure. These are also given by the mentioned EN 15232 standard for various categories of control and monitoring functions. Therefore, future work will be first of all focused on the verification of the potential energy savings by applying energy efficiency strategies to the case study building. In the next stage of work the authors will consider an extension of the lighting control strategies proposed in this paper as well as the integration of other control functions within advanced control strategies, organized according to guidelines from the EN 15232 standard. In particular, experimental validation of these requirements for specific case studies will be included. Moreover, taking into account similar research [40,47], implementation of energy 
management functions with the distributed BACS, ready for both Demand-Side Management (DSM) and Demand-Side Response (DSR) strategies, are considered by the authors for future studies.

Author Contributions: A.K. proposed the concept of the experiments, performed the measurements and data analyses, reviewed and refined the paper, and supported the editing work. A.O. wrote and organized the paper, provided theoretical knowledge and related work review, proposed the lighting control strategies investigated in experiments, presented the EN 15232 standard guidelines, analyzed potential energy savings, and discussed the results.

Funding: This research received no external funding.

Acknowledgments: This study is based upon work supported by the National Centre for Research and Development in the context of the Innovative Economy Program under grant no. POIG.02.02.00-00-018/08. This work was also supported by the 2017 Poznan University of Technology funds transferred from the Ministry of Science and Higher Education.

Conflicts of Interest: The authors declare no conflict of interest. The funders had no role in the design of the study; in the collection, analyses, or interpretation of data; in the writing of the manuscript; or in the decision to publish the results.

\section{References}

1. International Energy Agency. Energy Efficiency Market Report 2016; International Energy Agency: Paris, France, 2016.

2. International Energy Agency. Worldwide Trends in Energy Use and Efficiency Key Insights from IEA Indicator Analysis; International Energy Agency: Paris, France, 2008.

3. Ożadowicz, A. A New Concept of Active Demand Side Management for Energy Efficient Prosumer Microgrids with Smart Building Technologies. Energies 2017, 10, 1771. [CrossRef]

4. Van De Meugheuvel, N.; Pandharipande, A.; Caicedo, D.; Van Den Hof, P.P.J. Distributed lighting control with daylight and occupancy adaptation. Energy Build. 2014, 75, 321-329. [CrossRef]

5. Martirano, L.; Senior, I. A Sample Case of an Advanced Lighting System in a Educational Building. In Proceedings of the 2014 14th International Conference on Environment and Electrical Engineering (EEEIC), Krakow, Poland, 10-12 May 2014; pp. 4-9.

6. Zhou, X.; Yan, D.; Hong, T.; Ren, X. Data analysis and stochastic modeling of lighting energy use in large office buildings in China. Energy Build. 2015, 86, 275-287. [CrossRef]

7. Yu, X.; Su, Y.; Chen, X. Application of RELUX simulation to investigate energy saving potential from daylighting in a new educational building in UK. Energy Build. 2014, 74, 191-202. [CrossRef]

8. Kocabey, S.; Ekren, N. A new approach for examination of performance of interior lighting systems. Energy Build. 2014, 74, 1-7. [CrossRef]

9. Jovanović, A.; Pejić, P.; Djorić-Veljković, S.; Karamarković, J.; Djelić, M. Importance of building orientation in determining daylighting quality in student dorm rooms: Physical and simulated daylighting parameters' values compared to subjective survey results. Energy Build. 2014, 77, 158-170. [CrossRef]

10. Li, D.H.W.; Wong, S.L. Daylighting and energy implications due to shading effects from nearby buildings. Appl. Energy 2007, 84, 1199-1209. [CrossRef]

11. Qi, F.; Wang, Y. A new calculation method for shape coefficient of residential building using Google Earth. Energy Build. 2014, 76, 72-80. [CrossRef]

12. Katafygiotou, M.C.; Serghides, D.K. Analysis of structural elements and energy consumption of school building stock in Cyprus: Energy simulations and upgrade scenarios of a typical school. Energy Build. 2014, 72, 8-16. [CrossRef]

13. Hashemi, A. Daylighting and solar shading performances of an innovative automated reflective louvre system. Energy Build. 2014, 82, 607-620. [CrossRef]

14. Li, D.H.W. A review of daylight illuminance determinations and energy implications. Appl. Energy 2010, 87, 2109-2118. [CrossRef]

15. Chen, Y.; Liu, J.; Pei, J.; Cao, X.; Chen, Q.; Jiang, Y. Experimental and simulation study on the performance of daylighting in an industrial building and its energy saving potential. Energy Build. 2014, 73, 184-191. [CrossRef] 
16. Díaz-Vilariño, L.; Lagüela, S.; Armesto, J.; Arias, P. Indoor daylight simulation performed on automatically generated as-built 3D models. Energy Build. 2014, 68, 54-62. [CrossRef]

17. Pellegrino, A.; Blaso, L. Lighting Control Strategies and Energy Efficiency Benefits. In Sustainable Indoor Lighting, Green Energy and Technology; Green Energy and Technology; Sansoni, P., Mercatelli, L., Farini, A., Eds.; Springer: London, UK, 2015; pp. 303-334, ISBN 978-1-4471-6632-0.

18. Lobaccaro, G.; Carlucci, S.; Löfström, E. A review of systems and technologies for smart homes and smart grids. Energies 2016, 9, 348. [CrossRef]

19. Hong, S.H.; Kim, S.H.; Kim, J.H.; Kim, Y.G.; Kim, G.M.; Song, W.S. Integrated BACnet-ZigBee communication for building energy management system. In Proceedings of the IECON 2013-39th Annual Conference of the IEEE Industrial Electronics Society, Vienna, Austria, 10-13 November 2013; IEEE: Piscataway, NJ, USA, 2013; pp. 5723-5728.

20. Ozadowicz, A.; Grela, J. Control application for Internet of Things energy meter-A key part of integrated building energy management system. In Proceedings of the 2015 IEEE 20th Conference on Emerging Technologies \& Factory Automation (ETFA), Luxembourg, 8-11 September 2015; IEEE: Piscataway, NJ, USA, 2015; pp. 1-4.

21. Kastner, W.; Neugschwandtner, G.; Soucek, S.; Newmann, H.M. Communication Systems for Building Automation and Control. Proc. IEEE 2005, 93, 1178-1203. [CrossRef]

22. Ilieva, S.; Penchev, A.; Petrova-antonova, D. Internet of Things Framework for Smart Home Building. In Digital Transformation and Global Society; Communications in Computer and Information Science; Chugunov, A.V., Bolgov, R., Kabanov, Y., Kampis, G., Wimmer, M., Eds.; Springer International Publishing: Cham, Switzerland, 2016; Volume 674, pp. 450-462, ISBN 978-3-319-49699-3.

23. Wang, J. Zigbee light link and its applicationss. IEEE Wirel. Commun. 2013, 20, 6-7. [CrossRef]

24. Salamone, F.; Belussi, L.; Danza, L.; Galanos, T.; Ghellere, M.; Meroni, I. Design and Development of a Nearable Wireless System to Control Indoor Air Quality and Indoor Lighting Quality. Sensors 2017, 17, 1021. [CrossRef] [PubMed]

25. Borile, S.; Pandharipande, A.; Caicedo, D.; Schenato, L.; Cenedese, A. A Data-Driven Daylight Estimation Approach to Lighting Control. IEEE Access 2017, 5, 21461-21471. [CrossRef]

26. Saraf, R.; Bhavani, R.G. Assessment of daylight performance of a commercial office space in hot, arid climate for enhanced visual comfort conditions. In Proceedings of the 2017 International Conference on Technological Advancements in Power and Energy (TAP Energy), Kollam, India, 21-23 December 2017; IEEE: Piscataway, NJ, USA, 2017; pp. 1-6.

27. Mathew, M.; Kini, S.G. Daylight analysis and enhancing strategies for a low rise office building in composite climate of India. In Proceedings of the 2017 IEEE International Conference on Circuits and Systems (ICCS), Thiruvananthapuram, India, 20-21 December 2017; pp. 21-25. [CrossRef]

28. Larsen, O.K.; Jensen, R.L.; Antonsen, T.; Strømberg, I. Estimation methodology for the electricity consumption with daylight- and occupancy-controlled artificial lighting. Energy Procedia 2017, 122, 733-738. [CrossRef]

29. Delvaeye, R.; Ryckaert, W.; Stroobant, L.; Hanselaer, P.; Klein, R.; Breesch, H. Analysis of energy savings of three daylight control systems in a school building by means of monitoring. Energy Build. 2016, 127, 969-979. [CrossRef]

30. Jung, S.; Yoon, S. Study on the Prediction and Improvement of Indoor Natural Light and Outdoor Comfort in Apartment Complexes Using Daylight Factor and Physiologically Equivalent Temperature Indices. Energies 2018, 11, 1872. [CrossRef]

31. Grela, J.; Ozadowicz, A. Building Automation planning and design tool implementing EN 15232 BACS efficiency classes. In Proceedings of the 2016 IEEE 21st International Conference on Emerging Technologies and Factory Automation (ETFA), Berlin, Germany, 6-9 September 2016; IEEE: Piscataway, NJ, USA, 2016; pp. 1-4.

32. Lehmann, M.; Mai, T.L.; Wollschlaeger, B.; Kabitzsch, K. Design approach for component-based automation systems using exact cover. In Proceedings of the 2014 IEEE Emerging Technology and Factory Automation (ETFA), Barcelona, Spain, 16-19 September 2014; IEEE: Piscataway, NJ, USA, 2014; pp. 1-8.

33. De Bakker, C.; Van De Voort, T.; Rosemann, A. The Energy Saving Potential of Occupancy-Based Lighting Control Strategies in Open-Plan Offices: The Influence of Occupancy Patterns. Energies 2017, 11, 2. [CrossRef] 
34. Ozadowicz, A.; Grela, J. Impact of building automation control systems on energy efficiency-University building case study. In Proceedings of the 2017 22nd IEEE International Conference on Emerging Technologies and Factory Automation (ETFA), Limassol, Cyprus, 12-15 September 2017; IEEE: Piscataway, NJ, USA, 2017; pp. 1-8.

35. EU. Directive 2010/31/EU of the European Parliament and of the Council of 19 May 2010 on the energy performance of buildings (recast). Off. J. Eur. Union 2010, 13-35. [CrossRef]

36. Ahmed, K.; Carlier, M.; Feldmann, C.; Kurnitski, J. A new method for contrasting energy performance and near-zero energy building requirements in different climates and countries. Energies 2018, 11, 1334. [CrossRef]

37. European Parliament. Directive 2012/27/EU of the European Parliament and of the Council of 25 October 2012 on energy efficiency. Off. J. Eur. Union Dir. 2012, 1-56. [CrossRef]

38. European Commission Energy Roadmap 2050. 2012. Available online: https:/ / ec.europa.eu/energy/sites/ ener/files/documents/2012_energy_roadmap_2050_en_0.pdf (accessed on 19 August 2018).

39. EN 15232. Energy Performance of Buildings_-Impact of Building Automation, Controls and Building Management; European Committee for Standardization: Brussels, Belgium, 2012.

40. Favuzza, S.; Ippolito, M.G.; Massaro, F.; Musca, R.; Sanseverino, E.R.; Schillaci, G.; Id, G.Z. Building Automation and Control Systems and Electrical Distribution Grids: A Study on the Effects of Loads Control Logics on Power Losses and Peaks. Energies 2018, 11, 667. [CrossRef]

41. Beccali, M.; Bonomolo, M.; Ippolito, M.G.; Lo Brano, V.; Zizzo, G. Experimental validation of the BAC factor method for lighting systems. In Proceedings of the 2017 IEEE International Conference on Environment and Electrical Engineering and 2017 IEEE Industrial and Commercial Power Systems Europe (EEEIC/I\&CPS Europe), Milan, Italy, 6-9 June 2017; IEEE: Piscataway, NJ, USA, 2017; pp. 1-5.

42. Beccali, M.; Bonomolo, M.; Galatioto, A.; Ippolito, M.G.; Zizzo, G. A laboratory setup for the evaluation of the effects of BACS and TBM systems on lighting. In Proceedings of the 2015 International Conference on Renewable Energy Research and Applications (ICRERA), Palermo, Italy, 22-25 November 2015; IEEE: Piscataway, NJ, USA, 2015; Volume 5, pp. 1388-1393.

43. Sanseverino, E.R.; Zizzo, G.; La Cascia, D. Economic Impact of BACS and TBM Systems on Residential Buildings. In Proceedings of the 2013 International Conference on Clean Electrical Power (ICCEP), Alghero, Italy, 11-13 June 2013; pp. 591-595.

44. Rospi, G.; Cardinale, N.; Negro, E. Energy Performance and Economic Feasibility Study of Historical Building in the City of Matera, Southern Italy. Energies 2017, 10, 2009. [CrossRef]

45. EN 12464. Light and Lighting_Lighting of Work Places—Part 1: Indoor Work Places; European Committee for Standardization: Brussels, Belgium, 2012.

46. Kamaruzzaman, S.N.; Edwards, R.; Zawawi, E.M.A.; Che-Ani, A.I. Achieving energy and cost savings through simple daylighting control in tropical historic buildings. Energy Build. 2015, 90, 85-93. [CrossRef]

47. Miceli, R. Energy Management and Smart Grids. Energies 2013, 6, 2262-2290. [CrossRef]

(C) 2018 by the authors. Licensee MDPI, Basel, Switzerland. This article is an open access article distributed under the terms and conditions of the Creative Commons Attribution (CC BY) license (http:/ / creativecommons.org/licenses/by/4.0/). 
Article

\title{
An Optimal Air-Conditioner On-Off Control Scheme under Extremely Hot Weather Conditions
}

\author{
Mohammed Al-Azba ${ }^{1,2}$, Zhaohui Cen ${ }^{1, *}$, Yves Remond ${ }^{2}$ and Said Ahzi ${ }^{1}$ \\ 1 Qatar Environment and Energy Research Institute, Hamad Bin Khalifa University, Doha 5825, Qatar \\ 2 ICube Laboratory, Université de Strasbourg-CNRS, 67000 Strasbourg, France \\ * Correspondence: zcen@hbku.edu.qa
}

Received: 24 December 2019; Accepted: 20 February 2020; Published: 25 February 2020

\begin{abstract}
Being reliant on Air Conditioning (AC) throughout the majority of the year, desert countries with extremely hot weather conditions such as Qatar are facing challenges in lowering weariness cost due to AC On-Off switching while maintaining an adequate level of comfort under a wide-range of ambient temperature variations. To address these challenges, this paper investigates an optimal On-Off control strategy to improve the AC utilization process. To overcome complexities of online optimization, a Elman Neural Networks (NN)-based estimator is proposed to estimate real values of the outdoor temperature, and make off-line optimization available. By looking up the optimum values solved from an off-line optimization scheme, the proposed control solutions can adaptively regulate the indoor temperature regardless of outdoor temperature variations. In addition, a cost function of multiple objectives, which consider both Coefficient of Performance (COP), and AC compressor weariness due to On-Off switching, is designed for the optimization target of minimum cost. Unlike conventional On-Off control methodologies, the proposed On-Off control technique can respond adaptively to match large-range (up to $20^{\circ} \mathrm{C}$ ) ambient temperature variations while overcoming the drawbacks of long-time online optimization due to heavy computational load. Finally, the Elman NN based outdoor temperature estimator is validated with an acceptable accuracy and various validations for AC control optimization under Qatar's real outdoor temperature conditions, which include three hot seasons, are conducted and analyzed. The results demonstrate the effectiveness and robustness of the proposed optimal On-Off control solution.
\end{abstract}

Keywords: Air-Conditioning; On-Off control; desert climate; optimization; Elman Neural Networks

\section{Introduction}

Famous for its unique geographic location between desert and sea, Qatar has significant desert climate and extremely hot weather conditions with a high temperature, humidity, and sand storms. The Air Conditioning (AC) systems in this region are highly mandatory for daily life through the year. The local weather has some significance of representing a unique desert and coastline climate, which attract a lot of research interests in various fields such as building efficiency, health, urban environment, and renewable energy [1-11]. As elaborated in [12], in GCC region such as Saudi Arab, more than $60 \%$ of the electricity consumed by local buildings goes to Air conditioning (AC). This is particularly valid for other GCC countries like Qatar, which is reliant on AC throughout the majority of the year. Due to the persistent heavy cooling load caused by high temperature weather conditions and desert climate, heating, ventilation, and Air Conditioning (HVAC) equipment degrades faster compared to HVAC facilities in other areas and also requires higher reliability while maintaining energy efficiency and adequate levels of quality and comfort.

An Air Conditioner system is made up of a compressor as key part, and other parts such as a condenser and an evaporator, where the air conditioning capacity depends on the compressor power. 
In space cooling scenarios, indoor temperature will oscillate at a set-point because the compressor work in switch modes of on or off [13]. The component used to implement this control philosophy is known as an Air-Conditioner On-Off (or bang-bang) controller or thermoset. The On-Off compressor works as either being $\mathrm{ON}$ or OFF depending on the set-point boundary and the measured outdoor temperature. There is a dead range of $1.5^{\circ} \mathrm{C}$ to $2.0^{\circ} \mathrm{C}$ to prevent the too frequent On-Off fluctuations of the compressor that could lead to a reduced lifespan. Especially under hot weather conditions, long-term running with extra cooling load could degrade AC systems in a faster way, in particular if a very slight tracking error is imposed. Due to its simplicity, On-Off control is widely applied for temperature regulation application scenarios. However, On-Off control also have eminent drawbacks, which include temperature oscillation and non-optimal operation which negatively impacts the AC moving parts and its energy consumption [13-21].

Currently, a body of research work has been reported in the literature in response to challenges around HVAC control optimization. Several research works have been proposed to optimize building HVAC system [22,23], and address On-Off control issues under different constraints. The related research work can be summarized as two approaches: low-complexity optimization, and dynamic optimization. In low-complexity optimization approaches, Hysteresis controllers and Pulse Width Modulation (PWM) are widely utilized [13,24]. A hysteresis controller has a simple structure to implement in practice, so it is widely used in HVAC applications. Because the AC systems by hysteresis control work under fixed hysteresis conditions/parameters (with a set-point temperature boundary), the hysteresis controller does not perform well if ambient parameters/working conditions change. PWM control is usually used in PWM actuated split air conditioners. The PWM controller tunes the duty ratio of the AC On-Off control signal in a continuous way to allow continuous change of the manipulated variable. It can accommodate some work conditions change based on its fast feedback control, which is similar to Proportional-Integral-Derivative (PID) control. However, PWM controller has to change under very fast switching frequency at the initial stage of the control process or scenarios of large variations in the working conditions. This fast change could degrade the AC compressor, and it has also been proven not to be optimal for all the HVAC control cases. Both of these optimized controllers treat control process time horizon as a long-term general process in one day, one week, one month or one year. Thus, the full control process needs to rely on the temperature profile of an entire period and also the controller parameters can only be set and tuned in advance. For instance, the hysteresis control parameters are pre-set with a hysteresis result under certain working conditions. The controllers cannot accommodate ambient temperature variations in an adaptive way.

As a dynamic optimization approach, Model-Predictive Control (MPC) is employed to accommodate disturbances and variations of the operating conditions such as time-variant parameters and target [14,24-31]. The MPC's optimization performance is subjected to the prediction horizon length. In theory, a longer prediction horizon can helps the solution be closer to the global optimum; therefore, it needs more computational power and resources. However, a proper prediction horizon is difficult to determine in practice, and MPC optimization will consume a lot of online computation loads. As such, online optimization and offline optimization need to be integrated and balanced for a trade-off between the computational load and AC control adaptiveness. In [32], a scheme of AC On-Off control and optimization is proposed for variant ambient conditions, but the transition between off-line optimization and online control is not integrated, and the part of outdoor temperature prediction is missing because it is assumed. Moreover, demonstrations under Qatar climatic conditions for three hot seasons of a full year are missing.

Motivated by the heavy cooling demand and its associated HVAC components wearing issues under harsh weather conditions in desert countries such as Qatar, an optimal AC On-Off Control Scheme is presented to improve cooling performance under heavy cooling load scenarios. In this scheme, optimizations are performed offline to generate the optimal solutions under certain conditions and range of outdoor temperatures, and then, a static data table of optimized parameters is generated. Based on the proposed lookup algorithm for online optimization, On-Off control parameters are tuned 
online to accommodate outdoor temperatures variation. As opposed to the existing AC hysteresis On-Off control schemes, the proposed On-Off control and optimization scheme can process more complex cooling scenarios such as large range and fast temperature variations. This is because the controller can adaptively accommodate the variations by tuning the parameters optimization online. Compared to the MPC control, the proposed control scheme performs optimization work offline which does not require a huge computational power in a real-time way like the online optimization technique.

This paper is structured as follows: a typical Qatar outdoor temperature profile is analyzed and the thermal model of a simple house is built in Section 2; Section 3 presents the proposed optimal On-Off control scheme, which includes off-line optimization and online adaptive tracking scheme. The validation results under different cooling scenarios are presented in Sections 4 and 5 concludes the paper.

\section{Problem Formulation}

Due to Qatar's unique geographical environment, the outdoor environment shows a very unique desert climate with high temperature. According to the data from local weather records shown in Figure 1, the average outdoor temperature varies from $15{ }^{\circ} \mathrm{C}$ to $46^{\circ} \mathrm{C}$ throughout the four seasons. The daily temperature variation range could be up to $20^{\circ} \mathrm{C}$ during autumn or spring. This makes it necessary to study a typical thermal dynamics of a house with the local outdoor temperature profile analysis.

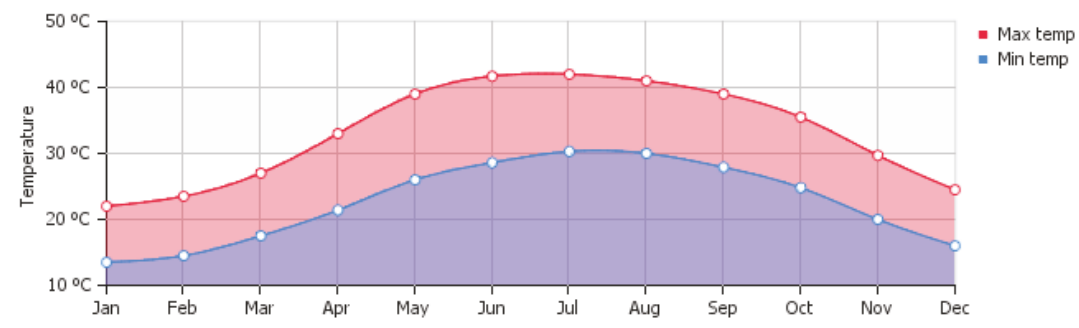

Figure 1. Average Min and Max Temperatures in Doha, Qatar for 2018.

\subsection{Outdoor Temperature Measurement under Qatar Weather Conditions}

A three-day outdoor temperature profile in October 2017, which represents typical weather characteristics of Qatar, is measured in real time using thermal sensors at the outdoor test facilities of Qatar Environment and Energy Research Institute (QEERI). The facilities are shown in Figure 2, and the three-day profile is shown in Figure 3. The outdoor facilities site mainly host renewable energy facilities such as $200 \mathrm{~kW}$ PV farm, $250 \mathrm{~kW} / 500 \mathrm{kWh}$ Lithium-ion battery storage system, and other micro-grid components. Moreover, a weather measurement station for solar irradiance and other weather parameters, which include the outdoor temperatures, is deployed for research purposes. The daily outdoor temperature variations in October, shown in Figure 3, have a very uniform probability distribution, which means that the weather prediction can be performed with higher accuracy. In practice, one day ahead prediction horizon is available for outdoor temperature prediction based on existing weather measurement and prediction technologies. Thus, it is feasible to have enough time to do offline optimization in advance based on predicted weather conditions, which can prevent the complexities of online optimization. 


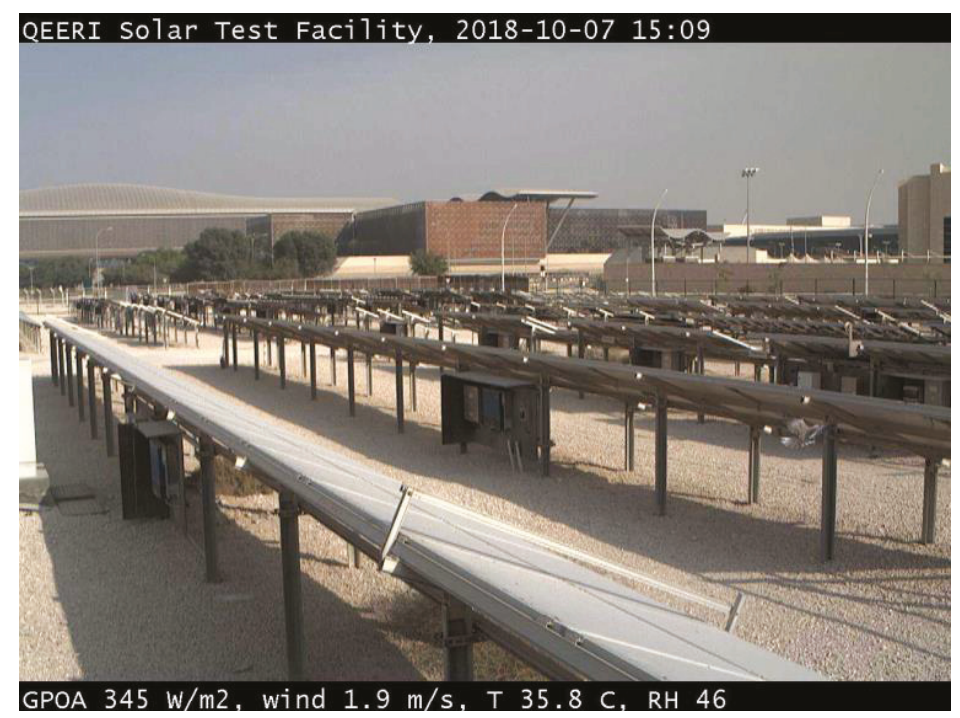

Figure 2. QEERI Outdoor Test facilities.

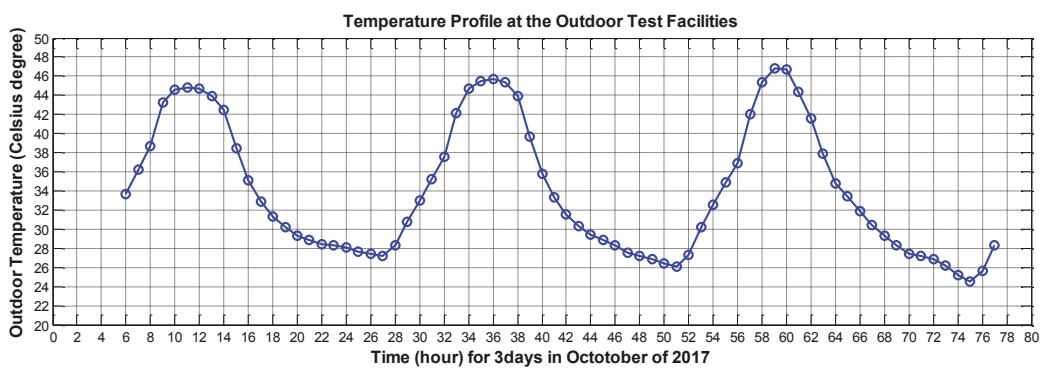

Figure 3. Three-day outdoor profile in October of 2017.

\subsection{House Thermal Model}

To mimic thermal dynamics of a house, an Air-Conditioned house with heat exchange diagram is shown in Figure 4 [32]. The model thermal dynamics related equations can be denoted as follows:

$$
\begin{gathered}
\frac{d T_{\text {indoor }}}{d t}=\frac{\dot{Q}_{d}-\dot{Q}_{e}}{C_{p} m} \\
\dot{Q}_{d}=\frac{T_{\text {outdoor }}-T_{\text {indoor }}}{R}
\end{gathered}
$$

By combining Equations (1) and (2), the house thermal dynamics are derived as follows:

$$
\frac{d T_{\text {indoor }}}{d t}=\frac{-1}{C_{p} m R} T_{\text {indoor }}+\frac{-1}{C_{p} m} \dot{Q}_{e}+\frac{1}{C_{p} m R} T_{a m b}
$$

The parameters in the above equations are defined in reference to the Table 1 of [32]. For simplicity, several assumptions need to be clarified to formulate the HVAC control problem. These assumptions are

- The AC system works only in cooling mode, which removes heat from the room; 
- The AC house is modelled as a first-order state-space physical system;

- Only heat transferred from the outdoors is considered;

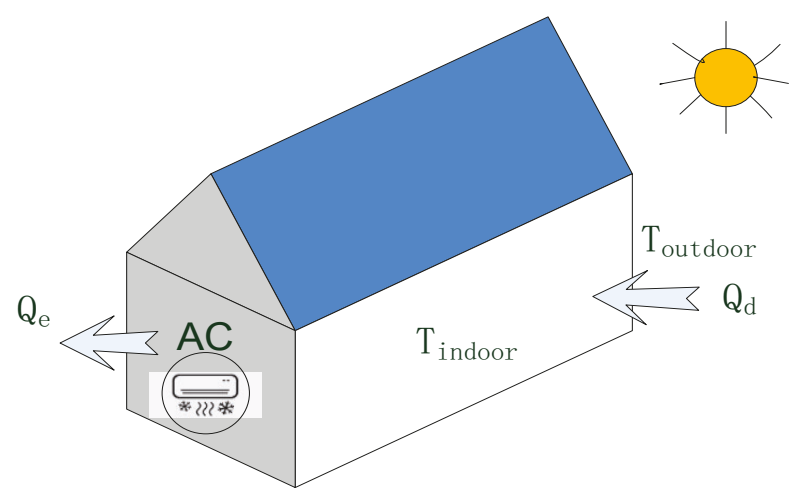

Figure 4. Thermal model of a house.

Remark 1. The assumption of only cooling mode fits Qatar's weather conditions because for most of the year (three hot seasons), the daytime outdoor temperature is over $25^{\circ} \mathrm{C}$, and only $A C$ cooling is needed. The last two assumptions are general for AC control and optimization studies, but it could be different in parameter setting for representing various application scenarios. For example, the indicator for extremely hot weather outdoor temperatures are considered as be time-variant in the studied house model.

Based on the assumptions mentioned above, the house thermal model can be reformulated as a state-space model structure shown in Figure 5.

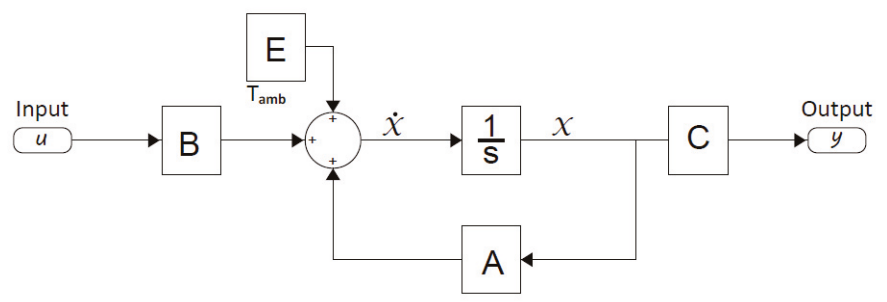

Figure 5. Block diagram representation of state space model.

The mathematical format of the state-space model shown in Figure 5 can be represented as follows:

$$
\begin{aligned}
\dot{x}(t) & =A x(t)+B u(t)+E \\
y(t) & =C x(t)
\end{aligned}
$$

In Equation (4), the variables and parameters have the following physical meaning: $x$ is the vector of the state, $\dot{x}$ is the corresponding time derivative, $u$ and $y$ is the input vector and the output vector, respectively, $A, B, C$, and $E$ is the corresponding state-space coefficient matrixes. Of note, $x$ represents 
the state $T_{\text {indoor }}$, which is measurable, and therefore $C=1$. If $P_{A C}$ denotes the rated power consumed by AC when the status is $\mathrm{ON}, u$ represents the On-Off control input $u=\dot{Q}_{e}=P_{A C}$, and it holds:

$$
u= \begin{cases}P_{A C} & t \in T_{o n} \\ 0 & t \in T_{o f f}\end{cases}
$$

By converting Equation (3) into the format of equations (4), the coefficients $A, B, E$ can be expressed as follows:

$$
\left\{\begin{array}{l}
A=\frac{-1}{C_{p} m R} \\
B=\frac{-1}{C_{p} m} \\
E=\frac{1}{C_{p} m R} T_{a m b}
\end{array}\right.
$$

Remark 2. In Equations (6), the term $E$ is a function of $T_{a m b}$, which means that if $T_{\text {amb }}$ is changes as a variable and is not constant, the state-space model of Equation (4) could be more complex. Because the dynamics in (4) will be changed as first-order state-space model with time-variant parameters. Therefore, the control input (shows in u) needs to adaptively accommodate the disturbance E due to time-varying outdoor temperature. Moreover, with a full consideration on both comfort level and On-Off switch weariness, multiple optimizations are needed to improve the control strategy to achieve an optimal solution for multiple objectives.

\section{Optimal On-Off Control for Time-Variant Outdoor Temperature}

In this section, an optimal AC On-off control methodology is presented for stabilizing the room temperature regardless of the outdoor temperature variations. The optimal control scheme can be divided into an offline phase and an online phase. The offline phase is to solve optimum solutions by corresponding solvers for multiple objectives, while the online phase is to regulate the indoor temperature by tuning the controller with the updated parameters from the offline optimization.

\subsection{Offline Multiple-Objective Optimization}

\subsubsection{Dynamics Subjected to On-Off Control}

Because the control variable $u$ in Equation (4) indicates On-Off binary variables, the solution of Equation (4) is different from the analytical solution of the standard state-space equation. The time-domain analytical solution can be written as follows:

$$
\left\{\begin{array}{l}
x_{1}(t)=\frac{B u_{o n}(t)+E}{-A}+\left(x_{1, \text { init }}+\frac{B u_{o n}(t)+E}{A}\right) e^{A t} \\
x_{2}(t)=\frac{B u_{o f f}(t)+E}{-A}+\left(x_{1, \text { end }}+\frac{B u_{o f f}(t)+E}{A}\right) e^{A(t-\alpha T)}
\end{array}\right.
$$

Figure 6 presents the physical meanings of the variables and parameters in by a diagram of oscillation period dynamics. Based on the temporal relationship shown in Figure 6, the following mathematics must hold:

$$
\left\{\begin{array}{l}
x_{1, \text { end }}=x_{2, \text { init }} \\
x_{1, \text { init }}=x_{2, \text { end }} \\
T=T_{o n}+T_{o f f} \\
T_{o n}=\alpha T
\end{array}\right.
$$


In addition, $x_{1, \text { init }}$ and $x_{1, \text { end }}$ can be derived from Equation (7) under $t=0, t=\alpha T$, and $t=T$. The solution is shown as follows:

$$
\left\{\begin{array}{l}
x_{1, \text { init }}=\frac{\frac{B u_{o n}+E}{-A}+\frac{B u_{o n}-B u_{o f f}}{-A} e^{A \cdot T_{o f f}}+\frac{B u_{o n}+E}{A} e^{A \cdot T}}{1-e^{A \cdot T}} \\
x_{1, \text { end }}=\frac{\frac{B u_{o n}+E}{-A}+\frac{B u_{o f f}+E}{A} e^{A \cdot T}+\frac{B u_{o n}-B u_{o f f}}{A} \cdot e^{A \cdot T_{o n}}}{1-e^{A \cdot T}}
\end{array}\right.
$$

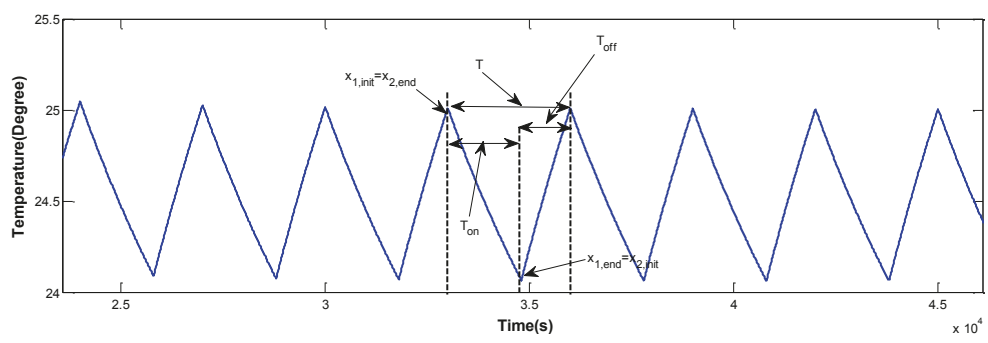

Figure 6. Diagram of oscillation period dynamics.

\subsubsection{Cost Function for Multiple-Objective Optimization}

Considering lowering the weariness cost due to AC On-Off switching while maintaining an adequate level of comfort in Coefficient of Performance (COP) value, the cost function can be defined as follows:

$$
\begin{aligned}
& J\left(T_{o n}, T_{o f f}\right)= \\
& \frac{Q \cdot \int_{0}^{T_{o n}}\left(x_{1}-x_{r e f}\right)^{2} d t Q \cdot \int_{T_{o n}}^{T_{o n}+T_{o f f}}\left(x_{1}-x_{r e f}\right)^{2} d t}{T_{o n}+T_{o f f}} \\
& +\frac{R J_{s w}}{T_{o n}+T_{o f f}}
\end{aligned}
$$

where $J$ denotes the cost of one period of oscillation in one cycle and $J_{s w}$ denotes the switching cost leading to weariness and is assumed to be constant. $Q$ and $R$ denote the weight coefficients of the COP cost and the switching cost. $x_{r e f}$ is the target indoor temperature. To convert this optimization as a convex optimization problem, the optimization variables of $T_{o n}, T_{\text {off }}$ can be converted into $T$ and $\alpha$, their boundary conditions holds:

$$
\left\{\begin{array}{l}
T \in[100,10000] \\
\alpha \in[0,1]
\end{array}\right.
$$

The optimization process leads to the following optimal solution:

$$
\left[T_{o p t}, \alpha_{o p t}\right]=\arg \min _{T, \alpha} J
$$

\subsubsection{Optimization Result Lookup Table Generation}

With the proposed cost function in (10) constrained by the boundary conditions in (11), an offline optimization algorithm is designed to generate the optimum parameters for online adaptive On-Off Control. The flow chart of the offline optimization is shown in Figure 7, and the details can be referred in the justification of Figure 3 in [32]. 


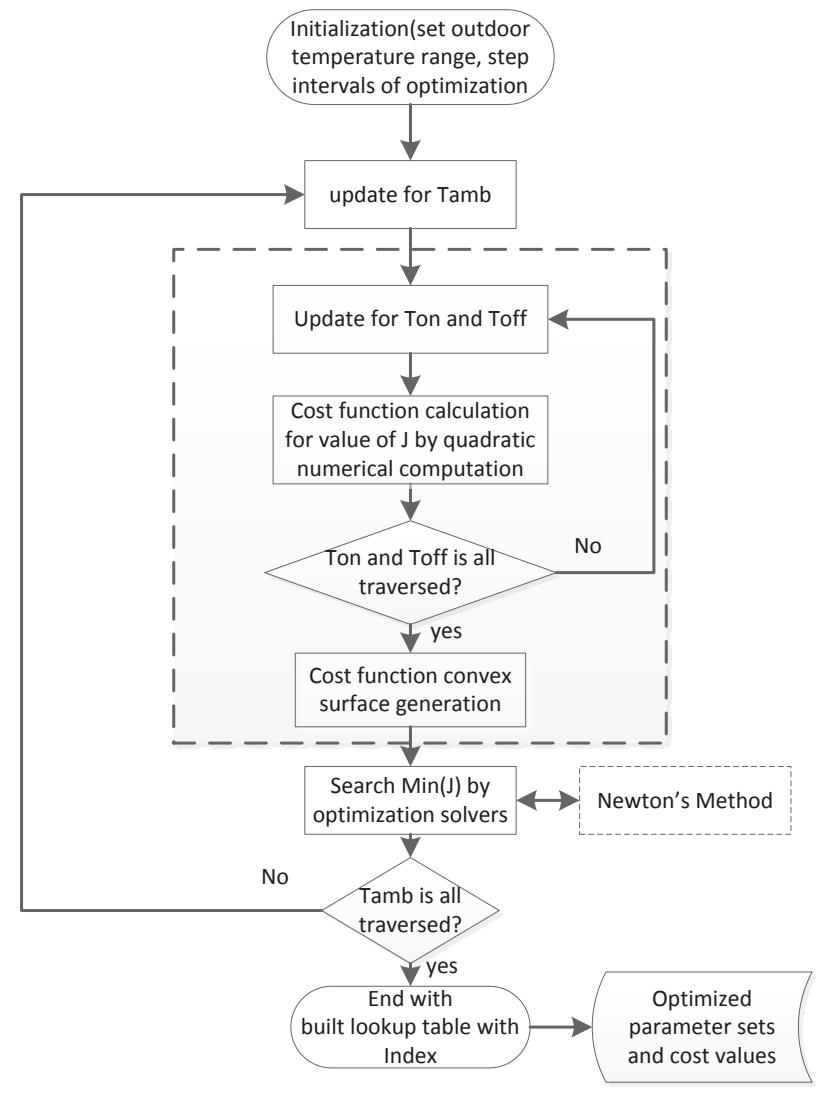

Figure 7. Flowchart of offline optimizations.

\subsection{Online Adaptive Control for Variable Outdoor Temperature}

By looking up the optimum parameters generated by offline optimizations, the online control scheme can adaptively accommodate outdoor temperature variations with a light computational load. The online control scheme is comprised of three parts: outdoor temperature profile discretion, online adaptive control, and Outdoor temperature prediction.

\subsubsection{Temperature Profile Discretion}

Normally, the outdoor temperature is recorded by one-hour intervals. However, if we analyze the temperature profile of Qatar, it shows slow variation properties. Thus, variation within one hour is not notable. Moreover, it is not necessary to use hourly outdoor weather data because more hour points in a day means more duplicate or similar optimization procedures are needed. Therefore, to simplify the proposed online control scheme, the one-hour temperature profile needs to be discretized as longer intervals such as 2-h interval or 4-h interval. To implement the discretion, some interpolation algorithms such as nearest neighbor interpolation and linear interpolation can be applied, Interpolation results for the example of a 3-day profile with different intervals are shown in Figure 8. 


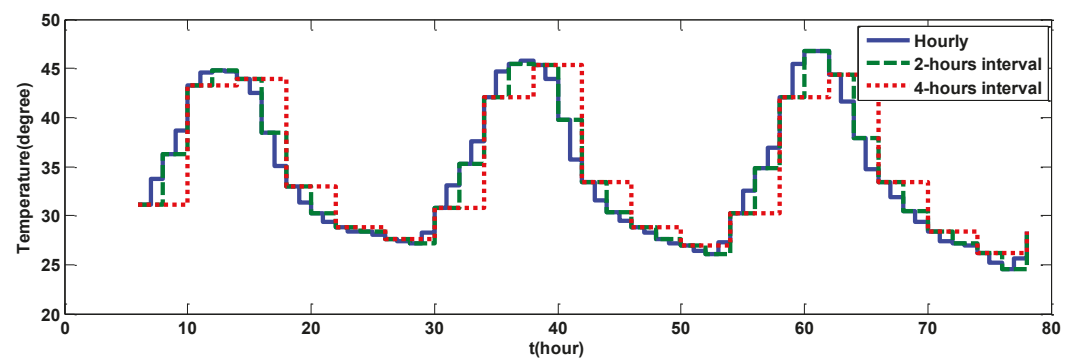

Figure 8. Temperature profile discretization.

\subsubsection{Online Adaptive Control Scheme}

After the outdoor temperature profile is discretized, the online adaptive control scheme is employed to stabilize the indoor temperature and accommodate the temperature variations adaptively. Figure 9 shows a flowchart of the proposed online AC control scheme. Firstly, the normal On-Off control is initiated to enable the AC system to converge into the target temperature range. Secondly, two cascaded control loops are executed. The outer control loop works to tune the On-Off controller parameters with the optimized solution. The inner loop works on the search for optimum parameter set by the index in a lookup table, which is previously generated online, in one horizon period. Finally, the scheme ends if all the time horizon of a full day is scanned.

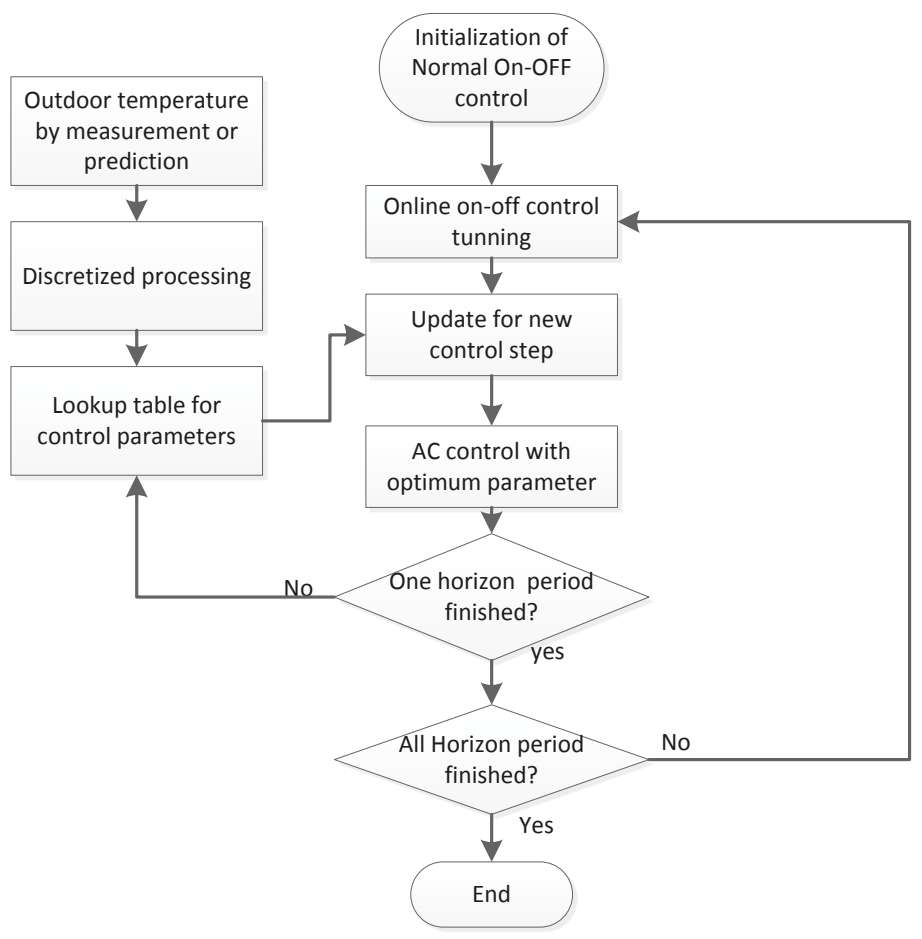

Figure 9. Online AC control flowchart. 


\subsubsection{Outdoor Temperature Prediction}

Temperature prediction is important for AC optimization control because the optimization objectives cover a period of control step processes. In the studied optimal control scenarios, at least $2 \mathrm{~h}$ ahead, prediction is mandatory for the online control process. With the consideration on a trade-off between prediction accuracy and computational complexities, a typical Elman Neural Network (NN) model shown in Figure 10 is employed as an estimator for outdoor temperature prediction.

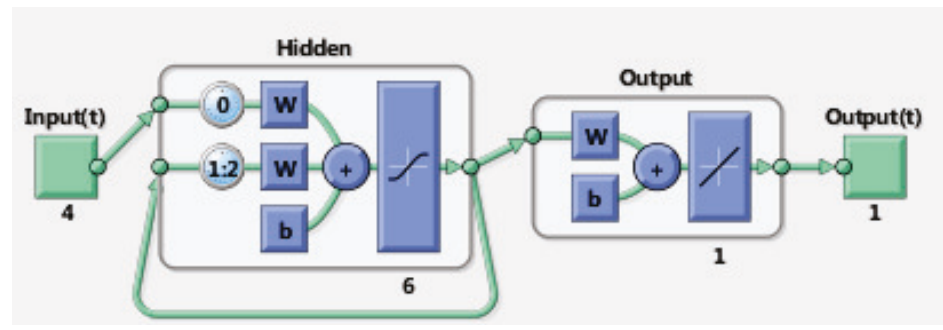

Figure 10. Proposed Elman Neural Network Model structure.

To represent the prediction process, the outdoor temperature series can be denoted as follows:

$$
T_{a m b}(i),\left(i=1, \ldots, N_{P}\right)
$$

Due to the temporal relativity of the outdoor temperature in the time domain, the outdoor temperature variation process can be denoted as follows:

$$
T_{a m b}(k)=F\left(T_{a m b}(k-1), \ldots, T_{a m b}(k-1-M)\right)
$$

where $T_{a m b}(k)$ denotes the outdoor temperature at current instant $k, F($.$) is the function between$ $T_{a m b}(k)$ and the temperature series at previous instants $k-1, \ldots, k-1-M$. For temperature time series prediction, a mapping model is needed to build firstly for approximating the mapping function $F($.$) . In this paper, a two-layer (second order) Elman neural network model in Figure 10$ is proposed to approximate the mapping function $F($.$) . The mathematical representation of Elman network can be$ denoted as follows:

$$
\left\{\begin{array}{c}
h_{t}=\sigma_{h}\left(W_{h} x_{t}+\sum_{i=1}^{N_{d}} W_{t, i} h_{t-i}+b_{h}\right) \\
y_{t}=\sigma_{y}\left(W_{y} h_{t}+b_{y}\right)
\end{array}\right.
$$

where $W_{h}, W_{t, i}\left(i=1, \ldots, N_{d}\right), W_{y}$ are weight coefficients, $b_{h}, b_{y}$ are offset coefficients, $\sigma_{h}$ and $\sigma_{y}$ are activation functions for hidden layer output and output layer respectively. For the outdoor temperature prediction, different prediction windows configurations exist. Depending on the availability of data series length, the input data vector length $M$ could be $2,3, \ldots, 24$. Thus, the cascaded prediction equations can be represented as follows:

$$
\left\{\begin{array}{c}
\hat{T}_{a m b}(k)=N N\left(T_{a m b}(k-1),\right. \\
\left.\ldots, T_{a m b}(k-1-M)\right) \\
\vdots \\
\hat{T}_{a m b}\left(k+N_{L}\right)=N N\left(T_{a m b}(k-1),\right. \\
\left.\ldots, T_{a m b}(k-1-M)\right)
\end{array}\right.
$$


Here, $\hat{T}_{a m b}(k)$ denotes the predicted or estimated value by the Elman NN model, $N_{L}$ denotes the prediction step number based on $M$ existing temperature serial data. To get an effective and high-accuracy estimation model, the Elman NN offline training based on a set of sample data is essential and needs to be done in advance. Thus, the training problems of the Elman NN can be denoted as follows: Given a set of sample dataset as

$$
\text { TrainingSet }\left(T_{a m b}(\text { in }), T_{a m b}(\text { out })\right)
$$

By selected training algorithms, the Elman NN model parameters such as $W_{h}, W_{t, i}\left(i=1, \ldots, N_{d}\right)$, $W_{y}$ and weight coefficients, $b_{h}, b_{y}$ can be determined based on the following criteria:

$$
\begin{aligned}
& \exists \operatorname{parameterset}(W, b) \\
& T_{a m b}(\text { out }) \doteq \hat{T}_{a m b}(\text { out })=N N\left(T_{a m b}(\text { in })\right)
\end{aligned}
$$

\section{Validation Results}

To validate the proposed On-Off control and optimization scheme, the studied house model parameters are referred from [13], and the AC power is changed as $1500 \mathrm{~W}$ but not $300 \mathrm{~W}$ because It is more reasonable due to the hot climate in Qatar. Moreover, to the best of our knowledge, the HVAC facilities in Qatar is prone to easily degrading and failing and normally need a high maintenance cost. Considering the fact that the AC weariness under hot climate is more serious, the weight factors of $\mathrm{Q}$ in (10) are reduced to reflect the factor of cost impact. The updated parameters are shown in Table 1.

Based on the AC parameter model list in Table 1, the proposed control and optimization scheme is demonstrated, which includes offline optimization and online AC control performance, in different cooling scenarios.

Table 1. Parameters values of the thermal model and optimization.

\begin{tabular}{ccccccccc}
\hline Parameter & A & B & E & R $\left({ }^{\circ} \mathrm{C} / W\right)$ & $J_{S W}$ & $\mathbf{C p}\left(\mathbf{J} / \mathbf{K g}{ }^{\circ} \mathrm{C}\right)$ & $\mathbf{m}(\mathbf{k g})$ & $\mathbf{Q}$ \\
\hline Value & $-2.00123 \times 10^{-4}$ & $4.4028 \times 10^{-6}$ & 0.002 & 0.022 & 2 & 1005 & 222 & 300 \\
\hline
\end{tabular}

\subsection{Offline Optimization Results}

To verify the offline optimization performance, a simplified AC cooling scenario is assumed for comparison in a quantitative way. In this cooling scenario, the indoor temperature is expected to be $24{ }^{\circ} \mathrm{C}$ by different controller parameter settings with the outdoor temperature set as $34{ }^{\circ} \mathrm{C}$. For comparison, the temperature profiles subjected to three types of controller settings are shown in Figure 11, and the corresponding COP values are compared. Figure 11 shows that the COP value of the proposed optimum control is the lowest at $J=1.5416$ among optimization and non-optimization scenarios. Based on the cost value comparison, the optimum case has lower cost value, which means that it achieves better multiple-objective optimizations than other control scenarios. The cost-saving performance among the three cooling scenarios are presented in Table 2. As shown in Table 2, 30.16\% can be saved from the case under outdoor temperature $29^{\circ} \mathrm{C}$ while $55.45 \%$ can be saved from the case under outdoor temperature $38^{\circ} \mathrm{C}$ by comparing with the optimum case. 
Table 2. Performance comparison by Cost-saving.

\begin{tabular}{ccccc}
\hline AC Cooling Control Strategies & Initial House Temperature & Period & Cost Value & Cost Saving \\
\hline Case1 & 38 & 1595 & 3.4606 & $55.45 \%$ \\
Case2 & 29 & 300 & 2.2074 & $30.16 \%$ \\
Optimum Case & 34 & 595 & 1.5416 & N/A \\
\hline
\end{tabular}

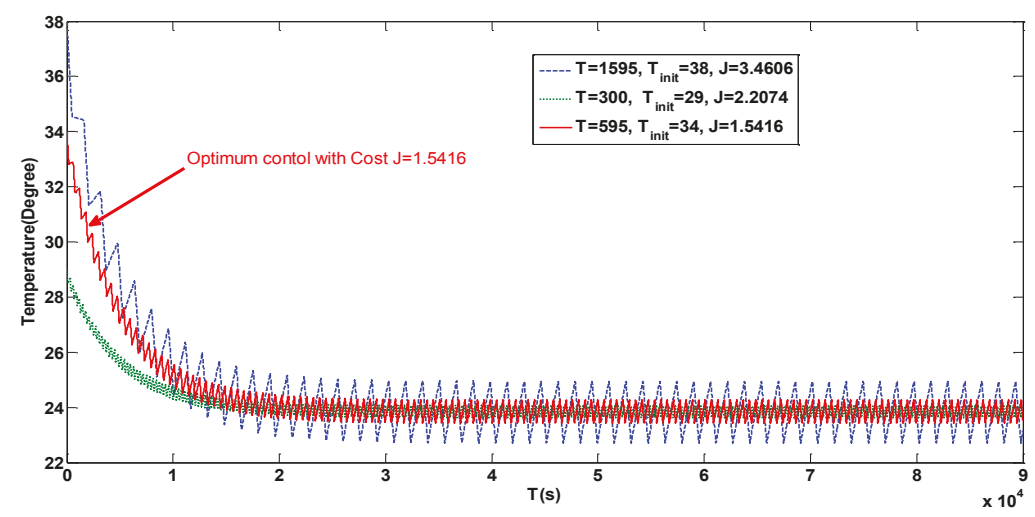

Figure 11. Cooling control profile comparison for different controller parameter settings.

To verify the effectiveness of offline optimization under different ambient temperature scenarios, a set of offline optimizations subjected to outdoor temperature range between $30{ }^{\circ} \mathrm{C}$ and $44{ }^{\circ} \mathrm{C}$ was performed. The target indoor temperature is expected to $24^{\circ} \mathrm{C}$. The offline optimization parameters are shown in Table 3, and the optimization convex surface results are shown in Figure 12, which presents eight subfigures for eight temperature profiles with $2{ }^{\circ} \mathrm{C}$ intervals. As can be seen from Figure 12 , all the optimization surfaces are convex, which means that the global optimum solution for the studied optimization case exists and only one solution exists and is unique. Due to the impact of the ambient temperature, the optimization surface shapes and cost values vary in a large range. As an input for the online adaptive control, the corresponding optimization control parameters are also generated in a list of a lookup table.

Table 3. Optimization control parameters.

\begin{tabular}{cccc}
\hline Outdoor Temperature & Minimum Cost $J$ & Period $(\mathrm{T})$ & Duty Ratio $(\alpha)$ \\
\hline 28 & 0.8585 & 1585 & 0.1090 \\
30 & 0.5927 & 2400 & 0.2780 \\
32 & 2.1917 & 595 & 0.2575 \\
34 & 1.5416 & 595 & 0.3070 \\
36 & 1.4318 & 595 & 0.3565 \\
38 & 1.9049 & 595 & 0.4060 \\
40 & 3.0049 & 595 & 0.4555 \\
42 & 3.8368 & 595 & 0.5545 \\
44 & 3.0705 & 595 & 0.6040 \\
\hline
\end{tabular}



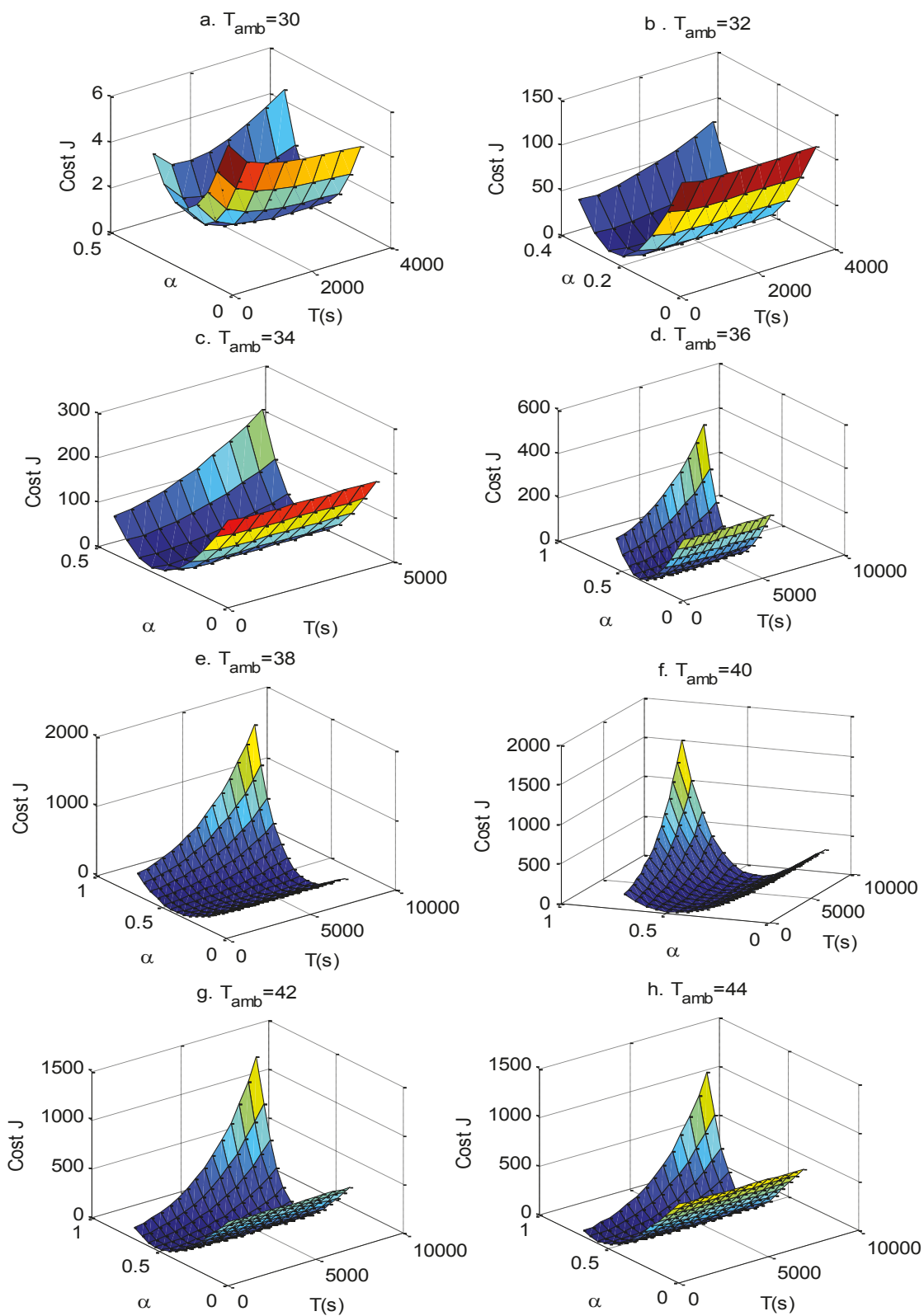

Figure 12. Offline optimization results under different outdoor temperatures.

\subsection{Outdoor Temperature Prediction Results}

To validate the effectiveness of the proposed Elman NN based outdoor temperature prediction, the 3-day outdoor profile shown in Figure 3 was used for Elman NN model training and test. The 3-day temperature has 72 points of hourly temperature in total. The prediction window is set as 4 so the total data point number for NN test set is $72-4=68$, and it is divided into two groups, 34 
points each. After selecting the proper values for the Elman Training algorithm, we can get the training results in Figure 13a,b. From Figure 13a we can see that the Elman model output value is very close to the true value by the training procedure regression. From Figure $13 \mathrm{~b}$ we can see that the error is less than 0.6 degrees, which means the model regression accuracy is very high. To test the generalization ability of the proposed Elman NN model, the other group of 34 points data is used for validation, and the results are shown in Figure 13c,d. From Figure 13c, we can see that the estimation value is also very close to the true value. From Figure $13 \mathrm{~d}$ we can see that the estimation error is within the acceptable range (less than 2 degrees) although it is a little higher than training error. Based on the training regression and test validation results, we can conclude that the Elman NN model can generate an effective prediction of outdoor temperatures.

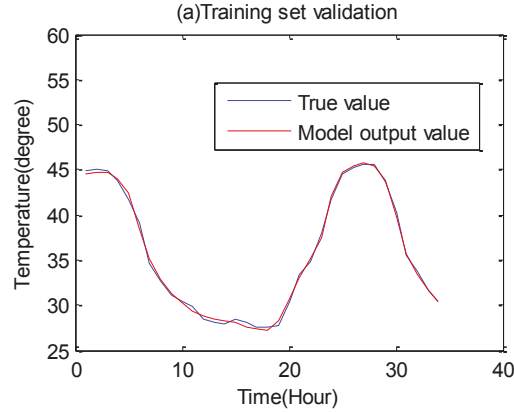

(c) Test Set Validation

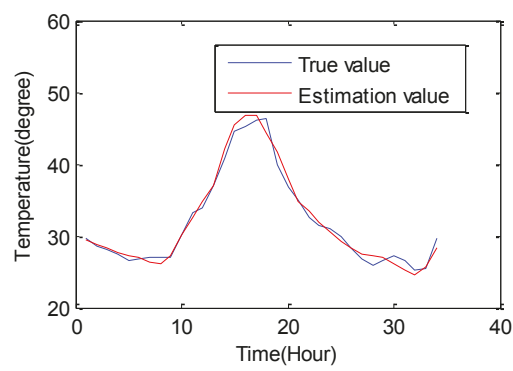

(b) Training Error

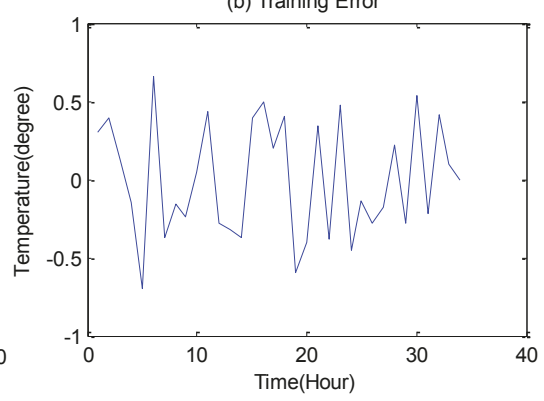

(d) Validation Error

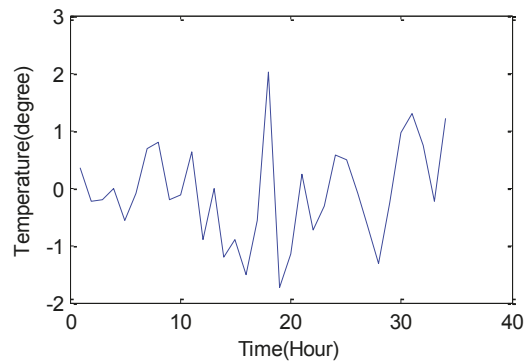

Figure 13. Outdoor Temperature prediction results.

\subsection{Online Adaptive Control Results}

To validate the proposed online adaptive control scheme for the AC cooling under Qatar weather conditions, daily indoor temperature control scenarios are considered in the this paper. In general, the comfort indoor temperature is set as $25^{\circ} \mathrm{C}$ with an acceptable variation range of $2{ }^{\circ} \mathrm{C}$. Moreover, due to the slow time-variant characteristics of the outdoor temperature, a two-hour interval is a reasonable time zone to evaluate the weather temperature in existing weather measurement applications.

\subsubsection{Case 1- One-Day Typical Case in Doha of Qatar}

To verify the effectiveness of the proposed scheme, the second day outdoor temperature profile in Figure 3, which is a typical day with large temperature variations, was selected for the data analysis. To compare the performance between the proposed optimal control and the PWM control mentioned in the literature review, comparative experiments are also conducted to re-implement the PWM control in the same house model as the proposed optimal control under same Qatar outdoor condition. The implemented PWM control has an adjustable pulse-width based PID control structure. In this PWM 
control, the PWM period is constant while the duty ratio/pulse width is adjusted as a continuous control variable to avoid the fast switching in the On-Off control. In addition, a PID module is added to make the tracking control to converge in a smooth way with acceptable oscillation error. The PWM control diagram is shown in Figure 14, and the period time of the PWM pulse is set as $500 \mathrm{~s}$, which is similar as the minimum period of the optimal control. The detailed AC control process variables such as AC consumption power, period time and duty ratio are shown in Figure 15, and the temperature control results are presented in Figure 16.

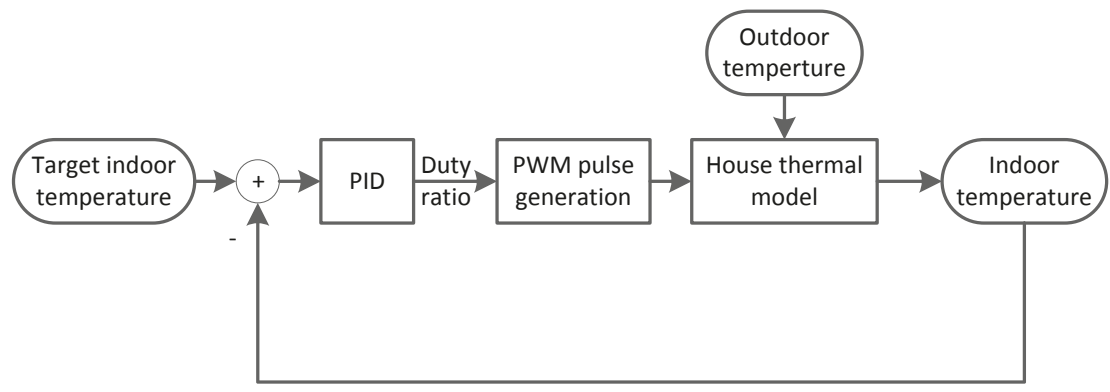

Figure 14. PWM control diagram.
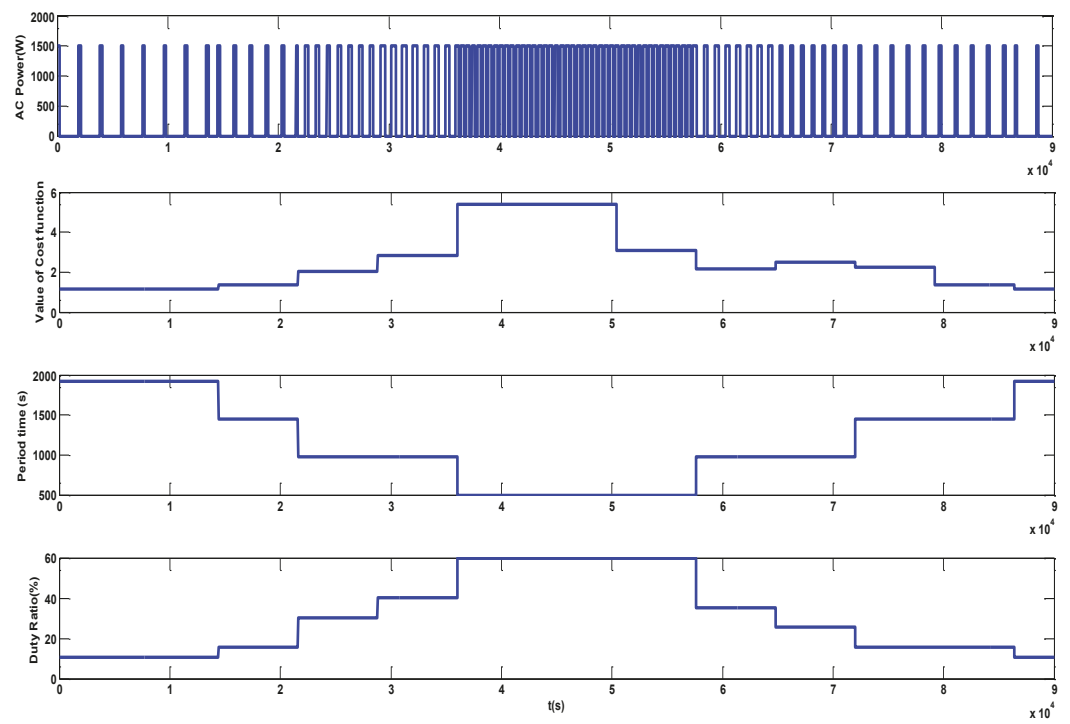

Figure 15. Online optimal control variables and parameters.

Figure 15 shows a modulated pulse output of the AC control power, cost value, period time, and duty ratios respectively. As can be seen from the plot of the AC power output, the AC consumption power is fixed at rated $1500 \mathrm{~W}$ while the pulse width is variable due to different cooling loads. The cost value plot also changes with time and shows a peak when the corresponding pulse width is the shortest. This means that a high outdoor temperature generates a heavier cooling load and more frequent AC On-Off switching actions and thus, the AC cooling overall cost is higher. From the period plot, we can see that the period time shows a valley point which it corresponds the closet pulse. From the duty ratio plot we can see that the duty ratio also reaches a peak point when the heavy load is 
highest. In a summary of all the plots, the results demonstrate that the control process can track the ambient temperature change and respond with appropriate control action in a real-time manner.

Figure 16 shows that the optimization AC cooling results under a typical day in summer. From the indoor temperature profile, we can see that the control scheme is effective to stabilize the indoor temperature at $25{ }^{\circ} \mathrm{C}$ and be within the temperature fluctuation range although the curve is a little offset from the target curve at some instances. From the outdoor temperature profile, we can see that the outdoor temperature changes from $27^{\circ} \mathrm{C}$ to $46^{\circ} \mathrm{C}$ at a gap almost $20^{\circ} \mathrm{C}$, but the control process can adaptively accommodate these variations by adjusting the control optimization parameters. Therefore, it is demonstrated that the proposed scheme applies for a typical large-variation scenario.

In addition, Figure 16 also shows the temperature profile comparison between the PWM control and the proposed optimal control. Both the PWM control and the optimal control can stabilize the target indoor temperature at $25^{\circ} \mathrm{C}$, although the outdoor temperature changes in a large daily range of $20^{\circ} \mathrm{C}$. The PWM control oscillates at the transition time of the outdoor temperature's rapid change while the proposed optimal control can accommodate this rapid transition in a smoother way. Moreover, the rapid transition causes the PWM control taking a large range of oscillation (between $23^{\circ} \mathrm{C}$ to $27^{\circ} \mathrm{C}$ ) to converge into the target indoor temperature $25^{\circ} \mathrm{C}$, while the optimal control can stabilize at a smaller range (between $24{ }^{\circ} \mathrm{C}$ to $26^{\circ} \mathrm{C}$ ). Through this comparison, it can be demonstrated that the optimal control has a better control performance than the PWM control in both stabilization accuracy and transition convergence speed.

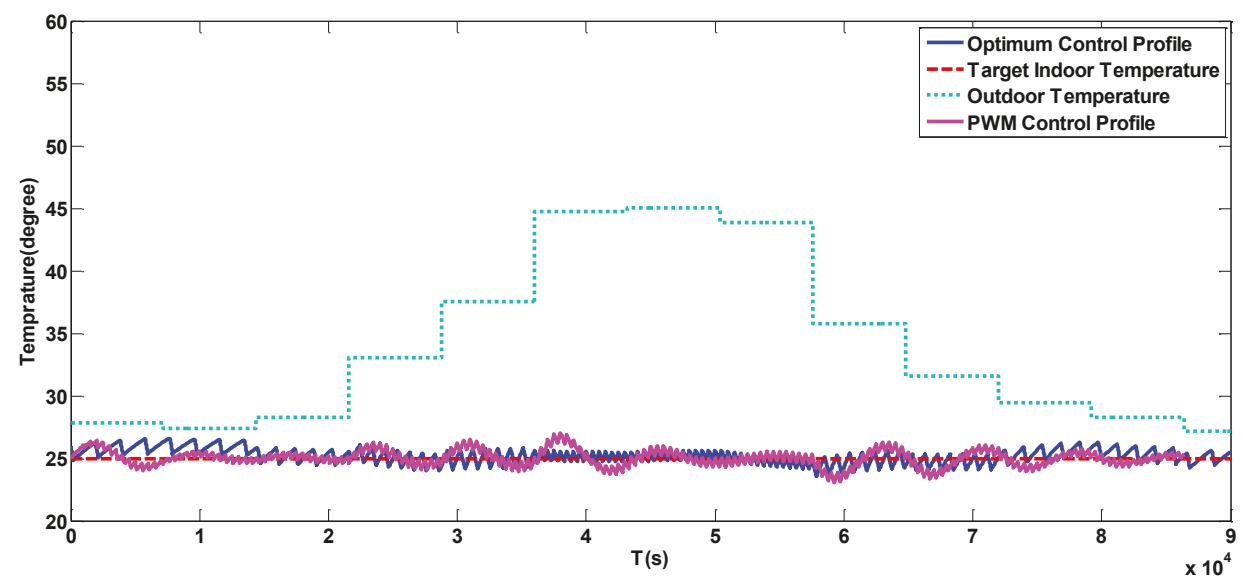

Figure 16. Temperature control profile in a typical day with large temperature variations.

By investigating more details about the control variables of the control approaches, the power profile for the optimal control and the PWM control is shown in Figure 17a,b, respectively. The duty ratio profile of the PWM control is shown in Figure 17c. By comparing Figure 17a,b, it can be concluded that the optimal control has less pulses than the PWM control, and the cost due to switch is obviously reduced. From Figure 17c, we can see that the duty ratio is a continuous variable which changes according to the outdoor temperature profile, which means that the PWM control can smooth the transition in a better way that the conventional On-Off control, although it is not the optimum comparing to the proposed optimum control.

Based on the fixed period value (500 s) and corresponding duty ratio sampling in each time duration, the cost in terms of COP in (10) is calculated, and the cost comparison in the corresponding time period is shown in Table 4. From the table, we can see the proposed optimal control has less cost than the PWM control in each time duration, which means the proposed optimal control has a better 
control performance for multiple objectives, which consider both Coefficient of Performance, and AC compressor weariness due to On-Off switching.
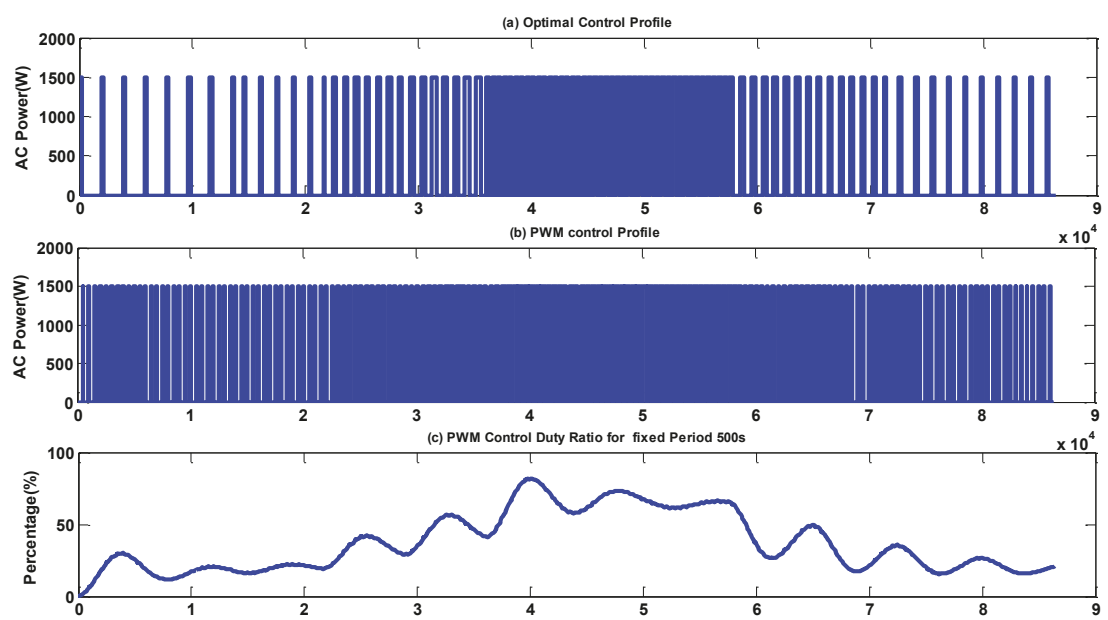

Figure 17. PWM control process variables.

Table 4. Cost comparison between PWM control and the proposed optimal control.

\begin{tabular}{cccc}
\hline Time Duration & Duty Ratio $\boldsymbol{\alpha}(\mathbf{\%})$ & Cost of PWM Control & Cost of Optimal Control \\
\hline 0 AM-2 AM & 1 & 14.85 & 1.1420 \\
2 AM-4 AM & 11.76 & 7.77 & 1.1420 \\
4 AM-6 AM & 16.02 & 1.75 & 1.3436 \\
6 AM-8 AM & 37.90 & 4.59 & 2.0533 \\
8 AM-10 AM & 45.61 & 11.06 & 2.8456 \\
10 AM-12 AM & 64.68 & 6.26 & 5.3890 \\
12 AM-2 PM & 66.02 & 14.67 & 5.3890 \\
2 PM-4 PM & 61.07 & 112.89 & 3.0836 \\
4 PM-6 PM & 33.24 & 56.58 & 2.1577 \\
6 PM-8 PM & 19.01 & 10.07 & 2.5015 \\
8 PM-10 PM & 16.43 & 12.33 & 2.2423 \\
10 PM-12 PM & 17.02 & 29.89 & 1.3436 \\
\hline
\end{tabular}

\subsubsection{Case 2- Cases through Three Hot Seasons in Doha of Qatar}

To demonstrate the validity of the proposed scheme under three hot seasons in Qatar, complete yearly outdoor temperature data taken from our measurements, from June of 2018 to June of 2019, were used. As can been from Figure 18, the outdoor temperature is fully or partially over 25 degrees except from December to March. In other words, spring, summer, and autumn are all hot seasons in Qatar. Thus, AC cooling is essential and the proposed optimization scheme can be applied. Considering this diversity of the Qatar desert climate, profiles of three typical days in each season, which include 2019/8/6, 2018/11/6,2019/5/6, were selected to validate the proposed scheme for the three seasons of summer, autumn, and Spring, respectively.

As the hottest month of the year in Qatar, August is a typical for AC cooling scenarios due to its high outdoor temperature and resulting cooling load. The validation results for the selected summer day in August are presented in Figure 19. The outdoor temperature profile shows that it varies from $32{ }^{\circ} \mathrm{C}$ to $46^{\circ} \mathrm{C}$ in one summer day, which means that the gap between peak point and valley point is large and the AC needs to adaptively accommodate the corresponding time-variant cooling load. 
From the optimum temperature control profile, we can see that the oscillation period time at the peak point of the outdoor temperature is the shortest because it needs to generate the highest cooling effort. With 2-h interval time zone moving, the duty ratio and period time are both set as the best optimized value by the offline optimizations. Although the profile is not always close to the central of the target temperature of $25^{\circ} \mathrm{C}$, the temperature variation range is within $1^{\circ} \mathrm{C}$. This result demonstrates that the proposed AC control scheme can achieve the optimal cooling effect within the comfort level need of AC cooling.

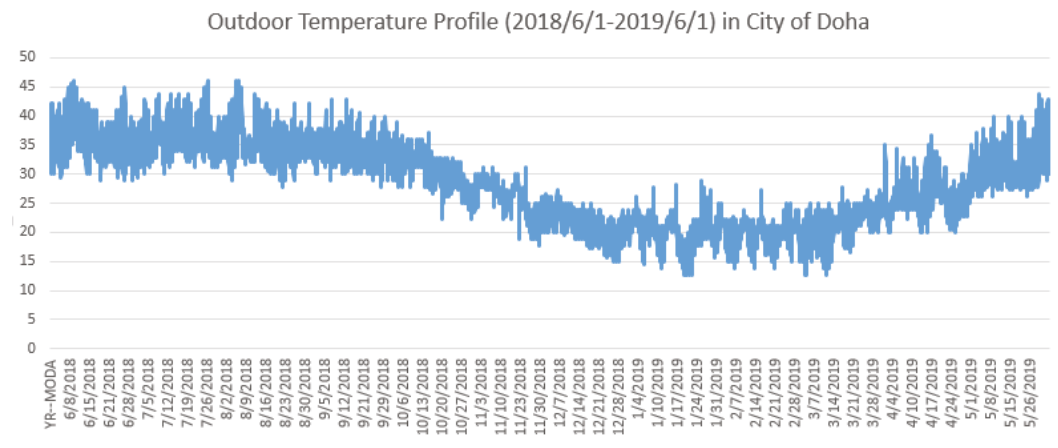

Figure 18. Yearly Outdoor Temperature Profile from June of 2018 to June of 2019.

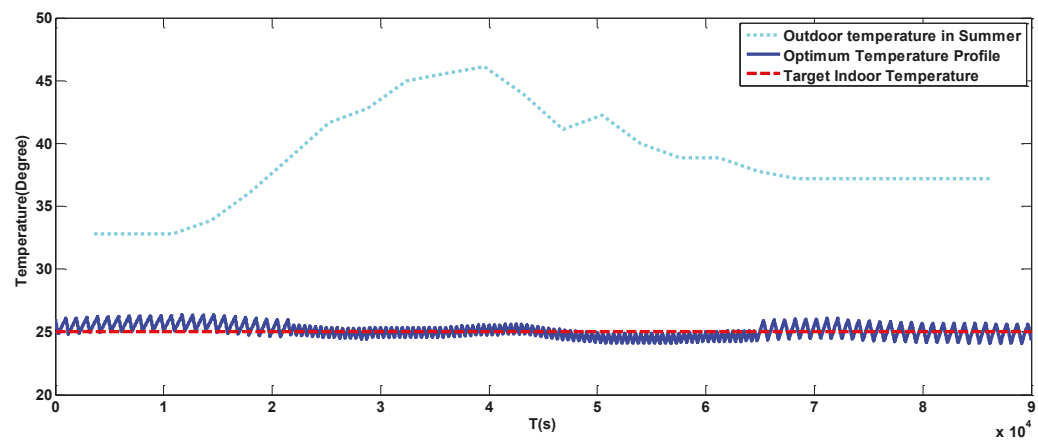

Figure 19. Optimal control for typical summer day in Aug of 2018.

The validation result for a typical autumn day, in November of 2018, is presented in Figure 20. As shown in the outdoor temperature profile, it changes from $27^{\circ} \mathrm{C}$ to $29^{\circ} \mathrm{C}$ in one autumn season day. This means that the gap during the same day is very small, and also, the cooling load is lighter compared to summer days. From the optimum temperature control profile, we can see that the oscillation period time is longer than the summer case because of the lighter cooling load. The control profile is always kept to approach the target $25^{\circ} \mathrm{C}$ with $1{ }^{\circ} \mathrm{C}$ variation range. These results demonstrate the effectiveness of the proposed scheme during autumn days in Qatar.

The validation result for a typical spring day, in May of 2019, is shown in Figure 21. The outdoor temperature changes from $27^{\circ} \mathrm{C}$ to $37^{\circ} \mathrm{C}$ in one spring season day. This means the gap during the same day is smaller compared to summer days, and also the cooling load is changing quickly within same day. From the optimum temperature control profile, we can see that the oscillation period time is set with a very different value range because the cooling load is changing with large value range. The control profile is a little off the target $25^{\circ} \mathrm{C}$ due to the large variations of AC control parameter 
tuning, but it still satisfy the comfort level by approaching the target $25^{\circ} \mathrm{C}$ within $2{ }^{\circ} \mathrm{C}$ variation range. These results demonstrate the effectiveness of the proposed scheme under spring season days in Qatar.

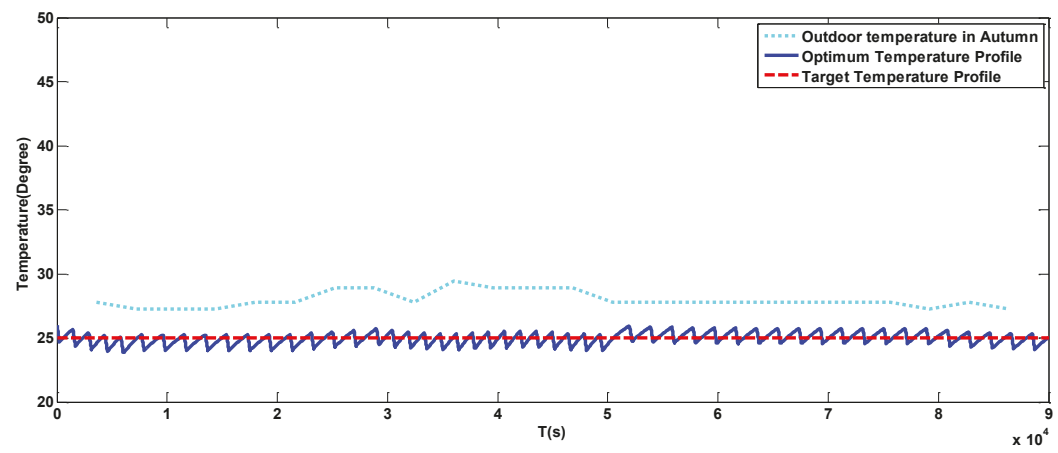

Figure 20. Optimal control for typical autumn day in November 2018.

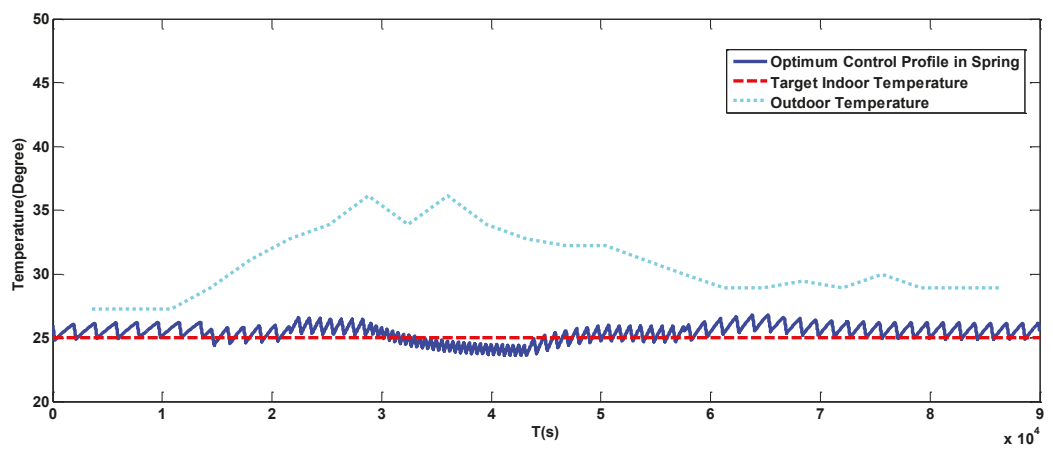

Figure 21. Optimal control for typical spring day in May of 2019.

Remark 3. Because of the significance of Qatar's extremely hot weather conditions, AC Optimization and control in this region is very different from other regions. Particular challenges such as large daily temperature peaks, heavy cooling load through the year, AC Group Consumption peaks, and local resident electricity consumption behaviours, also need to be considered. To address these challenges, effective optimization solutions are supposed to be applied for multiple objectives but not only COP and switching cost. Therefore, the needed optimization process will be more complicated, and accordingly, more advanced optimization theories such as optimization feasible zone, and tools including cloud-computing and super-computing are needed for future studies.

\section{Conclusions}

This paper presents a new optimal control strategy that improves existing conventional On-Off control for air conditioning under the harsh desert climate of Qatar. The optimum AC control is achieved based on an integration between off-line optimization and online adaptive control. The optimization process involves the use of a multiple objectives methodology driven by a cost function for maximum energy efficiency (COP) and minimized compressor weariness. This control scheme offers a practical trade-off between online and off-line optimization methods where the multiple objective optimization is achieved without excessive computational needs. By simulating several AC cooling scenarios, based on our experimental weather data from the outdoor test facility at Qatar Environment and Energy Research Institute, the resulting performance is very promising. More 
optimization objectives and factors such as sizing AC unit numbers, AC power fluctuations and impact to grid will be considered for future work.

Author Contributions: Conceptualization: M.A.-A., Z.C., S.A., Y.R.; Methodology, Validation, Data Curation: M.A.-A., Z.C.; Writing-Original Draft Preparation: M.A.-A., Z.C.; Writing-Review and Editing: M.A.-A., Z.C., S.A., Y.R. All authors have read and agreed to the published version of the manuscript.

Funding: This research received no external funding.

Acknowledgments: The authors thank the QEERI Outdoor Facility Team (Energy Center), particularly Dr. Benjamin Figgis, for the local weather data and technical support.

Conflicts of Interest: The authors declare no conflict of interest.

\section{References}

1. Shaaban, K.; Muley, D. Investigation of weather impacts on pedestrian volumes. Transp. Res. Procedia 2016, 14, 115-122. [CrossRef]

2. Guo, B.; Javed, W.; Figgis, B.; Mirza, T. Effect of dust and weather conditions on photovoltaic performance in Doha, Qatar. In Proceedings of the IEEE 2015 First Workshop on Smart Grid and Renewable Energy (SGRE), Doha, Qatar, 22-23 March 2015; pp. 1-6.

3. Perez-Astudillo, D.; Bachour, D. Variability of measured global horizontal irradiation throughout Qatar. Solar Energy 2015, 119, 169-178. [CrossRef]

4. Touati, A.; Hussain, S.J.; Touati, F.; Bouallegue, A. Effect of atmospheric turbulence on hybrid FSO/RF link availability under Qatar harsh climate. Int. J. Electron. Commun. Eng. 2015, 9, 902-908.

5. Sim, L.F. Numerical modelling of a solar thermal cooling system under arid weather conditions. Renew. Energy 2014, 67, 186-191. [CrossRef]

6. Ayoub, N.; Musharavati, F.; Pokharel, S.; Gabbar, H.A. Energy consumption and conservation practices in Qatar-A case study of a hotel building. Energy Build. 2014, 84, 55-69. [CrossRef]

7. Touati, F.; Al-Hitmi, M.; Chowdhury, N.A.; Hamad, J.A.; Gonzales, A.J.S.P. Investigation of solar PV performance under Doha weather using a customized measurement and monitoring system. Renew. Energy 2016, 89, 564-577. [CrossRef]

8. Sofotasiou, P.; Hughes, B.R.; Calautit, J.K. Qatar 2022: Facing the FIFA World Cup climatic and legacy challenges. Sustain. Cities Soc. 2015, 14, 16-30. [CrossRef]

9. Shaaban, K.; Muley, D.; Elnashar, D. Evaluating the effect of seasonal variations on walking behaviour in a hot weather country using logistic regression. Int. J. Urban Sci. 2018, 22, 382-391. [CrossRef]

10. Saffouri, F.; Bayram, I.S.; Koç, M. Quantifying the cost of cooling in qatar. In Proceedings of the 2017 9th IEEE-GCC Conference and Exhibition (GCCCE), Manama, Bahrain, 8-11 May 2017; pp. 1-9.

11. Teather, K.; Hogan, N.; Critchley, K.; Gibson, M.; Craig, S.; Hill, J. Examining the links between air quality, climate change and respiratory health in Qatar. Avicenna 2013, 2013, 9. [CrossRef]

12. Kassas, M. Modeling and simulation of residential HVAC systems energy consumption. Procedia Comput. Sci. 2015, 52, 754-763. [CrossRef]

13. Deng, H.; Larsen, L.; Stoustrup, J.; Rasmussen, H. A novel method for control of systems with costs related to switching: Applications to air-condition systems. In Proceedings of the IEEE 2009 European Control Conference (ECC), Budapest, Hungary, 23-26 August 2009; pp. 1148-1154.

14. Godina, R.; Rodrigues, E.M.; Pouresmaeil, E.; Catalão, J.P. Home HVAC energy management and optimization with model predictive control. In Proceedings of the 2017 IEEE International Conference on Environment and Electrical Engineering and 2017 IEEE Industrial and Commercial Power Systems Europe (EEEIC/I\&CPS Europe), Milan, Italy, 6-9 June 2017; pp. 1-5.

15. Di Felice, M.; Piroddi, L.; Leva, A.; Boer, A. Adaptive temperature control of a household refrigerator. In Proceedings of the IEEE 2009 American Control Conference, St. Louis, MO, USA, 10-12 June 2009; pp. 889-894.

16. Martínez-Rosas, E.; Vasquez-Medrano, R.; Flores-Tlacuahuac, A. Modeling and simulation of lithium-ion batteries. Comput. Chem. Eng. 2011, 35, 1937-1948. [CrossRef]

17. Dong, J.; Olama, M.; Kuruganti, T.; Nutaro, J.; Winstead, C.; Xue, Y.; Melin, A. Model predictive control of building on/off HVAC systems to compensate fluctuations in solar power generation. In Proceedings 
of the 2018 9th IEEE International Symposium on Power Electronics for Distributed Generation Systems (PEDG), Charlotte, NC, USA, 25-28 June 2018; pp. 1-5.

18. Rehrl, J.; Horn, M. Temperature control for HVAC systems based on exact linearization and model predictive control. In Proceedings of the 2011 IEEE International Conference on Control Applications (CCA), Denver, CO, USA, 28-30 September 2011; pp. 1119-1124.

19. Huang, G. Model predictive control of VAV zone thermal systems concerning bi-linearity and gain nonlinearity. Control. Eng. Pract. 2011, 19, 700-710. [CrossRef]

20. Elliott, M.S. Decentralized Model Predictive Control of a Multiple Evaporator HVAC System. Master's Thesis, Texas A \& M University, College Station, TX, USA, 2008.

21. Chen, W.; Chan, M.Y.; Deng, S.; Yan, H.; Weng, W. A direct expansion based enhanced dehumidification air conditioning system for improved year-round indoor humidity control in hot and humid climates. Build. Environ. 2018, 139, 95-109. [CrossRef]

22. Jabarullah, N.H.; Shabbir, M.S.; Abbas, M.; Siddiqi, A.F.; Berti, S. Using random inquiry optimization method for provision of heat and cooling demand in hub systems for smart buildings. Sustain. Cities Soc. 2019, 47, 101475. [CrossRef]

23. Esmaeilzadeh, A.; Zakerzadeh, M.R.; Koma, A.Y. The comparison of some advanced control methods for energy optimization and comfort management in buildings. Sustain. Cities Soc. 2018, 43, 601-623. [CrossRef]

24. Afram, A.; Janabi-Sharifi, F. Theory and applications of HVAC control systems-A review of model predictive control (MPC). Build. Environ. 2014, 72, 343-355. [CrossRef]

25. Moroşan, P.D.; Bourdais, R.; Dumur, D.; Buisson, J. Building temperature regulation using a distributed model predictive control. Energy Build. 2010, 42, 1445-1452. [CrossRef]

26. Ma, Y.; Borrelli, F.; Hencey, B.; Coffey, B.; Bengea, S.; Haves, P. Model predictive control for the operation of building cooling systems. IEEE Trans. Control. Syst. Technol. 2011, 20, 796-803.

27. Martinčević, A.; Vašak, M.; Lešić, V. Model predictive control for energy-saving and comfortable temperature control in buildings. In Proceedings of the IEEE 2016 24th Mediterranean Conference on Control and Automation (MED), Athens, Greece, 21-24 June 2016; pp. 298-303.

28. Xu, M.; Li, S. Practical generalized predictive control with decentralized identification approach to HVAC systems. Energy Convers. Manag. 2007, 48, 292-299. [CrossRef]

29. Silva, J.M.; Godina, R.; Rodrigues, E.M.; Pouresmaeil, E.; Catalão, J.P. Residential MPC controller performance in a household with PV microgeneration. In Proceedings of the 2017 IEEE Manchester PowerTech, Manchester, UK, 18-22 June 2017; pp. 1-6.

30. Ascione, F.; Bianco, N.; De Stasio, C.; Mauro, G.M.; Vanoli, G.P. A new comprehensive approach for cost-optimal building design integrated with the multi-objective model predictive control of HVAC systems. Sustain. Cities Soc. 2017, 31, 136-150. [CrossRef]

31. Péan, T.; Costa-Castelló, R.; Salom, J. Price and carbon-based energy flexibility of residential heating and cooling loads using model predictive control. Sustain. Cities Soc. 2019, 50, 101579. [CrossRef]

32. Al-Azb, M.; Cen, Z.; Ahzi, S. Air-Conditioner On-Off optimization Control under Variant Ambient Condition. In Proceedings of the IEEE 2018 6th International Renewable and Sustainable Energy Conference (IRSEC), Rabat, Morocco, 5-8 December 2018; pp. 1-5.

(C) 2020 by the authors. Licensee MDPI, Basel, Switzerland. This article is an open access article distributed under the terms and conditions of the Creative Commons Attribution (CC BY) license (http:/ / creativecommons.org/licenses/by/4.0/). 

Article

\title{
Designing Intelligent MIMO Nonlinear Controller Based on Fuzzy Cognitive Map Method for Energy Reduction of the Buildings
}

\author{
Farinaz Behrooz ${ }^{1, *}$, Rubiyah Yusof ${ }^{1,2}$, Norman Mariun ${ }^{3,4}$, Uswah Khairuddin ${ }^{1,2}$ and \\ Zool Hilmi Ismail ${ }^{1,2}$ \\ 1 Malaysia -Japan International Institute of Technology (MJIIT), Universiti Teknologi Malaysia, \\ Kuala Lumpur 54100, Malaysia \\ 2 Centre for Artificial Intelligence and Robotics (CAIRO), Universiti Teknologi Malaysia, Kuala \\ Lumpur 54100, Malaysia \\ 3 Department of Electrical and Electronic Engineering, Faculty of Engineering, University Putra Malaysia, \\ Serdang 43400, Selangor, Malaysia \\ 4 Centre for Advanced Power and Energy Research, Faculty of Engineering, University Putra Malaysia, \\ Serdang 43400, Selangor, Malaysia \\ * Correspondence: fery_behrooz@yahoo.com; Tel.: +60-10-4315144
}

Received: 27 May 2019; Accepted: 9 July 2019; Published: 16 July 2019

\begin{abstract}
Designing a suitable controller for air-conditioning systems to reduce energy consumption and simultaneously meet the requirements of the system is very challenging. Important factors such as stability and performance of the designed controllers should be investigated to ensure the effectiveness of these controllers. In this article, the stability and performance of the fuzzy cognitive map (FCM) controller are investigated. The FCM method is used to control the direct expansion air conditioning system (DX A/C). The FCM controller has the ability to do online learning, and can achieve fast convergence thanks to its simple mathematical computation. The stability analysis of the controller was conducted using both fuzzy bidirectional associative memories (FBAMs) and the Lyapunov function. The performances of the controller were tested based on its ability for reference tracking and disturbance rejection. On the basis of the stability analysis using FBAMS and Lyapunov functions, the system is globally stable. The controller is able to track the set point faithfully, maintaining the temperature and humidity at the desired value. In order to simulate the disturbances, heat and moisture load changed to measure the ability of the controller to reject the disturbance. The results showed that the proposed controller can track the set point and has a good ability for disturbance rejection, making it an effective controller to be employed in the DX A/C system and suitable for a nonlinear robust control system.
\end{abstract}

Keywords: intelligent control (IC); fuzzy cognitive map (FCM); direct expansion air-conditioning (DX A/C); nonlinear; coupling effect; MIMO; stability analysis; Lyapunov function; fuzzy bidirectional associative memories (FBAMs)

\section{Introduction}

Heating, ventilating, and air-conditioning (HVAC) systems are a major cause of energy consumption in the building automation systems (BAS). Because of the depletion of energy sources in the world and the worsening fuel crisis, the need for advanced, improved control systems designs aimed at less energy usage and better performance are becoming a significant challenge for control engineers [1]. Because of the critical influence of HVAC systems on energy and power consumption, it is imperative to be familiar with the operation as well as the structure of HVAC systems [2]. 
Nowadays, the use of different controllers on HVAC systems is considered as an important issue, with the aim of increasing the system performance, by reason of their high installation demand in buildings and their huge energy consumption.

The direct expansion air-conditioning (DX A/C) system is considered as a subgroup of HVAC systems. The mentioned system has two types of units, window units and split units, which are frequently employed in small- to medium-sized buildings by reason of having a simple configuration, low cost maintenance, and higher energy efficiency [3,4].

According to the authors of [2], energy efficacy and indoor thermal conditions are the main objectives one needs to take into account when designing HVAC or A/C systems. Because of the complicated features of HVAC and A/C systems, attaining the mathematical model of HVAC and air conditioning systems is intricate and difficult. Likewise, designing an appropriate controller becomes a big challenge [5,6]. HVAC system is a multiple-Input and multiple-output (MIMO) system, sometimes with coupled parameters [7]. Moreover, it is a complex nonlinear system, which makes deriving the exact mathematical model a challenging task [3]. Consequently, the control system must be able to deal simultaneously with cross-coupling effects, nonlinearity, and uncertainty of the system. The high energy consumption of these systems calls for an intelligent control system that can adjust the parameters according to the systems' demands, so that it could prevent energy loss and balance between the energy consumption and thermal comfort. Needless to say, an appropriate controller could prevent a significant amount of energy loss [8]. However, designing a suitable controller for the air conditioning system as a nonlinear, complex MIMO system with coupled parameters is still a challenging task [8]. According to the authors of [9], a small increase in system operating proficiency causes major energy savings. Therefore, various studies have investigated different control methods and optimizations in the HVAC areas.

In the case of the DX A/C system, although one can utilize single-input and single-output (SISO) control approaches to control each parameter separately, by decoupling the effects of control variables, yet it degrades the performance of the system [10]. For solving the aforementioned problems and improving the system performance, the control of the DX A/C system can be viewed as a MIMO control system [4]. However, based on the literature, MIMO control systems have mostly been employed on the linearized model of the systems. Thus, the control system is locally stable. In other words, the controller has the ability to stabilize the system around a certain operating point, and stability of the system is not guaranteed outside of a small vicinity of the operating point [11]. Therefore, nonlinear control techniques have been introduced into the DX A/C systems [12,13]. Owing to the complexity and uncertainty of the system, the application of nonlinear control techniques to the system is limited. Complicated mathematical analysis, stability analysis, and the need for the entire states are some aspects that hinder the use of nonlinear control methods [13]. Feedback linearization, Lyapunov stability theory, and adaptive control are some of the approaches that have been applied to the system [14].

Some other control methods like model predictive control (MPC) are successfully applied in this field, but it is necessary to identify a suitable model for the system [15] and installation could be expensive. This means that an accurate dynamic model from the system is required and, considering the uncertainties, makes the problem very difficult to solve.

On the other hand, conventional controllers like a proportional-integral-derivative (PID) controller, usually implemented in a feedback control scheme, are the most applied controllers in this area because of the simplicity of the control law and easy implementation in continuous-time [16-18]. The feedback control scheme depends heavily on feedback sensors, which have some disadvantages such as cost, reliability, and noise, which can affect the output of the system. Moreover, control systems using PID controllers usually have overshoot or undershoot, which means they put sudden pressure on the actuators and reduce the actuators performance over time. This method suffers from disadvantages such as long testing times and limited performance. Tuning the parameters of the controller is cumbersome, and choosing the improper gains of PID controllers can cause the system to 
be unstable [19]. Considering the above mentioned challenges, a simple control method that can deal with the complexity of the system and be practically feasible is of interest.

\section{Construction of the DX A/C System}

The DX A/C system is constructed from two parts, including an air-distribution subsystem and DX refrigeration plant [3]. The refrigeration plant contains a condenser, oil separator, DX evaporator, electronic expansion valve (EEV), receiver, and variable speed compressor. The structure of air-distribution sub-system is composed of an air conditioned room, ductwork, and centrifugal supply fan (variable-speed) [3]. This system is characteristically complex, time varying, and nonlinear, with very effective coupled parameters of supplied air humidity and temperature in the evaporator. Controlling this system was done by varying the speed of the motor compressor and speed of supply fan, in order to attain the preferred humidity and temperature. Figure 1 shows the diagram of the DX $\mathrm{A} / \mathrm{C}$ framework.

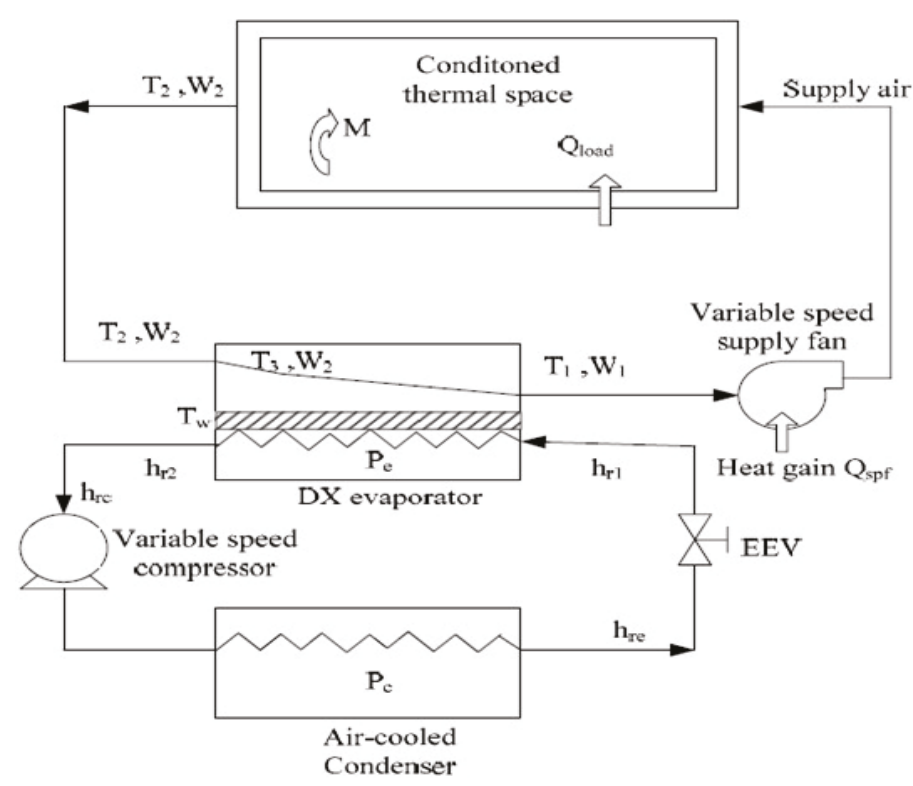

Figure 1. Direct expansion air-conditioning (DX A/C) framework. EEV, electronic expansion valve.

The system mathematical model is extracted from the mass and energy balance conservation principals and the dynamic system equations [3]. The working fluid of the refrigeration plant is Refrigerant 22 (R22), which operates on the basis of the vapor compression cycle's principle [20,21]. On the basis of the work of [3], the evaporator acts as an air cooling coil to simultaneously dehumidify and cool down the passing air through the evaporator. The emerging moisture and temperature content to the air side of the DX evaporator are $W_{2}$ and $T_{2}$, respectively, by the no fresh air assumption [22]. The passing air temperature through the evaporator has declined to the $T_{3}$ value along the dry-cooling section [3]. Because of the small area of the dry-cooling section, the entire evaporator wall temperature could be assumed to have the same temperature $\left(T_{w}\right)$. Equation (1) is obtained using the energy balance principle for the dry-cooling section [3].

$$
C_{p} \rho V_{h 1}\left(\frac{d T_{3}}{d t}\right)=C_{p} \rho f\left(T_{2}-T_{3}\right)+\alpha_{1} A_{1}\left(T_{w}-\left(\frac{\left(T_{2}+T_{3}\right)}{2}\right)\right.
$$


The temperature of evaporator tube wall is shown by $T_{w}$. The airflow rate is $f$. The volume of the dry-cooling section is illustrated by $V_{h 1}$, and the area of the heat transfer for dry-cooling region is demonstrated by $A_{1}$. Oppositely, the heat transfer and the sensible heat transfer obtained in the wet-cooling section between the evaporator wall and air. It is worth mentioning that the coupling effect of air cooling and air dehumidification mostly occurs in the wet-cooling section. The supplied air temperature by evaporator is shown by $T_{1}$, the wet-cooling section volume is $V_{h 2}$, the wet-cooling section area in air side is $A_{2}$, and the vaporization of water's latent heat is shown by $h_{f g h}$. Thus, the wet-cooling section energy balance is attained as follows:

$$
C_{p} \rho V_{h 2}\left(\frac{d T_{1}}{d t}\right)+\rho V_{h 2} h_{f g h}\left(\frac{d W_{1}}{d t}\right)=C_{p} \rho f\left(T_{3}-T_{1}\right)+\rho f h_{f g h}\left(W_{2}-W_{1}\right)+\alpha_{2} A_{2}\left(T_{w}-\frac{\left(T_{3}+T_{1}\right)}{2}\right) .
$$

The DX evaporator outlet supply air is assumed to be $95 \%$ saturated. As a result, the coupled supplied air humidity and air temperature by evaporator could be extracted using curve fitting and plotting $[3,4]$ as follows:

$$
\left(\frac{d W_{1}}{d t}\right)-\left(2 \times 0.0198 T_{1}+0.085\right)\left(\frac{d T_{1}}{d t}\right)=0 .
$$

As the division of the refrigerant side and air side is the evaporator wall, and because of the considerable different thermal inertia of the air and refrigerant, dynamics responding to changes on the refrigerant side are considerably faster than on the air side. In other words, it is quite time consuming for a full response on the air side compared with the refrigerant side. Consequently, using the same mass flow rate assumption for both inlet refrigerant to evaporator and outlet refrigerant from evaporator, the energy balance equation of the evaporator wall can be considered as follows:

$$
\left(C_{p} \rho V\right)_{w}\left(\frac{d T_{w}}{d t}\right)=\alpha_{1} A_{1}\left(\left(\frac{T_{2}+T_{3}}{2}\right)-T_{w}\right)+\alpha_{2} A_{2}\left(\left(\frac{\left(T_{3}+T_{1}\right)}{2}\right)-T_{w}\right)-\frac{\left(s V_{c o m}\right)}{v_{s}\left(\frac{P_{c}}{P_{e}}\right)^{\frac{1}{\beta}}}\left(h_{r 2}-h_{r 1}\right) .
$$

The calculation of the rotor compressor swept volume $\left(V_{\text {com }}\right)$ was done by applying the geometric parameters of compressor. The parameter $s$ is speed of motor compressor and superheated refrigerant specific volume is $v_{s}$. According to the energy conservation principle for the air-conditioned room, the sensible energy balance equation is as follows:

$$
C_{p} \rho V\left(\frac{d T_{2}}{d t}\right)=C_{p} \rho f\left(T_{1}-T_{2}\right)+Q_{s p l}+Q_{\text {load }} .
$$

The feedback of air temperature $\left(T_{2}\right)$ and air humidity $\left(W_{2}\right)$ from the air-conditioned room are considered equal to the thermal condition of the air-conditioned room. The volume of the flow rate is demonstrated by $f$. The supply fan heat gain is shown by $Q_{s p l}$. The volume of the air-conditioned room is $V$, and sensible load of room is illustrated by $Q_{\text {load }}$. Referring to the temperature, by increasing the air flow rate, the supply fan heat gain increases as follows:

$$
Q_{s p l}=K_{s p l} f .
$$

On the other hand, for humidity, the air-conditioned room's moisture mass balance is as follows:

$$
\rho V\left(\frac{d W_{2}}{d t}\right)=\rho f\left(W_{1}-W_{2}\right)+M
$$

The humidity load generation in the air-conditioned room is M. According to the authors of [3], Equations (1)-(5) and (7), which are all first order differential equations, could form the dynamic model of the DX A/C system. On the basis of the work done by Qi [22], Equations (1)-(7) are used as a dynamic mathematical model of the experimental DX A/C system and have been experimentally validated. 
Hence, these equations can be used as the DX A/C system model to design a multivariable control system. In order to design the multivariable nonlinear controller on the DX A/C system, the mentioned differential equations should be re-written in the state-space format. Therefore, the state-space form of the model can be expressed in the following compact format:

$$
\dot{X}=D^{-1} \cdot g_{1}(X, U)+D^{-1} \cdot g_{2}(Z) .
$$

The state variables are as vector $X,\left(X=\left[T_{1} ; T_{2} ; T_{3} ; T_{w} ; W_{1} ; W_{2}\right]^{T}\right)$, control variables are as vector $U,\left(U=[f, s]^{T}\right)$, and disturbances of the system are as vector $\boldsymbol{Z},\left(\mathbf{Z}=[\text { Qload; } M]^{T}\right) . g_{1}$ and $g_{2}$ are functions defined as follows:

$$
\begin{aligned}
& g_{1}(X, U)=\left[\begin{array}{c}
C_{p} \rho f\left(T_{3}-T_{1}\right)+\rho f h_{f g h}\left(W_{2}-W_{1}\right)+\alpha_{2} A_{2}\left(T_{w}-\frac{T_{3}+T_{1}}{2}\right) \\
C_{p} \rho f\left(T_{1}-T_{2}\right)+K_{s p l} f \\
C_{p} \rho f\left(T_{2}-T_{3}\right)+\alpha_{1} A_{1}\left(T_{w}-\frac{T_{2}+T_{3}}{2}\right) \\
\alpha_{1} A_{1}\left(\frac{T_{2}+T_{3}}{2}-T_{w}\right)+\alpha_{2} A_{2}\left(\frac{T_{3}+T_{1}}{2}-T_{w}\right)-\frac{\left(s V_{c o m}\right)}{v_{s}\left(\frac{P_{c}}{P_{e}}\right)}\left(h_{r 2}^{\frac{1}{\beta}}-h_{r 1}\right) \\
0 \\
\rho f\left(W_{1}-W_{2}\right)
\end{array}\right] \\
& g_{2}(Z)=\left[\begin{array}{c}
0 \\
Q_{\text {load }} \\
0 \\
0 \\
0 \\
M
\end{array}\right] \\
& D=\left[\begin{array}{cccccc}
C_{p} \rho V_{h 2} & 0 & 0 & 0 & \rho V_{h 2} h_{f g h} & 0 \\
0 & C_{p} \rho V & 0 & 0 & 0 & 0 \\
0 & 0 & C_{p} \rho V_{h 1} & 0 & 0 & 0 \\
0 & 0 & 0 & \left(C_{p} \rho V\right)_{w} & 0 & 0 \\
\frac{-\left(2 \times 0.0198 T_{1}+0.085\right)}{1000} & 0 & 0 & 0 & 1 & 0 \\
0 & 0 & 0 & 0 & 0 & \rho V
\end{array}\right] .
\end{aligned}
$$

\section{Open Loop Response of the Linearized Model of the System}

With the purpose of model validation, the results of the open loop system with step input for the fan flow rate as well as for compressor speed were compared to the same results of the work of [22]. The results of [22] are based on the experimental model of the DX A/C system, in which the experimental results were compared with the simulation results of the system according to the extracted dynamic mathematical model of the system. According to the results of [22], the dynamic mathematical model of the system was validated and represented as a multivariable control-oriented modelling of a direct expansion (DX) air conditioning (A/C) system. In this work, the open loop simulation results of the DX A/C model based on the dynamic mathematical equations of the system are compared to the simulation and experimental results of the same model, which was done by the authors of [22]. First, the results of the step response for the fan flow rate were compared. At $290 \mathrm{~s}$, the fan flow rate decreased by changing the speed of the fan motor from $2448 \mathrm{rpm}$ to $2160 \mathrm{rpm}$ or from $41 \mathrm{~Hz}$ to $36 \mathrm{~Hz}$. The block diagram of the open loop system in response to a step change in fan flow rate is indicated in Figure 2. 


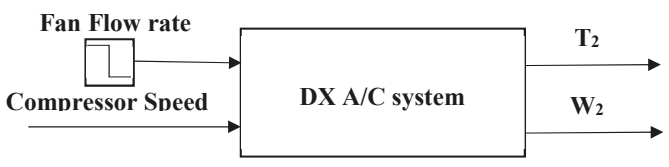

Figure 2. Block diagram of the open loop in step response of fan flow rate.

The results of Figure 3 show that the temperature of the air-conditioned room after $290 \mathrm{~s}$ started to increase to $24.15^{\circ} \mathrm{C}$ and stabilized at $600 \mathrm{~s}$. By reducing the fan flow rate, the effect of the flow rate of the fan on declining the temperature of the room decreased. As is clear from Figure 3, the temperature of the air conditioned room $\left(T_{2}\right)$ by decreasing the speed of the fan and, consequently, decreasing the flow rate of the fan, started to increase from $24^{\circ} \mathrm{C}$ at $290 \mathrm{~s}$ to $24.15^{\circ} \mathrm{C}$ and stabilized at $600 \mathrm{~s}$ and remained stable in this condition.

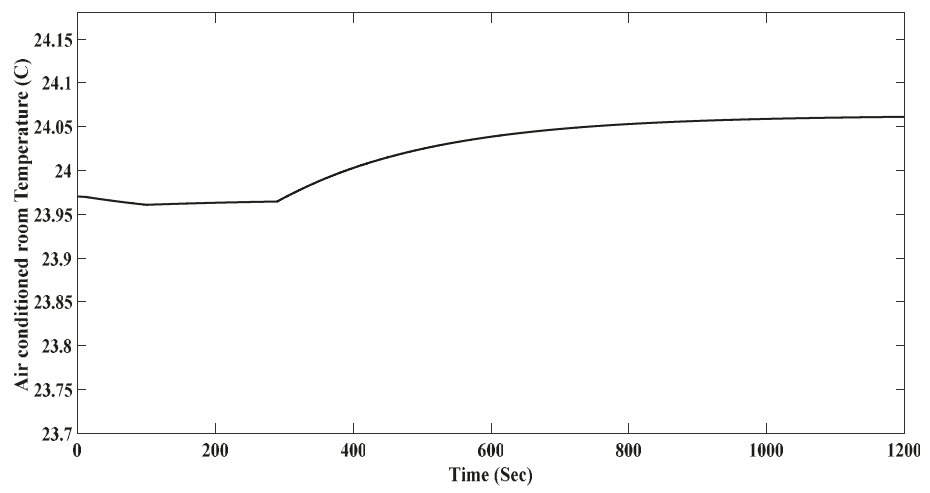

Figure 3. Open loop redo simulation result of air conditioned room temperature in step response of fan flow rate.

Also, the results of Figure 4 indicate that the humidity ratio of the air conditioned room after $290 \mathrm{~s}$ declined from $0.01130 \mathrm{~kg} / \mathrm{Kg}$ to $0.01113 \mathrm{~kg} / \mathrm{Kg}$ and remained stable after $600 \mathrm{~s}$. As the humidity of the air-conditioned room is affected less by varying the fan flow rate, by reduction of the fan flow rate, the humidity of the air-conditioned room was affected more by compressor speed, and consequently decreased.

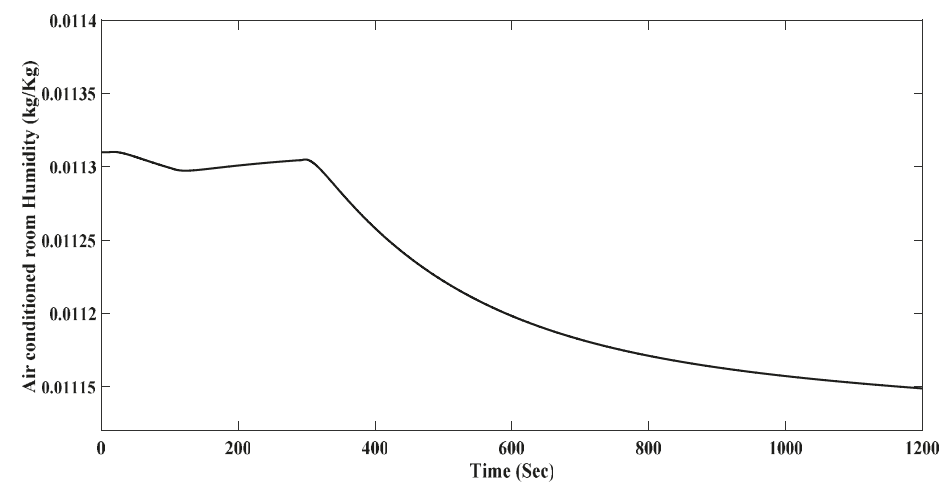

Figure 4. Open loop redo simulation result of air conditioned room humidity ratio in step response of fan flow rate. 
In the next step, the results of the step input for the compressor speed were investigated. The block diagram of the open loop system in step response of compressor speed is illustrated in Figure 5.

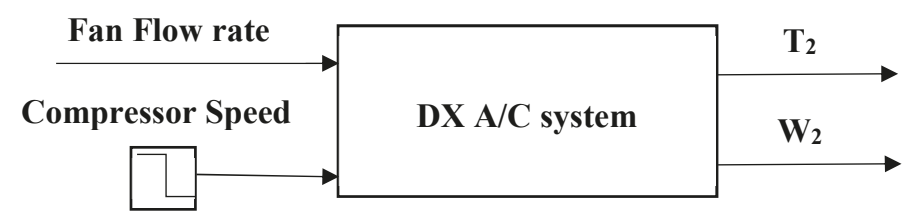

Figure 5. Schematic diagram of the open loop in step response of motor compressor speed.

At $400 \mathrm{~s}$, the speed of compressor increased from $3960 \mathrm{rpm}$ to $4488 \mathrm{rpm}$ or from $66 \mathrm{~Hz}$ to $75 \mathrm{~Hz}$. The results of Figure 6 display the temperature of the air-conditioned room. Because of the increasing speed of the compressor at $400 \mathrm{~s}$, as is obvious in Figure 6, the room temperature decreased from $24^{\circ} \mathrm{C}$ to $23.75^{\circ} \mathrm{C}$ and was maintained. In addition, the results of Figure 7 illustrate that the humidity ratio of the air-conditioned room. As is obvious from Figure 7, by increasing the compressor speed at $400 \mathrm{~s}$, the humidity of the room reduced from $0.0113 \mathrm{~kg} / \mathrm{Kg}$ to $0.0111 \mathrm{~kg} / \mathrm{Kg}$ and stabilized after $500 \mathrm{~s}$.

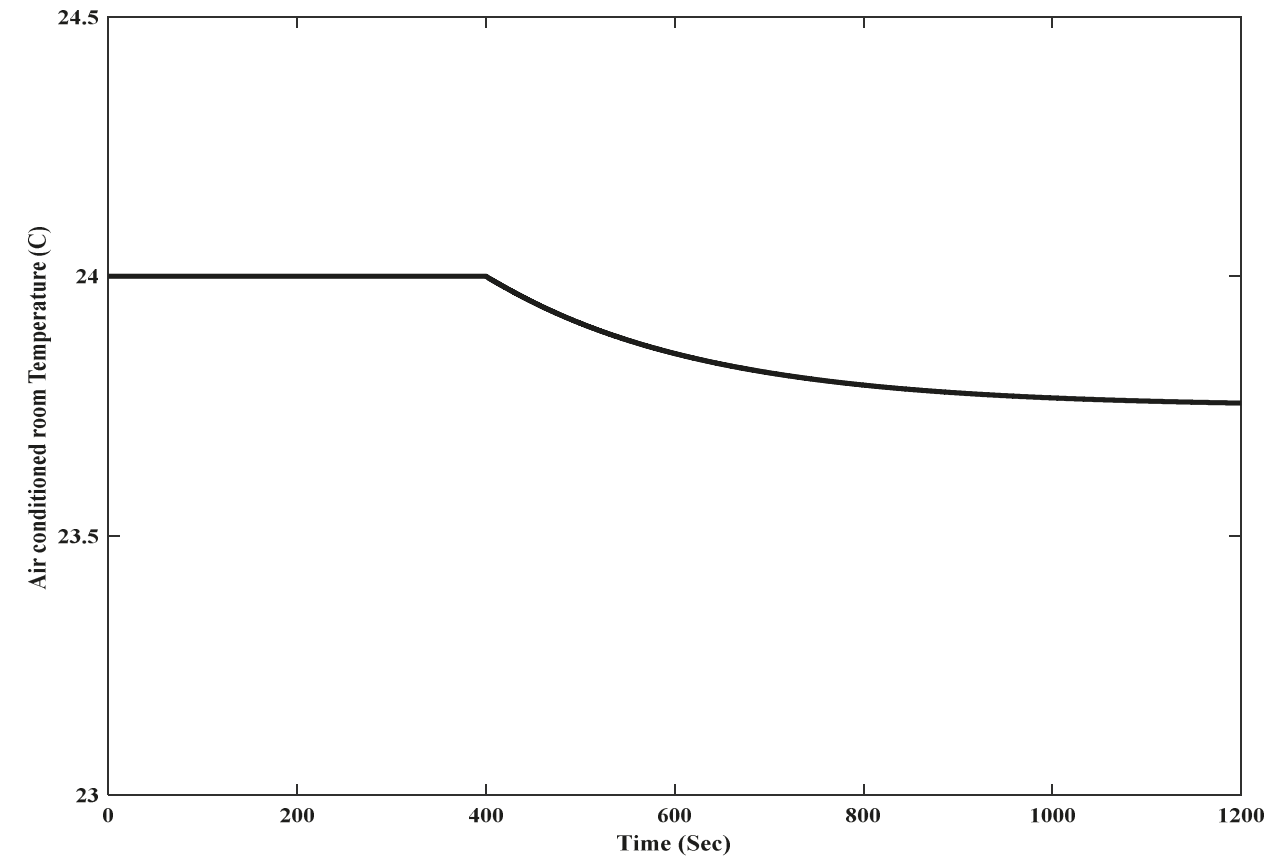

Figure 6. Open loop redo simulation result of air conditioned room temperature in step response of compressor speed.

The comparison between results by [22] and simulation results of this work shows that the dynamic mathematical model used in this research has almost similar behavior to the experimental model and validated simulation model in the work of [22]. Thus, the mathematical model used in this work represents the experimental model of the DX A/C system. 


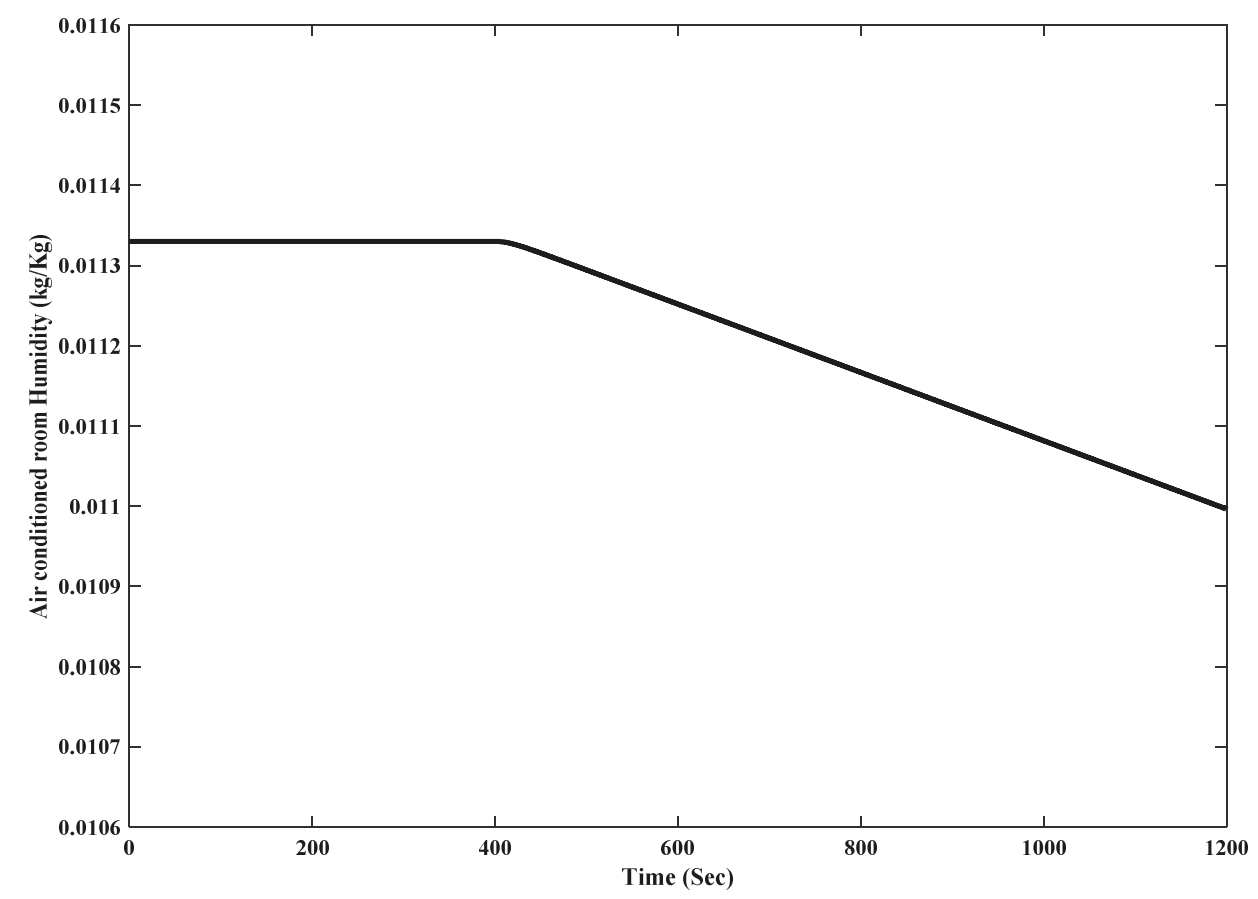

Figure 7. Open loop redo simulation result of air conditioned room humidity ratio in step response of compressor speed.

\section{Fuzzy Cognitive Map}

The development of technologies increases complex and nonlinear behaviours in the systems. Therefore, the requirement for new methods for analysing the systems is feasible [23]. The fuzzy cognitive map (FCM) method has been used in various areas such as economy, virtual reality simulation in place of expert systems [24], sociology, or knowledge-based expert systems with the purpose of analysing the complex systems [25]. The developed cognitive maps method was first presented and used for modelling the causal relationship among the concepts by the authors of $[25,26]$. Prior to that, this method was introduced by a political scientist [27], for decision making in the social sciences and political areas. The mechanism of this method is based on replacing the actual values of concepts with fuzzy values [28-30]. On the basis of a specified scenario, the fuzzy-graph structure of FCM illustrates the parameters of the system by nodes or concepts, and the system characteristics, condition, and effects of parameters on each other are depicted by causal reasoning [25,26,31]. In actual fact, the structure of the FCM method works based on the combination of fuzzy logic and neural networks methods. The robust properties of both methods are evident in the FCM method [28,31].

The FCM structure is formed according to the requested outcome from the system [28]. Consequently, the FCM method has the potential to form as a nonlinear MIMO control system possibly even with some coupled parameters. Choosing the required and effective parameters from the system as nodes and assigning the suitable weights among them leads to the design of a convenient controller for the HVAC or A/C system. This method is able to keep the structure of the system like MIMO, as well as coupled parameters; accordingly, an improvement in the accuracy and sensitivity of the controller can be obtained. Moreover, application of this control method on the nonlinear dynamic model of the system makes the results as similar as possible to the real results from the actual system. 
FCMs consist of concepts or nodes and edges between the nodes. The mathematical structure of FCM contains a $1 \times n$ state vector $(C)$ (it includes the values of the nodes) and an $n \times n$ weight matrix $\left(W_{i j}\right)$ (it includes the relationship between concepts), and the number of the nodes is shown by $n$ [32]. The value of every concept depends on the related concepts, with their weights and the concept's previous value. Value of nodes $\left(C_{i}\right)$ at a particular time reflect the activation degree of nodes in the system. The nodes values are attained by transformation of the real values of concepts into the interval $[0,1]$. Edges between the nodes $\left(W_{i j}\right)$ show the influence of each node on the other ones. Each weight gets a value in the range from -1 to $1[31,33] . W_{i j}>0$ shows the same effect of $C_{i}$ value on $C_{j}$ value. $W_{i j}=0$ depicts no relationship between $C_{i}$ and $C_{j}$. $W_{i j}<0$ shows an opposite effect of $C_{i}$ value on $C_{j}$ value $[23,28,31,33]$. Figure 8 indicates the diagram of the FCM method as a graph structure.

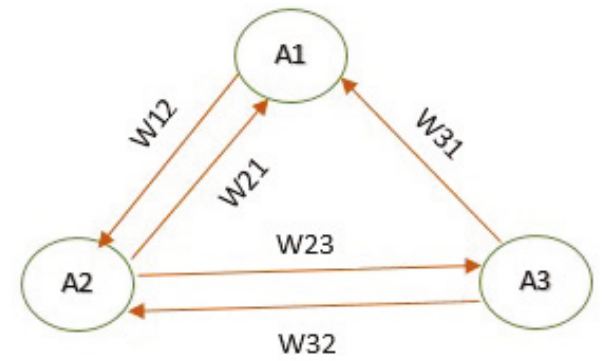

Figure 8. Schematic of the fuzzy cognitive map (FCM) method.

\section{MIMO Nonlinear Controller Based on FCM Method}

In order to design the controller for the DX/AC system according to the FCM method, the required concepts can be defined as follows [34]:

Concept 1: supply air temperature by evaporator $\left(T_{1}\right)$.

Concept 2: air conditioned room's temperature $\left(T_{2}\right)$.

Concept 3: supply humidity by evaporator $\left(W_{1}\right)$.

Concept 4: air conditioned room's humidity $\left(W_{2}\right)$.

Concept 5: supply fan flow rate $(f)$.

Concept 6: compressor speed $(S)$.

In spite of the advantageous of FCMs in design to control the complicated and complex nonlinear systems, the most considerable weak point of FCMs is their potential to converge to the undesired steady state. Thus, the initial weights were defined based on the predetermined information of the system around an operating point. Thus, to allocate proper initial weights for the FCM network, the generalized predictive control (GPC) method was chosen to find the minimum required values for control signals, which is suitable for the MIMO structure [20]. The relationships of concepts 1 to 4 were extracted from the direct and indirect relationships between the concepts in the linearized model of system. In other words, the matrixes $A$ and $B$ of the linearized model were applied to describe the concept weights. For the concepts where the relationship exists directly in matrix $A$ and $B$, the mentioned values were used as a direct relationship. For the other concepts with zero values, the indirect relations with the other concepts were considered and employed. For the actuators concepts, the relationships of them with other concepts are defined on the basis of the $K$ matrix calculation for the GPC model of the system. As the DX A/C system is MIMO, the steady space model of the system was employed for finding the $K$ matrix of the GPC model [31]. Regarding the use of these values as weights for the FCM method, they have to be normalized to the range of $[-1,1][34]$. 
Thus, the linearized dynamic model of the DX A/C system in state-space representation, which is more appropriate for designing MIMO control, can be written as Equation (9):

$$
\left\{\begin{array}{c}
\dot{X}=A x+B u \\
y=C x+d
\end{array}\right.
$$

where the output variables $y=\left[d T_{2}, d W_{2}\right]^{T}$ are the dynamic deviations of air-conditioned room temperature and humidity from their set points, respectively; and $A, B$, and $C$ are the coefficient matrices. According to the GPC method, the compact prediction notation is Equation (10) [35].

$$
\begin{aligned}
& \underset{\rightarrow k+1}{x}=\underbrace{\left[\begin{array}{c}
A \\
A^{2} \\
\vdots \\
A^{n}
\end{array}\right]}_{P_{x}} x_{k}+\underbrace{\left[\begin{array}{cccc}
B & 0 & \cdots & 0 \\
A B & B & \cdots & 0 \\
\vdots & \vdots & \ddots & \vdots \\
A^{n-1} B & A^{n-2} B & \cdots & B
\end{array}\right]}_{H_{x}} \underbrace{\left[\begin{array}{c}
u_{k \mid k} \\
u_{k+1 \mid k} \\
\vdots \\
u_{k+n-1 \mid k}
\end{array}\right]}_{\stackrel{u}{\rightarrow}} \\
& \underset{\rightarrow k+1}{x}=P_{x} x_{k}+H_{x} \underset{\rightarrow k}{u} .
\end{aligned}
$$

The output predictions follow a similar method, as shown in Equation (11). The $P_{x}$ depends on past and the $H_{x}$ depends upon decision variables [35].

$$
\begin{aligned}
& \underset{\rightarrow}{y}=\underbrace{\left[\begin{array}{c}
C A \\
C A^{2} \\
\vdots \\
C A^{n}
\end{array}\right]}_{P} x_{k}+\underbrace{\left[\begin{array}{cccc}
C B & 0 & \cdots & 0 \\
C A B & C B & \cdots & 0 \\
\vdots & \vdots & \ddots & \vdots \\
C A^{n-1} B & C A^{n-2} B & \cdots & C B
\end{array}\right]}_{H} \underbrace{\left[\begin{array}{c}
u_{k \mid k} \\
u_{k+1 \mid k} \\
\vdots \\
u_{k+n-1 \mid k}
\end{array}\right]}_{\underset{u}{\rightarrow}}+\underbrace{\left[\begin{array}{c}
d_{k} \\
d_{k} \\
\vdots \\
d_{k}
\end{array}\right]}_{L d} \\
& \underset{\rightarrow k+1}{y}=P x_{k}+L d_{k}+\underset{\rightarrow k}{H} .
\end{aligned}
$$

These are defined relative to an expected steady-state. Implicitly, their values assume a best estimate of the system disturbance, and of course knowledge of the desired target.

$$
\begin{aligned}
& r=y_{s s}=C x_{s s}+d_{k} ; \quad x_{s s}=A x_{s s}+B u_{s s} ; \\
& \hat{x}_{k}=x_{k}-x_{s s} ; \hat{u}_{k}=u_{k}-u_{s s} ; \\
& \left.\begin{array}{l}
\hat{x}_{k}=0 \\
\hat{u}_{k}=0
\end{array}\right\} \Rightarrow \hat{x}_{k+1}=A \hat{x}_{k}+B \hat{u}_{k}=0 ;
\end{aligned}
$$

The predictions are given as Equation (12) [35]:

$$
\underset{\rightarrow}{\stackrel{e}{\rightarrow}}=\underset{\rightarrow k+1}{\hat{y}}=P_{x} \hat{x}_{k}+H_{k} \underset{\rightarrow k}{\stackrel{\wedge}{u}}
$$

Finally, by minimising the performance index and rewriting in terms of the actual state and input, one gets Equation (13) [35]:

$$
u_{k}-u_{S S}=-\underbrace{E_{1}^{T}\left[H_{x}^{T} Q H_{x}+R\right]^{-1} H_{x}^{T} Q P_{x}}_{K}\left[x_{k}-x_{S S}\right] .
$$


By minimizing the $\left(u_{k}-u_{s s}\right)$, the least control efforts to reach to the defined set points are calculated. Equations (14)-(16) show the finalized equations for finding the minimization of performance index. Entire values should normalize into the interval $[-1,1]$.

$$
\begin{gathered}
X_{k+1}-X_{s s}=A\left(X_{k}-X_{s s}\right)+B\left(u_{k}-u_{s s}\right) \\
Y_{k}-Y_{s s}=C\left(X_{k}-X_{s s}\right) \\
u_{k}-u_{s s}=-K\left(X_{k}-X_{s s}\right)
\end{gathered}
$$

Table 1 shows the numerical values and operating point of the DX A/C system based on the works of $[3,4,22,34]$, which are used for making the matrices $A, B, C$, and $K$ using the GPC method for weight matrix values between the concepts [34].

Table 1. Operating condition and numerical values of the system.

\begin{tabular}{cccccc}
\hline$T_{1}$ & $13.25^{\circ} \mathrm{C}$ & $W_{2}$ & $11.35 \mathrm{~g} / \mathrm{kg}$ dry air & $W_{1}$ & $9.03 \mathrm{~g} / \mathrm{kg}$ dry air \\
\hline$T_{2}$ & $24{ }^{\circ} \mathrm{C}$ & $p_{c}$ & $1.812 \times 10^{6} \mathrm{pa}$ & $Q_{\text {load }}$ & $4.49 \mathrm{kw}$ \\
\hline$T_{3}$ & $1{ }^{\circ} \mathrm{C}$ & $p_{e}$ & $0.486 \times 10^{6} \mathrm{pa}$ & $s$ & $3960 \mathrm{rpm}$ \\
\hline$T_{w}$ & $13{ }^{\circ} \mathrm{C}$ & $M$ & $0.96 \mathrm{Kg} / \mathrm{s}$ & $f$ & $0.347 \mathrm{~m}^{3} / \mathrm{s}$ \\
\hline$A_{1}$ & $4.14 \mathrm{~m}^{2}$ & $C_{\mathrm{p}}$ & $1.005 \mathrm{Kj} / \mathrm{kg}$ & $\mathrm{V}$ & $77 \mathrm{~m}^{3}$ \\
\hline$A_{2}$ & $17.65 \mathrm{~m}^{2}$ & $\rho$ & $1.2 \mathrm{~kg} / \mathrm{m}^{3}$ & $h_{f g h}$ & $2450 \mathrm{Kj} / \mathrm{kg}$ \\
\hline
\end{tabular}

The links among concepts are as follows [34]:

Connection 1: Connection of concept $1\left(T_{1}\right)$ to concept $1\left(T_{1}\right)$ with negative effect.

Connection 2: Connection of concept $1\left(T_{1}\right)$ to concept $3\left(W_{1}\right)$ with positive effect.

Connection 3: Connection of concept $1\left(T_{1}\right)$ to concept $4\left(W_{2}\right)$ with positive effect.

Connection 4: Connection of concept $1\left(T_{1}\right)$ to concept $5(f)$ with positive effect.

Connection 5: Connection of concept $1\left(T_{1}\right)$ to concept $6(S)$ with positive effect.

Connection 6: Connection of concept $2\left(T_{2}\right)$ to concept $1\left(T_{1}\right)$ with positive effect.

Connection 7: Connection of concept $2\left(T_{2}\right)$ to concept $2\left(T_{2}\right)$ with negative effect.

Connection 8: Connection of concept $2\left(T_{2}\right)$ to concept $5(f)$ with negative effect.

Connection 9: Connection of concept $2\left(T_{2}\right)$ to concept $6(S)$ with positive effect.

Connection 10: Connection of concept $3\left(W_{1}\right)$ to concept $1\left(T_{1}\right)$ with positive effect.

Connection 11: Connection of concept $3\left(W_{1}\right)$ to concept $6(S)$ with positive effect.

Connection 12: Connection of concept $4\left(W_{2}\right)$ to concept $3\left(W_{1}\right)$ with positive effect.

Connection 13: Connection of concept $4\left(W_{2}\right)$ to concept $4\left(W_{2}\right)$ with negative effect.

Connection 14: Connection of concept $4\left(W_{2}\right)$ to concept $5(f)$ with negative effect.

Connection 15: Connection of concept $4\left(W_{2}\right)$ to concept $6(S)$ with positive effect.

Connection 16: Connection of concept $5(f)$ to concept $1\left(T_{1}\right)$ with positive effect.

Connection 17: Connection of concept $5(f)$ to concept $2\left(T_{2}\right)$ with negative effect.

Connection 18: Connection of concept $5(f)$ to concept $3\left(W_{1}\right)$ with positive effect.

Connection 19: Connection of concept $5(f)$ to concept $4\left(W_{2}\right)$ with negative effect.

Connection 20: Connection of concept $5(f)$ to concept $5(f)$ with positive effect.

Connection 21: Connection of concept $6(S)$ to concept $1\left(T_{1}\right)$ with positive effect.

Connection 22: Connection of concept $6(S)$ to concept $2\left(T_{2}\right)$ with positive effect.

Connection 23: Connection of concept $6(S)$ to concept $3\left(W_{1}\right)$ with positive effect.

Connection 24: Connection of concept $6(S)$ to concept $4\left(W_{2}\right)$ with positive effect.

Connection 25: Connection of concept $6(S)$ to concept $6(S)$ with positive effect.

Figure 9 illustrates the graph structure of the controller [34]. 


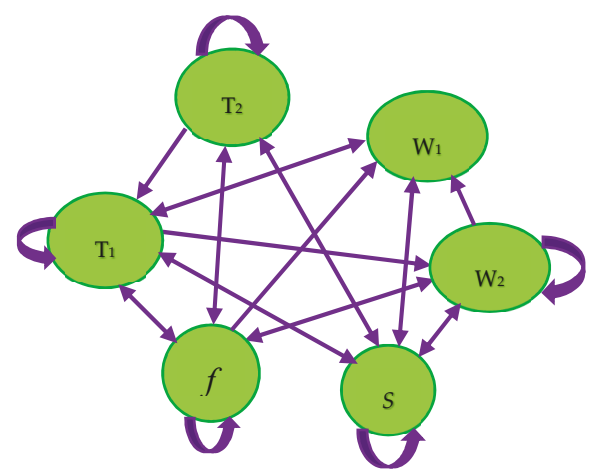

Figure 9. Schematic of graphical structure of the FCM controller.

This graphical structure of the controller follows the simple mathematical model of FCM, which contains a $1 \times n$ state vector $(C)$, which is the values of the $n$ concepts, and an $n \times n$ weight matrix, which is the weights $\left(W_{i j}\right)$ between the concepts. The number of concepts is six [32,34,36]. On the basis of the construction of FCMs, all nodes' real values are normalized in the range [0, 1] by Equation (17) as a transformation mechanism. Next, Equations (18) and (19) are employed for calculation of concepts activation level $\left(C_{i}\right)$ for every concept [23,32,34,36]. Lastly, Equations (18) and (19) were continued until the values do not change at all, and the control signal values were defuzzified and applied to the actuators [34].

$$
\begin{gathered}
g\left(S_{i}{ }^{t}\right)\left\{\begin{array}{cc}
0, & \text { if } S_{i}{ }^{t}<a_{i} \\
\left(S_{i}^{t}-a_{i}\right) /\left(2\left(m_{i}-a_{i}\right)\right), & \text { if } a_{i} \leq S_{i}{ }^{t}<m_{i} \\
0.5+\left(S_{i}{ }^{t}-m_{i}\right) /\left(2\left(b_{i}-m_{i}\right)\right), & \text { if } \mathrm{m}_{i}<S_{i}{ }^{t} \leq b_{i} \\
1, & \text { if } S_{i}{ }^{t}>b_{i}
\end{array}\right. \\
C_{i}^{\text {new }}=f\left(\Sigma C_{j}^{\text {old }} \times W_{i j}\right)+C_{i}^{\text {old }} \\
f(x)=\frac{1}{\left(1+e^{-\lambda x}\right)}
\end{gathered}
$$

The detected values of the $i$ th state at time $t$, max, min, and mean are shown by $S_{i}^{t}, b_{i}, a_{i}$, and $m_{i}$, respectively. $C_{i}^{\text {new }}$ depicts the activation level of concept $i$ at time $t+1$ and $C_{j}^{c o l d}$ discloses the activation level of concept $j$ at time $t$. The threshold function is shown by $f$.

FCM has been widely used in practice, mainly owing to its capabilities to deal with complex nonlinear systems. On the other hand, crucially big steady state error is one of the disadvantages of the FCM technique. To solve the aforementioned problem, one can deploy learnability of FCM. Updating the weights by applying supervised learning techniques on FCM makes the FCM technique more practical for online real-time control problems. Herein, the following supervised $\delta$ learning rules were chosen:

$$
\begin{gathered}
W_{i j}(K+1)=W_{i j}(K)+\Delta W_{i j}(K), \\
\Delta W_{i j}(K)=\eta A_{i}(K) C_{j}(K)\left(1-C_{j}(K)\right)\left(d_{j}-C_{j}(K)\right) .
\end{gathered}
$$

The expected value of input node $j$ is $d_{j}$; the state value of the nodes connected to this input node is $C_{i}$; the state value of input node $j$ is $C_{j}$; the learning rate is $\eta$, which it is in the range [0,1]; and the iteration step is $K$. The concepts' preferred values should be placed in matrix $d$. 


\section{Stability Analysis of Fuzzy Cognitive Map Method}

On the basis of the work of [37], the stability analysis test for the FCM method as a core control method on complex nonlinear MIMO systems should be done by considering the FCM as a one layer network fuzzy bidirectional associative memories (FBAMs). In other words, it can be taken into account as a special case of FBAMs, which has a one-layer network rather than a two-layer network. The FBAM is a two-layer neural network with two layers of $X$ and $Y$, where matrix $P$ displays the connection matrix from $X$ layer to $Y$ layer, and $R$ matrix specifies the connection matrix from $Y$ layer to $X$ layer. The number of fuzzy neurons are shown by $\mathrm{n}$ and $\mathrm{m}$ in the layers of $X$ and $Y$, respectively. The stability analysis of FBAMs is justified by applying the triangular norms [38]. Triangular norms ( $T$-norms) and $T$-conorm ( $S$-norm) generalize the AND operation and the OR operation for fuzzy system respectively. Any FBAM based on the $S-T$ composition is globally stable, if and only if the FBAMs are globally stable when the connection matrices are based on the max- $T$ composition, and the product of $S-T$ composition of them is convergent to the max- $T$ composition. This means that global stability of FBAMs is dependent on the matrix power convergence. In contrast, FCM is a one-layer network with one weight matrix. By decomposing the weight matrix $(W)$ to the two triangular matrices, it can be transferred to the two layer structure for stability analysis as follows [37]:

$$
W=L \cdot U
$$

The FCM weight matrix can be decomposed by the LU factorization method or the Gaussian elimination method. These methods show the matrix $W$, which is square, as a product of a permutation of a lower triangular matrix $(L)$, with ones on its diagonal and an upper triangular matrix $(U)$. If diagonal elements of $L$ or $U$ are non-zero, then such a factorization is unique for the given $W$. For being factorized into two matrices $L$ and $U$, the weight matrix $W$ for the given FCM must satisfy the following condition. As the weight matrix is a fuzzy matrix, it always has non-zero elements because of the interconnection of the parameters and its influence directly and indirectly through the other parameters on each other and, accordingly, non-zero principal minors. Thus, it can always be decomposed into two triangular matrices. By transforming the FCM structure to two-layer as layer $\mathrm{G}$ and layer $\mathrm{H}$, the relationship between the layers is presented by two factorized matrices. The $L$ matrix indicates the relationship between layer $\mathrm{G}$ and layer $\mathrm{H}$, and the $U$ matrix shows the relationship between layer $\mathrm{H}$ and layer $\mathrm{G}$. Both layers have the same number of concepts as FCM concepts. Figure 10 illustrates the transformation of the FCM method to two-layer as layer G and H [37].

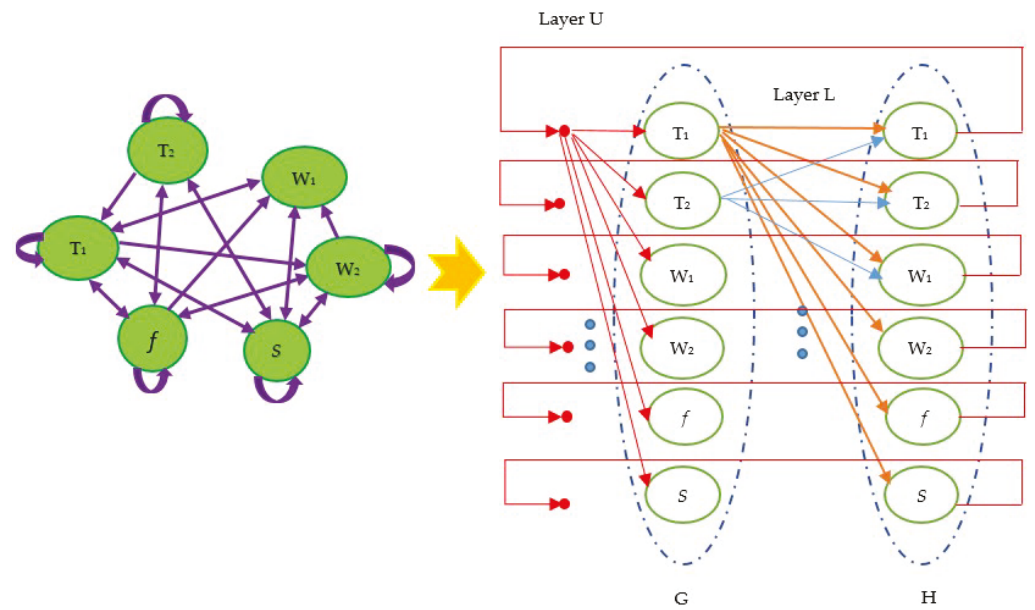

Figure 10. Transformation of the FCM method to two layers. 
The new style of FCM is written as follows:

$$
\left\{\begin{array}{l}
G^{(k+1)}=\left(G^{(k)} \cdot L\right) \cdot U \\
H^{(k+1)}=\left(H^{(k)} \cdot U\right) \cdot L
\end{array} .\right.
$$

For FCM stability analysis, a specific T-norm should exist, which is obtained from the $S-T$ composition of the triangular matrices from decomposition of the weight matrix. For this matter, this T-norm should be equal to the original weight matrix. If this $S$-T composition exists, the FCM is globally stable. The product of the $S-T$ composition for two matrices $L=\left(l_{i j}\right)_{m \times p}$ and $U=\left(u_{i j}\right)_{p \times n}$ should be as follows [37]:

$$
L \bigcirc U=\left(w_{i j}^{\prime}\right)
$$

where

$$
w_{i j}^{\prime}=\left(l_{i 1} T u_{1 j}\right) S\left(l_{i 2} T u_{2 j}\right) S \ldots S\left(l_{i p} T u_{p j}\right) .
$$

In other words, the following steps should be done for the stability analysis of FCMs:

1. Factorization of weight matrix to $L$ and $U$ matrices;

2. Getting a product of $S-T$ composition for $L$ and $U$ matrices based on Equation (25);

3. If such an $S-T$ composition exists for the $L$ and $U$ matrices, which is equal to $W$, then the assumed FCM structure is globally stable; otherwise, the proposed stability method cannot solve the stability problem of the assumed FCM method [37].

Following the above processes and the values from Table 1, the weight matrix and factorization to the matrix $L$ and $U$ for the proposed designed controller are as below. Accordingly, the designed controller is globally stable.

$$
\begin{aligned}
W & =\left[\begin{array}{cccccc}
-0.279 & 0.9 & 0.6 & 0 & 0.9295 & 0.94095 \\
0 & -0.9 & 0 & 0 & -0.26662 & 0.2267 \\
0.63795 & 0 & 0 & 0.45 & 0.7707 & 0.85448 \\
0.63795 & 0 & 0 & -0.9 & -0.7707 & 0.99689 \\
0.4238 & -0.417 & 0 & -0.7 & 1 & 0 \\
0.3471 & 0.4686 & 0.84 & 0.98 & 0 & 1
\end{array}\right], \\
L & =\left[\begin{array}{ccccccc}
-0.43734 & 0 & 0 & 0 & 0 & 0 \\
0 & & 1 & 0 & 0 & 0 & 0 \\
1 & & 0 & 0 & 0 & 0 & 0 \\
1 & & 0 & 0 & 1 & 0 & 0 \\
0.66432 & 0.46333 & 0 & 0.73996 & 0.98796 & 0 \\
0.54409 & -0.52067 & 1 & 0 & 0 & 0
\end{array}\right], \\
U & =\left[\begin{array}{ccccccc}
0.63795 & 0 & 0 & 0.45 & 0.7707 & 0.85448 \\
0 & -0.9 & 0 & 0 & -0.26662 & 0.2267 \\
0 & 0 & 0.84 & 0.73516 & -0.55815 & 0.65312 \\
0 & 0 & 0 & -1.35 & -1.5414 & 0.14241 \\
0 & 0 & 0 & 0 & 1.7735 & 1.0402 \\
0 & 0 & 0 & 0 & 0 & -1.8057
\end{array}\right] .
\end{aligned}
$$

Proof of stability analysis by the Lyapunov direct method can be written as below for the fuzzy cognitive map control method. Lyapunov design refers to the synthesis of control laws for the desired closed-loop stability properties, using the Lyapunov function for the mentioned nonlinear control system as follows $[39,40]$ :

$$
\dot{x}=f(x ; u(x))
$$


where $x \in R^{n}$ is the state and $u \in R^{m}$ is the control input. As a result, Equation (11) can be written in the following form [41]:

$$
C_{i}(x)\left\{\begin{array}{cc}
C_{i}=f\left(\sum C_{j}(x) w_{i j}(x)\right), & \text { for } \mathrm{x}=0 \\
C_{i}(x)=f\left(C_{i}(x-1)+\sum C_{j}(x) w_{i j}(x)\right), & \text { for } \mathrm{x} \neq 0
\end{array} .\right.
$$

The $f(x)$ is the unipolar sigmoid threshold function as Equation (15), which squashes the values in the range of 0 to 1 . As the fuzzy cognitive map is a nonlinear dynamical system, the system operates in the form of dynamics. As a result, the system can be evaluated in the way of the energy function reduction direction, and it reached a stable state. Therefore, a Lyapunov function for fuzzy cognitive map, which is called the energy function, by considering the $f$ function as the $S$ shaped continuous function like Equation (15), can be written as follows [41]:

$$
E(t)=-\frac{1}{2} \sum_{i=1}^{n} \sum_{j=1}^{n} w_{i j} f_{i}(x) f_{j}(x)-\sum_{i=1}^{n} \int_{0}^{f_{i(x)}} f^{-1}(x) d x .
$$

As for the activation function $f(x)$, the $S$-type threshold function is used. The energy function is bounded defined above, consequently, only proving of the $\frac{d E}{d x}$ will show the stability of the system.

$$
\frac{d E}{d t}=\sum_{i=1}^{n} \frac{\partial E}{\partial f_{i(x)}} \frac{\partial f_{i(x)}}{\partial t}
$$

The partial derivative is extracted from Equation (24) as follows:

$$
\frac{\partial E}{\partial f_{i(x)}}=-\sum_{j=1}^{n} w_{i j} f_{j}(x)+f^{-1}(x) .
$$

By combining Equations (29) and (30), Equation (29) can be rewritten as follows:

$$
\frac{d E}{d t}=-\sum_{i=1}^{n}\left(\sum_{j=1}^{n} w_{i j} f_{j}(x) \frac{d f_{i(x)}}{d t}+\left(\frac{d f_{i(x)}}{d t}\right)^{2} .\right.
$$

Because of the choice of the $S$-type threshold function, which is monotonic type as an activation function, the function is increasing. It is clear that its inverse function $f^{-1}(x)$ is also a monotonic type and increasing function. Therefore, the value $\frac{d f_{i(x)}}{d t} \geq 0$, so that $\frac{d E}{d t} \leq 0$. It is proven that the fuzzy cognitive map must be stable based on Lyapunov's theory [39,40].

\section{Performance Analysis Test and Results}

In order to performance validation of the designed control system, two performance measures are taken into account, namely reference tracking and disturbance rejection. Simulation was done in MATLAB/Simulink (R2017b, The MathWorks, Inc, Natick, MA, USA) and the results are presented below.

\subsection{Reference Tracking}

The reference tracking results emphasize the ability of the controller to stabilize the system to the new reference signal. To mimic tropical regions (i.e., Malaysia conditions), the mean temperature of $30{ }^{\circ} \mathrm{C}$ and mean humidity ratio of $80 \%$ were chosen for this simulation. The results clearly show that the comfort temperature and humidity based on tropical countries standards are obtained by the designed control system. Figure 11 displays the actual and reference of the air-conditioned room temperature. The reference temperature changed from $25^{\circ} \mathrm{C}$ to $2{ }^{\circ} \mathrm{C}$ at $1300 \mathrm{~s}$. The set initial room temperature is $30^{\circ} \mathrm{C}$. It can be obviously seen that the temperature stabilized at the desired set points with settling 
times of 380 and $1325 \mathrm{~s}$, respectively. The air-conditioned room humidity is shown in Figure 12. The set initial room humidity is $80 \%$ or $0.02157 \mathrm{~kg} / \mathrm{Kg}$. It is clearly seen that the humidity stabilized at the desired set points, with settling times of 1200 and $1357 \mathrm{~s}$, respectively. The humidity declines from $80 \%$ to $48 \%$ or $0.02157 \mathrm{~kg} / \mathrm{Kg}$ to $0.00948 \mathrm{~kg} / \mathrm{Kg}$ at about $1200 \mathrm{~s}$. After changing the set point at $1300 \mathrm{~s}$, the humidity reaches to $0.009977 \mathrm{~kg} / \mathrm{Kg}$ or $53.5 \%$ at $1357 \mathrm{~s}$ and stabilizes. The humidity is still in the tropical thermal comfort range, which is in the range of $40 \%$ to $60 \%$. The supply air temperature is depicted in Figure 13. The set initial supply air temperature is $11.25^{\circ} \mathrm{C}$. It is clear that the controller stabilized the supply air temperature at 180 and $1327 \mathrm{~s}$, respectively. After changing the set point, it increases at $1300 \mathrm{~s}$ to $15.85^{\circ} \mathrm{C}$ and then decreases gradually to $13.66^{\circ} \mathrm{C}$ at near $1327 \mathrm{~s}$ and is maintained. Figure 14 illustrates the supply humidity. The set initial supply humidity is $0.009 \mathrm{~kg} / \mathrm{Kg}$. It is evident that the controller stabilized the supply humidity at 1200 and $1329 \mathrm{~s}$, respectively. The humidity rises from $0.009 \mathrm{~kg} / \mathrm{Kg}$ to $0.01728 \mathrm{~kg} / \mathrm{Kg}$ at around $29 \mathrm{~s}$, becomes stable at $129 \mathrm{~s}$, and then declines to $0.006 \mathrm{~kg} / \mathrm{Kg}$ at almost $1200 \mathrm{~s}$, after which it increases a little to $0.006555 \mathrm{~kg} / \mathrm{Kg}$ and then stabilizes. After set point changing, the humidity increasingly changed to $0.009481 \mathrm{~kg} / \mathrm{Kg}$ at around $1329 \mathrm{~s}$, then decreased to $0.008754 \mathrm{~kg} / \mathrm{Kg}$ and maintained. The fan flow rate is illustrated in Figure 15. The set initial flow rate is $0.34 \mathrm{~m}^{3} / \mathrm{s}$. It is clear that the proposed controller stabilizes the flow rate at 1200 and $39 \mathrm{~s}$, respectively. It raises from $0.34 \mathrm{~m}^{3} / \mathrm{s}$ to $0.382 \mathrm{~m}^{3} / \mathrm{s}$ and stabilize at just about $1200 \mathrm{~s}$. After $1200 \mathrm{~s}$, it decreases rapidly to $0.325 \mathrm{~m}^{3} / \mathrm{s}$ and remains stable at $1300 \mathrm{~s}$. As a result of set point changing at $1300 \mathrm{~s}$, it is sharply increased to $0.8815 \mathrm{~m}^{3} / \mathrm{s}$, then dropped and finally stabilizes at $0.34 \mathrm{~m}^{3} / \mathrm{s}$ at around $1339 \mathrm{~s}$ and maintained. The compressor speed is demonstrated in Figure 16. The set initial compressor speed is $3000 \mathrm{rpm}$. It is obviously seen that the controller is able to stabilize the compressor speed at 1200 and $1334 \mathrm{~s}$, respectively. The speed raises from $3000 \mathrm{rpm}$ to $6359 \mathrm{rpm}$, and becomes more or less stable around $6320 \mathrm{rpm}$. Afterward, at around $1200 \mathrm{~s}$, it declined to $3960 \mathrm{rpm}$. From $1300 \mathrm{~s}$, the speed moved upwards to $6326 \mathrm{rpm}$, and from $1327 \mathrm{~s}$, it leveled off and maintained at $3180 \mathrm{rpm}$ at $1334 \mathrm{~s}$.

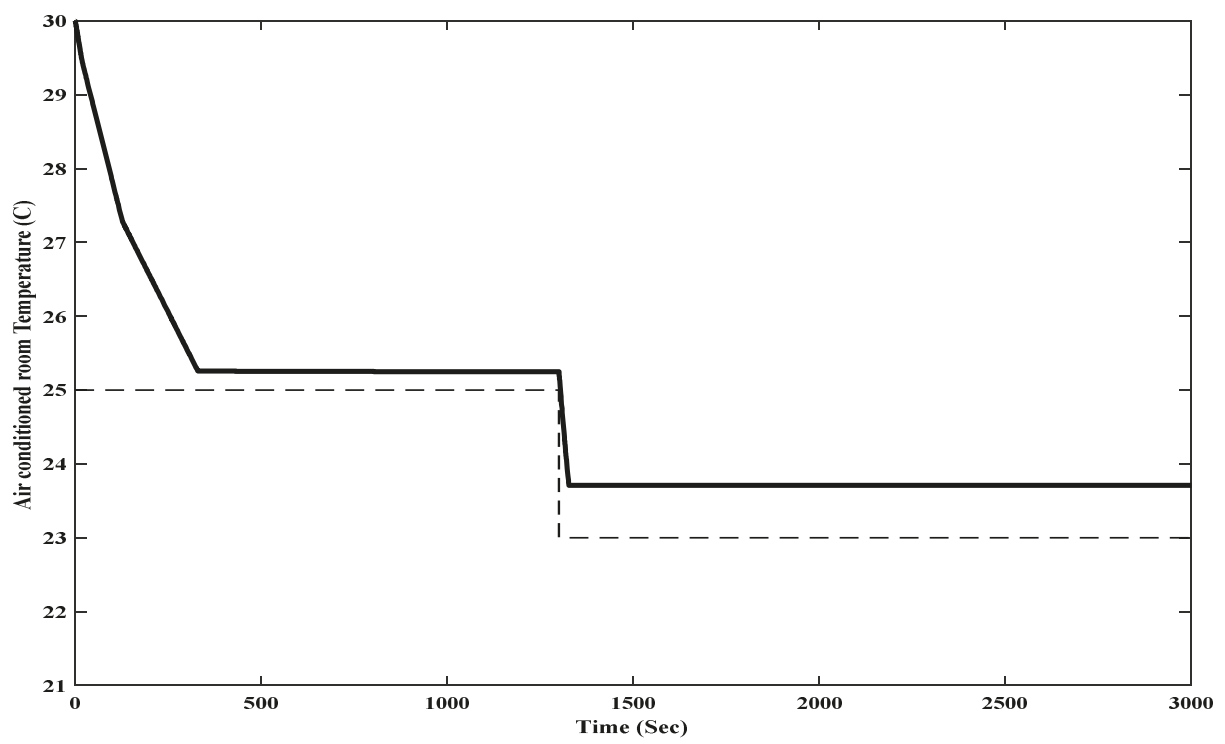

Figure 11. Temperature of the air-conditioned room in set point changing. 


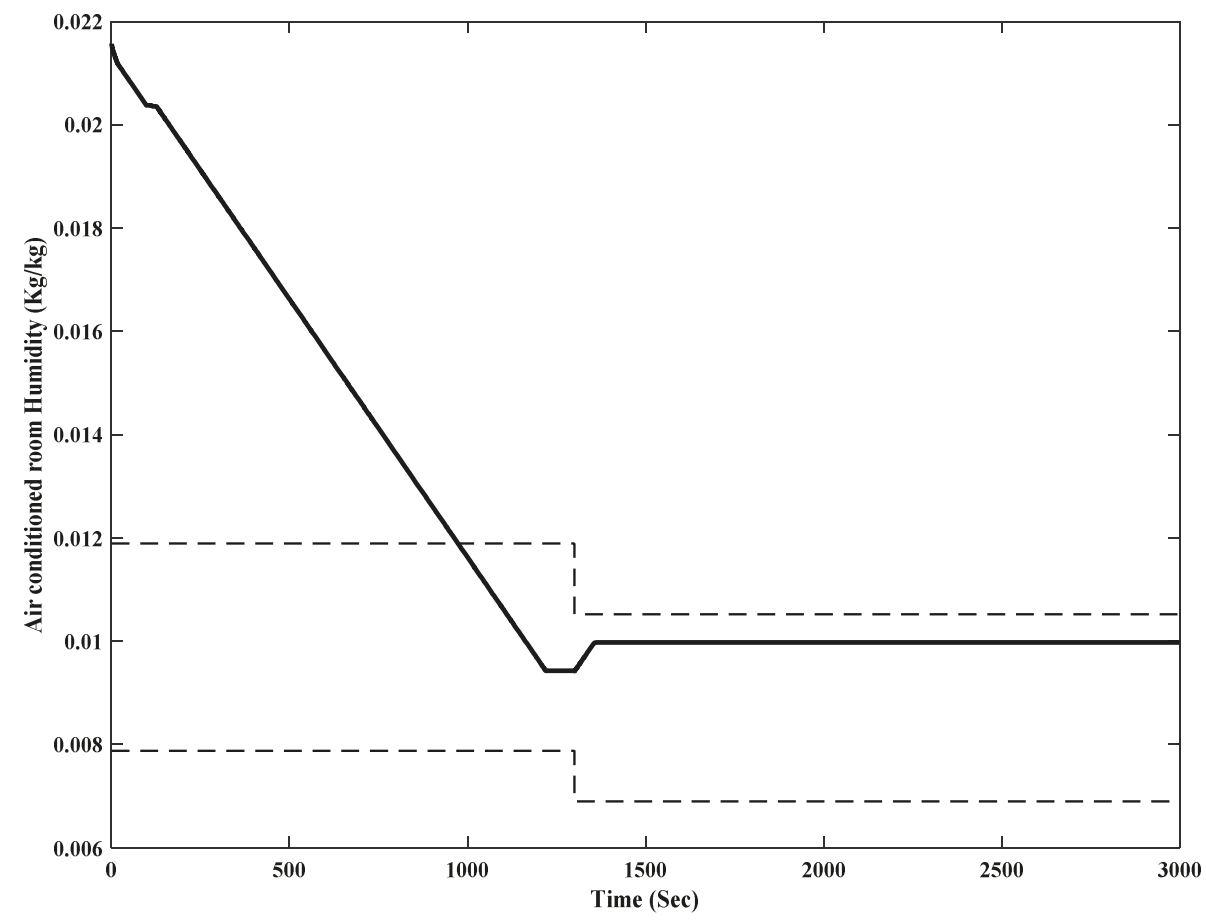

Figure 12. Humidity of the air-conditioned room in set point changing.

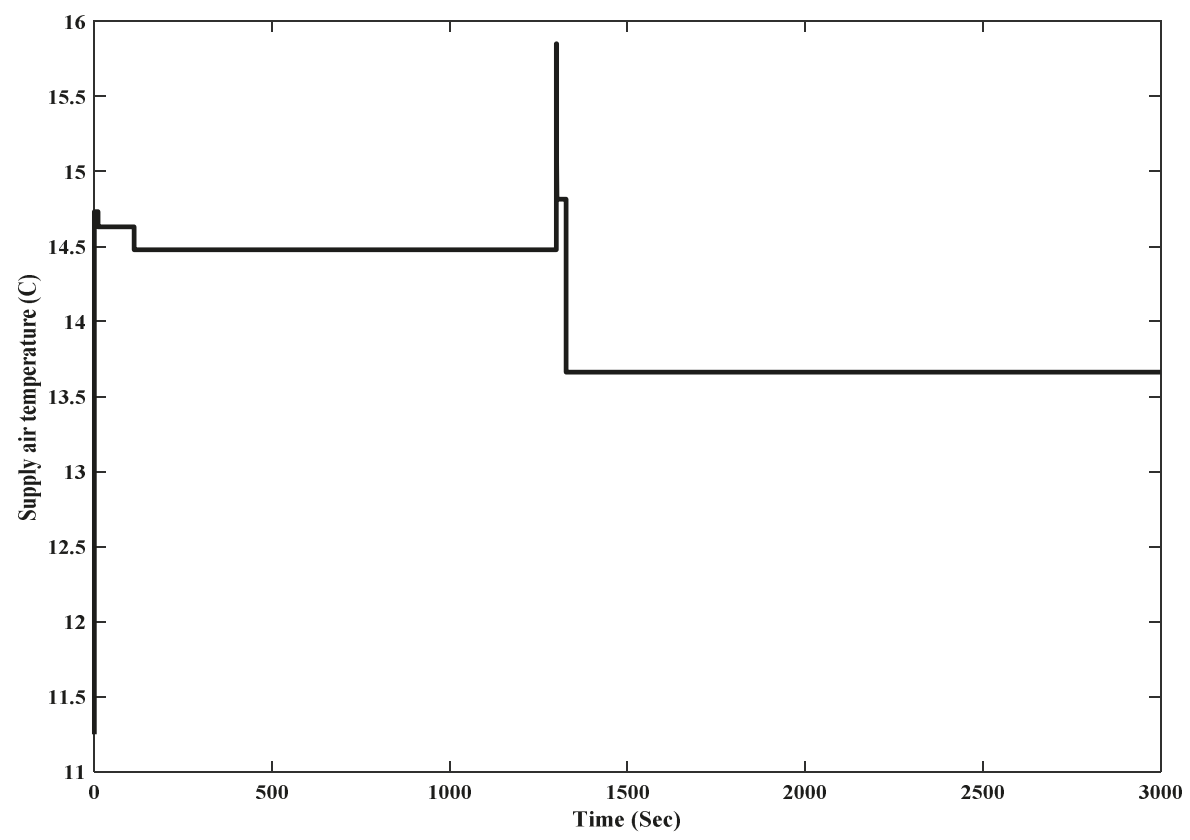

Figure 13. Supply temperature exit from evaporator in set point changing. 


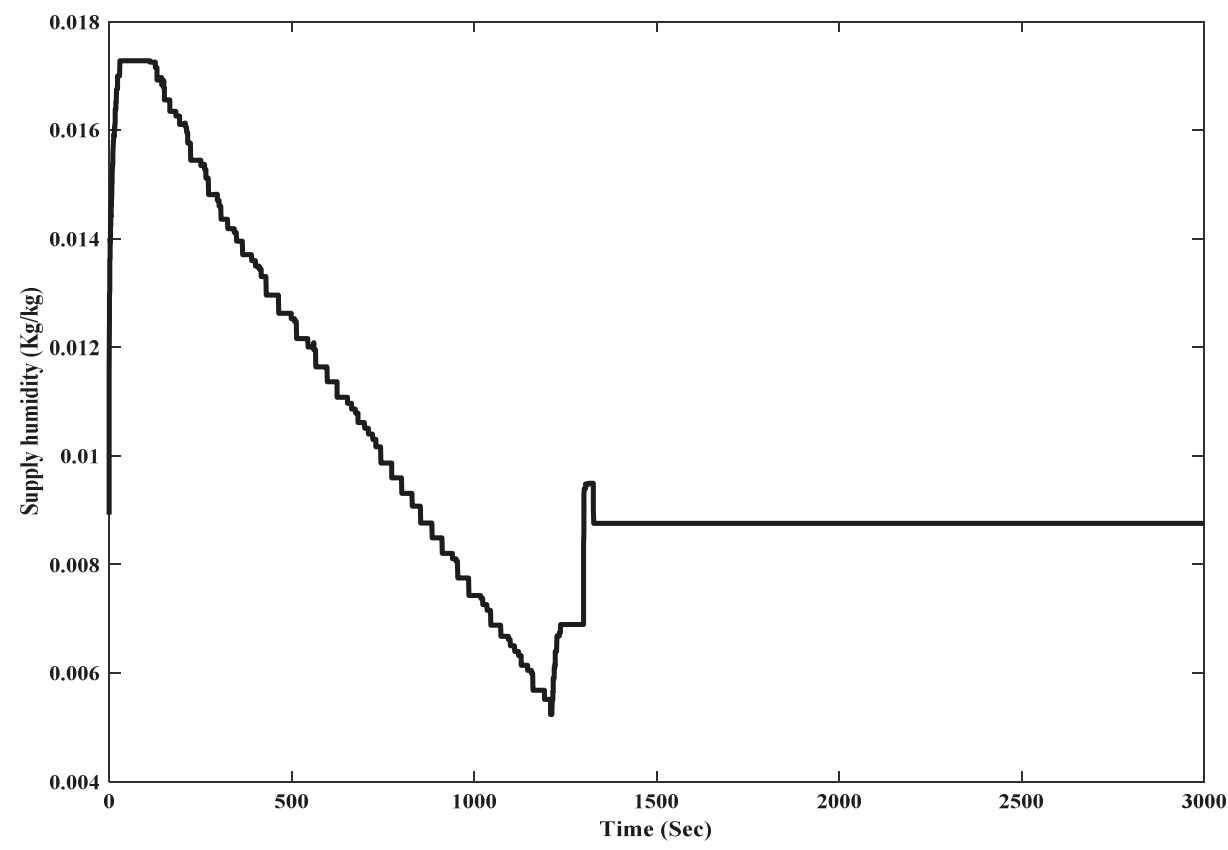

Figure 14. Supply humidity exit from evaporator in set point changing.

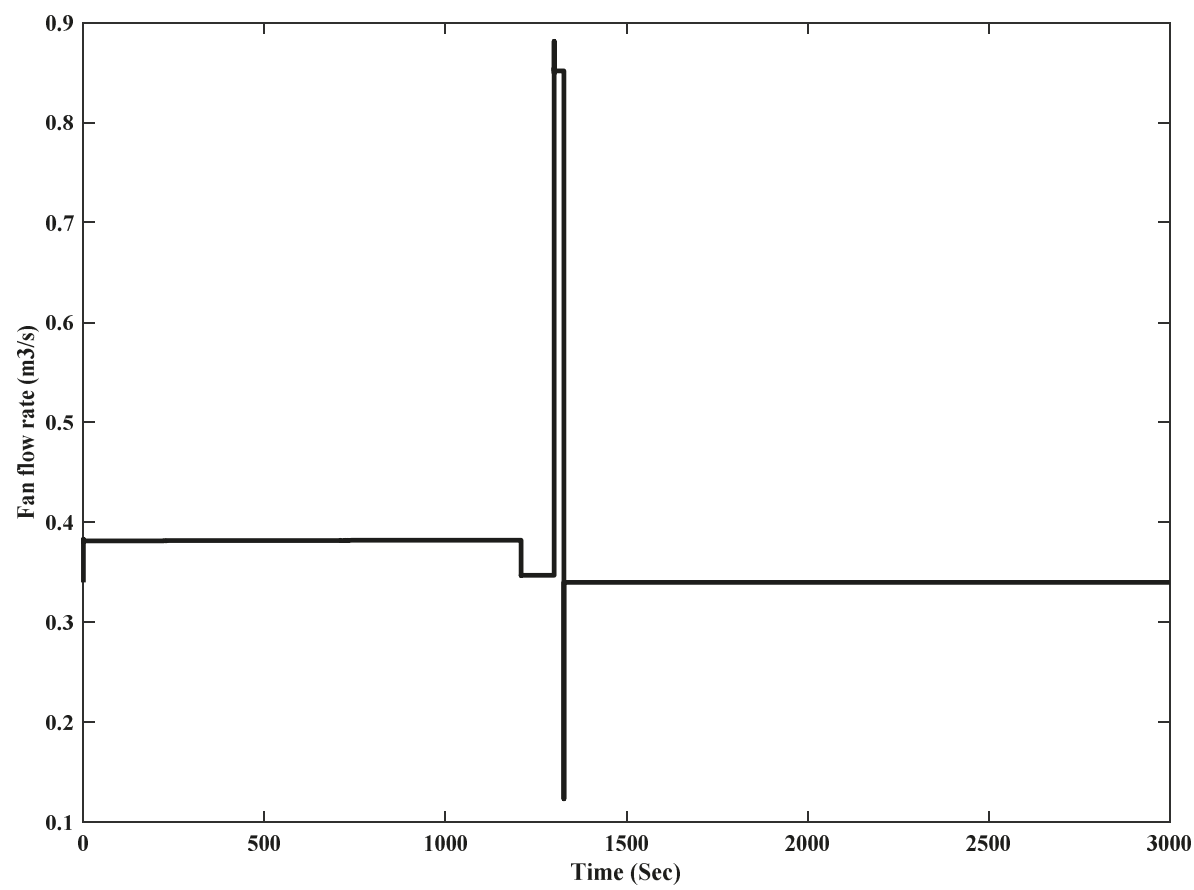

Figure 15. Supply flow rate by fan in set point changing. 


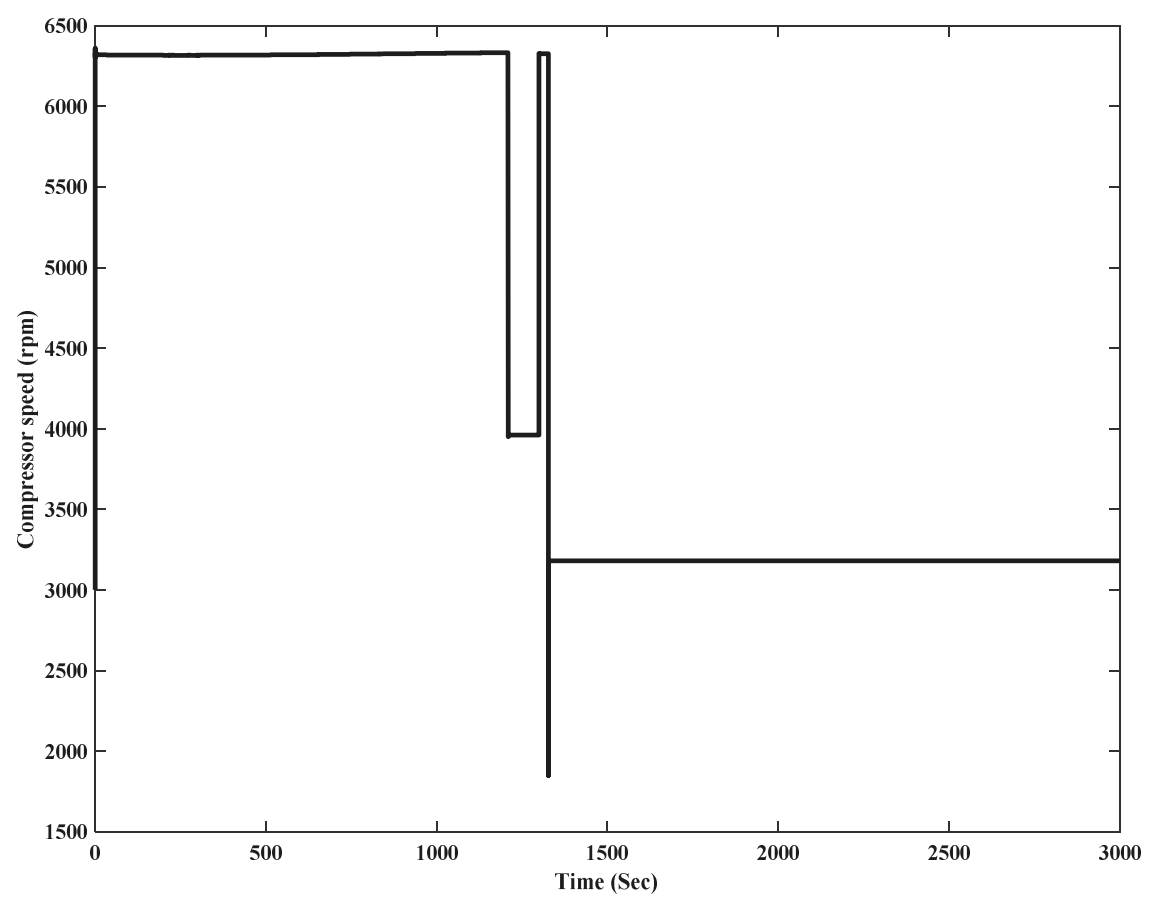

Figure 16. Compressor speed in set point changing.

\subsection{Disturbance Rejection}

The disturbance rejection test results emphasize the controller qualification for controlling the situation and maintaining the system outputs in preferred set points during the occurrence of disturbances. In this situation, changing the flow rate of the fan and speed of compressor allows for controlling the situation of the air-conditioned room temperature and humidity, and maintaining them at preferred set points. In order to test the disturbance rejection ability of the controller, at $2000 \mathrm{~s}$, the heat load changed from $4.49 \mathrm{~kW}$ to $5.49 \mathrm{~kW}$ and moisture load changed from $0.96 \mathrm{Kg} / \mathrm{s}$ to $1.6 \mathrm{Kg} / \mathrm{s}$. The temperature of the air-conditioned room in disturbance rejection test is indicated in Figure 17. At $2000 \mathrm{~s}$, the temperature of the air-conditioned room increased gradually; then at around $2050 \mathrm{~s}$, the temperature declined slowly; and finally, at $2100 \mathrm{~s}$, it stabled at $25.6^{\circ} \mathrm{C}$, which is in the thermal comfort range. As indicated in Figure 18, the humidity of the air-conditioned room went up gradually from $0.009863 \mathrm{~kg} / \mathrm{Kg}$ at $2000 \mathrm{~s}$ to $0.01008 \mathrm{~kg} / \mathrm{Kg}$ at around $2050 \mathrm{~s}$, then it picked up to $0.0101 \mathrm{~kg} / \mathrm{Kg}$ and then stabilized. Figure 19 shows the supply air temperature, which sharply reached $14.73^{\circ} \mathrm{C}$ in $4.03 \mathrm{~s}$, and then became stable until $2054 \mathrm{~s}$, where it sharply increased to $14.87^{\circ} \mathrm{C}$ and maintained. Supply humidity increased to $0.007986 \mathrm{~kg} / \mathrm{Kg}$ from $2000 \mathrm{~s}$ to $2061 \mathrm{~s}$, and then decreased gradually to 0.007735 at $2111 \mathrm{~s}$ and stabilized. Figure 20 illustrates the supply humidity. In Figure 21, the supply fan flow rate indicates that at $2000 \mathrm{~s}$, the flow rate by fan went upwards sharply from $0.341 \mathrm{~m}^{3} / \mathrm{s}$ to $0.4595 \mathrm{~m}^{3} / \mathrm{s}$ from $2046 \mathrm{~s}$ to $2066 \mathrm{~s}$ and maintained. Figure 22 shows the speed of the compressor, which increases from $4140 \mathrm{rpm}$ to $6332 \mathrm{rpm}$ at $2054 \mathrm{~s}$ to about $2066 \mathrm{~s}$ and stabilized. 


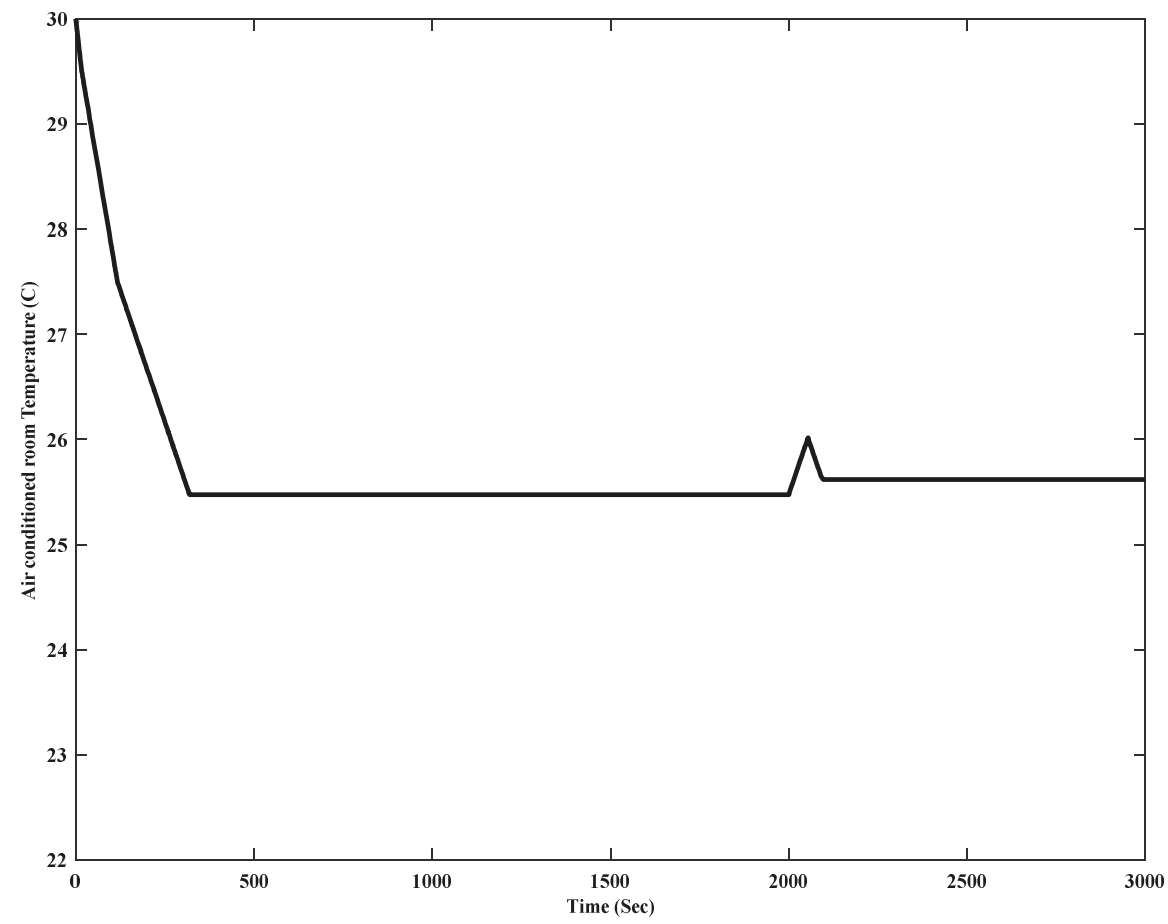

Figure 17. Temperature of the air-conditioned room in a disturbance rejection test.

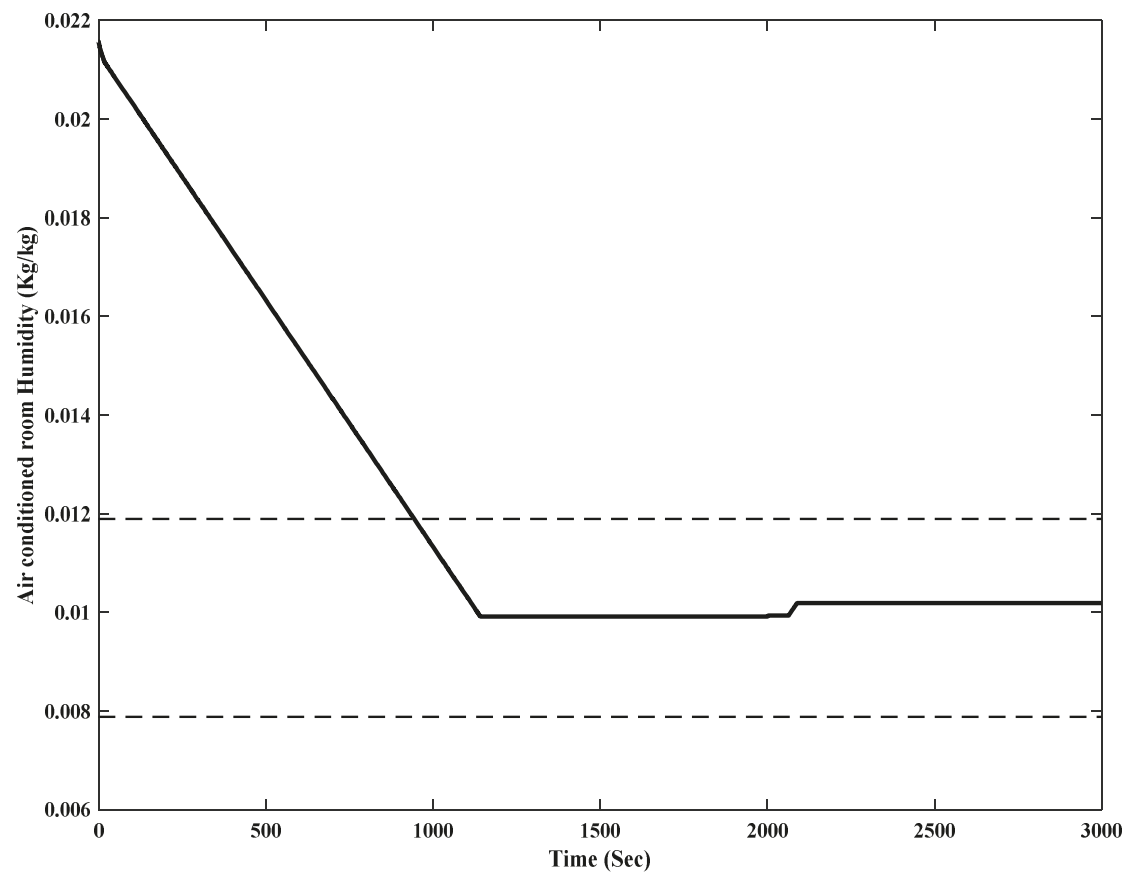

Figure 18. Humidity of the air-conditioned room in a disturbance rejection test. 


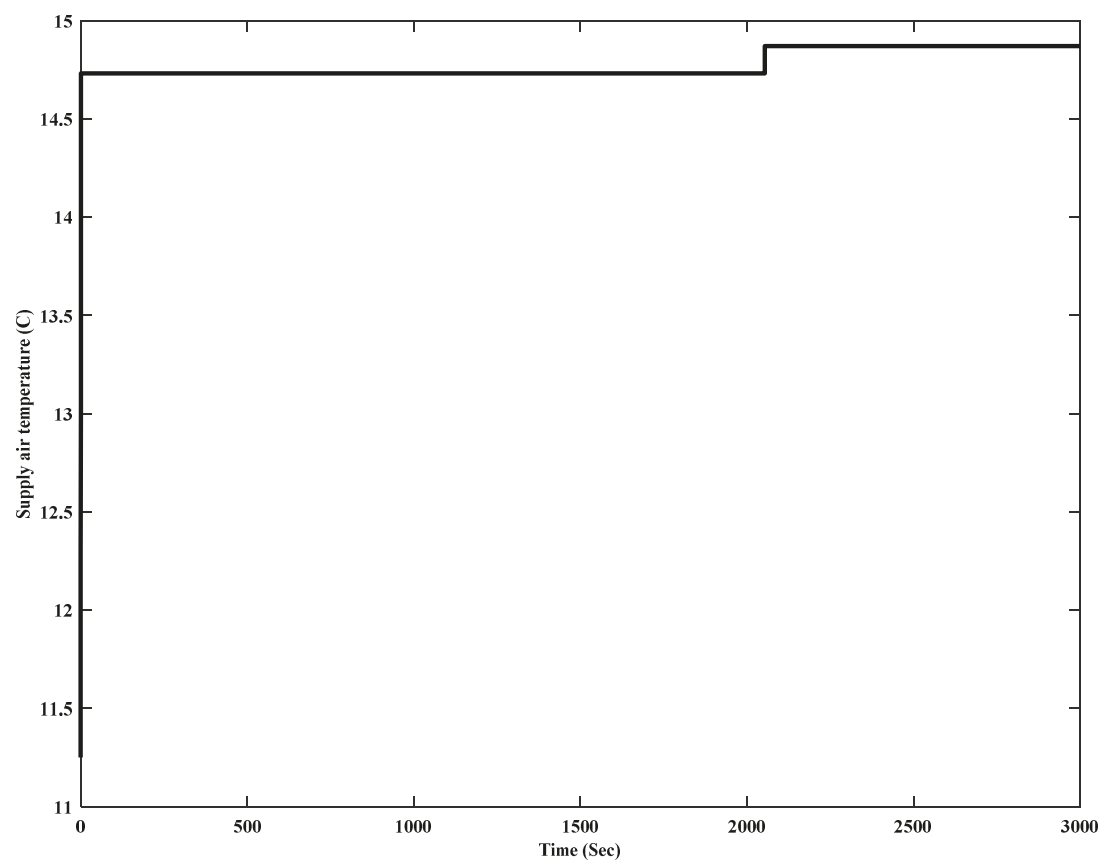

Figure 19. Supply air temperature exit from the evaporator in a disturbance rejection test.

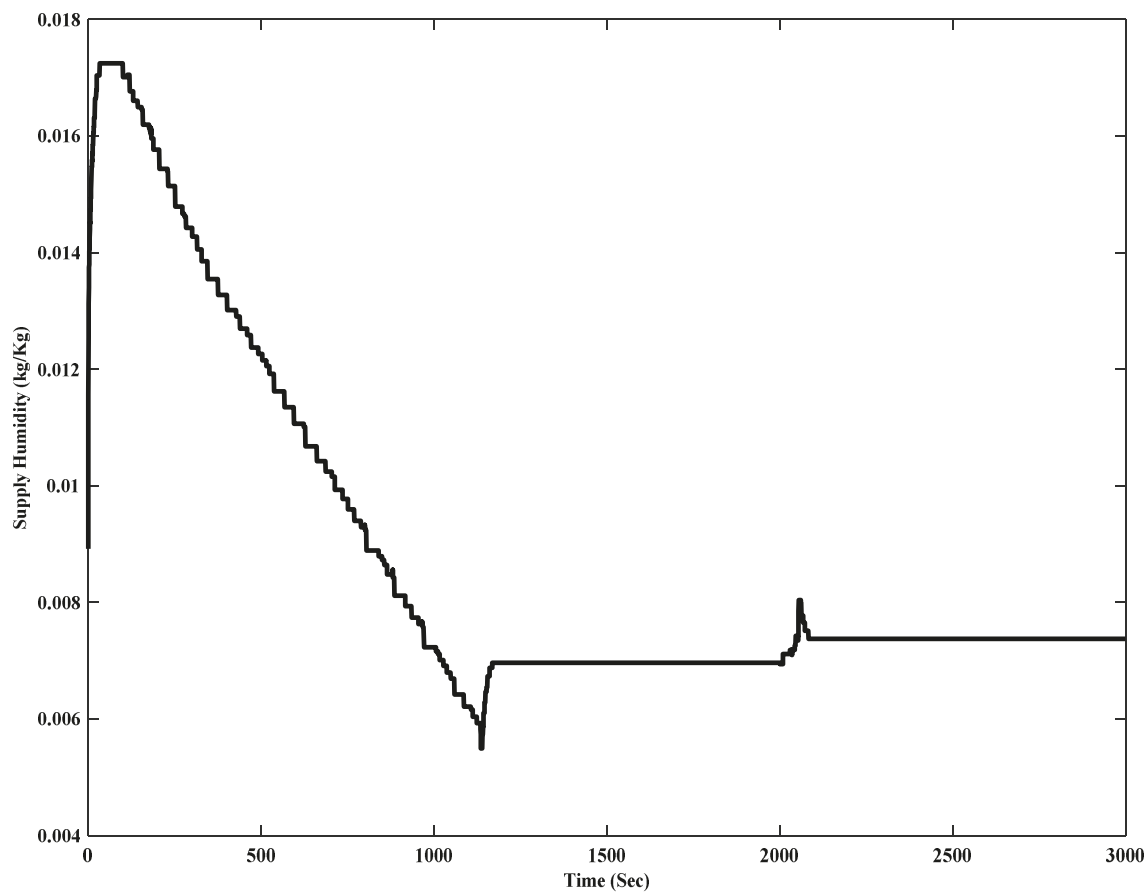

Figure 20. Supply humidity exit from the evaporator in a disturbance rejection test. 


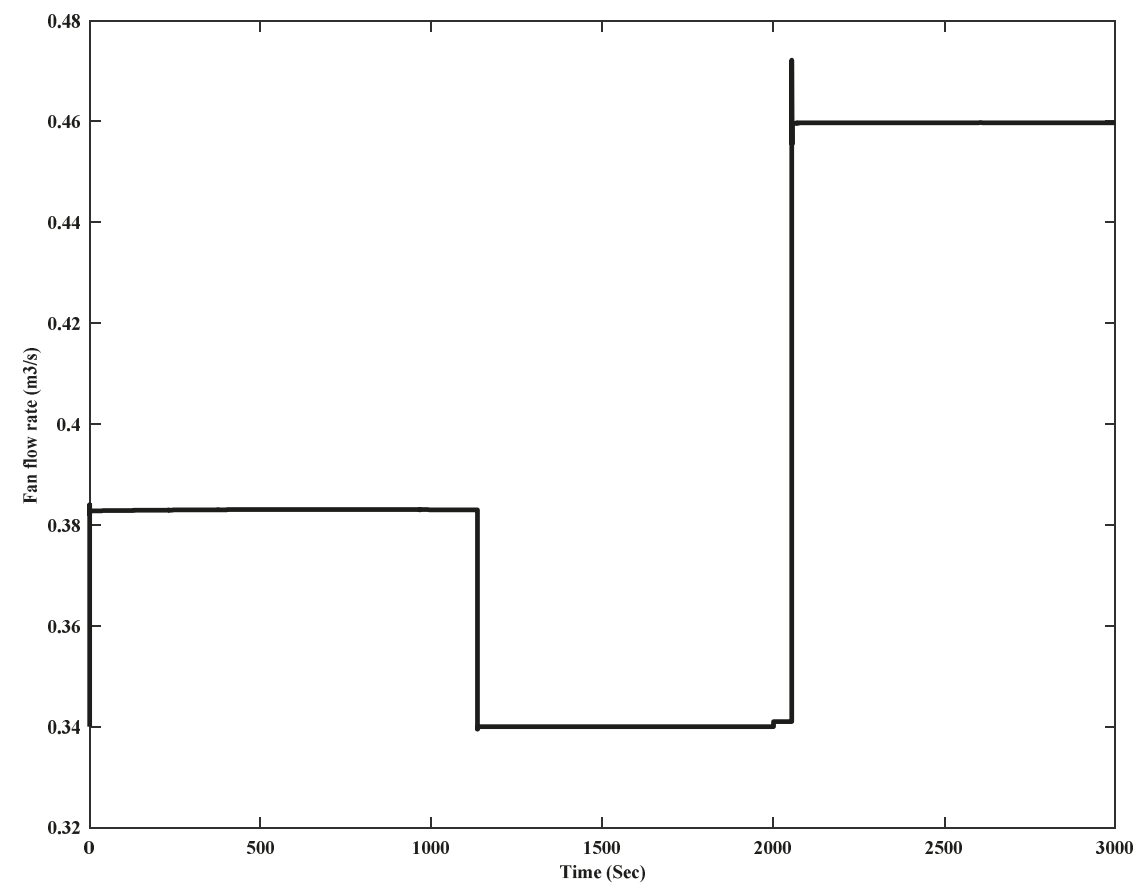

Figure 21. Flow rate of the fan in a disturbance rejection test.

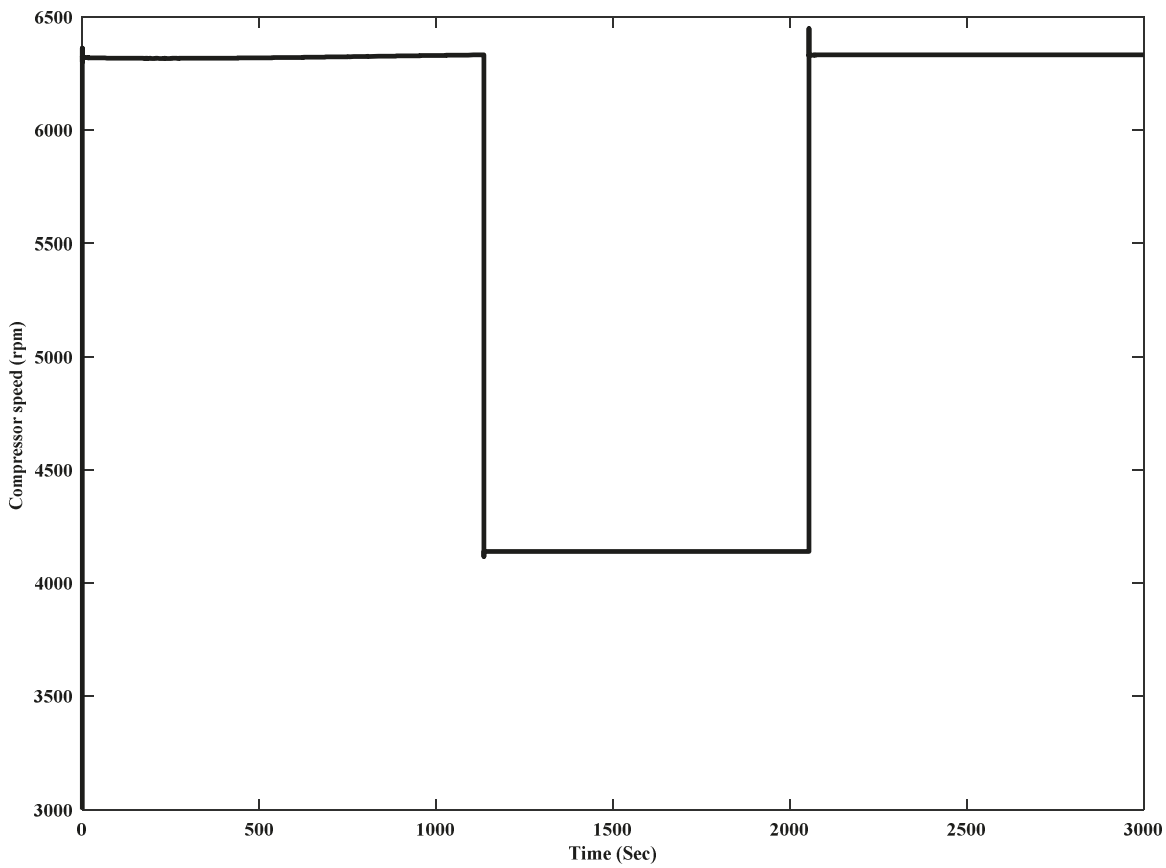

Figure 22. Speed of the motor compressor in a disturbance rejection test. 


\section{Discussion}

Because of the fact that the $\mathrm{A} / \mathrm{C}$ system is $\mathrm{MIMO}$, and in most previous works it is considered a SISO system, artificially decoupling two strongly coupled feedback loops results in poor transient control performance [22]. The FCM construction has the ability to consider the coupled variables, and accordingly, the accuracy and sensitivity of control can be increased. In addition, the MIMO controllers have frequently been applied on the linearized model of the system in neighbourhoods of the operating point for simplicity. In other words, the system can merely work and be stable nearby a specific operating point. Thus, the controller cannot be implemented in a wide operating range. In contrast, the MIMO FCM based controller works on a wide operating range. Subject to HVAC and A/C systems, applying the nonlinear control methods was restricted, because of the difficult procedure to find the Lyapunov function, using of the entire states of the system, which causes the nonlinear observer requirement and difficult mathematical analysis. Because of having the complex and uncertain system, the need for a simple control algorithm and simple mathematics is feasible. As regards to the FCM structure and its ability for designing the controller on the basis of the system requirements and what outcomes are expected from the system, not in what way it works, the FCM method could be an appropriate solution for HVAC and A/C systems. The FCM method is able to consider the mentioned requirements of the system such as coupled parameters, $\mathrm{MIMO}$, and nonlinear structures, in a single control structure. Not only the simple graph structure of the FCM method for designing the controller, but also the simple mathematics of the FCM method, are beneficial for obtaining the required control signals values with the intention of reaching the desired set points. In other words, the improvements by applying the FCM controller are avoiding complex and difficult mathematical analysis to design the controller; working in a wide operating range; making the nonlinear control design simple by observer elimination; decoupling cancellation and improving the sensitivity and accuracy of the system; reducing energy usage and improving energy efficiency; cancelling the overshoots and the undershoots due to the flexible and adaptable structure of the FCM; improving the transient behaviour of the system due to flexibility, adaptability, and convergence tendency of FCM; and using single control algorithm to integrate the DX A/C system's characteristics.

This section compares the results achieved by the designed FCM controller with the LQG controller by the authors of [22]. In order to compare the FCM controller with the LQG controller, the results of the both controller on the linear model of the system around $24^{\circ} \mathrm{C}$ are compared in this section. Both controllers were tested on the linear model of the DX A/C system in initial room temperature of $24{ }^{\circ} \mathrm{C}$ and $50 \%$ room humidity or $0.0095 \mathrm{~kg} / \mathrm{Kg}$ humidity. The results of the LQG controller were adopted from the work of [22]. Figure 23 shows the results of the air-conditioned room temperature by applying LQG controller on linear model of the system around the operating point, Figure 24 shows the air-conditioned room humidity, Figure 25 shows the compressor speed, and Figure 26 shows the supply fan speed.

The air-conditioned room temperature is indicated in Figure 23. It is clearly seen that the temperature of the air-conditioned room stabilized around $24^{\circ} \mathrm{C}$. When the set point changed at $500 \mathrm{~s}$ to $23.5^{\circ} \mathrm{C}$, the air-conditioned room temperature declined from $24^{\circ} \mathrm{C}$ to $23.5^{\circ} \mathrm{C}$. Increasing the supply fan flow rate and compressor speed, the temperature of the air-conditioned room decreased from $24^{\circ} \mathrm{C}$ in $520 \mathrm{~s}$ to $23.5^{\circ} \mathrm{C}$ at around $2000 \mathrm{~s}$. The temperature reaches the preferred set point in $25 \mathrm{~min}$ with no error and is maintained at the desired set point.

The air-conditioned room humidity is illustrated in Figure 24. As it is clear from the figure that the air-conditioned room humidity stabilized around $0.00965 \mathrm{~kg} / \mathrm{Kg}$. By changing the set point in $500 \mathrm{~s}$ to $23.5^{\circ} \mathrm{C}$, the humidity of the air conditioned room declined from $0.0095 \mathrm{~kg} / \mathrm{Kg}$ to $0.0093 \mathrm{~kg} / \mathrm{Kg}$. The humidity of the air-conditioned room decreased from $0.0095 \mathrm{~kg} / \mathrm{Kg}$ to $0.0093 \mathrm{~kg} / \mathrm{Kg}$ in about $2750 \mathrm{~s}$ with $2.5 \%$ error from $50 \%$ humidity by varying of the compressor speed and supply fan flow rate. Then, the air-conditioned room humidity stabilized at $0.0093 \mathrm{~kg} / \mathrm{Kg}$. 


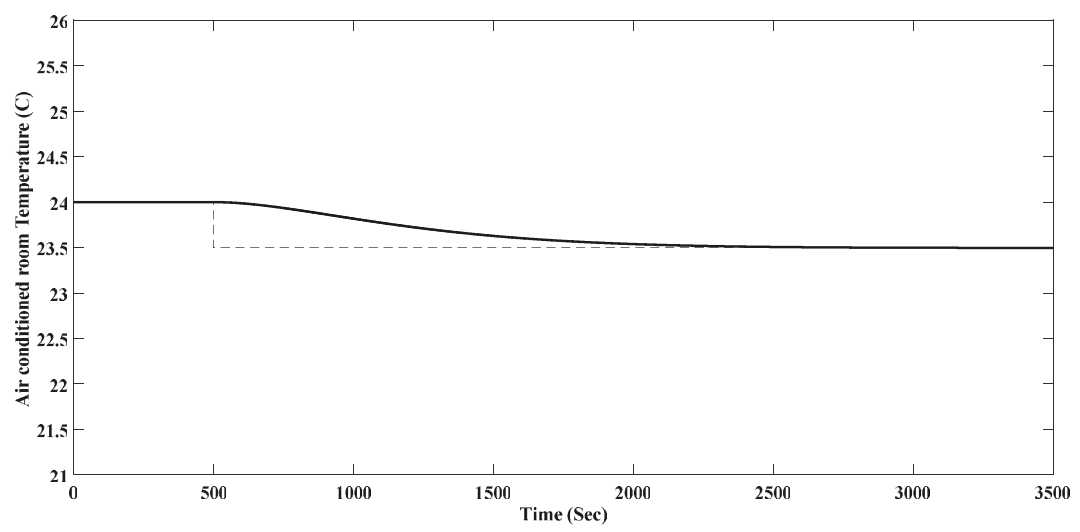

Figure 23. Simulation results of air-conditioned room temperature by applying the Linear Quadratic Gaussian (LQG) controller on the linear model of the system around the operating point.

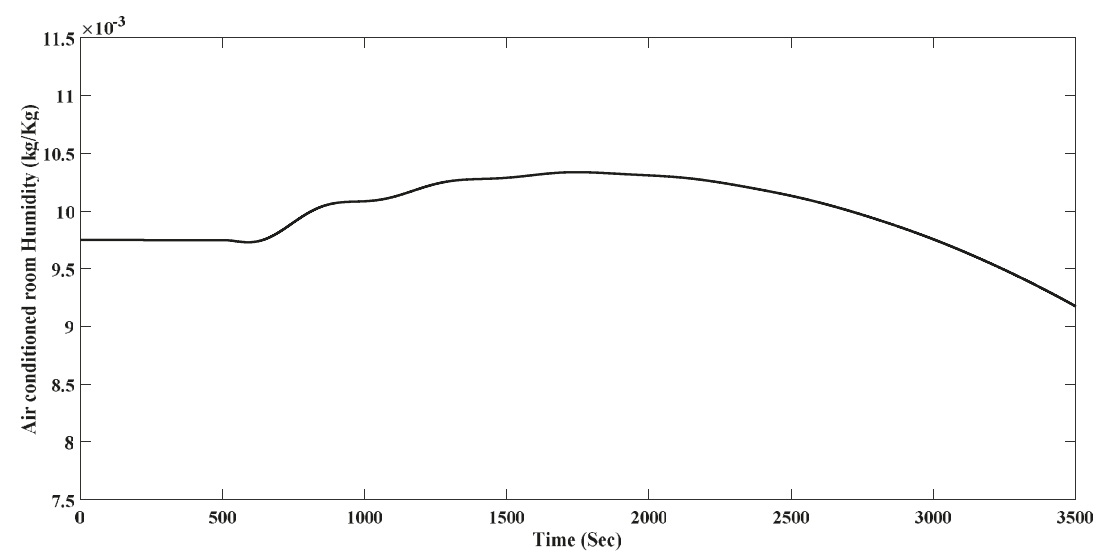

Figure 24. Simulation results of air-conditioned room humidity by applying the LQG controller on the linear model of the system around the operating point.

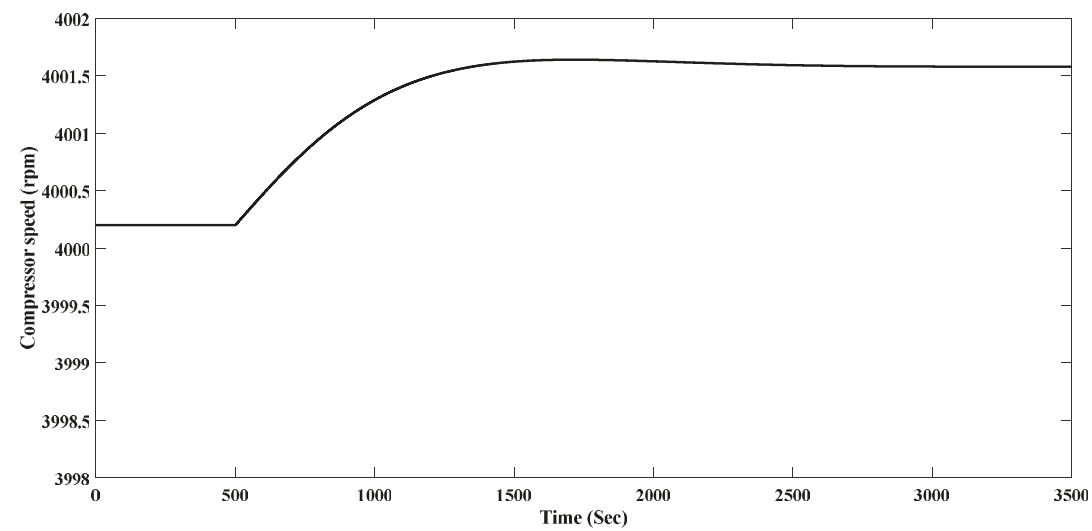

Figure 25. Simulation results of compressor speed by applying the LQG controller on the linear model of the system around the operating point. 
The speed of the compressor is depicted in Figure 25. Decline in the air-conditioned room temperature was obtained by increasing the compressor speed from $4000 \mathrm{rpm}$ to $4001.6 \mathrm{rpm}$ from $500 \mathrm{~s}$ to $1500 \mathrm{~s}$. In order to keep temperature and humidity of the room in preferred set points, the compressor speed maintained at $4001.6 \mathrm{rpm}$.

Figure 26 displays the supply fan speed. The fan speed raised from $2180 \mathrm{rpm}$ at $500 \mathrm{~s}$ to $2193 \mathrm{rpm}$ at $1500 \mathrm{~s}$ and maintained. While the air-conditioned room temperature achieved the preferred values, the fan speed maintained to the essential speed for keeping the air conditioned room humidity and temperature in chosen set points.

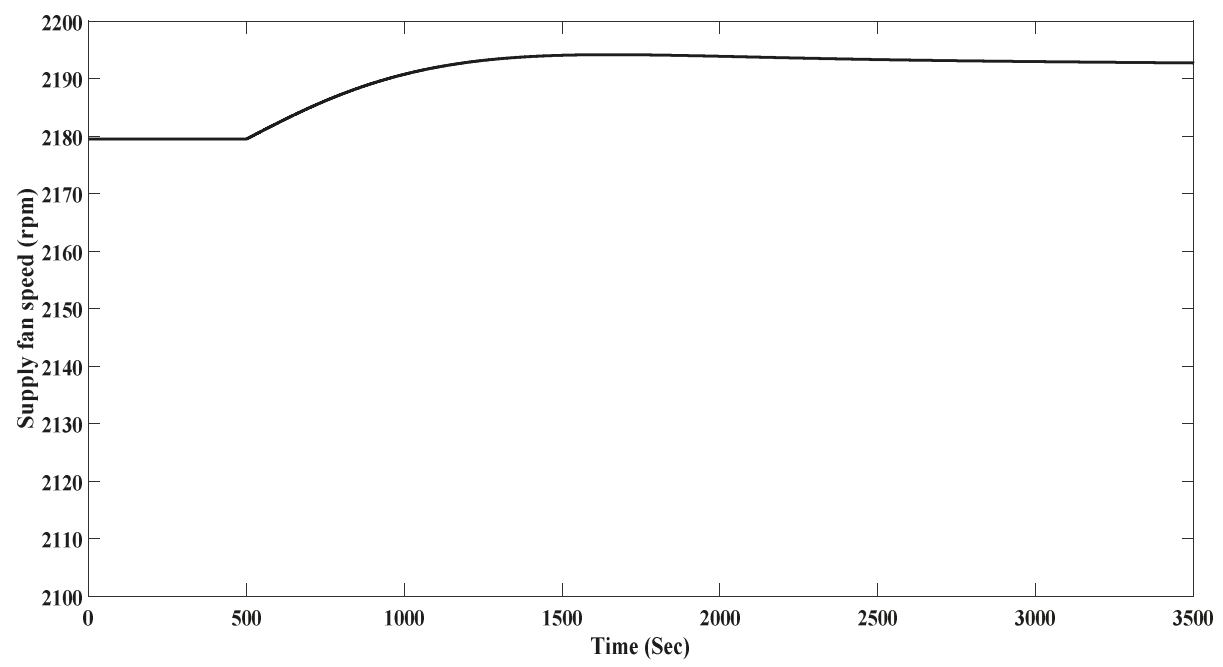

Figure 26. Simulation results of supply fan by applying the LQG controller on the linear model of the system around the operating point.

The results of the FCM controller on the linear model of the system around the operating point are shown as follows. The temperature of the air-conditioned room is indicated in Figure 27. It is clearly seen that the air-conditioned room temperature stabilized around $24{ }^{\circ} \mathrm{C}$. At $500 \mathrm{~s}$, the set point changed from $24{ }^{\circ} \mathrm{C}$ to $23.5^{\circ} \mathrm{C}$. Therefore, by changing the set point, the air-conditioned room temperature reduced by varying the supply fan flow rate and compressor speed. The air-conditioned room temperature reduced from $24{ }^{\circ} \mathrm{C}$ to $23.5^{\circ} \mathrm{C}$ at around $898 \mathrm{~s}$. The temperature stabilized in the preferred set point in $6.63 \mathrm{~min}$ with no error.

The humidity of the air-conditioned room is illustrated in Figure 28. As is clear from the figure, the air-conditioned room humidity stabilized around $0.0095 \mathrm{~kg} / \mathrm{Kg}$. By changing the set point in $500 \mathrm{~s}$ to $23.5^{\circ} \mathrm{C}$, the humidity of the air conditioned room declined from $0.0095 \mathrm{~kg} / \mathrm{Kg}$ to $0.0093 \mathrm{~kg} / \mathrm{Kg}$. By altering the supply fan flow rate and compressor speed, the air-conditioned room humidity reduced from $0.0095 \mathrm{~kg} / \mathrm{Kg}$ to $0.0093 \mathrm{~kg} / \mathrm{Kg}$ in about $1044 \mathrm{~s}$ with $1.25 \%$ error from $50 \%$ humidity. Then, the air-conditioned room humidity stabilized at $0.009257 \mathrm{~kg} / \mathrm{Kg}$ in about $1600 \mathrm{~s}$.

Figure 29 shows the speed of the compressor. For declining the temperature of the air-conditioned room, the compressor speed increased from $4000 \mathrm{rpm}$ to $4003 \mathrm{rpm}$ from $500 \mathrm{~s}$ to $689 \mathrm{~s}$. By declining the air-conditioned room temperature to the desired value, the speed of the compressor decreased and maintained at $4001 \mathrm{rpm}$ at $803 \mathrm{~s}$ to keep the air-conditioned room temperature and humidity in desired set points. 


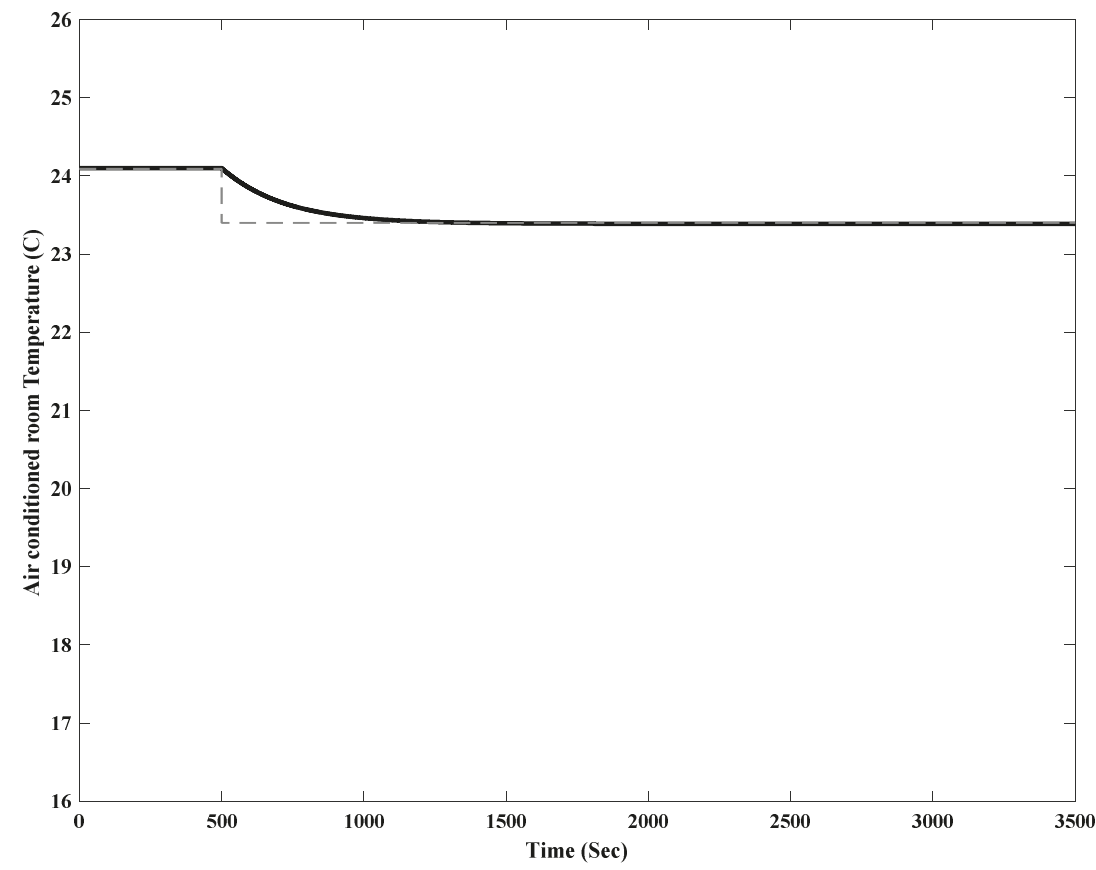

Figure 27. Simulation results of air-conditioned room temperature by applying the FCM controller on the linear model of the system around the operating point.

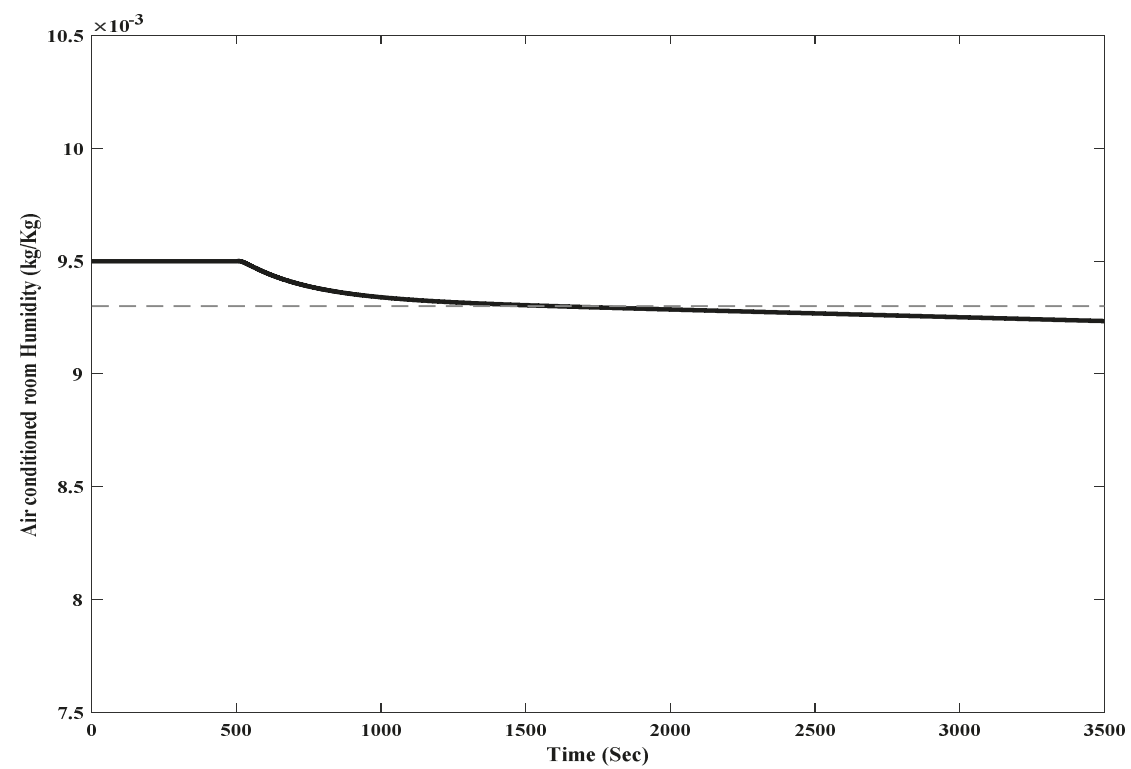

Figure 28. Simulation results of air-conditioned room humidity by applying the FCM controller on the linear model of the system around the operating point. 
The supply fan speed is shown in Figure 30. The fan speed increased from $2180 \mathrm{rpm}$ at $500 \mathrm{~s}$ to 2317 $\mathrm{rpm}$ at $1056 \mathrm{~s}$ and, after reaching the preferred values, it reduced to $2268 \mathrm{rpm}$ at $1779 \mathrm{~s}$ and maintained. After reaching the chosen set point for temperature of the room, at $1056 \mathrm{~s}$, the fan speed started to decline until it met the essential speed to keep the temperature and humidity of the air-conditioned room in chosen set points. The fan speed reduced to $2268 \mathrm{rpm}$ at $1779 \mathrm{~s}$ and maintained this speed.

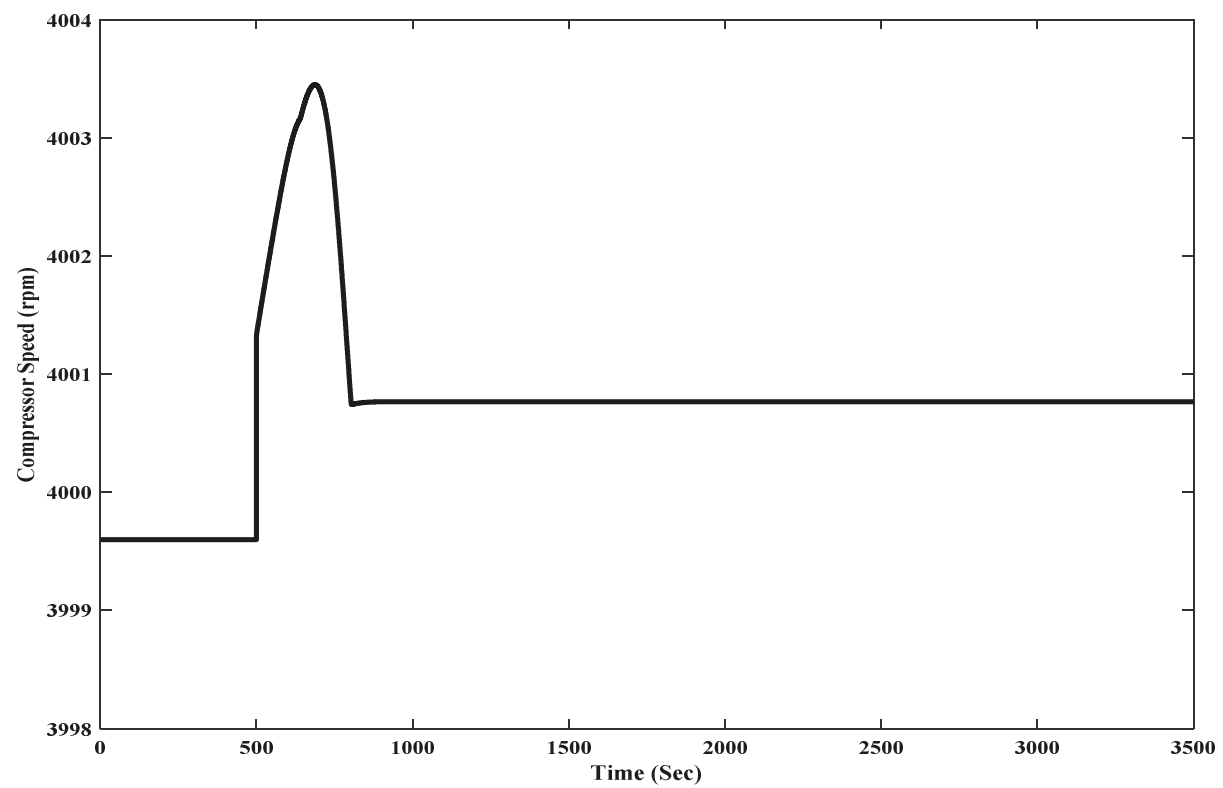

Figure 29. Simulation results of compressor speed by applying the FCM controller on the linear model of the system around the operating point.

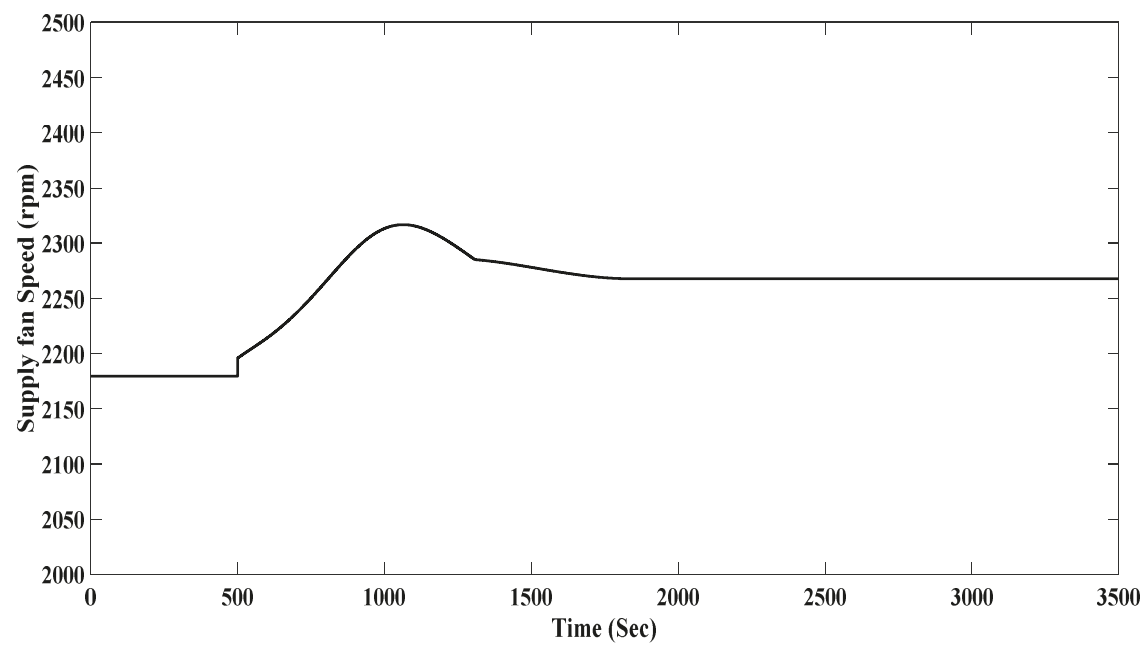

Figure 30. Simulation results of supply fan speed by applying the FCM controller on the linear model of the system around the operating point. 
The comparison between both controllers shows that the FCM controller managed to reach the desired set point for temperature of the air-conditioned room in $6.63 \mathrm{~min}$ with no error, while the LQG one managed to do so in 25 min with no error. In addition, the humidity of the air-conditioned room by the FCM controller reaches the desired set point after $544 \mathrm{~s}$ with $1.25 \%$ error, but the LQG one reaches the desired set point after $2250 \mathrm{~s}$ with $2.5 \%$ error. The benefits and shortcomings of both control methods are discussed as follows. Although the LQG controller has attractive features of thermal comfort and energy savings, rapid response [15], increased energy savings [15], and multivariable control [15], the disadvantages of the LQG control method are as follows.

- It was applied on the linear model of the system. Thus, it is not applicable for a wider operating range.

- $\quad$ The method is inherently complex. As a result, the optimal feedback control's evaluation and online implementation turn into a daunting challenge.

- The need to the model of the system. Thus, the appropriate model of the system should be identified [15].

On the other hand, the FCM controller avoids complex and difficult mathematical analysis to design the nonlinear controller. It works in a wide operating range and simplifies the designing of the nonlinear controller by removal of the observer. It improves the sensitivity and accuracy of the system by keeping the coupled structure of the system. The FCM controller is designed in a single control scenario for consideration of the system's characteristics. However, this control method is quite sensitive to the initial state values and to the type of concepts used in the FCM model, and it can give different results as they are different from and non-compatible with classic fuzzy.

The comparison of the two techniques' performances could be assessed by evaluating criteria such as integral square error (ISE), integral of absolute value of error (IAE), and integral of time-multiplied absolute value of error (ITAE). For these tests, the two controllers were tested under the same condition. The LQG controller was tested in initial room temperature of $30{ }^{\circ} \mathrm{C}$ and $80 \%$ room humidity or $0.02157 \mathrm{~kg} / \mathrm{Kg}$ humidity, and a desired temperature of $25^{\circ} \mathrm{C}$ and $50 \%$ humidity for comparison with the proposed FCM controller. Figure 31 shows the temperature of the air-conditioned room, which declined from $30{ }^{\circ} \mathrm{C}$ to $22.88{ }^{\circ} \mathrm{C}$ in $380 \mathrm{~s}$, and then after oscillation around $25^{\circ} \mathrm{C}$, it stabilized at $1200 \mathrm{~s}$ with small error. Figure 32 illustrates the humidity of the air-conditioned room, which reduced from $0.022 \mathrm{~kg} / \mathrm{Kg}$ to $0.008 \mathrm{~kg} / \mathrm{Kg}$, and it was unable to stabilize at certain values, which makes the system unstable. It is obviously seen that the LQG controller does not have the ability to control the humidity. The LQG controller is only able to control the condition around the operating point.

Table 2 shows the performance indexes results for both controllers based on the room temperature error. Table 3 indicates the performance indexes results for both controllers based on the room humidity error.

It is obvious from the results that the FCM controller has the ability to reduce the performance index. This means that for the real conditions of the room, the FCM controller works better than the LQG one. In other words, the FCM controller is more suitable and applicable. Also, the LQG controller was not able to control different conditions from the operating point and shows instability in the system. In other words, the LQG controller is severely dependant on the operating point, and changing the operating point makes the system unstable. In conditions where the controller is more dependent on the operating point, there is a need to design separate controllers for each operating point in the system. On the other hand, the Kalman filter in the LQG structure is suitable for linear systems or the linearized model of the nonlinear systems. As a result, working in wider operating points requires designing controllers for every operating point or using the multimodal controllers, or applying the extended Kalman filter (EKF) for calculating the operating point of the system in every second and extracting the required system parameters. By using each option in order to work in wider operating points, the volume of calculation is increased or designing the controller becomes complicated. The proposed FCM controller is not dependent on the operating point, and hence there is not a need to design more controllers at different operating points. 


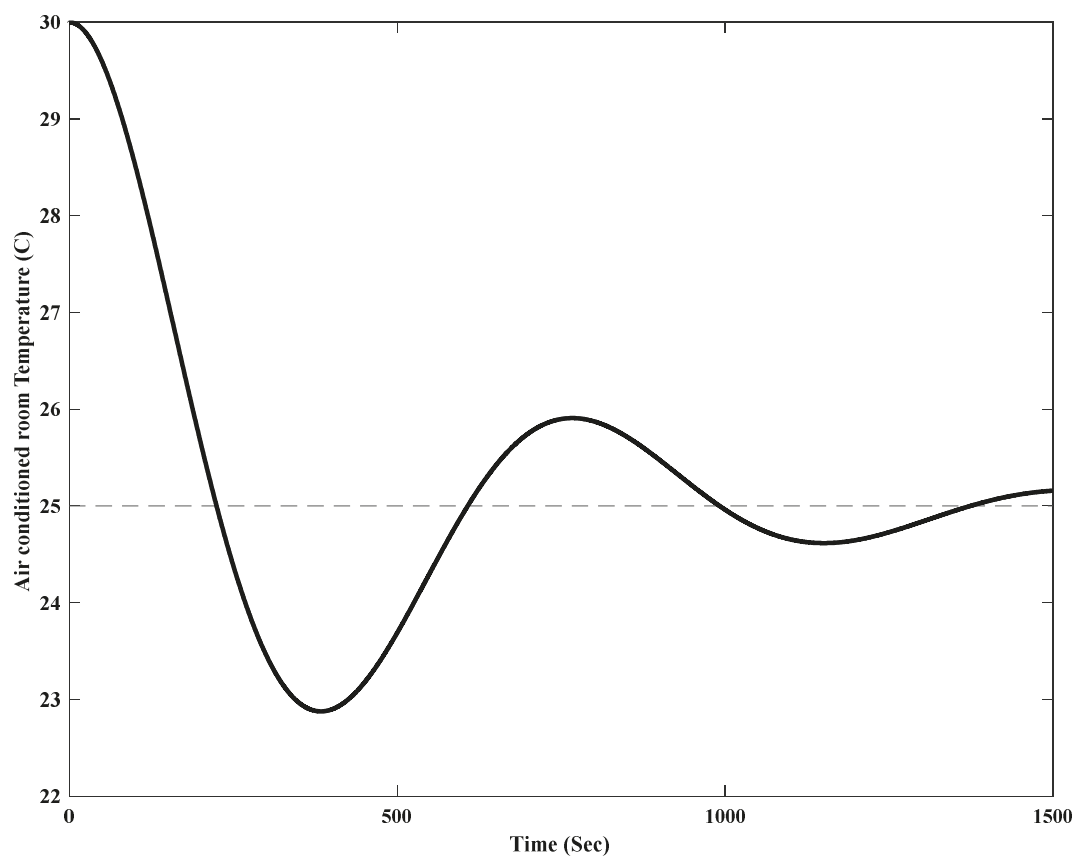

Figure 31. Temperature of the air-conditioned room by the LQG controller.

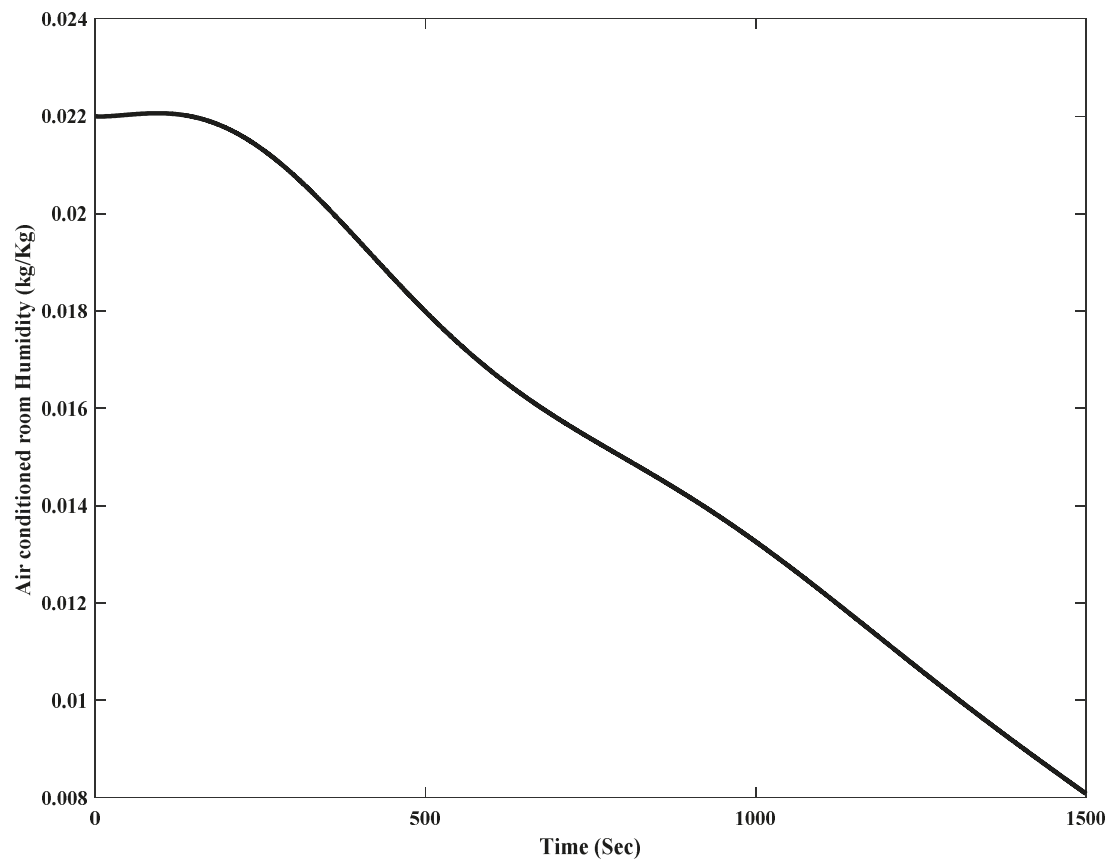

Figure 32. Humidity of the air-conditioned room by the LQG controller. 
Table 2. Results of the performance indexes for both controllers based on the room temperature error.

\begin{tabular}{cccc}
\hline \multirow{2}{*}{ Controller } & \multicolumn{3}{c}{ Performance index } \\
\cline { 2 - 4 } & ISE & IAE & ITAE \\
\hline LQG & $2.308 \times 10^{4}$ & 4096 & $1.763 \times 10^{6}$ \\
\hline FCM & 2857 & 1576 & $8.217 \times 10^{5}$ \\
\hline
\end{tabular}

Table 3. Results of the performance indexes for both controllers based on the room humidity error.

\begin{tabular}{cccc}
\hline \multirow{2}{*}{ Controller } & \multicolumn{3}{c}{ Performance index } \\
\cline { 2 - 4 } & ISE & IAE & ITAE \\
\hline LQG & 0.0602 & 7.719 & 3457 \\
\hline FCM & 0.05038 & 6.699 & 2674 \\
\hline
\end{tabular}

\section{Conclusion}

In this technical research, a new intelligent FCM control system was designed on the DX A/C system. This FCM control system could be easily designed for HVAC systems. The offered FCM controller is advantageous because of the graph structure of the FCM method, as well as its simple mathematics. Therefore, the FCM controller avoids the complex mathematical analysis, which is required to design the controller for a MIMO and nonlinear system with a complex structure. The proposed FCM controller met the requirements of designing a suitable controller for the DX A/C system. The requirements for designing the controller are consideration of nonlinearity, coupling effect, and MIMO structure, which are all considered in the offered FCM control method. The significance of this research is offering a new intelligent MIMO nonlinear controller for the DX A/C system. According to the results of the controller, the offered control system was designed easily and applied efficiently. The results obviously indicate the efficiency of the controller in the reference tracking signal. It is not successful only in the normal conditions but also in the occurrence of the heat and moisture loads disturbances works successfully. Designing the controller based on the FCM method does not require the mathematical model of the system and the suitable model of the system not like MPC method. It does not involve difficult mathematic analysis to derive the control law opposite to designing the nonlinear control or adaptive control methods. It has a wide operating range, unlike the MIMO controller, and it does not have overshoot and undershoot, which is different from the PID control method. Moreover, the stability of the FCM control method was proven by the Lyapunov function, as well as the FBAMs method. Therefore, the FCM control method can be designed for different nonlinear MIMO systems. Furthermore, it can be a suitable solution for designing the controller for the systems with no mathematical model or complex mathematical models.

Author Contributions: Conceptualization, Methodology, Software, Validation, Formal Analysis, Investigation, and Writing-Original Draft Preparation have done by F.B. Writing-Review \& Editing have done by R.Y., U.K. and Z.H.I. Supervision has done by N.M. Project Administration is R.Y. Funding Acquisition has done by U.K.

Funding: This research received no external funding.

Acknowledgments: The article has supported by the Universiti Teknologi Malaysia to publish in open access. I would like to thank Hossein Eliasi, Mohd Amran Mohd Radzi, Abdul Rahman Ramli and Mohammad Hamiruce Marhaban for their guidance in this research.

Conflicts of Interest: The authors declare no conflict of interest.

\section{References}

1. Tachwali, Y.; Refai, H.; Fagan, J. Minimizing HVAC Energy Consumption Using a Wireless Sensor Network. In Proceedings of the IECON 2007 33rd Annual Conference of the IEEE Industrial Electronics Society, Taipei, Taiwan, 5-8 November 2007; pp. 439-444. 
2. Tashtoush, B.; Molhim, M.; Al-Rousan, M. Dynamic model of an HVAC system for control analysis. Energy 2005, 30, 1729-1745. [CrossRef]

3. Qi, Q.; Deng, S.S. Multivariable control-oriented modeling of a direct expansion (DX) air conditioning (A/C) system. Int. J. Refrig. 2008, 31, 841-849. [CrossRef]

4. Qi, Q.; Deng, S.S. Multivariable control of indoor air temperature and humidity in a direct expansion (DX) air conditioning (A/C) system. Build. Environ. 2009, 44, 1659-1667. [CrossRef]

5. Wang, J.; An, D.; Lou, C. Application of Fuzzy-PID Controller in Heating Ventilating and Air-Conditioning System. In Proceedings of the 2006 International Conference on Mechatronics and Automation, Luoyang, China, 25-28 June 2006; pp. 2217-2222.

6. Lei, J.; Hongli, L.; Cai, W. Model Predictive Control Based on Fuzzy Linearization Technique FOR HVAC Systems Temperature Control. In Proceedings of the 2006 1ST IEEE Conference on Industrial Electronics and Applications, Singapore, 24-26 May 2006; pp. 1-5.

7. Matysko, R. Theoretical model of the operation parameters regulated by the MIMO and SISO system in a cooling chamber. Int. J. Refrig. 2015, 58, 53-57. [CrossRef]

8. Huang, G. Model predictive control of VAV zone thermal systems concerning bi-linearity and gain nonlinearity. Control Eng. Pract. 2011, 19, 700-710. [CrossRef]

9. Lü, H.; Jia, L.; Kong, S.; Zhang, Z. Predictive functional control based on fuzzy T-S model for HVAC systems temperature control. J. Control Theory Appl. 2007, 5, 94-98. [CrossRef]

10. Mirinejad, H.; Ghasemian, M.; Torab, H.; Sadati, S.H. Control Techniques in Heating, Ventilating and Air Conditioning (HVAC) Systems. J. Comput. Sci. 2008, 4, 777-783. [CrossRef]

11. Venkatesh, S.; Sundaram, S. Intelligent Humidity Control for Healthy Home to Wealthy Industry: A Review. Res. J. Inf. Technol. 2012, 4, 73-84. [CrossRef]

12. Kassai, M. Experimental investigation on the effectiveness of sorption energy recovery wheel in ventilation system. Exp. Heat Transf. 2017, 31, 106-120. [CrossRef]

13. Gruber, P.; Balemi, S. Overview of non-linear control methods; Technical Report; Swiss society for automatic control: St. Gallen, Switzerland, 2010.

14. Afram, A.; Janabi-Sharifi, F. Theory and applications of HVAC control systems-A review of model predictive control (MPC). Build. Environ. 2014, 72, 343-355. [CrossRef]

15. Perera, D.W.U.; Pfeiffer, C.F.; Skeie, N.O. Control of temperature and energy consumption in building-A review. Int. J. Energ. Environ. 2014, 5, 471-484.

16. Kozák, Š. State-of-the-art in control engineering. J. Electr. Syst. Inf. Technol. 2014, 1, 1-9. [CrossRef]

17. Kassai, M.; Kajtar, L.; Nyers, J. Experimental optimization of energy consumption for DC refrigerator by PID controller tuning and comparison with On-Off refrigerator. Therm. Sci. 2019, 23, 941-952. [CrossRef]

18. Raut, K.H.; Vaishnav, S.R. A study on Performance of Different PID Tuning Techniques. In Proceedings of the International Conference on Electrical Engineering and Computer Science, Trivandrum, India, 12 May 2012; pp. 250-254.

19. Yan, Y.; Song, Y.; Wu, S. Control strategies for indoor environment quality and energy efficiency-A review. Int. J. Low-Carbon Technol. 2013, 10, 305-312.

20. Shiming, D. A dynamic mathematical model of a direct expansion (DX) water-cooled air-conditioning plant. Build. Environ. 2000, 35, 603-613. [CrossRef]

21. Shiming, D. The application of feedforward control in a direct expansion (DX) air conditioning plant. Build. Environ. 2002, 37, 35-40.

22. Qi, Q. Multivariable Control of Air Temperature and Humidity in a Space Served by Direct Expansion Air Conditioning System. Ph.D. Thesis, Polytechnic University, Hong Kong, China, 2009.

23. Papageorgiou, E.; Stylios, C.; Groumpos, P.; Papageorgiou, E. Active Hebbian learning algorithm to train fuzzy cognitive maps. Int. J. Approx. Reason. 2004, 37, 219-249. [CrossRef]

24. Huerga, A. A Balanced Differential Learning Algorithm in Fuzzy Cognitive Maps. In Proceedings of the 16th International Workshop on Qualitative Reasoning, Barcelona, Spain, 2 June 2002; pp. 10-12.

25. Khor, S.; Khan, M. Scenario Planning Using Fuzzy Cognitive Maps. In Proceedings of the ANZIIS2003 8th Australian and New Zealand Intelligent Information Systems Conference, Sydney, Australia, 10-12 December 2003; pp. 311-316.

26. Kosko, B. Fuzzy cognitive maps. Int. J. Man. Mach. Stud. 1986, 24, 65-75. [CrossRef] 
27. Axelord, R. Structure of Decision: The Cognitive Maps Political Elites; Princeton University Press: Princeton, NJ, USA, 1976.

28. Stylios, C.; Groumpos, P. Fuzzy cognitive maps in modeling supervisory control systems. J. Intell. Fuzzy Syst. 2000, 8, 83-98.

29. Kim, M.C.; Kim, C.O.; Hong, S.R.; Kwon, I.H. Forward-backward analysis of RFID-enabled supply chain using fuzzy cognitive map and genetic algorithm. Expert Syst. Appl. 2008, 35, 1166-1176. [CrossRef]

30. Bertolini, M. Assessment of human reliability factors: A fuzzy cognitive maps approach. Int. J. Ind. Ergon. 2007, 37, 405-413. [CrossRef]

31. Aguilar, J. A survey about fuzzy cognitive maps papers (Invited paper). Int. J. Comput. Cogn. 2005, 3, 27.

32. Behrooz, F.; Ramli, A.R.; Samsudin, K. A survey on applying different control methods approach in building automation systems to obtain more energy efficiency. Int. J. Phys. Sci. 2011, 6, 2308-2314.

33. Stylios, C.; Groumpos, P. Modeling Complex Systems Using Fuzzy Cognitive Maps. IEEE Trans. Syst. Man Cybern. Part A Syst. Hum. 2004, 34, 155-162. [CrossRef]

34. Behrooz, F.; Mariun, N.B.; Marhaban, M.H.; Radzi, M.A.; Ramli, A.R. New Design Approach to MIMO Nonlinear Controller for Direct Expansion Air Conditioning System in Building Automation System. In Proceedings of the 2015 IEEE 15th International Conference on Environment and Electrical Engineering (EEEIC), Rome, Italy, 10-13 June 2015; pp. 1706-1712.

35. Rossiter, A. Generalised Predictive Control, Videos on model predictive control. Creative Commons, USA: Attribution 2.0 UK: England \& Wales Licence, 2014; Vol. chapter 2. Available online: https: //www.sheffield.ac.uk/acse/people/jar/mpcmaster (accessed on 12 July 2019).

36. Behrooz, F.; Ramli, A.R.; Samsudin, K.; Eliasi, H. Energy saving by applying the fuzzy cognitive map control in controlling the temperature and humidity of room. Int. J. Phys. Sci. 2017, 12, 13-23.

37. Martchenko, A.S.; Ermolov, I.L.; Groumpos, P.P.; Poduraev, J.V.; Stylios, C.D. Investigating Stability Analysis Issues for Fuzzy Cognitive Maps. In Proceedings of the 11th Mediterranean Conference on Control and Automation MED'03, Rhodes, Greece, 18-20 June 2003.

38. Cheng, Q.; Fan, Z.T. The stability problem for fuzzy bidirectional associative memories. Fuzzy Sets. Syst. 2002, 132, 83-90. [CrossRef]

39. Ge, S. Lyapunov Design, in Knowledge Foundations; Eolss Publishers: Oxford, UK, 2004.

40. Cannon, M. Nonlinear systems. Lectures Hilary Term 2016. Available online: https://markcannon.github.io/ teaching/ (accessed on 12 July 2019).

41. Li, H.; Yang, B.; Xie, Y.; Qian, W.A. Multi-relationship Fuzzy Cognitive Map Stability Method. J. Inf. Comput. Sci. 2013, 10, 5373-5379. [CrossRef]

(C) 2019 by the authors. Licensee MDPI, Basel, Switzerland. This article is an open access article distributed under the terms and conditions of the Creative Commons Attribution (CC BY) license (http://creativecommons.org/licenses/by/4.0/). 
Article

\title{
A Pathway to Reduce Energy Consumption in the Thermal Stabilization Process of Carbon Fiber Production
}

\author{
Srinivas Nunna *, Maxime Maghe, Seyed Mousa Fakhrhoseini, Bhargav Polisetti \\ and Minoo Naebe * \\ Institute for Frontier Materials, Carbon Nexus, Deakin University, 75 Pigdons Road, Waurn Ponds, \\ Victoria 3217, Australia; maxime.maghe@deakin.edu.au (M.M.); sfakhrho@deakin.edu.au (S.M.F.); \\ bharg@deakin.edu.au (B.P.) \\ * Correspondence: srinivas.nunna@deakin.edu.au (S.N.); minoo.naebe@deakin.edu.au (M.N.)
}

Received: 11 April 2018; Accepted: 3 May 2018; Published: 4 May 2018

\begin{abstract}
Process parameters, especially in the thermal stabilization of polyacrylonitrile (PAN) fibers, play a critical role in controlling the cost and properties of the resultant carbon fibers. This study aimed to efficiently handle the energy expense areas during carbon fiber manufacturing without reducing the quality of carbon fibers. We introduced a new parameter (recirculation fan frequency) in the stabilization stage and studied its influence on the evolution of the structure and properties of fibers. Initially, the progress of the cyclization reaction in the fiber cross-sections with respect to fan frequencies $(35,45$, and $60 \mathrm{~Hz})$ during stabilization was analyzed using the Australian Synchrotron-high resolution infrared imaging technique. A parabolic trend in the evolution of cyclic structures was observed in the fiber cross-sections during the initial stages of stabilization; however, it was transformed to a uniform trend at the end of stabilization for all fan frequencies. Simultaneously, the microstructure and property variations at each stage of manufacturing were assessed. We identified nominal structural variations with respect to fan frequencies in the intermediate stages of thermal stabilization, which were reduced during the carbonization process. No statistically significant variations were observed between the tensile properties of fibers. These observations suggested that, when using a lower fan frequency $(35 \mathrm{~Hz})$, it was possible to manufacture carbon fibers with a similar performance to those produced using a higher fan frequency $(60 \mathrm{~Hz})$. As a result, this study provided an opportunity to reduce the energy consumption during carbon fiber manufacturing.
\end{abstract}

Keywords: microstructure; carbon fibers; polyacrylonitrile; thermal stabilization; recirculation fan frequency; IR imaging

\section{Introduction}

Carbon fibers play a significant role in the manufacture of lightweight composite structures for automotive, aerospace, and defense applications, mainly due to their high specific strength and high specific stiffness [1-4]. The manufacture of carbon fibers includes (1) polyacrylonitrile (PAN) fiber spinning, (2) the stabilization of PAN fibers in an air atmosphere between $200-300{ }^{\circ} \mathrm{C}$, (3) the low temperature carbonization of stabilized fibers in an inert atmosphere from $450-1000{ }^{\circ} \mathrm{C}$, and (4) the high temperature carbonization of fibers in an inert atmosphere up to $1600{ }^{\circ} \mathrm{C}$ or above [5-8]. Out of these steps, stabilization is considered to be the most time- and energy-consuming step, as it consumes almost $48 \%$ of energy during PAN to carbon fiber conversion $[7,9,10]$. It is accepted that along with precursor fiber quality, the stabilization of precursor fibers is critical in defining the carbon 
fiber properties [11]. The thermal stabilization process is an exothermic process in which the linear polymer structure in the precursor fibers transforms to the ladder structure [12,13], which is essential to withstand high temperatures during the carbonization process [14].

This process is predominantly controlled by three parameters; temperature, time, and fiber tension [15]. From an industrial perspective, recirculation air fan frequency is another parameter that plays a substantial role in the stabilization stage as it helps to precisely maintain the oven set temperatures by the recirculation of heated air at a targeted velocity. Also it provides a fresh mixture of air to fibers and helps to remove exothermic heat from the fibers through gentle filament shaking [16] (Figure 1). Not only does it help to control the process, it also contributes to the energy consumption, hence a deeper understanding of its influence on the resultant carbon fiber properties would be beneficial to identify ways to reduce the energy consumption in carbon fiber manufacturing. In the past, Dunham et al. [17] validated the importance of air velocity in the stabilization oven in order to maintain adequate heat transfer conditions to avoid fiber degradation. However, this study was confined to a conceptual approach rather than identifying its influence on the structure and properties of fibers during carbon fiber manufacturing. To the best of our knowledge, it is the only study that showed the importance of air flow velocity in the stabilization of PAN fibers. On the other hand, it is essential to understand its role in controlling the progress of the exothermic reaction in the fiber cross-sections during thermal stabilization before any informed decisions are made. As was demonstrated earlier, the dissipation of extra heat from the fiber during thermal treatment plays a critical role in establishing homogeneous radial structure [18].

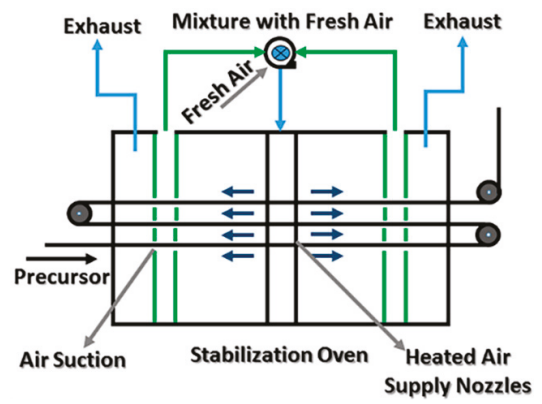

Figure 1. Schematic representation of a stabilization oven with air flow from the center to ends.

Hence, in the current study we sought to understand the influence of recirculation fan frequency on the development of the structure and properties of stabilized fibers and subsequent carbon fibers manufactured under industrial standards. Samples were obtained by processing fibers using a continuous process research line, equipped with center-to-ends ovens, by considering three recirculation fan frequencies in the stabilization ovens. Initially, the chemical structure evolution was assessed with Australian Synchrotron-high resolution IR micro-spectroscopy on the fiber cross-sections. Later, the development of microstructure and variation of properties at each stage of the carbon fiber manufacturing process were studied with respect to fan frequencies. Finally, suggestions were given in order to reduce the energy consumption during carbon fiber manufacturing without losing the quality of the final carbon fibers.

\section{Experimental}

\subsection{Sample Preparation}

PAN fibers ( $24 \mathrm{k}$ tow) with an average diameter of $\sim 12.9 \pm 0.89 \mu \mathrm{m}$ were acquired from BlueStar co Ltd., Beijing, China, and were used for carbon fiber processing using a research scale line (see schematic 
in Figure 2), located at Carbon Nexus, Australia. Three sets of samples were prepared by varying only the recirculation fan frequencies in the stabilization ovens, as shown in Table 1 . In order to maintain uniform temperatures throughout the stabilization oven, a minimum fan frequency is required. Based on our initial work, we identified that a minimum of $35 \mathrm{~Hz}$ was required for the considered oven volume to maintain acceptable conditions for thermal stabilization. Moreover, the maximum fan frequency was considered to be $60 \mathrm{~Hz}$ in order to avoid any physical damage to fibers due to violent vibrations at higher air flow velocities. The air flow velocity inside the oven at various fan speeds were measured at $50{ }^{\circ} \mathrm{C}$ by placing an Anemometer (testo 435) approximately $20 \mathrm{~cm}$ downstream from the central supply nozzle on either side (see Figure 1). The air velocities were registered as $2.01 \pm 0.09,2.97 \pm 0.04$, and $4.05 \pm 0.05 \mathrm{~m} / \mathrm{s}$ for fan frequencies $35 \mathrm{~Hz}, 45 \mathrm{~Hz}$, and $60 \mathrm{~Hz}$, respectively. Samples were collected at the end of each zone of stabilization, and at the output of the low temperature (LT) and high temperature (HT) carbonization stages.

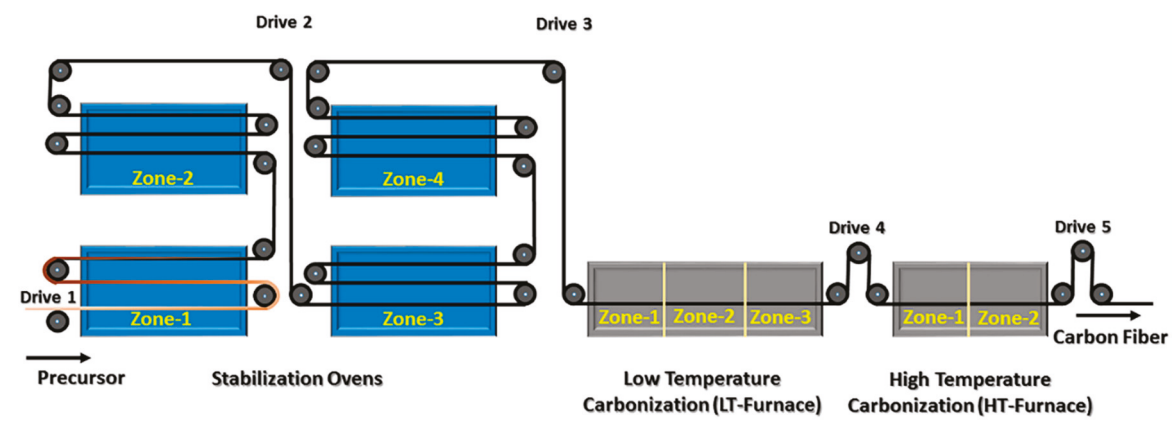

Figure 2. Schematic representation of the research production line at Carbon Nexus.

Table 1. Process parameters used for sample preparation.

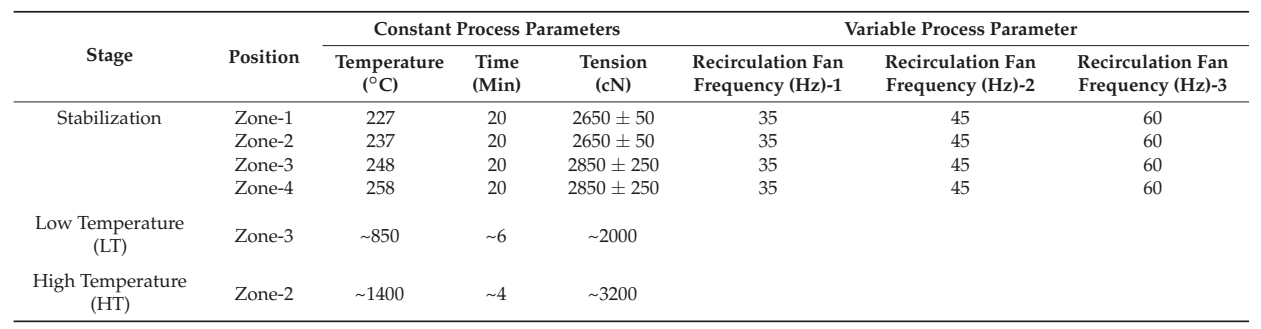

\subsection{X-ray Diffraction Studies}

Wide angle X-ray diffraction studies were conducted on fiber samples using an X-pert pro XRD machine, PANalytical, equipped with a Cu-K $\alpha$ radiation source (wave length of X-rays $\lambda=1.5406 \AA$, operating conditions $40 \mathrm{kV}$ and $30 \mathrm{~mA}$ ). The apparent crystallite size and d-spacing of the samples were obtained using Equations (1) and (2) [19].

$$
\begin{aligned}
& L_{c}=\frac{K \lambda}{B \cos \theta} \\
& n \lambda=2 d \sin \theta
\end{aligned}
$$

\subsection{Tensile Testing}

The single filament tensile testing was conducted by Textechno Favimat, which was mounted with a load cell of $210 \mathrm{cN}$. A gauge length of $25 \mathrm{~mm}$ and a pretension of $0.5 \mathrm{cN}$ were considered for 
the tests. For testing precursor and stabilized fibers a speed of $12.5 \mathrm{~mm} / \mathrm{min}$ was used, whereas for carbon fibers $2 \mathrm{~mm} / \mathrm{min}$ was used. At least 50 filaments were tested for each sample.

\subsection{IR Studies}

IR imaging on the fiber cross-sections was performed in Attenuated Total Reflectance (ATR) mode at the infrared micro-spectroscopy beam line, Australian Synchrotron. Initially, the samples were mounted in epoxy blocks and polished up to 1- $\mu \mathrm{m}$ precision before proceeding with imaging. The samples were brought to focus using $20 \times$ objective lens and areas of measurement were selected. This is followed by bringing the Ge crystal in to focus the measurement of the background in air at a resolution of $4 \mathrm{~cm}^{-1}$ and 128 co-added scans. After background measurement, samples were brought into contact with the crystal for the spectral acquisition from the selected area. The spot size considered for acquiring IR spectra was $3.1 \mu \mathrm{m}$, and the selected area was scanned at a step size of $1 \mu \mathrm{m}$. IR spectra at each spot were collected between 700 and $3800 \mathrm{~cm}^{-1}$ wavenumbers at a resolution of $4 \mathrm{~cm}^{-1}$. Each spectrum is a representation of 32 co-added scans. The acquired data was post-processed and baseline corrected using OPUS software. Since the resolution is high, we were not able to achieve a decent signal-to-noise ratio for wavenumbers below $1200 \mathrm{~cm}^{-1}$. Based on the absorbance intensity corresponding to nitrile and $\mathrm{C}=\mathrm{N}$ functional groups, the cyclization index was calculated using Equation (3) [18].

$$
\text { Cyclization index }=\frac{0.29 \times A b s(1584)}{(A b s(2243)+0.29 \times A b s(1584))}
$$

\section{Results and Discussion}

\subsection{Chemical Structure Transformation during Thermal Stabilization}

The thermal stabilization of PAN fibers involves three major reactions: cyclization, dehydrogenation, and oxidation [20]. Out of these reactions, it is well known that cyclization is an exothermic reaction [21]. During thermal stabilization any imbalance between the process parameters could lead to an uncontrolled exothermic reaction that results in the rapid degradation of fibers. Moreover, it has been determined that the cyclization reaction plays a critical role in the formation of radial heterogeneity in fibers [18,22]. The cyclization reaction is associated with the conversion of $\mathrm{C} \equiv \mathrm{N}$ to $\mathrm{C}=\mathrm{N}$ [21]. The variation of these functional groups in the fiber cross-section with respect to fan frequencies assists in the understanding of the influence of heat transfer conditions inside the oven in controlling the progress of the cyclization reaction and the evolution of the cross-sectional structure. Figure 3 shows $\mathrm{C} \equiv \mathrm{N}$ and $\mathrm{C}=\mathrm{N}$ functional group distribution in the fiber cross-sections. The blue regions shown in Figure 3 are associated with epoxy and the green region represents the transition region from epoxy to fibers. In the current study, only the central regions of fibers were considered for further analysis. Overall, Figure 3 shows that the $\mathrm{C} \equiv \mathrm{N}$ functional groups are uniformly distributed in PAN and thermally stabilized fibers. Moreover, no clear distinction in the distribution of $\mathrm{C}=\mathrm{N}$ in the fiber cross-sections is observed in Zone- 4 samples irrespective of the fan frequency, as shown in Figure 3 (right-hand side). This homogenous distribution could indicate the end of the thermal stabilization stage, where most of the nitrile groups are transformed to $\mathrm{C}=\mathrm{N}$.

It is also evident that there are some fibers which showed a higher absorbance intensity of the respective functional groups compared to others, indicating the heterogeneity in the progress of reaction between the fibers. However, relative variation in the measurement of these two functional groups at various stages of thermal stabilization could potentially assist in reducing the effects of surface unevenness in the fiber cross-sections and provides clear evidence of the progress of cyclization at each stage with respect to the fan frequencies. 
a)
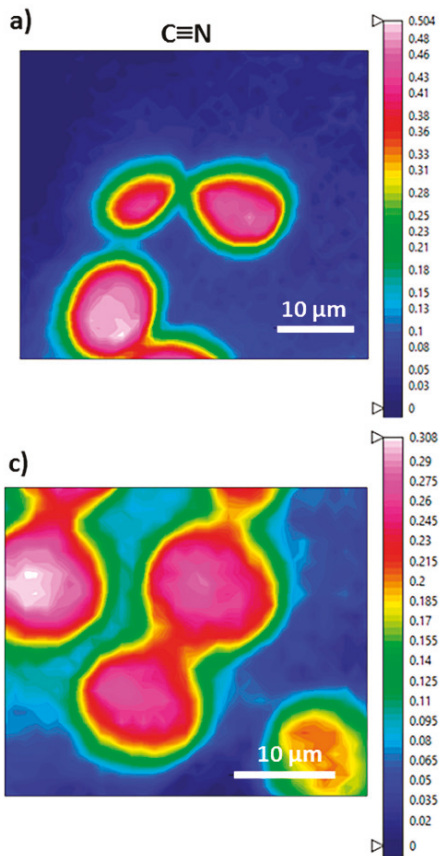

d)

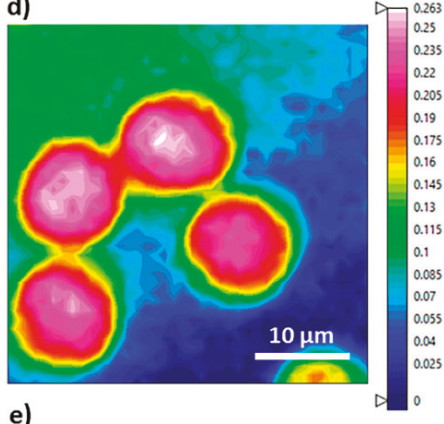

e)

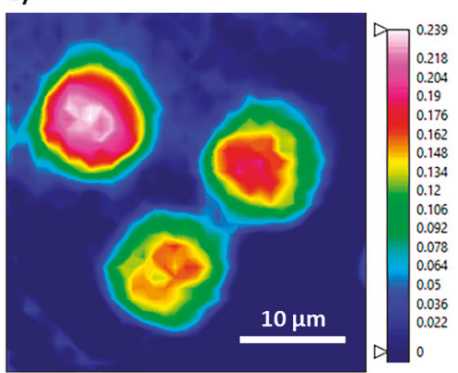

b)
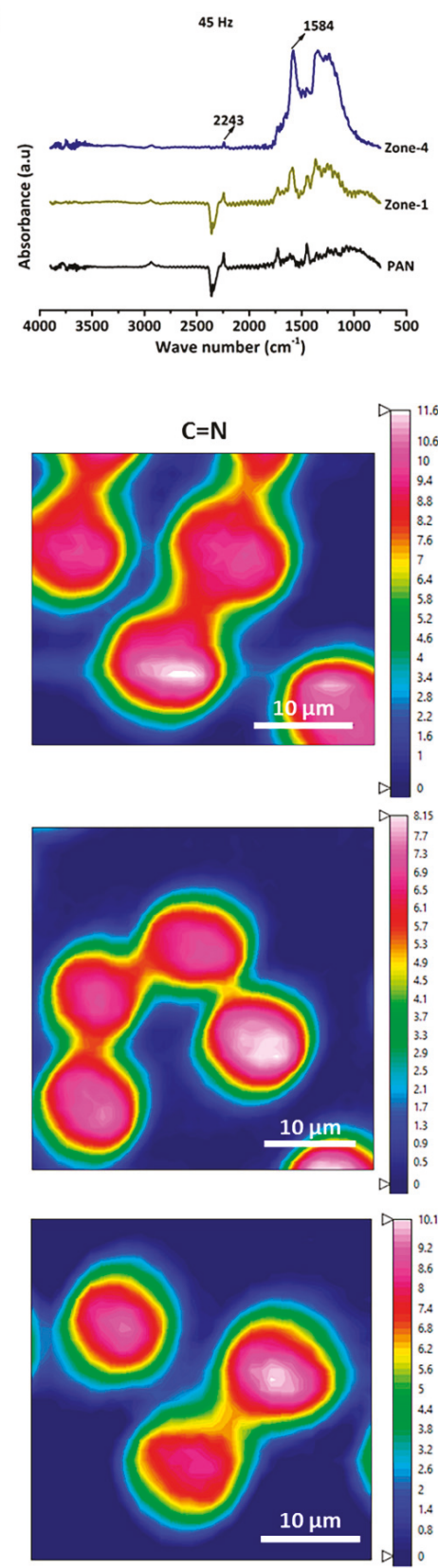

Figure 3. Distribution of nitrile $(\mathrm{C} \equiv \mathrm{N})$ and $\mathrm{C}=\mathrm{N}$ functional groups in the fiber cross-sections of PAN and Zone-4 samples with respect to fan frequencies. (a) PAN, (b) FTIR spectra, (c) $35 \mathrm{~Hz}$, (d) $45 \mathrm{~Hz}$, and (e) $60 \mathrm{~Hz}$. (Note: the left column indicates $\mathrm{C} \equiv \mathrm{N}$ distribution and the right column indicates $\mathrm{C}=\mathrm{N}$ distribution in thermally treated fibers). 
In Figure 3b, absorbance peaks at wavenumbers 2243 and $1584 \mathrm{~cm}^{-1}$ correspond to $\mathrm{C} \equiv \mathrm{N}$ and a combination of $\mathrm{C}=\mathrm{N}, \mathrm{C}=\mathrm{C}$, and $\mathrm{N}-\mathrm{H}$ functional groups [23]. There is an overall decrease in the absorbance intensity of peak at $2243 \mathrm{~cm}^{-1}$ and an increase in the intensity of peak at $1584 \mathrm{~cm}^{-1}$ from PAN to the final stabilization stage (Zone-4) samples, representing the progress of the cyclization reaction. Also, each spectrum was extracted from measured positions across the fiber diameter and assessed to understand the progress of the cyclization reaction in the fiber cross-sections with respect to the fan frequencies in early stage (Zone-1) and final stage (Zone-4) samples. The relative variation in these functional groups at each position in the fiber diameter is represented quantitatively in Figure 4 using Equation (3). Interestingly, fibers in Zone-1 showed a parabolic trend in the cyclization index across the fiber cross-sections irrespective of the fan frequency used, indicating that the progress of cyclization is higher in the central region compared to the surface regions of the fiber. On the surface region of fibers, the extra heat generated by the exothermic reaction is dissipated by means of heat exchange with the surrounding air. However, in the central region of fibers there is no route for the heat dissipation, which further triggers the progress of cyclization [22,24]. Moreover, this heterogeneity in the progress of cyclization in the fiber cross-section is diminished at the end of Zone- 4 for all samples, which supports our previous claim that most nitriles are converted to $\mathrm{C}=\mathrm{N}$ by this stage. In addition, no statistically significant difference in the cyclization index of samples treated at various fan frequencies is observed (Figure 4). Overall, it can be deduced that the lowest fan frequency $(35 \mathrm{~Hz})$ is sufficient to sustain the heat transfer conditions inside the oven.

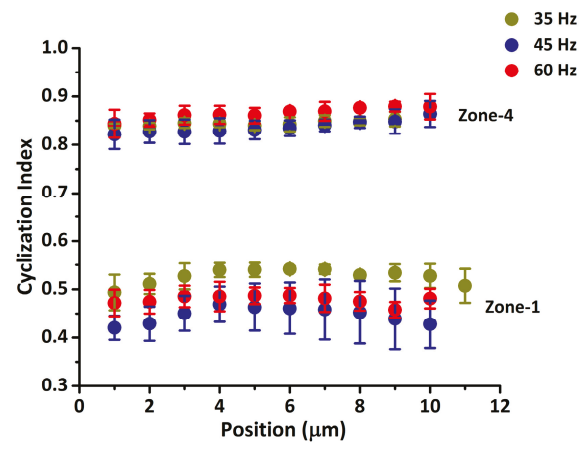

Figure 4. Cyclization index variation across the fiber cross-section in thermal stabilization.

\subsection{Microstructure and Property Variation in Fibers}

In the diffraction graphs shown in Figure $5 \mathrm{a}, \mathrm{b}$, three peaks were observed and located at $\sim 17^{\circ}$, which was associated with crystal plane (100) [25,26], at $\sim 26^{\circ}$, related to crystallographic plane (002), and at $\sim 29.5^{\circ}$, assigned to crystal plane (110) $[25,27,28]$. In PAN fibers a small hump at $\sim 26^{\circ}$ appeared to be associated with some weak systematically arranged units in the amorphous regions. The apparent crystallite size associated with the peaks at $17^{\circ}$ and $26^{\circ}$ was calculated using Equation (1). From Figure $5 c$, a sharp increase in crystallite size was observed for Zone- 1 fibers compared to PAN fibers for all fan frequencies. However, from Zone-2, fibers experienced a gradual decrease in crystallite size. Simultaneously, a sharp decrease in apparent crystallite size associated with the partial systematic units in the amorphous regions (Figure $5 \mathrm{~d}$ ) was perceived for all fibers until Zone- 2 of the stabilization process in comparison with PAN fibers (i.e., from $\sim 2.15 \mathrm{~nm}$ to $1.22 \mathrm{~nm}(35 \mathrm{~Hz}), 1.07 \mathrm{~nm}$ $(45 \mathrm{~Hz})$, and $1.49 \mathrm{~nm}(60 \mathrm{~Hz}))$. This was followed by a gradual increase in the apparent crystallite size associated with crystallographic plane (002) to $\sim 1.9 \mathrm{~nm}$ in carbon fibers irrespective of the fan frequency used during thermal stabilization. 


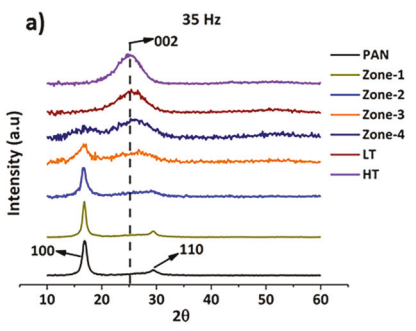

d)

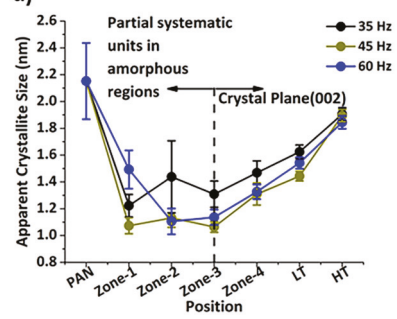

b)

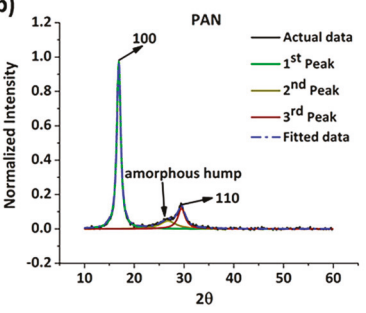

c)

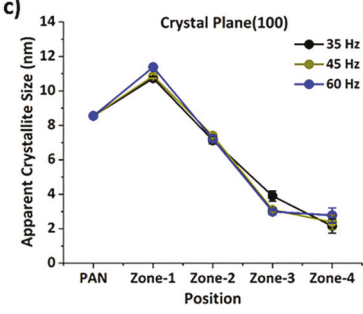

e)

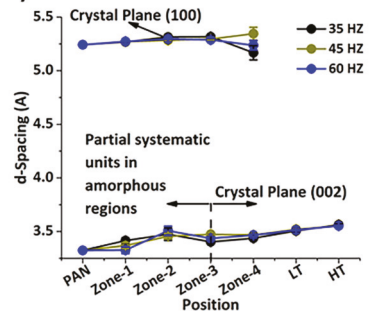

Figure 5. (a) X-ray diffraction peaks of fibers, (b) Lorentz curve fitting of PAN fibers, (c) apparent crystallite size variation in stabilization stage, (d) apparent crystallite size variation throughout the carbon fiber manufacturing, (e) d-spacing trends corresponding to crystal planes (100) and (002).

Initially, the functional groups of amorphous regions are more easily involved in stabilization reactions compared to highly crystalline regions [28,29]. During these procedures, some of the molecular chains in the amorphous phase arranged themselves into crystalline regions induced by improved dipole interactions between nitrile groups, assisted by segmental mobility [28,29]. This phenomenon helped in the crystallite growth. In support of this proposal, a sharp increase in crystallite size (Figure 5c) coupled with a decrease in the size of systematically arranged units from the amorphous regions (Figure 5d) was noticed in fibers from Zone-1 compared to PAN fibers. However, at higher temperatures, the energy was sufficient enough to initiate the stabilization reactions in the crystalline regions, which further ruptured the molecular rod structure and thereby reduced the crystallite size (Figure 5c) [28,29].

An evolution of turbostratic graphitic structure associated with crystallographic plane (002) (Figure 5d) was also discerned from Zone-3 to HT. This was developed by the rupture of crystallites in PAN fibers during thermal stabilization and the simultaneous progress of intermolecular crosslinking and removal of heteroatoms in the polymer [25]. Although there was a difference in the apparent crystallite size between fibers treated at different fan frequencies from Zone-2, these differences steadily decreased with the progress of thermal treatment.

In addition, the d-spacing of the crystallites was calculated using Equation (2). From Figure 5e, with the progress of thermal treatment, crystallites associated with (100) displayed an increase in planar spacing from $\sim 5.24$ to $5.34 \AA$. Similar to this trend, the partial systematics units and crystallites related to (002) showed an increment in the d-spacing from $\sim 3.32$ to $\sim 3.5 \AA$ and from $\sim 3.4$ to $3.56 \AA$. In case of crystallites corresponded to (100) and partial ordered units in the amorphous region, the progress of stabilization reactions from the ends of the molecular rods and in the amorphous regions might have led to a gradual increase in d-spacing, whereas in case of crystallites related to (002) after Zone-3, a steady increase in crosslinking atoms between the layers of a planar structure could be the reason for a gradual increase in the d-spacing. Moreover, unlike crystallite size, the d-spacing trends demonstrated no significant differences with respect to the recirculation fan frequencies in the intermediate stages of carbon fiber manufacturing. 
Supporting the structural data, no significant difference in the fiber properties were observed, as shown in Figure 6. Overall, the percentage elongation of fibers increased with thermal stabilization (Figure 6a), while decreasing trends were observed in strength and modulus (Figure $6 \mathrm{~b}, \mathrm{c}$ ). The loss of the tensile strength of fibers was attributed to the reduction of cohesive energy in the molecular chains with the progress of the cyclization reaction in the fiber structure [30]. Interestingly, the trend of tensile modulus (Figure 6c) was similar to the apparent crystallite size trend shown in Figure 5c, supporting the argument related to the improvement of morphological order at the initial stages. From Figure $6 \mathrm{~d}, \mathrm{f}$, after carbonization, an overall rapid decrease in the \% elongation of fibers and a rapid increase in the tensile strength and modulus of fibers was observed compared to stabilized fibers. This was attributed to an improvement in the growth of the planar carbon network with the promotion of intermolecular crosslinking and an increase in the stacking size $\left(L_{c}\right)$ and its orientation. Given the observations, it can be concluded that the stimulation of fiber tow at the lowest fan frequency during thermal stabilization should be sufficient enough to dissipate the exothermic heat from the considered fiber tow size. Moreover, considering the volume of the oven, the amount of extra heat generated by the stabilization reactions from a single tow might not be sufficient enough to create noticeable changes in the reaction front. However, the influence of varying the tow size on the generation of local exothermic heat inside the tow with respect to various fan frequencies during thermal stabilization needs to be further analyzed; this work is under progress. Moreover, in the future we will work on identifying the balance between air flow conditions and treatment temperature in order to reduce the residence time in stabilization without reducing the quality of the carbon fibers.

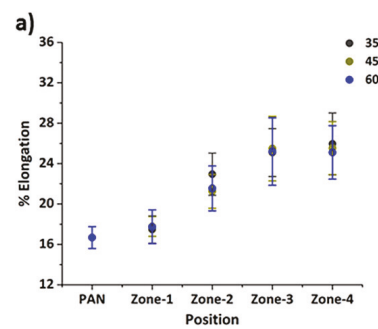

d)

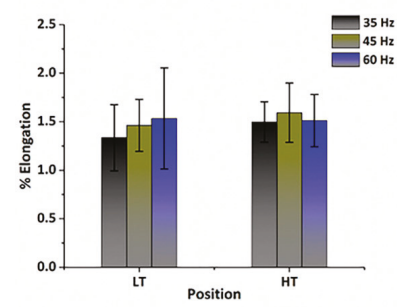

b)

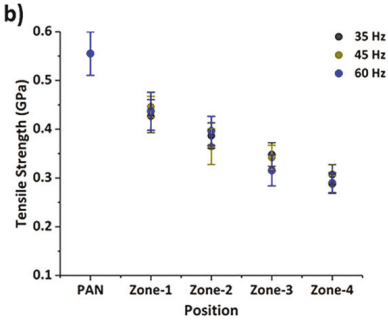

e)

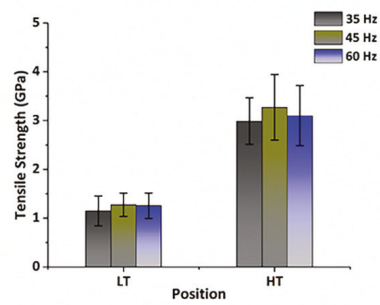

c)

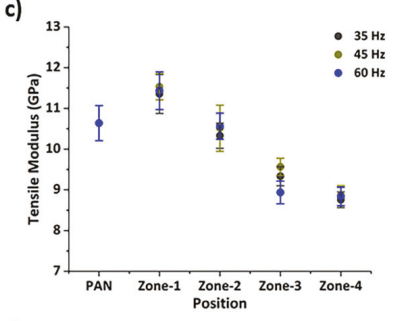

f)

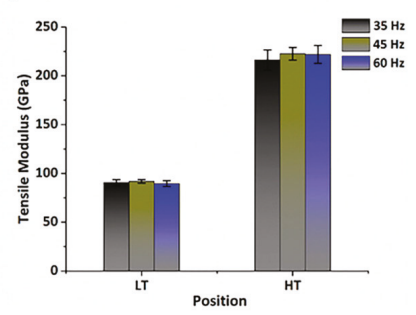

Figure 6. Tensile properties of stabilized and carbon fibers (LT and HT) with respect to recirculation fan frequencies with the (a) \% elongation at break, (b) tensile strength, (c) tensile modulus of stabilized fibers, and the (d) \% elongation at break, (e) tensile strength, and (f) tensile modulus of carbon fibers extracted from LT and HT furnaces.

\section{Conclusions}

The influence of recirculation fan frequency on the structure and property variations in fibers during thermal stabilization as well as the resultant carbon fibers was systematically examined. Overall analysis showed that no significant differences were observed in terms of structure or properties of stabilized and carbonized fibers for the chosen recirculation fan frequencies. The possible reason for this could be that the stimulation of fiber tow at the lowest fan frequency during thermal stabilization 
was sufficient enough to dissipate the exothermic heat from the fiber tow while allowing adequate stabilization conditions for fibers. Hence, it may be possible to use lower recirculation fan frequencies (e.g., $35 \mathrm{~Hz}$ ) during thermal stabilization to manufacture carbon fibers with similar properties to those produced using a higher fan frequency (e.g., $60 \mathrm{~Hz}$ ). This study shows a possible path towards potential approaches to the reduce energy consumption of carbon fiber production and develop a low cost carbon fiber, which is of high importance for automotive lightweight structural applications.

Author Contributions: S.N. and M.N. conceived and designed the experiments, conducted major analysis, wrote the paper; S.N. and M.M. performed IR imaging at Australian synchrotron; M.M. and B.P. worked on sample preparation and performed initial analysis; S.M.F. performed X-ray diffraction analysis on the samples.

Acknowledgments: This research was supported by the Australian Research Council World Class Future Fiber Industry Transformation Research Hub (IH140100018) and the Australian Research Council Training Center for Lightweight Automotive Structures (ATLAS). We also thank the Carbon Nexus operational team for the manufacture of fibers. We are thankful to Australian Synchrotron for providing access to the IRM beamline for fiber characterization.

Conflicts of Interest: The authors declare no conflicts of interest.

\section{References}

1. Jasjeet, K.; Keith, M.; Shaun, S. Producing high-quality precursor polymer and fibers to achieve theoretical strength in carbon fibers: A review. J. Appl. Polym. Sci. 2016, 133, 43963.

2. Liu, J.; Yue, Z.; Fong, H. Continuous nanoscale carbon fibers with superior mechanical strength. Small 2009, 5, 536-542. [CrossRef] [PubMed]

3. Zhao, W.; Lu, Y.; Zhou, L.; Jiang, J.; Wang, J.; Chen, Q.; Tian, F. Effects on the oriented structure and mechanical properties of carbon fibers by pre-irradiating polyacrylonitrile fibers with $\gamma$ ray. J. Mater. Sci. 2016, 51, 7073-7084. [CrossRef]

4. De Santis, V.; Campi, T.; Cruciani, S.; Laakso, I.; Feliziani, M. Assessment of the induced electric fields in a carbon-fiber electrical vehicle equipped with a wireless power transfer system. Energies 2018, 11, 684. [CrossRef]

5. Ko, T.H. The influence of pyrolysis on physical properties and microstructure of modified PAN fibers during carbonization. J. Appl. Polym. Sci. 1991, 43, 589-600. [CrossRef]

6. Nunna, S.; Naebe, M.; Hameed, N.; Fox, B.L.; Creighton, C. Evolution of radial heterogeneity in polyacrylonitrile fibres during thermal stabilization: An overview. Polym. Degrad. Stab. 2017, 136, 20-30. [CrossRef]

7. Maghe, M.; Creighton, C.; Henderson, L.C.; Huson, M.G.; Nunna, S.; Atkiss, S.; Byrne, N.; Fox, B.L. Using ionic liquids to reduce energy consumption for carbon fibre production. J. Mater. Chem. A 2016, 4, 16619-16626. [CrossRef]

8. Khayyam, H.; Naebe, M.; Bab-Hadiashar, A.; Jamshidi, F.; Li, Q.; Atkiss, S.; Buckmaster, D.; Fox, B. Stochastic optimization models for energy management in carbonization process of carbon fiber production. Appl. Energy 2015, 158, 643-655. [CrossRef]

9. Golkarnarenji, G.; Naebe, M.; Badii, K.; Milani, A.S.; Jazar, R.N.; Khayyam, H. Production of Low Cost Carbon-Fiber through Energy Optimization of Stabilization Process. Materials 2018, 11, 385. [CrossRef] [PubMed]

10. Golkarnarenji, G.; Naebe, M.; Badii, K.; Milani, A.S.; Jazar, R.N.; Khayyam, H. Support vector regression modelling and optimization of energy consumption in carbon fiber production line. Comp. Chem. Eng. 2018, 109, 276-288. [CrossRef]

11. Ko, T.H.; Li, C.H.; Hu, C.H. The characterization of high-performance PAN-based carbon fibers developed by continuous carbonization and air oxidation. J. Mater. Res. 1995, 10, 1529-1538. [CrossRef]

12. Fitzer, E.; Frohs, W.; Heine, M. Optimization of stabilization and carbonization treatment of PAN fibres and structural characterization of the resulting carbon fibres. Carbon 1986, 24, 387-395. [CrossRef]

13. Khayyam, H.; Fakhrhoseini, S.M.; Church, J.S.; Milani, A.S.; Bab-Hadiashar, A.; Jazar, R.N.; Naebe, M. Predictive modelling and optimization of carbon fiber mechanical properties through high temperature furnace. Appl. Therm. Eng. 2017, 125, 1539-1554. [CrossRef] 
14. Wang, L.; Lu, W.; Zhang, L.; Xue, L.; Ryu, S.K.; Jin, R.G. Effects of positive and negative stretching on the structure and properties of polyacrylonitrile fibers in the pre-oxidation process. Carbon Lett. 2011, 12, 107-111. [CrossRef]

15. Nunna, S.; Naebe, M.; Hameed, N.; Creighton, C.; Naghashian, S.; Jennings, M.J.; Atkiss, S.; Setty, M.; Fox, B.L. Investigation of progress of reactions and evolution of radial heterogeneity in the initial stage of thermal stabilization of PAN precursor fibres. Polym. Degrad. Stab. 2016, 125, 105-114. [CrossRef]

16. Badii, K.; Naebe, M.; Golkarnarenji, G.; Dhami, N.; Atkiss, S.; Buckmaster, D.; Fox, B.; Khayyam, H. Energy Saving in Electric Heater of Carbon Fiber Stabilization Oven. In Proceedings of the 2014 4th International Conference on Artificial Intelligence with Applications in Engineering and Technology (ICAIET), Kota Kinabalu, Malaysia, 3-5 December 2014; pp. 109-114.

17. Dunham, M.G.; Edie, D.D. Model of stabilization for pan-based carbon fiber precursor bundles. Carbon 1992, 30, 435-450. [CrossRef]

18. Nunna, S.; Creighton, C.; Fox, B.L.; Naebe, M.; Maghe, M.; Tobin, M.J.; Bambery, K.; Vongsvivut, J.; Hameed, N. The effect of thermally induced chemical transformations on the structure and properties of carbon fibre precursors. J. Mater. Chem. A 2017, 5, 7372-7382. [CrossRef]

19. Liu, F.; Wang, H.; Xue, L.; Fan, L.; Zhu, Z. Effect of microstructure on the mechanical properties of PAN-based carbon fibers during high-temperature graphitization. J. Mater. Sci. 2008, 43, 4316-4322. [CrossRef]

20. Zhao, J.; Zhang, J.; Zhou, T.; Liu, X.; Yuan, Q.; Zhang, A. New understanding on the reaction pathways of the polyacrylonitrile copolymer fiber pre-oxidation: Online tracking by two-dimensional correlation FTIR spectroscopy. RSC Adv. 2016, 6, 4397-4409. [CrossRef]

21. Khayyam, H.; Naebe, M.; Zabihi, O.; Zamani, R.; Atkiss, S.; Fox, B. Dynamic Prediction Models and Optimization of Polyacrylonitrile (PAN) Stabilization Processes for Production of Carbon Fiber. IEEE Trans. Ind. Inform. 2015, 11, 887-895. [CrossRef]

22. Layden, G.K. Retrograde core formation during oxidation of polyacrylonitrile filaments. Carbon 1972, 10, 59-63. [CrossRef]

23. Arbab, S.; Mirbaha, H.; Zeinolebadi, A.; Nourpanah, P. Indicators for evaluation of progress in thermal stabilization reactions of polyacrylonitrile fibers. J. Appl. Polym. Sci. 2014, 131, 40343. [CrossRef]

24. Kikuma, J.; Warwick, T.; Shin, H.J.; Zhang, J.; Tonner, B.P. Chemical state analysis of heat-treated polyacrylonitrile fiber using soft X-ray spectromicroscopy. J. Electron Spectrosc. Relat. Phenom. 1998, 94, 271-278. [CrossRef]

25. Hameed, N.; Sharp, J.; Nunna, S.; Creighton, C.; Magniez, K.; Jyotishkumar, P.; Salim, N.V.; Fox, B. Structural transformation of polyacrylonitrile fibers during stabilization and low temperature carbonization. Polym. Degrad. Stab. 2016, 128, 39-45. [CrossRef]

26. Yu, M.J.; Bai, Y.J.; Wang, C.G.; Xu, Y.; Guo, P.Z. A new method for the evaluation of stabilization index of polyacrylonitrile fibers. Mater. Lett. 2007, 61, 2292-2294. [CrossRef]

27. Karacan, I.; Erdoğan, G. The role of thermal stabilization on the structure and mechanical properties of polyacrylonitrile precursor fibers. Fibers Polym. 2012, 13, 855-863. [CrossRef]

28. Wang, B.; Xiao, S.; Cao, W.; Shi, X.; Xu, L. Evolution of aggregation structure of polyacrylonitrile fibers in the cyclization reaction. J. Appl. Polym. Sci. 2012, 124, 3413-3418. [CrossRef]

29. Ko, T.H.; Lin, C.H.; Ting, H.Y. Structural changes and molecular motion of polyacrylonitrile fibers during pyrolysis. J. Appl. Polym. Sci. 1989, 37, 553-566. [CrossRef]

30. Gupta, A.; Harrison, I.R. New aspects in the oxidative stabilization of PAN-based carbon fibers: II. Carbon 1997, 35, 809-818. [CrossRef]

(c) 2018 by the authors. Licensee MDPI, Basel, Switzerland. This article is an open access article distributed under the terms and conditions of the Creative Commons Attribution (CC BY) license (http:/ / creativecommons.org/licenses/by/4.0/). 


\title{
Robust Speed Control of PMSM Using Sliding Mode Control (SMC)—A Review
}

\author{
Fardila Mohd Zaihidee ${ }^{1,2}$, Saad Mekhilef ${ }^{1,3, *}$ and Marizan Mubin ${ }^{4}$ \\ 1 Power Electronics and Renewable Energy Research Laboratory (PEARL), Department of Electrical \\ Engineering, Faculty of Engineering, University of Malaya, Kuala Lumpur 50603, Malaysia; \\ farrmz@yahoo.com \\ 2 Faculty of Technical and Vocational, Sultan Idris Education University, Tanjong Malim 35900, Perak, Malaysia \\ 3 Center of Research Excellence in Renewable Energy and Power Systems, King Abdulaziz University, \\ Jeddah 21589, Saudi Arabia \\ 4 Department of Electrical Engineering, Faculty of Engineering, University of Malaya, Kuala Lumpur 50603, \\ Malaysia; marizan@um.edu.my \\ * Correspondence: saad@um.edu.my; Tel.: +6-037-967-6851
}

Received: 22 November 2018; Accepted: 18 January 2019; Published: 1 May 2019

\begin{abstract}
Permanent magnet synchronous motors (PMSMs) are known as highly efficient motors and are slowly replacing induction motors in diverse industries. PMSM systems are nonlinear and consist of time-varying parameters with high-order complex dynamics. High performance applications of PMSMs require their speed controllers to provide a fast response, precise tracking, small overshoot and strong disturbance rejection ability. Sliding mode control (SMC) is well known as a robust control method for systems with parameter variations and external disturbances. This paper investigates the current status of implementation of sliding mode control speed control of PMSMs. Our aim is to highlight various designs of sliding surface and composite controller designs with SMC implementation, which purpose is to improve controller's robustness and/or to reduce SMC chattering. SMC enhancement using fractional order sliding surface design is elaborated and verified by simulation results presented. Remarkable features as well as disadvantages of previous works are summarized. Ideas on possible future works are also discussed, which emphasize on current gaps in this area of research.
\end{abstract}

Keywords: permanent magnet synchronous motor; sliding mode control; motion control; fractional order; sliding surface; composite controller

\section{Introduction}

Permanent magnet synchronous motors (PMSMs) are widely used in low- to mid-power applications and high performance drives, e.g., robotics, electric vehicles and machine tools. They are preferred over brush-type motors and are gradually replacing induction motors in various fields of application due to their advantages such as compact structures, high air-gap flux density, high power density, high torque to inertia ratio, and high efficiency. However, PMSM systems are nonlinear and consist of time-varying parameters with high-order complex dynamics [1,2].

In recent years, field oriented control (FOC) of PMSMs has been a focus for many researchers. This technique simplifies the motor modeling, hence resulting in a simplified controller design. The stator current is decoupled into torque and flux producing components to allow independent control of both parameters as in DC motors [3]. FOC is used to achieve smooth starting, good acceleration and fast four-quadrant operation $[4,5]$. Compared to the direct torque control (DTC) method, the FOC approach results in lower torque and current ripple $[6,7]$. The FOC approach is the focused in this review. 
For speed regulation of PMSMs, control structures usually employ cascaded control loops, which consist of an outer speed control loop and two inner current loops. The advantages of cascaded control architecture lie in better disturbance resistance ability and better set point response performance. In the speed-current cascaded control structure, the relationship between the speed output and the reference quadrature axis current are usually described by a first-order model. However, considering the possibility that the closed-loop performance might degrade due to vanishing relative differences in control periods between both loops, second-order model relationships were also proposed [8]. For high speed applications of PMSMs, integrated speed and current controllers were used to solve the problem of nonlinear coupling between speed and current [9].

High performance PMSM applications require their speed controllers to provide a fast response, precise tracking, small overshoot and strong disturbance rejection ability. Linear control algorithms, e.g., PI controllers, have been widely used for speed control of PMSMs, but the performance was unsatisfactory in terms of tracking ability and robustness [10]. Various nonlinear control methods have been proposed to enhance the speed control performance of PMSMs in different aspects. These methods include sliding mode control [1,4,8,11,12], predictive control [13,14], backstepping control [15], adaptive control [15-19], Ho control [20], automatic disturbance rejection control [21] and artificial intelligence incorporated controllers [12,22].

Sliding mode control (SMC) is a class of variable structure system (VSS). Its theory was originated in the late 1950s in the former USSR by Prof. V. I. Utkin and Prof. S. V. Emelyanov [23,24]. The key idea of SMC is to reduce the complexity of high order systems to first order state variables, namely the sliding function and its derivative [25]. Sliding mode control is well known as a robust control method, which can guarantee excellent tracking although the system is exposed to internal parameter variations and external disturbances. Other than that, remarkable features of SMC are its high accuracy and simplicity. SMC has been successfully implemented in many fields [26-32] and applied to many other machine types other than PMSMs, e.g., induction motors [26,33-35], linear variable reluctance motors [36] and brushless DC motors [37,38]. SMC is applied to PMSMs directly for speed control, position control and efficiency as well as indirectly for observer design. This literature focuses on the application of SMC for speed control of PMSMs.

Challenges in SMC design are to ensure convergence of system state to sliding surface, to ensure control target achieved on sliding surface (i.e., error converge to zero, ensure robustness), to minimize reaching phase and to reduce chattering during sliding mode. Several sliding surface designs have been introduced for speed control of PMSM.

Lyapunov stability analysis is widely used to analyse the stability of the developed SMC controllers, where the stable convergence property is proven and evaluated. Several Lyapunov functions were chosen by researchers to be employed in the analysis, but typically the function in Equation (1) is chosen $[4,39]$ with the conditions given in Equation (2). Reaching condition is satisfied when the condition in Equation (3) is met:

$$
\begin{gathered}
V(t)=(1 / 2) s^{2}(t) \\
V(0)=0 \text { and } V(t)>0 \text { for } s(t) \neq 0 \\
\dot{V}(t)=s(t) \dot{s}(t)<0
\end{gathered}
$$

The main disadvantage of SMC methods is the chattering phenomenon they produce and high frequency switching near the sliding surface caused by the discontinuous control law in SMC design and from parasitic dynamics interactions $[1,4,40]$. Chattering can affect the system in terms of reducing control accuracy, resulting in high heat losses in electrical power circuits and high wear of moving mechanical parts [41].

This paper is organized as follows: Section 2 elaborates on various methods proposed by previous researchers to enhance SMC for PMSM speed control. The effectiveness of SMC enhancement using fractional order sliding surface design is verified through simulation results in Section 3. Discussion and conclusion are presented in Sections 4 and 5, respectively. 


\section{Sliding Mode Controller Enhancement in PMSM Speed Control}

Chattering phenomena in sliding mode control are said to be the main obstacle for SMC to become one of the significant discoveries in modern control theory [40]. Researchers have proposed various methods to overcome this issue, whereby in doing so, the controller's performance in terms of disturbance rejection properties and/or tracking was also affected, either in an advantageous or deteriorative way. Conversely, the proposed controller enhancement methods also determine the resulting chattering phenomena.

\subsection{Sliding Surface Design Modification}

New sliding surface designs have been proposed in many previous works in order to enhance the performance of SMC instead of using the conventional linear sliding surface. Linear sliding surface as in Equation (4) guarantees sliding mode asymptotic stability with convergence rate depending on the value of c [11]. The remarkable advantage of this design is its simplicity. However, the performance of conventional linear sliding surfaces is proven to be less satisfactory in terms of convergence rate and settling time [1,42]. The dynamic response of a closed loop system can be improved by using nonlinear sliding surfaces. For PMSM speed control, several works have been executed using linear SMC, but the drawbacks of linear SMC were overcame by means of hybridization to develop a composite SMC controller $[8,43,44]$ or modification of the reaching law [11].

$$
s=c x_{1}+x_{2}, c>0
$$

\subsubsection{Integer Order Integral SMC}

In conventional sliding mode control, the robustness of the system against parameters variation and external disturbances is achieved in the sliding phase. Prior to that, i.e., during the reaching phase, the robustness is not guaranteed. Integral sliding mode control (ISMC) (Equation (5)) was introduced by Utkin and Jingxin [45] to overcome this problem by eliminating the reaching phase, therefore, sliding phase is enforced throughout the entire system response. Furthermore, the order of motion equation in integral SMC is not reduced by the control input dimension, but equal to the order of the original system. The control law consists of a continuous nominal control and discontinuous control, which is responsible for the performance of the nominal system without perturbations and for rejecting perturbations respectively. Hence, with this type of SMC, system robustness can be guaranteed from the initial time. In addition, smaller maximum control magnitude is required for ISMC than conventional SMC since the value is usually bigger during the reaching phase $[46,47]$.

$$
s=e(t)-\int_{0}^{t}(a+b K) e(\tau) d \tau
$$

In PMSM speed control, constructing a conventional sliding surface requires both speed and acceleration signals. However, acceleration signals are usually immeasurable and difficult to accurately estimate due to noise and parameter uncertainties. Integral sliding mode solves this issue and avoids degradation of the system closed loop performance [48]. As far as speed control of PMSM is concerned, ISMC has proven to result in better speed response compared to conventional PI in terms of overshoot, response time and steady-state error. In their simulation, Song and Jia [49] proved a smaller speed drop by $87.5 \%$ under load disturbance when using ISMC, compared to a PI controller. Similarly, an improved speed drop by almost $29 \%$ was observed in experiment executed by Zaky [50]. However, a better result of $4 \%$ speed drop was obtained when using a tuned ISMC, concluding that good robustness is only ensured when ISMC gain is carefully tuned. The drawback of ISMC is the difficulty of parameter tuning of its gain, which is required to ensure good balance between disturbance rejection property of the controller and the chattering phenomenon. This drawback was overcome using various techniques such as using linear varying gain and extended state observer [48] or using gain tuning methods [50]. 


\subsubsection{Fractional Order SMC}

Another nonlinear sliding surface design method is the fractional order sliding surface, which utilizes fractional calculus in constructing its sliding surface. Fractional calculus emerged theoretically over 300 years ago, but in recent decades it has been applied practically in a wide range of science and engineering disciplines [51]. It is a generalization of the traditional integer order integration and differentiation to the non-integer order. Fractional order terms have the property of attenuating old data and storing new data, hence they are more stable or at least as stable as their integer order counterparts [4,52]. Fractional calculus has been extensively integrated with classical PID control theory to come out with fractional PID controllers indicated as $\mathrm{PI}_{\lambda} \mathrm{D}_{\mu}$ [53-55]. Other fractional order controller includes fractional adaptive controllers [56,57], fractional order compensators [58-61], fractional order sliding mode observer [62] and fractional order sliding mode control (FOSMC) [62-65].

Various hybrid FOSMC methods have been implemented in nonlinear systems. Delavari, et al. [64] compared the performance of their genetic-based Fuzzy FOSMC with integer order SMC in their simulation study. It was proven that the proposed FOSMC resulted in faster tracking response, improved tracking performance, smoother control action, minimized chattering, minimized root mean square errors, robust against model parameter uncertainties and is appropriate for practical application for nonlinear systems with parameter variations. Zhang, et al. [4] have experimentally implemented FOSMC with fuzzy logic tuning for speed control of PMSM. The extra degree of freedom of integral and differential operators can improve the controller's performance further compared to traditional integer order SMC (IOSMC). Theoretically, fractional order sliding surfaces experience slower energy transfer during switching, hence resulting in smaller chattering compared to integer order sliding surfaces which decay towards zero exponentially (Figure 1). Experimentally, FOSMC has been proven to achieve better control performance with smaller chattering and robust against external load disturbance and parameter variations compared to IOSMC. Similar simulation result was obtained by Huang, et al. [5]. The superior performance of FOSMC for applications in induction motors was elaborated and compared with a few other SMC controllers in Sun, et al. [66].

$$
\begin{gathered}
s=k_{p} e(t)+{ }_{0} D_{t}^{r} e(t) \\
k_{p}>0 ;{ }_{0} D_{t}^{r}(\cdot) \text { is fractional order integral with } 0<r<1
\end{gathered}
$$

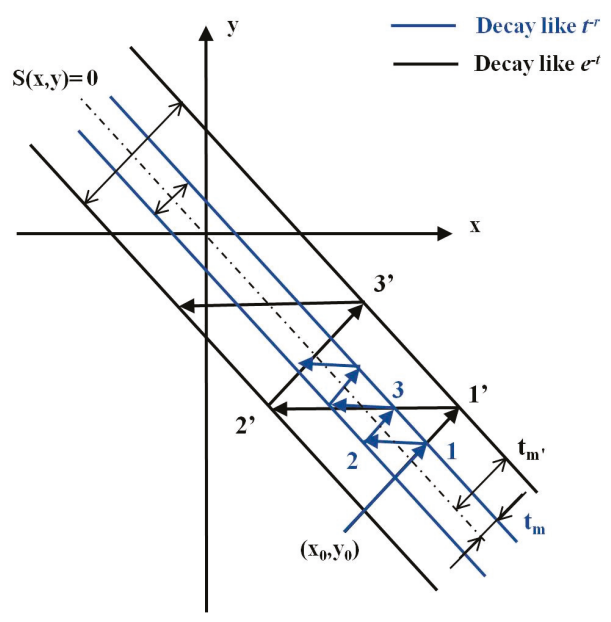

Figure 1. Comparison of energy transfer during sliding motion of FOSMC (blue line) and IOSMC (black line) [4]. 
Taking advantage of both integral SMC and fractional order integral, composite integral SMC was developed for position control of PMSM. The feedforward compensation part that consists of a fractional integral term in the control law ensures timely generation of control output during start-up or load disturbance by eliminating the integral saturation effect without having to increase the switching gain. In addition to the ability to suppress disturbance, the additional term also weakens the chattering phenomenon during sliding mode [52]. Implementation of similar composite controller for speed control of PMSM would also result in a robust, chattering-free system. Implementations of FOSMC in PMSM speed control need to consider the proper selection of the order, which lies between 0 and 1 , proper determination of the frequency band for fractional order operator approximation and selection of either fractional integration, fractional differentiation or both as a sliding surface design method.

\subsubsection{Terminal SMC}

Terminal sliding mode (TSM) control utilizes terminal sliding surfaces in which fractional power is introduced to ensure fast and finite-time states convergence during the sliding mode phase. Near the equilibrium point, rate of convergence will speed up which makes this controller preferred for high precision control. The drawback of conventional terminal SMC Equation (7) is its singularity problem that might occur if the initial conditions are not carefully selected, due to the recursive structure of switching manifolds $[1,67,68]$. Singularity in TSM was overcame by using two-phase control strategy by inducing the system state into a non-singular region, prior to finite time convergence control [69]. In [70], non-singularity was ensured by switching between TSM and linear hyperplane based sliding mode. However, as the boundary decreases to zero, the singularity effect is not fully eliminated. Furthermore, when the boundary is relatively large, the advantage of TSM is diminished [67].

$$
\begin{gathered}
s=x_{2}+\beta x_{1}^{\frac{q}{p}} \\
\beta>0 ; p, q \text { are positive odd integers, } \\
p>q
\end{gathered}
$$

Non-singular terminal sliding mode controller (NTSM, Equation (8)) was proposed to completely eliminate the singularity problem of conventional terminal sliding mode control and applied to robot manipulators $[30,67,71]$ and other systems [28,72]. Mu and He [73] proposed two new definitions and theorems to specifically characterize the dynamic behavior of TSM and NTSM for nonlinear systems. NTSM was applied for PMSM speed control and resulted in shorter settling time, better anti-disturbance ability, smaller tracking error and smaller chattering compared to conventional SMC $[1,74,75]$. However, controller enhancement still involves setting the switching gain at higher value as per in SMC. Hence, large chattering cannot be avoided due to discontinuity term in order to improve the controller performance. Another drawback lies in providing good convergence especially when the system state is far from equilibrium [42]. Furthermore, improper parameter selection of NTSM can affect its convergence rate when its performance approaches that of linear SMC [76]. Several works aimed to overcome these drawbacks using composite NTSM controllers [77,78] as well as proposing a design principle of controller variables based on homogeneity analysis [77].

$$
\begin{gathered}
s=x_{1}+\frac{1}{\beta} x_{2}^{\frac{p}{q}} \\
\beta>0 ; p, q \text { are positive odd integers, } \\
1<\frac{p}{q}<2
\end{gathered}
$$

Another type of terminal sliding mode controller proposed in the literature is the non-singular fast terminal sliding mode (NFTSM) controller Equation (9). NFTSM retains the advantages of NTSM but with a faster state convergence. Near equilibrium, the convergence rate of linear sliding mode remains constant while the convergence rate of NFTSM grows exponentially [76]. NFTSM has been 
applied for various control applications [79-84] and has shown not only faster convergence, but also stronger robustness, higher precision and lower chattering compared to conventional SMC.

$$
\begin{gathered}
s=x_{2}+\alpha x_{1}+\beta x_{1}^{\frac{q}{p}} \\
\alpha, \beta>0 ; p, q>0 \text { (odd numbers); } \\
p>q
\end{gathered}
$$

NFTSM implemented for speed control of PMSM has proven that singularity problem of TSM is successfully avoided and faster convergence is obtained [85]. Thorough research on application of NFTSM for PMSM speed control is still unavailable. However, for position control of PMSM, NFTSM was used to reduce the chattering phenomenon and provide better position tracking and convergence rate compared to a PD controller [42]. Similar result is expected if NFTSM is applied for PMSM speed control. This style of numbering is only for equations and none are given

\subsection{Higher Order SMC}

Higher order sliding mode control (HOSMC) was first introduced by Levant [86]. In first order $\mathrm{SMC}$, the selected sliding surface has a relative degree one with respect to the control input. The control input acts on the first derivative of the sliding surface. In higher order SMC, the control input acts on higher derivatives of the sliding surface. A sliding mode is defined as $n$-th order sliding mode when Equation (10) is satisfied [39]. The $n$-th derivative is predominantly supposed to be discontinuous or nonexistent [87]. The sliding order characterizes the system dynamics in the vicinity of the sliding mode in terms of degree of smoothness [88]. In literatures, various sliding surface types were used in designing their HOSMC. Reset numbering

$$
s(t)=\dot{s}(t)=\cdots=s^{(n-1)}(t)=0
$$

When compared to first order SMC, HOSMC provides smoother control, better performance in terms of switching delays in control implementation, eliminates the requirement of derivative information for the control and is better in chattering effects suppression while still preserving the main advantages of the conventional SMC approach in terms of robustness and easiness of implementation [86, 89,90]. On the other hand, the challenge in HOSMC lies in the usage of differentiators, where their practical behavior requires particular care in real implementation due to measurement noise. The deteriorative effects on overall closed loop performance increase dramatically with the number of differentiation stages [91].

For second order SMC (SOSMC), the main idea is not only to have the sliding surface at zero but also its first order derivative. Its control acts on the first derivative of the sliding surface. Second order sliding mode satisfies Equation (11). Different algorithms were proposed in literature to ensure SOSMC finite time convergence to zero such as suboptimal [92], twisting, super-twisting [93,94], drift and algorithm with a prescribed law of variation of $s$ [88]. Robustness issues of second order SMC, e.g., influences of measurement noise and unmodelled fast actuator dynamics, have been described in details by Levant and Fridman [95] and Bartolini, et al. [91].

$$
s(t)=\dot{s}(t)=0
$$

Second order SMC has been implemented in many systems [96-101] and for many machine types, e.g., DC motors [39,93,102], PMSM [103], induction motors [104] and induction generators [105,106]. For speed control of PMSM, SOSMC resulted in smaller output deviations, smaller convergence time of up to $66 \%$ and smaller chattering in comparison with first order SMC [94,107,108]. Chattering reduction property of SOSMC has been detailed by Bartolini, et al. [109]. Chattering analysis approach for systems with second order sliding mode control has been proposed by Boiko, et al. [110], where it 
can be applied to perform an in depth analysis of chattering for better comparison between SOSMC and first order SMC.

\subsection{Reaching Law Method}

The reaching law method aims to eliminate the chattering caused by non-ideal reaching at the end of the reaching phase. In sliding mode control, reaching law is the differential equation that specifies the dynamics of a switching function. Several types of reaching law proposed in the literature are constant rate, constant plus proportional rate, power rate, etc., where each has its own merits and demerits [111]. Conventionally, a constant rate signum function is used as reaching law. The boundary layer method is a common approach to alleviate chattering, where a smooth approximation is used to replace the sign function when the state trajectories lie within a certain boundary layer of the switching surface e.g., using saturation function [64] or hyperbolic tangent function [39]. However, this method sacrifices the system's anti-disturbance performance $[1,40]$ and causes steady-state error depending on boundary layer selection [99].

A novel exponential reaching law was proposed by Ke, et al. [9] for PMSM speed control. Absolute value of system state variable was incorporated in conventional exponential reaching law, so that the speed of convergence depends on how far the state variable is from the sliding surface. When sliding surface is reached, trajectory will become stable and allow the chattering phenomenon to be suppressed during sliding phase. However, the robustness of the controller is reduced and reaching time increases as the state approaches the sliding surface [11].

Chattering can also be minimized by decreasing the switching gain, but then the controller's reaching time will be jeopardized. To find a balance between these two parameters in different phases of SMC, Xiaoguang, et al. [11] proposed a novel reaching law that still uses the typical signum function as reaching law, but with a modified gain. Instead of a constant gain, the proposed gain adapts to sliding mode surface and system states variations. In the early stage of the reaching phase, a higher gain expedites the reaching time. As the system trajectory approaches the sliding surface, the gain will gradually decrease to zero in order to suppress chattering. The width of sliding mode band decreases as the system state decreases until it reaches an equilibrium point, hence effectively limiting the chattering effect of the SMC (Figure 2). Under disturbance-free conditions, chattering suppression of more than $50 \%$ was recorded. Moreover, the convergence rate of the proposed SMC can be improved by about $90 \%$. For a similar purpose, Zhang, et al. [112] eliminated the usage of the signum function in the reaching law and incorporated a terminal attractor term. However, chattering reduction was not clearly quantitatively documented and compared. A modified gain sliding surface was proposed by Jamoussi, et al. [113] for application in induction motors and their experimental results show a significant reduction in chattering. 


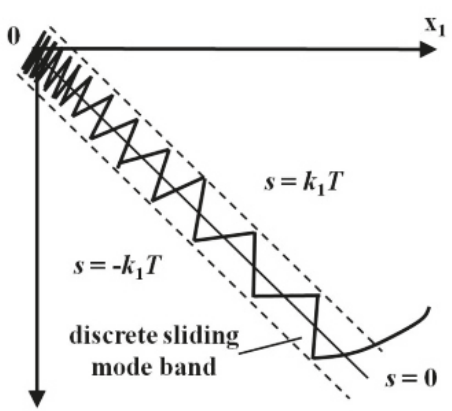

(a)

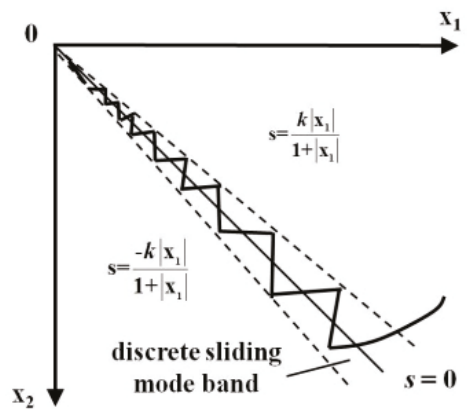

(b)

Figure 2. Sliding mode band of (a) conventional reaching law and (b) novel reaching law [11]. Reproduced with permission from Xiaoguang et al., Nonlinear speed control for PMSM system using sliding mode control and disturbance compensation techniques; published by IEEE Transactions on Power Electronics, 2013.

\subsection{Disturbance Compensation}

In standard sliding mode control theory, disturbances can be rejected completely when the selected switching gain is larger than the upper bound of disturbances. However, in practical applications, the upper bound of disturbances is hard to obtain. Hence, a high value of the switching gain is normally chosen to ensure disturbance rejection, which case will result in unwanted large chattering [8]. Several methods have been proposed to deal with this issue. An adaptive algorithm was incorporated to SMC for position control of PMSM in order to estimate the bound of disturbances and reduce the control effort [114]. However, the aforementioned proposed solution suffers from chattering problems [8]. Another proposal was by using a boundary layer method [115], which unfortunately sacrifices the disturbance rejection properties of the SMC to some extent.

In addition to the conventional feedback part, a feed-forward compensation part is introduced into the controller to improve the disturbance rejection performance of a system. The compensation part needs to be estimated, since direct measurement of the disturbances in PMSM applications is usually impossible. The estimated disturbance can be conventionally fed to the controller but fast suppression is not guaranteed. Moreover, in cases of severe disturbances, the system's performance can degrade tremendously [1]. Disturbance compensation allows a smaller value of switching gain to be selected since the gain value needs to be more than the bound of the disturbance compensation error instead of the lumped disturbances, which is usually larger. Hence, the disturbance rejection property of the controller is ensured without worsening the chattering effect [8]. Various disturbance estimation techniques as detailed in Chen, et al. [116] have been proposed in other fields [117-119], but for PMSM applications, disturbance observer and extended state observer are widely used.

In extended state observers (ESO), the lumped disturbances of the system (external disturbances and internal dynamics) are regarded as a new state of the system. ESO can estimate the states as well as the disturbances and its order is one magnitude higher than usual state observer. ESO was proposed by Jingqing [120] and has been incorporated in SMC for control in various fields [121]. SMC + ESO for speed control of PMSM has been experimentally proven to result in low speed drop (about $1 \%$ ) under load disturbance of $2 \mathrm{Nm}$ and lower chattering (by nearly 40\%) compared to pure SMC controller [8].

The disturbance observer (DOB) was originated by Ohnishi [122]. Disturbance observer-based SMC has been widely applied in various fields to improve the SMC in terms of disturbance rejection performance and chattering [123-125]. Implementing sliding mode theory into the observer, extended sliding mode disturbance observer (ESMDO) was developed by Xiaoguang, et al. [11] to provide the compensation part of their SMC. Estimated disturbance suffers from chattering since the observer is designed based on sliding mode and in this work it was suppressed using a low pass filter. 
The proposed composite SMC + ESMDO controller has better disturbance rejection ability than PI controller, with smaller overshoot and shorter settling time. Quantitatively, under a load disturbance of $4 \mathrm{Nm}$, the speed drop of the proposed controller was reduced by $75 \%$, overshoot was reduced by $50 \%$ and settling time was $60 \%$ shorter. A more relevant comparison was made between SMC with sliding mode-based disturbance observer and SMC without observer, where the composite SMC showed smaller overshoot, better robust stability and reduction in the effect of random load on the system [78]. Similarly, performance of a DOB-based nonsingular terminal SMC controller was compared with a conventional nonsingular terminal SMC by Mu, et al. [77]. Both their simulation and experimental approaches showed that their proposed composite DOB-based SMC controller resulted in faster speed response with a tracking error of less than $0.2 \%$ and overshoot reduction by $50 \%$. Furthermore, a remarkable improvement in current chattering was recorded, speed ripple was reduced by $80 \%$ as well as better robustness against step load torque compared to a conventional NTSM controller. The success in SMC controller enhancement through observers depends on how exact and quick the estimation can be obtained, which requires the parameters to be selected carefully. Furthermore, the parameters selected must also ensure that the reachability condition of the main SMC controller is satisfied.

\subsection{Artificial Intelligence}

Integration of artificial intelligence (AI) into SMC provides breakthrough to the sliding mode theory that has been studied for over 50 years. Composite SMC and computational intelligence controllers have been summarized technically by Kaynak, et al. [126], who discussed the possible ways of integration and by Xinghuo and Kaynak [90], who emphasized the individual advantages and disadvantages of integrating various AI methods into SMC. AI was incorporated in sliding mode controllers for various purposes such as combined with SMC to enhance the controller's performance, utilized the advantage of SMC to enhance main AI controllers, used for online or offline parameter tuning of a SMC controller, as uncertainties estimator and also to eliminate chattering. Several works have combined SMC, $\mathrm{AI}$ and adaptive control technique. The main advantages of adaptive control technique that researchers tried to bring to their controllers are their independence from precise prior knowledge of dynamic parameters, ability to effectively suppress errors caused by parameter uncertainties and also the ability to neutralize to some extent the influence of SMC high-frequency switching [127].

\subsubsection{Fuzzy Logic}

Fuzzy logic was proposed by Zadeh [128]. The main advantage of fuzzy logic is that it uses linguistic information instead of mathematical tools to model complex industrial processes. Memory capacity remains its biggest drawback, especially when high sensitivity of output is required. Composite fuzzy sliding mode controllers have been implemented in various fields [129-135]. For speed control of PMSM, fuzzy logic inference scheme (FLIS) was implemented to obtain the switching control gain of a fractional order SMC. This online tuning method has proven reliable in resulting a robust controller under various disturbance conditions [4]. Usage of fuzzy logic inference systems to obtain a gain requires the membership function to be sufficiently intensive for precision as well as wide enough to minimize the sensitivity to noise [136]. On the other hand, a composite fuzzy SMC was designed, whereby sliding mode properties can improve the performance of a conventional pure fuzzy controller. The control output was obtained by FLIS, depending on three sliding conditions (approaching, sliding and stable). Since the input variable of the fuzzy inference rules is defined by the sliding surface, the number of fuzzy rules can be minimized [137]. The proposed controller improved the performance of a pure fuzzy controller in terms of eliminating overshoot, settling time, tracking precision and steady-state error elimination [138]. Fuzzy adaptive mechanism was used to tune an integral SMC controller switching gain with respect to load torque. This method resulted in speed output without overshoot, steady-state error and chattering compared to a conventional ISMC without 
fuzzy [139]. For comparison purposes, similar performance was obtained when applying fuzzy SMC to an induction motor [140].

\subsubsection{Neural Network}

Neural networks have characteristics of fault tolerance, parallelism and learning [141]. NN-based SMC has been widely used as controller in various applications [142-146]. Wavelet NN (WNN) is developed by combining wavelets with neural networks. It combines online learning ability of artificial NN and identification ability of wavelet decomposition [12]. Its remarkable characteristics are quick convergence and high precision with reduced network size [147]. For speed control of PMSM, uncertain system dynamics was predicted using a WNN bound observer to solve the issue of uncertainty bound in SMC design. This composite WNN-SMC controller allowed a robust speed control for a wide range of operating conditions as compared to a conventional PID controller [12].

\subsubsection{Fuzzy Neural Network}

Both fuzzy logic and neural networks are universal approximators. However, the different characteristics of each of them are complementary. Fuzzy neural network (FNN) improves fuzzy logic-based controllers in terms of the learning abilities. Hence, it is capable of fuzzy reasoning in handling uncertain information as well as has capability of artificial NN in learning from processes. A combination of fuzzy and neural network theories was also incorporated in SMC controllers in various fields [141,148]. A recurrent fuzzy neural network (RFNN) intelligent observer was designed and combined with a SMC controller to facilitate real-time adjustment of the upper bound of the lumped uncertainty for position control of PMSM. The proposed controller resulted in a robust position control with less chattering and smaller control effort compared with a total SMC without RFNN observer [149]. Elmas and Ustun [43] proposed a hybrid controller that consists of a SMC and a neuro-fuzzy controller (NFC) connected in parallel for PMSM speed control. The error band method is used to determine which controller needs to be activated at a certain time. SMC is active during transient mode to get a fast dynamic response whereas the NFC is active during steady-state mode to get a smooth, less chattering dynamic response. The idea is to use the advantages of both SMC and NFC as well as to eliminate the disadvantageous factors of both. Current chattering of the proposed hybrid controller is reduced by around $92 \%$ compared to a conventional SMC. However, speed response of hybrid controller is faster by more than $7 \%$ compared to a conventional SMC. The hybrid controller is also less sensitive to parameter variations and external load disturbances compared to a conventional NFC.

The wavelet fuzzy neural network (WFNN) estimator combines fuzzy and wavelet neural network theories to estimate the unknown lumped uncertainties online to enhance the performance of a sliding mode controller. When applied to a six-phase PMSM, experimental results proved that the WFNN can perform a reliable estimation of lumped uncertainty and WFNN-SMC controller resulted in smaller tracking errors compared to a PI controller and SOSMC without WFNN lumped uncertainty estimator [99] and an adaptive backstepping SMC without WFNN [150].

\section{Fractional Order SMC for Speed Control of PMSM}

This section elaborates on a fractional order sliding mode control with PID sliding surface design (FOSMC-PID) proposed for speed control of PMSM using FOC approach, as illustrated in Figure 3. 


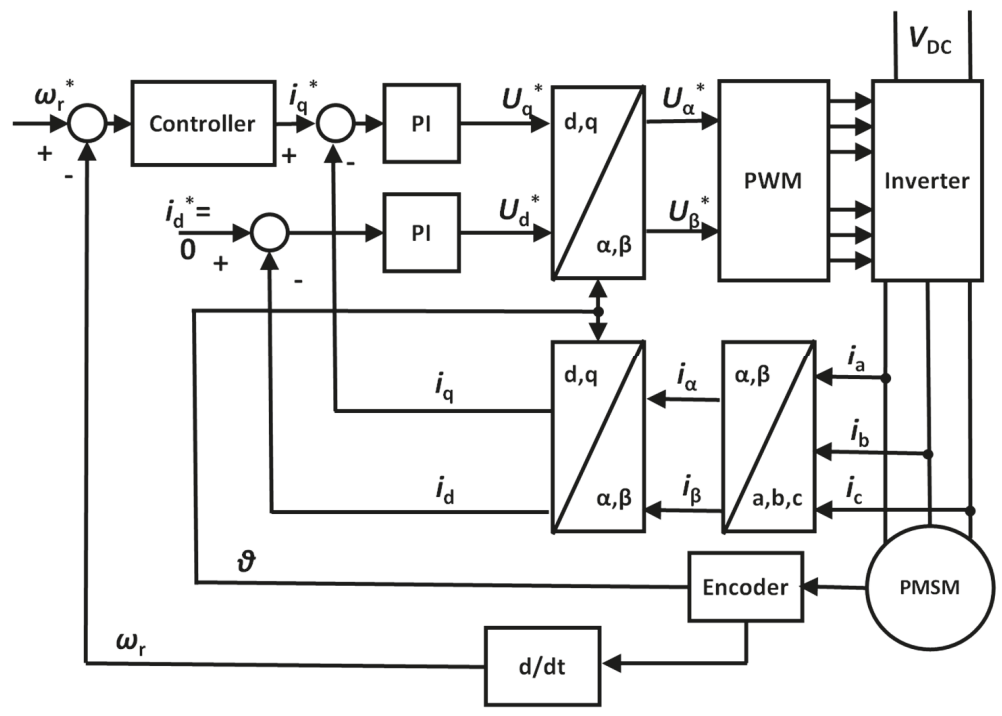

Figure 3. Typical block diagram of field oriented control of PMSM.

\subsection{Field Oriented Speed Control of PMSM}

In rotor rotating reference frame, the mathematical model of a PMSM is defined as below [4]:

$$
\begin{gathered}
u_{d}=R_{s} i_{d}+\dot{\lambda}_{d}-\omega_{f} \lambda_{q} \\
u_{q}=R_{s} i_{q}+\dot{\lambda}_{q}+\omega_{f} \lambda_{d} \\
T_{e}=1.5 p\left[L_{m d} I_{d f} i_{q}+\left(L_{d}-L_{q}\right) i_{d} i_{q}\right] \\
\omega_{f}=p_{n} \omega_{r}
\end{gathered}
$$

where $u_{d}, u_{q}$ are the $d, q$-axis stator voltages, respectively; $i_{d}, i_{q}$ are the $d, q$-axis stator currents, respectively; $L_{d}, L_{q}$ are the $d, q$-axis stator inductances, respectively; $T_{e}$ is the electric torque; $I_{d f}$ is the equivalent $d$-axis magnetizing current; $L_{m d}$ is the $d$-axis mutual inductance; $p_{n}$ is the pole pair; $\omega_{f}$ is the inverter frequency and $\omega_{r}$ is the rotor speed. $\lambda_{d}, \lambda_{q}$ are the $d, q$-axis stator flux linkages, which are defined as:

$$
\begin{gathered}
\lambda_{q}=L_{q} i_{q} \\
\lambda_{d}=L_{d} i_{d}+L_{m d} I_{d f}
\end{gathered}
$$

Motor dynamics is described as:

$$
T_{e}=J \dot{\omega}_{r}+B_{m} \omega_{r}+T_{L}
$$

where $J$ is the moment of inertia, $B_{m}$ is the viscous friction coefficient and $T_{L}$ is the load torque. In constant torque angle (CTA) control strategy $\left(\delta=90^{\circ}\right), d$-axis stator current command, $i_{d}$ is set to be zero. Electromagnetic torque in Equation (14) can be simplified as in Equations (19) and state equation of the servo drive is obtained as per Equations (20).

$$
T_{e}=1.5 p\left[L_{m d} I_{d f} i_{q}\right]=k_{t} i_{q}
$$




$$
\dot{\omega}_{r}=-\left(\frac{B_{m}}{J}\right) \omega_{r}+\left(\frac{k_{t}}{J}\right) i_{q}-\frac{T_{L}}{J}
$$

In this study, the main control problem is to ensure the motor speed, $\omega_{\mathrm{r}}$ to track the desired speed command, $\omega_{\mathrm{r}}^{*}$ asymptotically. For this purpose, the main speed controller i.e., the sliding mode controller provides an output in terms of q-axis stator current command, $i_{q}$ as the control input for the inner $q$-axis current controller. The speed tracking error, $e(t)$ and its derivative are defined in Equations (21) and (22) respectively, where $a, b, c, \varnothing(t)$ and $\delta(t)$ are defined as in Equations (23)-(26) with $\Delta a, \Delta b, \Delta c$ as the time-varying value of the system parameters and $\delta(t)$ is lumped uncertainty:

$$
\begin{gathered}
e(t)=\omega_{r}^{*}(t)-\omega_{r}(t) \\
\dot{e}(t)=-a e(t)-b i_{q}(t)+\varnothing(t)+\delta(t) \\
a=B_{m} / J, b=k_{t} / J, c=T_{L} / J \\
\varnothing(t)=a \omega_{\mathrm{r}}^{*}(t)+c(t)+\dot{\omega}_{\mathrm{r}}^{*}(t) \\
\delta(t)=\Delta a \omega_{\mathrm{r}}(t)-\Delta b i_{q}(t)+\Delta c(t) \\
|\delta(t)| \leq \Omega, \Omega \in R^{+}
\end{gathered}
$$

\subsection{Design of FOSMC-PID}

The proposed fractional PID $\left(\mathrm{PI}^{\alpha} \mathrm{D}^{\beta}\right)$ sliding surface is defined in Equation (27), where ${ }_{0} \mathrm{D}_{t}^{-\alpha}($.$) is$ a fractional integration of order $\alpha$ and ${ }_{0} \mathrm{D}_{t}^{\beta}$ (.) is a fractional differentiation of order $\beta$. By selecting $\alpha=\beta=1$, a classical integer order PID sliding surface is obtained. The control law in Equation (28) is adopted in this study. The equivalent control law in Equation (29) is obtained. Stability analysis of the proposed controller is detailed in [151]:

$$
\begin{gathered}
s(t)=k_{p} e(t)+k_{i_{0}} D_{t}^{-\alpha} e(t)+k_{d_{0}} D_{t}^{\beta} e(t) \\
k_{p}, k_{i}, k_{d}>0,0<\alpha<1,0<\beta<1 \\
\dot{s}=-w s-k_{s} \operatorname{sign}(s), w, k_{s} \in R^{+} \\
i_{q}^{*}(t)=\left(b k_{p}\right)^{-1}\left[\begin{array}{c}
k_{i_{0}} D_{t}^{1-\alpha} e(t)+k_{d_{0}} D_{t}^{\beta+1} e(t) \\
+(w-a) k_{p} e(t) \\
+k_{p} \varnothing(t)+w k_{i_{0}} D_{t}^{-\alpha} e(t) \\
+w k_{d_{0}} D_{t}^{\beta} e(t)+k_{s} \operatorname{sign}(s)
\end{array}\right]
\end{gathered}
$$

\subsection{Simulation Results}

The proposed controller is simulated in MATLAB/Simulink environment to evaluate its performance as a speed regulator. The PMSM model used primarily in this simulation is a three-phase Y-connected $1.93 \mathrm{~kW}$ motor with parameters as listed in Table 1. Fractional orders $\alpha$ and $\beta$ of the designed controller was chosen to be 0.35 and 0.3 , respectively. 
Table 1. Parameters of PMSM.

\begin{tabular}{cc}
\hline Parameter & Value \\
\hline Stator resistance, $R_{s}$ & $1.2 \Omega$ \\
$d$-axis stator inductance, $L_{d}$ & $6.35 \mathrm{~m} \mathrm{H}$ \\
$q$-axis stator inductance, $L_{q}$ & $6.75 \mathrm{~m} \mathrm{H}$ \\
Moment of inertia, $J$ & $2.31 \times 10^{-4} \mathrm{~kg} \mathrm{~m}^{2}$ \\
Viscous friction coefficient, $B_{m}$ & $0.0002 \mathrm{Nm} \mathrm{s}$ \\
Flux linkage, $\lambda_{f}$ & $0.15 \mathrm{~Wb}$ \\
Pole pair, $p_{n}$ & 4 \\
\hline
\end{tabular}

3.3.1. Performance Comparison of Fractional Order SMC with Conventional Integer Order SMC

The performance of the proposed fractional order SMC is compared with the conventional integral SMC. Reference speed of $500 \mathrm{rpm}$ was set and load torque of $2 \mathrm{Nm}$ was applied at $t=0.5 \mathrm{~s}$. Results in Figure 4 show that drive system with SMC speed controller experienced $9.22 \%$ overshoot, which was 10 times higher than the overshoot of the FOSMC system.

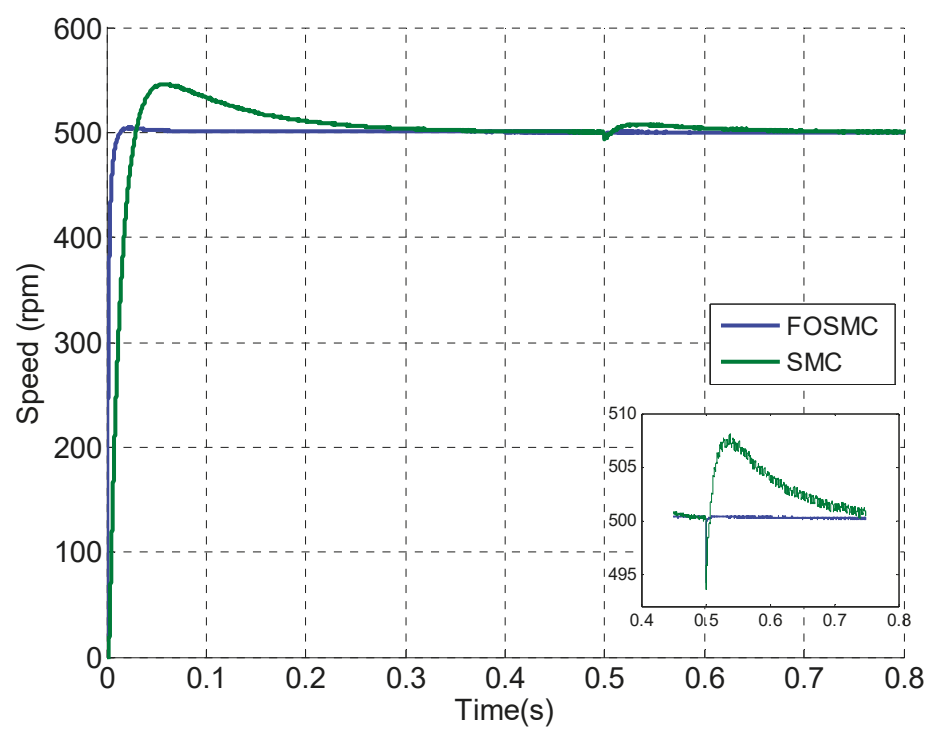

Figure 4. Speed response of the proposed FOSMC and conventional SMC speed controller.

The recorded settling time for SMC system was more than 30 times longer than FOSMC system. When load torque was applied, SMC system suffered from speed drop of almost the same as those of FOSMC system. However, it took almost 25 times longer time to settle back to the reference speed compared to the FOSMC system. Furthermore, in system with conventional SMC, 10 times higher speed ripple was observed.

\subsubsection{Performance Comparison of Fractional Order SMC with Different Sliding Surface Designs} (PI, PD and PID)

In this work, a fractional PID sliding surface was proposed to construct the sliding mode controller. The performance of the proposed sliding surface design is compared with PI and PD sliding surface. Similarly, reference speed was set at $500 \mathrm{rpm}$ and load torque of $2 \mathrm{Nm}$ was applied at $t=0.5 \mathrm{~s}$. From Figure 5, it can be seen that FOSMC with PID sliding surface resulted in better speed response with almost 19 times less overshoot than FOSMC with PI sliding surface. When load torque of $2 \mathrm{Nm}$ 
was applied, FOSMC-PI experienced a speed drop of $4 \%$, which is $2.8 \%$ more than the speed drop of a FOSMC-PID system. After the speed drop, the FOSMC-PI system settled back to reference speed after almost $0.01 \mathrm{~s}$, whereas the FOSMC-PID settled faster by one fifth. In terms of torque ripple, the performance of FOSMC-PID was prominent, where the resulting torque ripple was half of the torque ripple of FOSMC-PI. Figure 6 shows that FOSMC-PD resulted in no overshoot compared to FOSMC-PID. However, its settling time was almost nine times longer than FOSMC-PID. In addition to that, FOSMC-PD had a larger steady state error of almost three times higher than FOSMC-PID. Torque ripple of both controllers was almost equal.

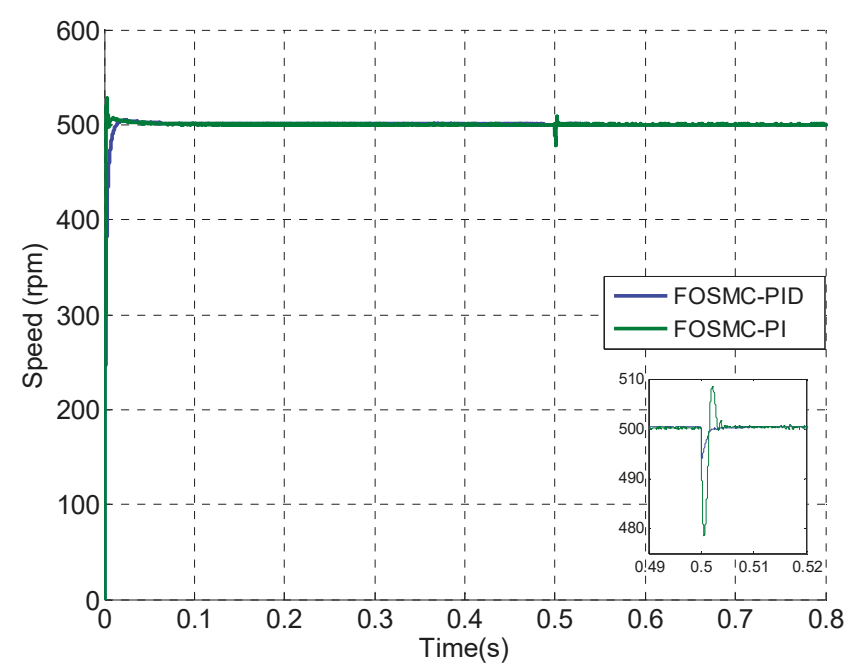

Figure 5. Speed response of FOSMC-PID and FOSMC-PI speed controller.

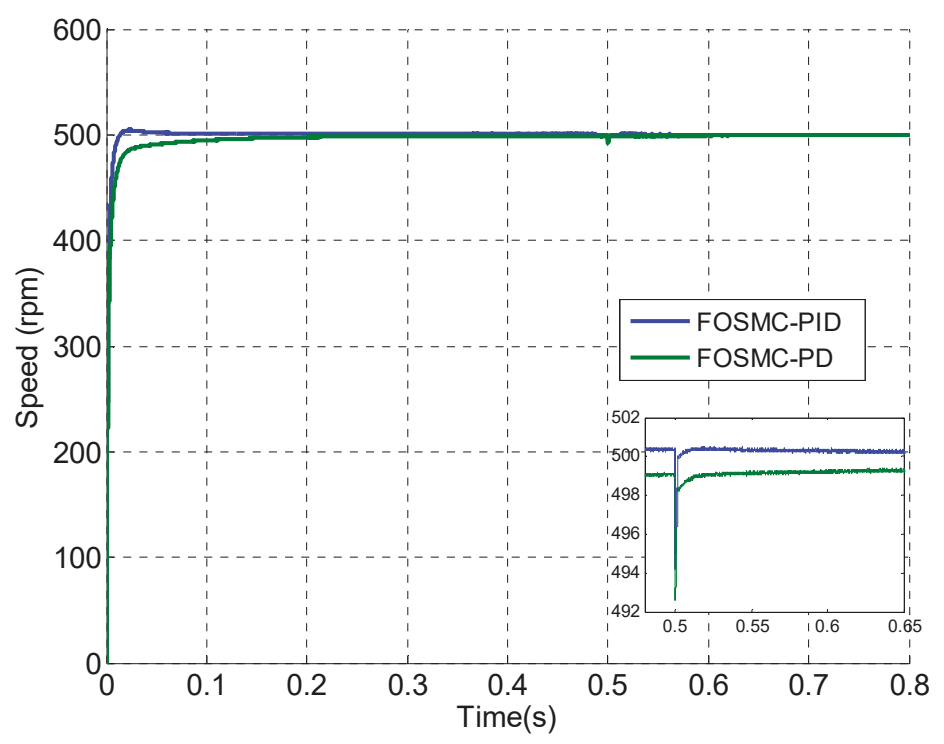

Figure 6. Speed response of FOSMC-PID and FOSMC-PD speed controller. 
In its own fractional order SMC group, the proposed FOSMC-PID has shown its advantages in balancing the individual strength and weaknesses of FOSMC-PI and FOSMC-PD. The proposed FOSMC reduced the overshoot contributed by the integral portion and at the same time reduce the steady state error contributed by the differential portion. The combination of PID also reduces the speed drop when the load torque was applied. Small speed and torque ripple proves that chattering phenomenon has been successfully suppressed. Performance indices of the proposed controller and the controllers in comparison are summarized in Table 2.

Table 2. Comparison of performance indices of the proposed controller with previous works.

\begin{tabular}{ccccc}
\hline Performance Indices & SMC & FOSMC-PI & FOSMC-PD & $\begin{array}{c}\text { FOSMC-PID } \\
\text { (Proposed) }\end{array}$ \\
\hline Overshoot (\%) & 9.22 & 5.52 & 0 & 0.8593 \\
Settling time (s) & 0.288 & 0.0094 & 0.096 & 0.0096 \\
Speed drop (\%) & 1.28 & 4.3 & 1.5 & 1.16 \\
Steady state error (\%) & 0.02 & 0.04 & 0.06 & 0.02 \\
Torque ripple (\%) & 12 & 11 & 10 & 10 \\
Speed ripple (\%) & 0.16 & 0.12 & 0.014 & 0.014 \\
\hline
\end{tabular}

\subsubsection{Performance of the Proposed FOSMC Speed Controller for Various Conditions}

The controller is then tested for various conditions to ensure its robustness. Figure 7 shows the controller performance when reference speeds of 100 to $3000 \mathrm{rpm}$ are given. The reference speed is tracked with settling time not more than $0.2 \mathrm{~s}$ and with overshoot not more than $2 \%$. On a different case, various load torque $(2-6 \mathrm{Nm})$ are given to the system at $t=0.5 \mathrm{~s}$ and the speed reference is fixed at $500 \mathrm{rpm}$. Figure 8 shows that the controller is able to track back the reference speed after speed drop of less than $13 \%$. The proposed controller is also tested for a few motor sizes. As shown in Figure 9, the proposed controller ensures successful speed tracking for motors between $1.67 \mathrm{~kW}$ and $13.22 \mathrm{~kW}$. The performance of the proposed controller is further validated and compared with performance of SMC under different scenarios. Figure 10 shows the speed response of system when positive loads of $2 \mathrm{Nm}$ (at $t=0.5 \mathrm{~s}$ ) and $6 \mathrm{Nm}$ (at $t=1.0 \mathrm{~s}$ ) as well as negative loads of $-4 \mathrm{Nm}$ (at $t=1.5 \mathrm{~s}$ ) are applied. The speed response for both positive and negative reference speeds of $500 \mathrm{rpm},-500 \mathrm{rpm}$ and $-1000 \mathrm{rpm}$ at $t=0.0 \mathrm{~s}, t=0.5 \mathrm{~s}$ and $t=1.0 \mathrm{~s}$ respectively is shown in Figure 11. In this case, a load torque of $2 \mathrm{Nm}$ is applied at $t=1.5 \mathrm{~s}$. 


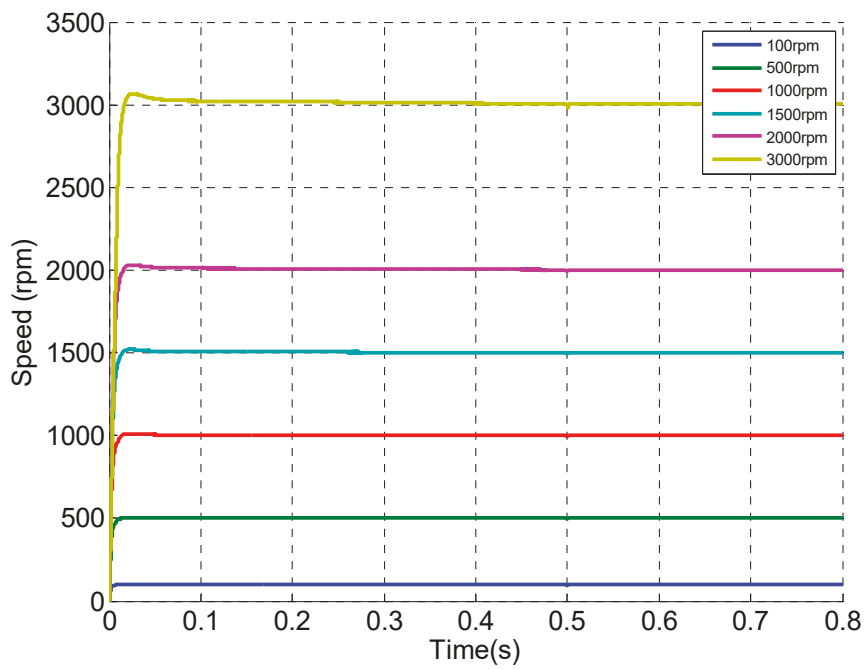

Figure 7. Speed response of FOSMC-PID speed controller for various speed references.

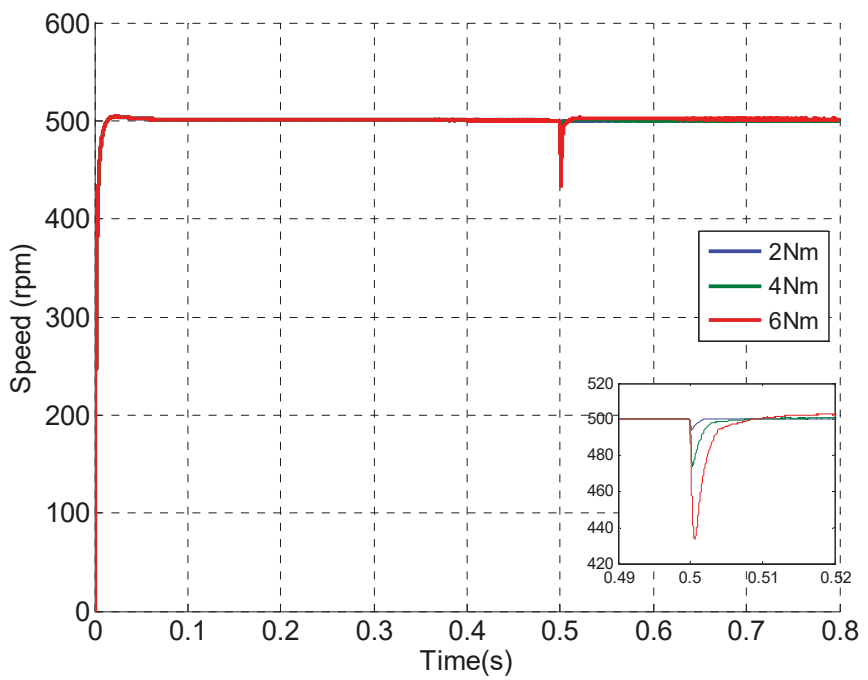

Figure 8. Speed response of FOSMC-PID speed controller for various load torque. 


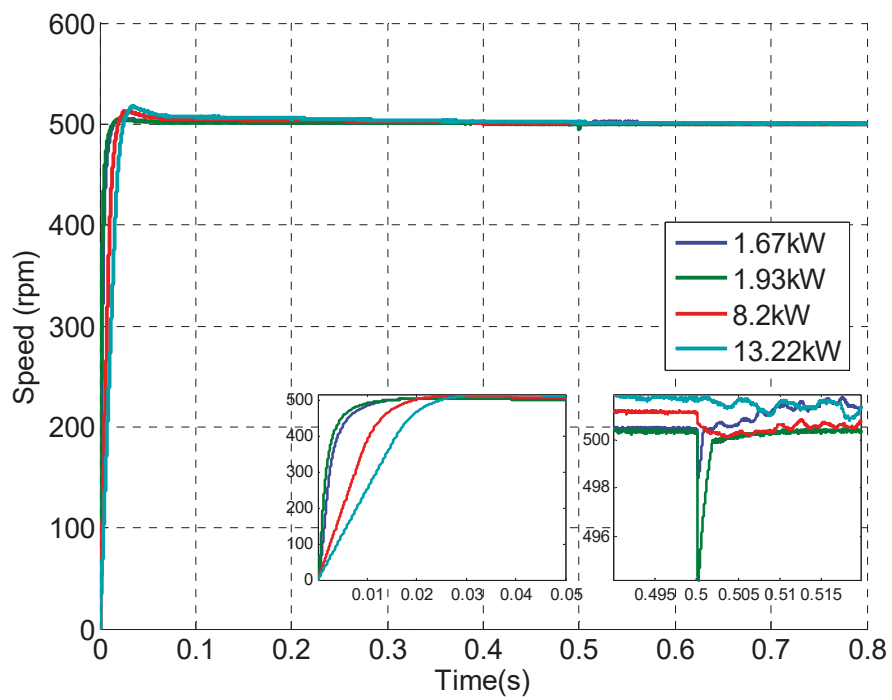

Figure 9. Speed response of FOSMC-PID speed controller for motor sizes.

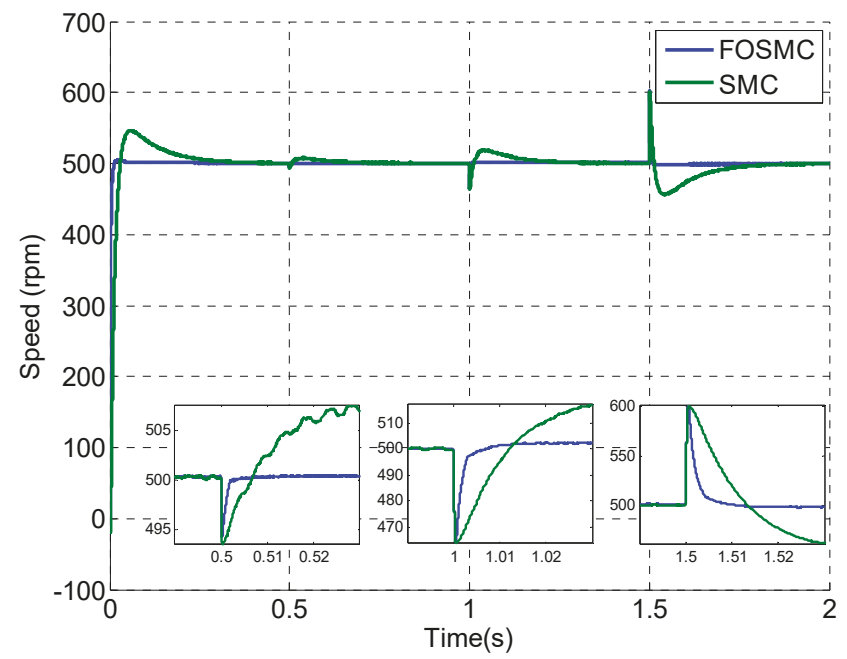

Figure 10. Comparison of speed response of FOSMC and SMC speed controller when positive and negative loads are applied. 


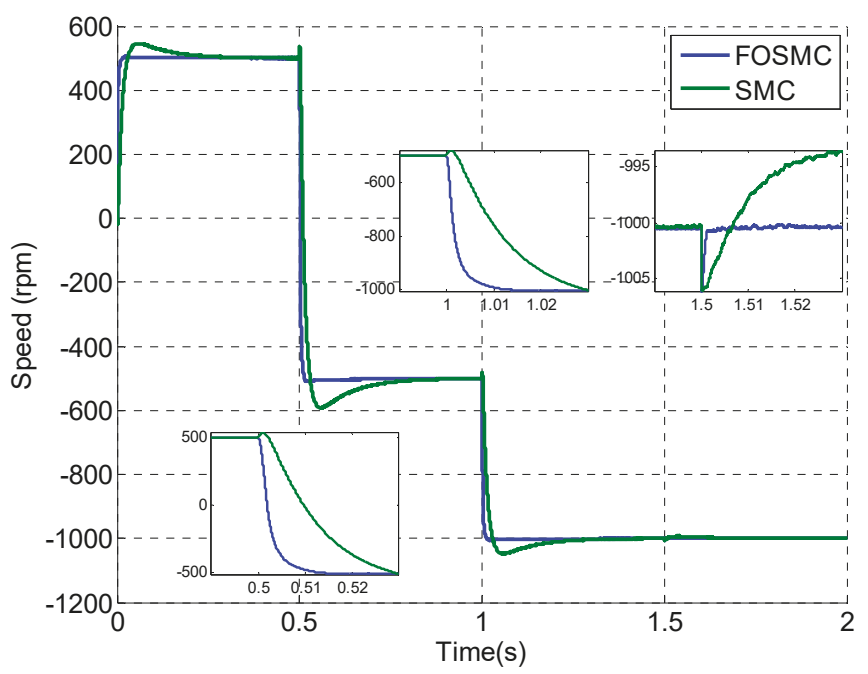

Figure 11. Comparison of speed response of FOSMC and SMC speed controller when positive and negative reference speeds are given.

Simulation results proved that the proposed FOSMC speed controller performs as a robust and fast anti-disturbance controller to regulate the speed of a PMSM and proven its advantages against SMC controllers. The proposed sliding surface design also improves the FOSMC in terms of torque ripple reduction, chattering reduction and anti-disturbance properties, compared to FOSMC with PI or PD sliding surface. Its robustness is also proven for various conditions such as for various speed references, various load torques and various motor sizes.

\section{Discussion}

Sliding mode control possesses many remarkable features to be applied in designing a robust and precise speed controller for permanent magnet synchronous motors. Sliding surface design and the chosen reaching law determine the equivalent control law that should result in system state's convergence to sliding surface and control target achieved on the sliding surface as well as should ensure that reaching phase is minimized and chattering effect is attenuated. In addition to pure SMC controllers, controller's performance is enhanced in terms of robustness and chattering reduction by many means. The main idea behind composite SMC is to have an adaptive sliding surface design based on uncertainties and disturbances, compared to a sliding surface design with fix values in conventional SMC. State-of-the-art implementation of SMC for speed control of PMSM is summarized in Figure 12 and compared in terms of their remarkable properties and disadvantages in Table 3. 


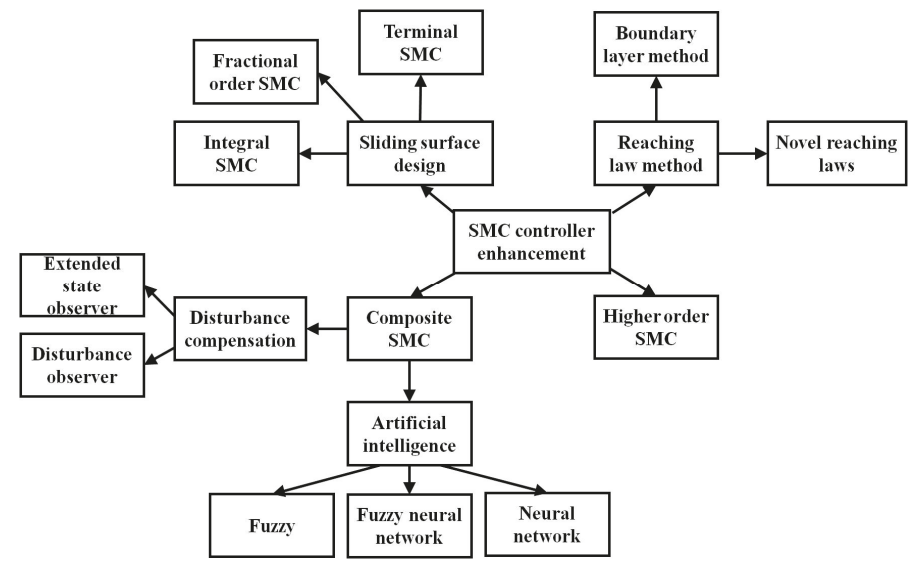

Figure 12. Summary of SMC enhancement methods.

As far as SMC for PMSM speed control is concerned, opportunities of future works are still wide open. The lists of sliding surface designs and composite sliding mode controllers that have been successfully applied to other machines but have not been applied for speed control of PMSM are endless. Similar good results are not guaranteed but they are worth to be researched on. It is also necessary to mention that more research works is required in the area of SMC parameter tuning, since many researchers still based their work on conventional trial and error method. A new promising approach to optimise parameter tuning is by using adaptive SMC to ensure a dynamical adaptation of the control gain in order to be as small as possible whereas sufficient to counteract the uncertainties/perturbations [152-158]. For PMSM application, disturbance estimation techniques are mostly constraint at either $\mathrm{DOB}$ or state observer. This situation opens the opportunity for future researchers to explore the combination of SMC with other estimation techniques.

Many composite SMC controllers have been proposed, but researchers should not compromise one of the remarkable features of SMC namely its simplicity. Integration of artificial intelligence into SMC in order to produce an 'intelligent' controller should not jeopardize the controller's speed. Information on chattering effect is crucial in presenting SMCs. In several literatures, the chattering effect of their proposed controllers was not carefully presented or simply not addressed. It can be concluded that what remains in designing a SMC for speed control of PMSM is to find balance between the robustness and the chattering for wide range of operating conditions. 
Table 3. Comparison of state-of-the-art implementation of SMC for speed control of PMSM.

\begin{tabular}{|c|c|c|}
\hline Controller Description & Remarkable Properties & Disadvantages \\
\hline Linear SMC & Simplicity & $\begin{array}{l}\text { Unsatisfactory convergence rate and settling } \\
\text { time (can be improved by composite SMC or } \\
\text { reaching law modification) }\end{array}$ \\
\hline Integral SMC & $\begin{array}{l}\text { - } \quad \text { Reaching phase eliminated } \\
\text { - Order of motion equation is equal to order of } \\
\text { the original system }\end{array}$ & $\begin{array}{l}\text { Controller gain must be carefully tuned to } \\
\text { ensure balance between robustness and } \\
\text { chattering }\end{array}$ \\
\hline Fractional order SMC & $\begin{array}{l}\text { - Improve controller performance with extra } \\
\text { degree of freedom (fractional operator) } \\
\text { Small chattering due to slower energy transfer } \\
\text { compared to integral SMC }\end{array}$ & Careful tuning of fractional operator required \\
\hline Higher order SMC & $\begin{array}{l}\text { - Control input acts on higher derivatives of the } \\
\text { sliding surface } \\
\text { - Sliding order characterizes the system dynamics } \\
\text { in the vicinity of the sliding mode in terms of } \\
\text { degree of smoothness }\end{array}$ & $\begin{array}{l}\text { Usage of differentiators, where their practical } \\
\text { behavior requires particular care in real } \\
\text { implementation due to measurement noise. }\end{array}$ \\
\hline Boundary layer SMC & $\begin{array}{l}\text { Smooth approximation to replace sign function to } \\
\text { alleviate chattering }\end{array}$ & $\begin{array}{l}\text { - Sacrifices the system's } \\
\text { anti-disturbance performance } \\
\text { Steady-state error depending on } \\
\text { boundary layer selection }\end{array}$ \\
\hline SMC with AI & $\begin{array}{l}\text { Areas of AI utilization with SMC: } \\
\text { - } \quad \text { combined with SMC to enhance the } \\
\text { controller's performance } \\
\text { - utilize the advantage of SMC to enhance main } \\
\text { AI controllers } \\
\text { - used for online or offline parameter tuning of a } \\
\text { SMC controller } \\
\text { - } \quad \text { as uncertainties estimator } \\
\text { - to eliminate chattering. }\end{array}$ & Computational load \\
\hline
\end{tabular}

\section{Conclusions}

An extensive review of the state-of-the-art implementation of SMC for speed control of PMSMs is presented in this paper. Various sliding surface designs and composite controller designs with SMC implementation have been highlighted. The remarkable properties as well as the disadvantages of previous works are summarized. Ideas on possible future works are also discussed, which emphasize on current gaps in this area of research. Certain areas have not been thoroughly covered in this study and deserve a separate thorough review e.g. the application of sliding mode controllers in sensorless speed control of PMSMs, the application of SMC for DTC control of PMSMs and sliding mode observers in combination with other controllers for speed control of PMSMs. 
Author Contributions: Conceptualization, F.M.Z., S.M. and M.M.; Methodology, F.M.Z., S.M. and M.M.; Software, F.M.Z.; Validation, F.M.Z., S.M. and M.M.; Formal Analysis, F.M.Z., S.M. and M.M.; Investigation, F.M.Z., S.M. and M.M.; Resources, F.M.Z., S.M. and M.M.; Data Curation, F.M.Z., S.M. and M.M.; Writing-Original Draft Preparation, F.M.Z.; Writing-Review \& Editing, F.M.Z., S.M. and M.M.; Visualization, F.M.Z., S.M. and M.M.; Supervision, S.M. and M.M.; Project Administration, F.M.Z; Funding Acquisition, S.M. and M.M.

Funding: The authors would like to acknowledge the financial support received from the University of Malaya, Malaysia, through FRGS Grant No. FRGS/1/2018/TK07/UM/01/3, Frontier Research Grant No. FG007-17AFR and Postgraduate Research Grant (PPP) No. PG192-2015B.

Conflicts of Interest: The authors declare no conflict of interest.

\section{References}

1. Shihua, L.; Mingming, Z.; Xinghuo, Y. Design and Implementation of Terminal Sliding Mode Control Method for PMSM Speed Regulation System. IEEE Trans. Ind. Inform. 2013, 9, 1879-1891. [CrossRef]

2. Pillay, P.; Krishnan, R. Modeling, simulation, and analysis of permanent-magnet motor drives. I. The permanent-magnet synchronous motor drive. IEEE Trans. Ind. Appl. 1989, 25, 265-273. [CrossRef]

3. Abu-Rub, H.; Iqbal, A.; Guzinski, J. High Performance Control of AC Drives with Matlab/Simulink Models; Wiley: Hoboken, NJ, USA, 2012.

4. Zhang, B.; Pi, Y.; Luo, Y. Fractional order sliding-mode control based on parameters auto-tuning for velocity control of permanent magnet synchronous motor. ISA Trans. 2012, 51, 649-656. [CrossRef] [PubMed]

5. Huang, J.; Li, H.; Teng, F.; Liu, D. Fractional order sliding mode controller for the speed control of a permanent magnet synchronous motor. In Proceedings of the 24th Chinese Control and Decision Conference (CCDC), Taiyuan, China, 23-25 May 2012; pp. 1203-1208.

6. Maleki, N.; Pahlavani, M.R.A.; Soltani, I. A Detailed Comparison Between FOC and DTC Methods of a Permanent Magnet Synchronous Motor Drive. J. Electr. Electron. Eng. 2015, 3, 92-100. [CrossRef]

7. Garcia, X.D.T.; Zigmund, B.; Terlizzi, A.A.; Pavlanin, R.; Salvatore, L. Comparison between FOC and DTC Strategies for Permanent Magnet Synchronous Motors. Adv. Electr. Electron. Eng. 2011, 5. Available online: http://advances.utc.sk/index.php/AEEE/article/view/179 (accessed on 20 January 2019).

8. Li, S.; Zong, K.; Liu, H. A composite speed controller based on a second-order model of permanent magnet synchronous motor system. Trans. Inst. Meas. Control 2011, 33, 522-541. [CrossRef]

9. Ke, Z.; Xiao-guang, Z.; Li, S.; Chang, C. Sliding mode control of high-speed PMSM based on precision linearization control. In Proceedings of the International Conference on Electrical Machines and Systems (ICEMS), Beijing, China, 20-23 August 2011; pp. 1-4.

10. Gou-Jen, W.; Chuan-Tzueng, F.; Chang, K.J. Neural-network-based self-tuning PI controller for precise motion control of PMAC motors. IEEE Trans. Ind. Electron. 2001, 48, 408-415. [CrossRef]

11. Xiaoguang, Z.; Lizhi, S.; Ke, Z.; Li, S. Nonlinear speed control for PMSM system using sliding mode control and disturbance compensation techniques. IEEE Trans. Power Electron. 2013, 28, 1358-1365. [CrossRef]

12. El-Sousy, F.F.M. Robust wavelet-neural-network sliding-mode control system for permanent magnet synchronous motor drive. IET Electr. Power Appl. 2011, 5, 113-132. [CrossRef]

13. Huixian, L.; Shihua, L. Speed Control for PMSM Servo System Using Predictive Functional Control and Extended State Observer. IEEE Trans. Ind. Electron. 2012, 59, 1171-1183. [CrossRef]

14. Errouissi, R.; Ouhrouche, M.; Wen-Hua, C.; Trzynadlowski, A.M. Robust nonlinear predictive controller for permanent magnet synchronous motors with an optimized cost function. IEEE Trans. Ind. Electron. 2012, 59, 2849-2858. [CrossRef]

15. Zhou, J.; Wang, Y. Adaptive backstepping speed controller design for a permanent magnet synchronous motor. IEE Proc.-Electr. Power Appl. 2002, 149, 165-172. [CrossRef]

16. Vu, N.T.-T.; Choi, H.H.; Jung, J.-W. Certainty equivalence adaptive speed controller for permanent magnet synchronous motor. Mechatronics 2012, 22, 811-818. [CrossRef]

17. Shihua, L.; Zhigang, L. Adaptive speed control for permanent magnet synchronous motor system with variations of load inertia. IEEE Trans. Ind. Electron. 2009, 56, 3050-3059. [CrossRef]

18. Han Ho, C.; Nga Thi-Thuy, V.; Jin-Woo, J. Digital Implementation of an Adaptive Speed Regulator for a PMSM. IEEE Trans. Power Electron. 2011, 26, 3-8. [CrossRef]

19. Wang, Q.; Yu, H.; Wang, M.; Qi, X. A Novel Adaptive Neuro-Control Approach for Permanent Magnet Synchronous Motor Speed Control. Energies 2018, 11, 2355. [CrossRef] 
20. Hsien, T.L.; Sun, Y.Y.; Tsai, M.C. H $\infty$ control for a sensorless permanent-magnet synchronous drive. IEEE Proc. Electr. Power Appl. 1997, 144, 173-181. [CrossRef]

21. Su, Y.X.; Zheng, C.H.; Duan, B.Y. Automatic disturbances rejection controller for precise motion control of permanent-magnet synchronous motors. IEEE Trans. Ind. Electron. 2005, 52, 814-823. [CrossRef]

22. Han Ho, C.; Jin-Woo, J. Discrete-Time Fuzzy Speed Regulator Design for PM Synchronous Motor. IEEE Trans. Ind. Electron. 2013, 60, 600-607. [CrossRef]

23. Utkin, V. Variable structure systems with sliding modes. IEEE Trans. Autom. Control 1977, 22, $212-222$. [CrossRef]

24. Hung, J.Y.; Gao, W.; Hung, J.C. Variable structure control: A survey. IEEE Trans. Ind. Electron. 1993, 40, $2-22$. [CrossRef]

25. Fallaha, C.J.; Saad, M.; Kanaan, H.Y.; Al-Haddad, K. Sliding mode robot control with exponential reaching law. IEEE Trans. Ind. Electron. 2011, 58, 600-610. [CrossRef]

26. Rong-Jong, W.; Kun-Lun, C.; Jeng-Dao, L. On-Line Supervisory Control Design for Maglev Transportation System via Total Sliding-Mode Approach and Particle Swarm Optimization. IEEE Trans. Autom. Control 2010, 55, 1544-1559. [CrossRef]

27. Chih-Lyang, H.; Li-Jui, C.; Yuan-Sheng, Y. Network-Based Fuzzy Decentralized Sliding-Mode Control for Car-Like Mobile Robots. IEEE Trans. Ind. Electron. 2007, 54, 574-585. [CrossRef]

28. Syuan-Yi, C.; Faa-Jeng, L. Robust nonsingular terminal sliding mode control for nonlinear magnetic bearing system. IEEE Trans. Control Syst. Technol. 2011, 19, 636-643. [CrossRef]

29. Bao-Lin, Z.; Qing-Long, H.; Xian-Ming, Z.; Xinghuo, Y. Sliding Mode Control With Mixed Current and Delayed States for Offshore Steel Jacket Platforms. IEEE Trans. Control Syst. Technol. 2014, 22, 1769-1783. [CrossRef]

30. Chuan-Kai, L. Nonsingular Terminal Sliding Mode Control of Robot Manipulators Using Fuzzy Wavelet Networks. IEEE Trans. Fuzzy Syst. 2006, 14, 849-859. [CrossRef]

31. Jeng-Dao, L.; Suiyang, K.; Zhi-Bin, W. DSP-based sliding mode control for electromagnetic levitation precise position system. IEEE Trans. Ind. Inform. 2013, 9, 817-827. [CrossRef]

32. Han, S.I.; Lee, J.M. Balancing and Velocity Control of a Unicycle Robot Based on the Dynamic Model. IEEE Trans. Ind. Electron. 2015, 62, 405-413. [CrossRef]

33. Faa-Jeng, L.; Chih-Kai, C.; Po-Kai, H. FPGA-Based Adaptive Backstepping Sliding-Mode Control for Linear Induction Motor Drive. IEEE Trans. Power Electron. 2007, 22, 1222-1231. [CrossRef]

34. El-Sousy, F.F.M. Adaptive Dynamic Sliding-Mode Control System Using Recurrent RBFN for High-Performance Induction Motor Servo Drive. IEEE Trans. Ind. Inform. 2013, 9, 1922-1936. [CrossRef]

35. Veselic, B.; Perunicic-Drazenovic, B.; Milosavljevic, C. Improved Discrete-Time Sliding-Mode Position Control Using Euler Velocity Estimation. IEEE Trans. Ind. Electron. 2010, 57, 3840-3847. [CrossRef]

36. Pupadubsin, R.; Chayopitak, N.; Taylor, D.G.; Nulek, N.; Kachapornkul, S.; Jitkreeyarn, P.; Somsiri, P.; Tungpimolrut, K. Adaptive Integral Sliding-Mode Position Control of a Coupled-Phase Linear Variable Reluctance Motor for High-Precision Applications. IEEE Trans. Ind. Appl. 2012, 48, 1353-1363. [CrossRef]

37. Yaonan, W.; Xizheng, Z.; Xiaofang, Y.; Guorong, L. Position-Sensorless Hybrid Sliding-Mode Control of Electric Vehicles With Brushless DC Motor. IEEE Trans. Veh. Technol. 2011, 60, 421-432. [CrossRef]

38. Jian-Bo, C.; Bing-Gang, C. Fuzzy-Logic-Based Sliding-Mode Controller Design for Position-Sensorless Electric Vehicle. IEEE Trans. Power Electron. 2009, 24, 2368-2378. [CrossRef]

39. Eker, İ. Second-order sliding mode control with experimental application. ISA Trans. 2010, 49, $394-405$. [CrossRef] [PubMed]

40. Young, K.D.; Utkin, V.I.; Ozguner, U. A control engineer's guide to sliding mode control. IEEE Trans. Control Syst. Technol. 1999, 7, 328-342. [CrossRef]

41. Utkin, V.I. Sliding mode control design principles and applications to electric drives. IEEE Trans. Ind. Electron. 1993, 40, 23-36. [CrossRef]

42. Qi, L.; Shi, H. Adaptive position tracking control of permanent magnet synchronous motor based on RBF fast terminal sliding mode control. Neurocomputing 2013, 115, 23-30. [CrossRef]

43. Elmas, C.; Ustun, O. A hybrid controller for the speed control of a permanent magnet synchronous motor drive. Control Eng. Pract. 2008, 16, 260-270. [CrossRef]

44. Yan, L.; Ju-Beom, S.; Jang-myung, L. PMSM speed controller using switching algorithm of PD and Sliding mode control. In Proceedings of the ICCAS-SICE, Fukuoka, Japan, 18-21 August 2009; pp. 1260-1266. 
45. Utkin, V.; Jingxin, S. Integral sliding mode in systems operating under uncertainty conditions. In Proceedings of the 35th IEEE Conference on Decision and Control, Kobe, Japan, 11-13 December 1996; pp. 4591-4596.

46. Castanos, F.; Fridman, L. Analysis and design of integral sliding manifolds for systems with unmatched perturbations. IEEE Trans. Autom. Control 2006, 51, 853-858. [CrossRef]

47. Liang, Y.W.; Ting, L.W.; Lin, L.G. Study of Reliable Control Via an Integral-Type Sliding Mode Control Scheme. IEEE Trans. Ind. Electron. 2012, 59, 3062-3068. [CrossRef]

48. Xia, C.; Wang, X.; Li, S.; Chen, X. Improved integral sliding mode control methods for speed control of PMSM system. Int. J. Innov. Comput. Inf. Control 2011, 7, 1971-1982.

49. Song, Q.; Jia, C. Robust Speed Controller Design for Permanent Magnet Synchronous Motor Drives Based on Sliding Mode Control. Energy Procedia 2016, 88, 867-873. [CrossRef]

50. Zaky, M. Adaptive and robust speed control of interior permanent magnet synchronous motor drives. Electr. Eng. 2012, 94, 49-58. [CrossRef]

51. Efe, M.O. Fractional Order Systems in Industrial Automation-A Survey. IEEE Trans. Ind. Inform. 2011, 7, 582-591. [CrossRef]

52. Huang, J.; Cui, L.; Shi, X.; Li, H.; Xiang, Z. Composite integral sliding mode control for PMSM. In Proceedings of the 33rd Chinese Control Conference (CCC), Nanjing, China, 28-30 July 2014; pp. 8086-8090.

53. Hongsheng, L.; Ying, L.; Yang Quan, C. A Fractional Order Proportional and Derivative (FOPD) Motion Controller: Tuning Rule and Experiments. IEEE Trans. Control Syst. Technol. 2010, 18, 516-520. [CrossRef]

54. Lanusse, P.; Oustaloup, A.; Sabatier, J. Robust factional order PID controllers: The first generation CRONE CSD approach. In Proceedings of the International Conference on Fractional Differentiation and Its Applications 2014 (ICFDA), Catania, Italy, 23-25 June 2014; pp. 1-6.

55. Zhang, B.T.; Pi, Y. Robust fractional order proportion-plus-differential controller based on fuzzy inference for permanent magnet synchronous motor. IET Control Theory Appl. 2012, 6, 829-837. [CrossRef]

56. Efe, M.O. Fractional Fuzzy Adaptive Sliding-Mode Control of a 2-DOF Direct-Drive Robot Arm. IEEE Trans. Syst. ManCybern. Part B Cybern. 2008, 38, 1561-1570. [CrossRef]

57. Ladaci, S.; Charef, A. On Fractional Adaptive Control. Nonlinear Dyn. 2006, 43, 365-378. [CrossRef]

58. Luo, Y.; Chen, Y.; Ahn, H.-S.; Pi, Y. Fractional order robust control for cogging effect compensation in PMSM position servo systems: Stability analysis and experiments. Control Eng. Pract. 2010, 18, 1022-1036. [CrossRef]

59. Salehtavazoei, M.; Tavakoli-Kakhki, M. Compensation by fractional-order phase-lead/lag compensators. IET Control Theory Appl. 2014, 8, 319-329. [CrossRef]

60. Luo, Y.; Chen, Y.Q.; Ahn, H.S.; Pi, Y.G. Fractional Order Periodic Adaptive Learning Compensation for State-Dependent Periodic Disturbance. IEEE Trans. Control Syst. Technol. 2012, 20, 465-472. [CrossRef]

61. Ying, L.; Yang Quan, C.; Hyo-Sung, A.; Youguo, P. Fractional order periodic adaptive learning compensation for cogging effect in PMSM position servo system. In Proceedings of the American Control Conference (ACC), St. Louis, MO, USA, 10-12 June 2009; pp. 937-942.

62. Mujumdar, A.; Tamhane, B.; Kurode, S. Observer-Based Sliding Mode Control for a Class of Noncommensurate Fractional-Order Systems. IEEE/ASME Trans. Mechatron. 2015, PP, 1-9. [CrossRef]

63. Calderón, A.J.; Vinagre, B.M.; Feliu, V. Fractional order control strategies for power electronic buck converters. Signal Process. 2006, 86, 2803-2819. [CrossRef]

64. Delavari, H.; Ghaderi, R.; Ranjbar, A.; Momani, S. Fuzzy fractional order sliding mode controller for nonlinear systems. Commun. Nonlinear Sci. Numer. Simul. 2010, 15, 963-978. [CrossRef]

65. Abdelhamid, D.; Bouden, T.; Boulkroune, A. Design of Fractional-order Sliding Mode Controller (FSMC) for a class of Fractional-order Non-linear Commensurate Systems using a Particle Swarm Optimization (PSO) Algorithm. J. Control Eng. Appl. Inform. 2014, 16, 46-55.

66. Sun, G.; Wu, L.; Kuang, Z.; Ma, Z.; Liu, J. Practical tracking control of linear motor via fractional-order sliding mode. Automatica 2018, 94, 221-235. [CrossRef]

67. Feng, Y.; Yu, X.; Man, Z. Non-singular terminal sliding mode control of rigid manipulators. Automatica 2002, 38, 2159-2167. [CrossRef]

68. Liu, J.; Wang, X. Advanced Sliding Mode Control for Mechanical Systems: Design, Analysis and MATLAB Simulation; Springer: Berlin/Heidelberg, Germany, 2012.

69. Wu, Y.; Yu, X.; Man, Z. Terminal sliding mode control design for uncertain dynamic systems. Syst. Control Lett. 1998, 34, 281-287. [CrossRef] 
70. Man, Z.; Xing Huo, Y. Terminal sliding mode control of MIMO linear systems. IEEE Trans. Circuits Syst. I Fundam. Theory Appl. 1997, 44, 1065-1070. [CrossRef]

71. Jin, M.; Lee, J.; Chang, P.H.; Choi, C. Practical Nonsingular Terminal Sliding-Mode Control of Robot Manipulators for High-Accuracy Tracking Control. IEEE Trans. Ind. Electron. 2009, 56, 3593-3601. [CrossRef]

72. Zhang, R.; Dong, L.; Sun, C. Adaptive nonsingular terminal sliding mode control design for near space hypersonic vehicles. IEEE/CAA J. Autom. Sin. 2014, 1, 155-161. [CrossRef]

73. $\mathrm{Mu}, \mathrm{C}$; $\mathrm{He}, \mathrm{H}$. Dynamic Behavior of Terminal Sliding Mode Control. IEEE Trans. Ind. Electron. 2018, 65, 3480-3490. [CrossRef]

74. Xu, W.; Jiang, Y.; Mu, C.; Yue, H. Nonsingular terminal sliding mode control for the speed regulation of permanent magnet synchronous motor with parameter uncertainties. In Proceedings of the 41st Annual Conference of the IEEE Industrial Electronics Society, IECON, Pattaya, Thailand, 9-12 November 2015; pp. 001994-001999.

75. Liu, X.; Yu, H.; Yu, J.; Zhao, L. Combined Speed and Current Terminal Sliding Mode Control With Nonlinear Disturbance Observer for PMSM Drive. IEEE Access 2018, 6, 29594-29601. [CrossRef]

76. Xinghuo, Y.; Man, Z. Fast terminal sliding-mode control design for nonlinear dynamical systems. IEEE Trans. Circuits Syst. I Fundam. Theory Appl. 2002, 49, 261-264. [CrossRef]

77. Mu, C.; Xu, W.; Sun, C. On Switching Manifold Design for Terminal Sliding Mode Control. J. Frankl. Inst. 2016, 353. [CrossRef]

78. Lu, E.; Li, W.; Yang, X.; Xu, S. Composite Sliding Mode Control of a Permanent Magnet Direct-Driven System For a Mining Scraper Conveyor. IEEE Access 2017, 5, 22399-22408. [CrossRef]

79. Xu, S.S.D.; Chen, C.C.; Wu, Z.L. Study of Nonsingular Fast Terminal Sliding-Mode Fault-Tolerant Control. IEEE Trans. Ind. Electron. 2015, 62, 3906-3913. [CrossRef]

80. Gudey, S.K.; Gupta, R. Recursive fast terminal sliding mode control in voltage source inverter for a low-voltage microgrid system. IET Gener. Transm. Distrib. 2016, 10, 1536-1543. [CrossRef]

81. Komurcugil, H. Fast terminal sliding mode control for single-phase UPS inverters. In Proceedings of the IEEE International Symposium on Industrial Electronics, Gdansk, Poland, 27-30 June 2011; pp. 277-282.

82. Mobayen, S. Fast terminal sliding mode tracking of non-holonomic systems with exponential decay rate. IET Control Theory Appl. 2015, 9, 1294-1301. [CrossRef]

83. Al-Ghanimi, A.; Zheng, J.; Man, Z. Robust and fast non-singular terminal sliding mode control for piezoelectric actuators. IET Control Theory Appl. 2015, 9, 2678-2687. [CrossRef]

84. Mu, C.; Sun, C.; Xu, W. Fast sliding mode control on air-breathing hypersonic vehicles with transient response analysis. Proc. Inst. Mech. Eng. Part I J. Sys. Control Eng. 2015, 230, 23-34. [CrossRef]

85. Shuo, Z. Nonsingular fast terminal sliding mode control method and its application on permanent magnet synchronous motor. In Proceedings of the 34th Chinese Control Conference (CCC), Hangzhou, China, 28-30 July 2015; pp. 3383-3386.

86. Levant, A. Sliding order and sliding accuracy in sliding mode control. Int. J. Control 1993, 58, 1247-1263. [CrossRef]

87. Levant, A. Principles of 2-sliding mode design. Automatica 2007, 43, 576-586. [CrossRef]

88. Bartolini, G.; Ferrara, A.; Levant, A.; Usai, E. On second order sliding mode controllers. In Variable Structure Systems, Sliding Mode and Nonlinear Control; Young, K.D., Özgüner, Ü., Eds.; Springer: London, UK, 1999; pp. 329-350.

89. Khan, M.K.; Spurgeon, S.K. Robust MIMO water level control in interconnected twin-tanks using second order sliding mode control. Control Eng. Pract. 2006, 14, 375-386. [CrossRef]

90. Xinghuo, Y.; Kaynak, O. Sliding-Mode Control With Soft Computing: A Survey. IEEE Trans. Ind. Electron. 2009, 56, 3275-3285. [CrossRef]

91. Bartolini, G.; Pisano, A.; Punta, E.; Usai, E. A survey of applications of second-order sliding mode control to mechanical systems. Int. J. Control 2003, 76, 875-892. [CrossRef]

92. Matraji, I.; Laghrouche, S.; Jemei, S.; Wack, M. Robust control of the PEM fuel cell air-feed system via sub-optimal second order sliding mode. Appl. Energy 2013, 104, 945-957. [CrossRef]

93. Damiano, A.; Gatto, G.L.; Marongiu, I.; Pisano, A. Second-order sliding-mode control of DC drives. IEEE Trans. Ind. Electron. 2004, 51,364-373. [CrossRef] 
94. Bounasla, N.; Hemsas, K.E. Second order sliding mode control of a permanent magnet synchronous motor. In Proceedings of the 14th International Conference on Sciences and Techniques of Automatic Control and Computer Engineering (STA), Sousse, Tunisia, 20-22 December 2013; pp. 535-539.

95. Levant, A.; Fridman, L. Robustness issues of 2-sliding mode control. In Variable Structure Systems: From Principles to Implementation; Institution of Engineering and Technology: Stevenage, UK, 2004; pp. 131-156. [CrossRef]

96. Bartolini, G.; Pisano, A.; Usai, E. Second-order sliding-mode control of container cranes. Automatica 2002, 38, 1783-1790. [CrossRef]

97. Ling, R.; Maksimovic, D.; Leyva, R. Second-Order Sliding-Mode Controlled Synchronous Buck DC-DC Converter. IEEE Trans. Power Electron. 2016, 31, 2539-2549. [CrossRef]

98. Ferrara, A.; Incremona, G.P. Design of an Integral Suboptimal Second-Order Sliding Mode Controller for the Robust Motion Control of Robot Manipulators. IEEE Trans. Control Syst. Technol. 2015, 23, 2316-2325. [CrossRef]

99. Lin, F.J.; Hung, Y.C.; Ruan, K.C. An Intelligent Second-Order Sliding-Mode Control for an Electric Power Steering System Using a Wavelet Fuzzy Neural Network. IEEE Trans. Fuzzy Syst. 2014, 22, 1598-1611. [CrossRef]

100. Ebrahimi, B.; Tafreshi, R.; Mohammadpour, J.; Franchek, M.; Grigoriadis, K.; Masudi, H. Second-Order Sliding Mode Strategy for Air-Fuel Ratio Control of Lean-Burn SI Engines. IEEE Trans. Control Syst. Technol. 2014, 22, 1374-1384. [CrossRef]

101. Shtessel, Y.B.; Shkolnikov, I.A.; Levant, A. Guidance and Control of Missile Interceptor using Second-Order Sliding Modes. IEEE Trans. Aerosp. Electron. Syst. 2009, 45, 110-124. [CrossRef]

102. Pisano, A.; Davila, A.; Fridman, L.; Usai, E. Cascade Control of PM DC Drives Via Second-Order Sliding-Mode Technique. IEEE Trans. Ind. Electron. 2008, 55, 3846-3854. [CrossRef]

103. Laghrouche, S.; Plestan, F.; Glumineau, A.; Boisliveau, R. Robust second order sliding mode control for a permanent magnet synchronous motor. In Proceedings of the American Control Conference, Denver, CO, USA, 4-6 June 2003; pp. 4071-4076.

104. Kommuri, S.K.; Rath, J.J.; Veluvolu, K.C.; Defoort, M.; Soh, Y.C. Decoupled current control and sensor fault detection with second-order sliding mode for induction motor. IET Control Theory Appl. 2015, 9, 608-617. [CrossRef]

105. Susperregui, A.; Martinez, M.I.; Tapia, G.; Vechiu, I. Second-order sliding-mode controller design and tuning for grid synchronisation and power control of a wind turbine-driven doubly fed induction generator. IET Renew. Power Gener. 2013, 7, 540-551. [CrossRef]

106. Beltran, B.; Benbouzid, M.E.H.; Ahmed-Ali, T. Second-Order Sliding Mode Control of a Doubly Fed Induction Generator Driven Wind Turbine. IEEE Trans. Energy Convers. 2012, 27, 261-269. [CrossRef]

107. Bitao, Z.; Youguo, P. Velocity Control of Permanent Magnet Synchronous Motor Based on Second-Order Sliding-Mode Technology. In Proceedings of the International Conference on Digital Manufacturing \& Automation (ICDMA), Changsha, China, 18-20 December 2010; pp. 893-897.

108. Qi, L.; Shi, H. A Novel Second Order Sliding Mode Control Algorithm for Velocity Control Permanent Magnet Synchronous Motor. In Intelligent Computing for Sustainable Energy and Environment: Second International Conference; Li, K., Li, S., Li, D., Niu, Q., Eds.; Springer: Berlin/Heidelberg, Germany, 2013.

109. Bartolini, G.; Ferrara, A.; Usani, E. Chattering avoidance by second-order sliding mode control. IEEE Trans. Autom. Control 1998, 43, 241-246. [CrossRef]

110. Boiko, I.; Fridman, L.; Pisano, A.; Usai, E. Analysis of Chattering in Systems With Second-Order Sliding Modes. IEEE Trans. Autom. Control 2007, 52, 2085-2102. [CrossRef]

111. Gao, W.; Hung, J.C. Variable structure control of nonlinear systems: A new approach. IEEE Trans. Ind. Electron. 1993, 40, 45-55. [CrossRef]

112. Zhang, X.; Zhao, K.; Sun, L. A PMSM sliding mode control system based on a novel reaching law. In Proceedings of the International Conference on Electrical Machines and Systems (ICEMS), Beijing, China, 20-23 August 2011; pp. 1-5.

113. Jamoussi, K.; Ouali, M.; Chrifi-Alaoui, L.; Benderradji, H.; Hajjaji, A.E. Robust Sliding Mode Control Using Adaptive Switching Gain for Induction Motors. Int. J. Autom. Comput. 2013, 10, 303-311. [CrossRef]

114. Faa-Jeng, L.; Sheng-Lyin, C.; Kuo-Kai, S. Novel sliding mode controller for synchronous motor drive. IEEE Trans. Aerosp. Electron. Syst. 1998, 34, 532-542. [CrossRef] 
115. In-Cheol, B.; Kyeong-Hwa, K.; Myung-Joong, Y. Robust nonlinear speed control of PM synchronous motor using boundary layer integral sliding mode control technique. IEEE Trans. Control Syst. Technol. 2000, 8, 47-54. [CrossRef]

116. Chen, W.H.; Yang, J.; Guo, L.; Li, S. Disturbance-Observer-Based Control and Related Methods-An Overview. IEEE Trans. Ind. Electron. 2016, 63, 1083-1095. [CrossRef]

117. Chiang, H.K.; Tseng, C.H. Integral variable structure controller with grey prediction for synchronous reluctance motor drive. IEE Proc.-Electr. Power Appl. 2004, 151, 349-358. [CrossRef]

118. SangJoo, K.; Wan Kyun, C. A discrete-time design and analysis of perturbation observer for motion control applications. IEEE Trans. Control Syst. Technol. 2003, 11, 399-407. [CrossRef]

119. She, J.H.; Fang, M.; Ohyama, Y.; Hashimoto, H.; Wu, M. Improving Disturbance-Rejection Performance Based on an Equivalent-Input-Disturbance Approach. IEEE Trans. Ind. Electron. 2008, 55, 380-389. [CrossRef]

120. Jingqing, H. The Extended State Observer of a Class of Uncertain Systems. Control Decis. 1995, 1. Available online: http://en.cnki.com.cn/Article_en/CJFDTOTAL-KZYC501.019 (accessed on 20 January 2019).

121. Wang, J.; Li, S.; Yang, J.; Wu, B.; Li, Q. Extended state observer-based sliding mode control for PWM-based DC-DC buck power converter systems with mismatched disturbances. IET Control Theory Appl. 2015, 9, 579-586. [CrossRef]

122. Ohnishi, K. A new servo method in mechatronics. Trans. Jpn. Soc. Electr. Eng. 1987, 107, 83-86.

123. Zhang, J.; Liu, X.; Xia, Y.; Zuo, Z.; Wang, Y. Disturbance Observer-Based Integral Sliding-Mode Control for Systems With Mismatched Disturbances. IEEE Trans. Ind. Electron. 2016, 63, 7040-7048. [CrossRef]

124. Cao, Y.; Chen, X.B. Disturbance-Observer-Based Sliding-Mode Control for a 3-DOF Nanopositioning Stage. IEEE/ASME Trans. Mechatron. 2014, 19, 924-931. [CrossRef]

125. Mu, C.; Zong, Q.; Tian, B.; Xu, W. Continuous sliding mode controller with disturbance observer for hypersonic vehicles. IEEE/CAA J. Autom. Sin. 2015, 2, 45-55. [CrossRef]

126. Kaynak, O.; Erbatur, K.; Ertugnrl, M. The fusion of computationally intelligent methodologies and sliding-mode control-a survey. IEEE Trans. Ind. Electron. 2001, 48, 4-17. [CrossRef]

127. Chen, Z.; Shan, C.; Zhu, H. Adaptive Fuzzy Sliding Mode Control Algorithm for a Non-Affine Nonlinear System. IEEE Trans. Ind. Inform. 2007, 3, 302-311. [CrossRef]

128. Zadeh, L.A. Fuzzy sets. Inf. Control 1965, 8, 338-353. [CrossRef]

129. Mohanty, A.; Patra, S.; Ray, P.K. Robust fuzzy-sliding mode based UPFC controller for transient stability analysis in autonomous wind-diesel-PV hybrid system. IET Gener. Transm. Distrib. 2016, 10, 1248-1257. [CrossRef]

130. Zhang, X.; Wang, Y.; Liu, G.; Yuan, X. Robust Regenerative Charging Control Based on T-S Fuzzy Sliding-Mode Approach for Advanced Electric Vehicle. IEEE Trans. Transp. Electrif. 2016, 2, 52-65. [CrossRef]

131. Dasmahapatra, S.; Sarkar, B.K.; Saha, R.; Chatterjee, A.; Mookherjee, S.; Sanyal, D. Design of an Adaptive Fuzzy-Bias SMC and Validation for a Rugged Electrohydraulic System. IEEE/ASME Trans. Mechatron. 2015, 20, 2708-2715. [CrossRef]

132. Yin, X.X.; Lin, Y.G.; Li, W.; Liu, H.W.; Gu, Y.J. Fuzzy-Logic Sliding-Mode Control Strategy for Extracting Maximum Wind Power. IEEE Trans. Energy Convers. 2015, 30, 1267-1278. [CrossRef]

133. Saghafinia, A.; Wooi Ping, H.; Nasir Uddin, M. Fuzzy sliding mode control based on boundary layer theory for chattering-free and robust induction motor drive. Int. J. Adv. Manuf. Technol. 2014, 71, 57-68. [CrossRef]

134. Elsayed, B.A.; Hassan, M.A.; Mekhilef, S. Fuzzy swinging-up with sliding mode control for third order cart-inverted pendulum system. Int. J. Control Autom. 2015, 13, 238-248. [CrossRef]

135. Elsayed, B.A.; Hassan, M.; Mekhilef, S. Decoupled third-order fuzzy sliding model control for cart-inverted pendulum system. Appl. Math. 2013, 7, 193-201. [CrossRef]

136. Lee, C.C. Fuzzy logic in control systems: Fuzzy logic controller. I. IEEE Trans. Syst. ManCybern. 1990, 20, 404-418. [CrossRef]

137. Guo, Y.; Long, H. Self organizing fuzzy sliding mode controller for the position control of a permanent magnet synchronous motor drive. AIN Shams Eng. J. 2011, 2, 109-118. [CrossRef]

138. Kuo, C.-F.; Hsu, C.-H.; Tsai, C.-C. Control of a permanent magnet synchronous motor with a fuzzy sliding-mode controller. Int. J. Adv. Manuf. Technol. 2007, 32, 757-763. [CrossRef]

139. Brock, S.; Deskur, J.; Zawirski, K. Robust speed and position control of PMSM. In Proceedings of Proceedings of the IEEE International Symposium on Industrial Electronics (Cat. No.99TH8465) (ISIE), Bled, Slovenia, 12-16 July 1999; pp. 667-672. 
140. Saghafinia, A.; Ping, H.W.; Uddin, M.N.; Gaeid, K.S. Adaptive Fuzzy Sliding-Mode Control Into Chattering-Free IM Drive. IEEE Trans. Ind. Appl. 2015, 51, 692-701. [CrossRef]

141. Faa-Jeng, L.; Po-Hung, S. Robust Fuzzy Neural Network Sliding-Mode Control for Two-Axis Motion Control System. IEEE Trans. Ind. Electron. 2006, 53, 1209-1225. [CrossRef]

142. Liangyong, W.; Tianyou, C.; Lianfei, Z. Neural-Network-Based Terminal Sliding-Mode Control of Robotic Manipulators Including Actuator Dynamics. IEEE Trans. Ind. Electron. 2009, 56, 3296-3304. [CrossRef]

143. Rossomando, F.G.; Soria, C.M. Adaptive Neural Sliding Mode Control in Discrete Time for a SCARA robot arm. IEEE Lat. Am. Trans. 2016, 14, 2556-2564. [CrossRef]

144. Wiest, J.H.; Buckner, G.D. Indirect Intelligent Sliding Mode Control of Antagonistic Shape Memory Alloy Actuators Using Hysteretic Recurrent Neural Networks. IEEE Trans. Control Syst. Technol. 2014, 22, 921-929. [CrossRef]

145. Castaneda, C.E.; Loukianov, A.G.; Sanchez, E.N.; Castillo-Toledo, B. Discrete-Time Neural Sliding-Mode Block Control for a DC Motor With Controlled Flux. IEEE Trans. Ind. Electron. 2012, 59, 1194-1207. [CrossRef]

146. Lin, F.J.; Hung, Y.C.; Chen, S.Y. Field-programmable gate array-based intelligent dynamic sliding-mode control using recurrent wavelet neural network for linear ultrasonic motor. IET Control Theory Appl. 2010, 4, 1511-1532. [CrossRef]

147. Pan, H.; Xia, L.Z. Efficient Object Recognition Using Boundary Representation and Wavelet Neural Network. IEEE Trans. Neural Netw. 2008, 19, 2132-2149. [CrossRef]

148. Wai, R.J.; Muthusamy, R. Fuzzy-Neural-Network Inherited Sliding-Mode Control for Robot Manipulator Including Actuator Dynamics. IEEE Trans. Neural Netw. Learn. Syst. 2013, 24, 274-287. [CrossRef]

149. Wai, R.J. Total sliding-mode controller for PM synchronous servo motor drive using recurrent fuzzy neural network. IEEE Trans. Ind. Electron. 2001, 48, 926-944.

150. Sheng, L.; Xiaojie, G.; Lanyong, Z. Robust Adaptive Backstepping Sliding Mode Control for Six-Phase Permanent Magnet Synchronous Motor Using Recurrent Wavelet Fuzzy Neural Network. IEEE Access 2017, 5, 14502-14515. [CrossRef]

151. Zaihidee, F.M.; Mekhilef, S.; Mubin, M. Fractional order SMC for speed control of PMSM. In Proceedings of the 6th International Electrical Engineering Congress (iEECON2018), Krabi, Thailand, 7-9 March 2018.

152. Plestan, F.; Shtessel, Y.; Brégeault, V.; Poznyak, A. New methodologies for adaptive sliding mode control. Int. J. Control 2010, 83, 1907-1919. [CrossRef]

153. Chen, Q.; Tao, L.; Nan, Y.; Ren, X. Adaptive Nonlinear Sliding Mode Control of Mechanical Servo System With LuGre Friction Compensation. J. Dyn. Syst. Meas. Control 2015, 138, 021003-021009. [CrossRef]

154. Roy, S.; Kar, I.N. Adaptive robust tracking control of a class of nonlinear systems with input delay. Nonlinear Dyn. 2016, 85, 1127-1139. [CrossRef]

155. Roy, S.; Kar, I.N. Adaptive sliding mode control of a class of nonlinear systems with artificial delay. J. Frankl. Inst. 2017, 354, 8156-8179. [CrossRef]

156. Roy, S.; Kar, I.N.; Lee, J.; Jin, M. Adaptive-Robust Time-Delay Control for a Class of Uncertain Euler-Lagrange Systems. IEEE Trans. Ind. Electron. 2017, 64, 7109-7119. [CrossRef]

157. Roy, S.; Kar, I.N.; Lee, J.; Tsagarakis, N.G.; Caldwell, D.G. Adaptive-Robust Control of a Class of EL Systems With Parametric Variations Using Artificially Delayed Input and Position Feedback. IEEE Trans. Control Syst. Technol. 2018, 1-13. [CrossRef]

158. Mobayen, S. An adaptive chattering-free PID sliding mode control based on dynamic sliding manifolds for a class of uncertain nonlinear systems. Nonlinear Dyn. 2015, 82, 53-60. [CrossRef] 

MDPI

St. Alban-Anlage 66

4052 Basel

Switzerland

Tel. +41616837734

Fax +41 613028918

www.mdpi.com

Energies Editorial Office

E-mail: energies@mdpi.com www.mdpi.com/journal/energies

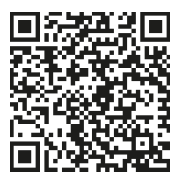



MDPI

St. Alban-Anlage 66

4052 Basel

Switzerland

Tel: +41 616837734

Fax: +41 613028918 